

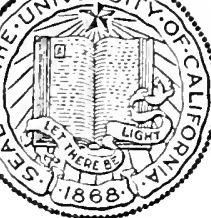

THE LIBRARY OF

THE UNIVERSITY OF CALIFORNIA LOS ANGELES

GIFI OF

SAN FRANCISCO COUNTY MEDICAL SOCIETY 






\title{
CANCER OF THE STOMACH
}

\author{
A CLINICAL STUDY OF 9zI OPERATIVELY \\ AND PATHOLOGICALLY DEMONSTRATED CASES
}

BY

FRANK SMITHIES, M. D.

Gastro-enterologist to Augustana Hospital, Chicago; formerly Gastro-enterologist to The Mayo Clinic, Rochester, Minn.; formerly Instructor in Internal Medicine and Demonstrator of Clinical Medicine in the University of Michigan, Ann Arbor; Fellow of the American Gastro-enterological Association, Etc.

With a Chapter on the

\section{SURGICAL TREATMENT OF GASTRIC CANCER}

\author{
BY
}

ALBER'T J. OCHSNER, M. D., LL. D., F. R. C. S. Professor of Clinical Surgery in the School of Medicine of the University of Illinois; Surgeon-in-Chief to Augustana Hospita1, Chicago; Consulting Surgeon to St. Mary's Hospital, Chicago

ILLLSTRATED

PHILADELPHIA AND LONDON

W. B. SAUNDERS COMPANY

I 9 I 6 
Copyright, 1916, by W. B. Saunders Company

Printed in America 


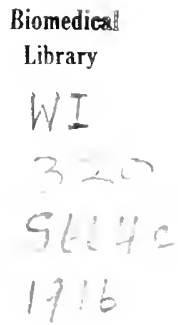

TO MY

FIRST TEACIER IN MEDICINE

DR. GEORGE DOCK

AND

MY FRIENDS

DRS. JAMES B. HERIRCK, RICHARD DEWEY,

IRA DEAN LOREE

AND

WARREN PLIMPTON LOMBARD 



\section{PREFACE}

THIs work attempts to set forth the facts which are considered valuable from a study of 921 operatively and pathologically demonstrated instances of gastric cancer.

The cases and their records comprised part of my services, extending over ten years, at the University Hospital (Ann Arbor, Mich.), the Mayo Clinic (Rochester, Minn.), and my present clinic at Augustana Hospital.

It is at least a decade since a momograph upon this subject appeared. The interim has been prolific in its contributions to our better clinical, pathologic and surgical knowledge of gastric cancer. It is to be hoped that the practical worth of the more important of these advances is sufficiently emphasized in the pages following.

For numerous courtesies, I am indebted to many. Particularly would I express my gratitude to Drs. William J. Mayo, Christopher Graham, William Carpenter MacCarty and Russell D. Camman of the Mayo Clinic, for permission to study cases and records, and for illustrations and material in the text. Dr. Frederick Hoffman, statistician of the Prudential Life Insurance Company, has generously allowed me to avail myself of the results of his valuable compilations. Dr. Albert J. Ochsner has kindly written the chapter entitled "The Surgical Treatment of Gastric Cancer." It has been ably illustrated from sketches made at the operating-table by Mr. Thomas Jones. Drs. Oscar Nadeau and John Nuzum and Mr. E. Schmidt have helped with the illustrations and the text. My secretaries, Misses Pearl Empey and Frances Horgan, have been most faithful and painstaking. From my wife, I gladly acknowledge much practical aid and encouragement. 
Finally, it should be understood that this monograph has been written during a moderately busy life. It is by no means the "last word" upon gastric cancer. It is simply one clinician's analysis of certain facts which he observed in a great group of patients affected with a like ailment.

Frank Silthies.

Chicago, Illivois,

January, 1916. 


\section{CONTENTS}

\section{CHAPTER I}

PAGE

General Distribution and Etiology.

CHAPTER II

Morbid Anatomy: Gross, Microscopic and Experimental . . . 51

CHAPTER III

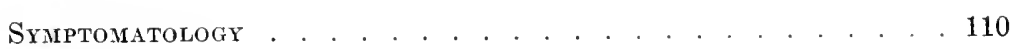

CHAPTER IV

Physical Abrormalities. . . . . . . . . . . . . . . . . . . 160

CHAPTER V

Exanination of Gastro-intestinal Function . . . . . . . . . . 193

CHAPTER VI

Roentgen Exanination in Gastric Cancer . . . . . . . . . . . 268

CHAPTER VII

The Blood in Gastric Cancer . . . . . . . . . . . . . . . 319

CHAPTER VIII

The Significance of Gastric Ulcer with Respect to Gastric

Cancer. . . . . . . . . . . . . . . . . 350

CHAPTER IX

Gastric Cancer in the Young. . . . . . . . . . . . . . . . 373

CHAPTER X

Differential Diagnosis 382 
Surgical Treitment . . . . . . . . . . . . . . . . . . . 417

By Albert J. OChsier, M. D., LL. D., F. R. C. S.

CHAPTER XII

Non-surgical Treathext . . . . . . . . . . . . . 472

Ixdex of XaMes. . . . . . . . . . . . . . . . 501

INDEX . . . . . . . . . . . . . . . . 505 


\section{CANCER OF THE STOMACH}

\section{CHAPTER I \\ GENERAL DISTRIBUTION AND ETIOLOGY}

Definition.-Gastric cancer is a disease of the stomach produced by the development of a neoplasm in the wall of that viscus. The affection is generally of a perniciously progressive nature, and occurs with the greatest frequency between the ages of 40 and 70 years. Fatal termination is the rule. The disease is characterized chinically by imperfect gastric function. This malfunction usually manifests itself by abdominal distress or pain, associated with loss of appetite and body weight, by weakness, anemia, cachexia, vomiting and the appearance of an epigastric tumor.

The gastric extracts or the vomitus frequently exhibit deficient gastric emptying power, diminished free hydrochloric acid, altered blood and the presence of foreign organic acids, microorganisms and ferments.

When the stomach is filled with substances opaque to the Roentgen ray, alterations from the normal contour can, not infrequently, be demonstrated upon examination with the fluoroscopic screen or the $x$-ray plate.

Frequency of Cancer in General.- Trustworthy statistics record that more than 75,000 deaths from cancer occurred in the United States during 1913. It is estimated that there were fully a half million deaths from malignant disease in the same period throughout the civilized world. Of the cancer deaths recorded in the United States Regis- 
tration Area their ratio to deaths from all other causes in 1911 was as $1: 19.06$. The differences in ratio geographically are demonstrated in Table 1.

Table 1

\begin{tabular}{|c|c|c|c|}
\hline State & $\begin{array}{l}\text { Deaths, all } \\
\text { causes }\end{array}$ & Cancer deaths & Cancer ratio \\
\hline Michigan.............. & 37,993 & 2,137 & $1: 13.05$ \\
\hline Vermont............. & 5,440 & 396 & $1: 13.98$ \\
\hline Wisconsin............ & 26,987 & 1,841 & $1: 14.65^{\prime}$ \\
\hline Washington............. & 10,187 & 679 & $1: 15.002$ \\
\hline Minnesota............. & 21,988 & 1,423 & $1: 15.24$ \\
\hline Maine ............... & 12,031 & $73 \mathrm{~S}$ & $1: 16.3$ \\
\hline Massachusetts.......... & 53,003 & 3,262 & $1: 16.55$ \\
\hline California............. & 34,010 & 2,053 & $1: 16.56$ \\
\hline Ohio................ & 65,466 & 3,936 & $1: 16.63$ \\
\hline Rhode Island . ......... & 8,644 & 510 & $1: 16.94$ \\
\hline New York.............. & 142,608 & $\$, 209$ & $1: 17.37$ \\
\hline Indiana $\ldots \ldots \ldots \ldots$ & 35,210 & 1,943 & $1: 18.1$ \\
\hline New Jersey............ & 37,779 & 2,054 & $1: 18.39$ \\
\hline Connecticut............ & 17,552 & 895 & $1: 19.5$ \\
\hline Pennsylvania.......... & 111,842 & 5,426 & $1: 20.61$ \\
\hline 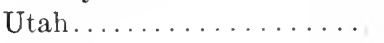 & 3,913 & 188 & $1: 20.81$ \\
\hline Maryland............. & 20,694 & 955 & $1: 21.5$ \\
\hline Colorado.............. & 10,740 & 459 & $1: 23.3$ \\
\hline North Carolina.......... & 6,672 & 219 & $1: 30.46$ \\
\hline Kentucky............. & 30,407 & $9 S 6$ & $1: 40.8$ \\
\hline
\end{tabular}

Ratio of Cancer Deaths to deaths from all other causes in United States Registration Area in 1911.

It is seen that 7 of the then registration states fell below the general average, while 13 states rose above such. The striking difference in ratio between cancer deaths in Michigan from those in North Carolina and Kentucky (more than 3 times the minimum) would seem to point to either errors in diagnosis or local increase from unsolved cause in the states evidencing the maximum.

Comparison between the ratio of cancer deaths in the United States and foreign countries is estimated on the same basis of mortality returns in Table 2 modified from Buday. 
Table 2

\begin{tabular}{|c|c|c|c|}
\hline Country & Ratio & Country & Ratio \\
\hline Brazil........ (1908) & 0.41 & U.S. A........(1908) & 7.65 \\
\hline Portugal ....... (1904) & 2.39 & England ....... (1907) & 9.08 \\
\hline Spain........ (1903) & 4.4 & New Zealand.... (1907) & 9.3 \\
\hline Japan. . . . . . . (1905) & 5.3 & Norway......... (1906) & 9.75 \\
\hline Belgium . . . . . (1907) & 6.02 & Holland... . . . . (1903) & 9.9 \\
\hline Victoria ....... (1907) & 6.09 & Sweden... . (1890-1898) & 9.96 \\
\hline Italy . . . . . . . (1906) & 6.09 & France.......... (1906) & 10.00 \\
\hline Hungary ....... (1904) & 7.00 & Denmark. . . . . (1907) & 12.2 \\
\hline Germany...... (1907) & 7.45 & Switzerland.... (1907) & 12.5 \\
\hline Austria....... (1905) & 7.5 & & \\
\hline
\end{tabular}

Comparison between ratio of Cancer Deaths in the United States and in other countries.

Wide variations in cancer death ratio are shown. These cannot be explained wholly upon the theory of inefficient government mortality records or unproved diagnoses.

Table 3

\begin{tabular}{|c|c|c|c|c|c|c|}
\hline \multirow{2}{*}{$\begin{array}{l}\text { Age period } \\
\text { all ages }\end{array}$} & \multicolumn{3}{|c|}{1911} & \multicolumn{3}{|c|}{1901} \\
\hline & Persons & Males & Females & Persons & Males & Females \\
\hline Crude rate. . & 83.9 & 64.2 & 104.0 & 65.8 & 48.7 & 83.0 \\
\hline Corrected rate. & 77.6 & 56.7 & 97.2 & 62.2 & 43.6 & 79.7 \\
\hline Under 5 years... & 3.0 & 3.1 & 3.0 & 3.4 & 3.8 & 3.1 \\
\hline 5 to 9 years.......... & 1.2 & 1.3 & 1.1 & 1.0 & 1.3 & 0.8 \\
\hline 10 to 14 years......... & 1.3 & 1.0 & 1.5 & 0.9 & 0.9 & 0.9 \\
\hline 15 to 19 years......... & 2.3 & 2.9 & 1.7 & 2.1 & 1.9 & 2.2 \\
\hline 20 to 24 years.... & 4.8 & 4.9 & 4.6 & 3.9 & 3.3 & 4.5 \\
\hline 25 to 34 years.... & 13.9 & 9.7 & 19.4 & 13.4 & 9.4 & 17.5 \\
\hline 35 to 44 years......... & 61.0 & 31.1 & 92.5 & 60.2 & 32.5 & 89.6 \\
\hline 45 to 54 years........ & 166.3 & 109.2 & 227.0 & 146.5 & 90.0 & 205.4 \\
\hline 55 to 64 years......... & 352.4 & 283.4 & 422.3 & 268.3 & 203.8 & 331.8 \\
\hline 65 to 74 years......... & 566.7 & 512.8 & 617.8 & 418.8 & 366.0 & 468.9 \\
\hline 75 years and over....... & 794.7 & 730.5 & 848.7 & 557.6 & 520.8 & 589.8 \\
\hline 25 years and over: & & & & & & \\
\hline Crude rate $\ldots \ldots \ldots \ldots$ & 155.7 & 117.7 & 195.0 & 124.5 & 90.9 & 158.7 \\
\hline Corrected rate......... & 159.0 & 117.9 & 195.9 & 127.2 & 90.4 & 160.3 \\
\hline
\end{tabular}

Mortality from Cancer in the United States, 1901-1911. (Registration States of 1900; Rates per 100,000 of Population by Age and Sex.) 
Increase in Cancer in General.-For much of our accurate knowledge that such increase is real and not apparent we are indebted to Hoffman. That this is so for the United States is exhibited in Table 3, compiled from the publications of the Department of Commerce. The increase is plotted for each set in Charts I and II (page 21).

Emphasis is to be placed upon the fact that there has been an actual increase in general cancer mortality rate in all decades above age 40. Hoffman has computed, from an enormous experience, that in the United States the number dying from cancer at the present time is approximately 25 per cent. greater than 10 years ago. He finds that of the total mortality from cancer in the regis-

Table 4

(Rates per 100,000 Population)

\begin{tabular}{|c|c|c|c|c|}
\hline & 1900 & 1909 & $\begin{array}{l}\text { Actual } \\
\text { increase }\end{array}$ & $\begin{array}{l}\text { Per cent. of } \\
\text { increase }\end{array}$ \\
\hline Switzerland. . . . . . . & 129.9 & 126.7 & $3.2^{2}$ & $2.5^{2}$ \\
\hline Netherlands......... & 91.7 & 102.6 & 10.9 & 11.9 \\
\hline Scotland............ & 79.0 & 101.6 & 22.6 & 28.6 \\
\hline England and Wales... & 82.9 & 96.1 & 13.2 & 15.9 \\
\hline Norway............. & 90.8 & 94.6 & 3.8 & 4.2 \\
\hline German Empire...... & 72.1 & 83.5 & 11.4 & 15.8 \\
\hline Ireland............. & 60.8 & 80.0 & 19.2 & 31.6 \\
\hline Austria............. & 70.9 & 78.6 & 7.7 & 10.9 \\
\hline New Zealand......... & 56.3 & 73.2 & 16.9 & 30.0 \\
\hline Australia............ & 62.6 & 72.3 & 9.7 & 15.5 \\
\hline Japan.............. & 45.4 & 65.2 & 19.8 & 43.6 \\
\hline Italy.................... & 52.2 & 63.5 & 11.3 & 21.6 \\
\hline Denmark........... & 46.2 & 56.1 & 9.9 & 21.4 \\
\hline Spain............... & 39.3 & 51.0 & 11.7 & 29.8 \\
\hline Hungary............ & 36.7 & 44.5 & 7.8 & 21.3 \\
\hline Jamaica............. & 16.5 & 18.3 & 1.8 & 10.9 \\
\hline Serbia............... & 9.4 & $12.6^{1}$ & 3.2 & 34.0 \\
\hline Ceylon............. & 6.6 & 4.0 & $2.6^{2}$ & $39.4^{2}$ \\
\hline Average ........ & 59.6 & 73.5 & 13.9 & 23.3 \\
\hline
\end{tabular}

${ }^{1} 1908 . \quad 2$ Decrease.

International Cancer Statistics, 1900-1909. 


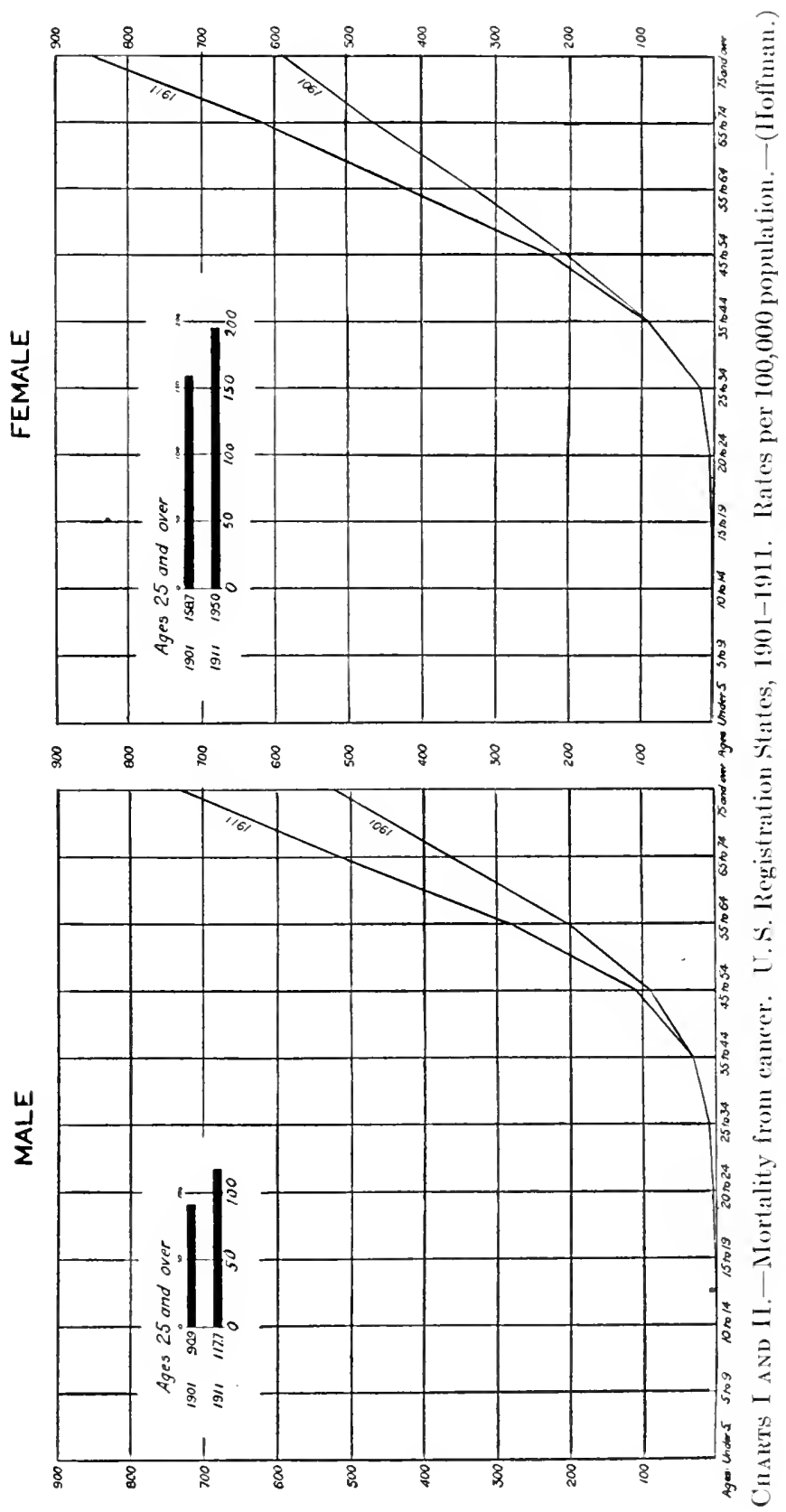


tration area during 1906 to 1910 , the proportion of such deaths at the age of 45 and over was 83 per cent.

In countries other than the United States this increase in cancer mortality practically is a constant finding, as shown by Table 4.

The relative increase in Germany, England and America is demonstrated graphically in Chart III. These statistics return the significant information that "the cancer death rate in the civilized portion of the earth has increased 23.3 per cent. in the past decade."

In Gastric Cancer, the ratio of occurrence to that of non-cancerous disease is to be determined mainly from the study of hospital admissions and mortality records. Information obtained from examination of hospital admissions is of but relative worth inasmuch as reports of this type are not representative of the status of the whole mass of population. The class of patients frequenting large general hospitals is often not a true index of the kind of folk inhabiting a given zone. Moreover, it should be observed that not infrequently the statistics reported are from hospitals largely surgical, to which patients go on account of the reputed skill of some operator.

Table 5 indicates the gastric cancer admission ratios in representative American and English hospitals. It is

Table 5

\begin{tabular}{|c|c|c|c|}
\hline Hospital & $\begin{array}{c}\text { Total } \\
\text { admissions }\end{array}$ & $\begin{array}{l}\text { Number of } \\
\text { gastric } \\
\text { cancers }\end{array}$ & Ratio \\
\hline Johns Hopkins Hospital. . & 8,464 & 150 & 1 to 56.4 \\
\hline Mass. General Hospital... & 11,812 & 129 & 1 to 91.5 \\
\hline Nontreal General Hospital. & 9,458 & 54 & 1 to 175.0 \\
\hline St. Thomas, London........ & 18,958 & 98 & 1 to 193.6 \\
\hline St. Bartholomew's, London... & 23,500 & 106 & 1 to 221.6 \\
\hline London Temperance Hospital. . & 4,643 & 20 & 1 to 231.2 \\
\hline
\end{tabular}

Proportion of Gastric Cancer admissions to total admissions of Representative Hospitals. 


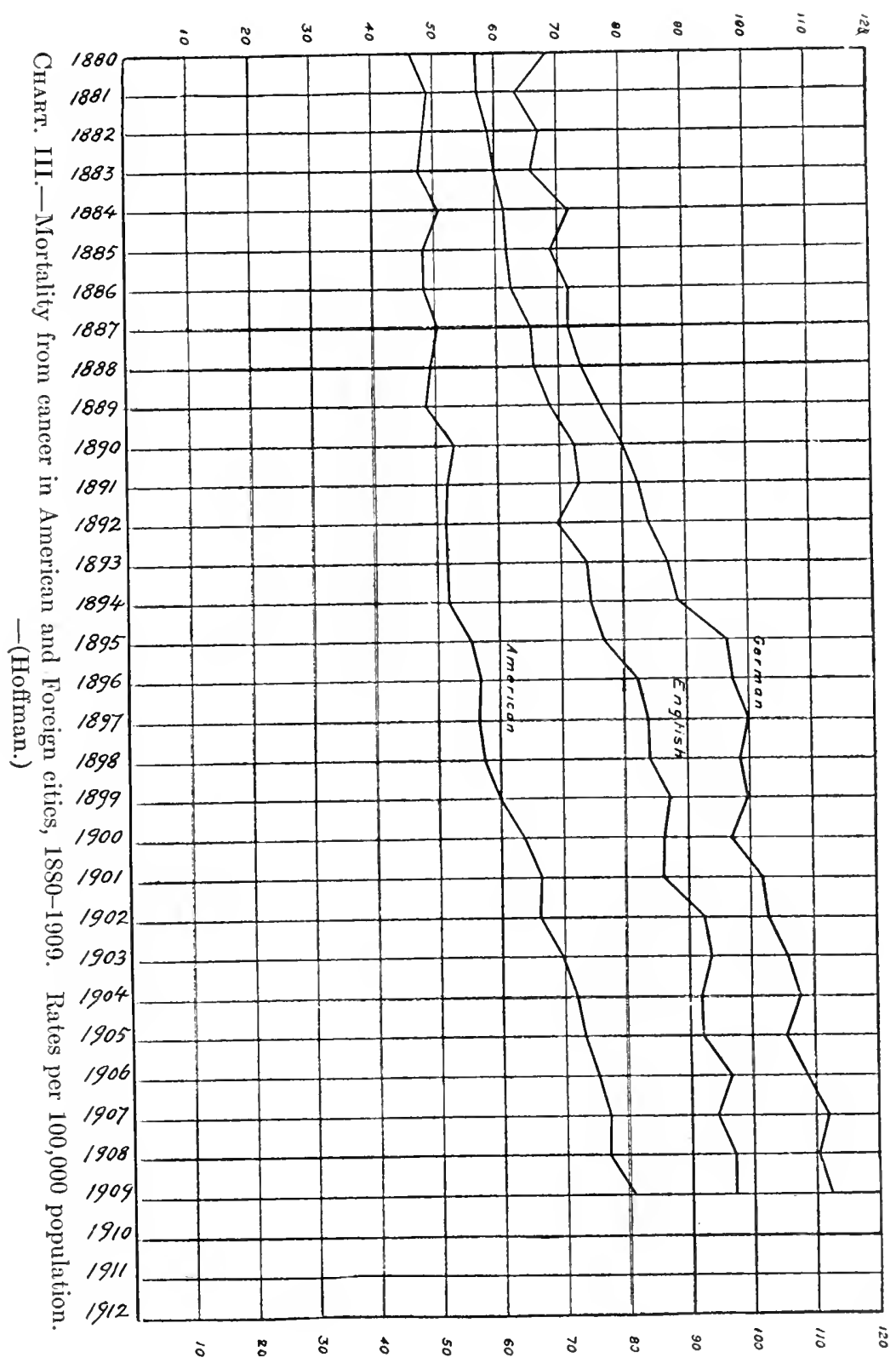


seen that while in general the above hospitals occupy similar positions in their respective communities, the admission rates exhibit a wide range in the frequency of gastric cancer. While in ratio, the London hospitals vary least, that of American hospitals along the Atlantic seaboard shows rather striking and unexplainable differences.

Our 921 instances of gastric malignancy occurred in approximately 82,000 clinical admissions for all forms of medical and surgical disease. The average age of these patients was about 40 years. These figures return a ratio of the frequency of gastric cancer of approximately 1 to 89 hospital admissions.

The information returned from the study of mortality records is of but relative value. Until recently, the American mortality statistics were partly fact and partly estimate, owing to there being but a limited area of registration. Even in registration areas the returns are open to question, inasmuch as they are based upon material which has been checked but rarely by pathologic reports. That mistakes in the diagnosis of gastric cancer commonly occur even in large city practice, is emphasized by Fenwick. He states that of 56 cases, admitted into London hospitals, under his care, with a diagnosis of gastric cancer, in only 31 (55.3 per cent.) was the disease proved to exist. In 25 instances (44.7 per cent.) the patients were free from the disease. These mistakes had occurred in spite of the fact that the arerage duration of the illness prior to hospital admission was nearly 4 months. An experience similar to this can be furnished by any American hospital that makes a practice of requiring a pathologic or laparotomy diagnosis, instead of one based upon purely clinical or chemical eridence. Cabot states that in 
his large experience correct clinical diagnosis of gastric cancer occurred in but 72 per cent. of instances.

Dependable mortality reports indicate that gastric cancer furnishes nearly 38 per cent. (or about 30,000 cases annually) of all cancer deaths. These figures indicate that malignant disease of the stomach is more common than is malignancy of any other organ. The rate is fully 10 per cent. higher in males than in females. In the latter, malignancy of the genitalia and peritoneum is more frequent. These various factors are well demonstrated in Table 6 .

\section{Table 6}

(Rates per 100,000 Population)

(Ages 10 and over)

\begin{tabular}{|c|c|c|c|}
\hline \multicolumn{4}{|l|}{ Males } \\
\hline & Number & Per cent. & $\begin{array}{c}\text { Rate per } \\
100,000\end{array}$ \\
\hline 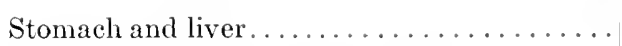 & 50,157 & 50.5 & 96.6 \\
\hline Rectum, intestines and peritoneum......... & 11,980 & 12.1 & 23.1 \\
\hline 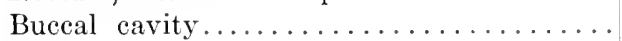 & 7,304 & 7.3 & 14.1 \\
\hline Skin ................... & 6,252 & 6.3 & 12.0 \\
\hline Other organs....... & 23,690 & 23.8 & 45.6 \\
\hline Total, ages of 40 plus........ & 99,383 & 100.0 & 191.4 \\
\hline Total, all ages............. & 109,299 & & \\
\hline
\end{tabular}

Females

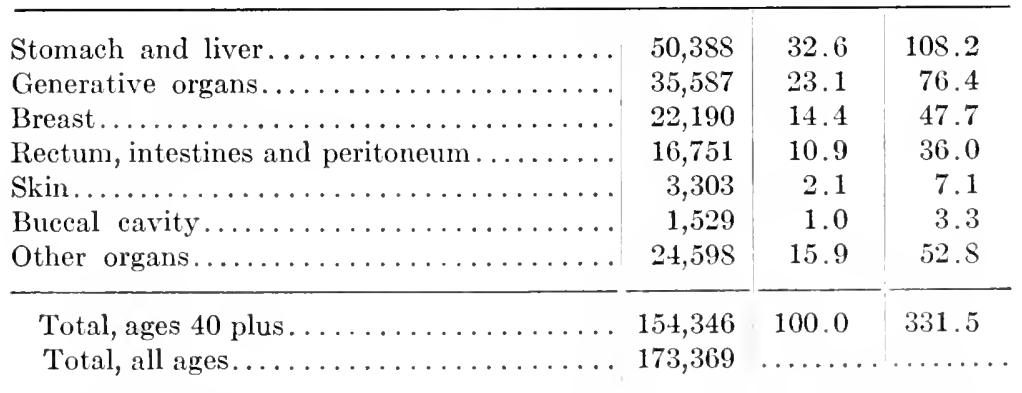

Cancer Mortality by Organs or Parts Affected; U. S. Registration Area, 1901-1910.-(Hoffman.) 
The increase in gastric cancer is real and alarming. At age 40 and above, it has increased more than 10 per cent. for both sexes. This is well brought out by Table 7 .

Table 7

(Rates per 10,000 Population)

\begin{tabular}{l|r|r|rc}
\hline \multicolumn{1}{c|}{ Ages } & Males & Females & $\begin{array}{c}\text { Actual excess in } \\
\text { female rate }\end{array}$ & $\begin{array}{c}\text { Excess } \\
\text { per cent. }\end{array}$ \\
\hline 30 to 39 & 7.8 & $10.2+$ & 2.4 & 30.8 \\
40 to 49 & 34.2 & $39.0+$ & 4.8 & 14.0 \\
50 to 59 & 85.7 & $99.7+$ & 11.0 & 12.4 \\
60 to 69 & 182.9 & $192.2+$ & 9.3 & 5.1 \\
70 to 79 & 262.2 & $282.0+$ & 19.8 & 7.6 \\
$80+$ & 207.6 & $201.2+$ & $\ldots \ldots \ldots \ldots \ldots \ldots \ldots$ \\
\hline $40+$ & 96.6 & 108.2 & 11.6 & 12.0 \\
\hline
\end{tabular}

Mortality from Cancer of the Stomach and Liver; U. S. Registration Area, 1901-1910.

Sex.-Clinical and pathologic data, compiled from hospital and dead-house reports of a half century ago, would appear to indicate, with few exceptions, that gastric cancer is of rather more frequent occurrence in males than in females. Certain groups of statistics are often quoted in support of this statement. In 1859, Brinton declared, after studying reports of 600 cases, that the disease occurred twice as frequently in males as in females. In 1872, Fox collected 1,303 instances of gastric cancer from the literature and his own practice. There were 680 males and 623 females. Welch's analysis of 2,214 cases of gastric malignancy (recorded mainly in continental literature during a period when microscopic pathology was in its infancy) demonstrates the ratio of males to females as 5:4. Fenwick claims a proportion of males over females of approximately $6: 4$. Friedenwald has recently collected 1,000 cases, clinically of gastric cancer (26.6 per cent. came to laparotomy) and records that there were 588 males and 412 females.' 
In our series of 921 cases, which were proven to be primary gastric cancer operatively and pathologically, there were 693 males and 228 females, or 3.04 males to 1 female.

This proportion is particularly interesting because it comes from controlled material; it is a distinctively American experience; it has been returned mainly from living and not dead subjects; it closely approximates the sex ratio which is associated with simple chronic gastric ulcer.

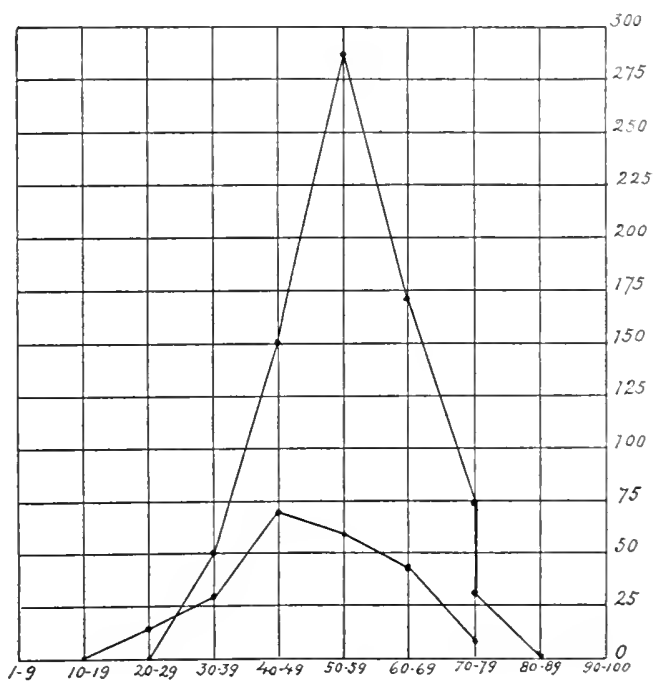

Chart IV.-Incidence of gastric cancer by age and sex. The upper line represents males, the lower, females.-(Author.)

Age.-According to modern writers, from 60 to 70 per cent. of the deaths from gastric cancer occur between the fortieth and seventieth years. In the living the incidence is commonly recorded as being greatest between the fiftieth and sixtieth years. Necropsy reports usually place the maximum age frequency rather higher than where such is determined from living patients. 
Table 8 shows the age incidence by decades and sex in the material which we have studied. Chart IV demonstrates graphically the age curves in relation to sex.

Table S

\begin{tabular}{c|rrr}
\hline \multicolumn{1}{c}{ Age } & Niale & Female & Total \\
\hline 1 to 9 & 0 & 0 & 0 \\
10 to 19 & 0 & 1 & 1 \\
20 to 29 & 4 & 5 & 9 \\
30 to 39 & 54 & 34 & 88 \\
40 to 49 & 159 & 73 & 232 \\
50 to 59 & 284 & 66 & 350 \\
60 to 69 & 160 & 40 & 200 \\
70 to 79 & 31 & 9 & 40 \\
$80+$ & 1 & 0 & 1 \\
\hline
\end{tabular}

Total Cases 921. Ratio Males to Females, 3.04: 1.

Sex and Age by Decades.-(Author's Series.)

Certain facts of interest are brought out. The greatest number of cases occurred in the sixth decade (350 cases between agos 50-59). Between ages 40-69 there occurred 84.9 per cent. of all our cases. Ten instances (1.20 per cent.) were tabulated below age 30. One case died of gastric cancer at age 19. In males the greatest number of cases fell between ages 50-59 (40.9 per cent. of the total for the sex), while 87 per cent. of all cases fell between ages 40 and 69 . In females the disease appeared to occur at a rather earlier period of life than in males. In this sex the greatest number of cases (73) were in the fifth decade, while 78 per cent. of all instances came between ages 40-69.

Race and Nationality.-Friedenwald states that 5.2 per cent. of his cases of gastric cancer occurred in negroes. In this race the greatest frequency of the disease was in the sixth decade. Hoffman has made a careful study of the 
difference in mortality from cancer of the stomach and liver in whites and colored (Table 9).

Table 9

(Rates per 100,000 Population)

\begin{tabular}{c|r|r|r|r}
\hline \multicolumn{1}{c|}{ Ages } & \multicolumn{2}{|c|}{ Males } & \multicolumn{2}{c}{ Females } \\
\cline { 2 - 4 } & White & Colored & White & Colored \\
\hline 30 to 39 & 9.7 & 15.2 & $\mathrm{S.7}$ & 11.3 \\
40 to 49 & 28.3 & 30.5 & 31.3 & 27.4 \\
50 to 59 & $\$ 9.5$ & 84.4 & 87.6 & 91.0 \\
60 to 69 & 221.0 & 166.4 & 151.6 & 82.0 \\
$70+$ & 268.3 & 152.3 & 168.5 & 160.6 \\
\hline $40+$ & 105.5 & 73.1 & 84.5 & 64.5 \\
\hline
\end{tabular}

Wortality from Cancer of the Stomach and Liver, by Races; District of Columbia, 1901-1910.-(Hoffman.)

He observes that the death rates for negroes exceed those for whites, in males at ages under 50 , and for females under 40. Above such ages, the white death rate is in excess of that of the negro. It would seem also that gastric cancer is becoming more prevalent among the American negroes. This is shown by the reports of the U. S. Dept. of Commerce, 1912-13.

Our investigations record no case of gastric cancer among negroes. This, doubtless, happens because we rarely see a negro patient.

While 40.3 per cent. of our patients were American born, yet nearly 80 per cent. were of foreign extraction. We have attempted to classify these facts in Table 10.

It has been stated frequently that gastric cancer is uncommon in Jews. From a relatively small proportion of Jewish patients, our records show 17 (1.S per cent.) cases in either American or foreign born Jews. There were 11 males and 6 females. It would appear that, living under similar conditions, Jews are as prone to the 
Table 10

\begin{tabular}{|c|c|c|}
\hline Nationality & Number of cases & Per cent. of total \\
\hline Americans................. & 372 & 40.3 \\
\hline $\operatorname{Irish} \ldots \ldots \ldots \ldots \ldots \ldots \ldots \ldots \ldots$ & 170 & 18.0 \\
\hline 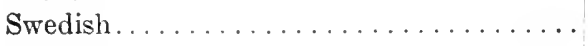 & 143 & 15.5 \\
\hline 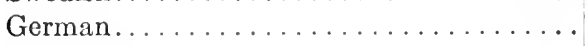 & 108 & 11.7 \\
\hline 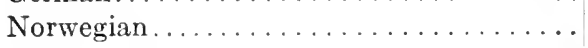 & 73 & 7.8 \\
\hline Austrian . . . . . . . . . . . . . . & 18 & 1.9 \\
\hline 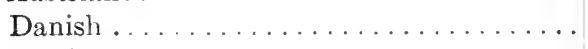 & 12 & 1.3 \\
\hline Russiar . . . . . . . . . . . . . . . & 11 & 1.02 \\
\hline English . . . . . . . . . . . . . & 4 & 0.43 \\
\hline Belgian ................... & 3 & 0.32 \\
\hline French $\ldots \ldots \ldots \ldots \ldots \ldots \ldots \ldots$ & 2 & 0.22 \\
\hline Italian $\ldots \ldots \ldots \ldots \ldots \ldots \ldots \ldots$ & 2 & 0.22 \\
\hline 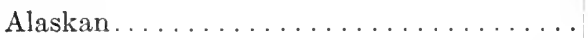 & 1 & 0.10 \\
\hline Mexican...................... & 1 & 0.10 \\
\hline Portuguese.$\ldots \ldots \ldots \ldots \ldots \ldots \ldots$ & 1 & 0.10 \\
\hline 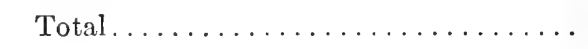 & 921 & 100 \\
\hline
\end{tabular}

Incidence of Gastric Cancer by Nationality-(Author.)

disease as are other human families. It has been advanced that the explanation for the former supposition of the lessened liability of Jews to cancer lay in the fact that Jewish families were usually large-hence the proportion of young Jews to old was relatively great. At present it is not uncommon to find many small Jewish families (particularly in America), probably as the result of prosperity, heterodoxy, and altered mode of life. This may explain the evident increase of gastric cancer among modern Jews.

While we have no specific data to offer, our observations would appear to indicate the relative infrequency of carcinoma of the stomach among those races far removed from modern civilization, so called. It is quite possible, however, that accurately compiled statistics from the lands inhabited by such folk will eventually demonstrate the ubiquity of the disease. Certainly cases are not 
infrequently being reported from Japan (Miyake). Dr. Hie Ding Lin of Foo Chow, China, personally assures me that not uncommonly well-to-do Chinese die from chronic gastric disease, often of a type associated with cachexia and anemia.

Occupation.-At the present state of our knowledge, it cannot be said definitely that any one type of work predisposes to gastric cancer. It has been observed by Tatham that the general cancer mortality during the period 1851-1S90 was twice as great among the financially prosperous, having no particular occupation, as it was among occupied males with diverse vocations. Aschoff seems to have shown that Berliners living on incomes or pensions furnished the greater part of the cancer mortality in their section. Roger Williams makes similar comments. These general studies are interesting, but by no means conclusive. It is perhaps possible that the explanation for the evident frequency of cancer in the retired, wellto-do part of the population may be found in the observation that this class is mainly made up of individuals at the cancer age. Relatively few persons succeed in amassing sufficient material wealth before the fifth decade to enable them to stop working. It will be recalled that approximately 8 out of 10 cases of gastric cancer occur above age 40 .

We have grouped our material, according to occupation, in Table 11.

A very striking feature of the Table is the preponderance of farmers. The series demonstrates that 32.4 per cent. of our instances of the disease occurred in farmers, active or retired. The proportion of patients of this type is increased by 11.3 per cent. on analysis of the group labeled "housework." These patients were farm-dwellers, living under similar conditions to the males furnishing the 
Table 11

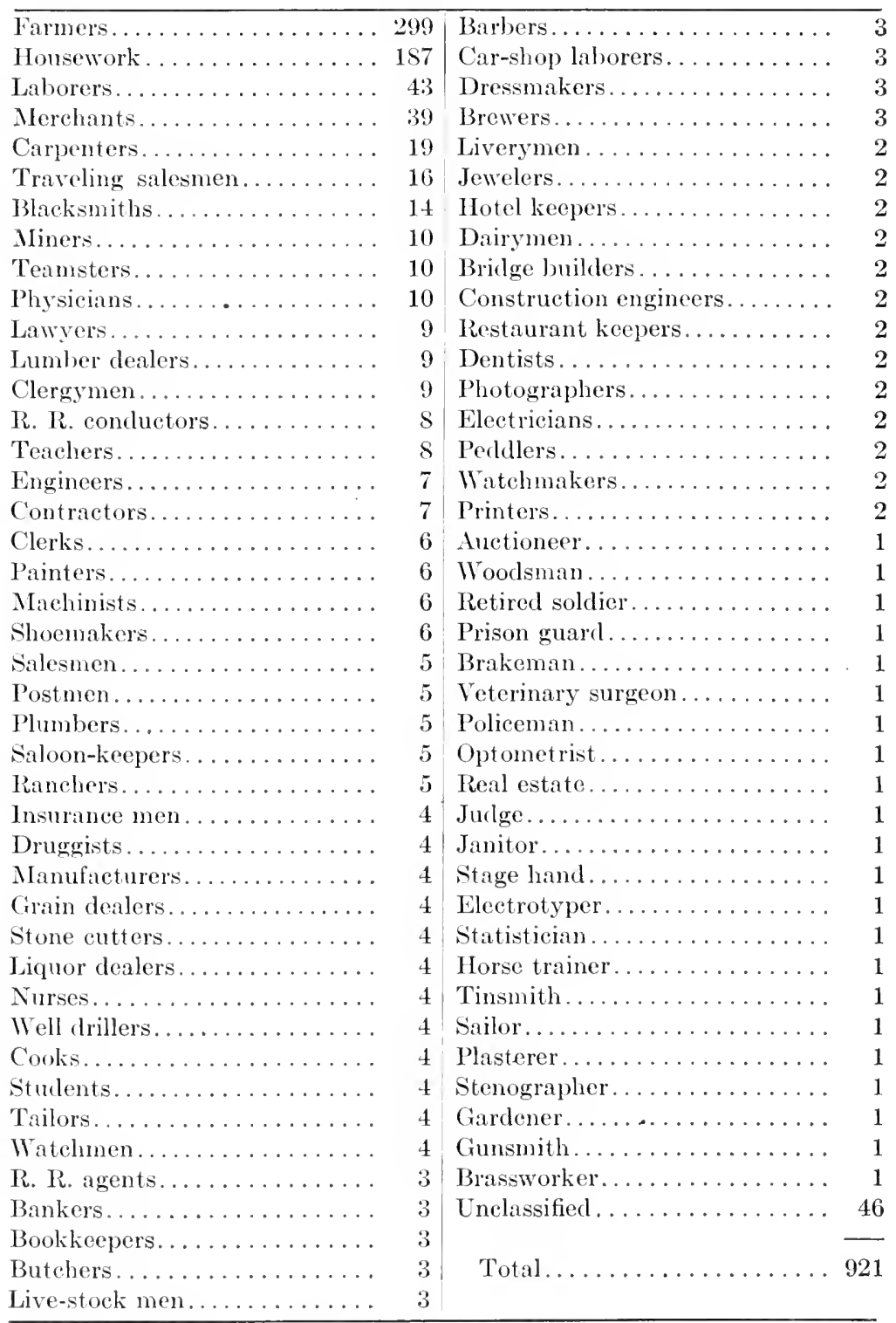

Occurrence of Gastric Cancer According to Occupation.-(Author's series.)

"farmer" class. Certain other so-called "out-door" occupations largely contributing, actually or relatively, 
to the list are laborers, traveling salesmen, teamsters, ranchers, stockmen, liverymen, construction engineers, well-drillers, contractors and gardeners. These occupations make up nearly 55 per cent. of our total. Even when it is considered that many of the patients seeking relief at the clinics, where our work was done, are from rural communities, yet the figures are sufficiently remarkable to warrant consideration. In the study of general cancer etiology, Williams (loc. cit.) has observed that while much of the material which he studied came from Londoners following sedentary occupations, there was a strikingly large proportion of cancer cases among the group engaged in out-of-door work. Ochsner has frequently called attention to the fact that many individuals affected with gastric cancer are liberal consumers of uncooked or unwashed regetables and fruits. This is not an uncommon and preponderant diet at certain periods of the year in rural communities. Urban populations, especially the well-to-do, are able also to indulge liberally in similar delicacies and frequently all the year round. In cities there is an increasing consumption of head lettuce, radishes, cucumbers, young onions, melons, plums, peaches, grapefruit, etc. It may be that irritants, parasitic or chemical, are ingested with such unwashed or uncooked foods, which influence atypic metabolism.

Our analysis fails to show the immunity of printers, miners, quarry-men or industrial workers to gastric cancer. It cannot be said that there is a peculiar susceptibility to the disease among merchants, brewers, cooks, hotelkeepers, domestics, metal-workers or sailor's. In this series carpenters, blacksmiths and shoemakers furnish a comparatively high proportion of instances of gastric cancer when one recalls the limited number of men, 
per population, actually engaged in such labor. Hard physical effort, the swallowing of nails, bits of wood, etc., combined with liability to abdominal injury, may be a not unimportant contributing factor in the production of the disease.

Social Status.-It has been said that a high cancer mortality is an indication of general prosperity (Williams). Material abundance in some way appears to favor the derelopment of malignancy. The highest cancer incidence is found in those communities where people are well housed, well fed and free from the cares associated with the struggle for bare existence. Where poverty is prevalent, as in center's of dense population, prisons, asylums and almshouses, cancer is relatively infrequent. Williams has noted that Ireland has a much lower cancer death rate than has England. In the former country riches and the concomitant ease and abundance of food are somewhat rare. Noreover, in those parts of Ireland where the conditions of life are hardest and the people are uniformly under fed (e.g., Kerry), the cancer mortality is lowest. In an extremely interesting study of life insurance statistics, Hoffman proves that the proportionate mortality from cancer is measurably less among risks representative of the wage-earning population than it is among those risks from the prosperous and well-to-do group, even though the latter class is subjected to more rigid medical examination on entrance. At age 40-50, the proportionate mortality from cancer for males was 5.1 per cent. in the wage-earning group and 6.7 per cent. in the well-to-do division. For females at the same decade, the proportionate mortality was 13.7 per cent. for the wage-earning and 18.5 per cent. for the prosperous.

The patients comprising our series of gastric cancers 
were largely from that great middle class making up the bulk of the population of the Mississippi Talley, the northwest United States and southern Canada. There were few people extremely poor or uncomfortably rich. The great number was in easy circumstances; certainly the cases actually fighting for mere existence were rare. A large part of the group was made up of folk whose parents were foreign born, or who themselves had emigrated from other shores when young. While the early years of many had doubtless been strenuous, the later life had seen the majority installed in comfortable homes and surrounded with many material luxuries. It has been pointed out by numerous writers that just this type of population furnishes the mass of the nerer-ending cancer army. The environmental change, the unaccustomed abundance of meat and drink, combined with sedentary life at the cancer age and the urbanization of a class of people previously largely rural, appear to stimulate the metabolism of cells that are perhaps already of perrerted tendency.

Conjugal State.-Of our entire series $\$ 06$ patients (87.5 per cent.) were married or were widows or widowers. Fifty-two were single. The status of 63 was not noted. In our series there were 7 instances where gastric cancer had developed in man or wife within 5 months of its inception or the fatal issue in the other.

Diet and Nutrition.-Accumulated evidence fails to demonstrate that especial frequency of gastric cancer can be attributed to orer-indulgence in any particular kind of food. Except in rare instances, chronic over-eating appears to be more detrimental to the stomach than does the kind of viands ingested. Statistical evidence in proof of this statement is not lacking. Reviewing the investigations of the Actuarial Society of America, Hoffman states that an 
analysis of the relation of the physique of life insurance applicants at entry to causes of death was made. Those applicants who were over-weight at entry returned cancer death rates (age 15-19) of 0.9 per 10,000, while the underweights in the same group had a cancer mortality of 0.8 per 10,000 . At ages $30-44$, the cancer mortality of over-weights was $3 . \overline{7}$ per 10,000 and of under-weights 2.4 . At age 45 and orer, the cancer mortality of orer-weights was 15.6 and of under-weights 12.0 per 10,000 .

That excessive feeding puts unnecessary work upon the digestive glands is quite within reason. That this highly specialized type of gland may be stimulated to chronic orer-activity by excessive amounts of food is well within the bounds of known physiology. What effect upon intraglandular metabolism this demand for special products may have we can but conjecture. It might be suggested that should these excessive demands ultimately leave an exhausted cell-that is, exhausted in so far as its specific function is concerned - such cell might not only be more susceptible to trauma (mechanical, chemical or parasitic), but reasonably might be expected to proliferate in a vegetative, undifferentiated manner due to the stimulus of its individual over-nourishment.

Careless mastication or the indulgence in hot or cold liquids appears to have little significance apart from the possibilities of intragastric trauma.

Upon extremely slender evidence, it has been held that excess of proteid food was responsible for the prevalence of gastric cancer. While such excess may be a contributory factor to a cause of the disease, yet it will be recalled that gastric cancer is rarely found in the North American Indian or the Esquimaux. The great laboring classes in the United States consume astonishingly 
large amounts of proteid food, and yet in these groups cancer has a low ratio of incidence. Our investigations demonstrate that less than 7 per cent. of the patients were excessive proteid eaters, while 58.8 per cent. subsisted upon a mixed diet. Dietetic information was not obtained regarding 29 per cent. of cases.

Certain sects have in some unexplained way convinced themselves that abstinence from proteids (meats, eggs, etc.) prevents alimentary cancer. Rather more than 5 per cent. of our cases either had refrained from meat for years or had been strict and life-long regetarians. As we have shown above, more than half of our cancer cases came from people living in small towns or rural communities, where an abundance of regetables and fruit is eaten. Among many such people fresh meat is eaten rarely more than three times weekly. Whether or no the ingestion of an excess of raw or unwashed regetables or fruits acts unfarorably upon the gastric lining has not yet been proven. However attractive may be the theory, there is certainly no experimental evidence that a parasite causing gastric cancer is introduced into the body in this way. Fenwick, quoting Hendly, states that of 102 patients who were operated upon for general carcinoma at Jeypore, India, between 1880 and 1888, 59.8 per cent. were life-long vegetarians.

Alcohol.-While there is much carelessly expressed opinion upon the matter, there is no proof that the partaking of alcoholic liquors predisposes to gastric cancer. In this relation, Williams calls attention to the following significant facts: Of several hundred female cancer patients, the majority had led sober lives; of 116 male cancer patients, aged from 40-65, 50.7 per cent. had been total abstainers or habitually temperate, 25.7 per cent. had 
been irregular drinkers, while 22.3 per cent. had been constant indulgers; the increase in alcoholism among women has been relatively greater than among men, yet cancer has proportionately increased among males; while it has been stated that those whose occupations permit of special facility in obtaining alcoholic drinks (hotel keepers, brewers, traveling salesmen, etc.) have a relatively high cancer mortality, it has also been noted that individuals engaged in certain rocations where drinking is very common (printers, iron and steel workers, compositor's, paper makers, miners, etc.) appear to be less prone to cancer than do their fellows in the same community; and lastly, although cancer mortality is high in certain alcohol-consuming countries (Bavaria, Saxony), other sections equally intemperate (Spain, Italy, West Ireland) return a relatively low cancer death rate. Fenwick claims that nearly 40 per cent. of his patients at the London Temperance Hospital were total abstainers. Reviewing 150 cases of the disease, Osler and McCrae state that 51.3 per cent. had used alcoholic drinks, but only 5.33 per cent. gave history of excessive indulgence.

We have definite figures with reference to 258 males of our 921 gastric cancer cases. In females the question was frequently not put when taking the history. Of this group 53 (20.6 per cent.) were total abstainers. Of the 205 remaining cases 11 (5.3 per cent.) were pronounced topers. The balance claimed either an occasional debauch or took small quantities of beer, light wines, "hard" cider or whiskey, as the desire visited them.

Habits. - Tobacco.- Of 423 males from whom we were able to obtain information, 272 or 64.3 per cent. used tobacco in some form. Of these, 41 or 15.1 per cent. were excessive smokers. 
Venereal Disease.-Definite information was possible in 504 males. Of this number 43 ( 8.5 per cent.) admitted gonorrhea and 6 (1.2 per cent.) had had hard chancres. It is quite possible that the proportion of those actually having had venereal disease is higher than our figures indicate. While the incidence of gonorrhea and syphilis among these gastric patients appears to have little, if any, direct etiologic bearing upon the disease, yet the admission of such infection is a certain useful index of the patient's general habits.

Traumatism. - Such may be mechanical, chemical, biochemical or parasitic. Injury, mechanically, to the gastric lining may result from ingestion of hard chunks of food or foreign bodies. Only rarely do such injure normal gastric mucosa. When such a lesion as peptic ulcer, atrophic gastritis or neoplasm already exists, the consequence of frequent temporary irritation (as improper food) or a constant irritant (foreign body) cannot be overlooked. Carcinomatous change may be incited in benign gastric ulcer, as shown by Fütterer, or acceleration in the rate of growth of a neoplasm already present might be expected. It is quite possible, although as yet unproved, that chemical irritants (alcohol, tobacco juice, etc.) or biochemical bodies (as the end digestion products of certain foods or substances formed as result of their deterioration) may act similarly. That parasites ingested in food are capable of actually causing certain atypic growths appears to have been shown recently by Fibiger. As a consequence of his feeding rats upon roaches infested with a certain nematode, growths of a cancerous type developed in a large number of the stomachs of such rats.

That external traumata of mechanical type sometimes influence the development of gastric malignancy appears 
proved. One camnot always state that such injury definitely caused cancer to derelop, but there would seem to be abundant evidence that latent processes may be aroused to actirity following certain injuries.

We had 21 patients $(2.6$ per (ent.) where traumata had occurred shortly before the onset of gastric malignancy. In 14 of these (1.5 per cent.), injuries occurred in the early history of the affection and were shortly followed by the appearance of simptoms. The traumata were usually blows, falls or kicks in the abdomen, or crushing accidents from such events as being run over by a vehicle. The following case is illustrative:

F. V.-Male, age 52, teamster. Comes on account of epigastric distress, weakness, weight loss and recent hematemesis and melena.

Family history negative. Denies venereal. Up to 2 years ago was in perfect health. At that time was thrown from a wagon. Fell hard upon his back, and was confined to bed for a week. On getting about again noted burning sensation in epigastrium, usually several hours post cibo and at night. This distress occurred on and off and was never severe. Relief was gained by rest, diet, and medicine given him by physician (alkali?). Appetite and bowels remained negative. There was some bloating and flatulence. Nothing different from the above was observed until about 3 months ago. At that time he received a severe blow in the "pit" of the stomach. His "wind was knocked out" and he suffered rather acute distress for a half hour. Several hours afterward he vomited about a pint of blood mixed with food. He felt "sick all over" and perspired rather profusely. For 2 or 3 days afterward he passed black stools. He has "never been himself since." He has continuous burning in epigastrium and no appetite.

Condition on examination revealed weight loss of 40 pounds (in about 2 months); hemoglobin, 87 per cent.; considerable emaciation, and a movable nodule in the upper, mid-epigastrium.

Test-meal revealed free $\mathrm{Hcl}, 0$; total acidity, 64 . There 
were lactic acid and altered blood present. Glycyltryptophan test was positive. Pyloric obstruction was proved by persistent 12-hour food retention.

Microscopically, Oppler-Boas bacilli were seen in abundance.

Laparotomy disclosed an irremorable carcinoma involving the entire pyloric region of the stomach. The perigastric lymph glands were involved. Anterior gastro-enterostomy was performed. The patient died 11 days afterward.

Hygiene.- We have called attention to the fact that approximately half of our patients came from rural communities or small towns. The majority of these people were farmers or had lived upon farms. Many subjects were foreign born or of alien parentage. While the plane of life of the American farmer is relatively high, yet it must be admitted that both general and personal hrgiene are susceptible of improvement. This is especially so among immigrant farmers. One not infrequently sees housing conditions for horses and stock practically as perfect as those for the landsman himself. The residence is generally built after other things have been adequately taken care of. Consequently, over-crowding, with lack of fresh air and sunlight, are quite as common in country homes as in those located in towns. Bath-rooms are comparatively rare in the homes of the arerage foreign-born farmer. Privies of the disgusting and dangerous well-trpe are generally located near the farm dwelling, and lavishly hospitable to hosts of infection-spreading insects. Poorly covered drinking-wells are the rule. Cattle-yards are generally conveniently adjacent to kitchen-gardens and milk-houses, and hence are a constant source of pollution.

While it cannot be said that this existing state of affairs is directly responsible for the prevalence of gastric disorders, particularly ulcer and cancer, among farmers, it must 
be recognized that the stage is perfectly set for the enaction of any disease drama to which a rural population may be susceptible. The home environment would seem not infrequently to counteract whatever tendency toward longerity the farming element might acquire by virtue of its physically active, open-air life. Orer-eating and the sedentary habit is common during at least a third of the year among farmers. During the relatively inactive winter months, their diet is not reduced from that upon which they worked during the periods of tilling, seed time and harvest. Not rarely, added richness of food is supplied by a home-cured porker, a beef or by the multitudinous examples of peasant culinary art whose creation a full larder tempts. As a result of this prolonged gastronomic debauch, late winter, spring and early summer are seasons during which digestive disturbances among inhabitants of rural communities are so prevalent as to be almost fashionable, i.e., they are an index of prosperity.

Teeth.-Relatively few foreign-born farmers and many of native parentage pay any attention whatever to oral cleanliness. In our experience with this class of citizen, gastric cancer and filthy mouths go hand in hand. Dirty, decaying, infected teeth, loosely imbedded in soggy, swollen, pus-laden gums, not only prevent proper mastication of food, but also act as reservoirs from which a constant supply of poison passes into the lymph-and, perhaps, blood-streams. From such mouths can be isolated virulent cocci, bacilli, spirochætæ, leptothrix, flagellate protozoa (cercomonads, trichomonads), and amœbæ. Pyorrhea alveolaris is a visible, local evidence of the damage which this infection may cause. We have no means of knowing at present what effect upon gastric mucosa such organisms may have as result of their passing into the circulation or 
from their lodgment and growth upon a damaged gastric lining. What upset of gastric physiology may result from the constant ingestion of millions of microorganisms and the products of their growths we can but conjecture. Certainly it is difficult to see how such intake can be in any way beneficial.

From microscopic study of the gastric extracts of 2,406 different individuals affected with "stomach trouble," I have shown that irrespective of the degree of acidity of such gastric extracts, bacteria could be isolated in $\$ 7$ per cent. Degree of gastric acidity is by no means an index of the stomach's cleanliness bacteriologically. In my studies, cocci and diplococei were present in 83 per cent., short and long rods (often of the colon group) in 58 per cent., streptococci in 17 per cent., and Leptothrix buccalis in 24 per cent. In cultural studies of the saliva from more than 300 dyspeptic patients, I showed that streptococei and staphylococci could be obtained in $\$ 0$ per cent., bacilli in 66 per cent., and leptothrix in 14 per cent. It would seem that many mouth organisms thrive in gastric juice, and only rarely is the degree of hydrochloric acid sufficiently high to act as a germicide or to retard bacterial proliferation. I also showed that saliva from the dirty mouths of dyspeptics not infrequently contains the amino-acid tryptophan, and that more than 90 per cent. of such salivæ hold a ferment (?) capable of cleaving the dipeptid glycyltryptophan.

Heredity.-In our series, a family or blood-relationship history of cancer, generally, was obtained from 78 individuals ( 8.5 per cent.). Of this number, an hereditary history of gastric cancer was noted in $46,58.9$ per cent. of the group and 4.9 per cent. of the total number of cases (921). 
Our material fails to demonstrate a predisposition to gastric cancer among children of the same sex as the affected parent, a view which has been advanced by some clinicians (e.g., Fenwick). In 11 instances where sons were cancerous the mother had had a like ailment; of such mothers there were 2 cancerous daughters. There were 11 instances where fathers had succumbed to gastric malignancy. In this group there were 9 males and 2 females. There were recorded 15 cases where brothers and sisters were affected with the disease. One male was observed both of whose parents had died from gastric malignancy. Another patient, aged 39, reported the recent death from cancer of the stomach of a brother and a sister. There were 2 females whose uncles had died with the disease and 2 males whose grandfathers had been likewise affected.

While heredity appears to play a part in the predisposition to cancer in certain families, yet we feel from the analysis of our material that the exact significance of such influence is at present unknown. It may be that the transmission by parents to offspring of a mode and conduct of life is a factor not without importance, Galton's famous twins to the contrary notwithstanding.

Certain classic cancer families are frequently mentioned. Napoleon I, his father, his brother and two sisters (Pauline and Caroline) are said to have died of gastric cancer. Monichon states that of 23 families with whose history he was conversant, there were 69 cancerous members; of these, 57 were affected with gastric cancer. Fenwick mentions a family where a father, his sister and three brothers all succumbed to the disease. Williams gives the following interesting analysis of a cancer family in which certain other cancer lesions were associated with 142 consecutive uterine cancers. 
Table 12

\begin{tabular}{|c|c|}
\hline Member of family & Part affected \\
\hline Father's brother (in 1 family) .. & $\begin{array}{l}\text { Face } \\
\text { Stomach (brother) }\end{array}$ \\
\hline Father's brother and 3 cousins (in 1 family).. & $\begin{array}{l}\text { Breast (female cousin) } \\
\text { Neck (male cousin) } \\
\text { Foot (male cousin) } \\
\text { Uterus }\end{array}$ \\
\hline Father's sister (in 3 families) ............. & $\begin{array}{l}\text { Intestine } \\
\text { Arm }\end{array}$ \\
\hline Maternal grandmother (in 1 family) ... & $\begin{array}{l}\text { Uterus } \\
\text { Uterus (2) } \\
\text { Breast }(2)\end{array}$ \\
\hline Mother (in 9 families) $\ldots \ldots \ldots \ldots \ldots \ldots$ & $\begin{array}{l}\text { Stomach (2) } \\
\text { Intestine } \\
\text { Abdomen } \\
\text { Face } \\
\text { Uterus (2) }\end{array}$ \\
\hline Mother's sister (in 6 families) ........... & $\begin{array}{l}\text { Breast (2) } \\
\text { Not stated (2) }\end{array}$ \\
\hline $\begin{array}{l}\text { Mother's brother and child of mother's } \\
\text { sister (in } 4 \text { families) } \ldots \ldots \ldots \ldots \ldots \ldots \ldots \ldots\end{array}$ & $\begin{array}{l}\text { Stomach (brother) } \\
\text { Face (brother) } \\
\text { Not stated (brother) } \\
\text { Stomach (sister's child) }\end{array}$ \\
\hline Patient's sister (in 8 families) ............. & $\begin{array}{l}\text { Uterus }(6) \\
\text { Breast }(2)\end{array}$ \\
\hline $\begin{array}{l}\text { Patient's brother (in } 1 \text { family) ........... } \\
\text { Patient's daughter (in } 1 \text { family) . . . . . . }\end{array}$ & $\begin{array}{l}\text { Stomach } \\
\text { Uterus }\end{array}$ \\
\hline
\end{tabular}

Summary.-Seats of hereditary disease, 38. Groups: uterus 13 , breast 7 , stomach 6 , locality not stated 3 , intestine 2, abdomen, arm and foot, each 1.

The transmission of the same type of malignant tumor to homologous organs has been emphasized by Virchow in the case of anal melanosis in horses. The susceptibility to tumor implantation in strains of laboratory animals is common knowledge (Slye). Similarly in humans, the hereditary features have been brought out with reference to cancer of the penis, melanotic sarcoma, glioma and sarcoma of the kidney. 


\section{ASSOCIATION WITH OTHER GASTRIC DISEASE}

1. Gastric Ulcer.-(a) Its relation to gastric cancer. Chapter VIII considers this question in detail. It is proper to remark here that there has been much ancient and recent controversy regarding the question of the frequency of the association of benign gastric ulcer, of the type pathologically chronic, and carcinoma of the stomach. It is somewhat striking that those internists, pathologists and surgeons who have only meager evidence to present most staunchly proclaim that such transition but rarely occurs. Pathologists and clinicians acquainted with and practising modern methods admit that the question is still unsettled in many of its phases. They maintain, however, that until we are thoroughly acquainted with the facts bearing upon the cause and life history of gastric ulcer and of gastric cancer there is but little hope of scientifically proving how many benign gastric ulcers become malignant and how many continue to pursue a benign course. At present neither pathologist nor clinician can prognose the future course, histologically or clinically, of any gastric ulcer, acute or chronic. In so far as many ulcera carcinomatosa and early gastric cancers present symptoms which it is common to associate with benign peptic ulcer, it behooves us to cease a ralueless controversy over the frequency with which gastric ulcers become cancers, and to devote our energies to the discovery of diagnostic procedures which will enable us to recognize early malignancy, whether such arise in ulcer or primarily, from a previously healthy gastric lining. By so doing we can serve humanity and increase the sum of histologic and clinical knowledge.

(b) Benign peptic ulcers are found sometimes in asso- 
ciation with gastric carcinomata. Our observations revealed 12 instances where such combination occurred. In these cases it is often impossible to separate the pictures, clinically, until the neoplasm has brought about definite constitutional changes due to local digestive disturbances and malignant intoxication. Our series also demonstrated the concomitance of benign duodenal ulcer and gastric cancer 8 times.

2. Gastric Syphilis.-Unless negative Wassermann reaction is returned, it is never possible to exclude gastric syphilis in patients with history of lues and chronic dyspepsia associated with weight loss, anemia, achylia gastrica or epigastric tumor. There were 9 such cases among our patients. It is quite likely that more extensive serologic investigations upon those so-called "atypical" gastric neoplasms will demonstrate an increasing number of tumors which have a luetic etiology.

3. Tuberculosis. - The stomach is rarely involved in tuberculous processes. Only 1 of our cases had associated with gastric cancer an ulcer which appeared grossly and histologically tuberculous.

4. Achylia Gastrica.-That the absence of gastric secretions alone does not predispose to carcinoma of the stomach appears to be borne out by the clinical and laboratory evidence that patients with "primary" achylia can exist quite comfortably for many years without cancer resulting. Also, in the various secondary and essential anemias there is almost uniformly an absence of gastric secretion, without the succeeding development of a neoplasm.

5. Actinomycosis.-Microscopic examination of the tumor mass in 1 of our cases revealed medullary cancer in association with abundant growth of ray fungi. The 
patient was a male, farmer, without oral, nasal, pulmonary or body surface focus of actinomycosis.

\section{ASSOCIATION OF GASTRIC CANCER WITH EXTRAGASTRIC DISEASE}

1. Malignancy Primary in Other Organs. - In 5 female patients gastric cancer occurred as a metastatic process from cancer of the breast. In 3 females primary cancer of the genitalia was followed by stomach involvement. Three males had gastric neoplasms in association with hepatic cancer. In 2 females cancer of the gall-bladder resulted in succeeding gastric involvement. In 3 instances (2 males and 1 female) malignancy of the colon was responsible for the stomach lesion. Adenomata of the thyroid were concomitant in 3 instances.

2. Cholelithiasis and Cholecystitis.-Gall-stones had previously been removed or were noted at laparotomy in 18 of our patients. Cholecystitis in recognizable degree was observed in 28 of 214 patients where note was made. In 46 cases the appendix had previously been removed or was removed at the operation for cancer.

3. Infectious Diseases.--(a) Tuberculosis.-Many writers have observed the frequency of healed tubercles in the lungs at autopsies of those who have died from gastric cancer. Fenwick noted such in 15.8 per cent. It has also been advanced that various forms of tuberculosis are more than ordinarily common in families where cancer later develops. Williams states that 50 per cent. of his cases of uterine and mammary cancer had tuberculous family history; Osler reports such in more than 31 per cent. and Fenwick 26 per cent. of gastric cancers. In our series there was a family or blood-relationship history of tuberculosis in 26 instances $(4.3$ per cent.) where definite 
facts were obtainable. It is suggested that inasmuch as the great mass of cancer deaths occurs after the fifth decade, age alone may account for the prevalence of healed tubercles in the lungs of 15 per cent. or more of these subjects. Their presence at necropsies of adults is by no means uncommon in individuals dying from diseases other than gastric cancer.

(b) Rheumatism.-In 38 cases (4.1 per cent.) chronic rheumatoid affections were recorded. There were cardiac leakages in 14 instances.

(c) Malaria.-It has been claimed that carcinoma is not common in regions where malaria is endemic. Table VI shows that our lowest cancer death rates occur in North Carolina and Kentucky, sections of the United States where malaria is frequently endemic and active. Study of our records of gastric cancer patients demonstrates that of 732 cases where definite information was possible 17 instances (2.3 per cent.) were recorded. This is a relatively high figure inasmuch as comparatively few of our cases come from the South.

(d) Typhoid Fever.-A remarkably large proportion of our patients gave a history of having had typhoid fever. Of 689 instances where data were available 92 patients (14.8 per cent.) had been affected with the disease.

(e) Other Infectious Diseases.-Yellow fever was noted in 1; gonorrhea in 43; asiatic cholera in 1; erysipelas in 2; pleurisy in 3 ; smallpox in 3 ; mumps in 12 ; scarlet fever in 18; tonsillitis or quinsy in 20; diphtheria in 22; pneumonia in 29; measles in 34 ; la grippe in 54 .

4. Parasitic Infections.-Seven patients had passed tape-worms at some period of their lives. In one case a tape-worm passed 6 weeks before the patient came under observation, appeared to precipitate malignant symptoms. 
Protozoa were isolated from the stools in 11 cases. They were found as follows: trichomonas hominis 4, cercomonas hominis 2, endamœbæ 4 , and balantidium coli 1 .

5. Constitutional Diseases.-Asthma was observed in 31 instances; Bright's disease 18 times; cirrhosis of the liver 4 times; gout, eczema and diabetes each 3 times.

\section{REFERENCES}

Buday: Conference Internationale du Cancer, Paris, 1910, p. 89.

Hoffman, F. L.: Transactions of the American Gynecological Society, 1913; and Surg., Gyn. and Obst., 1914, June, p. 726.

Fenwick, S.: "Cancer and Other Tumors of the Stomach," Philadelphia, 1903.

CABot, R.: Jour. Amer. Med. Assn., 1912, Dec. 28, p. 2295.

Brinton, W. W.: "Diseases of the Stomach," 1859; and Brit. and For. Chir. Rev., 1857, January.

Fox, W.: "The Diseases of the Stomach," London, 1872.

WeLCH, W.: "Cancer of the Stomach," Am. Syst. Med., II.

Friedenwald, J.: Am. Jour. Med. Sc., 1914, Nov., p. 660.

Miyake: Sei-I-Kwai Med. Journ., Tokio, 1914, May 10, No. 5.

TAтнam: Supplement to Registrar-General's Fifty-fifth Annual Report, 1897 (London).

Aschoff: Cited by Williams.

Williams, R.: "The Natural History of Cancer," New York, 1908.

Osler and McCrae: "Cancer of the Stomach," Philadelphia, 1900.

Fǘterer, G.: Quoted by Mayo Robson and Moynihan, "Diseases of the Stomach," 1904.

Fibiger: Berl. Klin. Wochnschr., 1913, Feb. 17, p. 289.

Galton: Journal of the Anthropological Institute, 1876, vol.v, p. 391.

Monichon: Thèse de Paris, 1896, No. 415.

VIrchow: Onkologie, II, p. 352.

SLYE: Interstate Medical Journal, 1915, July.

Smituies: Arch. Int. Med., 1912, Dec., p. 1. 


\section{CHAPTER II}

\section{MORBID ANATOMY: GROSS, MICROSCOPIC AND EXPERIMENTAL}

\section{CLASSIFICATION OF GASTRIC NEOPLASMS}

By clinicians, gastric tumors are usually grouped as benign or as malignant.

The benign neoplasms are those that arise primarily within the wall of the viscus and confine their growth to the stomach. It is rare for them to invade adjacent viscera by contiguous extension or by metastases through blood- or lymph-channels. They are generally of relatively slow growth, but may bring about death of their host. 'The fatal termination most commonly ensues from the production of various types of stenoses. Starvation and malnutrition then result. Tumors of this type are myomata, fibromata, papillomata, syphilomata, tuberculomata, lipomata, cysts, osteomata, myxomata and aneurysms.

Malignant gastric neoplasms most frequently arise at some part of the gastric wall. They may, however, secondarily involve the stomach as a consequence of metastasis from an extragastric tumor (uterus, liver, breast, etc.). This group includes carcinomata and sarcomata. Clinically, both may be styled cancer. These tumors are malignant in the sense that they are progressive in growth, bring about destruction of normal tissue, produce useless malformations of the viscus, often involve adjacent organs by direct extension or distant organs by metastases, and, if not disturbed, generally cause death of the individual by that metabolic derangement whose consequences we recognize as cachexia. 
Of the malignant gastric tumors, sarcoma is of rare occurrence. The first authentic example was described by Virchow. Since then an occasional specimen has been mentioned in theliterature. Eleven years ago the Fenwicks estimated that sarcomata constitute more than 5 per cent. of all primary neoplasms of the stomach. Interesting summaries of the affection have been made by Yates, Clendenning, Frazier and Campbell. They are infrequently diagnosed before laparotomy or necropsy, inasmuch as symptomatically they resemble carcinomata.

Gastric carcinoma-commonly styled "cancer"-comprises nearly 94 per cent. of all clinically or pathologically known forms of neoplasm primarily arising in the stomach wall. Pathologically this type of tumor manifests itself as a group of malignant histo-pathologic processes. It is necessary to consider both gross and microscopic deviations from the normal.

1. Gross Deviations from the Normal.-While the line of demarcation between types of carcinomata of the stomach is a rather indefinite one, the classic descriptions of such embodied in the writings of Waldeyer and Rokitansky comprise the most practical macroscopic classification of the disease. These pioneers recognized 3 general forms of gastric cancer. We have added a fourth group. The following classification is adopted from their descriptions:

(a) Fibrous cancer; scirrhus; carcinoma fibrosum. Usually a dense, well-delimited, poorly vascularized growth, generally forming circumscribed nodules, or arranged as a tumor of annular type. The stomach-wall is thickened, stiffened and puckered. Nodules project into its lumen. The tendency to ulceration is not great. The growth can be moderately well outlined by the fingers. It is firm and 
unyielding to the touch until secondary changes have taken place.

(b) Medullary cancer; "fleshy," "cauliflower," cellular and vascular type; carcinoma medullare. Essentially a rapidly growing tumor primarily of the structures of the mucosa and submucosa. This results in soft, nodular protuberances which invade the lumen of the stomach,

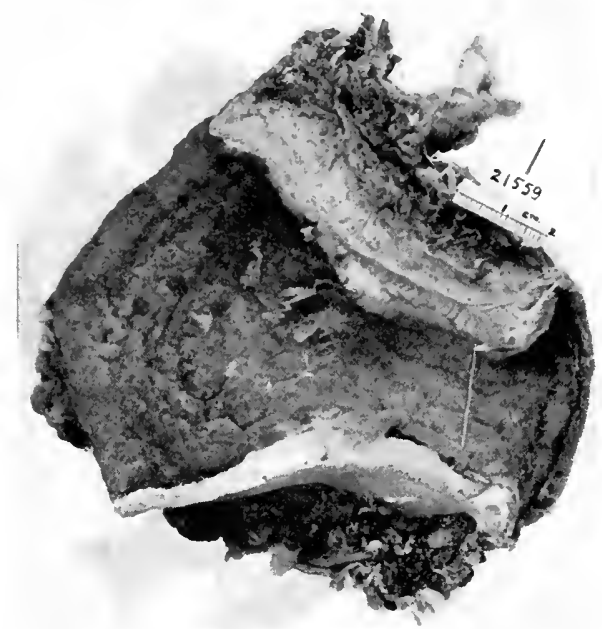

FIg. 1.-(Case No. 21,559).-Primary carcinoma involving the pyloric third, scirrhus type, pyloric obstruction. Great thickening of gastric wall, gland involvement, free fluid in abdomen. Specimen obtained at laparotomy.-(Courtesy of Mayo Clinic.)

spread rapidly and ulcerate early. The actively proliferating cancer cells quickly penetrate all layers of the stomachwall; vascularization is generally free; adjacent organs are invaded by continuity or lymph and blood-stream metastases; perigastric lymph glands harbor the growth and distant organs early suffer malignant change. This cellular and vascular type of growth renders both primary and secondary tumors soft to the palpating fingers. 
(c) Ulcerating cancer; ulcus carcinomatosum. This may result from cancerous change in a pre-existing calloused ulcer, or may occur from the secondary ulceration and sloughing of carcinoma medullare or carcinoma fibrosum. At laparotomy this type is of most common occurrence. The growth may be but $1 \mathrm{~cm}$. in diameter, but may in-

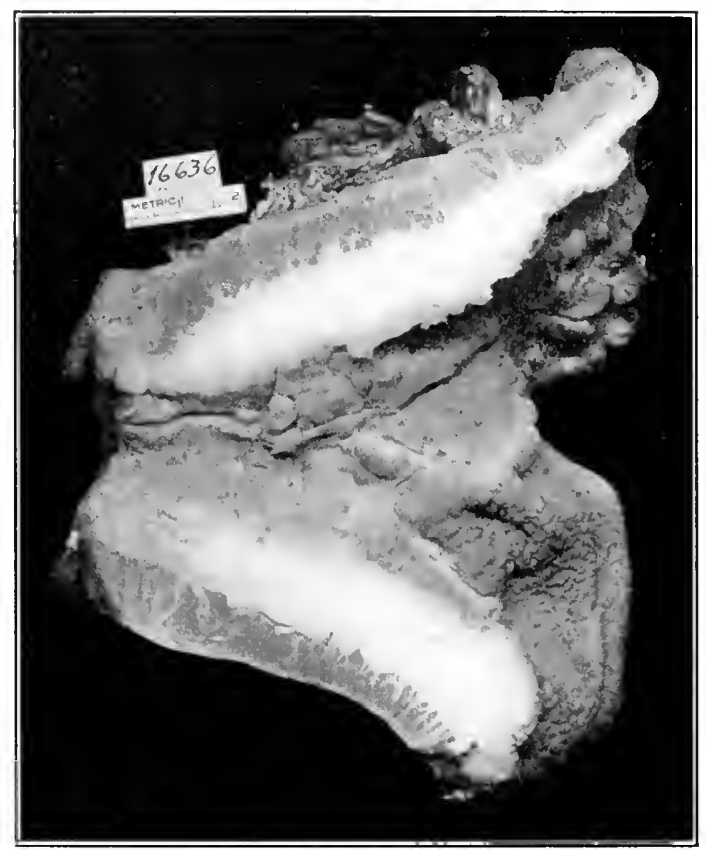

FIG. 2.-(Case No. 16,636).-Cancer of the pyloric end. Clinically scirrhus type. Great thickening of stomach wall, multiple nodules, perigastric gland involvement. No history of antecedent dyspeptic disturbance. Specimen obtained at laparotomy.-(Courtesy of Mayo Clinic.)

volve as much as a fourth of the viscus. Its base is commonly firm. Its edges are not infrequently soft, ragged, vascular and undermined (but may be smooth and shiny). The process is often well delimited. Malignant perigastric lymphatic gland enlargements are common. Metastases out of all proportion to the size of the local 


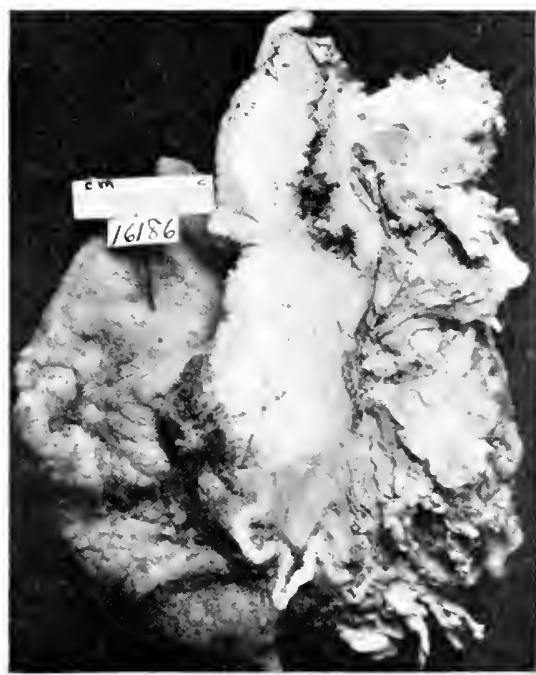

FIG. 3.-(Case No. 16,186).-Primary carcinonıa involving pyloric end of stomach. Medullary type with numerous points of ulceration. Extensive infiltration of gastric wall. Specimen obtained at laparotomy.(Courtesy of Mayo Clinic.)

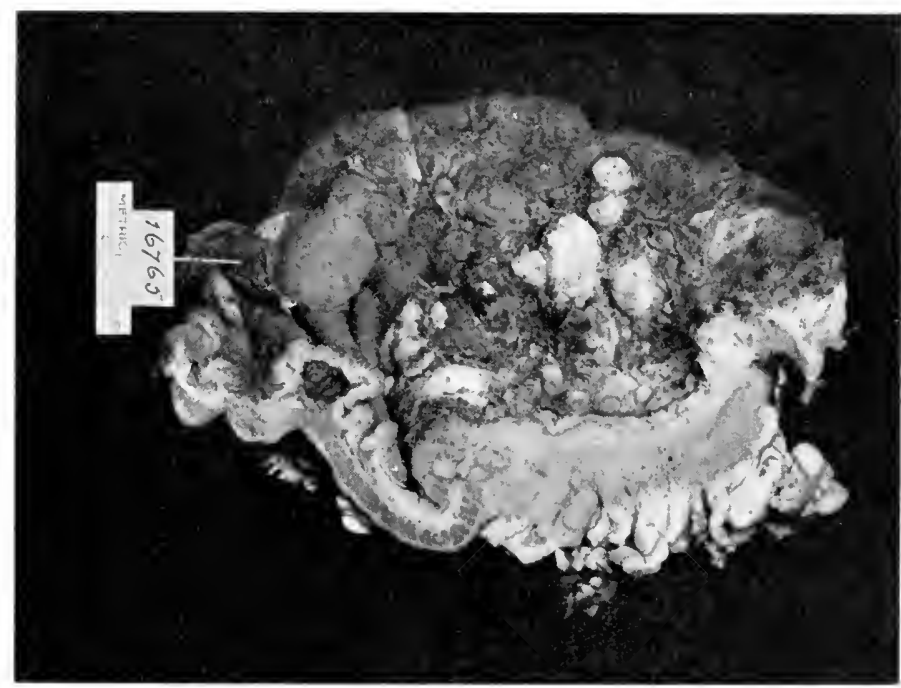

FIG. 4.-(Case No. 16,765).-Multiple gastric carcinoma of the medullary type, involving pyloric third of stomach. Numerous nodules and points of surface ulceration. Thickening of gastric wall, perigastric gland involvement. Specimen obtained at laparotomy.-(Courtesy of Mayo Clinic.) 


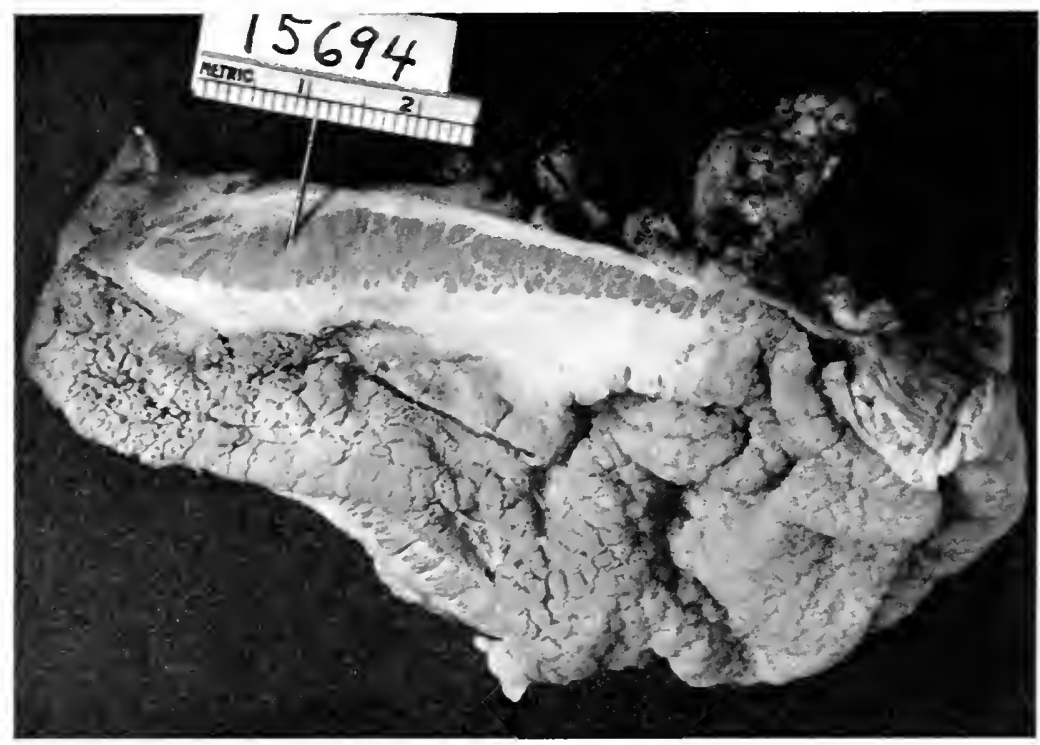

F1G. 5.-(Case No. 15,694).-Large carcinoma of the medullary-scirrhus type involving the pyloric third of the stomach. Enormous infiltration and hyperplasia of stomach walls; superficial erosions; pyloric obstruction. Patient had precarcinomatous history, clinically that of peptic ulcer. Specimen obtained at laparotomy.-(Courtesy of Mayo Clinic.) 


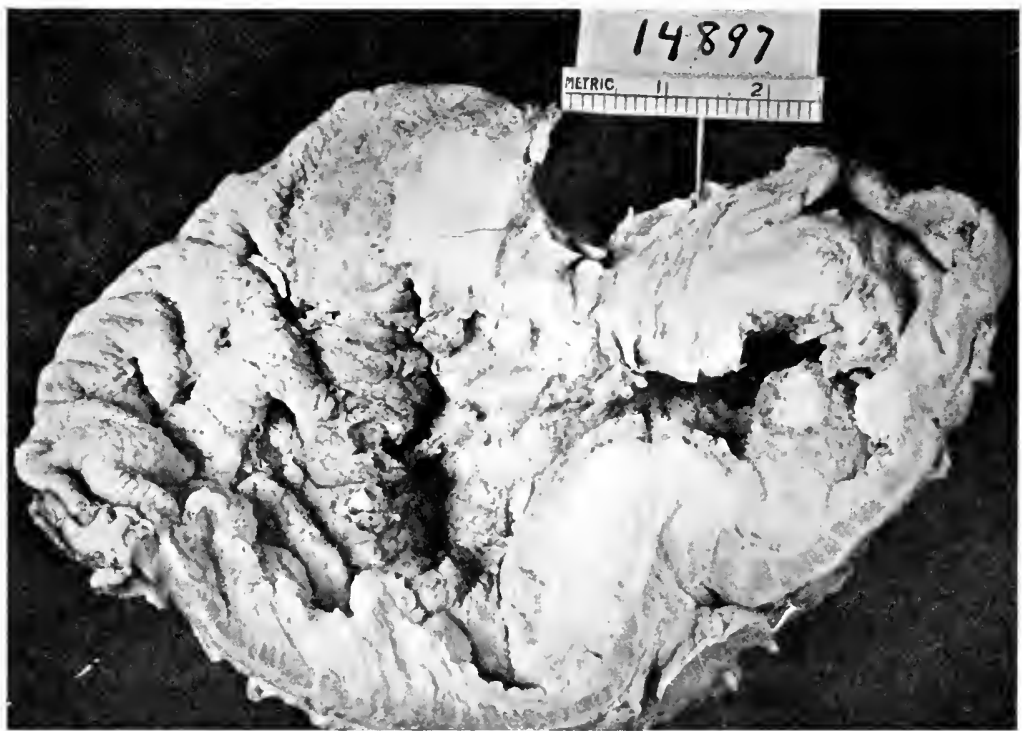

FIG. 6.-(Case No. 14,897).-Large carcinoma of the posterior wall and pyloric end of the stomach; medullary-scirrhus type; extensive invasion of the gastric wall; superficial neerosis, sloughing and hemorrhage; pyloric obstruction. Patient had precarcinomatous history, clinically that of peptic ulcer. Specimen obtained at laparotomy.-(Courtesy of Mayo Clinic.) 
gastric process may be discovered in adjacent glands or organs.

(d) Colloid cancer; gelatinous cancer, mucoid cancer; carcinoma colloides. The diffusely infiltrating type, which, due to mucoid degeneration of the cells composing it, as well as to their excretion of a substance resembling clouded

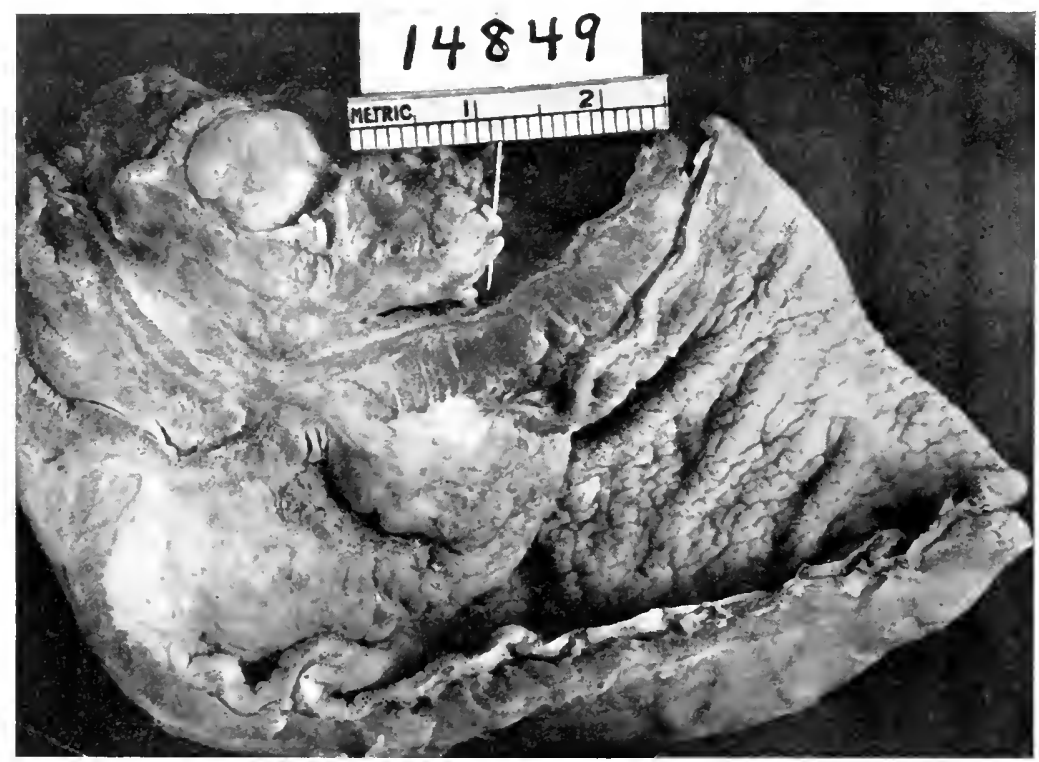

FIG, 7.-(Case No. 14,849).-Carcinoma involving the pylorus and lesser currature. Extensive tumor of the medullary-scirrhus type with small areas of colloid degeneration. Enormous infiltration of stomach wall, pyloric obstruction; perigastric gland invasion. Patient had precarcinomatous history, clinically that of peptic ulcer. Specimen obtained at laparotomy--(Courtesy of Mayo Clinic.)

egg-white, presents a water logged, translucent, jelly-like appearance. It ulcerates rarely, spreads extensively, and may form metastases. It is the least frequent type of gastric cancer (excluding sarcoma).

2. Histologic Deviations from the Normal.-While the above macroscopic classification of gastric cancer is a practical clinical grouping, it must not be supposed that the 


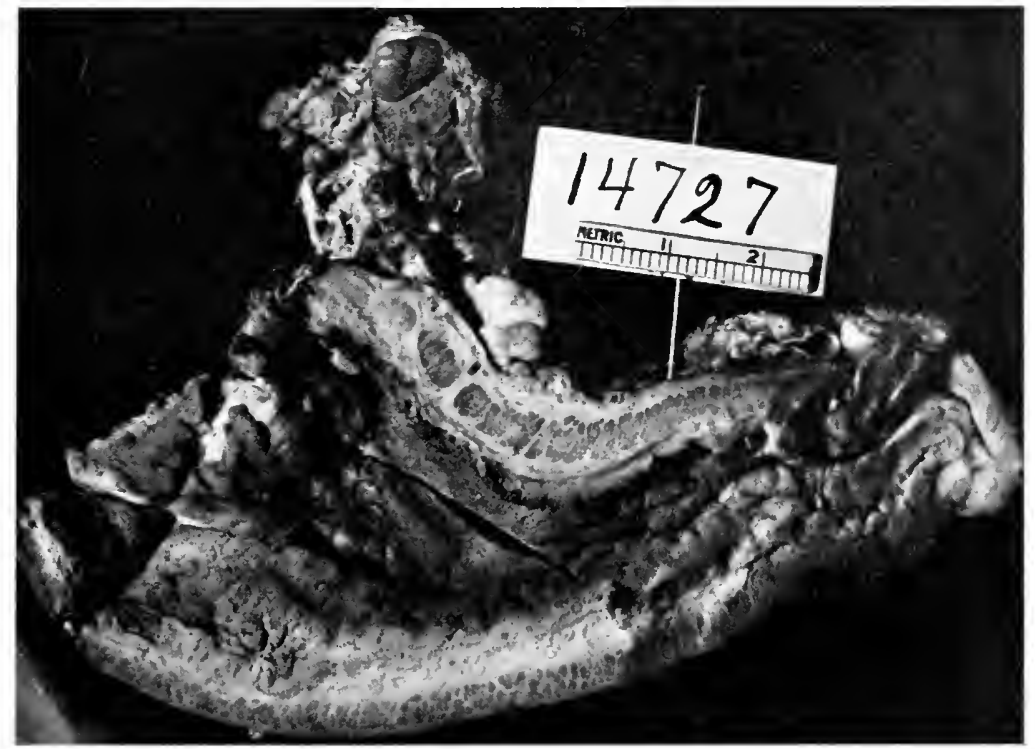

FIG. 8.-(Case No. 14,727).-Extensive primary carcinoma of the medullary-scirrhus type involving pyloric half of stomach; enormous thickening of stomach wall; pyloric obstruction; small gastric lumen ("leather-bottle type"); perigastric gland involvement. Specimen obtained at laparotomy.(Courtesy of Mayo Clinic.) 


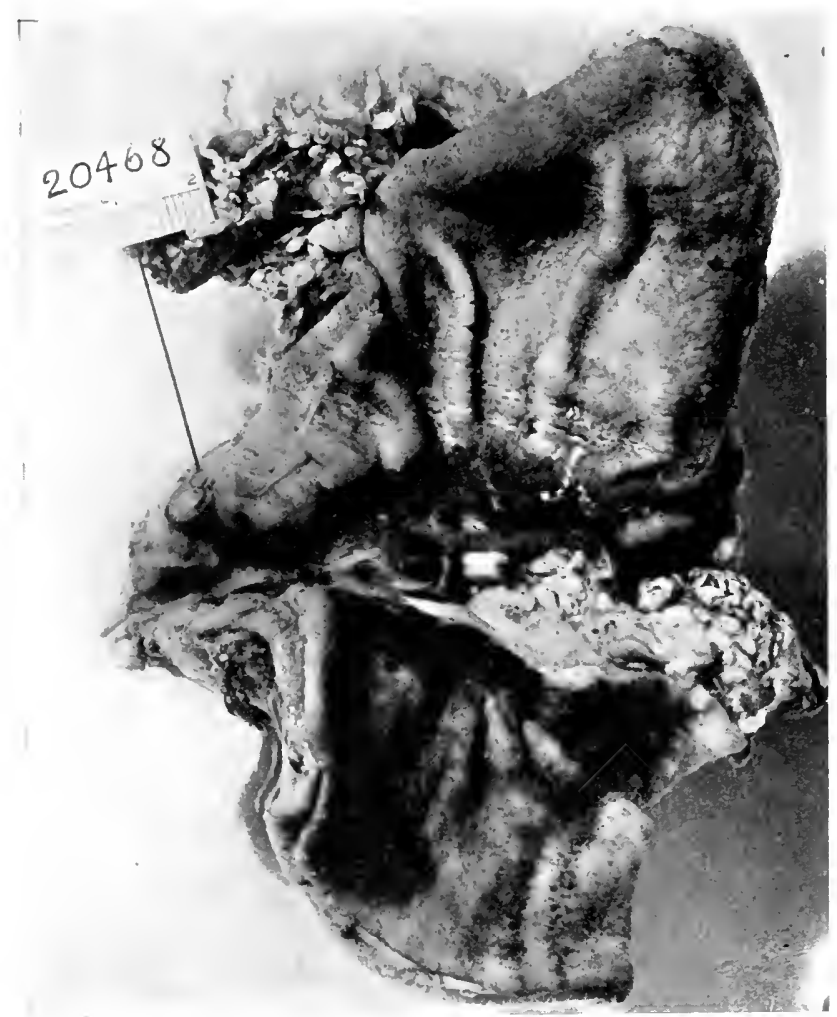

FIG. 9.-(Case No. 20,468).-Ring cancer of the medullary-scirrhus type involving the pylorus; moderate hyperplasia of the stomach wall; superficial ulceration; perigastric gland involvement. Patient had precarcinomatous history, clinically that of peptic ulcer. Specimen obtained at laparotomy.-(Courtesy of Mayo Clinic.) 


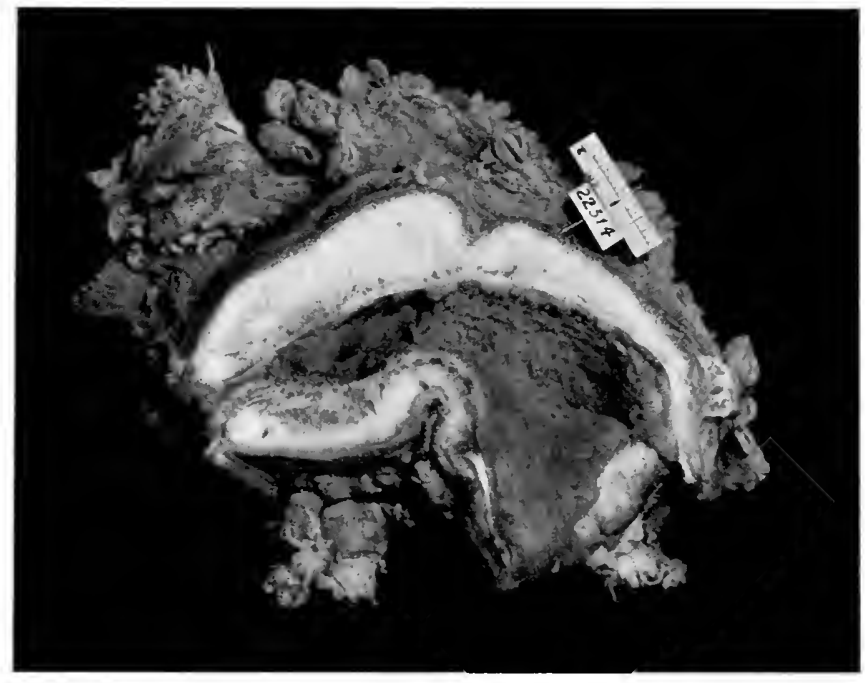

Fig. 10.-(Case No. 22,314).-Carcinoma of the scirrhus type involving the pyloric half of the stomach; enormous hyperplasia of gastric wall; pyloric stenosis; small gastric lumen ("leather-bottle type"). Specimen obtained at laparotomy.-(Courtesy of Mayo Clinic.) 


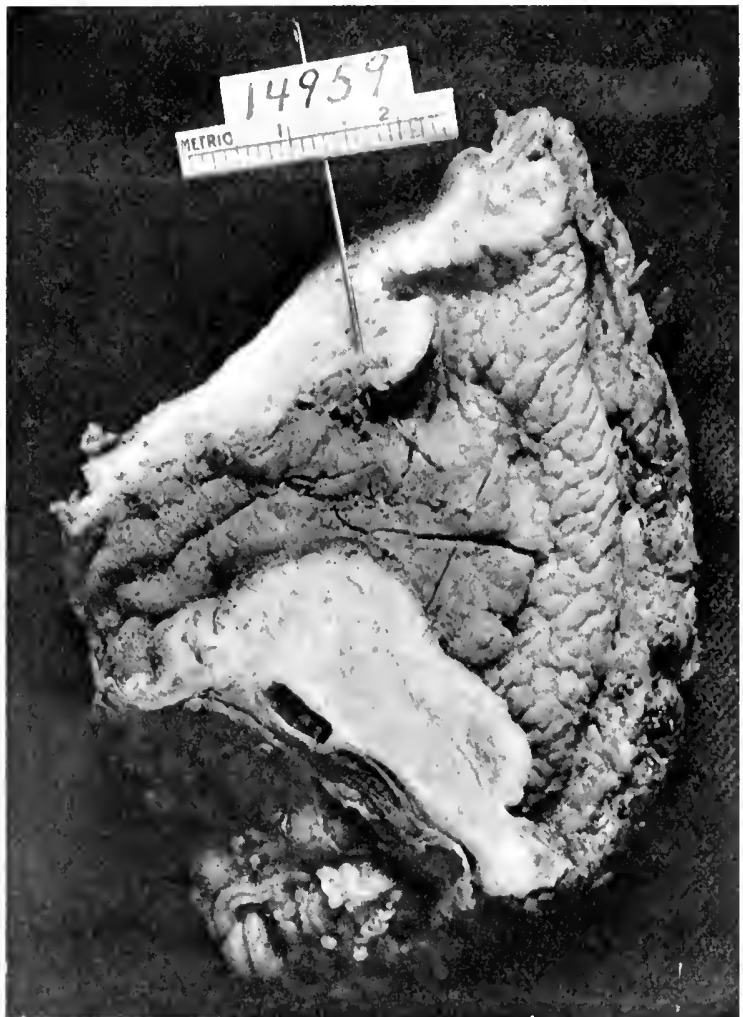

Fig. 11.-(Case No. 14,959).-Ring carcinoma of the scirrhus type involving the pylorus and antrum; extensive infiltration of stomach wall; pyloric obstruction. Patient had precarcinomatous history, clinically that of peptic ulcer. Specimen obtained at laparotomy.-(Courtesy of Mayo Clinic.) 


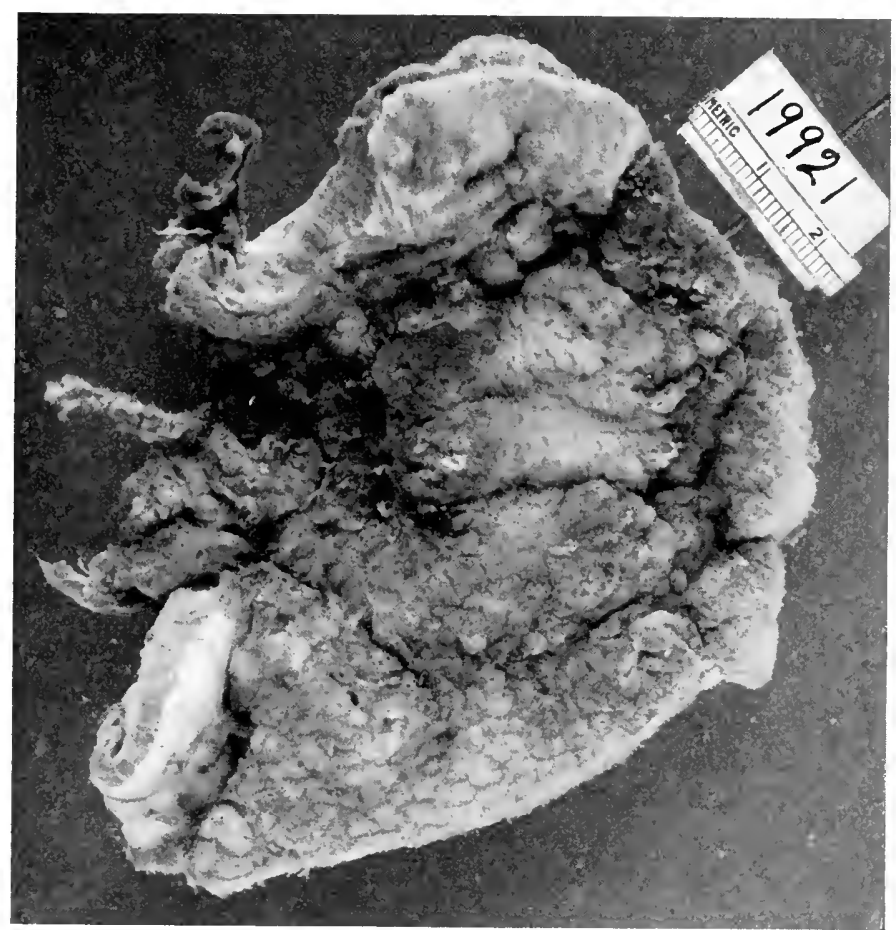

FIG. 12.-(Case No. 19,921).-Extensive carcinoma of the medullary type involving pyloric third of stomach; moderate pyloric obstruction; areas showing necrosis, sloughing and hemorrhage; moderate invasion of the gastric wall. Patient had precarcinomatous history, clinically that of peptic ulcer. Specimen obtained at laparotomy.-(Courtesy of Mayo Clinic.) 


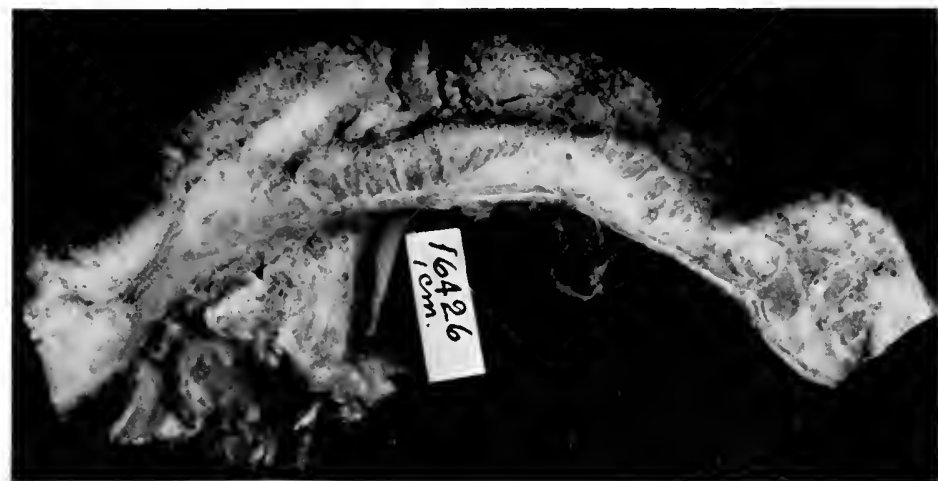

FIG. 13.-(Case No. 16,426).--Large medullary carcinoma of the papillomatous type; superficial erosions; enormous hyperplasia and infiltration of the gastric wall. Patient had precarcinomatous history, clinically that of peptic ulcer. Specimen obtained at laparotomy.--(Courtesy of Mayo Clinic.)

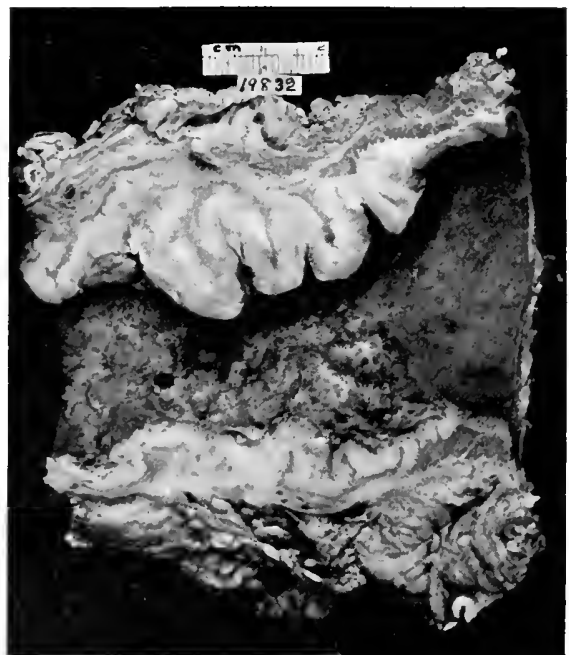

FIG. 11.-(Case No. 19,832).-Medullary carcinoma involving the pylorus; enormous hyperplasia of gastric wall; pyloric obstruction; secondary ulceration of tumor. Patient had a precarcinomatous history, clinically that of peptic ulcer. Specimen obtained at laparotomy.-(Courtesy of Mayo Clinic.) 


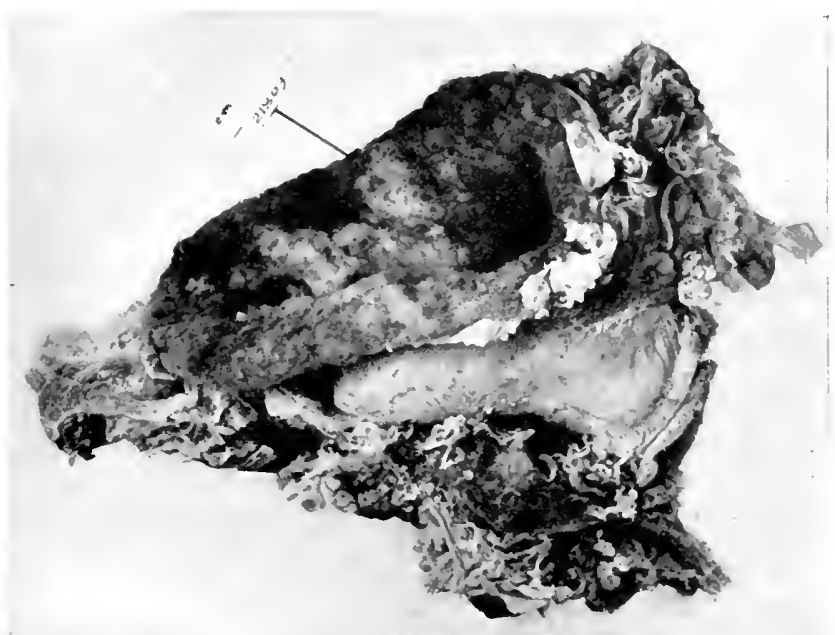

Fig. 15.-(Case No. 21,503).-Ring carcinoma of the medullary type involving pyloric end of stomach. Patient had precarcinomatous history of the peptic ulcer type. Specimen shows canalization of tumor obstructing pylorus; enormous hyperplasia of stomach wall; perigastric gland involvement. Specimen obtained at laparotomy.-(Courtesy of Mayo Clinic.)

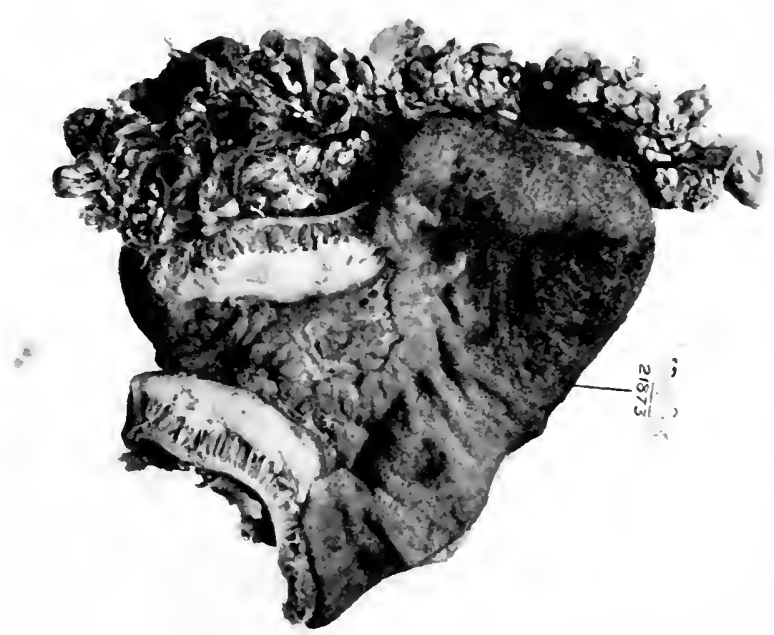

FIG. 16.-(Case No. 21,873).--Ring carcinoma involving the pylorus; superficial erosions; great hyperplasia of gastric wall, locally; pyloric obstruction; perigastric gland involvement. Patient had precarcinomatous ! history clinically that of peptic ulcer. Specimen removed at laparotomy.-(Courtesy of Mayo Clinic.) 


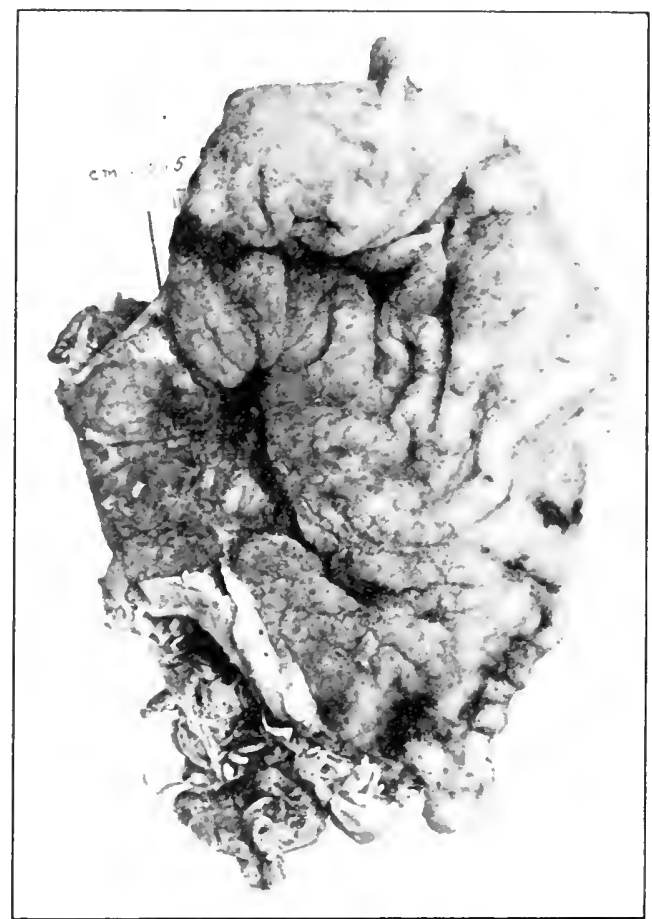

FIG. 17.-(Case No, 21,555).- Stereogram showing carcinoma associated with a large ulcer of the lesser curvature.-(Wilson and MacCarty.) 


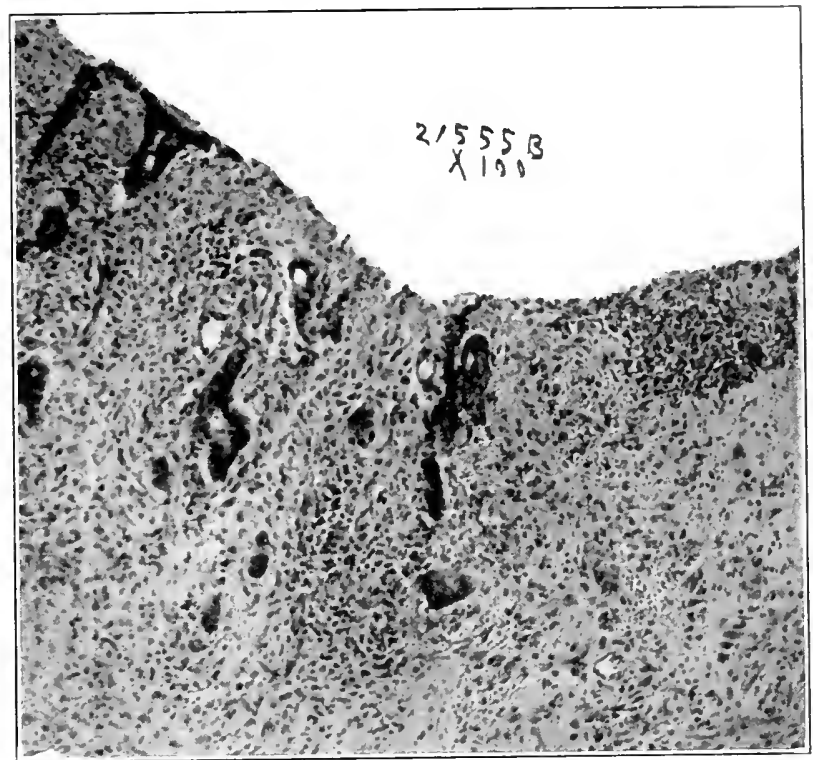

FIG. 18.-(Case No. 21,555).-Photomicrogram from the edge of the ulcer, bases of tubular glands show early carcinomatous change ( $X 100)$. (Wilson and MacCarty.)

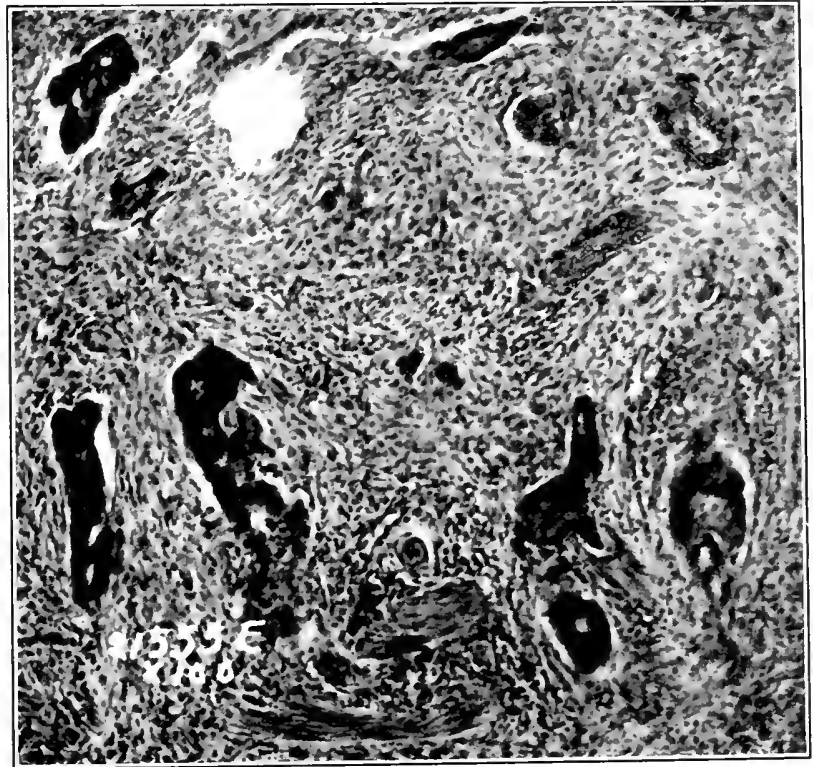

FIG. 19.-(Case No. 21,555).-Photomicrogram of a section of the base of the ulcer, showing scirrhus cancer $(X 100)$.- (Wilson and MacCarty.) 


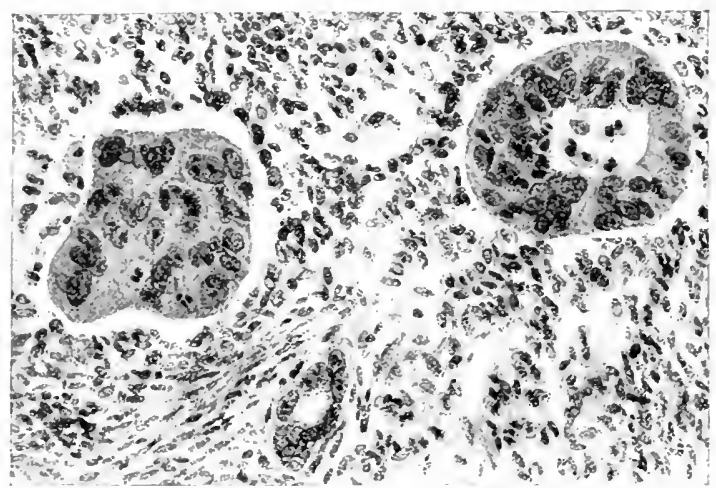

FIG. 20.-(Case No. 21,555).-Islands of carcinoma in the submucosa.-(MacCarty.)

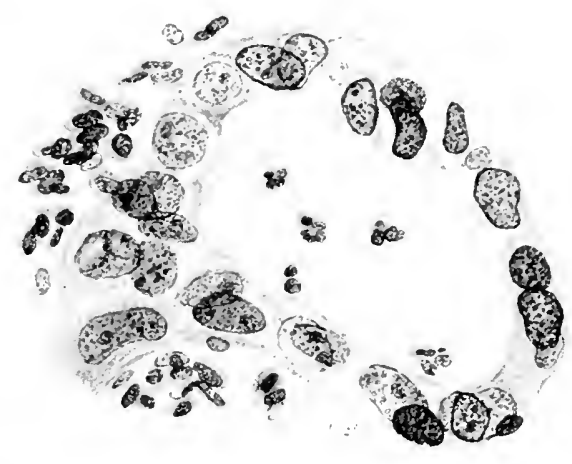

FIG. 21.-(Case No. 21,555).--Irregularly shaped epithelial cells near the muscularis mucosæ.-(MacCarty.) 


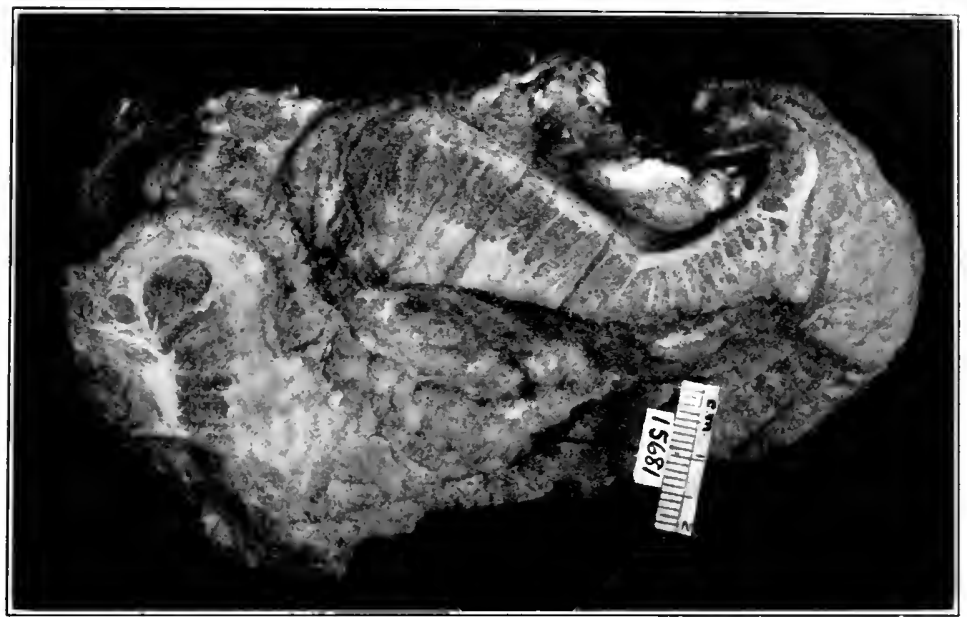

FIG. 22.-(Case No. 15,681).-Stereogram of a portion of the pyloric half of the stomach, showing carcinoma involving the lesser curvature and the pylorus.-(Wilson and MacCarty.)

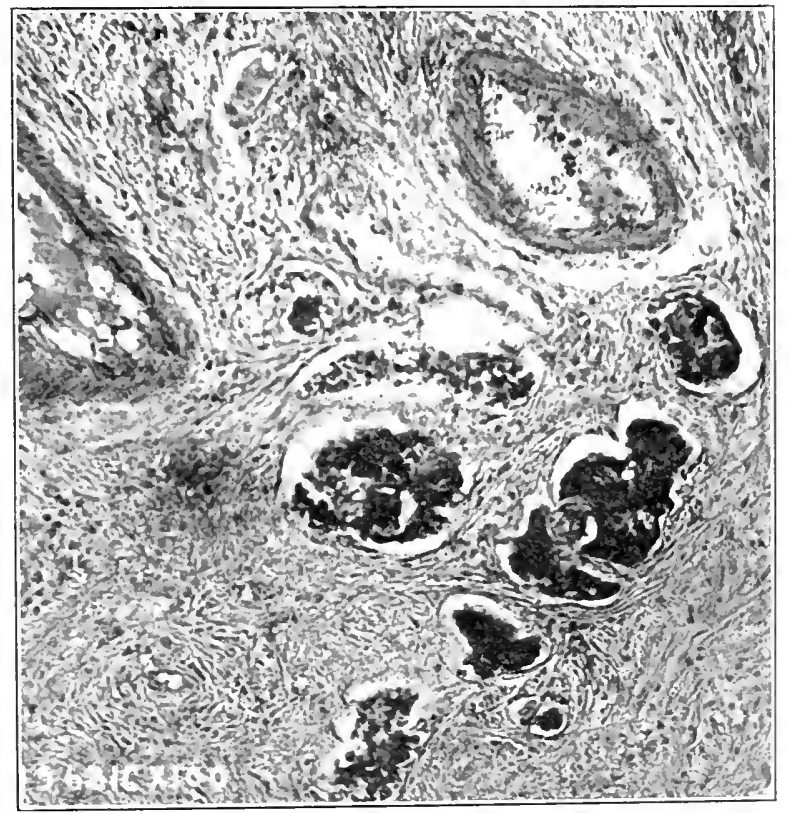

FIG. 23.-(Case No. 15,681),-Photomicrogram showing hyperplastic epithelium in the swollen mucosa of the edge of the ulcer ( $\times 100)$. (Wilson and MacCarty.) 


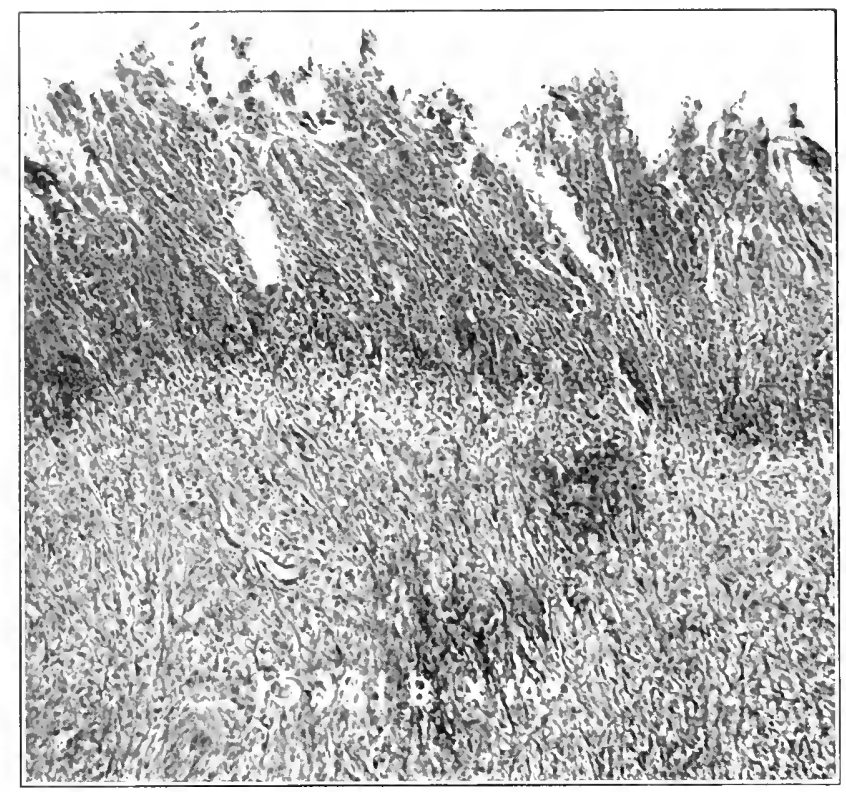

Fig. 24.-(Case No. 15,681).-Photomicrogram showing atypical epithelial proliferation $(\times 100)$. - (Wilson and MacCarty.)

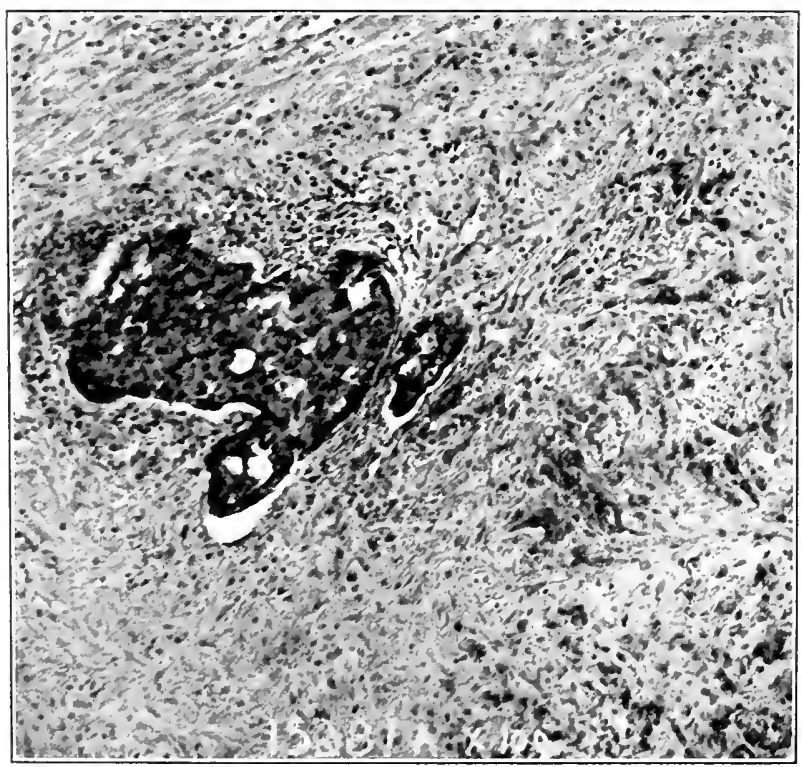

Fig. 25.-(Case No. 15,681).-Photomicrogram from the base of the malignant ulcer $(\times 100)$. - (Wilson and MacCarty.) 


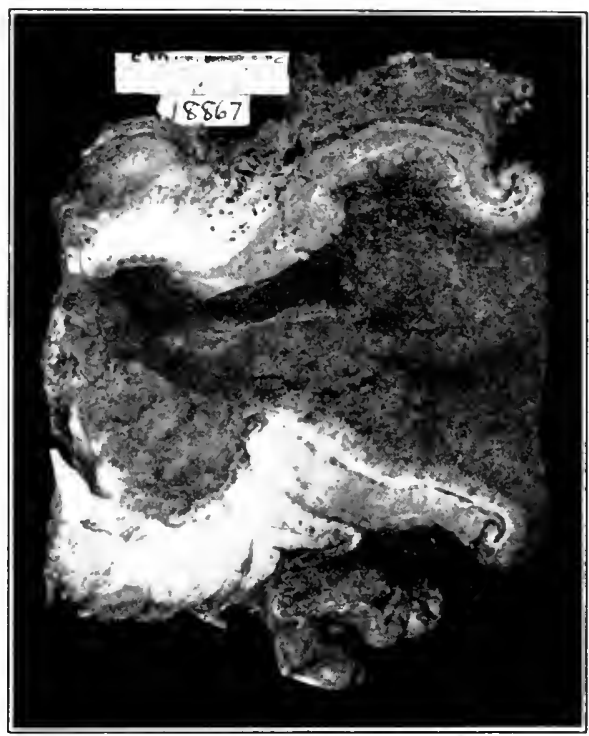

FIG. 26.-(Case No. 18,867).--Stereogram of the lesser curvature, showing proliferation of the muscularis associated with ulcus carcinomatosum. (Wilson and MacCarty.)

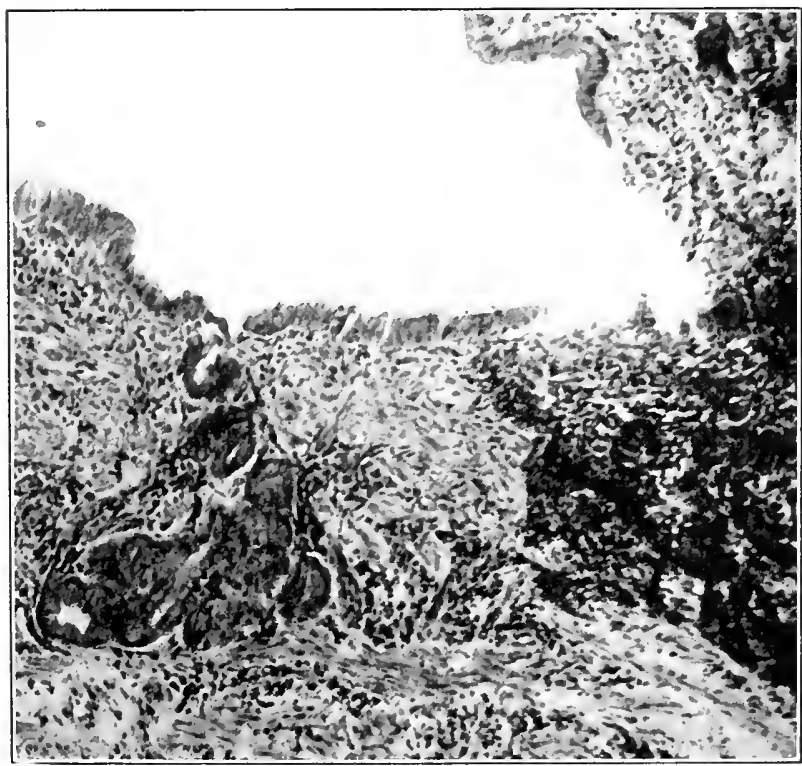

FIg. 27.-(Case No. 18,867).-Photomicrogram of epithelial cells partially cut off from the surface, actively proliferating but not infiltrating the surrounding tissues ( $\left.X^{\prime} 100\right)$.- (Wilson and MacCarty.) 


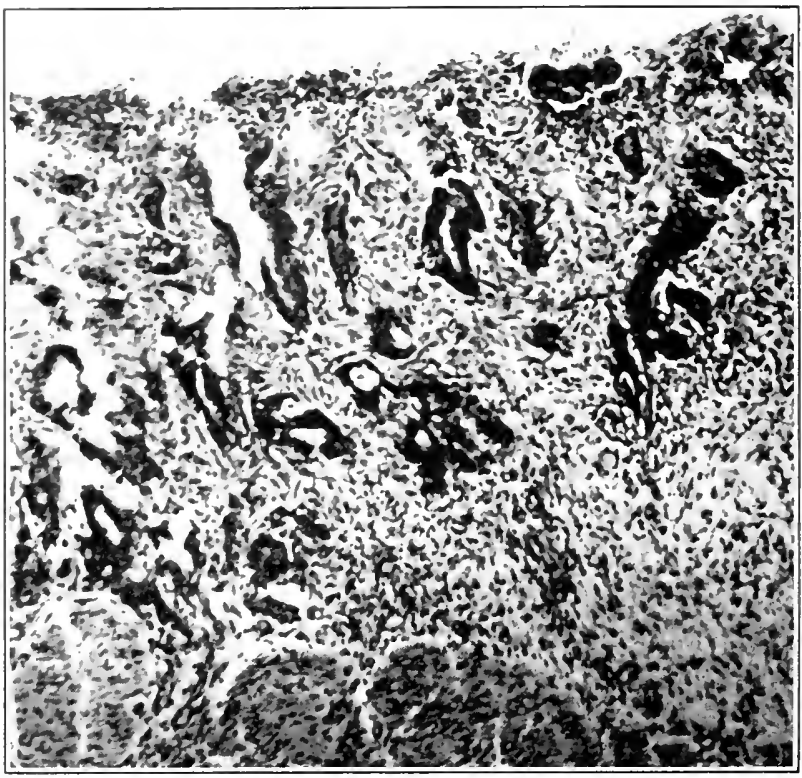

FIG. 2S.-(Case No. 18,867).-Photomicrogram showing area of typical carcinomatous tissue $(\times 100)$. - (Wilson and MacCarty.)

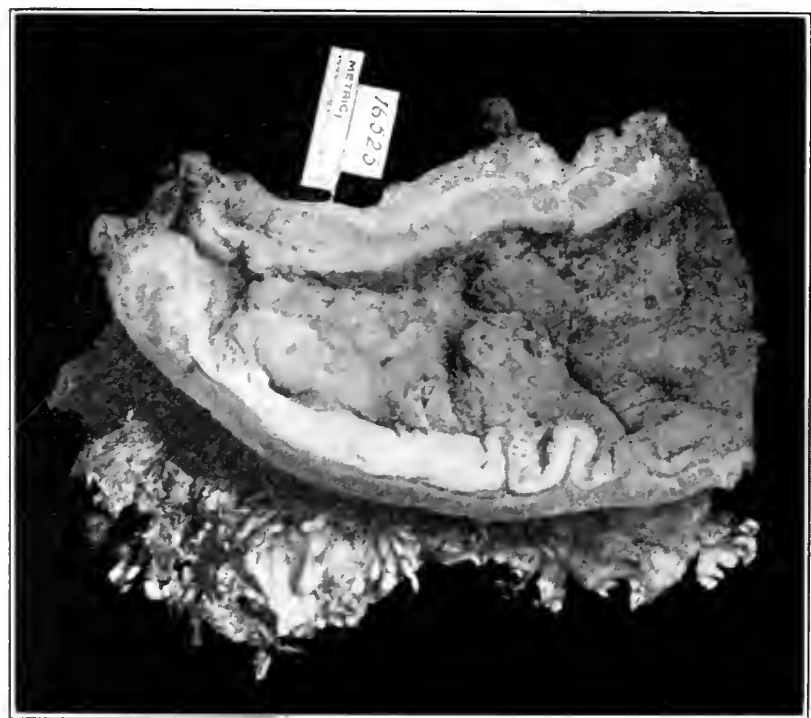

FIG. 29.-(Case No. 16,525).--Stereogram of the pyloric two-thirds of the stomach; carcinomatous ulcer beginning on the lesser curvature.-(Wilson and MacCarty.) 


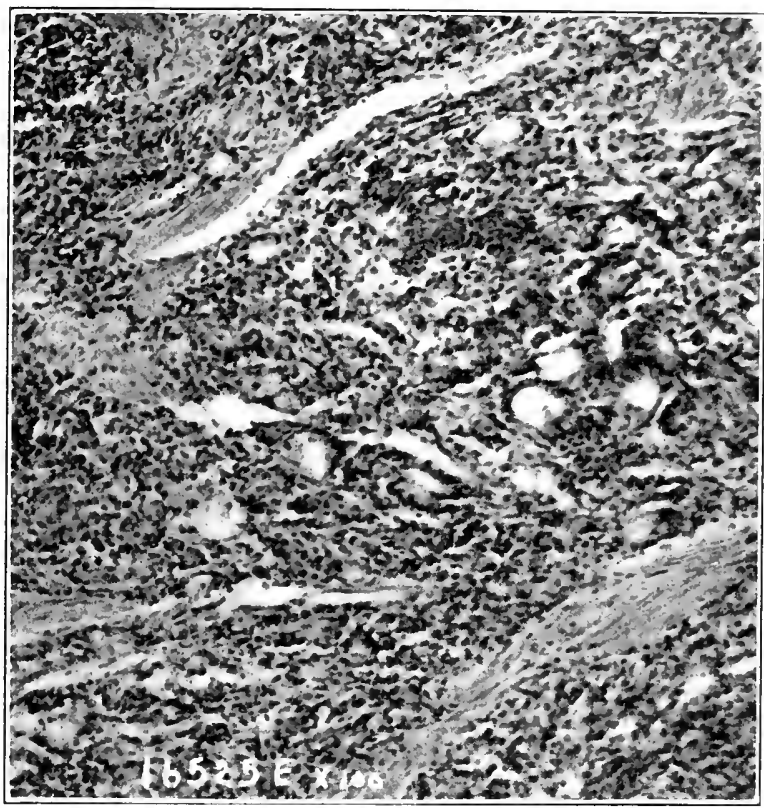

Fig. 30.-(Case No. 16,525).-Photomicrogram showing areas of scirrhus carcinoma ( $\times 100)$.- (Wilson and MacCarty.)

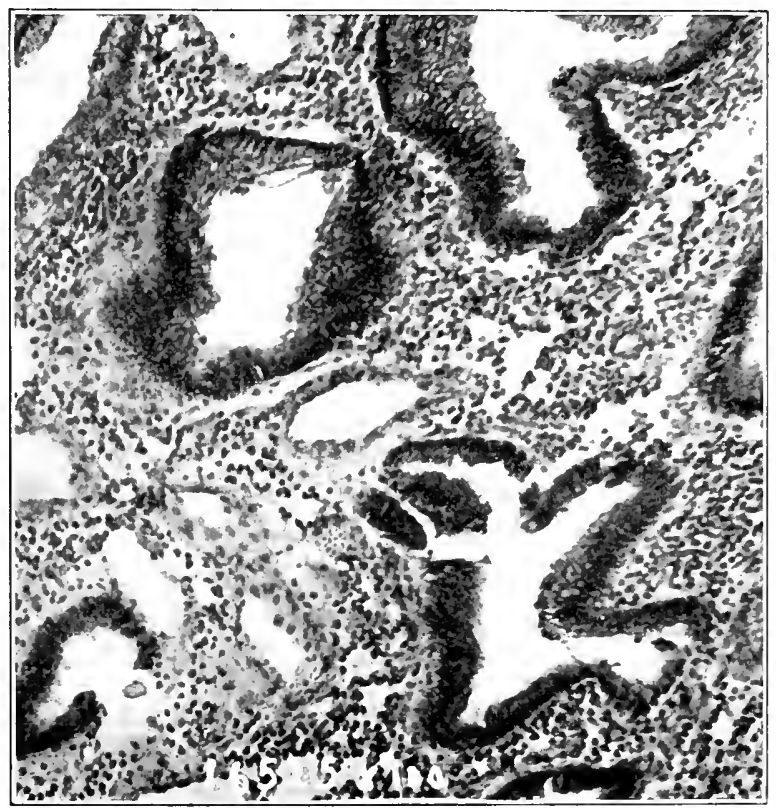

FIG. 31.-(Case No. 16,525).-Photomicrogram showing a cross-section of the hyperplastic epithelial elements with round-cell infiltration between the gland tubules ( $\times 100$ ).- (Wilson and MacCarty.) 


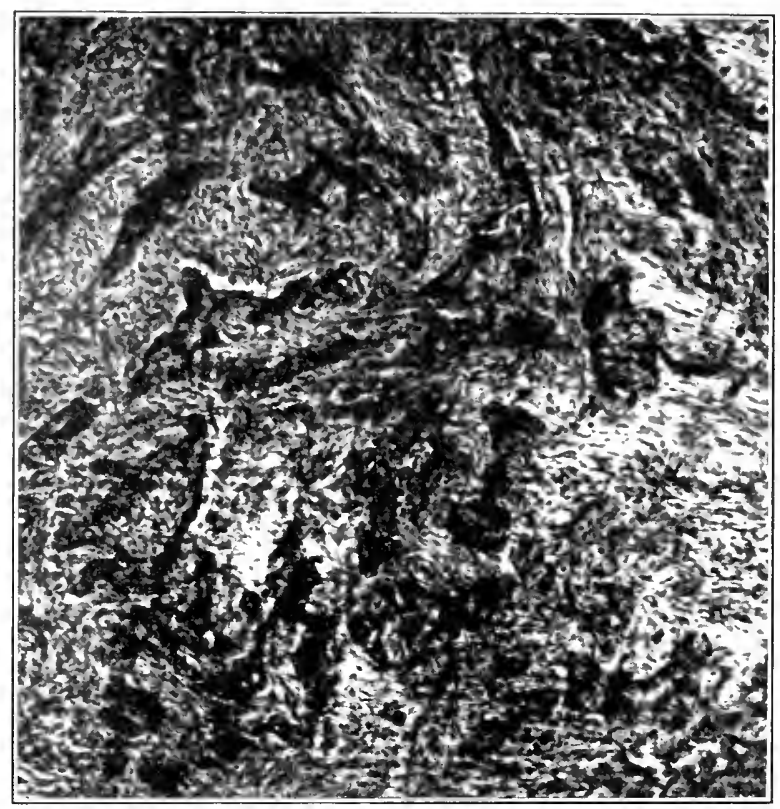

FIG. 32.-(Case No. 16,525).-Photomicrogram showing the bases of the glands clipped off by sear tissue $(\times 100)$. - (Wilson and MacCarty.)

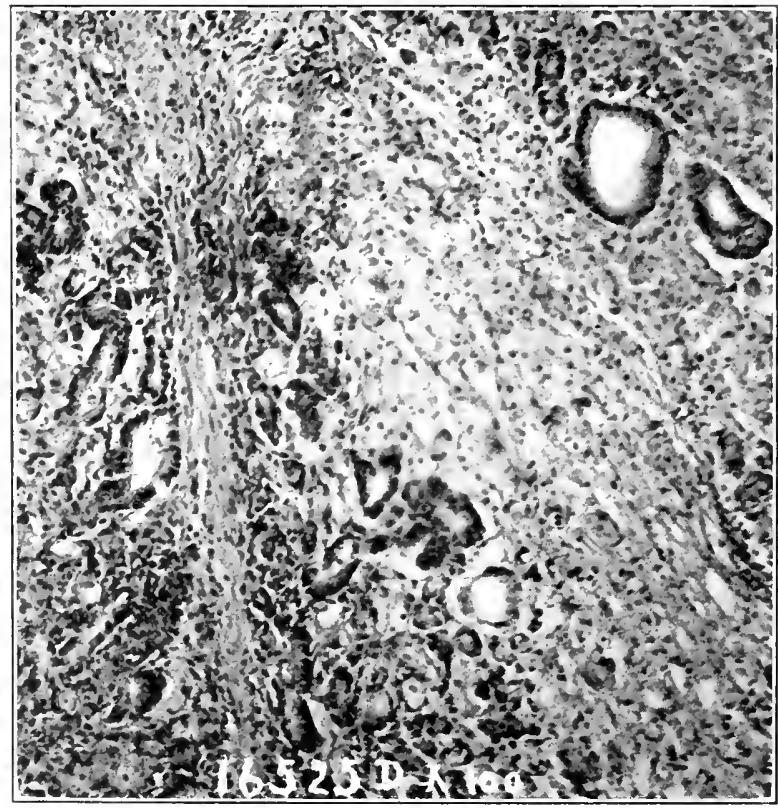

Fig. 33.-(Case No. 16,525),-Photomicrogram showing active malignant proliferation with much round-cell infiltration $(\times 100)$. - (IVilson
and MacCarty.) 


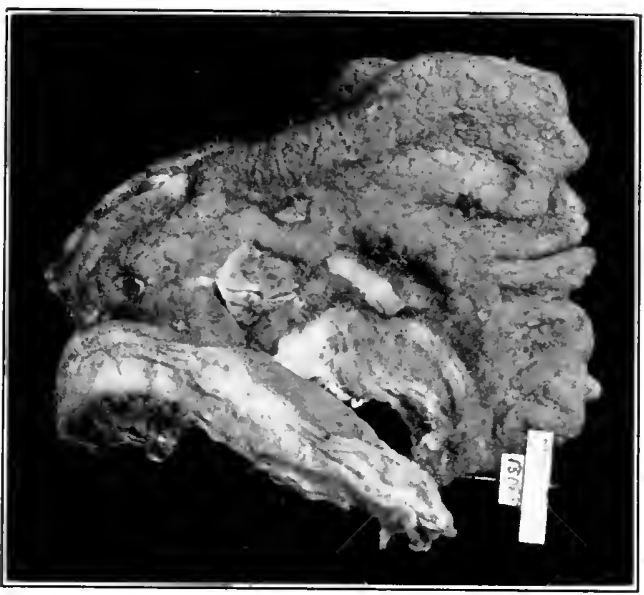

Fig. 34.-(Case No. 1S,0SS).-Stereogram of the pyloric third of the stomach; multiple ulcers of malignant type.--(Wilson and MacCarty.)

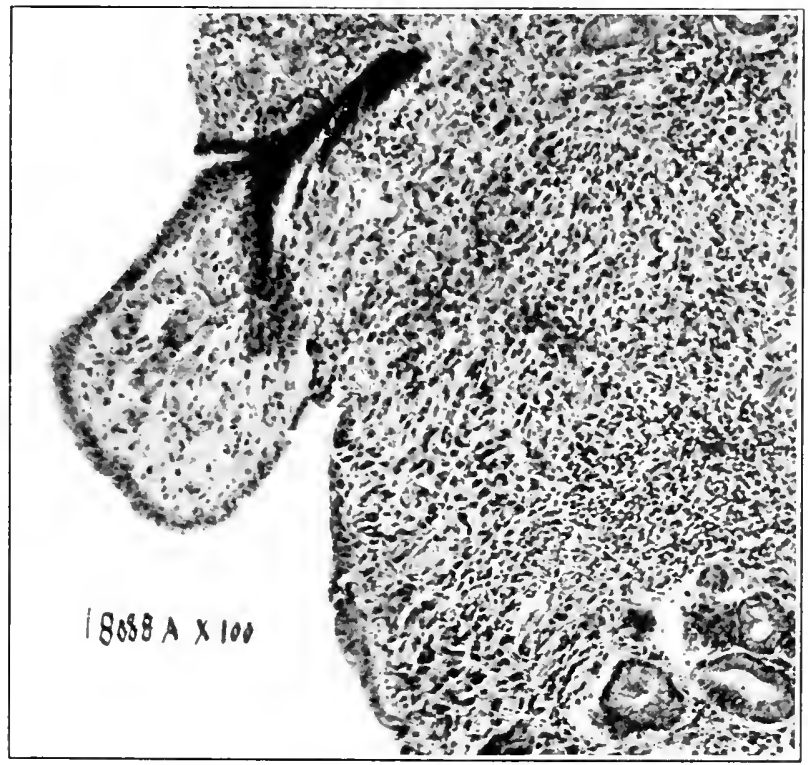

FIG. 35.-(Case No. 18,0SS).-Photomicrograph of the overhanging ulcer border (X 100).-(IVilson and MacCarty.) 


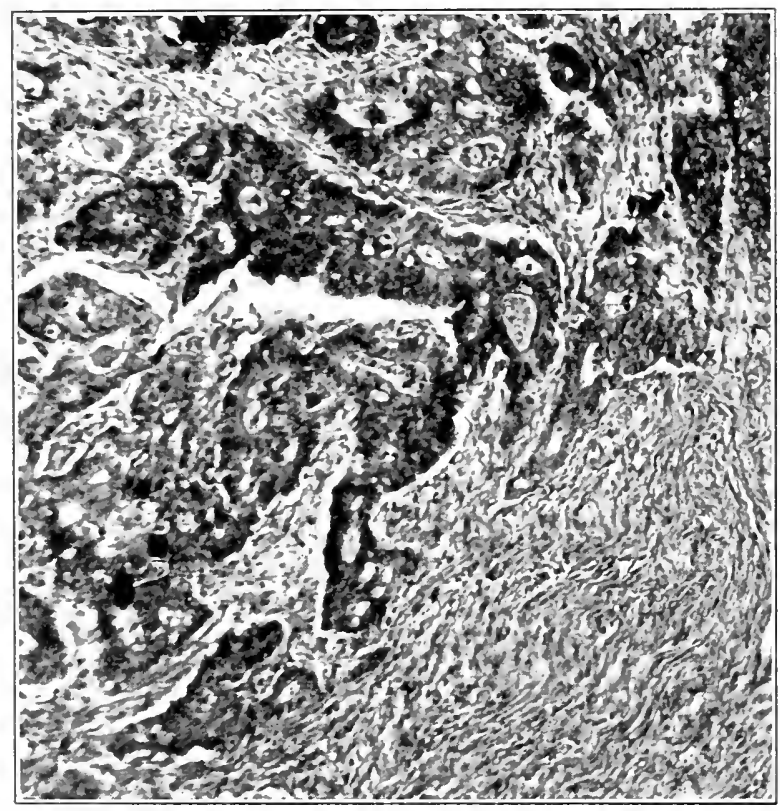

FIG. 36.-(Case No. 18,088).-Photomicrograph from submucosa showing aberrant proliferation and infiltration $(\times 100)$. - (Wilson and MacCarty.) 


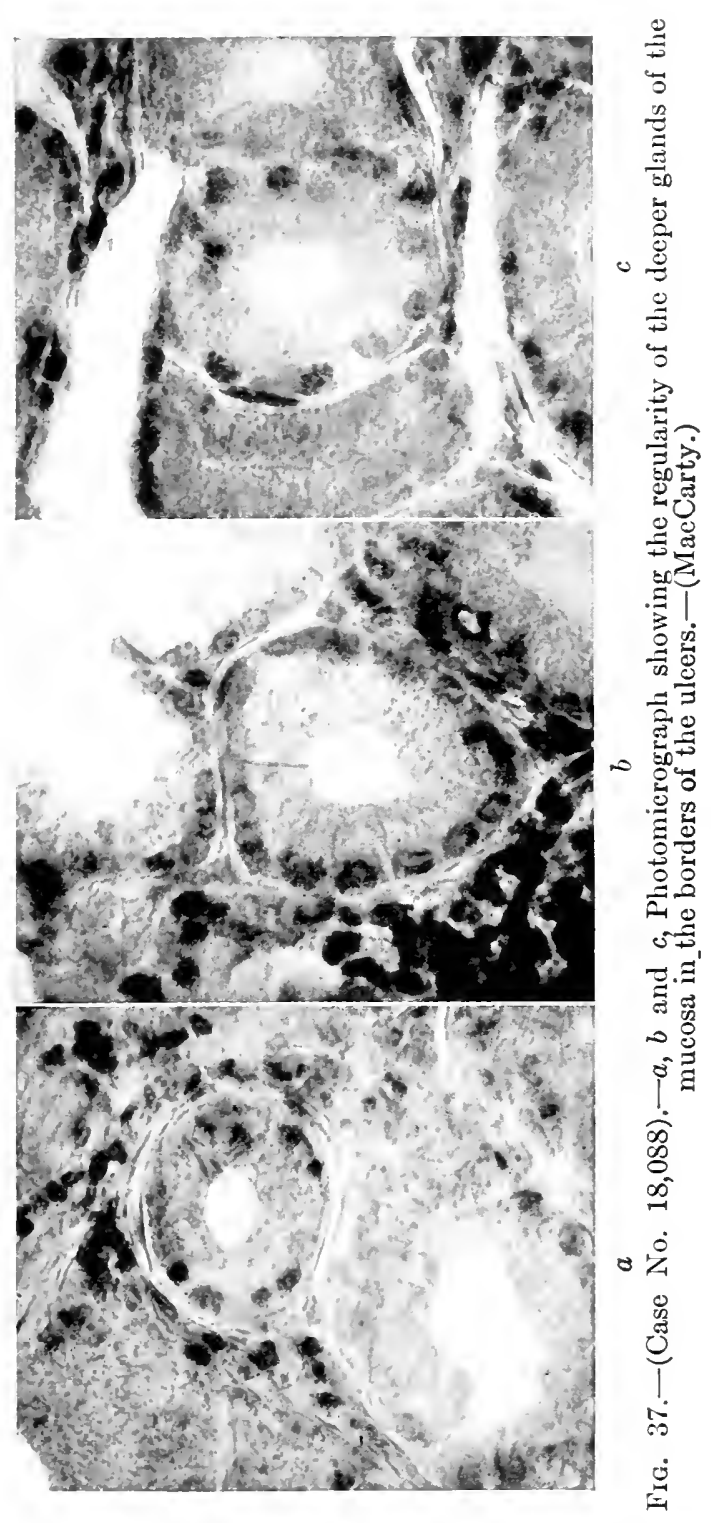




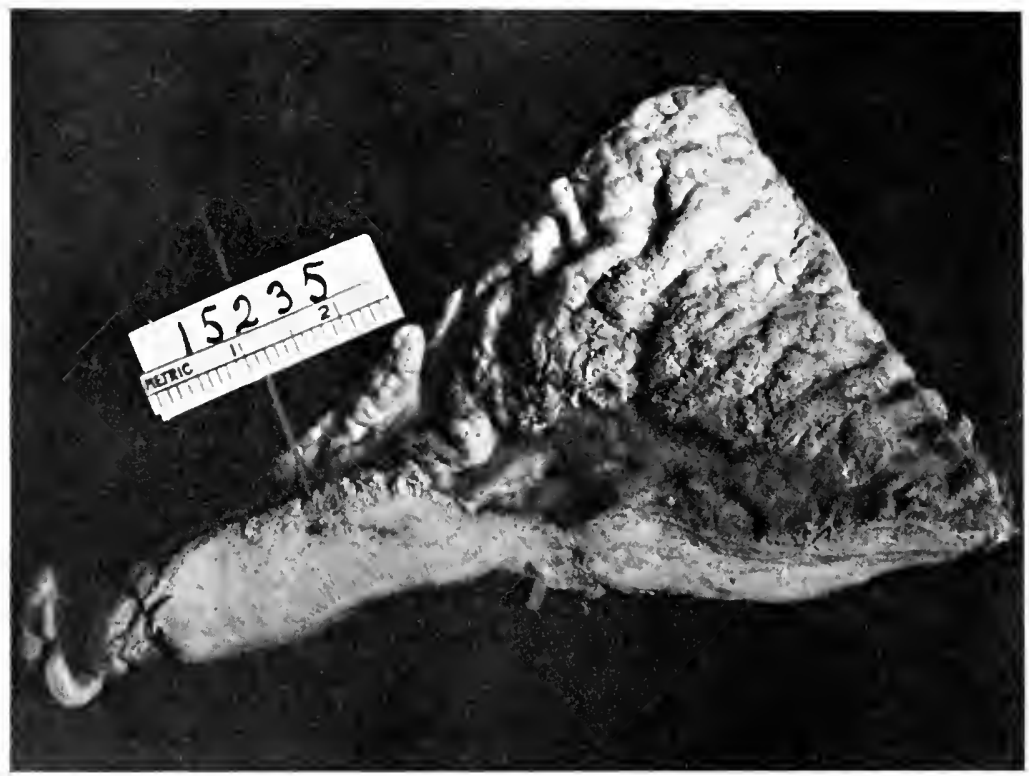

Fig. 38.-(Case No. 15,235).--Uleus carcinomatosum of the antrum. Ulcer has smooth terraced borders and is surrounded by actively proliferating carcinoma of the medullary type. Enormous infiltration and hyperplasia of gastric wall. Patient had precarcinomatous history, elinically that of peptic ulcer. Specimen obtained at laparotomy.-(Courtesy of Mayo Clinic.) 
types described include definite and single histologic entities. It should be emphasized that all gastric cancers have in and about them histologic changes demonstrating attempts at resistance and repair on the part of the invaded tissues. Consequently, it is possible to define scirrhus, medullary, ulcerating, or even colloid areas, locally, in the majority of growths, where the process has existed for a considerable time. This is especially the case when specimens are examined from necropsies. Growths received from laparotomies contain fewer examples of retrograde changes. The latter type furnishes the most satisfactory material upon which to study cancer invasion and from which to offer a classification of the disease from the histologic standpoint.

Various histologic groupings of gastric carcinomata have been advanced. Most of them are artificial, incomplete, confusing and unnecessary.

Inasmuch as carcinoma is an atypical epithelial neoplasm (Waldeyer), and inasmuch as in the stomach, the neoplasm originates from the cylindrical epithelia making up the glands of the mucosa, it is evident that but few elementary factors can enter into the histopathologic picture presented by its development. The facts to be considered are: (a) the abnormal proliferation of gland cells; $(b)$ the rate of their growth; $(c)$ the direction of that growth; $(d)$ the reaction of adjacent tissue to the presence of atypically proliferating and physiologically undifferentiated epithelium; and (e) retrograde changes in either epithelia or connective tissue or both.

\section{THE ABNORMAL PROLIFERATION OF GLAND CELLS}

No one has ever observed the earliest beginnings of any malignant process, histologically, in the human. 
No one has ever experimentally produced a cancer in a human being, hence it is impossible to say just what intracellular change or what primary intracellular fault constitutes the beginnings of malignancy. The large majority of histologic descriptions of malignancy, particularly of gastric cancer, are not those where the disease is in its inception, but are views of a pathologic process well under way. This fact is to be strongly emphasized with respect to those histopathologic reports from necropsies where patients have succumbed to gastric cancer. In such beginning cellular change cannot be described. The cellular battle is largely over. The primary disposition of the warring forces is a histologic secret. Unfortunately such end-results were painstakingly described nearly a half century ago, and have become rooted in the general mind and form much of the pathologic conception of the disease, whereas they only constitute the least interesting phase of it, namely, the end-result. From the viewpoint of cancer prophylaxis, their chief value lies in pointing out the moral-to emphasize the consequences of tumor growth.

It is now generally accepted that early malignant processes are in the nature of hyperplasia of already existing structural elements. Through some intracellular deviation from the normal, a tissue reaction takes place which results in undifferentiated growth of a particular cell group. In the majority of instances the initial structure of the part affected is reproduced, but commonly the specific function of the cells making up that structure is lost. Thus, so far as our knowledge extends, the earliest evidences of gastric malignancy occur in what were previously normal cells lining gland tubules. At this stage of the process, there is as yet very little, if any, recognizable periglandular tissue reaction. 
Just what causes this intracellular fault in the cells involved, we are not at present able to state. It may be reaction to any irritant or group of irritants. These irritants may be chemical, biochemical, mechanical or parasitic. It would appear that in addition to the presence of cellular irritants one must consider the possibilities of an inborn or acquired susceptibility on the part of an individual cell, or a group of cells, to a metabolic upset from such source. Certainly, the same types of traumatic agents, offered to like tissue in different hosts, cause widely varying cell reactions.

Certain experimental evidence tends to support the above general description of the earliest phase of malignancy. At this stage rarely is a microscopic picture presented, nor is it proper to term the early intracellular change a precancerous lesion. There would seem to be abundant proof that not infrequently similar early alterations occur in cell-groups and yet do not progress to malignancy. To Rous and Murphy we are indebted for our microscopic conception of slightly developed neoplasms. Working with a chicken sarcoma implanted in developing chick embryos, they noted that first, the transplant occurred along the line of the tissue which had been injured by the inoculating needle. After 16 days this growth was well defined and had the appearance of scar-tissue. Vascularization took place in 4 days and shortly later than when the appearance of secondary nodules had occurred along the line of the vessels. Microscopically, very actively proliferating cells were seen enclosed in a loose mesh-work of supporting tissue. The early sarcomatous cells appeared larger than those of normal connective-tissue elements. That there seemed to be a definite intracellular difference was demonstrated by the fact that the cytoplasm stained 
much more deeply with methylene blue than did that in normal cells. Nuclei were large, oval or elongated or resicular. They contained a fine chromatin network and had a well-defined, deeply staining nucleolus. Proliferation was extremely active, as many as 69 per cent. of the cells being noted in various stages of division at one time. The growth took place mainly by amitosis. In the surrounding tissue the first influences of growth were those occurring as result of simple pressure. The neoplasm developed along the line of least resistance and metastases took place through the lymph spaces. Very early there developed about the growing tumor fragment a moderate amount of edema and exudation. Polymorphonuclear cells appeared coincidentally. These were soon replaced by a small round-cell lymphocyte infiltration which usually became most marked where the invading cells were in actual contact with the cells of the host. This was followed by the appearance of fibroblasts, macrophages and newly formed capillaries at these points. A few giant cells often appeared. Sometimes the vascularization was tardy and before it occurred the tumor cells had invaded the tissue of the host at the points of contact with it. The growth proved to be most rapid along the normal blood-vessels and the connective tissue already present. At the advancing margin there was not the tissue reaction as is seen in inflammation. Lymphocytes were few and often practically absent. Of course, these investigators were dealing with embryos and in them few protective bodies had as yet been developed. When this histologic picture was presented the transplanted growth might be considered well established. The resultant great tumor was made up of accumulations of cells similar to the few which originated the process. Vascularization and progressive 
changes resulted. If, however, the host was not susceptible or there were some factors in resistance which are not as yet understood, retrograde changes occurred and the initial tumor soon disappeared.

Working with an adenocarcinoma of the white rat described by Flexner and Jobling, Levin claims to have produced certain local tissue reactions in non-susceptible animals which, later on, permitted the transplantation of a neoplasm. In other words, by chemical or mechanical means, or both, he claims to have so altered the inherent cellular resistance of tissue as to bring about a so-called "precancerous" state which later on permitted the growth of an implanted tumor. Levin injected into the testicle of a rabbit 2 or 3 minims of Scharlach Roil. These injections produced a local tissue reaction simulating simple inflammatory hyperemia. There may have been some other local fault. When placed into this inflamed and hyerplastic tissue, tumors developed in a considerable proportion of instances. That the injection of the irritant does not itself produce anything more than a granulomatous tumor has been shown by the experiments of Snow.

Recently, Erwin Smith has shown that if plant cells are submitted to a local irritant, cancer-like growths result. Working with a bacillus isolated from galls of a daisy, he has succeeded in producing at will similar tumors in such plants. The bacillus is known as Bacterium tumefaciens. If healthy plants are inoculated with this organism, rapidly developing growths result which erentually destroy the plant. Other plants are also susceptible. Certain metastasis-like secondary growth develop from the primary tumor. While many of the tumors described by Smith are of the granulomatous type, it would seem that 
his experiments are not without value as showing in what manner normal tissue reacts to a constantly multiplying irritant. The reaction is essentially one of hyperplasia of certain cell groups. Not infrequently it is impossible to state where benign hyperplasia ends and malignant hyperplasia begins.

With regard to the experimental production of gastric cancer in animals, few facts are available. The most striking investigations are those of Fibiger of Copenhagen. This investigator noted that not infrequently certain laboratory rats died as the result of cancer-like tumors developing in their stomachs. On investigating some of these tumors, he discovered that while they were histologically carcinoma-like many of them contained cysts in which were lodged nematodes. Further investigations disclosed that these rats fed upon roaches which infested a certain sugar refinery from which the majority of the laboratory rats were obtained. These roaches on examination were found to be infested with nematodes. Fibiger then collected a large number of these roaches and fed them to non-infected laboratory rats. In the stomachs of many of these, carcinoma-like growths developed. While there is some doubt of the true carcinomatous nature of Fibiger's rat tumors, the investigation is of value in showing the reaction of gastric mucosa to a parasitic irritant. It also shows the histologic difficulty of differentiating hyperplasia of a granulomatous type from the hyperplasia of true malignancy. It is quite conceivable that the ingestion of such food as roaches acted as traumata to the gastric lining, and furnished a locus minoris resistentia, at which point the nematode could act mechanically, could bring about hyperplasia as result of undetermined infection of 
this parasite itself, or as a consequence of a chemical excretion, secretion or ferment.

To the painstaking and brilliant work of MacCarty, we owe practically all our useful knowledge regarding the early histologic changes which are associated with the development of gastric cancer. This investigator has patiently studied the microscopic deviations from the normal of gastric ulcers and gastric cancers obtained at laparotomy. His observations are of particular worth, because they cover an experience extending over many years in handling the largest number of specimens ever recorded; are upon fresh, and not dead-house, material; include both gross and microscopic studies (the latter with oil immersion amplification); have been carried on in a uniform manner and checked by comparative photographs and the clinical courses of the affected patients. It is to be expected that MacCarty's results are as yet not generally appreciated. They are too highly specialized to come within the understanding of the pathologist with ordinary material for study because they are based upon a vast material and comprise a histopathology essentially cellular. They bear a similar relation to routine pathologic investigations that such maintain toward hand glass or gross pathology. It is significant that the intracellular deviations and the intercellular faults described by MacCarty are generally comparable to such recognized by research workers engaged in tumor implantation (Rous, Murphy, Gay) or cell culture studies (Carrel).

MacCarty has shown that in gastric ulcer two basic histologic processes are in evidence:

(a) Hyperplasia of the elements of the gastric mucosa and (b) hyperplasia of adjacent tissue; (2) retrograde changes in both locations. 
1. Hyperplasia.- - (a) This occurs in the gland structures of the ulcer edges or those lying superficial or deep in the submucosa.

Such exuberant growth manifests itself by both cellular and intracellular change. The cellular change consists in multiplication of cylindrical cells in the stratum germinativum. The cells assume regetative qualities rather than specific function. At first the overgrowth is confined to

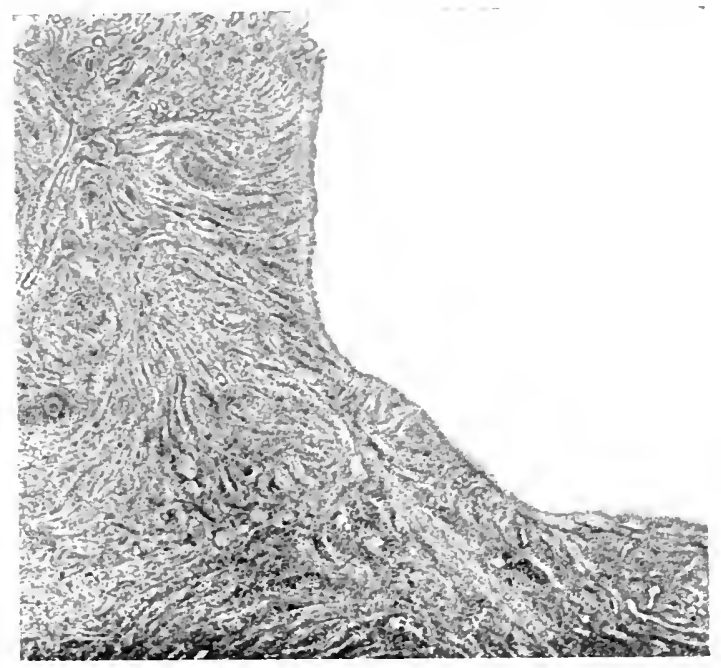

Fig. 39.-(Case No. 27,919). - Section through a portion of the border of a benign chronic peptic ulcer- - (IIacCarty.)

the primary cell layer. The indiridual cells increase in number, swell and often stain abnormally with methylene blue or hematoxylin and eosin. The process may stop here. If not, the next step consists of such definite hyperplasia as to result in the appearance of an inner, secondary layer of cells arising from rapidly multiplying cells of the basal layer. These, in turn, may be succeeded by such increase in cells as to completely fill the space formerly held as a gland lumen. Variations in cell shape and size 
appear, perhaps, as a consequence of some altered, intercellular relation (Fig. 37). Thus far the process is adenomatoid.

Depending upon the directions in which histologic sections are cut, and their location with respect to. mucosa and subnucosa, isolated areas ("cell inchusions," often so-called) or filled gland-tubules may be described. Thus far there has been no alteration in the relation of the basal layer of cells to the surrounding structures. The hyper-

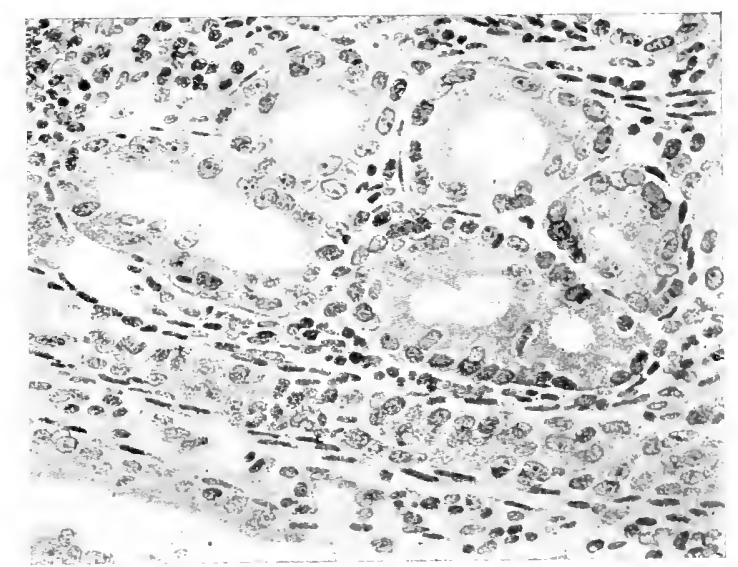

Frg. 40.-(Case No. 30,040).-Photomicrogram showing hyperplasia in the mucosa at the border of a peptic ulcer.-(MacCarty.)

plastic gland elements are well delimited. After the second gland-cell layer has developed the study of intracellular change reveals important variations from the normal. They must be looked for with oil-immersion objective. The individual cells vary much in size and form, while the basal layer remains cylindrical, cuboid or flattened. The secondarily developing layers may be oblong, oval, rhomboid, rounded or irregular. The whole gland is often distended and distorted. At this time distinct nuclear changes are visible (Figs. 21, 42 et seq.). Nuclear hy- 
pertrophy occurs. This is associated with structural alterations in the nuclei themselves. Their increase in size is noticeable. Whereas, in normal gland cells, the relative proportion between nucleus and cytoplasm is approximately as $1: 3$ or $1: 4$, increase in the nucleus occurs to such extent that practically it may equal in volume the cytoplasm. A striking phenomenon is that of great variation in size, shape and staining reaction of these nuclei. In place of dense nuclei

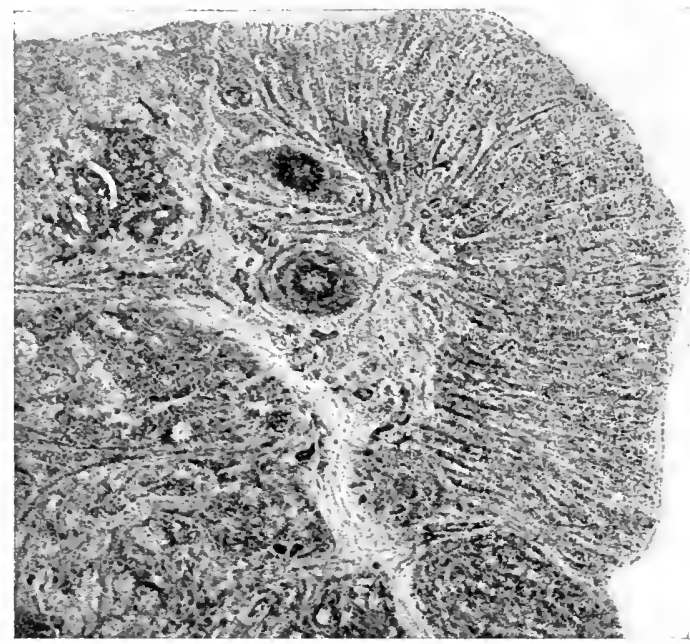

FIG. 41.-(Case No. 27,919).-Photomicrogram of a portion of the border of a malignant peptic ulcer showing islands of carcinoma in the submucosa.-(MacCarty.)

staining deeply with Unna's polychrome blue or with hematoxylin, the nuclei stain much less densely and are poorly differentiated structurally. If the biochemical alterations associated with the gland hyperplasia are maintained, the nuclei divide so rapidly as to render mitotic figures visible. At any rate, proliferation appears accelerated. At this phase, the mass of hyperplastic cells may readily simulate adenoma. The most marked change occurs at the ulcer borders. 
It would appear that the line of demarcation between simple hyperplasia and a malignant type of the same process is very indefinite at this stage. If the stimulation to the outgrowth is not stopped, however, the succeeding histologic picture often reveals alterations in the basal layer of gland-cells. At some point, or generally, they may wander through the "basement membrane." This is the first actual evidence, histologically, of what seemed to be a simple, progressive hyperplasia assuming malignant characteristics. Such occurrence is most common where

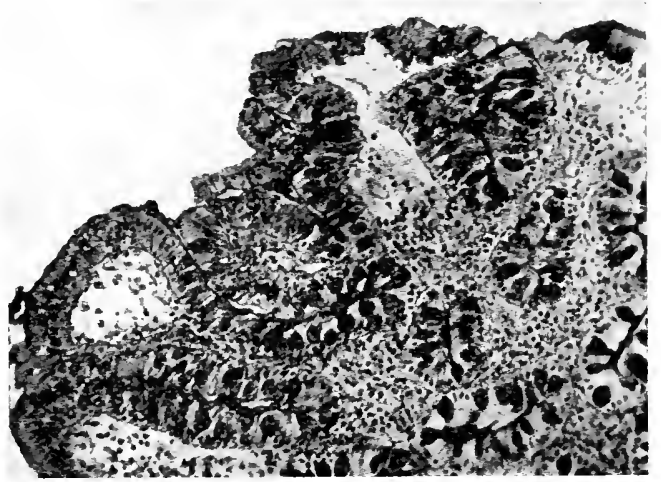

FIG. 42.-(Case No. 97,051).-Densely staining mucin within columnar cells. High amplification.-(WVilson.)

hyperplasia is most active, i.e., at the edges of ulcers: the points where attempts at resistance and repair are being most persistently carried on. The phenomena occur less strikingly (and often later) in the scar-tissue forming the base of the ulcer-crater. Depending on the extent of the ulcerated area, the severity of the agent tending to destroy tissue and the ability of such tissue to oppose this, small or large areas showing these alterations may be described in the mucosa and submucosa. The discovery of a single gland tubule, where actively hyperplastic cells have penetrated beyond the normal limiting membrane, is, however, 
all that is required for a diagnosis of malignancy. Previous to this malignancy is questionable. The subsequent fate of such atypically growing cells depends upon their number, their relative position in the gastric wall, their stimulation to growth, the rapidity of their proliferation, and the degree of success which surrounding tissues have in repelling their invasion. There may be variation in any or all of these factors. Consequently, the above-described process may be halted at any stage of its advance. The mass of malignant hyperplastic epithelial elements, together with

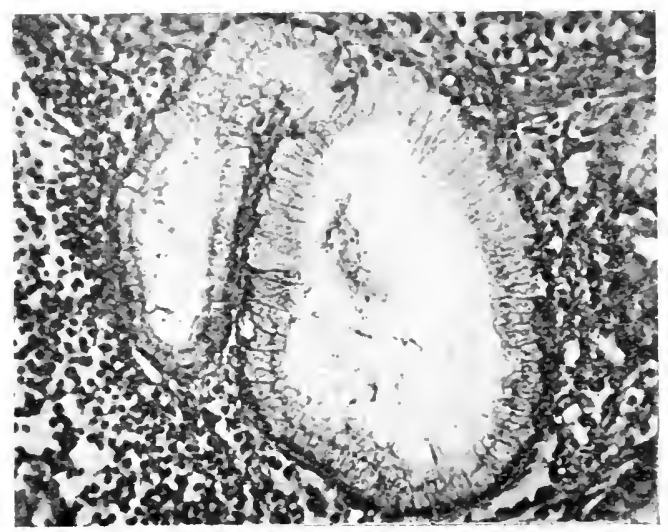

FIg. 43.-(Case No. 94,647).-Photomicrogram (high power amplification) of section across a cyst of a pyloric gland in early gastric cancer. Columnar cells filled with mucin; crescentic nuclei.—(Wilson.)

surrounding stroma, makes up the neoplasm. Wide variation in size is possible. The relation existing between volume of epithelial cells and connective tissue determines the clinical type of tumor resulting. It furnishes the basis of a rough histologic grouping.

(b) Hyperplasia of the extraglandular structures constitutes eridences of tissue resistance. It is grossly a local index of the strength of the stomach's defensive forces. These may be rigorous during the early stages of the ulcerative process and then apparently become negligible, 
or, late in the derelopment of a rapidly advancing ulcer, energetic resistance may be put forth and the process stopped.

As we have stated, in the early stages of simple ulcer, hyperplasia is most active in the glands of the edges. This increase causes overhanging borders. There is frequently a similar hyperplasia in the base. Tissue resistance is manifested by an abundant infiltration of small lymphocytes. Not infrequently polynuclear leucocytes are also present. The hyperplastic gland process is thus well walled

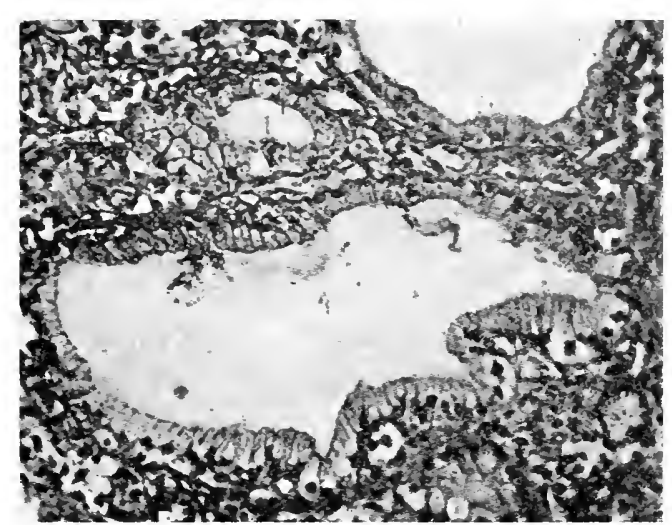

FIg. 44.-(Case No. 94,647).--Same tissue as in Fig. 43, showing cells freely discharging mucin; spherical nuclei.-(Wilson.)

off. There is little evidence of vascularization. Surface necrosis often keeps pace with the epithelial hyperplasiathe sloughing thereby giving the ulcer a characteristic crater-like form. In instances of moderate activity, the gland hyperplasia is checked apparently by the leucocyte invasion. Hyperplasia of scar tissue then occurs, and typical, protected, callous ulcer results. If, however, a source of stimulation keeps the epithelial hyperplasia active, and if the process becomes moderately extensive, the hypertrophied gland tubules push aside the con- 
nective-tissue cells and diminish the number of avenues along which protective cells or fluids may be conveyed to

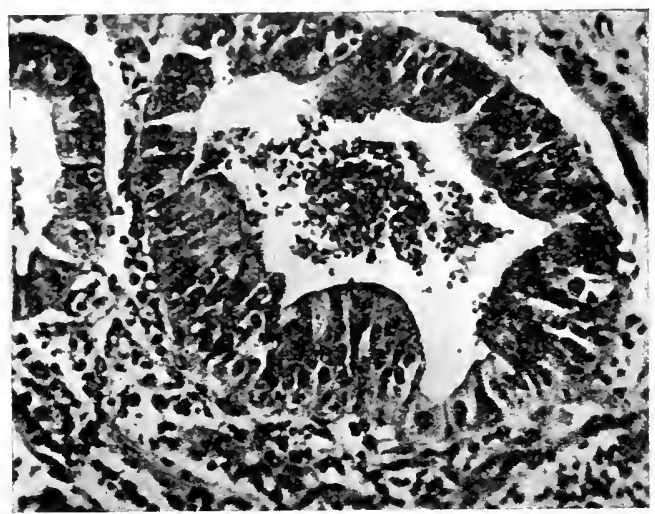

Fig. 45.-(Case 53,784).-Hyperplasia of the epithelial gland elements with marked piling up of epithelium. High amplification.-(Wilson.)

the pathologic area. There is, however, always evidence of an active resistance being carried on at the limits of the hyperplastic epithelial process. As a consequence,

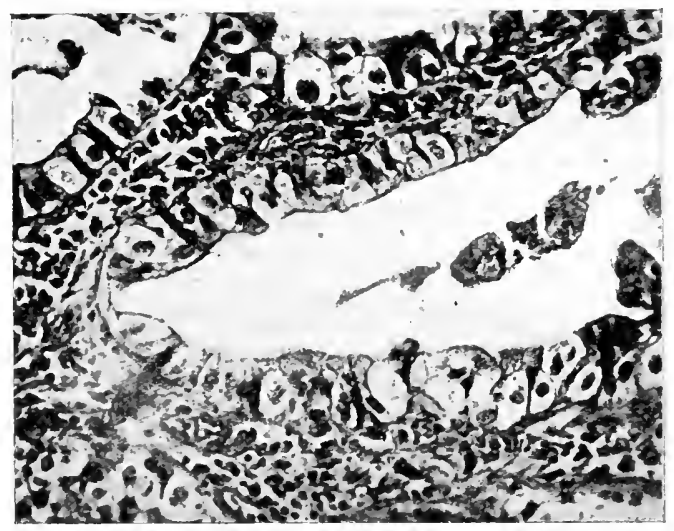

Fig. 46.-(Case No. 80,863).-Photomicrograph showing beginning multiplication of layers of gland epithelium. High amplification.(Wilson.)

not only may submucosa show hyperplastic connectivetissue changes, but such may be made out in the muscularis 
and subserosa. The tendency is everywhere toward the production of dense, undifferentiated scar tissue. Deepening of the ulcer crater by surface sloughing together with extension of rapidly enlarging gland tubules may accelerate the process or increase its extent.

The above attempt to resist invasion appears to be carried on by connective tissue concomitantly with epithelial increase until direct intrusion of the epithelial tissue occurs. After that essential, histologic, structural fault, some yet unknown influence seems to diminish the strength of the connective-tissue resistance. This is shown, histologically, by diminution of lymphocytes and leucocytes, by tendency to vascularization, more active growth of atypically located gland cells and by retrograde changes of tissue. These are frequently manifested early. When once this balance between tendency of gland cells to exuberant growth and inherent property of surrounding tissue to prevent such has been upset, the tide of cancer cells flows forward seemingly opposed very weakly.

2. Retrograde Changes.-In ulcerating gastric mucosa progressive and retrograde changes are constantly interacting. While hyperplasia is yet simple, the surface epithelium undergoes cloudy swelling, granulation, mucoid degeneration and liquefaction necrosis. Microscopic or gross sloughs result. These changes form a part of the attempt at repair which is being carried on, and of which the process of hyperplasia of gland and connective-tissue elements is a part.

If the agent exciting epithelial elements to attempt normal repair is not removed, and the false metaplasia which results in tumor formation ensues, retrograde changes are manifested in the neoplasm itself. They are most actively carried on toward the lumen of the stomach, but 
may be exhibited at any part of its altered wall. These retrograde changes are resisted by the cancer cells just as normal epithelium resisted them. The altered physiologic units, however, not infrequently fail to prolong such resistance. As a consequence, the cells at the surface of the neoplasm may rapidly undergo granulation, mucoid or colloid change and necrosis. The free vascularization of the more cellular types of growth permits extensive blood extravasation, cyst formation and frequently extensive sloughing. The rapidity with which cell proliferation, necrosis and sloughing occur and cause the death of the individual rarely permit the retrograde changes going beyond the colloid stage. Occasionally, small calcified areas are seen in slow-growing tumors located deep in the submucosa.

These progressive and retrograde tumor changes and the reaction of surrounding tissues have much to do with the gross characteristics of gastric tumors. Their evidences are largely responsible for the common clinical classification of such neoplasms into carcinoma medullare, carcinoma fibrosum, ulcus carcinomatosum, and carcinoma colloides. They also influence the histologic picture, so that we not infrequently see such groupings of the disease as spheroidal-celled carcinoma, cylindrical-celled carcinoma (adenocarcinoma) and mucous-celled (colloid) carcinoma. From the preceding attempt to explain the early changes constituting gastric cancer, it will be seen that any such classification is incomplete and misleading. So-called "types" of carcinoma occur entirely because cancer cells grow differently in relation to connective tissue and they are influenced by both progressive and retrograde changes in themselves and in surrounding tissue. These neoplasms are derived from gastric gland epithelium and 
are thus essentially of the same parentage. At some stage in the development of any gastric cancer, it is quite likely that practically all so-called "types" of cells could have been recognized and that degenerative processes could have been seen. The confused histologic and gross classifications that have been presented are accounted for largely because they have been based upon examination of specimens removed from patients who have died from the disease. In comparison we would mention that a full-grown flower rarely resembles its bud nor yet does the bud recall the seed which gave both, as well as the rine, their birth.

\section{THE RATE OF GROWTH OF GASTRIC NEOPLASMS}

That neoplasms in general may develop with astonishing rapidity is experimentally shown by the work of Rous. Fifteen days after transplanting minute fragments of chicken sarcoma into chick embryos, visible and tangible tumors were present. Histologically 69 per cent. of the tumor cells were shown to be dividing by amitosis.

In human gastric cancer, the rate of growth varies widely. Those tumors confining themselves to the mucous membrane and the loose submucosa grow with the greatest speed. Such rapid advance occurs most commonly in so-called "primary" gastric cancer (without antecedent ulcer, benign or malignant). In such location connectivetissue barriers are weak and few. Clinically, this type of tumor may cause death of the host, in our experience, within 7 weeks of the onset of symptoms. We have seen epigastric tumor increase in size threefold in rather more than a month. Tumors derived from gland-cell groups located deep in the submucosa and progressing in it or toward the muscularis increase in size with comparative slow- 
ness. We have observed instances where epigastric nodules have existed in epigastria for more than a year and symptoms for as long as 3 years, and yet at laparotomy tumors have weighed less than 60 grams. Epithelial elements in such are generally well split up with scar tissue. Not infrequently, it would seem that atypically growing epithelia exist in the edges or bases of ulcers for many months without progressing far. Sloughs may remove many such and be a prophylactic measure. These hosts often have ulcer symptoms for more than 5 years, but refuse surgical relief until stenoses or perforation occur. Examination of fresh tissue obtained at laparotomy reveals numerous areas of malignancy, frequently with extensive perigastric lymph-gland invasion or secondaries in adjacent or distant organs. Colloid cancers appear to spread rapidly because they are very rich in epithelial elements. That the age of the individual is a factor of relative value in the determination of rate of growth of gastric cancer seems to be well supported by clinical observation. We have observed 18 instances of the disease in patients below age 31 . The average duration of life of 12 of these, after symptoms had appeared, was less than 9 months. Of our gastric carcinomata over age 50, the average length of life after the beginning of the complaint was rather longer than $1 \frac{1}{2}$ years. In experimental animals where tumors have been transplanted a similar factor influencing "takes" and growth rate has been emphasized by Bashford.

The state of body nutrition appears to have an influence upon the rate of progress of gastric cancer. It has been our experience that such neoplasm developing in wellfed, robust patients of the erythremic type advanced rapidly not only locally, but also by metastasis. Lean, 
spare, sallow folk appear to tolerate gastric cancer relatively well. Tumors grafted experimentally upon underfed hosts would seem to grow more slowly than when hosts were supplied with an abundance of food. Such observations have been made by Moreschi and by Rous for mice and rats.

The location of gastric cancer in the wall of the viscus only relatively influences growth rate. At the orifices where traumata are possible during chymification and passage of food, neoplasms in general grow very rapidly. At such points (especially in the pyloric third of the stomach) peristaltic movements are frequent and vigorous and digestive secretions have their highest potency. However, variation in epithelial cell content of the tumor mass would seem to be a not negligible factor in the rate of the development of the disease even at these points.

Location of Gastric Neoplasms. - It is a waste of time to speculate upon the location of gastric cancers unless one is doing so for the purpose of determining the correctness of clinical signs or of symptoms in cases upon which laparotomy or necropsy is to be subsequently performed. That those most proficient in the diagnostic art not uncommonly fail in attempts at localization of the neoplasm can be demonstrated by a sojourn at any busy surgical clinic or in a modern morgue. Not rarely cases clinically diagnosed gastric cancer prove to be primary affections of adjacent viscera. Consequently, the only tables of worth regarding the situation of gastric carcinomata are those determined from post-mortem examinations or at laparotomy, and those where the growths have been grossly and microscopically proven to be cancer. From dead-house material it is impossible to say at what part of the gastric wall cancer began. One can only note what portions of the stomach are then involved. 
Such figures are of but relative value. That they do not correspond with location of neoplasms removed at laparotomy is seen by comparing groups of collected statistics. The statistics for situation of gastric cancer commonly quoted in literature (even recent) follow.

In $1859 \mathrm{~W}$. Brinton analyzed 360 clinical cases and necropsies. He stated that in these the pylorus was involved in 60 per cent. and the cardia in 10 per cent. In 1871 Luton summarized the cases of Lebert, Dittrich and Louis. His study comprised 102 instances of the disease. The situations were given thus: pylorus 57.8 per cent.; lesser curvature 16.6 per cent.; cardia 7.8 per cent.; anterior or posterior wall 4.8 per cent.; greater curvature 1.9 per cent.; general 0.9 per cent. and in various parts 6.8 per cent. Welch later collected 1,300 cases of gastric cancer mainly from the continental literature. His tabulation is compiled largely from necropsy reports. He states that in 60.8 per cent. the pylorus was involved; in 11.3 per cent. the lesser curvature; in 8 per cent. the cardia; in 5.2 per cent. the posterior wall; in 4.6 per cent. the whole or greater part of the stomach; in 2.6 per cent. the greater curvature; in 2.3 per cent. the anterior wall and in 1.4 per cent. the fundus. In 3.4 per cent. there were multiple tumors. In 1903 the Fenwicks analyzed 263 of their own cases. They state that the pylorus was involved in 65.3 per cent. These investigators also submit an analysis of 1,850 instances of the disease collected from necropsies (?) in various countries. Of these, the pylorus was affected in 58 per cent.; the lesser curvature in 11.5 per cent.; the cardia in 9.8 per cent.; the posterior wall in 5 per cent.; the greater curvature in 2.8 per cent.; the anterior wall in 2.2 per cent.; the fundus in 1.5 per cent.; general involvement in 6 per cent. In 2.9 per cent. there were multiple growths. 
The above summaries demonstrate that necropsy reports exhibit relatively slight variations in the parts of the stomach involved by neoplasms that have, in the main, caused death of those affected.

In our series of 921 gastric cancers, operatively and pathologically proved (but an occasional case was studied at necropsy), there were 854 instances where accurate localization of the disease could be determined or was recorded. The summary is shown in Table 13 .

Table 13

\begin{tabular}{|c|c|c|c|c|c|c|c|c|c|}
\hline Situation & $\frac{0}{3}$ & 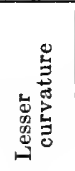 & 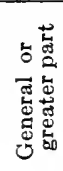 & 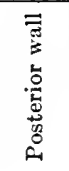 & 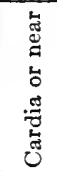 & 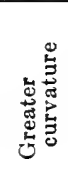 & 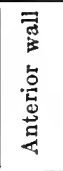 & 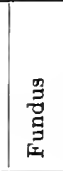 & 题题 \\
\hline No. of cases. & 359 & 201 & 102 & 79 & 30 & 20 & 20 & 8 & 26 \\
\hline Percentages. . & 42 & 24.7 & 12 & 9.3 & 3.5 & 2.3 & 2.3 & 0.9 & 2.9 \\
\hline
\end{tabular}

Analysis of Author's 854 Cases of Carcinoma of the Stomach Showing the Frequency of the Neoplasm in Various Parts of the Viscus.

These figures are to be contrasted with those derived from study of post-mortem or unproved clinical material. The following table demonstrates that the situation of gastric cancer as shown at laparotomy corresponds rather strikingly to the location of chronic, calloused gastric ulcer.

Table 14

\begin{tabular}{|c|c|c|}
\hline & Number of cases & Per cent. \\
\hline Pyloric $\ldots \ldots \ldots \ldots \ldots \ldots \ldots \ldots \ldots \ldots$ & 65 & 46.4 \\
\hline Prepyloric.$\ldots \ldots \ldots \ldots \ldots \ldots \ldots \ldots \ldots$ & 5 & 3.5 \\
\hline Lesser curvature ................... & 43 & 30.7 \\
\hline Near cardia .................. & 9 & 6.4 \\
\hline Posterior wall...... & 12 & 8.5 \\
\hline Anterior wall... & 2 & 1.4 \\
\hline 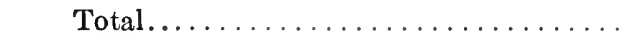 & 136 & \\
\hline
\end{tabular}

Showing Location of Benign, Non-retention Gastric Ulcers (Author). 
Welch's figures for location of gastric ulcer also closely approximate those of the author for benign ulcer and for gastric cancer as here given.

\section{COMPLICATIONS OF GASTRIC CANCER (General)}

In our series many of the text-book complications of carcinoma of the stomach are missing, because a large number of instances were operated upon early. As a consequence numerous terminal complications of the disease were lacking.

A. Pyloric Obstruction.-This occurred in some grade in nearly 72 per cent. of instances. When it is recalled that fully 3 out of 5 of all ulcers and cancers are located in the pyloric third of the stomach, it is quite easy to realize the reason for this frequency of stenosis. When the pyloric obstruction was caused by ulcer in whose edge was demonstrated malignant tissue microscopically, or when the gastric affection was ulcus carcinomatosum, marked dilatation of the stomach was an apparently early manifestation of this obstruction. Where the pylorus was intruded upon by a nodular growth, dilatation of the stomach was rarely so marked as in the case of ulcer. The average amount of gastric extract removed from the fasting stomach of instances of pyloric obstruction due to malignant ulcer was $350 \mathrm{cc}$. The average amount from cases where a large tumor occluded the pyloric channel was $200 \mathrm{cc}$. Of course there were great variations from the figures in both illustrations.

Pyloric obstruction is recognized readily by persistent vomiting of retained food, of copious vomitus and can usually be demonstrated at the Roentgen examination.

B. Obstruction at Cardia.-This occurred in 17 instances or (1.8). It usually resulted from malignant ulcer at or 
near the cardia, tumor situated at the fundus or high on the lesser curvatire or from the pressure of gland metastases about the cardia. It is recognized clinically by dysphagia, vomiting soon after food ingestion, rapid emaciation, scanty urine, obstruction to the passage of a stomach tube with or without free bleeding and by $x$-ray examination.

C. Malignant Hour-glass.-This is a frequent complication in instances where a "saddle ulcer" has become malignant or where large tumor masses project into the gastric lumen. While it may be suspected from the clinical history, the vomiting soon after eating, the nature of the abdominal tumor, the appearance of the stomach upon inflation with air and the auscultation of the abdomen, it is most conclusively demonstrated at the Roentgen examination. In our cases malignant hour-glass occurred in some degree, approximately in 1 out of 15 cases.

D. Hemorrhage is a sign in from 15 to 25 per cent. of all cases. Hemorrhage occurred in more than 19 per cent. of our cases irrespective of the type of the lesion. In the instance where malignancy has supervened upon a previous gastric affection, usually of the type clinically classed as peptic ulcer, hemorrhage may be copious and associated with symptoms of shock. When malignancy has become well established, sudden, severe, copious hemorrhages are not the rule. There is in the large majority of instances, however, constant seepage. This may be recognized by chemical tests for altered blood in the gastric extracts and the feces. Such tests were positive in nearly 9 out of 10 of our cases of gastric cancer.

E. Perforation.-In advanced cancer of the stomach perforation occurs in from 2.5 per cent. to 6 per cent. of all instances. It may be sudden or gradual. Sudden perforation is as a rule readily recognized, if there is a history 
of a malignant gastric affection rather chronic. This is associated with epigastric tumor in more than 3 out of 4 instances. Pain has generally been not so severe as to be classed as "colicky." After vomiting, a fall, a heavy meal or some sudden exertion, sudden sharp lancinating abdominal pain may come on. This is associated with shock, subnormal temperature, abdominal distention and great prostration. Temperature generally rises fairly rapidly and terminal evidences of septic peritonitis become apparent.

Gradual perforation of a cancer of the stomach sometimes occurs. There may be some attacks of colicky pain but the leakage from the stomach is so slight that opportunity for walling it off occurs. As a consequence, perigastric abscess or localized abdominal abscess between the stomach and other viscera develop. The following case illustrates these features:

Perforating Gastric Cancer; Perigastric Abscess; Few symptoms:

Mrs. L. N.-Age 54, Swedish, housekeeper.

Family History.-Mother died from carcinoma mamma.

Personal History.-Always well and strong to about 1 year ago; then began to lose weight gradually, but without symptoms. Two months since unaccountable anorexia developed. One month ago began to belch much gas following food intake. Two weeks since, had a sudden sharp pain in the left loin. It came on at night and distress lasted 3 days. It then practically disappeared. A few days ago noted a tender swelling along the left rib edge. This increased in size and gradually advanced across the epigastrium to the mid-line. It became much larger and very tender. Thinks she had fever; had chilly sensations daily and sweated rather freely.

Appetite.-Capricious. Never vomited.

Bowels.-Costive.

Urine.-Negative. 
Weight.-One year ago, 180; three months ago, 170; present, 154.

Examination.-Temp., 101; pulse, 100; resp., 24. Cheerful, comfortable appearing woman; mucosæ pale, but cheeks pink.

Throat and Neck.-Negative.

Thorax.-Heart sounds weak; lungs, negative.

Traube's Space.-Dull on percussion.

Splenic dulness at eighth rib in axilla.

Abdomen.-Tense swelling from left rib edge across lower epigastrium to right of navel. Fluctuation (?)

Rectum.--Negative.

Blood.-Hg., 70 per cent.; r.b.c., 3,880,000; w.b.c., 16,000.

Operation.-Incision over most prominent part of epigastric tumor. A quantity of creamy, thick fluid came out under tension. Drainage.

Subsequent Course:

Abdominal Examination.-After abscess had been drained revealed a firm, fixed mass occupying the entire left epigastrium.

Test-meal.-Marked 12-hour retention. Total acidity, 50 ; free $\mathrm{Hcl}, 0$; lactic acid+; altered blood+; Wolff + , microscopic examination revealed large numbers of bacilli of the Oppler-Boas type.

$X$-ray.-Entire pylorus, pars media and greater curvature involved with a fungoid growth. Almost complete pyloric obstruction.

F. Fistulæ usually result from perforation with a resultant abscess cavity or direct connection between the stomach or the large bowel or develop from necrosis of a contiguously extending tumor. Gastrocolic fistulæ occurs in from 1 to 4 per cent. of late cases of gastric cancer. Clinically they are recognized by a previous malignant gastric disease later showing evidences of fæcal contents in the vomitus, in diarrhea of the lienteric type, hemorrhage, the speedy appearance of a test capsule of carmine in the stool or by the Roentgen ray. 
Gastric Cancer, Involving Transverse Colon, Pancreas and Liver; Hemorrhage; Metastases to Pelvis; Anemia; Few Clinical Symptoms:

Mr. L. J.-Age 57, Swedish, cabinet maker.

Family History.-Mother died of cancer (pelvic). Father and sister died of pulmonary tuberculosis.

Personal History.-Malaria many years ago. Denies venereal.

Present Trouble.-Nearly 2 years ago began to have attacks of diarrhœa. These came on at intervals of from 2 weeks to 3 months. Occasionally passed bright red blood in stool. Three months ago fainted away while at work, and was taken to a hospital. Since then has gradually become weaker and has lost weight.

Appetite.-Gradual failure.

Bowels.-Occasional diarrhœic stool.

Weight.-One year ago 170 pounds; present, 117.

Examination.-Tired looking, pale, emaciated blonde.

Mouth.-Teeth poor; tongue coated.

Neck.-Enlarged left supraclavicular glands.

Thorax.-Heart, weak muscle sounds.

Abdomen.-Irregular mass in high and middle thorax, moving on respiration, tender. Small amount free fluid in peritoneal sac.

Rectal.-Several nodules in Douglas pouch. Bleeding hemorrhoids.

Laboratory Examinations:

Blood.-Hg., 40 per cent.; r.b.c., 3,490,000; w.b.c., 5,100.

Wassermann and Luetin Tests.-Negative.

Test-meal.-Twelve-hour retention. Total acidity, 20; free Hcl, 0; lactic acid+; altered blood+; Oppler-Boas bacilli.

$X$-ray Examination.-Filling defect involving pyloric half of stomach, and invading the transverse colon, pancreas and liver (?).

Laparotomy.-High median incision. Mass involving pyloric half of stomach and extending along coronary artery to esophagus. Transverse colon, omentum and head of pancreas also involved. Few nodules in liver.

Operation.-Explored.

Pathologic Report.-(Post-mortem) Adenocarcinoma. 
G. Metastases occur most commonly to the lymphatics in gastric cancer. We have tabulated their significance in Chapter IV. In more than 70 per cent. of our cases of gastric cancer, metastases to the perigastric lymph chains were demonstrated. As has been pointed out by MacCarty and Blackford, the extent of perigastric lymph-gland invasion bears no relation to the size of the primary process in the stomach wall; the gross size of the individual lymph nodes is no definite evidence as to whether or no they contain malignant tissue; malignant glands are usually hard upon palpation and their cut surface has a glistening white color. Upon the degree of lymph-gland invasion depends absolutely the prognosis in a given case, wholly irrespective of the size of the primary growth.

Metastases to the liver occur in from 15 to 35 per cent. of all instances of late gastric cancer. In our series (which comprises many cases where the diagnosis of malignant disease of the stomach was made microscopically) the liver was involved in 18 per cent. of cases. With the involvement of the liver one not infrequently notices the development of ascites. This is due to interference with the portal circulation and also to direct involvement of the peritoneum. The ascites usually develops very gradually, but there are cases where a rapid accumulation of ascitic fluid occurs. In such instances a fatal termination supervenes in a short time.

Metastases to the Lungs.-If the disease is allowed to progress without interference, metastatic involvement of the pleura, pericardium, the lung tissue and the mediastinal lymphatics is noted in from 5 to 11 per cent. of all instances. If the pleura is involved serous exu dation may appear rapidly. The puncture fluid not infrequently shows blood cells, cells with atypical mitotic forms and an increase in the 
incoagulable nitrogen (Roger Morris). If lung tissue is actually invaded, malignant consolidation or necrosis with abscess result.

H. Jaundice may result from direct extension of the gastric growth to the liver or the gall tract, from pressure of a pyloric tumor upon the bile passages, from malignant invasion at the head of the pancreas and consequent obstruction of the common bile duct or from pressure upon the biliary passages by malignant lymph nodes. In early cases of gastric cancer, jaundice is present in about 3 per cent. of instances. Where the growths are extensive, jaundice occurs in 12 to 15 per cent. of cases.

I. Thrombosis is a terminal complication of gastric cancer. It is relatively infrequent. It may be arterial or venous. The vessels most commonly involved are the femoral, saphenous, the external iliacs, the subclavian or the brachial. If the patients are not too far gone, thrombosis is evidenced by local pain, edema or discoloration of tissues.

J. Enteritis occurs concomitantly with the majority of gastric cancers. It varies greatly in degree or extent. Not rarely tenderness over the bowels, colicky abdominal pains, diarrheic stools or the appearance of hemorrhoids indicate deficient function on the part of the small or large bowel.

K. Nephritis.-It is difficult to estimate whether anomalies on the part of the kidneys are due to the disease or are malfunctions to be expected in individuals past middle life. In our series albumin was noted in 17 instances and casts in 19.

L. Nervous complications are comparatively rare occurrences in gastric cancer. A certain melancholy frame of mind not unnaturally develops in individuals who real- 
ize that they are fatally ill with a disease around which clings much popular dread. Fenwick claims that this may amount to insanity in 1.6 per cent. of instances. We have no case of insanity as a complication in our series. Neuritis, paralyses or sometimes general tonic spasms of the tetanoid type have been described associated with this disease. Whether such complications are directly caused by the neoplasm or are evidences of functional or toxic disturbances apart from the gastric cancer, it is difficult to state.

\section{ADDENDUM}

\section{A RAPID METHOD OF CUTTING AND STAINING SECTIONS OF TISSUE (WILSON)}

Place a piece of tissue no thicker than $3 \mathrm{~mm}$. on freezing stand and add enough dextrin solution of the consistency of molasses to cover. Then turn nozzle allowing the $\mathrm{CO}_{2}$ to escape until the tissue is just hard enough so that sections may be cut without crumbling. Under proper conditions such as a sharp knife, proper density of tissue, proper temperature, etc., sections may be cut as thin as 5 microns. Keep the knife continually flooded with water while cutting the sections. Use ball of the finger to remove section from the knife and place section in a dish of water. Pieces of tissue that have been hardened for 12 to 24 hours in 10 per cent. formalin can be cut with much greater ease than when not so treated. The sections so made may be stained either by the hematoxylin-eosin method and permanently mounted or stained by the following method which is much more rapid but less permanent.

Rapid Method of Staining.-Place the section in a small dish containing about a dram of Brun's polychrome methylene-blue (Grubler's) solution for 10 to 15 seconds 
and transfer immediately to water. After rinsing a few seconds, place in Brun's glucose medium to clear the sections.

Formula for Brun's Glucose Medium.-(a) Glucose, 240 cc. Hot distilled water, 840 cc. Mix thoroughly.

(b) Spirits of camphor, 60 cc. Glycerine, $60 \mathrm{cc}$. Mix thoroughly.

$\operatorname{Mix}(a)$ and (b) together and filter.

After section has been in above solution a few seconds it is floated upon a microscopic slide, the excess fluid allowed to drip off and a cover slip placed in position. It is ready for examination. Under ordinary conditions the slide will keep 2 to 3 weeks. By this method one may have a well-stained section of tissue ready for microscopical examination within 3 minutes. Either the Spencer or Leitz freezing microtomes are suitable for this work. Either may also be used for the cutting of celloidin or paraffin blocks.

\section{REFERENCES}

VIrchow: "Pathologie des Tumeurs," vol. vii, p. 236.

Fenwick, S.: "Cancer and Other Tumors of the Stomach," Philadelphia, 1903.

YATES: Annals of Surgery, 1906, Oct., p. 599.

Clendenning: Am. Jour. Med. Sc., 1909, Aug., p. 1.

Frazier: Am. Jour. Med. Sc., 1914, June, p. 781.

Campbell: Surg. Gyn. and Obstets., 1915, Jan., p. 66.

WALDEYer: "Arbeiten über den Krebs," Virchow's Archiv., 41, p. 470. Rokitansky: Lehrbuch der pathol. Anatomie, 3 Aufl., iii, p. 171.

Rous: Jour. Exp. Ned., 1910, vol. xii, No. 5; and Proc. Am. Philosophical Soc., 1912, vol. li, No. 205.

Rous And Jones: Jour. Exp. Med., 1914, vol. xx, No. 4.

Rous, Murphy and Tytler: Jour. Am. Med. Assn., 1912, June 8, p. 1751.

Murphy: Jour. Exp. Med., 1913, vol. xvii.

Levis: Jour. Exp. Med., 1912, No. 12, p. 149.

Sxow: Journ. of Infect. Dis., vol. iv, No. 3, p. 385.

Siritr: Bulletins Nos. 213 and 255, U. S. Dept. of Agriculture, 1912. 
Fibiger: Berl. Klin. Wchnschr., 1913, Feb. 17, p. 289.

MacCarty, W. C., ANd Wilson: Am. Jour. Med.Se., 1909, Dec., p. 846.

MacCarTy, W. C.: Surg. Gyn. and Obstets., 1910, x, p. 449.

MacCarty and Broders: Arch. of Int. Med., 1914, xiii, p. 208.

MacCarty, W. C.: Jour. of Iowa State Med. Soc., 1914, iv, p. 1.

MacCartr, W. C.: Am. Jour. Med. Sc., 1915, No. 4, p. 469.

Rous AND Murphy: Jour. Exp. Med., 1914, vol. xx, No. 4.

GAY: Jour. Med. Research, 1909, Feb., p. 175.

CARrel: Jour. Exp. Med., 1912, vol. xvi, No. 2.

Moreshi: Ztschrft. f. Immunitätsforch, 1909, vi, p. 651.

Rous: Proceedings of the Society for Experimental Biology and Medicine, 1911, viii, p. 128; and Jour. Exp. Med., 1914, vol. xx, No. 5.

Brinton: "Diseases of the Stomach," 1859.

Luton: "Magenkrebs," Noureau diction de Med., Paris, 1871.

Welch: "Cancer of the Stomach," Am. Syst. of Med., ii.

Fenwick, S.: (Loc cit.)

MacCartTy and Blackford: Annals of Surg., 1912, June, p. 811.

Morris, Roger: Arch. of Int. Med., 1911, Oct., p. 457.

Wilson: Jour. Am. Med. Assn., 1905, Dec. 2. 


\section{CHAPTER III}

\section{SYMPTOMATOLOGY}

The work of the surgeon and of the cellular pathologist has demonstrated that the cases of gastric cancer which are cured, or given any considerable lease of life, are those where such early diagnosis of malignancy has been made as to permit of the resection of the neoplasm while.it is still a localized process.

The pathologist, familiar with fresh tissue examination, has proved that the highest proportion of operatively cured cases of gastric cancer makes up that group where the early diagnosis consisted in the recognition of malignancy (with the highest power microscope) in extirpated sections of the gastric wall in patients where the disease seemed clinically, surgically and macroscopically chronic, calloused gastric ulcer.

The early diagnosis of malignancy in these curable cancer cases involved the histologic demonstration of intracellular progressive nuclear hypertrophy, undifferentiated (functionally) gland-cells, exuberant hyperplasia of gland structures and beginning invasion of hyperplastic epithelial cells into non-epithelial portions of the stomach wall.

The cases deriving the greatest benefit from operation are those in which the fewest areas of malignant epithelial hyperplasia are found, or those where active gland-cell hyperplasia exists, which permit of a presumptive histologic diagnosis of malignancy. 
Specimens of extirpated tissue microscopically revealing the least advanced epithelial cellular faults are generally, but not always, associated with the minimum of perigastric lymph-gland malignancy.

In gastric cancer, the extent of lymph-gland metastasis controls most certainly the prognosis. The size of the local neoplasm, in the stomach wall, is but a relative index of the possible extent of perigastric lymph-gland invasion. The size of gastric lymph glands, themselves, is no criterion of the degree to which they may be malignantly invaded. Minute glands may contain a greater number and more perniciously active cancer cells than do large glands. Hence, the diagnosis (and the prognosis) of the stage of a gastric cancer is primarily in the hands of the surgical pathologist.

From the foregoing facts it would appear that the past decade has contributed the most radical clinical information regarding gastric cancer that has come to us since its classic description by Virchow and Waldeyer. It would seem that a great prophylactic advance were now possible. Previously, the clinician's complaint has been that the gastric cancer case came to him for aid only when the disease was so far advanced that the diagnosis and prognosis stood revealed on the face of the patient. One could run and read. Early diagnosis mainly implied detecting gastric malignancy before general carcinosis and early death threatened. Medical treatment but prolonged misery. The surgeons who operated on these patients offered little. But they were a bold lot: scientific vikings, who, by persistent exploration, have come back to the less venturesome members of their clan with an epoch-making discovery. We can now state that the early diagnosis of gastric cancer is a microscopic one; it is possible from 
histologic study of freshly removed tissue; such tissue is seen least developed in those patients whose gastric history has been that of chronic, recurring peptic ulcer and in whom to eye and hand such ulcer appears at laparotomy.

As is to be expected, this view of the situation is not as yet generally accepted. Healthy skepticism is a scientific virtue, but doubt, unsupported by facts, is a pernicious habit. This sluggishness of the professional mind might be discouraging to both investigators and trusting patients, had it not been shown that gastro-enterologic history is rich in similar examples. It is but a few years since "catarrhal gastritis," "hyperacidity," "Reichmann's disease" and "pyloric spasm" were considered definite disease entities. Only recently, and reluctantly, has the world medical accepted these pet ailments for their face value, as being symptoms of gastric malfunction associated with peptic ulcer or subinfections of the appendix or gall-bladder.

This introduction is necessary to support the grouping of symptom-complexes of gastric cancer which the study of the 921 instances comprising our series seems to establish.

Our material can be grouped under the following symptom-complex heads:

I. Gastric cancer in individuals who came to laparotomy for clinically benign gastric ulcer, and in whom cancer was diagnosed microscopically.

II. Gastric cancer clinically developing in patients with years of antecedent dyspepsia of the "peptic ulcer type," in whom malignancy subsequently appeared.

III. Gastric cancer in individuals who prior to the onset of a malignant disease had enjoyed perfect gastric health.

$I V$. Gastric cancer in individuals in whom malignancy followed periods of gastric disturbance of no clinical type. 
$V$. Gastric cancer in individuals who presented few clinical evidences of a malignant process primary in the stomach wall.

VI. Gastric cancer secondary to an extragastric malignant process.

Group I. Gastric Cancer in Patients Who Came to Laparotomy for Clinically Benign Gastric Ulcer and in Whom Cancer was Microscopically Diagnosed

This group comprised 72 cases (7.8 per cent.). There were 44 males and $2 S$ females. The average age was 56.5 years. The shortest period of gastric distress was $31 / 4$ years, the longest 42 years, the average 13.7 years. There was a family or blood relationship history of malignancy in 3 instances ( 4.1 per cent.).

Mode of Onset.-The disease had been periodic, without acute attacks in 42 cases (58.3 per cent.). Between attacks until complications ensued (stenoses, perforation, etc.) the patients generally enjoyed good gastric health. The periods of exacerbation varied in frequency from 1 every week or 10 days to one in 3 or 4 years. Peculiar seasonal relationship of the "spells" of dyspepsia were noted: fall or spring or both seemingly bringing such on, or aggravating an already present dyspepsia.

In 19 cases (26 per cent.) the disease had been chronic and continuous. There were frequently noted times when the gastric malfunction was of increased severity.

Eleven patients (15.2 per cent.) gave history of acute attacks of dyspepsia, either at infrequent periods or at some phase of a continuous ailment. These acute attacks usually implied severe, often colicky, abdominal pain, vomiting, hemorrhage with or without symptoms of shock, loss of appetite or strength, constipation, or occasionally jaundice. 
The Digestive Disorder.-Anorexia was generally evidenced only during the periods of abdominal discomfort. There was good appetite between such. Not infrequently, the fear of bringing on pain by ingestion of food, normal in amount and quality, the dread of precipitating hemorrhage, or oscillations between various types of "diet," resulted in the development of a poor appetite habit. Mild or marked degrees of stenosis at cardiac or pyloric orifices occurred in 30 instances (41.1 per cent.). In such cases poor appetite resulted from pain on ingestion, nausea or fear of precipitating vomiting.

Dysphagia was a late symptom in 3 instances (4.1 per cent.). It resulted from calloused ulcer partly occluding the cardia or from severe spasm at that orifice as a consequence of an irritated ulcer high on the lesser curvature of the stomach.

Water-brash with or without pyrosis often proved an annoying symptom. It was a major complaint at some stage of the disease in 55 cases ( 76.4 per cent.). In the ulcer patients exhibiting periodicity of complaint waterbrash and pyrosis were generally early warnings that a "spell" was coming on. The greatest discomfort from these sources occurred from 1 to 4 hours following food ingestion in the majority of instances. In cases.where the digestive disturbance was continuous, patients frequently stated that they were rarely free from "sour stomach." Prompt relief of these symptoms was commonly obtained by ingestion of food, the taking of alkalies, by vomiting, by gastric lavage, or by free catharsis.

Bowels.-Constipation was observed in 47 cases (66.9 per cent.). It was frequently periodic and heralded the approach of a dyspeptic storm. Diarrheic stools were described by 6 patients ( 8.3 per cent.). 
The tongue was described as "coated" in 62 instances (86 per cent.).

Nutrition.-There were 16 patients who had lost no weight and were in excellent flesh. Thirty-four cases had lost less than 10 pounds. In 22 the weight loss ranged from above 10 pounds to as great as 39 pounds. Weight was frequently noted as having been lost in the attacks or periods of exacerbation of the disease. It was commonly gained when the dyspeptic agony had passed. Associated even with marked and rapid weight loss, there was but rarely cachexia, such as is usually present in malignancy.

Blood.-The average hemoglobin was 80 (Tallqvist or Dare). The red-cell count averaged 4,200,000. Leucocytes were above 9,000 in but 2 instances. These were both ulcers in which perforation was subsequently demonstrated.

Strength was "poor" in 18 patients.

Vomiting.-This proved a troublesome symptom at some phase of the ailment in 58 cases ( 80 per cent.). The vomitus was the color of ingested food, white, green or yellowish. Food was not infrequently poorly chymified. Copious vomitus of the "delayed" type was a late manifestation in 32 instances ( 44 per cent.). Blood was vomited by 24 patients (34.5 per cent.). Emesis occurred with the greatest frequency from 2 to 4 hours following food ingestion. Five cases with cardiac or high lesser curvature ulcers vomited shortly after the swallowing of food. The vomitus was described as sour, bitter, or salt.

Hemorrhage.-Hematemesis was experienced by 24 patients (34.5 per cent.) and melena by 3 (4.2 per cent.). Melena without hematemesis occurred once.

The stool examined at some phase of the ailment had 
given chemical test for altered blood in 52 cases (71.1 per cent.) by the benzidin or guaiac reactions.

Pain.-Some form of abdominal distress was complained of by every patient in this group at some time during the disease. Only 3 instances were recorded where distress was constantly below the level of the navel. These were lesser curvature or anterior wall ulcers in rather lowlying stomachs.

There was usually general epigastric distress. In 23 cases (31.9 per cent.) more intense pain was a local complaint in addition to its being of widespread distribution. In 18 of these instances the severe complaint was at or to the left of the mid-abdominal line.

Type of abdominal distress varied. In 10 patients (14 per cent.) it was described as "colicky," "boring," "knife-like." Fifty-four cases (73 per cent.) complained of "burning," "soreness," "gnawing." In the remaining instances rague "full"_-"heary"_-_"bloated" or "pressure" sensations were complained of.

The time of occurrence of abdominal distress bore definite relation to food intake in 58 cases ( $\$ 1$ per cent.). In these patients pain came on from 2 to 5 hours after eating and disappeared, or was lessened by again taking food. Until complications ensued (especially stenoses) the quality of food eaten seemed to have little significance toward the stopping of the distress. Quantity frequently appeared to exert greater influence: longer relief was obtained after a heary meal than after a small one, but when pain recurred it was then generally of greater severity. Other forms of pain-relief were in the order of their usefulness: alkalies, vomiting or lavage. In about 13 per cent. opiates were required at some time.

There were 5 cases where pain was relieved by none of 
the routine remedies. It was continuous, rarely very sharp, and bore no relation to food intake. The patients had not been given opiates.

There were 7 instances where pain was of so violent a character that ulcer perforation was suspected. Hemorrhage was associated 3 times. At laparotomy, some grade of perforation was made out in 5 of these cases. A stone in the cystic duct was found once.

Test-meal Findings. - A complete discussion of this aspect of this group of gastric cancers is found in Chapter V. Briefly: Trelye-hour retention was demonstrated in 33 cases ( 46 per cent.); total acidity areraged 62 , and free hydrochloric acid 38. Lactic acid was demonstrated by the Uffelmann test twice. Blood by chemical test was noted in 18 gastric extracts (25 per cent.). Wolff-Junghans' test for increased soluble albumin was positive 5 times in 9 cases tested. Microscopic examination of gastric extracts revealed no instance where organisms of the OpplerBoas type were seen. In 84 per cent. of the stomachs showing gastric retention, yeasts and sarcinæ were observed.

\section{HISTORIES}

\section{Cases Illustrating Group I Type of Gastric Cancer}

1. Chronic, Recurring Peptic Ulcer of Non-stenosing TypeExcision; Pathologically Malignant:

Mr. F. B.-Age 53, German, farmer.

Family History.-Negative.

Personal History.-Denies venereal; married; 2 children; wife has had no miscarriages; measles at 12; never used tobacco or alcohol; always worked hard.

Digestive History.-For the past 15 years has had attacks of "stomach trouble." Up to 6 months ago these came on 2 or 3 times a year, being especially aggravating toward the Christmas holidays. The attacks consisted of ache or colics in the right upper abdominal quadrant or toward the rib 
edges. They would come on 3 to 4 hours p.c. or at night and last until he ate something or forced himself to vomit. His appetite was generally good, but he limited his eating at such times. Between the "spells" of dyspepsia he was perfectly well. Six months ago he began to have rather constant distress accompanied by acid eructations and some nausea. Appetite became less sharp. One night while unlacing his shoes, he felt "queer" and soon afterward vomited about a pint of red blood. Was in bed a week; then felt fairly well except for soreness in the pit of his stomach. Since the hemorrhage, has felt moderately comfortable except for some weakness. Appetite has been a bit capricious. Bowels which were formerly constipated have been loose for past 2 weeks. Never noted blood. Thinks he is about 15 to 20 pounds underweight.

Status Prosens.-Moderately well nourished, lanky male; sclera clear; tongue large and coated heavily; teeth poor and dirty; fetor ex ore.

Neck and Chest.-Negative.

Abdomen.- Somewhat full in epigastrium; tenderness with muscle spasm in right upper quadrant and at right rib margin; nothing abnormal palpated.

Rectal examination negative.

Blood.--Hg., 85 (Tallqvist); r.b.c., 5,340,000; w.b.c., 7,000 .

Test-meal.-Total acidity, 44. Free Hcl, 28. Combined acids and acid salts, 10 . No 12-hour retention.

Stool. - Trace of blood (benzidin) on milk diet.

Laparotomy.-Calloused ulcer size of a half dollar on lesser curvature in antral region; edges thickened; base clean; no enlargement of perigastric lymph glands; stomach moderately dilated.

Operation.-Mikulicz-Hartman, Billroth No. 2 with posterior gastro-enterostomy.

Pathologic Report.-Early carcinoma of glands of edge and base of ulcer. Numerous areas showing epithelial wandering through basement membrane. Extensive round cell infiltration.

2. Chronic Calloused Prepyloric Ulcer; Stenosis; "Delayed"

Vomiting; Laparotomy: Indurated Ulcer on Lesser Curvature; Microscopically-Adenocarcinoma with much Scar-tissue: 
Miss N. McD.-Age 42, stenographer.

Family History.-Negative.

Personal History.- - Scarlet fever twice when young girl; many attacks of tonsillitis; has severe headaches at irregular intervals.

Present Illness.-Gives account of "stomach trouble" for $2 \frac{1}{2}$ years. Onset was gradual but has never been free from dyspepsia since ailment began; has been worse for past 6 months.

Complaint.-Pain. Is located in epigastrium, especially in right upper quadrant. Character is "gripping" and aching; maximum distress is generally 2 to 3 hours p.c. or at night. There is no transmission of pain. Vomiting, diet and alkalies give relief; lying on left side, jolting or body movements aggravate pain.

Vomiting.-Began 11 months ago; for 3 to 4 weeks vomited after every meal, usually between 10 and 11 a.m. Eating or "crampy" pain appeared to precipitate vomiting. Later began to vomit at bed-time; material vomited frequently contained food eaten at breakfast. Vomitus was "blackish" once. Always felt relieved after vomiting. For past 6 months has vomited rarely up to 1 week ago. At that time had sharp epigastric pain and vomited until stomach was entirely empty; noted no blood in either vomitus or stool. Has been on liquid diet since.

Regurgitation, pyrosis and water-brash very annoying; came on mainly at night.

Appetite.-Good clesire, but afraid to eat.

Bowels.-Always costive.

Weight.-Eleven months ago lost 40 pounds; gained 20 pounds and held it to 2 months ago; is now 25 pounds under normal weight.

Menopause.-Eleven months ago.

Status Prosens.-Rather poorly developed maiden lady but fairly well nourished. Skin dry and loose; sallow. Sclera clear; mucosæ pale; tongue coated; teeth in fair state of repair.

Head, Neck and Chest.-Negative.

Abdomen.-Somewhat scaphoid. Abdominal aorta pulsated visibly and violently. Palpation: Tenderness in mid-epigastrium with slight rigidity of right rectus muscle. Stomach, splashy. 
Taginal and Rectal Examinations.-Negative. Laboratory Findings:

Blood.-Hg., 70; r.b.c., 4,020,000; w.b.c., 10,600.

Test-meal.-Twelve-hour retention. Total acidity, 68; free Hcl, 40. No blood. Microscopically, yeasts and small type sarcinæ.

Urine--Negative.

Laparotomy.-Calloused ulcer involving the pylorus and lesser currature in antral region; ulcer $4 \mathrm{~cm}$. in diameter; no gland involvement; diverticulum $1 \frac{1}{2} \mathrm{~cm}$. in diameter is seen on jejunum about 1 meter from the pylorus.

Operation.-Pylorectomy; posterior gastro-enterostomy.

Pathologic Report.-Indurated ulcer with adenocarcinoma in edges and base.

3. Acute Onset; Rapid Course; Hemorrhage Early; Weight Loss; Calloused Llcer at Laparotomy; Malignant Microscopically:

Mr. J. S.-Age 65, Swedish, laborer.

Family History.-Negative.

Personal History.-Always well up to onset of present trouble.

Present Trouble. - Three months ago had sudden and severe epigastric pain following food ingestion. Since then has never felt himself. Pain comes on immediately after eating and lasts about 4 hours; then it is relieved by vomiting. Recently pain has been particularly severe at night between $12 \mathrm{~m}$. and $3 \mathrm{a} . \mathrm{m}$. Cannot sleep until he vomits.

Tomiting.-Three to 8 hours following meals; vomitus consists of undigested food and blood. Is constantly nauseated.

Appetite.-Tery poor; is on milk diet.

Weight.-Thirty pounds loss in 3 months.

Bouels.-Constipated for past 25 years.

Urine.-Peculiar odor.

Status Prosens.-Moderately well developed, but soft tissues flabby. Color, good; skin, soft and smooth; sclera, clear; tongue, clean; teeth, fair but dirty.

Head, Neck and Chest.-Negative.

Abdomen.-Negative except for slight tenderness in upper epigastrium. 
Rectum.-Negative.

Blood. - -Hg., s0 per cent.

Test-meal.-Moderate 12-hour retention; blood-tinged gastric extract. Total acidity, 58; free $\mathrm{Hcl}, 34$; blood+ (benzidin test). Microscopically, few budding yeasts and red blood cells; many short, fat rods (colon bacilli).

Stool.-Blood+ (benzidin test).

Laparotomy.-A hard indurated ulcer on the anterior wall and the upper surface of the pylorus; partial pyloric stenosis; moderately dilated stomach ; no perigastric lymphgland involvement.

Operation.-Pylorectomy; posterior gastro-enterostomy.

Pathologic Report.-Indurated gastric ulcer; much scartissue; in base are areas of adenocarcinoma.

4. Short Duration; Few Symptoms, Hemorrhage; Malignant Anterior Wall, Ulcer:

Mr. P. L., age 66, German, lumber dealer.

Family History.-Mother died of a malignant growth of the pharynx.

Personal History.--Always well to 6 months ago, when he began to have abdominal fulness, particularly after eating, belched much gas. No pain or vomiting; occasionally nausea. Appetite and bowels normal. One week ago had severe abdominal cramps. Was nauseated and could not get bowels to move. The next day passed a large amount of black blood by rectum. Has had no recurrence.

Weight.-Thinks perhaps 10 pounds below normal.

Status Prosens.-Well nourished; good color; tongue clear.

Head, Neck and Chest.-Negative.

Abdomen.-Spasm in mid-epigastrium on palpation.

Rectal.-Negative.

Laboratory Examinations:

Blood.-Hg., 85.

Test-meal.-No retention. Free Hcl, 26. No blood.

Stool.-Blood+ (benzidin).

Laparotomy.-Calloused ulcer on anterior surface of stomach $4 \mathrm{~cm}$. from pylorus; no glands involved; serous surface adherent; cholecystitis. 
Operation.-Partial gastrectomy; posterior gastro-enterostomy; cholecystostomy.

Pathologic Report.-Indurated ulcer. Adenocarcinoma in edges and base; moderate amount of scar-tissue.

\section{Group II. Gastric Cancer, Clinically Developing in Patients with Years of} Antecedent "Dyspepsia" of the Peptic Ulcer Type

Our series demonstrates that this group includes the majority of cases of gastric carcinoma. There were 436 instances (47.3 per cent.) where patients appeared with a gastric ailment evidently malignant that had been superimposed upon a previously benign type of dyspepsia. The form of antecedent gastric malfunction satisfied the commonly accepted clinical complex of chronic peptic ulcer. The disturbance readily divides itself into two strikingly different periods: one benign and usually long drawn out, and the other malignant, continuous and progressing relentlessly toward a fatal issue. The anamnesis alone brings out the characteristics of the disease in each of its stages.

It is becoming more generally recognized that gastric diagnosis gains in accuracy in proportion to the diligence with which enquiry is made into the departures from proper stomach functioning previous to those which exist at the time when the patient appears for a solution of his dyspeptic dilemma. Accidental complications often of a mechanical nature not infrequently cause the gastric case to seek a doctor's aid. It is only by disengaging the patient (and often his physician) from the present state and endearoring to determine and analyze his early departure from gastric health that one is able to accurately judge what sequence of clinical events led up to the immediately existing digestive anomaly.

Study of our material has by no means shown that 
gastric cancer most commonly attacks individuals whose stomachs had previously been, as Napoleon told his physician Automanchi "like iron." On the contrary, the great number of these so-called ferruginoid stomachs have an important etiologic history behind them, even as did the classic gut of Napoleon. For, as we inquire into the early history of this oft-cited case, we find abundant evidence that the imperial stomach rebelled quite regularly and sometimes disastrously (vide battle of Leipsic.)

(a) The Precarcinomatous Period.-The average duration of all symptoms in the first stage of the ailment, that clinically benign, was 10.5 years. The shortest history of this phase extended over 2 years and the longest more than 45 year's. This long antecedent history of gastric malfunction proves that the majority of our cases of gastric cancer did not develop in patients who had previously been in good gastric health. The interpretation, clinically, that we place upon the nature of the early dyspepsia, depends, naturally, upon the conception which experience has given us regarding symptomatology of gastric disease, the value of facts making up the histories, and our observations of the subsequent course of the ailment.

The point of greatest import in the consideration of the precarcinomatous picture of the disease, is, that while certain histologic changes of a malignant type may have lain latent in the gastric wall for years, it was, at this first period, impossible to recognize such, clinically.

Symptomatically, this early period of the ailment satisfies the accepted clinical complex which we associate with chronic, recurrent gastric ulcer. This statement does not imply that the majority of gastric ulcers become cancers; it is simply a statement indicating that the greatest number of our proved cases of gastric cancer had had gastric ulcer 
symptoms prior to the time when their dyspepsias assumed malignant features. No one can offer opinion, based upon actual facts, as to the percentage of ulcers that undergo malignant transformation. Doubtless many ulcers heal or remain benign. The caution should be strongly urged, however, that in every gastric ulcer there exists the anlage of a future cancer, and that only by recognition of this possibility and by constantly watching the progress of individuals thus affected (especially as they pass beyond the fourth decade of life) can we hope to be of any actual service to those individuals, or to the race, with respect to cure or prevention of gastric malignancy.

The symptomatology of the precarcinomatous period is in every way similar to that above outlined as comprising the whole course of cases making up Group I. It is not necessary to repeat it. The cases give typical ulcer histories. And, indeed, if such patients come to laparotomy at any time during this clinically benign stage, ulcers without evidence of malignant hyperplasia are demonstrated surgically and often pathologically.

Briefly, the study of this early dyspeptic disturbance, established the following facts in our material:

Attacks or "spells" of indigestion occurred in $\$ 2$ per cent. of instances. Seasonal relationship was not infrequently demonstrated. Over-work, exposure and intercurrent infectious diseases appeared to play a considerable rôle in precipitating or aggravating symptoms. These "spells" of dyspepsia were characterized by epigastric distress or actual pain in 392 cases (90 per cent.). In 366 instances ( $\$ 4$ per cent.) the abdominal distress bore definite relation to the taking of food. Until complications supervened, this "food relief" of pain was quite constant and characteristic. Other forms of pain relief were alkalies, 
diet or romiting. Pain was generally located, symptomatically, in the upper two-thirds of the epigastrium. In 270 cases (62 per cent.) pain had a point of maximum intensity. This was most frequently located at or slightly to the right of the mid-line. In 224 patients (51.4 per cent.) pain was referred to some part of the back. The areas of referred distress were most commonly between the shoulders or to one or both scapular regions.

Food desire was usually strong, but appetite suffered from fear of precipitating digestive upsets by eating.

Weight was not infrequently lost in these attacks of limited food intake, only to be gained again when quiescence supervened. The average weight loss was $15 . \mathrm{S}$ pounds.

Constipation was noted in 209 instances (4S.2 per cent.). Twenty-seven patients (6.5 per cent.) had experienced diarrhea at some time during this precarcinomatous period.

Vomiting was recorded in 265 cases (63 per cent.). In 217 of these patients who vomited ( $\$ 2$ per cent.), the emesis was periodic. Retention romiting was noted in 98 cases (37 per cent.).

Bleeding (hematemesis or melena) was a sign in 92 instances (21 per cent.), during this stage of the disease. Of those bleeding, 47 cases (50.6 per cent.) had experienced hemorrhage at least 2 years before the time when they came under observation. Of those instances (45) where bleeding had occurred more recently than 2 years, 28 (62 per cent.) were patients whose dyspeptic history was longer than 10 years.

Anemia was rarely marked in the clinically, benign ulcer stage. The average hemoglobin was approximately 75 per cent. 
Before proceeding to a study of the second (malignant) phase of this class of patient we would emphasize the group type by the following histories.

1. Long Dyspeptic History; Ulcer Type; Early Hemorrhage, Supervening Malignancy; Free Abdominal Fluid:

Mr. A. L.-Age 52, German, carpenter.

Family History.-Negative.

Personal History.-Married twice, first wife died of tuberculosis.

Present Trouble.-Periodic dyspepsia from 18 to 21 years of age; this consisted of epigastric pain, sour eructations and belching, and occasionally vomiting of sour fluid. At age 22 vomited about a pint of blood; was in bed 2 weeks; since then has had only rare attacks of the dyspepsia until 17 months ago. He then began to have pain in the epigastrium. This was a gnawing and sore feeling and was worse about 2 hours after eating. Shortly afterward he began to vomit. Recently has had night pain, with vomiting about 1 o'clock in the morning. He frequently vomits up what he had eaten for breakfast, and occasionally material eaten the day previously.

Weight Loss.-Fifty pounds in 6 months.

Physical Examination.--Much emaciated, sallow complexion, skin dry; weak and tired-looking; tongue coated.

Thorax.-Negative.

Abdomen.-Moderately distended; small umbilical hernia; inguinal glands moderately enlarged. Percussion dulness in flanks, movable. No abdominal tumor palpable.

Rectal Examination.-Negative. Liver and spleen, not enlarged.

Laboratory Examinations:

Blood.-Hg., 82 per cent.; r.b.c., 4,980,000; w.b.c., 6,200.

Test-meal.-Total acidity, 28; free $\mathrm{Hcl}, 12$; combined acids, 8; altered blood+. Moderate 12-hour retention.

Microscopically.-Oppler-Boas bacilli (?). Yeasts.

Stool.-Altered blood+ (benzidin test). Schmidt bichlorid test: hydrobilirubin.

Laparotomy.-Long right-rectus incision. About 2,000 cc. of serofibrinous peritoneal fluid. Large carcinoma involving greater curvature, with extension into pancreas. Secondaries in liver. 
Operation.-Exploratory.

Pathologic Report.-Gland removed shows carcinoma.

2. Previous History Suggesting Ulcer at the Cardia Followed by

Partial Malignant Stenosis; Young Patient:

Mrs. H. S.-Age 26, Finnish, housewife.

Family History.-Negative.

Past History.-Negative.

Present Trouble.-During the past 5 years has had attacks of high epigastric pain coming on immediately after eating, and accompanied by vomiting of food that has been eaten shortly before. Sour stomach and acid eructations have been prominent in these attacks. Had no alteration in the symptoms until 3 or 4 months ago when noticed that solid food seemed to stick in the esophagus and would cause pain lasting from $1 / 3$ to 1 hour after eating. Relief of distress was obtained by forced vomiting. During the past 6 weeks the pain has been constant, and has been accompanied by constipation and marked vomiting if patient is on ordinary diet. Recently pain has been very marked in the "small of the back."

Appetite.-Good, but afraid to eat on account of pain.

Bowels.-Very constipated.

Weight.-Has lost 26 pounds in about 3 months.

Examination.-Patient poorly nourished; skin pale with acne-like eruption over face. Tongue coated; teeth dirty; breath heavy.

Thorax.-Negative.

Abdomen.-Flat; general tenderness in epigastrium; at the left costal arch a slightly movable tumor is palpated toward the pit, no tenderness.

Vaginal Examination.-Negative.

Laboratory Examination:

Blood.-Hg., 40 per cent. (Dare).

Test-meal.-Tube met resistance at cardia. There followed free bleeding. No contents obtained. Microscopic examination of blood clots in end of stomach tube showed chains of streptococci; many red blood cells and large, partly disintegrated, epithelial cells.

Stool.-Altered blood+ (benzidin test).

Laparotomy.-High median incision. At the cardiac end of the stomach is a carcinomatous mass encircling the 
orifice; it is very hard and about $2 / 3$ of an inch broad. The cardiac lumen barely admits a little finger. The cardiac glands are greatly hypertrophied.

Operation.-Exploratory; appendicectomy.

Pathologic Report.-Gland from near cardia. Adenocarcinoma with much scar-tissue.

\section{Carcinoma Following Uleer History: Hemorrhages; Ob- struction:}

Mr. R. J.-Age 4S, Swede, stone mason.

Family History.-Negative.

Personal History.-Pneumonia 3 years ago, otherwise well; denies venereal.

Present Illness.-Gastric disturbance for 6 years. Complaint usually epigastric pain, coming on generally 2 hours after eating and most serere just before eating. Food intake promptly relieved pain. Pain transmitted to right upper abdominal quadrant and to back, between shoulders. Frequently awakened at night by pain. Body movement aggravated distress. Usually worse in fall and has periods of remission.

Present Trouble.-Three weeks ago became nauseated and romited about a cupful of blood. Never vomited before or since. Since then has had constant belching, pyrosis and has noted since then that stools have been blackish in color. Pain has diminished in intensity, but has become constant.

A ppetite.-Poor; strength reduced.

Weight.-Lost 42 pounds in less than a month.

Physical Examination.-Well developed, but poorly nourished. Sallow color; tongue coated. Post-malleolar edema; skin loose and dry.

Head, Neck and Chest.-Negative.

Abdomen.-Marked rigidity of both recti muscles, particularly on left side. Stomach dilated, great curvature extends 2 f.b. below navel.

Laboratory Examination:

Blood.-Hg., 65; r.b.c., 3,400,000; w.b.c., 12,000.

Test-meal.-Twelve-hour retention. Total acidity, 76; $\mathrm{Hcl}$, trace; lactic acid+; altered blood+; Wolff test+ (400 units of precipitable albumin); pepsin and rennin, trace.

Stool.-Altered blood+ (guaiac test). 
Urine-Albumin trace; few coarse granular casts.

Laparotomy.-Carcinomatous mass, $10 \mathrm{~cm}$. in circumference, on lesser curvature; glands involved.

Operation.-Exploratory.

Pathologic Report.-Gland from lesser curvature: adenocarcinoma.

4. Gradual Onset, Ulcer Symptoms, Early Vomiting, Recent Rapid Weight Loss; Carcinoma of Pylorus with Large Cardiac Glands:

Mrs. M. B.-Age 48, German, housewife.

Family History.-Negative.

Past History.-Three years and 6 months ago, began to regurgitate food soon after eating. This seemed to be caused by a burning pain extending from the ensiform to the base of the tongue. Was worse after heary meals. Much sour regurgitation, nausea and pyrosis. Never romited blood, but frequently large amounts of partly changed food. A few months afterward noted a pain in the left costal arch. It came on at once after eating, and was quite severe. Has been fairly constant since. Took ulcer cure in a hospital. At present there is much gas and bloating for 3 or 4 hours after meals when patient is relieved by romiting. Of late some difficulty in swallowing large pieces of food; they seem to stick at the pit of the stomach.

Appetite.-Has been poor.

Bowels.-Constipated and stools of offensive odor.

Weight.-Has lost 115 pounds in 6 months.

Physical Examination.-Markedly emaciated; skin sallow; looks cachectic; slight edema about the ankles; teeth very poor; tongue coated; breath foul.

Chest.-Negative.

Abdomen.-No palpable tumor; tenderness to left of navel; stomach splashy.

Vaginal Examination.-Negative.

Blood.-Hg., 60; r.b.c., 3,600,000; w.b.c., S,400.

Differential White Cell Count.-Polynuclears, 77.3 per cent.; small lymphocytes, 20.7 per cent.; large lymphocytes, 1.1 per cent.; transitionals, 0.7 per cent.; eosinophiles, 0.3 per cent.; 1 normoblast seen.

Test-meal. - Stomach tube meets resistance at cardia; no bleeding. Gastric extract tan-brown in color, food poorly chymified; somewhat rancid odor, moderate 12- 
hour retention. Total acidity, 34; free Hcl, 0; lactic acid + ; altered blood + .

Stool.-Small, mushy, light brown. Altered blood+.

Laparotomy.-High right rectus incision. Tumor-mass the size of a croquet-ball involves the pylorus and antrum posteriorly. Tumor is freely movable; peripyloric glands enlarged. Very marked enlargement of glands at the cardia, especially where the gastric artery meets the lesser curvature.

Operation.-Partial gastrectomy; posterior gastroenterostomy.

Pathological Report.-Diffuse infiltrating carcinoma.

(b) The Clinically Carcinomatous Period.-The preceding histories convey a fairly representative impression of the sequence of events leading up to a malignancy from a clinically benign affection. The change in the character of the disease is often a very gradual one. When we recall the pathologic alterations that are going on in the ulcer areas in these cases, it would seem quite reasonable that a neoplasm should well establish itself before it could be expected to give any definite clinical warning of its existence.

When we analyze the mode of onset of the apparently malignant stage of the dyspepsia we are impressed by the great number of ways in which the patient or his medical attendant are made aware that some sort of alteration in the ailment is in progress. Bleeding, constant nausea, vomiting, gassy distention, persistent abdominal discomfort, loss of appetite, weight and strength, diarrhea or enlarging abdomen may usher in the pernicious phase of the disease. Whatever may be the form which the new type of affection assumes, the most characteristic manifestation is the appearance of a continuous and progressively downward gastric disorder in an individual whose previous dyspepsia had been periodic $(82+$ per cent.). This occurs with or without the onset of pyloric or cardiac 
obstruction or hindrance to the onward progress of food by loculation of the stomach from a neoplasm. Where previously there was abdominal discomfort in "spells" or attacks, frequently relieved by food or medicines, one observes food aggravation of such distress, persistent night pain, delayed vomiting, continuous presence of altered blood in the stools, diarrhea, anorexia, weight loss steadily maintained, weakness, anemia, peripheral edema, cachexia, sallow, dry, scaly skin, lack-luster eye and a peculiar hopeless apathy reflected in the face. There often seems to be a sense of impending evil. Melancholic symptoms may not be lacking. Introspective mental attitudes may be established in individuals who previously had been cheerful and optimistic. Rarely, suicidal tendencies are exhibited.

While the above is the common clinical picture presented as cancer intoxication evidences itself, no especial change may be observed (particularly by a busy individual with large interests) until an epigastric nodule or loose clothing bring him to a physician for an explanation. Not infrequently patients attribute an unexplainable vague "queerness" to over-work, physical or mental, and seek relief through the medium of a holiday, osteopathy, "christian science," patent "tonics," and such ever-baited snares, only to appear some months afterward with an extensive and hopeless malignancy. It is often astonishing how child-like may be the mental processes of otherwise mature and brilliant brains, when such occupy themselves with the consideration of bodily ills. Hence, the class of patients in whom gastric malignancy has its mild beginnings, most frequently come to a physician when the disease is farthest advanced. When constant pain, vomiting or diarrhea are suffered early they often serve a useful purpose. Such patients consult the physician earliest: the science and art 
of charlatan, the "wise" neighbor and the cure-all almanac are soon exhausted.

Duration of the Malignant Period.-At the time when patients came under observation, they had been affected with an evidently cancerous process for an arerage time of 6.3 months. In other words, more than a half year was consumed by the growing neoplasm in making it erident to the patient, or his adrisers, that he was not having a recurrence of his former dyspeptic disturbance. When we reflect that more than 4 out of 5 such patients had a continuous gastric malfunction succeed one previously periodic, it is quite evident that either the onset of the ailment has been insiduous or that professional advice has been timid or uncertain. Meanwhile the growth of the cancer has progressed so extensively that in but onethird of all our cases of the disease were the surgeon and the patient giren the advantage of a localized ailment when laparotomy was performed. Early abdominal exploration of the chronic dyspeptic at the cancer age is the only way to change such experience.

Abdominal Pain.-In but 74 of the cases (17 per cent.) comprising Group II was serere pain a complaint. It was usually described as "raw"-_sore"_- dull, heary ache" or "cramps." In 51 instances (11.6 per cent.) opiates were required to give relief of distress. "Gassy" and "bloated" sensations were quite generally complained of, particularly when the new-growth involved the orifices, and food was retained in either stomach or esophagus. Rarely was "food relief" of abdominal discomfort observed. Food aggravation was the rule. While intensity of pain was usually less than a given individual had suffered at some time in his premalignant history, yet the constancy of the distress frequently proved very annoying and 
exhausting. This was particularly the case where night pain prevented sleep or where aggravation of gastric discomfort prevented the taking of proper nourishment.

There was rarely a point of maximum epigastric distress. The complaint was commonly of a diffuse or general pain. In 35 cases ( $\$$ per cent.) in addition to epigastric discomfort, pains referred to the back, rib-edges, and the legs were recorded. Aggravation of abdominal pain by body movements was noted in 48 cases (11 per cent.). The common modes of pain relief in the order of their usefulness proved to be romiting, diet, lavage, alkalies, opiates and rest. In certain instances the resourcefulness of the medical attendant was sorely taxed after the exhibition of all these remedies.

Vomiting.--This symptom occurred in 390 instances (89 per cent.) when malignancy supervened upon what had been a clinically benign process. In 315 cases ( 74 per cent.) the romiting was of almost daily occurrence.

Vomitus was more copious in this phase than previously. Not infrequently sereral quarts of material were raised. The odor of the vomitus was quite characteristic in moderately advanced cases. Whereas this had commonly been of a sour or yeasty odor in the earlier months or years of the affection, when malignancy was established the odor most frequently was cheesy, rancid or penetratingly pungent. When sloughs of cancer tissue, decomposed blood or contents of adjacent viscera were present as result of fistulæ, the sickening, foul odor was almost pathognomonic.

The color of the vomitus ranged from white to dark brown or black. The color was imparted by decomposing food, blood or tissue. In but 88 cases (20.2 per cent.) was the romitus "coffee colored," as is so often de- 
scribed in connection with gastric cancer. When vomitus of this color was observed, the disease was far advanced in 83 per cent. of instances and irremovable surgically in more than 3 out of 4 such.

Excess of mucus, chunks of poorly chymified food and (rarely) blood-clots, tissue sloughs or puree-like, evilscented fluid composed the vomitus.

Group III. Gastric Cancer in Individuals who Prior to the Onset of a Malignant Disease had Enjoyed Perfect Gastric Health

Symptomatically, the patients comprising this group satisfy the popular conception of what constitutes gastric cancer clinically. But, in even this class, wide variations in symptomatology are possible. What is commonly described as gastric malignancy is doubtless the picture presented, clinically, when affected individuals come for examination as a consequence of symptoms arising from mechanical interference to the easy, onward progress of food, constitutional evidences of a grave ailment or the accidental discovery of an abdominal tumor. In private or hospital practice, but rarely is the beginning of this form of the disease appreciated or diagnosed. As we have previously quoted, the Fenwicks state that 31 cases of gastric cancer entering their services had previously been ill for an average time of nearly 4 months, and this time had elapsed apparently before such danger signals had been observed as to indicate a serious malady. The failure to differentiate between disease entities and the symptoms associated with such is largely responsible for gastric cancer in its early progress being clinically styled "acute" or "chronic gastritis," "gastric catarrh," "achylia gastrica," "chronic dyspepsia," "liver trouble," "nervous prostration" and the like. These terms are not fanciful: they are 
such as were actually given by patients to describe the early status of their ailments. These "diseases" were in the main, reflections of the viewpoint from which their medical attendants considered their early departure from gastric health.

While it is true that mild beginnings characterize many instances of this type of carcinoma of the stomach, it is a fact that the average duration of early symptoms is of sufficient length to permit a careful analysis of the anomaly presented. Here we rarely deal with an emergency. This digestive or constitutional fault is certainly such as might reasonably warrant more than passing attention. Its salient factors are these: A dyspepsia in an individual, generally past middle age (at the so-called "cancer age"), to whom such gastric disturbance is foreign. The malfunction is usually continuous. It is early manifested by anorexia, weakness, anemia, weight loss, cachexia, pain, vomiting, hemorrhage, diarrhea or abdominal tumor. While it is possible to associate many of these symptoms with benign gastric disorders, the professional mental attitude is seriously delinquent which does not regard all gastric malfunction in individuals past the age of 40 as malignant or potentially so, until it has been definitely proved to be benign. If pursued with common sense and tact, this course is quite possible without producing undue apprehension among patients. During the past decade there has been such widespread popular education upon malignant disease that reservations in diagnosis are often considered proper and necessary.

Of the 921 cases of gastric cancer forming the basis of this study, there were but 294 instances (31.9 per cent.) where a malignant gastric ailment had occurred in patients who previously had experienced no gastric com- 
plaint. The most exhausting anamnesis failed to elicit any symptoms pointing to a disease of the stomach before an average time of approximately 7 months prior to the patients' coming under observation. Because there had been no gastric complaint previous to this average figure, it should be emphasized that one is not justified in maintaining that in such individuals there had been no gastric pathology anteceding 7 months. On the contrary, the not infrequent post-mortem or laparotomy discovery of healed ulcers or eren neoplasms in subjects who have had no clinical pointings referable to such stomach anomaly should lead us to exercise caution with regard to insisting when or how malignant processes arise in the gastric wall. The longest history of dyspepsia in cases making up this group extended over about 314 years; the shortest barely 2 weeks.

(a) Mode of Onset.-Many so-called "primary" gastric cancers have histories extending over weeks of time, where the symptom-complex closely approximates that which is our present-day conception of peptic ulcer, of the continuously active and progressive type. The clinical picture is different, however, in one important respect. To the ulcer symptom-complex are early added the manifestations of a malignant systemic poisoning. There is a hurried progression through the clinical course of "gastritis" and "ulcer" to a definitely malignant disease. With regard to time, the whole course of the ailment forms a sort of "tabloid" from which careful analysis readily segregates parts closely approximating, clinically, those stretched out over years as exhibited by that great class of gastric cancers making up our Group II. The rapidity through which these different clinical stages of the disease may be passed seems to emphasize the necessity for our 
demanding a separate and not altogether unimportant recognition of the term "chronicity" in its pathologic sense and in its time-duration application (i.e., meaning years, months or weeks) of the existence of an ailment. It is reasonably possible that the whole gamut of injury to the stomach lining, local or general gastritis, ulceration and cancer may be run in a few weeks or months. The rapidity of change occurring in experimentally produced tumors and in embryonic, undifferentiated tissue certainly would permit this conception of the development of gastric cancer. Such view explains why the mode of onset of "primary" gastric neoplasms varies widely.

The onset in the ordinary case (there is no "typical" case) is rarely acute. The transition from complete gastric well-being is commonly gradual. Arersion to certain kinds of food (meat, sweets, legumes) or beverages (beer, coffee, milk) is often an early manifestation. While the desire for some viands may be keen, the aversion to others may be marked and, for the individual, radical. Eructations, sometimes of disagreeable taste, may be troublesome from the first. Gaseous distention of the abdomen is frequently annoying. Unexplainable nausea may cause concern. Diarrhea or diarrheic stools appeared without apparent cause in 40 of our cases (13.5 per cent.). Tomiting may be the first symptom and its copiousness, odor, taste and color distressing and alarming. Pain or abdominal discomfort of some degree is a frequent indication that all is not well. It appeared early in 214 instances (73 per cent.) of this group. Sudden hemorrhage not infrequently startles a previously well individual and leads to his seeking medical care. This happened in 12 cases (4.2 per cent.), comprising this group. Again, rapid weight 
loss, with weakness and anemia may bring patients under observation.

The following histories emphasize certain features of the onset and course of the disease in cases comprising Group III.

1. Gastric Malignancy with Gradual Onset and without Severe Symptoms:

Mr. C. B.-Age 60, Swedish, farmer.

Family History.-Negative.

Past Illness.-Denies venereal; had measles. Has been constipated for 15 years, and on account of irregular dyspepsia has been taking medicine for past 2 years.

Present Complaint.-About 2 years ago began to lose strength and weight. This was very gradual until a year ago, when appetite began to fail and on account of weakness patient was confined to bed for 2 weeks. At the end of that time was able to get up and be about, but did not feel quite himself. Appetite has remained poor and for 3 months he has been on liquid diet. Food does not distress him, but more liberal diet causes vomiting about a half hour after meals. Never vomited blood. Never passed blood in stools. For past few months has had vague soreness in epigastrium but never real pain. Strength has diminished and for 2 weeks has had edema of the feet and ankles. Recently constipation has become obstinate.

Physical Examination.-Poorly nourished; moderate anemia; 20 pounds under weight; skin dry and scaly; edema almost to knees.

Chest.-Negative except for slight cardiac arrhythmia.

Abdomen.-Liver dulness extends 2 finger breadths' below level of ribs in nipple line. On palpation, a mass may be made out running from 2 finger breadths' below the ensiform cartilage to the left and just below the navel. It moves downward on deep inspiration. No adenopathy. Laboratory Examinations:

Blood.-Hg., 65 per cent. (Dare); r.b.c., 3,800,000; w.b.c., 11,600 .

Test-meal.-Total acidity, 36; free $\mathrm{Hcl}, 0$; combined acid, 18; lactic acid + ; altered blood + (benzidin test); formol index, 26 (Sorenson-Schiff method). Moderate retention. 
Microscopic Examination.-Bacilli of the Oppler-Boas type; few chains of streptococci; few small yeast colonies.

Stool.-Altered blood+ (benzidin test)-on milk diet.

Laparotomy.-High right rectus incision; carcinoma involving the greater curvature, pylorus and one-half of lesser curvature. Secondaries in the liver. Peritoneum extensively involved with masses resembling fish-eggs.

Operation.-Explored only.

Pathologic Report.-Nodule from peritoneum shows carcinoma of encephaloid type.

2. Carcinoma Developing without Previous Gastric Malfunction; Diarrhea at Onset:

Mrs. M. L.-Age 50, Norwegian, housewife.

Family History.-Negative. First husband died of pulmonary tuberculosis; three children well and strong; no miscarriages; has always performed hard manual labor.

Past History.-Ten years ago had malaria. After attacks of chills and fever, did not menstruate for 6 months. Five years ago had attack of dysentery which lasted 6 weeks.

Present Trouble.-Five months ago the patient for the first time in her life began to have trouble with her stomach. Noted gassy eructations and pyrosis, together with burning, epigastric pain coming on soon after eating. Was never quite free from epigastric pain. For past 3 months has had attacks of diarrhea coming on every 2 or 3 weeks. Two months ago began to feel nauseated in the middle of the afternoon; 6 weeks ago began to vomit every day in late afternoon; vomiting resulted from epigastric pain and bloating. Never vomited blood, but frequently vomited material eaten for breakfast.

Appetite.-Poor.

Bowels.-Rather diarrheic; never dark colored.

Weight.-Has lost 22 pounds in about 3 months, and feels much weaker than formerly.

Physical Examination.-Anemic, poorly nourished, complexion sallow, slight peripheral edema, looks tired; tongue, coated; teeth, dirty.

Chest.--Negative except for slight muscular weakness of heart. 
Abdomen.--A hard, nodular, movable mass, size of adult fist in mid-epigastrium; is somewhat tender to the touch. No gland enlargements palpable.

Pelvic Examination.-Negative.

Laboratory Examinations:

Blood.-Hg., 70 per cent.; r.b.c., 4,000,000; w.b.c., 6,200.

Test-meal.-Total acidity, 74; free Hcl, 6; combined acids, 58 ; lactic acid + ; altered blood+ (guaiac test). Retention moderate.

Microscopical Examination.-Occasional long bacillus of Oppler-Boas type; few leptothrix and short fat rods.

Laparotomy.- - High right-rectus incision; mass the size of grape fruit on lesser curvature near pyloric end. Tumor circumscribed. slight gland enlargement.

Operation.-Mikulicz-Hartman, Billroth No.2. Posterior gastroenterostomy.

Pathologic Report.-Adenocarcinoma.

3. Alcoholism; Chronic Gastritis; Umbilical Metastasis:

Mr. C. F.-Age 46, American, merchant.

Family History.-Negative.

Personal History. - Typhoid fever 9 years ago, very ill, since then has had scarlet ferer, measles and whooping cough; denies renereal disease. Was a hard drinker up to a year and a half ago, since then temperate.

Present Illness.-Gastric disturbance 1 year, previous to that says he not infrequently had indigestion, especially following drinking bouts.

Complaint.-Dull aching pain, moderately constant in epigastrium; reaches its maximum about 3 hours after meals. Has found nothing that would relieve pain except rest and romiting.

Nausea, prominent symptom, comes on after every meal and is followed by vomiting. Vomits large amounts of finely divided food, whitish in color and sour to taste. Sometimes delayed romitus. Never romited blood.

Belching almost constantly with much regurgitation of food, even on liquid diet.

Bowels.-Constipated; never blood or dark stools.

Weight Loss.- Seventy pounds in 8 months.

Physical Examination.--Moderately well developed, but poorly nourished; color rather florid. Tongue coated. 
Head, Neck and Chest.-Negative.

Abdomen.-Scaphoid, no tenderness on deep pressure in any part. On inspiration, a hard, irregular mass is felt high in the epigastrium to right of median line. Is about 4 inches long and not tender.

Laboratory Examinations:

Blood.-Hg., 60 per cent.

Test-meal. - Tube meets slight resistance at cardia with rather free bleeding. Small amount of retained food. Total acidity, 16; free $\mathrm{Hcl}, 0$; combined acids, 10 ; lactic acid, trace; Wolff-Junghans' test for soluble albumin + (300 units); altered blood + (benzidin test).

Microscopic Examination.-Oppler-Boas bacilli; few budding yeasts; streptococci and short, fat rods in chains.

Stool.-Altered blood + (benzidin test).

Laparotomy.- Stomach is involved in one mass of cancer, adherent to transverse colon and jejenum. There is a malignant metastasis to umbilicus.

Operation.--Exploratory.

Pathologic Report.-Perigastric gland shows cancer cells.

4. Slow Onsetwith Vomiting a Prominent Symptom. Abdominal Tumor Noted for 8 Months:

Mrs. A. D.-Age 46, American, housewife.

Family History.-One sister died of "quick consumption."

Personal History.-Pleurisy 5 years ago, lumbago for many years, otherwise well.

Present Illness.- "Indigestion" for past 21/2 years; this has been characterized by pain in mid-epigastrium constantly present and of gnawing character; pain transmitted to back between shoulders. Pain has been constant and not relieved by diet, medicine or posture.

Nausea.-A marked symptom, prostrating and comes any time of day or night; worse when lying down.

Vomiting.-For the past month vomiting after every meal; vomitus contains food but no blood; never bad taste; vomitus watery, never colored; relieves some of the distress; been on liquid diet but no relief.

Belches gas constantly; never sour; no relief by soda.

Constipation.-NIarked; never blood.

Flatulence.-Marked. 
Weight Loss. - Twenty-four pounds in 2 years.

Genito-urinary.-No urinary symptoms. Menopause 1 year ago.

Physical Examination.-Fairly well developed and nourished; moderate pallor.

Tongue.-Slightly coated.

Head, Neck and Chest.-Negative.

Abdomen.-Diastasis of recti muscles with dilatation of superficial cutaneous veins. Just above navel is a semilunar, freely movable mass $12 \mathrm{~cm}$. long and $5 \mathrm{~cm}$. wide, not tender; can be displaced up and down but not freely from side to side. Tenderness over gall-bladder.

Vaginal.-Perineum lacerated.

Laboratory Examinations: 7,800 .

Blood.-Hg., 70 per cent.; r.b.c., 4,200,000; w.b.c.,

Test-meal.-Resistance to tube at cardia; no bleeding. Slight 12-hour retention. Total acidity, 24; free $\mathrm{Hcl}, 2$; combined acids, 18; lactic acid +; altered blood + (guaiac test); Wolff-Junghans' test for soluble albumin + (more than 200 units). Glycyltryptophan test +; formol index, 22 (Sorenson-Schiff method).

Microscopic Examination.-Few bacilli of Oppler-Boas type.

Stool.-Altered blood + (benzidin test).

Laparotomy.-Pylorus and entire lesser curvature carcinomatous; stomach thickened, small (leather bottle type).

Operation.-Explored.

Pathologic Report.-Gland shows carcinoma.

5. Rapid Onset and Few Symptoms. Cancer in Family:

Mrs. E. S.-Age 79, German, housewife.

Family History.-One sister dead, cancer of the stomach;

1 daughter dead, cancer of the rectum.

Personal History.-Past negative.

Present Illness.-Vague gastric disturbance for 4 months.

Complaint.- Heavy feeling in abdomen immediately after eating; lasts 1 to 2 hours.

Distress Relief.-When bowels move or when belches. Few eructations, no nausea or vomiting at any time.

Bowels.-Constipated, never melena. 
Appetite.-Fair.

Physical Examination.-Well developed and moderately well nourished. Rather pale.

Tongue.-Clean.

Head and Neck.-Negative.

Chest.-Heart; slight arrhythmia.

Lungs.-Negative.

Abdomen.-Small hard movable mass $1 \mathrm{~cm}$. to left and $2 \mathrm{~cm}$. above navel. Mass painful on pressure. Examination otherwise negative.

Laboratory Examinations:

Blood-Hg., 50 per cent.; r.b.c., 2,800,000; w.b.c., 12,000 .

Test-meal.-Moderate 12-hour retention. Total acidity, 46; free Hcl, 8; combined acids, 22; lactic acid, trace; altered blood + (guaiac test); Wolff-Junghans' test + (300 units).

Microscopic Examination.-Oppler-Boas bacilli, many cocci and small type sarcinæ.

Laparotomy.-Carcinoma of pylorus and antrum extending to first part of duodenum. Roux operation.

Pathologic Report.-Adenocarcinoma.

6. Acute Onset, Severe Pain a Prominent Symptom; Carcinoma Involving Pylorus; Gall-stones:

Mr. C. L.-Age 68, American, engineer.

Family History.-Negative.

Personal History.-Usual diseases of childhood; had pneumonia 3 times, 40,14 and 7 years ago; had typhoid fever 35 years ago. Claims to have had 3 attacks of appendicitis, one 53 years ago, one 56 years ago and one 6 years ago; in bed each time for from 5 days to 2 weeks. Had 7 attacks of severe la grippe. Denies venereal disease.

Present History.-Three months ago was taken with severe cramp in lower abdomen, immediately after eating; it lasted 15 minutes. Twelve hours later again very severe cramp with nausea. No chills, fever, sweats or jaundice. Since then patient has constantly had hard epigastric ache with nausea, water-brash and eructations. Vomits immediately after eating and frequently vomitus 
contains food eaten 24 to 48 hours previously. Never vomited blood or noted dark stools.

Appetite.-Fair.

Bowels.-Constipated.

Weight Loss.-Twenty pounds in 2 months.

Physical Examination.-Emaciated, anemic. Skin dry and scaly; post-malleolar edema; no adenopathy.

Eye Reaction.-Normal. Eye grounds negative.

Tongue.-Coated.

Thorax.-Apices rather dull to percussion.

Heart.-Slightly enlarged; second aortic tone roughened. Moderate arteriosclerosis.

Abdomen.--Visible peristaltic wares running from left to right in region of navel.

Stomach.-By percussion reaches almost to symphysis. No tumor palpable. Resistance in right upper quadrant to deep palpation.

Laboratory Examinations:

Urine.-Trace of albumin.

Blood.-Hg., 40 per cent.; r.b.c., 3,060,000; w.b.c., 9,000.

Test-meal.-Marked 12-hour retention. Total acidity, 84; free Hcl, 12 ; combined acid, 3S; lactic acid+; altered blood + (benzidin test).

Microscopic Examination.-Great numbers of OpplerBoas bacilli; colonies of yeasts.

Stool.-Altered blood + (benzidin test).

Laparotomy.-High right-rectus incision. Gall-bladder 6 times normal size. Adherent to omentum and contains several large stones. Stomach dilated. Carcinoma involving pylorus and antrum with marked stenosis. Appendix adherent to anterior abdominal wall. Glands along lesser curvature carcinomatous.

Operation.--Posterior gastroenterostomy; separation of gall-bladder adhesions; appendicectomy, cholecystostomy. Pathologic Report.-Gland: carcinoma.

7. Acute Onset with Pain and Vague Dyspepsia; Rapid Weight Loss; Gastric Carcinosis:

Mr. W. B.-Age 62, German, miller.

Family History.-Negative.

Personal History.-Patient was never sick in his life 
until 6 weeks ago. Unaccountably, he began to lose appetite and to experience pain from the epigastrium into the left flank. This pain was best described as a "pulling sensation;" a week later began to have soreness in the right hypochondrium, was never severe, but has been fairly constant. Five weeks ago troubled with bitter eructations and water-brash 1 hour after eating; never vomited; appetite has been rather poor; heavy meals aggravate the pyrosis.

Bowels.-Constipated.

Strength.-Poor; tires easily.

Weight.-Has lost 40 pounds in less than 5 weeks.

Physical Examination.-Patient poorly nourished; anemic; mucous membranes pale; tongue coated; teeth poor; chest, negative.

Abdomen.-Marked peristaltic waves extending across the epigastrium to the right of the umbilicus. No tumor mass palpable. No abdominal tenderness.

Laboratory Examinations:

Blood.-Hg., 30 per cent.; r.b.c., 2,400,000; w.b.c., 13,500.

Test-meal.-Bleeding as tube passes cardia. Marked 12-hour retention. Total acidity, 32; free $\mathrm{Hcl}, 0$; combined acids, 22 ; lactic acid, trace; altered blood+ (guaiac test).

Microscopic Examination.-Numerous bacilli of the Oppler-Boas type.

Stool.-Altered blood + (guaiac test).

Laparotomy.-High median incision. Carcinoma involving the entire stomach except cardiac end. Appeared to be primary on posterior wall. Gall-bladder filled with stones (261). Extensive gland involvement.

Operation.-Gastrectomy, four-fifths of stomach removed; gastro-enterostomy; cholecystostomy.

Pathologic Report.-Adenocarcinoma.

8. Early Hemorrhage; Pain and Vomiting:

Mr. J. S.-Age 65, Swedish, laborer.

Family and Personal History.-Negative.

Comes for epigastric pain and vomiting of food and blood. Present Illness.-Three months ago moderately acute pain in high epigastrium. Pain now comes on at once after eating; lasts 3 to 4 hours. Pain relieved only by vomiting. 
Vomiting follows every meal; greater in amount after heavy meal. Almost daily vomits bright bloody fluid. Is constantly nauseated.

Appetite.-Poor; lives on milk and eggs.

Bowels.-Constipated 25 years.

Urinary System.-Negative except for peculiar odor of urine.

W'eight Loss.-Thirty pounds in 3 months. Is very weak.

Physical Examination.-Well developed and well nourished, color fair, tongue and sclera negative.

Head, Neck and Chest.-Negative.

Abdomen.-Slight, deep tenderness in pit of stomach, negative otherwise.

Laboratory Examinations:

Blood.-Hg., 60 per cent.; r.b.c., 4,000,000; w.b.c., 11,000.

Test-meal.-Dark brown liquid with small amount of retained food. Total acidity, 15; free $\mathrm{Hcl}, 10$; combined acids, 4; lactic acid, 0; altered + blood (benzidin test); Wolff-Junghans' test + (300 units of precipitable albumin).

Microscopic Examination.-Many short rods; few budding yeasts.

Stool.-Altered blood+ (benzidin test).

Laparotomy.-Ulcus carcinomatosum, anterior wall near pylorus.

Operation.-Pylorectomy, posterior gastroenterostomy.

Pathologic Report.-Adenocarcinoma.

(b) Symptomatology when the Cancer is Well Established. -Perusal of the foregoing histories demonstrates that certain symptoms are fairly common after the neoplasm has advanced beyond its incipient stage. Factors which modify clinical manifestations are the histologic type of growth, the location of the neoplasm, ulceration, perforation, the degree of involvement.

Delayed vomiting was a sign in 211 (54 per cent.) of all the cases where emesis occurred. Such vomiting was most commonly due to pyloric stenosis, malignant "hour-glass," "or obstruction at the cardia, with dilatation of the esophagus.

Appetite was "poor" in 353 instances (81 per cent.). 
It was "fair" in 70 cases ( 16 per cent.). In but 13 patients (2.9 per cent.) was appetite recorded "good" when the period of malignancy had come on.

Dysphagia was noted in 8 cases of this group (1.S per cent.). When such was present the neoplasm involved the cardia, the high lesser curvature or the fundus.

Hemorrhage.-Of the whole number bleeding in this group (92 cases), 21 instances (22.9 per cent.) of hemorrhage occurred within 1 year of the patient's coming under observation. Hematemesis was noted in 17 and melena in 4 cases.

"Occult" Hemorrhage.-Tests for the demonstration of altered blood in the feces were positive in 86 per cent. of cases in this group where the reaction was sought (vide Chapter V).

Temperature. - It has been stated that approximately one-third of gastric cancer patients have elevations in temperature during some portion of the malignant ailment (Fenwick). Ulceration, extensive metastases, perforation, sepsis of the cancer locally, or a systemic subinfection are considered causes of the increase. It is quite likely that the absorption of cancer proteid or products of its digestion may cause fever. Various observers, particularly Vaughan, have emphasized the fact that parenteral digestion of proteid by ferments in blood or body fluids may cause hyperpyrexia. It would seem that the presence of cancer proteid in the blood or lymphatic circulation, in the form of metastasizing cells, living or dead, or the products of their digestion by ferment activity, might account for fever, continuous or intermittent, in gastric cancer.

Of our cases, there were 79 instances where temperature records had been kept. In 12 cases (1.51 per cent.) temperatures above $99^{\circ} \mathrm{F}$. were recorded. The lowest 
was $99.2^{\circ} \mathrm{F}$. (a case of fungoid cancer involving the pars media); the maximum was $102.6^{\circ} \mathrm{F}$. (an instance of fulminant gastric carcinosis with extensive metastases). The average temperature of this group was $100.4^{\circ} \mathrm{F}$.

Weight Loss.-Definite information was possible in 197 cases in this group. Detailed information is given in Chapter IV. The minimum weight loss was 11 pounds; the maximum 72 pounds, and the arerage 22.7 pounds. Rapid decrease in weight was commonly noted when stenoses of the orifices of the stomach brought on obstinate vomiting, or where marked anorexia or pain on food ingestion limited nourishment.

Urine--One camnot always attribute changes in urine analysis to the nature of the disease. It must be remembered that the majority of cancer patients are past the fourth decade, i.e., at a time when faulty kidney functioning is by no means uncommon. In febrile cases, one expects to find albumin and casts in the urine, as happens similarly in febrile benign affections. Indican, urea, total nitrogen, and sulphates are said to be increased. Total 24-hour quantity and chlorides may be considerably below normal. We have definite information respecting the urine in patients making up this group, available in 141 cases. In 81 cases ( 57 per cent.) the 24-hour quantity of urine was 1,000 cc. or below. In 13 instances (9.2 per cent.) casts were noted. In 21 cases (14.9 per cent.) albumin was present alone or associated with casts.

Duration of the Disease.-Approximately 6 out of 10 cases were dead within $i$ year following their coming under observation. Not quite 2 out of 10 lived longer than 3 years and but rather more than 1 out of 7 patients were alive at the end of 5 years even when the most radical surgical procedures were carried out. 
Group IV. Gastric Cancer in Individuals in Whom Malignancy followed Periods of Gastric Disturbance of Irregular Clinical Type

There were 84 cases (9.12 per cent.) in this group. There were 38 males and 46 females. The average period of gastric disturbance preceding the evidently malignant disease was 9.1 years.

As in cases making up Group II, there were two definitely separate types of dyspepsia in these patients, namely, the first, an indigestion of the clinically benign type, and second, a superrening gastric disturbance, easily recognized as malignant. The malignant stage of the disease areraged 6.2 months.

(a) The Precarcinomatous Stage of Dyspepsia.-During an average period of more than 9 years, patients in this group have been affected with digestive disturbances suggestive of gastritis, cholecystitis, cholelithiasis, chronic appendicitis, pancreatitis or dyspepsia seemingly of functional foundation. But rarely did histories present a cleancut symptomatology. The dyspepsia was continuous in type in 12 cases. Questionable hemorrhage had been noted in 7 instances ( 8.3 per cent.).

Vomiting, usually intermittent, occurred in 30 cases (36 per cent.).

Abdominal pain was complained of by 70 patients ( $\$ 3$ per cent.). Severe colicky attacks were recorded 8 times (9.5 per cent.).

Jaundice had occurred in 5 patients.

Gall-stones had been removed in 3 instances. They were demonstrated at the laparotomy for the malignant affection in 4 other cases.

Water-brash, eructations and pyrosis occupied a prominent place in the symptomatology of the affection at this stage. They had not infrequently formed a basis for the 
early diagnosis of "hyperacidity with probable gastric ulcer," but had not been associated with other clinical evidences of such disease.

Weight loss had averaged 13.1 pounds in 77 cases, where definite data were recorded. Anemia and cachexia had been rare, except in several of the nervous patients.

Appetite was usually good, except in the "spells" of more or less pronounced dyspepsia.

Constipation was noted in 51 cases (63 per cent.), diarrhea was observed 4 times.

(b) The Carcinomatous Period.-Its onset was rarely acute. In 2 cases severe hematemesis ushered in the stage of malignancy. In the majority of cases weight-loss, weakness, anemia, anorexia, continuous dyspepsia, abdominal discomfort, vomiting and epigastric tumor came on gradually. The course of this period of the ailment was in every way similar to that forming the second part of cases in Group II or to the whole course of cases making up Group III. Only the history of the early years of the dyspepsia identified the group.

The following cases are representative of this class:

1. Gastric Malignancy Following 12 Years of Irregular Dyspepsia-Ulcer (?), Gall-Bladder (?):

Mrs. A. V.-Age 68, American, housewife.

Family History.-Negative.

Personal History.- When young had scarlet fever, diphtheria, pneumonia. Ovariotomy 6 years ago.

Present Trouble.-Up to 6 months ago had for 12 years irregular attacks of indigestion. These were characterized by abdominal pain soon after eating, flatulence and occasional vomiting of green, watery fluid. In the attacks constipation was marked. Attacks lasted from 2 to 4 weeks. Abdominal discomfort was relieved by vomiting diet and food ingestion (?).

Six months ago began to notice gradual loss in weight 
and gastric distress became more or less continuous. Vomiting came on daily soon after eating, and nausea, eructations and pyrosis were constant.

Appetite.-Poor.

Bowels.-Constipated.

Weight.-Lost 40 pounds in 4 months.

Examination.-Small, pale, poorly nourished female.

Head, Neck and Chest.-Negative.

Abdomen.-Tender to pressure throughout epigastrium; suggestion of a high transverse ridge just below the ensiform.

Rectal Examination.-Negative.

Laboratory Examinations:

Blood.-Hg., 84 per cent.; r.b.c., 4,480,000; w.b.c., 17,500 .

Test-meal.-No 12-hour retention. Total acidity, 4; free Hcl, 0; altered blood +; lactic acid (?); Wolff test +.

Stool.-Altered blood (?).

$X$-ray.-Filling defect on the lesser curvature near the cardia; malignant hour-glass.

Laparotomy.-High median incision, irremovable tumor of the body of the lesser curvature of the stomach; extensive perigastric gland involvement.

Pathologic Report.-Gland removed showed carcinoma.

2. Irregular Previous Gastric History-Ulcer (?), Pancreatitis (?), Cholecystitis (?)-with Supervening Malignancy:

Mr. E. H. A.-Age 57, Hebrew, salesman.

Family History.-Negative.

Personal History.-Negative, denies venereal.

Duration of Present Illness.-For the past 20 years has suffered from epigastric pain associated with severe diarrhea. Until 10 months ago this trouble occurred in spells. Pain was usually in the right epigastrium and was most marked 2 hours after eating. It would be relieved by vomiting, alkalies, diet or the belching of gas. The diarrhea was watery in type and frequently contained undigested food. Never passed blood.

Ten years ago began to have constant diarrhea and epigastric distress associated with loss of weight and strength. Appetite became poor and anemia developed. 
Bowels.-Four to 6 movements a day; watery and without pain.

Weight.-Sixty pounds loss in a year.

Examination.-Sallow, anemic male.

Throat, Neck and Thorax.-Negative.

Abdomen.-Scaphoid in shape; movable mass in the right upper quadrant from the mid-line to the rib edge.

Rectal.-Negative.

Laboratory Examinations.-Hg., 32 per cent.; r.b.c., $4,000,000$; w.b.c., 5,000. Small lymphocytes 25.3 per cent.; large lymphocytes, 8.7 ; polynuclears, 60.7 ; transitionals, 1 ; eosinophiles, 2.6 ; basophiles, 1.7 .

Test-meal.-No retention. Total acidity, 9; free Hcl, 0; lactic acid + ; altered blood + .

Stool.-Altered blood+.

Laparotomy.-High right rectus incision. Cancer the size of a cocoanut, involving the stomach; metastases to the liver and abdominal lymphatics.

Pathologic Report.-Gland showed carcinoma.

\section{Previous Dyspepsia, Ulcer Type; Large Supraclavicular}

Fossa Metastasis; Few Recent Gastric Symptoms:

Mr. E. K. B.-Age 61, American, traveling salesman.

Family History.-Negative.

Personal History.-Uses tobacco and alcohol in moderation.

Duration of Present Trouble.-Up to 5 years ago had dyspeptic attacks characteristic of gastric ulcer. Epigastric pain 2 to 5 hours after eating which was relieved by food intake, alkalies or whiskey; these attacks occurred in spells several times a year; 2 hemorrhages from the stomach 30 years ago; moderate constipation.

These attacks continued until about 5 years ago when dyspepsia disappeared. Patient felt well until 6 months ago when he began to feel vague abdominal distress soon after eating, experienced nausea, began to lose weight and noted a gradually enlarging tumor above the left clavicle. This tumor was examined 4 days previous to his coming under our observation and pronounced Hodgkin's disease. Under $x$-ray treatment this tumor became smaller but is again rapidly enlarging. 
Appetite.-Poor.

Bowels.-Slightly constipated.

Weight.-One year ago 200 pounds; 6 months ago 190; present, 157.

Examination:

Throat.-Hypertrophied adenoid tissue.

Neck.-Firm, nodular, tender tumor size of small grape fruit in left supraclavicular space. Tumor is fixed and below it are several smaller nodules. In left axilla lymph gland size of a walnut.

Thorax.-Negative.

Abdomen.-Rounded; tenderness on deep pressure over right and mid-epigastrium. Dulness on percussion. Greater curvature of stomach 1 f.b. below navel.

Rectal Examination.-Negative.

Laboratory Examinations:

Blood.-Hg., 50 per cent.; r.b.c., 3,600,000; w.b.c., 13,200 .

Test-meal.-Stomach tube meets resistance at cardia with free bleeding. Total acid, 62 ; free $\mathrm{Hcl}, 38$; altered blood +; lactic acid, 0; Wolff +.

Microscopic Examination.-Yeasts; sarcinæ; many diplococci and short fat bacilli.

Stool.-Altered blood+.

$X$-ray Examination.-Filling defect involving pars pylorica; moderate gastric retention.

Operation.-Block dissection of neck tumor showed secondary carcinomatous metastasis from the stomach.

Pathologic Report.-Carcinoma.

Group V. Gastric Cancer in Individuals Who Presented Few Clinical Evidences of a Malignant Process Primary in the Stomach Wall

Such cases numbered 19, or 2.1 per cent. of our entire series. This class of case is extremely difficult to recognize clinically. The symptoms are very vague. Unless tumor appears the clinical picture is apt to be that of "nervous" dyspepsia, arteriosclerosis, pernicious anemia, Bright's disease, Addison's disease, chronic gastritis, achylia gastrica, colitis, tabes or extragastric neoplasn. 
In our series there were, however, certain ear-marks of malignancy even though such did not appear to primarily involve the stomach. All the patients had lost weight. The average loss was 18.2 pounds.

Anemia was never pronounced. In only 1 case was hemoglobin below 50 per cent. and red cell count lower than $3,000,000$ cells. The color index areraged 0.72 .

Abdominal tumor was palpable in 5 cases. Its common situation was to the left of the mid-line.

Diarrhea was a symptom in 7 cases (43.7 per cent.).

Blood was noted in the stools twice. Abdominal distress was complained of in but 3 cases. In but 1 instance was it severe. Vague "uneasy" sensations were not uncommon. Unaccountable nausea or anorexia, sudden belching of gas or the regurgitation of bitter, salt, or "rotten" fluid not rarely led to the visit to a physician. Dizziness, mental confusion, shortness of breath or profuse perspiration on even moderate exertion were not uncommon complaints. Signs of mental depression, almost of melancholic grade, developed in 1 patient. Laparotomy, testmeal analyses or Roentgen examination usually renclered positive a diagnosis previously but suspicious for malignant disease of the stomach.

Some of the peculiarities of this group are emphasized in the histories below.

\section{Rapid Onset, Few Symptoms, Irremovable Carcinoma of} the Greater Curvature:

Mrs. I. M.-Age 50, Swedish, housewife.

Family History.-Negative.

Personal History. - For the past 6 weeks has had a bloated sensation in abdomen a few hours after meals. No belching, eructations, or vomiting; no nausea. For the past 2 weeks has had soreness in epigastrium and the left lumbar region almost continuously, and a week ago discovered an epigastric "lump." 
Appetite.-Fair.

Bowels.-Diarrheic during the last 3 weeks.

Weight Loss.-Twenty pounds in 1 month.

Physical Examination.-Well developed, but poorly nourished; mucosæ anemic, tongue slightly coated; chest negative.

Abdomen.-At the left costal margin a mass the size of a hen's egg; it is hard and tender and moves downward on respiration. Abdomen otherwise negative.

Laboratory Examinations:

Blood.-Hg., 70 per cent.; r.b.c., 3,600,000; w.b.c., 11,600 .

Test-meal.-Slight 12-hour retention. Gastric contents blood stained; total acidity, 14; free Hcl, 0; lactic acid +; altered blood +; Wolff test + .

Stool.-Altered blood.

Roentgen Examination.-Filling defect in pars media of greater curvature; malignant hour-glass; peristalsis absent; pylorus gaping; stomach fixed.

Laparotomy.-High right rectus incision; tumor mass involves whole of greater curvature; extensive gland involvement.

Operation.-Explored.

Pathology.-Gland showed carcinoma.

2. Primary Carcinoma of Pyloric End of Stomach; Few Symptoms:

Mr. A. S.-Age 49, Hebrew, tailor.

Family History.-Had "quinsy" as a boy. Denies venereal disease.

Present Trouble.-Has been constipated for 2 years. Four weeks ago, while bathing, noticed tumor size of a lemon in left abdomen. Since then has had sensation of tightness and pressure in the epigastrium; usually most severe 1 to 2 hours p.c. Belches gas occasionally. Thinks strength is somewhat diminished.

Appetite.-Fair.

Bowels.-Move once a week, with cathartics.

Weight.-One year ago 129 pounds (normal); 6 months ago 125 (summer weight); present, 112. 
Examination.-Thin, spare, neurotic Hebrew; skin sallow; slight edema about ankles.

Throat, Neck and Chest.-Negative.

Abdomen.-Flat; panniculus thin; no tenderness. In left navel region is a freely movable mass the size of a tangerine orange. Stomach is moderately dilated.

Rectal Examination.-Hypertrophy of prostate.

Laboratory Examinations:

Blood.-Hg., 75 per cent.; r.b.c., 4,500,000; w.b.c., 11,000.

I'rassermann.-Negative.

Urine-Negative.

Stool.-Brownish-yellow; partly formed; hydrobilirubin + ; altered blood + .

Test-meal.-Slight 12-hour retention. Contents, pale yellow; free bleeding on lavage. Total acidity, 25; free $\mathrm{Hcl}, 3$; lactic acid, 0 ; $\mathrm{Wolff}+$; altered blood + .

Microscopic.-Several groups of acid fast bacilli of Oppler-Boas type.

X-ray.-Fluoroscopy demonstrates filling defect of pylorus and part of antrum. Mass is freely movable. Stomach atonic and moderately dilated.

Laparotomy.- - High right rectus incision; large tumor involving pyloric third of stomach; glands moderately involved.

Operation.--Partial gastrectomy; posterior gastro-enterostomy.

Pathology.-Adenocarcinoma.

3. Primary Gastric Cancer with Few Symptoms:

Dr. C. H. B.-Age 59, American, physician.

Family History.-Nothing important.

Personal History.-Typhoid fever 25 years ago; very ill. Leg lost by accident. No venereal disease.

Present Trouble.-Had "nervous" strain 5 months ago. To that time had had no gastric disturbance. Four months ago began to belch a little gas and noted a slight gnawing in the epigastrium 4 to 5 hours after taking food. This was not distressing.

Appetite.-Remained very good.

Bowels.-Negative to a week or so ago, when movements became rather loose; never any blood. 
Strength and endurance remained excellent. 140 .

Weight.-One year ago $165 ; 6$ months ago 155 ; present,

Examination.-Tired looking, but well-nourished male. Tanned skin, but mucosæ rather pale.

Throat.-Negative; neck negative.

Thorax.-Negative.

Abdomen.-Deep tenderness over gall-bladder. Sensation of deep resistance over mid-epigastrium, with dulness on percussion.

Rectal Examination.-Negative.

Laboratory Examinations:

Blood.-Hg., 80 per cent.; r.b.c., 4,200,000; w.b.c., 6,000.

Urine.-0.

Stool.-Altered blood, trace.

Test-meal.-Moderate 12-hour retention. Total acidity, 8; free Hcl, 0; lactic acid, trace; Wolff + ; altered blood + ; several bacilli of Oppler-Boas type seen.

X-ray Examination.-Filling defect of pars media and posterior wall; malignant hour-glass.

Laparotomy.-High right rectus incision. Large carcinoma involving pars media; lesser curvature; posterior wall and pancreas. Irremovable.

Pathologic Report.-Gland showed carcinoma.

Group VI. Gastric Cancer Secondary to an Extragastric Malignant Process

Such instances are rare. Our series comprises 16 cases (1.7 per cent.). The disease secondarily invades the stomach either by contiguity or by metastases through the lymph stream. We have records of 5 cases where gastric cancer followed cancer of the breast, 3 instances following malignant disease of the female genitalia, 3 cases of extension to the stomach of a hepatic cancer, 2 where cancer of the gall-bladder spread to the stomach, and 3 cases (2 males and 1 female) of gastric malignancy associated with neoplasms of the colon. For reasons, often purely scientific, it is essential to differentiate actual metastases to the 
stomach from a previously existing lesion, e.g., ulcer, which has become malignant.

History of previous operation upon malignant disease of breast, pelvic organs or bowel usually direct attention toward the stomach. The course of the ailment is similar to that when the disease has occurred primarily in the stomach. Clinically it is often distressing on account of its association with recurrent tumors at the original focus.

The cases below bring out the essential features of this group.

1. Cancer of the Liver, Jaundice, Secondary Invasions of the Stomach:

Mr. C. H.-Age 53, German, railroad man.

Family History.- Negative.

Personal History.-Negative.

Present Complaint.-Patient claims that he was perfectly well until 3 months ago; then became jaundiced. This has persisted and has changed from a canary yellow to a greenish-yellow tint. Two months ago began to vomit food, lost appetite, became drowsy and sleepy and markedly constipated. Urine has been of very dark color.

Weight Loss.-Eighteen pounds in 2 months.

Pain.-Has had no pain but feeling of fullness in epigastrium.

Abdominal Examination.-Patient emaciated, jaundiced deeply, mucosa rather pale.

Bowels.-At present loose. The liver is enlarged extending downward to within one finger breadth of the navel, it is very tender. The tumor moves up and down on respiration.

Laboratory Examinations:

Blood.-Hg., 75 per cent.; r.b.c., 4,000,000; w.b.c., 14,500.

Test-meal.-Moderate 12-hour retention; total acidity, 22 ; free Hcl, 0 ; lactic acid (?); altered blood + ; formol index, 26.2 .

Stool.-Altered blood.

Laparotomy.--Large carcinomatous mass involving twothirds of the liver; gall-bladder much distended and filled 
with a whitish pus-like fluid; pancreas and lesser curvature of stomach invaded by a hard, nodular growth.

Operation.-Cholecystostomy.

Pathology.-Gland showed carcinoma.

\section{Lymphosarcoma of Colon:}

Mr. J. N.-Age 27, Swede.

Family History.-Negative.

Personal History.- - Well until 2 years ago, at that time began to complain of pain in the epigastrium which lasted 2 or 3 days and was usually followed by diarrhea. He had several of these spells during the first year and rather more than this during the last 9 months. Bowels were always constipated but he never passed blood. Abdomen not infrequently distended and at that time patient says he can hear gurgling sounds. The abdominal distention and the pains are brought on by solid foods. Two and a half weeks ago he felt a tumor the size of a hen's egg in left hypochondrium.

Weight Loss.-Twenty pounds in last 6 months.

Abdominal Examination.--Patient undernourished, anemic.

Heart and Lungs.-Negative.

Abdomen.-Hernia of the linea alba midway between navel and xyphoid. On standing a small tumor protrudes at this point. Just above the level of the navel in the left hypochondrium is a tumor the size of one's fist. It is fixed and tender on palpation. Percussion of the abdomen reveals a moderate amount of ascites. No external lymph tissue metastases palpable.

Blood.-Hg., 60; r.b.c., 3,200,000; w.b.c., 9,600.

Stool.-Altered blood + .

Laparotomy.-Low median incision; the abdomen is partly filled with free chylous fluid. There is a large adherent tumor involving the large intestine just below the splenic flexure, adherent to the stomach, several loops of small bowel run through it.

Operation.-Entero-enterostomy.

Pathology.-Gland showed lymphosarcoma. 


\section{CHAPTER IV}

\section{PHYSICAL ABNORMALITIES}

The General Appearance.-In the group of cases where gastric cancer is shown to exist only upon microscopic examination of extirpated sections of the stomach wall at laparotomy, frequently there are very few evidences of the disease on inspection. If the patient should be seen at a time when he is having acute exacerbation of his ailment, evidences of malnutrition are usually not lacking.

When we recall that the clinical appearances of such cases simulate those of chronic gastric ulcer, we can readily understand why alterations that indicate systemic intoxication of the malignant type are absent.

In instances where early cardiac or pyloric stenosis has occurred, weight loss incident to the failure of nutrition as a result of vomiting is apparent in the disappearance of subcutaneous fat, in the loose skin and a certain pallor of the mucous surfaces.

In patients where nausea is a common symptom, distress is plainly indicated in the face by constant or intermittent pallor associated with profuse perspiration. The annoyance from pyrosis is frequently reflected in the face. Belching may be constant and audible.

If pain is a prominent symptom, the appearance and attitude of the patient may be characteristic. A peculiar drawn look about the corners of the mouth, the facial expression indicating discomfort, or an unusual body posture (bent over, hands over epigastrium, lying on one side, 
etc.), may show that some departure from the normal is taking place.

When malignancy has become well established the proof that such exists may be evident to even the casual onlooker. There is a peculiar apprehensive, or again resigned, hopelessness in the general appearance of such patients. This is shown by the dull eye, the sunken cheek, and the posture. Such patients seem to partly collapse upon sitting down, or they lie in bed passively. The gait may be shuffling. Physical exertion generally leads to rapid exhaustion.

In addition to the evidence of weight loss, as shown in the scaly skin, disappearance of normal fat accumulations about the body, the sunken eyes and the scaphoid appearance of the abdomen, there are external evidences of anemia which impart a grayish-white to yellowish-tan cast to the skin. It is unusual for a patient's skin to assume canary-yellow coloration, or for it to be moist or oily, associated with the presence of a nearly normal amount of subcutaneous fat such as one sees in essential anemias. Occasionally, however, the general appearance is strikingly similar.

The mucous surfaces are often pale and dry. The tongue is generally heavily coated. It frequently shows fissures and hypertrophied papillæ. There is usually marked fetor ex ore. This is due partly to accumulation of foul material in the stomach, the presence of microorganisms and food detritus in the mouth and bad teeth. The last are sometimes strikingly noticeable.

Many instances of gastric cancer have decaying teeth and pronounced pyorrhea alveolaris. While in such cases bad teeth have existed for years, it sometimes seems as though the onset of malignancy were associated with 
rapid deterioration of the teeth, together with the appearance of pyorrhea. From mouths containing poor or dirty teeth may frequently be isolated amœbæ, flagellate protozoa, leptothrix or a multitude of pathogenic microorganisms. It would be difficult to assume that the swallowing of such foreign substances is entirely beneficial to injured gastric mucosæ.

Edema not uncommonly occurs in the late stages of gastric cancer. It is rarely general. Usually it is evident by slight puffiness beneath the eyes or about the ankles. Where anemia has developed rapidly, one can often detect so-called "false edema" behind the malleoli. We have noted only a few instances where the edema reached above the knees. In such instances it is not uncommon to find interstitial nephritis associated with the malignant disease.

External Evidences of Metastasis.-These are observed in rather definite locations. Perhaps the most common external evidence of secondary malignancy is seen in the left supraclavicular space. Here the deep (or, more rarely, the superficial) lymph glands enlarge. We have seen 1 patient in which the metastasis was as large as a mediumsize grape fruit. Usually, however, the glands are as large as marbles or walnuts. They are commonly discrete, but may be confluent. It would seem that the reason for the frequent metastasis on the left side lies in the fact that the thoracic duct empties into the subclavian vein near where this gland chain is located. Metastases are apparently directly carried to this gland chain, and implanted there quite early in the case of growths where there is abundant and rapid cellular proliferation. In many such instances examination of the mediastinal lymph spaces shows no evidence of metastasis involvement.

Where there has been metastasis to the peritoneum one 
may observe early evidences of ascites. In such abdomens, bulging in the flanks, when the patient is recumbent, may be noted. This is in rather striking contrast to the lack of subcutaneous fat and the general flatness of the abdomen. When such patient stands or lies upon the side, or assumes the knee-chest position, the filling out of the abdomen in the dependent portion may be observed.

Where there has been rather rapid emaciation, enlargement of the liver, due to secondary growths carried to it through the portal lymph stream, may be seen. We have noted several cases where small nodular infiltrations about the navel were prominent. Only rarely does one find visible evidence of metastasis in the inguinal glands.

Nervous Signs.-There has been much written about patients with gastric cancer exhibiting melancholia, insanity and other psychoses. We have never observed a case in which such were markedly present. It is true that almost every patient who appears with abdominal tumor, rapid weight loss, deficient strength and other evidences of malnutrition, is greatly concerned regarding his exact status. It is quite natural that such a person should be grievously depressed. In an individual whose temperament is rather unstable, one can readily conceive that such depression might amount to melancholia, with even suicidal manifestations. In highly neurotic Jews, mental conditions which render the patient utterly irresponsible, may be expected.

The Weight Loss.-As we have before stated, in the instances where the clinical picture is that of chronic, gastric ulcer, there may, or may not, be weight loss, accordingly as the patient comes under observation during a period of exacerbation, or when his process is quiescent. Even in circumstances where malignancy is established, 
one may see but slight physical evidences that such patients have lost much weight. Unless a careful check has been kept upon the weight, the general appearance may not lead one to suspect that there had been a pronounced decrease. This applies particularly to individuals who have been markedly over-nourished, or to patients of the large, "raw-bone" type who have not had much subcutaneous fat to lose. In the average case, however, it is possible to judge from the looseness of the clothing or from the flabbiness of the muscles that there has been weight loss.

Table 15.-Nutrition

\begin{tabular}{c|c|c}
\hline \multirow{2}{*}{ Months } & $\begin{array}{c}\text { Minimum weight loss, } \\
\text { pounds }\end{array}$ & $\begin{array}{c}\text { Maximum weight loss, } \\
\text { pounds }\end{array}$ \\
\hline 3 & 4 & 50 \\
4 & 8 & 72 \\
5 & 10 & 42 \\
6 & 8 & 100 \\
7 & 20 & 50 \\
8 & 11 & 60 \\
9 & 7 & 75 \\
10 & 25 & 50 \\
11 & 50 & 50 \\
12 & 3 & 60 \\
14 & 40 & 40 \\
15 & 75 & 75 \\
18 & 35 & 65 \\
24 & 20 & 50 \\
30 & 40 & 60 \\
36 & 20 & 75 \\
\hline
\end{tabular}

Showing Weight Loss in Gastric Cancer According to Duration of Symptoms.-(Author.)

We have instances where the disease had existed for 3 months, 6 months, 9 months and even 12 months, where the weight loss has been respectively but 4 pounds, 8 pounds, 7 pounds, and 3 pounds; in other cases where the disease has made itself evident for such periods, the weight 
loss has been $50,100,75$, and 60 pounds respectively. Table 15 shows the average minimum and maximum weight losses in individuals whose ailments have been evident for from 3 months to 3 years.

It will be noted that there is a striking variation in the rate in which cancer patients lose weight. Certain factors are responsible for this:

1. The Age at Which the Disease Occurs.-While no general rule can be formulated, it would seem that cancer patients past the age of 60 lose weight less rapidly than do those who are attacked by the disease below the age of 40. In our experience, cancer in the young has always been attended by relatively rapid weight loss and other evidences of cachexia. We have seen, however, old people lose as much as $S 0$ pounds in 6 months.

2. Failure to Take Sufficient Food. - This may be due to the loss of appetite which early appears in gastric cancer. Food desire may be greatly altered so that aversion may be manifested toward those things which have previously been eaten. As a result of this, the patient may eat very little, not knowing that with a change of diet food may be taken with comfort and relish. Constant, or intermittent, severe pain is also responsible for rapid weight losses. Many individuals are afraid to eat on account of bringing on or aggravating pain by so doing.

3. Vomiting not Infrequently Precipitates Rapid Weight Loss.-If pyloric obstruction, obstruction at the cardia, or malignant hour-glass are developed, then early vomiting or constant nausea may result. In these instances the accumulation of foul material in the stomach is a not altogether negligible factor in bringing on early cachexia.

4. Condition of the Mouth.-Poor teeth, missing teeth or sore mouth may render it impossible for a patient to 
properly masticate food, or to take food which has definite strength-giving composition.

5. The Poor-appetite Habit.-Many individuals with gastro-intestinal troubles are literally "dieted to death." In the attempt to work out various novel theories regarding nutrition and metabolism, well-meaning individuals not infrequently suggest dietetic regimes which are entirely foreign to the human family. Some of these are sufficiently distasteful to "gag a buzzard." If the individual has a long precancerous, gastric history (frequently that of gastric ulcer) the poor-appetite habit may be already well developed at the time when the cancerous process begins.

6. Mental Attitude.-It is not uncommon to find individuals who, upon learning that there is a possibility of a malignant disease developing, or having developed, assume such an attitude of general disinterestedness in life and the things which pertain to it that not only are dietetic principles unheeded, but the general condition-the care of the teeth, skin, kidneys, bowels and the like-is entirely neglected. In such individuals, it seems that weight loss progresses very rapidly.

\section{THE ABDOMINAL EXAMINATION}

General Considerations.-Experience has impressed upon us the necessity of properly preparing patients for abdominal examination, and also of carrying on such investigation under favorable circumstances.

Whenever possible, abdominal examination should be conducted with the hollow viscera empty. If the stomach and bowels are filled with feces and gas, even those most expert in diagnosis may fail to detect abnormalities. Many a so-called clever diagnosis has its foundation upon 
thorough gastric lavage and vigorous catharsis previous to the carrying out of the abdominal examination.

Our practice is to administer 2 ounces of castor oil, in a half glass of beer, malt-extract or acid fruit-juice, 12 hours before the patient appears for study. If the bowels have not moved freely after this interval, an enema of soap-suds and warm water is then administered. A few obstinate cases require more potent enemata. We have found that in such instances the old-fashioned glycerin and salts clystra usually answers very well. But rarely has it been necessary to use so formidable an enema as that composed of milk and molasses, warmed (milk 600 cc.; New Orleans molasses 400 cc.; mix thoroughly; warm to $37^{\circ} \mathrm{C}$.; inject in the usual manner). If the lower bowel is clogged with hardened feces, this enema is generally effective where other types have failed.

Just previous to the abdominal examination, the patient's stomach is emptied with the aid of a stomach tube of large caliber. After a fruitless search for a tube that would enable us to free the stomach of the foul mixture which it often contains where the pylorus is stenosed, or when hourglass contraction has occurred, we devised the stomach tube described in Chapter V. Lavage with such tube, using 2 or 3 liters of warm normal salt solution, usually empties even the most dilated or malformed stomach in a short time.

Following lavage, the patient should be stripped of all clothing, at least to the waist. We prefer to have patients appear for examination clad in only a thin, readily removed night-shirt or kimono. Some improperly educated folkphysicians as well as laymen-still believe that dependable abdominal diagnoses can be made without exposing the area of suspected disease. This applies especially in the 
case of females. Yet such modestce regularly display their anatomical perfections, wherever these may exist, to the eyes of the multitude in order to partake of the psychic comfort supplied by the "fox trot" or the "bunny hug." Proper abdominal examination cannot be made unless the abdomen can be seen, felt and listened to. The ingenious American union-suit and its equally unsanitary rival, the fashionable corset, coupled with the "don't-letme-annoy-you" attitude of the popular attendant, with a medical license, are often responsible for gastric cancer reaching hopelessly inoperable stages before such is even suspected to exist.

Nervous or excitable individuals sometimes require a quieting potion before being examined. Thirty grains of sodium bromid in a wine-glass of angostura bitters usually aid these unfortunates. An attitude on the part of the physician that inspires confidence is better therapy, however.

We prefer routinely to examine the patient as he lies upon a table with his feet toward an unshaded window. His head should rest upon a small, firm pillow. The room should be clean, quiet, warm and not draughty. The physician's hands should be warm and unsoiled. In this age of motor cars, both these cautions are appropriate.

A general physical examination should always precede the special study of the abdomen. The type of "abdominal specialist" who can tell that an enlarged liver is not due to a tricuspid regurgitation or a nodular omentum not secondary to a pulmonary tuberculosis, without examination of the primary focus of disease, is fortunately becoming an extremely rara avis. All the information that it is possible to derive from chemic, microscopic, serologic or roentgenographic investigation should supplement the physical examination. 
1. Inspection may return no facts of clinical value in those cases where early carcinomatous change exists in the border of a peptic ulcer. Unless in severe pain, the patient lies at ease. The skin may be of good color and sleekly stretched over normal amounts of subcutaneous fat. The normal, bony boundaries of the belly may be well hidden. Cancerous change cannot exist long, however, before weight loss and cachexia are apparent. The fat of the abdominal parietes is usually diminished early. As a consequence, pale, dry, loose, wrinkled skin covers the belly and sags down in more or less marked folds in the flanks. Its hairy adnexa are often dry and brittle.

The rib margins and the pelvic bones may become strikingly prominent. If an extensive neoplasm is present, degenerative changes take place in the muscle layers of the abdominal wall, resulting in their loss of tone. As a consequence, the belly appears flat or scaphoid. Not rarely a scaphoid or flat abdomen may have local areas of prominence due to visible gastric tumor, enlarged liver or pancreas, or metastases to the navel, omentum, or inguinal lymph nodes. Where free fluid is present in the peritoneal sac, but the peritoneal sac not filled, bulging in the flanks may be observed. This usually shifts with change of the patient's position. If there is a great accumulation of fluid, the abdomen may appear rounded. The navel may bulge.

Should marked emaciation have taken place, pulsations of the abdominal aorta are readily observed. They were recorded in 129 instances (15.01 per cent.) of our series.

Local abdominal prominences are due to the presence of a primary tumor, its metastases, encapsulated collections of fluid or air, or visibility of normal-size, solid organs as a result of extreme emaciation (liver) or hardened feces. 
Respiratory movements cause certain alterations in the abdominal contour. Tumors of the stomach and intestines usually change their position, and frequently their shape during respiration. Such prominences as occur from enlargement of the pancreas, abdominal aneurysm, retroperitoneal sarcoma, hypernephroma or various nonmalignant tumors of the abdominal wall itself, may show no appreciable alteration during inspiration and expiration. Not infrequently, in an emaciated person, ripples or indefinitely outlined shadows are produced, by vigorous respiratory efforts, when free fluid exists in the peritoneal sac.

Change of position causes important alterations in the appearance of the abdomen. Frequently when a patient lies upon either side, stands, or assumes the knee-chest posture, local or general bulging may occur from intraabdominal tumors or fluid. This is a valuable maneuver and should not be neglected in doubtful cases.

Visible peristalsis of stomach or intestines, or both, is not rarely made out when obstruction occurs (complete or partial) at the pylorus or in the small bowel. Occasionally where there is a secondary or primary malignant involvement of the transverse colon, local out-pouchings, usually on the proximal side of the obstruction, may be made out. While these do not as a rule show active peristaltic waves, the variation in size of the bulging at different examinations suggests the complication.

When a malignant obstruction occurs at the outlet of the stomach the dilatation of the viscus is usually prompt and may be very extensive.

In the early stages of gastric cancer before there has been secondary degeneration of muscle fibers in the stomach wall the peristalsis remains active. A vigorous attempt 
is made to force gastric contents through the narrow outlet. If the abdominal wall is not too thick, one readily perceives rhythmie waves passing from the left rib edge across the epigastrium. Such waves are usually more evident toward the pyloric region. They may occur so vigorously that the pylorus is brought forward toward the anterior abdominal wall and may appear as an evanescent "phantom tumor."

Kussmaul first described this phenomenon in a case of non-malignant pyloric stenosis. It appeared in 12.2 per cent. of our cases where the pylorus was extensively involved. When the obstruction is marked, as in early scirrhus cancer developing in the edge of a chronic annular ulcer, reverse peristaltic waves may be observed. These pass from the right to the left across the epigastrium. They are rarely so well evidenced as are peristaltic waves in the normal direction. In a good light, with the observer's eyes at the level of the stomach, they may be seen to develop in the right upper quadrant and fade away indefinitely toward the mid-or left epigastrium. When seen they always indicate a marked grade of pyloric stenosis. We have observed reverse peristalsis in 9 cases. Sometimes visible peristalsis may be brought out by gently tapping the abdomen or by several rather sudden "dipping" movements upon the epigastrium.

If the obstruction occurs-and this is very uncommonin the small intestine, rather slowly forming, transient swellings, resembling the movements of an angle worm, may be made out in the low epigastrium or below the navel. It has been stated that such movements assume characteristic patterns depending upon the part of the small bowel obstructed. We have never been able to satisfy ourselves that these tumor patterns had any special diagnostic sig- 
nificance with regard to enabling us to accurately prophesy where the obstructive point would be at laparotomy.

Enlarged lymph nodes may be observed in the superficial inguinal chain. They rarely attain so great a size as do metastases to the glands of the neck or the axilla. Sometimes a metastasis occurs directly to the navel. This usually results in a retracted, malformed, fixed umbilicus. It occurred in 59 (6.4 per cent.) of our cases. Very rarely one sees a surface ulceration of such a metastasis. This is not apt to be extensive, but if secondary infection takes place, a granulomatous tumor may result. We have seen several instances where a perforated, anteriorwall ulcer resulted in accumulation of pus in the region of the navel. Such may closely simulate a metastasis from gastric malignancy.

2. Palpation.-From this maneurer we gain the greatest amount of information regarding the abdomen in malignant disease. One should guard against hasty and vigorous palpation. The patient should be accustomed to light movements, pressure of the fingers, or the flat of the hand, before attempts are made to palpate deeply lying structures with the tips of the fingers. Particularly in the case of the abdomen, where the history of the patient is not clear, or has not been obtained, or where it is suspected that such a complication as hemorrhage or perforation has taken place, light palpation is an essential requisite for safety. It is well to accustom the subject to pressure of the hand over areas in which the disease is not suspected, before exploring disease foci.

The tension of the abdominal wall in well-established malignancy is diminished unless there has been extensive involvement of the peritoneum with or without the accumulation of free fluid. In early malignancy, 
where a previously complicated ulcer is undergoing cancerous change, the abdominal wall tension may be normal or even increased locally or generally. This is particularly the case where a recent hemorrhage has occurred, perforation of an ulcer has taken place, or where great pain results in the patient's unconsciously tightening the abdominal muscles. In such event it is almost impossible to successfully examine the intra-abdominal organs without the patient being given an anesthetic, or unless he has lain for from 15 minutes to $1 / 2$ hour in a bath tub filled with water above $371 / 2{ }^{\circ} \mathrm{C}$. The latter diagnostic maneuver is one of a few real advances in abdominal examination that has been made in this country during the past 15 years. Its use has been emphasized particularly by Dock. We have found it an almost invaluable aid in the examination of hypersensitive individuals, or in instances where perforation or obstruction have been suspected. After the patient has been in the hot water for the required time, the abdomen can be examined while he is still submerged, or immediately after he has been removed from the bath, dried and placed upon an examining table in a rather warm room. We have never found it necessary to administer a general anesthetic in order to facilitate an abdominal examination. There may be cases, however, in which such a procedure is entirely justifiable.

The palpating hand in gastric cancer usually notes at once the temperature, dryness, scaliness and looseness of the skin covering the abdomen. Not infrequently, due to the rapid disappearance of subcutaneous fat, the skin may be raised by the fingers several inches above the level of the abdomen. It falls back slowly and in loose folds due to the diminution of its elasticity. Occasionally, between the skin and the muscular wall, or superficially in the mus- 
cular wall, one is able to feel small nodules or even tumors. These so-called "Nélaton" tumors are not infrequently confused with the tumor resulting from gastric malignancy. Lipomata are rarely delimited over the abdomen in this disease. Infiltration of the navel can be usually well made out if it is present. The navel remains more or less stiffened, fixed and adherent deeply. Sometimes inguinal glands that cannot be seen are palpable. They are rarely tender to pressure. The muscular wall in even moderately advanced cancer is loose and lax. If much weight has been lost it is apt to be very thin and the deeper structures easily made out through it.

In instances where an accumulation of fluid has distended the peritoneal sac, the muscular wall while evidently thin is stretched and tight. Such abdomens may be tender if there has been a very extensive accumulation of serum. Unless there is very great distention of the abdomen by fluid, or unless the fluid is accumulated in small, well walled-off pockets due to the extensive involvement of the omentum and mesentery, one can usually demonstrate by tapping, fluctuating waves of greater or less definiteness.

Deep palpation of the abdomen causes pain in the majority of instances. As a general rule, the epigastric distress on palpation is greater in early cancer developing upon ulcer, or in primary cancer which has later ulcerated, than it is where there has been an extensive or rapid growth of the neoplasm. In instances where a recent hemorrhage has occurred or perforation with inflammatory change in the peritoneum has taken place, the pain upon palpation may be as marked as in the acute abdomen due to other diseases (appendicitis, gall-stones and the like).

If there has been much emaciation, the normal organs may be palpated with greater ease than usual. This 
applies especially to the liver, kidneys, the abdominal aorta, the bowels or the pancreas. Some of these may be invaded by secondary growths.

The most important palpatory sign in gastric cancer is the determination of the presence or absence of an abdominal tumor associated with the stomach.

\section{THE ABDOMINAL TUMOR}

(a) Incidence.-In the observation of 150 cases clinically diagnosed gastric cancer, Osler and MacCrae report the presence of palpable tumor in 76 per cent. The Fenwicks studied 154 instances of the disease. In 69 per cent. there were definitely demonstrated nodules; in an additional 8 per cent. "ill-defined tumor" or "sense of resistance" were recorded. Earlier investigators (Brinton, Lebert, Leube, Hahn) place the tumor frequency at from 80 to 86 per cent. In a recent study of 1,000 cases clinically, gastric cancer (only 26.6 per cent. came to laparotomy) Friedenwald reports the presence of recognizable abdominal ridge or mass in 71.9 per cent.

The above figures exhibit a range of about 17 per cent. The stage during which the disease came under observation, and the skill possessed by the individual examiner, doubtless account for the seeming discrepancy in tumor incidence.

Of the 921 instances of operated and pathologically demonstrated gastric cancer making up our series, abdominal tumor or ridge was palpated in 609 (66.1 per cent.). From this analysis all such doubtful things as "ridges," "indurations" or "resistances" have been excluded.

There were 312 cases (33.7 per cent.) in which abdominal tumor was not recorded as being palpable. One source of failure was undoubtedly the earliness with which some of the cancers were diagnosed. Where the proof of gastric 
malignancy rests upon microscopic examination of extirpated tissue, only rarely is it possible to demonstrate tumor before laparotomy. Other reasons varying the possibility of tumor recognition are: the care with which the abdomen has been palpated; the skill of the examiner; the preparation of the patient; the position in which he has been studied (examination in the knee-chest, lateral or sitting positions, with or without the added advantage of deep breathing or the hot bath, often aid in the discovery of an abdominal mass); the relative accessibility of the growth (neoplasms near the cardia, at the fundus and on the posterior wall are delimited with difficulty); the type of growth (encephaloid cancers, confining themselves largely to the submucosa, are not so readily palpated as are localized, nodular, fibrous tumors); the tension or thickness of the parietes; the pain caused by palpation and the presence of metastases (tumor of the liver, omentum or colon or the existence of ascites).

(b) Position of the Tumor.-In 515 instances ( 85.7 per cent.) the palpable nodule or mass was located in the epigastrium proper. In 86 cases (13 per cent.) the growth

Table 16

\begin{tabular}{|c|c|c|}
\hline Location & Number of cases & Per cent. \\
\hline Right upper quadrant. . & 199 & 32.6 \\
\hline Left upper quadrant... . & 165 & 27.1 \\
\hline Mid-epigastrium..... . & 73 & 11.9 \\
\hline "Pit" of stomach..... & 47 & 7.7 \\
\hline Epigastrium, general.... & 31 & 5.1 \\
\hline At navel............. & 27 & 4.4 \\
\hline Right of navel........ & 38 & 6.2 \\
\hline Left of navel.......... & 21 & 3.4 \\
\hline Below navel.......... & S & 1.3 \\
\hline Total.... & 609 & 99.7 \\
\hline
\end{tabular}

Position of Abdominal Tumor in Gastric Cancer.-(Author's Cases.) 
was found in the region of the navel or at about its level. But 8 times (1.3 per cent.) was the tumor below the level of the navel. Table 16 gives in detail the locations of the growth with the patient in the dorsal position.

(c) Relation of Position of Abdominal Tumor to Part of Stomach Involved.-Our study shows that in 66.7 per cent. of instances the neoplasm involved the pylorus, antrum and the lesser curvature. Approximately 8 out of 10 such growths were palpated before laparotomy. In 12 per cent. the greater part of the stomach had been invaded. More than 9 out of 10 of these were palpable. The posterior wall was the seat of the disease in 9.3 per cent. In this group, tumor could be felt externally in but 5 out of 10. The anterior' wall was involved in 2.3 per cent. These growths were palpable in 9 out of 10 instances. In 3.5 per cent. of our cases the growth was at or near the cardia. But 3 out of 10 such tumors were palpable. The greater curvature was involved in 2.3 per cent. and 6 out of 10 such tumors were delimited through the abdominal wall. None of the tumors invading the fundus were palpable. Some part of the multiple tumors forming 2.9 per cent. of our cases was palpated in more than 9 out of 10 instances.

Factors other than anatomic location of the gastric neoplasm which modify its ease of palpation have already been enumerated. In addition, we would emphasize that very frequently tumors are rendered palpable and even visible by inflation of the stomach with air or gas, either by means of a Davidson double-bulb syringe through a stomach tube, or by the administration of 4 grams of tartaric acid in $50 \mathrm{cc}$. of water, followed by 4 grams of sodium bicarbonate in $50 \mathrm{cc}$. of water, or by filling the viscus with fluid. It should be likewise emphasized here 
that distention of the stomach or filling it with liquid not infrequently cause a tumor already palpable to disappear. This is particularly apt to occur in the event that the neoplasm involves the posterior wall, greater curvature or the antrum. It is good practice to examine all stomachs suspected to be the seat of malignant growths both before and after air distention, or filling with water.

(d) Size of the Tumor.-Ulcera carcinomatosa may be felt only as narrow finger-like ridges or small nodules, both before or after gastric filling. Other cancers range in size at palpation from that of a common marble to as great as a child's head, a medium-size squash or a discus. The tumor may fill the entire epigastrium and extend well into the left flank. It may be so large as to simulate a leukemic spleen. If invasion of the liver, pancreas or adjacent hollow viscera has taken place, a huge, irregular, plaque-like mass may occupy the entire upper epigastrium. Rarely does the growth extend below the navel, but if the omentum is involved, the tumor may be so extensive as to cast doubt upon its being primarily gastric in origin.

(e) Tenderness of palpable tumors varies in degree from vague discomfort to actual pain. With the exception of ulcera carcinomatosa it is uncommon to have gastric cancer patients experience so much distress upon palpation as do those affected with benign peptic ulcer. Even where malignant perforation has taken place, the resultant peritoneal invasion is accompanied by less pronounced evidences of inflammatory reaction than where this complication occurs in benign affections.

There are but few gastric cancers which fail to exhibit some tenderness upon palpation. Of 572 instances where definite information is available in our series, 34 patients (5.9 per cent.) complained of no discomfort upon the manual 
examination of the growth. Of the 538 cases remaining, in 401 instances (70.1 per cent.) there was some degree of discomfort exhibited; in 137 cases (24 per cent.) palpation was noticeably painful. Of our entire series of 921 cases furnishing the basis of this study, there were 122 instances (13.3 per cent.) where some grade of perforation existed at laparotomy, and an additional 81 cases (8.8 per cent.) where the serous surface of the stomach had been definitely involved in the malignant disease. From a consideration of these figures, it would seem that protected perforation or peritoneal invasion without perforation may be present and their existence not be recognized even upon careful abdominal palpation.

(f) Mobility of the Abdominal Tumor.-Factors influencing the demonstration of the mobility of the palpable tumor in gastric cancer are: the histologic type of tumor; the duration of its growth; the position it occupies in the wall of the viscus; its relation to complications (perforation, adhesions, contiguous extension, fluid in the peritoneal sac); the position in which the patient is examined; its behavior when the stomach or colon is filled with air or fluid; the thickness of the abdominal parietes and the respiratory movements.

While the majority of gastric cancers exhibit some freedom of movement, in only 437 instances ( 71.7 per cent.) of our cases was it possible to definitely move about the tumor with the palpating fingers. The remaining growths were either located in relatively fixed parts of the stomach or had been more or less immobilized during their development.

It is of considerable importance to demonstrate mechanical mobility (i.e., by the fingers) of gastric neoplasms, inasmuch as those in which the greatest freedom of move- 
ment is demonstrated are generally most successfully treated surgically.

The histologic type of the neoplasm qualifies its freedom of morement. Cancers dereloping in ulcer edges, or cancers rich in fibrous elements in which ulceration has occurred through surface necrosis are apt to be more mobile than so-called "primary" gastric neoplasms of the medullary or colloid types. It will be recalled, moreover, that 2 out of 3 tumors are located in the pyloric third-a portion of the stomach which, normally, has the greatest range of displacement. In this part of the stomach the muscle layers are also densest. It would seem that such might interpose a strong barrier to the rapid, external spread of the discase. General inrolvement of the stomach by a tumor of the clinically, scirrhus type, permits freedom of the viscus for the longest time. Extensive perigastric lymphgland invasion may limit such mobility.

The duration of the disease affects the mobility of gastric tumors only in a general way. We have already emphasized that cell proliferation may occur with astonishing rapidity in gastric cancer. Consequently, we have numerous instances where malignant symptoms have existed for less than 2 months, and yet the resultant tumors were definitely anchored by secondary involvement of adjacent structures. On the other hand, certain tumors may exist (particularly in the pyloric region, or upon the lesser curvature) for as long as nearly $1 \frac{1}{2}$ years, and upon palpation and at laparotomy be shown to be entirely free. Of tumors where the evidently malignant discase had existed for from 6 to 12 months, but 22 per cent. were mechanically mobile. In instances where a long precancerous history is obtained, not rarely the complications occurring during that period early limit mobility of the supervening tumor. This is very 
likely to be the case if the early gastric history has been that which clinically we recognize as peptic ulcer.

The position which a neoplasm occupies in the gastric wall greatly influences its freedom of movement. Carcinomata in the pyloric third of the stomach exhibit the greatest degree of possible displacement. Tumors of the anterior wall or of the greater currature long remain free. Growths upon the lesser currature proximal to the antrum remain relatively freely mobile, but on account of their tendency to metastasis to the pancreas, liver and adjacent thickly grouped lymph glands, they may quite early become only limitedly movable. Tumors of the posterior wall early become fixed by reason of extension to surrounding organs (especially to the pancreas). The anatomical limitation of movement at the cardia and fundus account for tumors in such locations being the least mobile of all gastric neoplasms.

Complications may limit the mobility of a tumor at any stage of its progress. Such limitations may occur very quickly. Acute or chronic perforations, contiguous extension to adjacent viscera, perigastric lymph-gland involvement, inflammatory adhesions or the rapid accumulation of free fluid in the peritoneal sac may immobilize a gastric cancer. Occasionally, such complications convey greater mechanical mobility-as, for example, when free fluid "floats up" a tumor, or when an adhesion to the bowel or omentum permits the dragging about of a stomach growth.

The degree of mobility of some gastric neoplasms is varied accordingly as palpation is carried on with the patient in different positions. Not infrequently a growth which was not palpable with the patient in the dorsal position, or was only indefinitely palpable or movable, becomes readily rec- 
ognizable if he is placed in either lateral or knee-chest posture. Tumors of the body or fundus may be shown to be mobile by having the patient sit or stand after the location occupied by the growth in the dorsal position has been previously outlined. Placing the subject in the Trendelenburg position occasionally causes tumors of the upper epigastrium to move upward beneath the liver, sternum or rib margins or to be lost through the change of position of free peritoneal fluid.

When the stomach or colon is distended with air or gas marked alterations in the location, the shape or size of gastric cancers may become evident. Upon gastric inflation we have seen carcinomata displaced as far as 9 inches from the place where they were palpated with the stomach empty. Pyloric tumors may travel from the left, upper quadrant to the mid-epigastrium, the right epigastrium, the region of the navel, the left hypochondrium, and rarely to the suprapubic region. We have observed one male pátient in whom an immense, dilated stomach permitted the pyloric cancer to drop into the pelvis upon gastric inflation. In female subjects with low-lying stomachs, normally, the pyloric tumor is usually displaced to a lower point upon inflation than is the case in the average male patient. Tumors of the lesser curvature, fundus or greater curvature are often displaced as much as 5 inches upon gastric inflation. Not infrequently the rotation of the distended stomach permits the ready palpation of a tumor which had before been indefinitely recognized. While gastric inflation usually increases the size of a palpable mass, there are instances where a tumor is barely felt or even lost altogether after this maneuver.

Most commonly, air inflation of the colon (through a rectal tube) displaces gastric cancers upward or to the right. 
A low-lying transverse colon adherent to an involved omentum, or to the gastric tumor itself, may drag down the neoplasm upon colon being inflated. Colon distention may push up under the edge of the ribs or beneath the margin of the liver, tumors which had been both visible and palpable.

The successive inflation of the stomach and the colon is an extremely valuable procedure in the differential diagnosis of obscure abdominal neoplasms.

The thickness of the abdominal parieties may vary the ease with which gastric tumors are palpable to such extent that one may or may not be in doubt regarding the mobility of such.

Respiratory movements displace practically all tumors of the gastro-intestinal system lying above the navel. Inspiration usually forces downward, or downward mesially or latterly, cancers of the pyloric third, the lesser curvature, the anterior wall or the greater curvature of the stomach. The extent of the displacement rarely exceeds 3 inches. Tumors of the fundus, high lesser curvature or the cardiac region may become palpable following deep inspiration. Inspiration not infrequently forces the stomach forward when the patient lies in the dorsal position, so that a tumor of greater extent may be felt at the end of deep inspiration than when the subject is in expiration or breathing quietly.

If at the end of inspiration a tumor can be held down while expiration takes place, the possibility of adhesions to or contiguous involvement of such solid organs as the liver, spleen, or left kidney is less than if the expiratory movement carried upward the palpable epigastric tumor. Such diagnostic maneurer does not always indicate the operability or the inoperability of a gastric cancer. Extensive, deeply lying adhesions (to pancreas, retroperitoneal tissues, 
bowel) or widespread invasion of the lymphatic system may occur wholly irrespective of the degree of mobility of the primary focus in the stomach.

Gland Involvement.-The observation that enlargement is taking place in groups of surface lymphatics has great value in indicating methods of therapy. Where there has been malignant invasion of superficial lymphatics, it can hardly be expected that surgical procedures are of any permanent worth, excepting in rare instances where operative measures are carried out for the relief of mechanical obstruction. The enlargement of such gland groups as those lying above the left clavicle (Virchow's gland), the inguinal nodes, about the narel or lying in the cul-de-sac posterior to the bladder, clearly indicates that the disease has been scattered far beyond its source of origin in the stomach, and it is but a question of time before fatal termination will result. Careful palpation should be carried on in a systematic manner in an effort to recognize these lymph-gland metastases. Sometimes the inguinal nodes are but slightly swollen. They may be as large as common marbles. They are usually the last of the lymphgland chains to show involvement of intra-abdominal malignant disease. The glands are as a rule discrete. They are rarely tender to the touch, and we have never seen a case where infiltration from such glands occurred in the surrounding or adjacent structures. We have never seen an instance of ulceration or necrosis in malignant involvement of such superficial lymph nodes. Invasion of the lymph tissue in the neighborhood of the navel is rather rare. It usually occurs when there has been quite active peritoneal involvement.

"Blumer's shelf" is not an infrequent site for the location of pelvic metastasis in malignant disease of the stom- 
ach. Upon rectal examination one is able to outline a small shelf-like or pouch-like projection posterior to the bladder. In a normal individual this is free, but where an extensive carcinoma has involved the stomach and freely metastasized, one can not infrequently delimit at the tip of the examining finger one or several discrete nodules lying in this pouch. Metastases to this location seem to occur with more frequency than to other lymph-gland areas. This is probably due to the fact that with the patient in an erect position cancer cells are carried into the pelvis along the peritoneal surface by force of gravity. There may be some other reason why there is an apparent predilection for gland invasion at this point.

We have never seen a case, where enlarged glands were palpated as "Blumer's shelf," that was operable, i.e., in the sense of permanent relief, even by the most radical resection. In our series of cases metastasis to this region was noted in 16 per cent.

Enlargement of the supraclavicular glands might logically be considered at this point. We noted such enlargement in 11.5 per cent. We have already mentioned why it is that gland metastases from cancers in the stomach occur with rather striking frequency above the left clavicle. We have noted but a few instances where coincident or independent enlargement of glands in the right supraclavicular space could be demonstrated. We have never seen an instance where the malignant invasion of these glands occurred without the primary focus being beyond the hope of permanent relief by surgical measures.

Another evidence of extensive gland involvement by cancer is shown upon palpation by the presence of free peritoneal fluid. The cancerous intoxication or irritation usually results in rather rapid accumulation of free ab- 
dominal fluid. Palpation frequently enables one to roughly estimate the volume of such exudate by noting alterations in the local or general laxness or tension of the abdominal wall. The character of the abdominal fluid varies. It is usually thin enough to permit of its rapidly shifting from one part of the abdominal cavity to the other. Further considerations of abdominal fluid are given in Chapter VII

A palpable liver may result from secondary involvement of that organ through the lymph stream or contiguous involvement from the primary tumor. When the liver can be well outlined by the palpating fingers, either along its entire lower margin, or locally at any point, one can safely say that the primary growth is irremovable. In our experience the liver was palpable in 23 per cent.

Lymph glands about the pylorus or the lesser curvature can occasionally be felt where the disease is extensive, where the parietes is not too thick, or where the presence of free fluid does not interfere with palpation. In certain instances these glands may be largely responsible for pyloric obstruction.

Where pyloric obstruction occurs early, while the peristaltic activity of the stomach is still vigorous, during palpation the stiffening and increased tension of the gastric wall may be felt beneath the palpating hand.

It is perhaps important here to emphasize the above seven signs of inoperability of gastric neoplasms. They are: evidence of gland enlargement above the left clavicle, Blumer's rectal shelf, to the umbilicus, local or general increase in size of the liver, presence of free peritoneal fluid, enlargement of the inguinal lymph nodes, and palpable lymphatic metastases about the pylorus or along the lesser curvature.

Even if the primary tumor is not itself large, or even though it may be freely movable, the discovery of any or 
all of the above external evidences of the spread of the disease seriously limits the prognosis from the various types of surgical operation.

In all these instances where lymphatic involvement is determined an attempt should be made to remove one of the enlarged nodes for the purpose of microscopic study. It is usually quite possible to remove one of these glands with the aid of a simple local anesthetic (ethyl chlorid or a 2 per cent. cocaine solution). Examination of the tissue removed may be rapidly made with the aid of an apparatus for cutting frozen sections. These can be readily stained with Unna's polychrome męthylene blue. It should be emphasized here that the judgment of an expert is required in many instances to determine the histologic changes occurring in these removed lymph nodes. Operative procedures should never be neglected solely upon the evidence furnished by the examination of extirpated glands.

Percussion.-This diagnostic maneuver is of but limited value. The greatest service it renders is in the determination of enlargements of solid organs (liver, spleen), the demonstration of the amount and mobility of peritoneal fluid, and occasionally in enabling one to form an approximate estimation of the size of the gastric cancer. Sometimes it is possible to outline a dilated or distended stomach by percussion. When such gastric limits are checked by operative procedure or $x$-ray examination, one generally discovers that the percussion outlines only roughly estimate the actual size or even the position of the viscus.

There are certain cases where no epigastric tumor can be definitely palpable, and yet percussion enables one to delimit areas of dulness in certain parts of the abdomen where one suspects that a neoplasm exists.

Percussion should be carried on with the use of both light 
and heary strokes. Not infrequently where light percussion demonstrates tympany, deeper percussion results in highpitched tones that suggest airless tissue lying below a gas-filled hollow viscus.

In cases where the peritoneal sac is not too greatly distended with fluid, one can delimit areas of dulness and tympany that frequently change their outlines upon the subject's assuming rarious positions.

If the accumulation of fluid is great and the gastrointestinal tract is empty, the entire abdomen may lack resonant tones upon light or even deep tapping.

Occasionally, hollow viscera are floated upon moderate accumulations of free peritoneal fluid. As a result, the major portion of the abdomen may be tympanitic to percussion with the patient in the dorsal position. In such instances, however, it is extremely uncommon not to find dulness in the flanks or in the dependent portions of the abdomen when the patient sits up, stands erect, or assumes the knee-chest posture.

We have frequently observed an interesting, and we believe a hitherto undescribed, percussion sign in cases where cancer involves the fundus or the anterior wall of the body of the stomach.

When the patient is in the dorsal position percussion of Traube's space is not uncommonly dull instead of nor'mally tympanitic. Examination by percussion of the same area at the end of deep inspiration, or upon the patient lying on the right side, or standing, sometimes results in the disappearance of the dull tones upon the percussion of Traube's space and the appearance of characteristic tympany. This sign we have observed in 18 out of 24 cases of carcinoma involving the superior portion of the stomach where an epigastric tumor could not be palpated. 
Auscultation is of relatively little diagnostic value in cases of gastric carcinoma.

Occasionally it is useful to prove that the contents of the esophagus pass into the stomach where a growth is known to exist in the region of the cardia. If the orifice is patent, auscultation over the region of the cardia, anterior or posterior, enables the recognition of the second swallowing sound when swallowed liquid passes from the esophagus into the stomach, should the cardiac orifice be patent. If the individual is obese, or the cardia be moderately elosed (particularly with a papillomatous growth) the second swallowing sound is usually greatly modified or entirely unrecognizable upon auscultation.

Auscultation over the epigastrium proper sometimes furnishes an index of the peristaltic activity in a given stomach. This is sometimes of value in determining stomach outline upon inflation of the viscus with air through a stomach tube or the patency of a ring cancer of the pylorus, in allowing one to ascertain the degree of obstruction by listening for gurgling sounds caused by the passage of air and fluid.

In cases where an extension of the malignant growth to an adjacent loop of the bowel is suspected, with greater or less obstruction of the lumen of the bowel, auscultation sometimes permits us to state whether or no the air or liquid has passed beyond the suspected area of constriction.

In rare instances, auscultatory evidences of the patency of a gastro-enterostomy stoma are established by listening over the region of the artificial opening, or over the bowel beyond.

\section{GASTROSCOPY}

During the last 3 decades a number of men have given their attention to the design of a practical gastroscope, 
among whom should be mentioned Mikulicz, Rosenheim, Kausch, Forametti, Jackson, Kuttner, Kelling, Elsner, Janeway and Hall.

As the result of the work of these men a number of instruments have been made. These have met with more or less success.

Sussmann Gastroscope.-Some 5 years ago, Dr. Martin Sussmann of Berlin constructed a crude model of a flexible gastroscope which seemed to fulfill the requirements in a

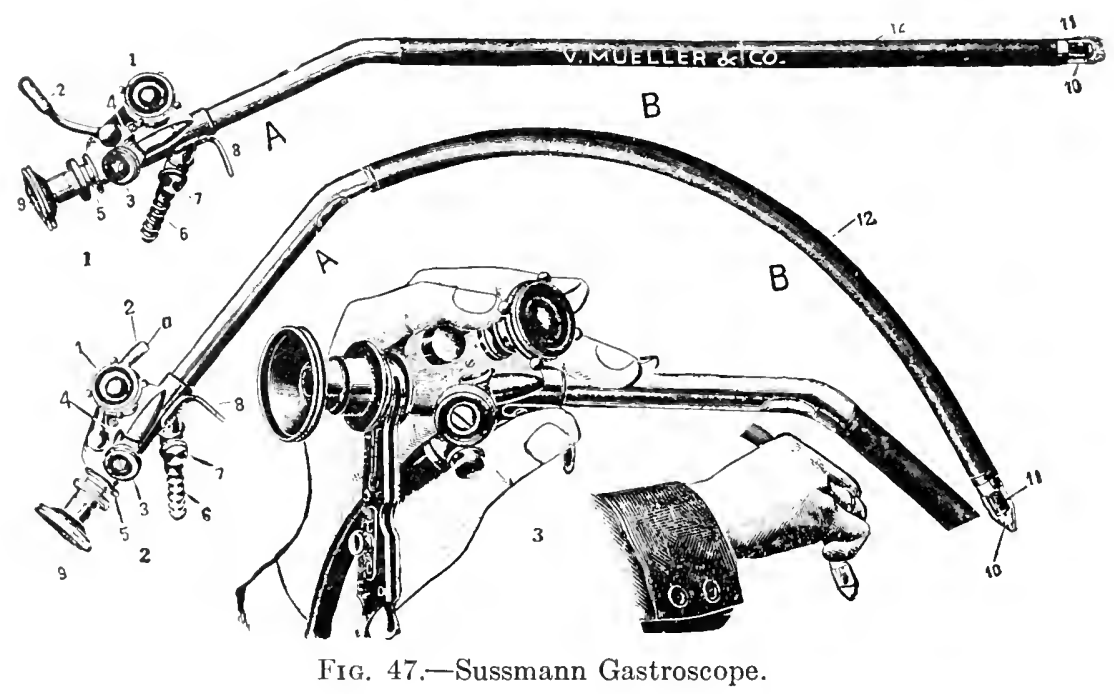

theoretical way, but he experienced difficulty in having the optical system and mechanical parts made. After 4 year's of experimenting, he finally succeeded in perfecting the instrument as shown in the illustrations (Fig. 47).

Contrary to the impression one might first have from these illustrations, the instrument is extremely simple in manipulation. The appliance consists primarily of 2 parts, the non-flexible part $A$ and the flexible part $B$. The total length from eyepiece to the lens at the distal end is $74 \mathrm{~cm}$. 
of which part $A$, non-flexible, is $24 \mathrm{~cm}$. and part $B 50 \mathrm{~cm}$. The diameter is $12 \frac{1}{2} \mathrm{~mm}$.

The non-flexible part $A$ is set at an angle of $150^{\circ}$ to part $B$, and in spite of this angle and the comparative great length of the instrument, a clear view can be had of the walls of the stomach when distended with air, due to the ingenious systems of lenses which have been adopted. On part $A$ is mounted the ocular (9). The turning device with indicator (3) for revolving the objective at the distal end of part $B$, lever (2) for placing the instrument in a flexible condition, tension wheel (1) for replacing the instrument in a rigid position, connection for compressed air tubing (6), with needle air control valve (7) and a hook or finger rest (8). Part $B$ is covered with a pure gum tube. At the distal end of the instrument is located the objective. Mounted on each side of the objective is a small flat tungsten filament electric bulb, the objective and bulb being covered by a hood or protector which is made of glass and metal, preventing the entrance of moisture and mucus, which might otherwise cause difficulty in the illuminating on account of short-circuiting of the current. In earlier forms of gastroscopes this turning feature offered quite an objection, in that the entire tube was turned, making the procedure a painful one to the patient.

Fig. 47 shows the gastroscope in a flexible position, and also in a rigid position, as set for the purpose of inspection. Fig. 47, 3 shows the method of introduction. Change from rigid to flexible is easily accomplished by simply throwing back lever 2 from position $A$ to $B$ and giving it a slight amount of pressure.

The system of lenses employed deserves special mention, from the fact that they are unusual in many respects. It is generally considered that in passing the instrument the 
shortest distance from objective to the posterior wall or entrance to the cardia is $2 \mathrm{~mm}$. and that the average distance in a well-inflated stomach is $7 \mathrm{~cm}$. from the objective to the part under inspection. The lens system, however, is universal in so far as an object will present a sharp, clear view, whether within $2 \mathrm{~mm}$. of the lens or at a distance of $7 \mathrm{~mm}$. The eyepiece (9) may be adjusted so as to accommodate itself to the lens system. The range of the field under observation at one time is sufficient so that an area measuring $6 \mathrm{~cm}$. across may be observed without any readjustment of the instrument. This makes it possible to observe the entire wall of the stomach, since the lens system can be turned so as to describe a complete circle and the instrument may be moved downward to any point.

The inventor claims that this instrument can be introduced without difficulty in every case where it is possible to introduce a stomach tube, and that the introduction is easier than with a straight, non-flexible instrument.

Since the above illustrations were made, the inventor has added an important improvement to the gastroscope, in the shape of a second ocular, which permits of two persons observing the conditions of the stomach at the same time.

There is an air inlet with automatic ralve. To this is connected a double balloon or foot bellows for the inflation of the stomach. In order to be absolutely sure of just how much pressure one is using, a manometer may be used in connection. 


\section{EXAMINATION OF GASTRO-INTESTINAL FUNCTION}

This concerns itself chiefly with analysis of gastric contents and feces. Of minor value are examination of vomitus, determination of the position, size and shape of the stomach and demonstration of the patency, mobility and situation of the small and large bowels.

(A) TEST-MEAL EXAMINATION OF GASTRIC FUNCTION

In well-established cancer of the stomach, there is no method of clinical investigation which yields more information of worth than does properly applied study of certain features of gastric function. There are few clinical methods - and certainly no clinical laboratory procedure-which enable one to estimate the status of a given case of gastric cancer so quickly, accurately and inexpensively as does intelligent scrutiny of test-meal data. There is no disease of the stomach, per se, which returns gastric-extract analyses so characteristic as those exhibited in instances of what is clinically advanced gastric cancer. In cases where dubious information is furnished by test-meal analyses, it can be demonstrated to be a fact that other clinical and laboratory aids are rarely conclusive and that in these cases final opinion generally rests upon exploratory laparotomy and the report of the cellular pathologist. These statements are based upon the author's personal analysis of 7,192 gastric extracts.

Our series of cases gives us 701 instances of proved gastric cancer in which test-meal examination of stomach function was carried out. It is our purpose to consider this phase of 
clinical examination in the light of the facts returned by such analyses. It has been our endeavor to discriminate between essentials and non-essentials.

Much opprobrium has been attached to test-meal work during recent years. This has resulted largely from the clinical laboratory being expected to do all the work relative to making a cliagnosis, and of ten from a few cubic centimeters of material brought or sent to it; from certain laboratory men failing to distinguish useful, practical, clinical tests from those chemically fascinating, but often entirely of an experimental nature; from the eager pursuit of "specific" tests for each disease or every symptom of it, and from the failure of patient, analyst, pathologist and surgeon to "get together" in an attempt to establish facts of worth from the mass of information collected independently.

From rather more than a casual acquaintance with the subject our records indicate that examination of gastric function has to do mainly with the investigation of (1) the emptying power of the stomach; (2) the chemical analysis of test-meal extracts or vomitus; and (3) the microscopic study of gastric contents or vomitus.

\section{INVESTIGATION OF GASTRIC EMPTYING POWER}

Scarcely a year passes without some "new" method for estimating gastric emptying power being exploited. The very fact that clinicians of reputedly large experience frequently put forth these procedures would appear to indicate either that there is no satisfactory method of determining the time of the stomach's emptying or that the emptying-rate of the so-called "normal" stomach is unsettled. It is a waste of space and energy to catalogue the various novel and often amusingly ingenious "motor-tests" 
that have been described. We have found the following procedure useful. It has limitations. A not altogether unimportant feature of the information returned by this motor-meal, in our series, lies in its having been administered to a large number of patients whose ailments were subsequently determined at laparotomy.

(a) Physiologic Method of Estimating Gastric Emptying Power.-Its object is to prove whether food can pass into or out of the stomach. The contraindications are few: recent severe hemorrhage, clinical evidences of perforation, coma, extreme asthenia, severe cardio-renal disturbances or mental upsets.

Method.-The patient's stomach should be washed free from whatever material it contains. Following the lavage (provided the clinical history of marked stenoses at the orifices has not been obtained) 2 ounces of castor oil are administered through the stomach tube or per oram. Three hours later the patient is allowed to eat a moderate-size meal of mixed food, in the manner that he ordinarily follows. This meal contains among other ingredients, at least 50 grams of cold meat, 2 leaves of head lettuce, and 20 raw raisins. Instructions are sometimes needed to insure the patient's swallowing the skins of the raisins. It is important that they should be eaten. Beverages are allowed, preferably water, milk, or weak tea. This meal has the advantages of being readily available, palatable and of sufficient bulk. The last consideration is of essential value in any motor-meal. It is impossible to establish evidence of the anatomic condition of the stomach's orifices by motor-meals of the baby pap type. An interval of from 8 to 12 hours is permitted to elapse before a stomach tube is passed and attempts at the recovery of remains of the motor meal are made. Experience has taught us that 
food remnants present constantly in a stomach after 8 hours generally indicate some mechanical hindrance to their free exit from the viscus. It is not uncommon in healthy individuals to demonstrate the presence of food in the stomach after 4 to 6 hours, i.e., the common time limit for estimation of gastric emptying power. In many instances of pyloric spasm, associated with peptic ulcer, disease of the

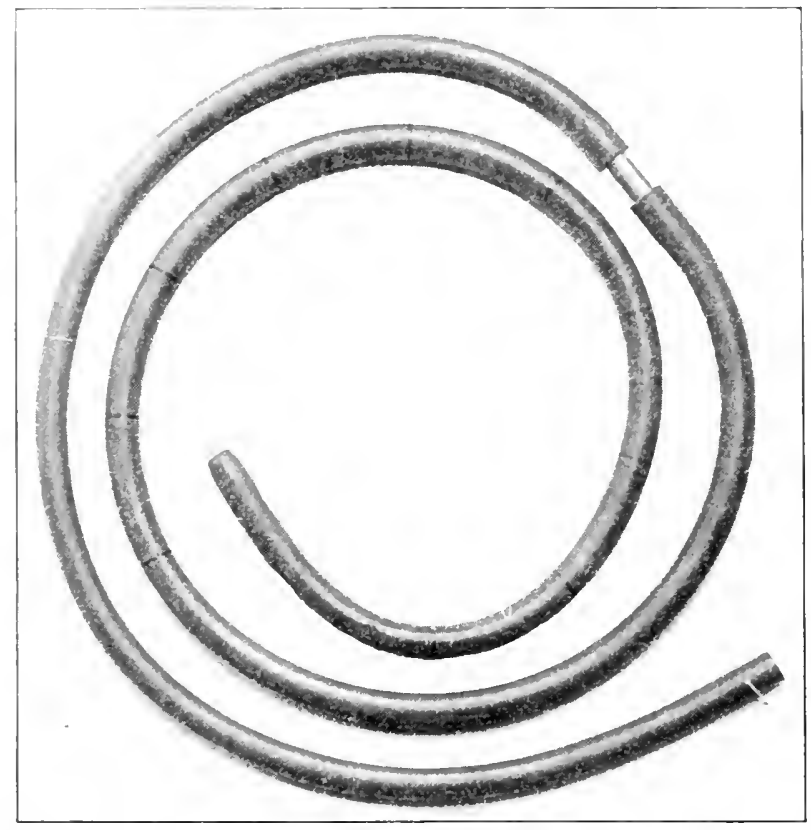

FIG. 45.-Motor-meal and lavage tube showing distal end, markings on tube and the aluminum mid-piece.-(Author.)

gall-bladder, the appendix, etc., intermittent (and sometimes marked) 6- to $S$-hour residues may be removed from the stomach. In pronounced atony, rather more than 4 per cent. of cases exhibit food retained longer than 6 hours.

For the purpose of readily removing gastric contents we some time since constructed the stomach tube illustrated by Figs. 48 and 49 . 
Motor-meal and Lavage Tube.-Stomach tubes, as ordinarily made, seem to have the following faults: (1) the inferior rubber in their construction, which prevents sterilization by boiling (the only efficient method); (2) small lumina, which frequently render unsatisfactory the aspiration or expression of motor-meals or poorly chymified test-breakfasts; (3) improperly placed, too few or too small fenestra at their distal ends; (4) the incorporation into the tubes of "aspirating bulbs" of questionable service, which are difficult to keep clean. In an effort to remedy some of these defects, we have had constructed tubes of the type to be described. They have given greater satisfaction

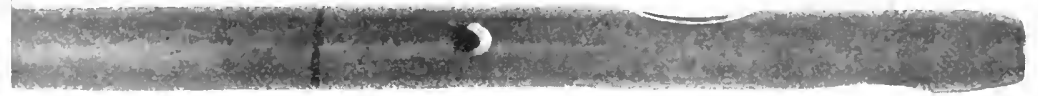

FIG. 49.-Motor-meal and lavage tube showing openings in distal end.(Author.)

than any other form in the last 1,800 cases examined in which test-meal examinations were made.

The tube is constructed of the best quality of red rubber. The wall is $2 \mathrm{~mm}$. thick and the lumen has a diameter of 9 $\mathrm{mm}$. The tube is made in two sections, distal and proximal, connected by a thin, but strong, aluminum mid-piece.

The distal segment of the tube is $90 \mathrm{~cm}$. long. It has a distal opening of $1 \mathrm{~cm}$. diameter. Beginning $1 \mathrm{~cm}$. from this tip is a lateral fenestrum (Fig. 49) of ellipsoid form, 2 $\mathrm{cm}$. long and $1 \mathrm{~cm}$. wide. On the opposite lateral surface to this is a second similar lateral fenestrum, which begins $3 \mathrm{~cm}$. from the tip of the tube. The fenestrum is placed at this point, not only to permit free siphonage of gastric contents, but also to facilitate the introduction of the tube. 
Its location, $3 \mathrm{~cm}$. from the tip of the tube, is the average distance in different individuals from the pharyngeal dip of the tongue to the introitus cesophagi. When the tip of the tube enter's the pharynx, with the second lateral fenestrum toward the tongue, the subject's swallowing motions promptly bend the tube at this fenestrum and the tip glides readily into the esophagus. Using a tube with a distal end of this type, we have not entered the larynx more than a dozen times in our last 5,000 cases. Eight centimeters from the tip of the tube are placed three round fenestra, of a diameter of $2 \mathrm{~mm}$. They pierce the tube at the same level. This distal segment of the tube, beginning $10 \mathrm{~cm}$. from its tip, is marked off by encircling black lines (Fig. 48), every $5 \mathrm{~cm}$. for a distance of $55 \mathrm{~cm}$. from the distal end. These markings permit of fairly accurate location of obstructions in the esophagus. The tube, in such instances, acts as a hollow sound through which the contents of esophageal sacculations and the like may be readily secured.

The connecting aluminum mid-piece is $5 \mathrm{~cm}$. long, with a wall $1 \mathrm{~mm}$. thick and a lumen of $1 \mathrm{~cm}$. diameter. It is slightly roughened and fits snugly into the rubber parts. It is non-rusting.

The proximal end of the tube is $60 \mathrm{~cm}$. long. It may be replaced, when necessary, by an aspiration bulb which fits onto the metal mid-piece.

These tubes appear to have many advantages. They are durable; they may be boiled for months and still retain their form. The lumen is large enough to permit free expression or aspiration of retention contents after the administration of a motor test-meal. The distal fenestra are so placed as to permit the easy passage of the tube and the rapid siphonage or aspiration of gastric contents. Free lavage is readily carried on, either for the purpose of de- 
termining retention (as in hour-glass stomach) or for therapeutic effect. The tube acts as a safe and convenient esophageal sound. The aluminum mid-piece replaces easily broken glass connections. It enables one rapidly to convert the straight siphon or lavage tube into one of bulb type for the purpose of inflating the stomach with air or when expression of gastric contents proves difficult.

In the passing of a stomach tube, we find it convenient to seat the subject upon a straight-backed chair. All clothing to the waist is removed, except an undergarment. The patient places his hands flat, across the region of the navel. The head should be tilted slightly forward. This widens the introitus asophagi. Appropriate rubber and linen covers protect the patient. It is rarely necessary to hold the subject's head, etc. After boiling, the stomach tube is kept until needed in a bowl of cracked ice. With the patient prepared, the end of the cold tube is rapidly passed to the pharynx, and as the patient swallows, it is advanced boldly and rapidly into the esophagus; then passed quickly, but carefully, into the stomach. Coughing, cyanosis and pain promptly develop if the tube has entered the larynx. When the tube reaches the stomach (recognized by gas, or contents coming from the tube or by the feeling of its passing the cardia) the patient is sharply commanded to press his hands upon his belly and to bend quickly forward. The gastric extracts usually spurt from the free end of the tube promptly. If none appear, the tube may be successively advanced and retracted cautiously, the patient instructed to cough, or an aspirating bulb used. We have rarely found it necessary to resort to such bulb. Sometimes the gastric contents are very thick or made up of large pieces of food. In this event one may have to dilute such or gradually wash the material out. 
Every attempt should be made to secure contents without resorting to this procedure, because analysis of the material removed from a "fasting stomach" returns most useful information (particularly upon microscopic examination).

Frequency of Gastric Retention in Cancer of the Stomach.Some degree of 12-hour retention was demonstrated in 483 cases (69 per cent.) in our series of 701 instances where test-meal data are available. Frequently the amount raried considerably upon repeated examinations, but there were but 20 per cent. of cases where the neoplasm was located at the antrum or distal to it where some trace of food retained for from 8 to 12 hours could not be persistently demonstrated.

\section{THE MACROSCOPIC STUDY OF RETENTION EXTRACTS IN GASTRIC CANCER}

If the pylorus is patent the wash-water may return clear. This is especially the case where so-called scirrhus or colloid cancers are present. Fully 95 per cent. of all gastric cancers contain areas where cell-arrangement is of the medullary type. It follows, consequently, that the wash-water is frequently turbid or tinged with blood.

Where retention exists from a few cubic centimeters to more than a liter of more or less altered test-food may be removed. Not rarely, large pieces of partly digested material, intermixed with mucus, together with puree-like fluid are secured.

Color.-Traumatic blood was noted in nearly 47 per cent. of 223 consecutive cases in our series. Bile coloring was observed in 56 instances ( 8 per cent.). It commonly occurred where a tough fibrous cancer held partly open the pylorus. Classic coffee-colored or dark brown extracts were 
noted in 149 cases (21.3 per cent.). Such growths were inoperable in more than 97 per cent. The color of gastric contents is quite as apt to be tan or brownish in nonmalignant stenoses as in cancer. Occasionally, almost black extracts are removed. Such are often due to disintegrated blood or necrotic tissue or medicines (bismuth, iron preparations).

Odor.-In more than 80 per cent. of our cases, acrid, rancid odors were recorded. They are usually produced by volatile, organic acids. Such odors are almost pathognomonic of malignancy when associated with 12-hour food retention. Putrefactive odor's, sometimes nauseatingly penetrating, may be encountered from sloughing of cancer tissue, deterioration of blood, perforation, or fistula to adjacent viscera.

Mucus.-An excess is observed in more than 2 out of 5 instances of malignant gastric retention. It is generally in tough, gelatinous strings or ropes, more or less intimately mixed with food, or the products of cancer growth.

Chymification of Test-food.-Where peristalsis is below the normal strength or frequency, free hydrochloric acidity low, the test-food poorly masticated and retention pronounced, chymification is greatly diminished. Commonly, test-food eaten 12 hours previously is little altered, particularly meat, lettuce or raisin skins.

Acidity of "Fasting Stomach" Extracts.-Methods.For practical clinical use sufficiently accurate relative results are obtained by the Toepfer technique of determining acidity. The gastric extracts should be filtered, if quantity permits. If analyzed unfiltered, the readings are apt to be higher than where filtered extracts are examined. Other errors may occur. 
"Free Hydrochloric" Acidity.--There is no absolutely reliable way of estimating such in retention contents, because, as Schryver and Singer have emphasized, hydrochlorids of amino-acids (end-digestion products) act as acids, and organic acids (lactic, butyric and acetic) likewise affect such indicators as dimethylamidoazobenzol and Günzberg's reagent (phloroglucin-vanillin solution). For comparative work, however, it appears useful to estimate free hydrochloric acidity with dimethyl as an indicator.

Method.-Ten cc. of gastric filtrate are placed in a white porcelain evaporating dish. To it are added 2 drops of 0.5 per cent. solution of dimethylamidoazobenzol in 95 per cent. alcohol. If free hydrochloric acid is present the mixture assumes a magenta red. The acidity is neutralized by slowly adding from a burette $n / 10$ solution of sodium hydroxide. The end point is reached when the mixture in the evaporating dish becomes lemon yellow in color. The number of cubic centimeters or parts thereof required to bring about this color change is read from the burette. This figure multiplied by 10 gives the acidity per hundred parts. If the acidity per cent. is desired, multiply the resultant figure by 0.00365 .

Degree of Free Hydrochloric Acidity in Retention Contents. - In 319 cases (66 per cent.) no free hydrochloric acid could be detected by dimethyl in the extracts from fasting stomachs. In the remaining 164 instances, free hydrochloric acid ranged from 2 to 70 . The average was 26.6. In other words, practically 1 out of every 3 cases of gastric cancer with retention of food have free hydrochloric acid above an average of 26 , in the fasting stomach contents.

Total Acidity.-This is a term of but relative worth. Its physiologic significance is open to question. Clinically, high total acidity is not rarely associated with abdominal discomfort, nausea, pyrosis and eructations. Not a small 
part of this discomfort would appear to be due to the presence of organic acids in stagnant, gastric contents.

Method of Determining Total Acidity.-Ten cubic centimeters of gastric filtrate are placed in a white enamel evaporating dish. Two drops of a 1 per cent. alcoholic solution of phenolphthalein are added. There is usually no color change unless the total acidity is very low or the extract alkaline. $n / 10$ sodium hydrate solution is added to the mixture from a burette. When the acidity has been neutralized by the soda, the contents of the evaporating dish take on a pinkish-red hue. The degree of acidity is computed as in the case of estimating free hydrochloric. Total acidity includes free hydrochloric, combined hydrochloric, organic acids and acid salts.

\section{Degree of Total Acidity in Retention Contents in Gastric} Cancer.- In none of the 483 cases in which 8- to 12-hour food retention was demonstrated was total acidity 0 . The average total acidity was 40.3 . The minimum was 4 ; the maximum 132.

"Combined" acidity as estimated by using sodium alizarin solution as an indicator is a finding of dubious value.

Method.-Ten cubic centimeters of gastric filtrate are placed in a white porcelain evaporating dish. To this are added 2 drops of sodium alizarin solution. The mixture is titrated against $n / 10$ sodium hydroxide solution until the contents turn violet. Quantitatively, the estimation is made as in the determination of free hydrochloric.

Degree of "Combined" Acidity in Retention Contents of Gastric Cancer.-Of the 483 cases in this series, combined acidity was never below 1.5. The average was 16.5. The minimum was 1.5 , and the maximum 126 .

Acid Salts.-Schryver and Singer (loc. cit.) have pointed out in their admirable critique of the methods employed in gastric analysis, that in malignant tumors affecting the pyloric region of the stomach, the amount of secreted chlo- 
ride is generally increased. Our 483 observations above detailed upon the relation of total, combined and free hydrochloric acidity would appear to add, indirectly, certain clinical corroboration of this fact.

Lactic Acid.-Tests for this and other organic acids are more commonly positive in retention contents than in gastric extracts where there has been no evidence of pronounced stagnation.

Method.-Six drops solution of liquor ferri sesquichlorati are added to 3 drops of 95 per cent. carbolic acid. The resultant mixture is diluted in distilled water until it assumes a lively amethyst blue. To this last solution are then added from 5 to 10 drops of gastric filtrate. If lactic acid is present the amethyst blue color is discharged upon the addition of the gastric filtrate and a canary-yellow color is seen.

Frequently gastric contents which have been extracted with ether, according to the method of Strauss, are employed in the above outlined test in place of the unextracted gastric contents.

By the adoption of this modification of the test a higher percentage of positives is returned in gastric cancer than where the original procedure was employed.

In our series of retention contents lactic acid was demonstrated in 66.8 per cent. In instances where lactic acid was demonstrated 95 per cent. were inoperable carcinomata. Of this number 58 per cent. had no precarcinomatous history of dyspepsia. In the cases where there had been precarcinomatous history of indigestion, the antecedent indigestion had been of the type clinically peptic ulcer in only 42 per cent.

Altered or "Occult" Blood.-Inasmuch as in 9 out of 10 instances of carcinoma of the stomach tumor areas are shown which pathologically contain tissue of the medullary type, 
it is to be expected that from time to time, or constantly, a certain amount of seepage of blood will occur. As we have already pointed out this can often be recognized in the wash-water, particularly after vigorous manipulation of the stomach tube. Where the hemorrhage has been moderately profuse, the gastric contents range in color from light tan to dark brown or almost black. In the majority of instances it is not necessary to test retention contents for blood chemically. Sometimes, however, the administration of blood-building or styptic medicines (iron, bismuth, tannic acid, etc.) imparts a color suspicious of blood to the gastric contents. Certain forms of food may also bring about such a change (red wines, soups, meats, chocolate, grape juice, etc.). It is, therefore, interesting in such an event to definitely prove whether or no the color is due to blood. Of course, it should be recognized that the resultant positive test may come not from the bleeding of cancerous tissue, but from the presence in retention contents of substances derived from food or medicine.

Method.-Many tests have been suggested. If properly controlled the majority of them have a certain place clinically. The most common ingredients used in the "occult" blood test are phenolphthalein (Boas) guaiac, benzidin or orthotoluidin (Ruttan and Hardisty).

The most reliable tests for the proof of blood, from a purely chemical standpoint, are the spectroscopic test or the demonstration of hemin crystals.

The tests depending upon color change of fluids are, however, more convenient of application than are those which require elaborate apparatus or demand more or less expert knowledge of crystallography.

Benzidin Test.-Clinically we have found that the altered blood test perfor'med with the use of powdered benzidin 
(pinkish-gray powder) is quite satisfactory. We have preferred benzidin to guaiac because we have found that positive tests to it are returned less frequently from food than in the event where guaiac is used. Our method of performing this test is as follows:

Method.-To 5 ce. of gastric extracts are added 5 drops of glacial acetic acid. This forms an acid hematin if blood be present. This acid hematin is then extracted by the addition of $5 \mathrm{cc}$. of chemically pure ether, by frequently pouring from test-tube to test-tube. To the ether extract are added 15 drops of a 1 per cent. solution of benzidin powder in 95 per cent. alcohol (or in strong glacial acetic acid). The mixture is again poured from one test-tube to another several times. From 15 to 25 drops of active hydrogen peroxide are finally added (commercial peroxide of 3 per cent. strength is satisfactory).

If blood is present in the gastric extracts a prompt color change takes place in the fluid. This color change varies from a lively emerald green to a greenish-blue or an indigo. If no blood is present, then the color change does not commonly appear. We find it of value to allow the tubes to stand 1 minute after the addition of the peroxide solution. If no greenish or bluish color has developed, it is safe to say that any blood which may be present has little clinical significance.

Instances of Altered or "Occult" Blood in Retention Contents.-Of the 483 cases in which 12 -hour retention was proven, the clinical test for altered blood was positive in 82 per cent. Of this number, as is noted above, lactic acid was present in 66.8 per cent.

\section{MICROSCOPIC EXAMINATION OF RETENTION CONTENTS}

This should never be neglected. Apart from the evidence of stagnation, in the large majority of instances of 
moderately advanced, or extensive gastric carcinoma, even if no abdominal tumor can be palpated externally, one is able to discover a rather characteristic picture upon microscopic examination of stained smears with high-power amplification. To the expert, examination of unstained smears is usually sufficient to return a diagnosis as to the type of retention contents. However, the examination should always be made with high power. Even those most experienced in the microscopic examination of gastric extracts cannot determine accurately the bacterial flora present using the 4-mm. or lower power objectives.

Method.-Various methods of staining are in clinical use. Not infrequently the desired information may be obtained by the so-called 3-drop method. This is performed by placing upon a clean glass slide 3 small drops of unfiltered retention contents. These drops are placed in a row about $3 / 4$ of an inch apart. The first one is simply covered with a clean cover slip; to the second is added a drop of Lugol's solution and then a cover slip; to the third is added a small drop of osmic acid and a cover slip. The unstained specimen permits of search for motile and non-motile organisms; the second drop, on account of the iodine stain, permits of examination for starch and regetable food rests, yeasts, leptothrix and the like; while the third drop allows the recognition of fat or some of its products. If the cover slips are placed firmly upon the drops, one can, with the addition of a small amount of immersion oil to each cover slip, quite satisfactorily examine with the high power.

This is a rough, clinical method, and is of limited use in an investigation of gastric contents from which information is desired regarding definite flora, food digestion and the like.

For purposes of studying, and convenience in examining, a large number of specimens in a short time, we devised the following method. We have found it quite dependable and simple in its application. 
Colored Agar Method for Staining Gastric Extracts.Agar Solutions.-Two per cent. agar solution is made by boiling up an appropriate quantity of best grade strip agar in distilled water. The solution is filtered several times while rery hot through double, hydrochloric-acid-washed filter paper. On cooling it solidifies as a firm jelly. It should be fractionally sterilized and refiltered on 3 consecutive days.

For purposes of convenience, 5 cc. of the molten agar are poured into each of numerous sterile test-tubes. The tubes are plugged with sterile cotton and set aside at room temperature. The agar jellies and may be used as desired.

Stains.-Thus far we have used coloring agents in combination with agar solutions for two purposes: (1) for the differentiation of bacteria, blood-cells, epithelial elements, tissue-bits and the like, we have found most satisfactory Unna's polychrome methylene blue; (2) for the differentiation of starch residues, regetable fibers, moulds, etc., we have employed freshly prepared Lugol's mixture.

Procedure.--For each specimen of gastric extract or feces emulsion, we make two agar-coloring matter combinations as follows: the 2 per cent. agar jelly is liquefied by heating orer a Bunsen flame, or in a water-bath. Two cubic centimeters of the solution are poured into each of two small testtubes (those measuring $10 \mathrm{~cm} . \times 15 \mathrm{~mm}$. answer very well). Into each of the tubes are then poured 15 drops of filtered staining agent. For staining bacteria and the like we have found that Unna's mixture gives good results, while Lugol's solution colors starch elements better than simple iodin. The added stains are intimately mixed with the agar and the tubes promptly placed in a beaker containing boiling water. The agar is thus kept as a solution.

Very thin smears of the gastric extracts or feces emulsions 
are made on cover slips. It is desirable to avoid getting gross particles of food in the smears. The smears may be dried in air or by rapidly passing through a gas flame.

Onto the smear to be examined for microorganisms and allied elements, by means of a pipette with a $1 \mathrm{~mm}$. bore, is placed 1 drop of the agar-methylene-blue mixture. The cover slip is promptly inverted on a clean glass slide. The agar-stain mixture rapidly spreads to the edges of the cover slip. As it cools the agar solidifies while the stain mixed with it permeates the smear. Similarly, to the smear to be examined for starch remnants and the like, is added 1 drop of the agar-Lugol's mixture. The cover slip is mounted at once on the same slide as the first preparation. About 1 minute suffices for firmly mounting and staining ordinary material. In a series, the first preparations are ready for examination by the time the last are made.

Examination.-The preparations are best examined by such light as comes from a Nernst, Schwann or tungsten lamp. If electricity is not available, a Welsbach mantle light suffices. When the specimens are properly focused, using an oil-immersion objective, it will be seen that bacteria are stained blue or deep purple; blood cells and nuclei are differentiated by pale pink and blue, as are also epithelial elements; tissue bits, if small, may show atypical, mitotic cells; muscle fibers are pink to red, with deeper striation; mucus is in bluish strips or whorls. Unless the preparation is over-stained, regetable fibers, leptothrix, moulds, etc., are but lightly colored. In the smear prepared with agar-Lugol's mixture, regetable fibers, leptothrix, moulds, and starch remnants are appropriately differentiated. The latter may be amethyst-blue, with deeper staining centers or laminæ, reddish-pink with 
mahogany-colored center's or faint pink or colorless. Budding yeasts, moulds, and occasionally bacteria take a brownish-blue tinge. Vegetable cells stand out prominently as blue to brown reticula. Large masses of amorphous material stain variously, but are readily distinguished from microorganisms.

Uncolored Preparations.-The agar without coloring agents added may be advantageously used for mounting smears. In fresh preparations, when the smears are not dried, motile organisms (bacteria, protozoa) may be observed for a long time. This is facilitated when the specimen is diluted with three volumes of warm normal saline solution before the smears are made.

Advantages of the Method.-A routine, differential report on two dozen smears, from half as many specimens of gastric extracts or feces, may be made in about 30 minutes. The specimens are simultaneously mounted and stained. The solidification of the agar gives so firm a mount that the specimens may be examined with high or low power, with the stage of the microscope at any angle. The mounting is sufficiently permanent to allow future study (we have preparations showing good fields after 10 weeks), drawings, photomicrographs or demonstration. Comparison of the smear's on the same slide, but differently stained, results in many interesting and instructive observations. There would seem to be advantages in using the method for the observation of motile bacteria, protozoa, or cells with ameboid activity, when uncolored agar solutions are used.

Significance of Microscopic Examination.--In malignant retention contents, food in the rarious stages of digestion can usually be recognized.

Food.-Unless food retention has been demonstrated 
macroscopically, the finding of food bits microscopically only has, in our experience, practically no diagnostic value.

Bacteria in Malignant Retention Contents.- The investigation has occupied laboratory workers for many years. It was early shown by Oppler working in Boas' Clinic that malignant retention extracts contained a large number of long, non-motile rods. This observer also noted that such organisms were of comparatively rare occurrence in gastric contents, either of the retention or non-retention type, in diseases other than carcinoma of the stomach. Later investigations upon the properties of these so-called "rather long bacilli" were carried on by Kauffmann. This observer proved that the bacteria described by Oppler and Boas were acid fast, fermented sugar, and were probably an altered form of Bacillus acidi lactici.

We have made observations upon 221 consecutive instances of gastric cancer for the presence of bacteria of this type. We were early impressed by the fact that the bacillus described by Oppler-Boas, Kauffmann and others appeared to be not a distinct type, but rather partook of the nature of a group of bacteria having similar morphological and cultural characteristics.

In addition to the microscopic examination of stained and unstained, wet and dry smears of gastric contents containing this organism, we undertook to attempt culture of the germ by perhaps a new method. A full report of the work is not yet available. Let it suffice to say that the information of the greatest worth which we are able to derive from our studies was derived from bacilli from malignant gastric contents grown in two types of media, namely: (a) a medium made from extracting fresh, sterile malignant tissue that had been ground up in a sterile meat grinder with a normal salt solution, and $(b)$ a similar 
medium made from beef extract. We are prepared to offer the following morphological observations.

Characteristics of Bacilli of the Oppler-Boas Group.The bacilli vary in length from 15 to 60 microns. The

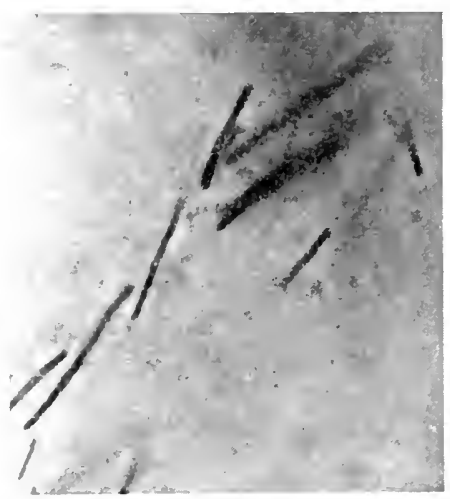

Fig. 50.-Typical long bacilli-Oppler-Boas. Photomicrogram.(Author.)

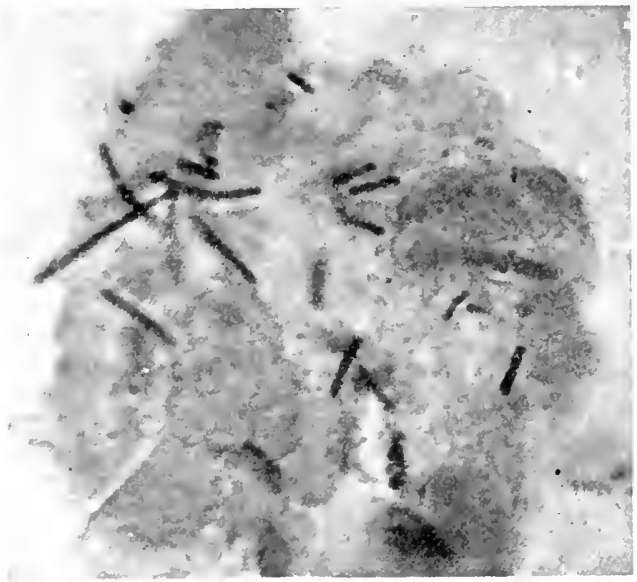

FIG. 51.-Bent and club-shaped forms of Oppler-Boas bacilli. Photomicrugram.-(Author.)

short forms are straight, slightly curved, wavy or occasionally comma shaped. As the forms grow larger, they rarely remain straight, but may be bent or wavy with a tend- 
ency to curl up like a J or "shinny"-stick at the end. In old retention contents the bacilli may assume U-shape, half circle forms, or may even have ring-like twists.

It is uncommon to find Oppler-Boas bacilli orer $20 \mathrm{mi}-$ crons long that are absolutely straight. When they are above this size the wavy or bent forms become numerous. In addition to being bent or wary, one frequently notes that an end of a bacillus is clubbed or bent at an oblique angle.

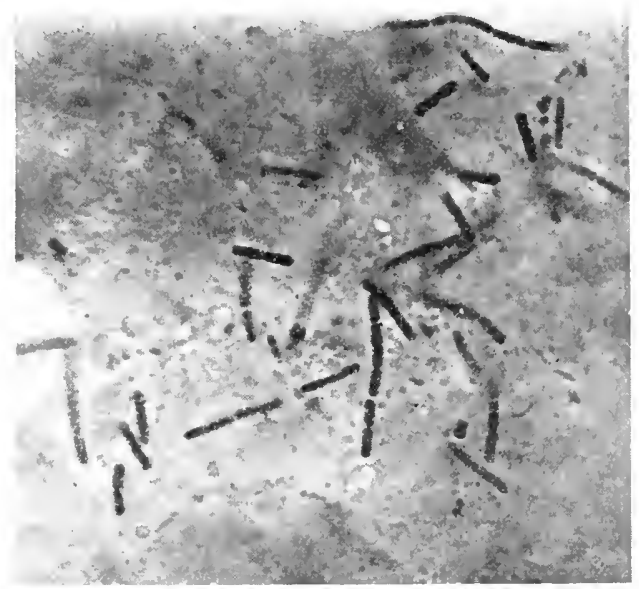

FIG. 52.-Oppler-Boas bacilli showing different groupings and variation in size of individual bacilli. Photomicrogram.-(Author.)

Long chains of organisms of this group are uncommon; short chains often occur. The individual bacilli making up the chains vary in length, but resemble each other, structurally. When the bacilli grow in chains they are usually blunt ended, but on account of the curved or wary outline of the individual bacillus the resultant chain often has an irregular zig-zag appearance. In old, stagnation contents, some of these chains form loops, nearly complete ellipses or circles.

Staining Properties.-The typical bacilli stain uniformly 
a bluish-purple with Enna's polychrome methylene blue. They absorb the blue about as deeply as do yeast cells. As the bacilli grow longer or form chains, they stain irregularly so that frequently there are alternate bands of deep and light color. This gives the organism a somewhat beaded appearance. It may be that this bizarre staining appearance is due to the fact that the organism

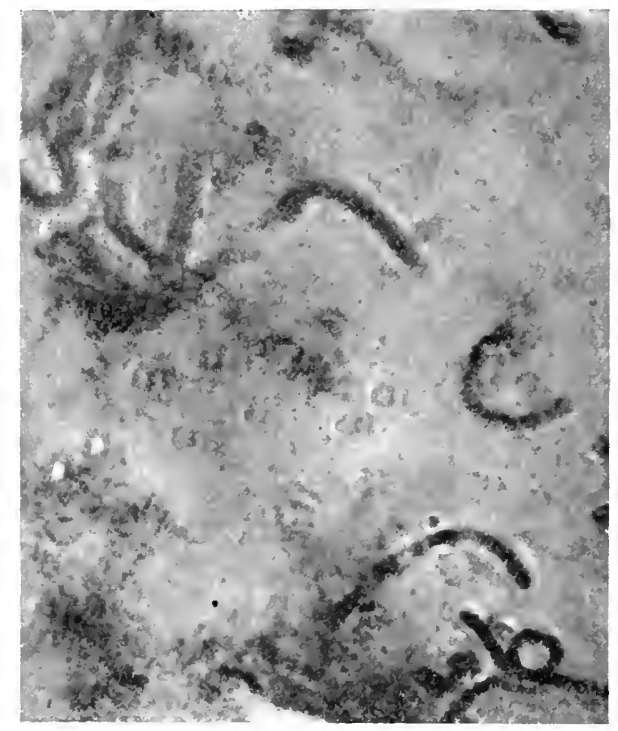

FIG. 53,-Oppler-Boas bacilli. Showing half-circle, "shinny stick" and ring forms. Photomicrogram of old retention contents.-(Author.)

is wavy or corkscrew-like. If this be so, different portions of the bacillus come into focus at different times. Certainly some of the more lightly stained areas appear darker upon focusing. The bacilli take the iodine stain evenly, and are colored a light amber-yellow by Lugol's solution. This perhaps helps to differentiate them from leptothrix forms and from ordinary lactic acid bacilli.

Artificial Culture.-Two types of culture media were used: (1) ordinary beef bouillon, and (2) bouillon made 
from fresh cancer tissue. The tubes were inoculated from a cáncerous retention contents. Its acidity was: total, 15 ; free $\mathrm{Hcl}, 0$.

Macroscopic Examination.-After 24 hours the tubes containing bacilli in the cancer extract medium were slightly opalescent and had a fine, granular, compact

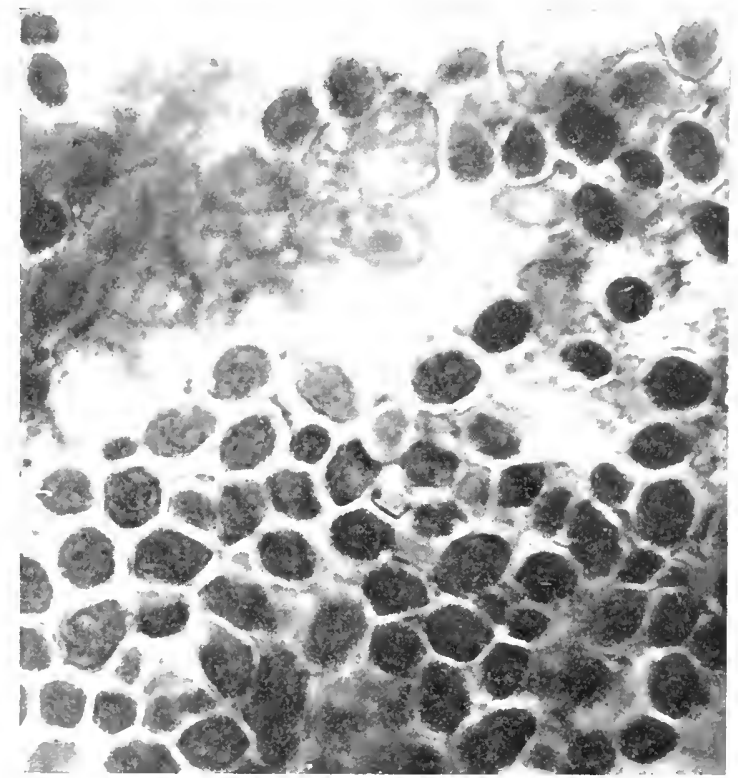

Frg. 54.-Mosaic of yeast cells from case of benign retention. Photomicrogram.-(Author.)

precipitate on the bottom. The cultures had a slightly yeast-like odor. Cultures made in the beef bouillon medium were densely opaque, and had a heary, flocculent precipitate with faint yeasty odor.

Microscopic Examination. - Smears made from the cancer extract bouillon exhibited an occasional yeast cell and from 3 to 6 bacilli of the Oppler-Boas type to a field. The bacilli were single or arranged in short chains in from 2 to 4 organisms. They were fairly even in length, the 
average being about 20 microns. They stained deeply and homogeneously with Unna's polychrome methylene blue. Some had rather club-shaped ends; those of others were slightly angulated. The ends were bluntly rounded. The individual bacilli joined each other in the chains at a broad oblique angle. In this medium were also seen numerous slender somewhat lance-shaped bacilli, arranged singly. They were occasionally curved at the middle, like a wide open $\mathrm{U}$. They stained rather faintly. With

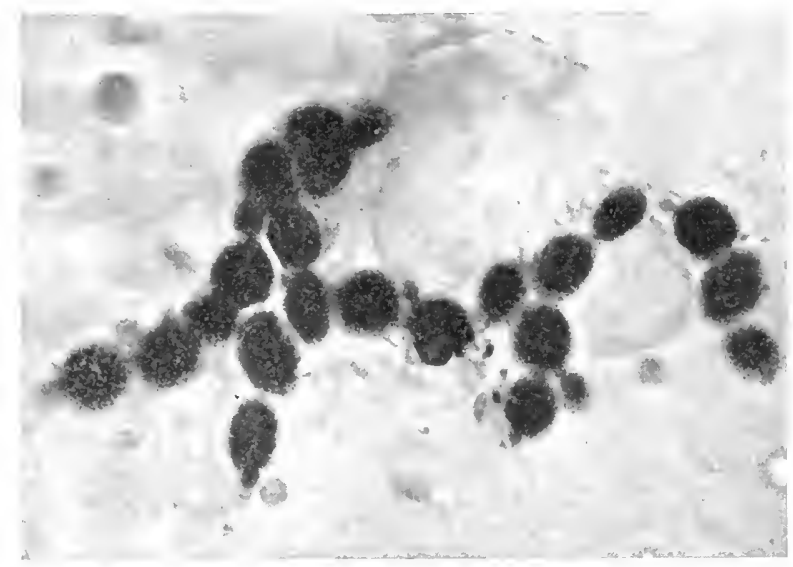

Fig. 55.- Yeast colony from case of benign gastric retention. Photomierogram.-(Author.)

Lugol's mixture, organisms of the Oppler-Boas type were colored light amber-yellow, as were also the lance-shaped bacilli above described. Occasionally one could see forms of the lance-shaped bacilli arranged singly and staining deeply. The average length of the small variety of these was three-fourths that of the diameter of a red blood cell. The large forms were twice as long as the diameter of a red blood cell.

C'ultures in Beef Bouillon.- The growth was very abundant. Great numbers of Oppler-Boas bacilli were seen. 
They raried very much in size ranging in length from half the diameter of a red blood cell to 3 times that diameter. They stained deeply. Their ends were bluntly rounded. The organisms were straight or somewhat bent in the middle or toward one end. Without regard to individual
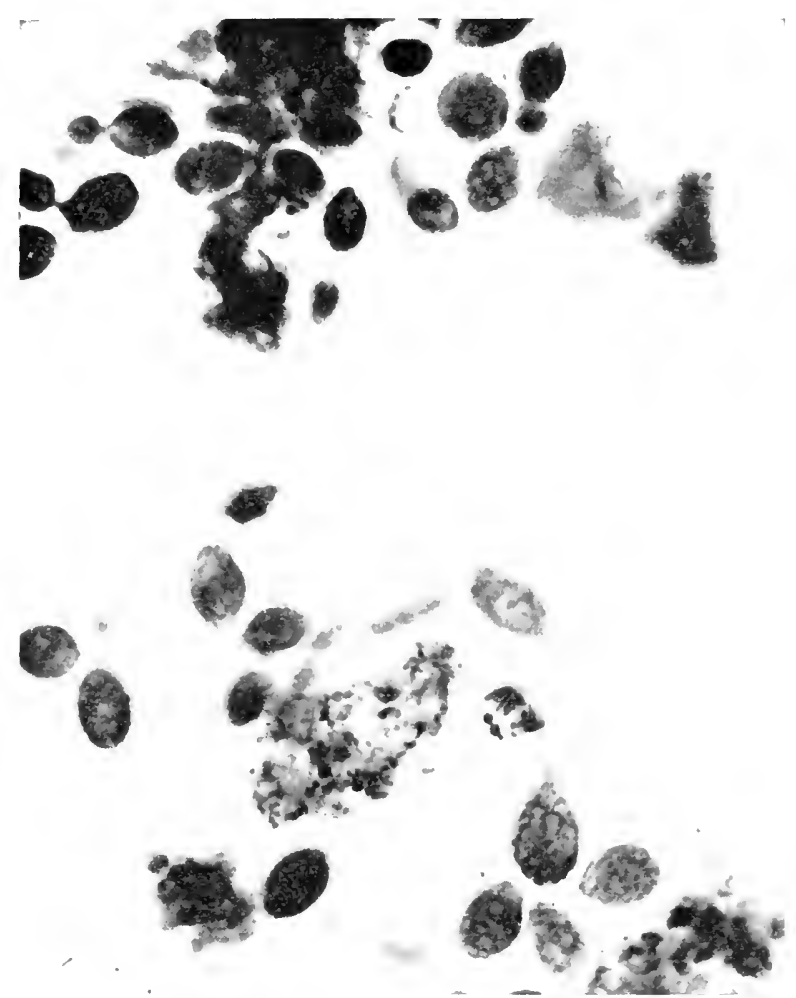

Fig. 56.-Old benign gastric retention. Yeasts and bacillus butyricus. Photomicrogram.--(Author.)

size they were arranged in short chains or pairs. When forming chains they rarely fitted squarely end to end, but usually at an angle. Sometimes the bacilli were joined at different or opposite angles so that irregular chains were formed. Occasionally, the angles were similar in direction. In this event curred chains resulted. Many 
long leptothrix chains were seen. These were much branched, bent, twisted and in irregular tangles. They stained more lightly than did the Oppler-Boas bacilli. The individual segments of these chains were usually narrower and longer, rarely being less than 30 microns. They

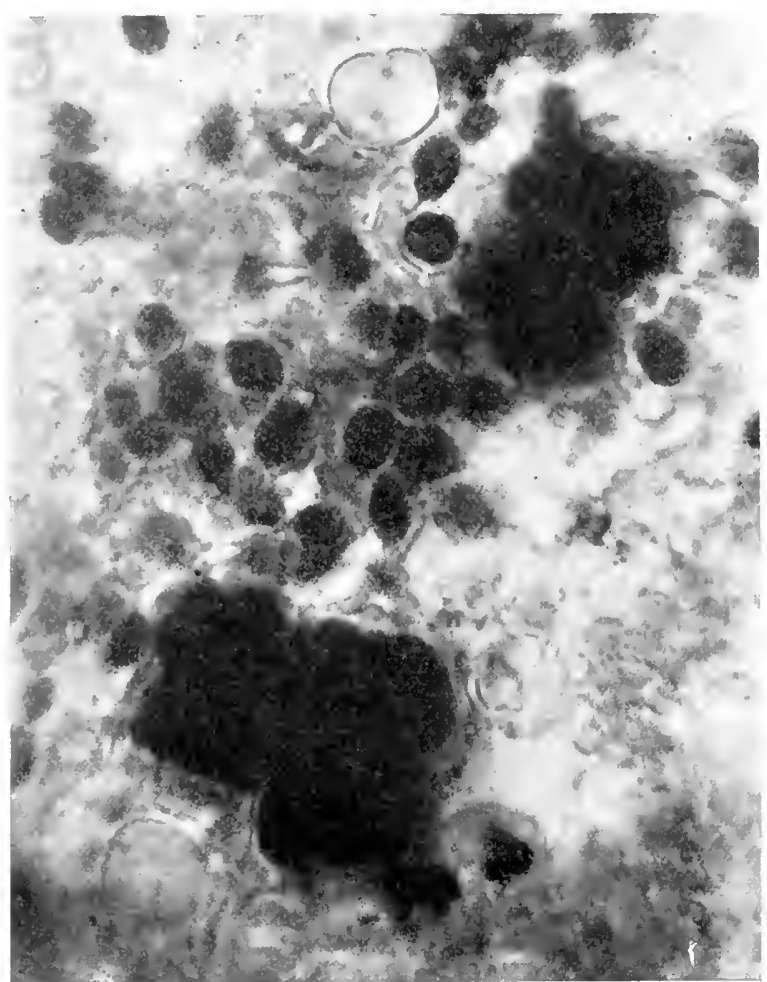

FIG. 57.-Yeasts and sarcine in case of benign retention. Photomicrogram.-(Author.)

took the stain not so evenly as did the Oppler-Boas type of organism, and their ends were rather acutely pointed or curved. Stained with Lugol's mixture, the Oppler-Boas bacilli in this medium were faintly amber. Leptothrix were more deeply colored.

After 48 hours, macroscopically, the culture on the 
cancer medium was so heary as to render the bouillon completely opaque. There was a heary, coarse, flocculent precipitate. On the surface of the medium was a thin, grayish-white, superficial pellicle. The culture had a musty odor. Microscopic examination of stained smears

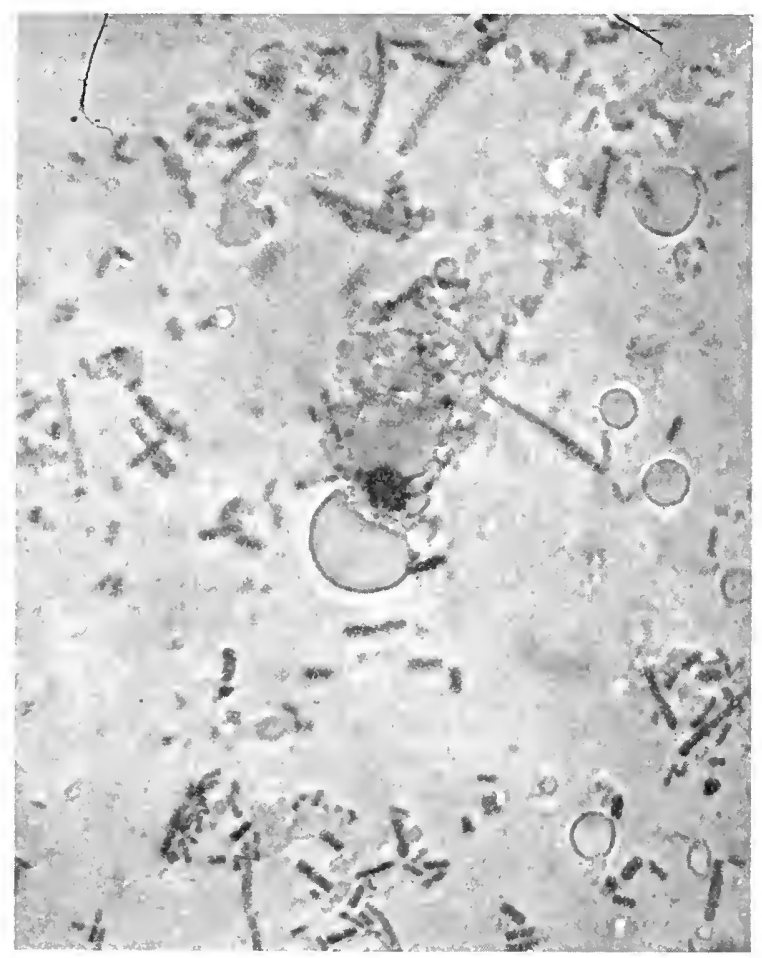

FIG. 55.-Oppler-Boas bacilli from artificial culture (cancer extract bouillon) showing variations in size, and different groupings. Photomicrogram.-(Author.)

showed an enormous number of short, thick bacilli in small groups and clumps. Their arerage length was 4 to 6 microns. They stained rather irregularly, some having beaded or club-shaped ends. They seemed different from another organism present, a bacillus staining regularly and 6 to 12 microns long. These occurred in 
short chains or singly. These forms were distinctly club shaped, the clubbed portion staining rather lightly (vacuole?). When club shaped, these bacilli were always single. The smear showed also many round or oval slight racuolated cocci about 2 microns in diameter. These resembled very much the clubbed ends of the bacilli above described. Occasionally, one could see a bacillus longer than 12 microns, in which the vacuolated clubbing appeared at each end. There were a few bacilli in which this vacuolation and clubbing occurred in the middle. The non-clubbed forms were straight or slightly bent rods with an average length of 8 microns. They were frequently in short chains, arranged at angles to each other, or forming small loops like a twisted whip-lash. Sometimes the long, straight variety was rather obliquely angulated at one end. Occasionally, what appeared to be separated clubbed ends of bacilli formed large colonies of coccuslike organisms. In these the transition from the clubbed end to the coccus form could be seen in all stages. Also it would seem that long, straight bacilli were gradually changed into the variety with clubbed ends. No yeasts or leptothrix were seen.

Cultures in Beef Bouillon after 48 Hours.-These cultures were slightly cloudy. There was a coarse, flocculent precipitate. They had a slightly musty odor.

Microscopic examination of stained smears showed enormous numbers of leptothrix in the form of large, single, wary segments, varying in length from 6 to 100 , plus, microns. Occasionally there was branching, but usually end-to-end union occurred. The segments joined each other at angles. Sometimes the leptothrix were arranged in coarse networks, loosely wound skeins, spirals or whorls. They stained very irregularly with resultant 
light and dark areas alternating. Occasionally, they presented a coarsely beaded appearance. Comparatively few long bacilli of the Oppler-Boas type were seen. These were usually arranged in small groups or occurred singly. They raried in length from 6 to 20 microns. They were usually straight or slightly curved. Their ends were rather blunt or angulated. No clubbed-or bulbed-shaped ends were seen. No racuolated forms were noted. They stained rather irregularly. Short chains occasionally occurred. These were made up of 2 to 4 organisms often arranged at sharp angles with each other. Stained with Lugol's solution, leptothrix were colored amber-yellow with no shade of blue, and took a color somewhat darker than do Oppler-Boas bacilli.

Cultures after 72 Hours. - The growths on cancer extract medium were densely opaque and had a faint musty odor. Stained specimens showed an abundant growth of rods which varied in length from 4 to 18 microns. They were arranged singly, in pairs and in angulated chains, of from 2 to 5 organisms, or in compact masses. The larger rods were often slightly curved or bent at the middle or toward one end. They stained deeply and usually homogeneously. Their ends were commonly bluntly rounded, but some were almost square. The medium-size or the small rods were most often club shaped or bulbed at one end. In this bulbed end was a spore-like body which did not stain so densely as does the shaft of the rod. These forms were thicker and usually single or closely arranged in clumps. A number of round or ovoid spore-like bodies appeared irregularly. A few curved chains or loop-like strings were seen. These were made up of curved or bent bacilli. No leptothrix or yeasts were seen. 
Seventy-two-hour Growth in Beef Bouillon.-The medium was rather hearily clouded. There was a coarse, granular precipitate. Microscopic examination revealed leptothrix in dense networks, skeins, whorls and bands. These chains were made up of irregularly branching strands or long organisms crossing each other in every direction. Occasionally a bacillus resembling the Oppler-Boas type was seen, but no spore-like forms or bacilli with clubbed ends were found. No yeasts were present.

Macroscopic Examination of Oppler-Boas Cultures after 96 Hours.-The cancer extract medium was somewhat opalescent. There was a coarse, heavy, granular sticky precipitate.

Beef bouillon cultures were cloudy. There was a thick, stringy, gelatinous sediment.

Microscopic Examination.-Stained smears from the cancer medium cultures revealed very few long rods. Those that were present were slightly bent or curved in the middle and were usually single. Some of the long rods were slightly club shaped at one end. They stained deeply and had blunt ends. There were many spore-like bodies that appeared larger than in the 72-hour culture. Many of them were elongated and a few were dumb-bell shaped. Sometimes these spore-like bodies were joined end to end and formed short chains of from 2 to 3 pairs of spores. An occasional leptothrix was seen. It usually formed a thin strand.

Cultures in the beef bouillon medium showed enormous numbers of leptothrix. These were single or grouped in skeins, whorls, or dense masses. They usually stained diffusely. There were no clubbed or dumb-bell shaped bodies nor any spore-like structures. With Lugol's solution they stained light amber. 
Macroscopic Examination of Oppler-Boas Cultures after 120 Hours.-Growths in the cancer medium were cloudy with abundant coarse, granular sediment and a rather yeastlike odor.

Cultures in the beef bouillon were very cloudy. On their surface was a slimy pellicle. There was a thick, flocculent sediment and a strong, rather acid odor.

Microscopic Examination of Cultures.-Smears from the cancer medium revealed an abundant growth of organisms from 6 to 20 microns long. They were arranged in long chains, loose groups or parallel bands. These organisms were bacilli, and while the majority of them were straight, the small ones were slightly curved. There were also long bacilli which were curved at the middle and occasionally wary. When they formed chains, the bacilli joined end to end at an angle. In the very large bacilli there were many with club-like endings. Short chains were occasionally seen. These had a zig-zag appearance or formed curves or loops. The smaller bacilli sometimes showed bipolar staining but as a rule they stained fairly deeply and uniformly. The longer bacilli rarely showed irregular staining. No spore-like bodies were seen.

Cultures in beef bouillon showed enormous growth of leptothrix arranged in chains, loops, skeins, twists, circles or long, single strands. There were many long, slender individual segments. These individual organisms appeared about half the width of the Oppler-Boas bacilli and varied greatly in length, so that without segmentation one might stretch across several microscopic fields (high power). Where segmentation occurred, the new segment frequently grew from the main one at a very sharp angle. These organisms stained uniformly. There were no bipolar bodies. 
Small individual bacilli were also seen. They were from 1 to 3 microns in length. Their ends were lance shaped. They were arranged in short chains. They never formed whorls or compact masses. There were no spore-like bodies or any organisms that showed bipolar staining. No yeasts were seen.

Table 17

\begin{tabular}{lrrrrrrrrrr}
\hline & Total Sarcinæ Yeasts & O.B. Lactic & $\begin{array}{c}\text { Abs. } \\
\text { Hcl }\end{array}$ & $\begin{array}{c}\text { Food } \\
\text { rem- } \\
\text { nants }\end{array}$ & Tumor $\begin{array}{c}\text { Occult } \\
\text { blood }\end{array}$ \\
& & & & & & & & & \\
& & & & & & & & & \\
Sarcinæ........ & 71 & 0 & 36 & 65 & 20 & 31 & 42 & 56 & 48 \\
Yeasts........ & 150 & 36 & 0 & 116 & 38 & 76 & 116 & 117 & 104 \\
Oppler-Boa.... & 221 & 65 & 116 & 0 & 50 & 132 & 178 & 186 & 172 \\
Lactic acid..... & 85 & 20 & 38 & 50 & 0 & 44 & 57 & 64 & 76 \\
Ab. free HCl... & 180 & 31 & 76 & 132 & 44 & 0 & 130 & 128 & 120 \\
Remnants..... & 194 & 42 & 116 & 178 & 57 & 130 & 0 & 146 & 142 \\
Tumor........ & 208 & 46 & 117 & 186 & 64 & 128 & 146 & 0 & 147 \\
Occult blood... & 202 & 48 & 104 & 172 & 76 & 120 & 142 & 147 & 0 \\
\hline
\end{tabular}

Showing the Clinical Interrelationship Existing between Important Testmeal Findings and the Presence of Abdominal Tumor.-(Author.)

Frequency of Occurrence of Oppler-Boas Bacilli in Gastric Cancer.- In 221 consecutive cases of the disease, in our series, organisms of the Oppler-Boas type were noted in 93.6 per cent. Yeasts were associated in 52 per cent. and sarcinæ in 29 per cent.

Table 17 has been constructed to summarize the relationship between the presence of Oppler-Boas bacilli, abdominal tumor and main test-meal data.

\section{EXAMINATION OF SECRETORY FUNCTION OF THE STOMACH IN GASTRIC CANCER}

A test-meal is employed. It should be easily prepared, palatable and contain essential food elements. We have found useful a meal consisting of

1. Sixty grams of second-day wheat flour bread and 500 ce. of luke warm water, or, 
2. Two shredded wheat biscuits and $500 \mathrm{cc}$. of luke warm water or weak tea (Dock), or,

3. Sixty grams of zweiback and $500 \mathrm{cc}$. of luke warm water or weak tea.

Generally, the meal is removed by means of a stomach tube at the end of 50 minutes. In instances where the stomach's emptying rate is rapid, the test-meal should be removed from 20 to 40 minutes after it has been eaten.

Macroscopic Study of Removed Contents.--In retention cases, unless the stomach has been thoroughly lavaged before the administration of the secretory meal, the test food may not be recognizable, on account of its admixture with stagnant gastric residue. In non-retention cases, the test-food appears poorly chymified, mixed with mucus and is often tinged with blood or bile.

The amount varies. Of course, if gastric retention exists, the quantity removed may be even greater than that of the test-meal given. In non-retention cases, from $50 \mathrm{cc}$. to 150 cc. are usually recovered. If a gristle-like cancer holds open a pylorus, the amount regained may be but a few cubic centimeters.

The odor is characteristic only where retention, hemorrhage or sloughing exists. The gastric extracts may then have rancid, fleshy, or putrid odors. In non-retention cases, the odor is not uncommonly of a peculiar, nauseating sweetness.

Acidity.-In retention cases, the arerage total acidity of the secretory meal was 32. The minimum was 2. The maximum was 67 . In non-retention cases, the arerage total acidity was 15 . The minimum was 2 . The maximum was 44.

Free hydrochloric acid averaged 9.2 in those cases where retention had been proved. The minimum was 0 and the 
maximum 29. Where retention did not exist, the arerage free hydrochloric acid was 17.4. The minimum was 0 and the maximum 73 .

"Combined" acidity areraged 13.2. The minimum was 0 and the maximum was 104. In the non-retention group, the average combined acidity was 7.1. The minimum was 0 and the maximum 27 .

Lactic acid was present in the secretory meal of 34.3 per cent. of the retention cases and 16 per cent. of the non-retention cases.

Altered ("occult") blood was demonstrated by the benzidin or guaiac tests in 68 per cent. of the retention cases and in 56.6 per cent. of cases of the non-retention group.

Microscopic examination of the secretory meal revealed bacilli of the Oppler-Boas type in $\$ 7.2$ per cent. of the retention group and in 36 per cent. of the non-retention class.

Gluzinski's Method of Determining Acidity.-In gastric cancer or in cases where a peptic ulcer is suspected of being carcinomatous, Gluzinski claims that the relative increase in acidity after different test-meals aids in the segregation of the two conditions. The method is particularly instructive in the cases in which an ulcer is beginning to develop cancerous degeneration. The contents of the fasting stomach are siphoned out in the morning and the findings recorded as to amount, color, odor, relics of food, blood, etc., litmus reaction, free acid with Congo paper and free acid with phloroglucin-vanillin; lactic acid with Strauss or Uffelmann tests, and occult blood with the guaiac-turpentine test. The amount of free acid and total acid is determined by titration. The microscopic findings are also recorded. Then the stomach is washed 
out clean with tepid water and an Ewald-Boas test breakfast is given. Forty-five minutes later the stomach contents are siphoned out again and the stomach thoroughly rinsed out anew. The test-dinner is then given: about 100 grams of chopped roast veal or boiled beef; 150 grams potato cooked with 20 grams fat and no fluid. The stomach contents are siphoned out anew after 2 hours. All this is done on one day. The finding of larger amounts of hydrochloric acid after the test-dinner speaks for ulcer; smaller proportions, for cancer. The insufficiency of the stomach mucosa is revealed by the lack of acid after the test-dinner, even when some acid was found after the test-breakfast. Fonio regards such findings as absolutely conclusive in dubious cases. He tabulates the findings in 26 ulcer cases and comments on the value of the information thus derived. In 4 other cases the findings proved dubious and the course of the cases showed that the ulcer at the time must have been just starting malignant degeneration. This procedure is one of much promise, and should be tried in doubtful cases as a matter of routine. Negative information should never, howerer, postpone laparotomy where the clinical history is suggestive.

Tests for Gastric Ferments in Gastric Cancer.-Rennin and Pepsin.-It would appear that there is still much uncertainty as to the inter-relationship existing between acidity and ferment activity in gastric ailments. This is particularly the case in gastric cancer. It appears that proteolysis is closely associated with the presence of free hydrochloric acid, so long as the acid concentration is below 0.4 per cent., and that milk-curdling ferment follows similar laws. In malignancy peptolysis appears to be increased at the expense of proteolysis. However, Pelosi states that in gastric cancer the excess of milk-curdling 
ferment and of the proteid digesting ferment are diagnostic in doubtful cases, where $\mathrm{Hcl}$ is absent. It would seem that lactic acid bacilli may exist in these cases of achlorhydria and that they may be responsible for increased milk curdling. As we show below (vide Glycyltryptophan Test), peptid-splitting, enzyme-like agents seem to be present in more than 40 per cent. of cases of cancer of the stomach.

\section{Edestin Test ("Peptic Index") of Fuld and Levison.-} According to these investigators the peptic power of gastric juice in cancer of the stomach is diminished. Confirmatory observations have been made by schryver and Singer. Edestin is very difficult to obtain pure, commercially, but may be made in a well-equipped laboratory. The latter's modification of the original test is as follows:

A pure preparation of edestin may be obtained in the laboratory by recrystallization from warm salt solution. From this is made a solution of 0.1 per cent. of edestin in 0.12 per cent. hydrochloric acid (i.e., 30 ec. normal $\mathrm{Hcl}$ in 1 liter distilled water. Such a solution of edestin need not be made freshly for each observation, but that if used as stock it must be stored at zero temperature). Into each of 10 small test-tubes $2.5 \mathrm{cc}$. of this solution are pipetted and left to take the temperature of the room $\left(10\right.$ to $20^{\circ} \mathrm{C}$.). Into each test-tube is now dropped $0.1,0.2,0.3,0.4, \ldots$ to $1.0 \mathrm{cc}$. (in ascending series) of the gastric juice under investigation previously diluted to one-tenth of its natural strength. Each test-tube is shaken and left to stand. After 30 minutes have elapsed $0.3 \mathrm{ce}$. of a saturated solution of sodium chlorid is added to each tube. If digestion has proceeded to a certain point, the solution remains clear, while with lower degrees of digestion a white cloudiness immediately develops.

The peptic index in any case is designated as the number of tenths of a cubic centimeter of a diluted juice added to the first clear test-tube and divided into 100 . This gives as a range of possible readings the ten numbers 100 (i.e., 100/1), 
50 (i.e., 100/2), 33 (i.e., 100/3), 25, 20, 17, 14, 12, 11 and 10. For greater accuracy intermediate amounts of diluted gastric juice may be added, as $0.125,0.150,0.175$ (between 0.1 and 0.2 ), etc., but with practice it becomes possible to read such intermediate numbers without recourse to actual experiment. The average of healthy cases is about 50 . Cases do occasionally present themselves with an index above 100 (i.e., in which even the test-tube containing only $0.1 \mathrm{cc}$. of diluted juice remains clear on the addition of the salt solution). In such instances recourse must be had to the use of more diluted juice. At the other extreme, however, it is seldom necessary to take readings below an index of 10 (i.e., in which even the test-tube containing $1.0 \mathrm{cc}$. of diluted gastric juice develops cloudiness on the addition of salt solution), for in such cases our experience seens to show that digestive power is probably almost absent.

We have examined 108 cases of gastric disease by the method of Fuld and Levison. The results thus far obtained would appear to indicate that early and adranced cases of carcinoma where free hydrochloric acid is low exhibit high peptolysis and low proteolysis. In benign peptic ulcers both peptolysis and proteolysis are low, if acids are reduced.

Formol Index. - Te have made observations upon 827 instances of gastric disease for the detection of specific ereptases in stomach juice. The have used the modification of the formaldehyde titration method of Sorenson and Schiff, suggested by Schryver and Singer.

Method.-20 cc. of a filtered 5 per cent. solution of Witte's peptone are mixed with $1 \mathrm{cc}$. of the filtered juice under examination. Another sample of a similar solution is mixed with $10 \mathrm{cc}$. $n / 10$ sodium hydroxide solution, to which is also added $1 \mathrm{cc}$. of the gastric juice. Similar mixtures without addition of juice are kept as controls, and all the 4 samples are incubated for 20 to 24 hours at $37^{\circ}$, with the addition of $1-2 \mathrm{ce}$. of toluene to prevent 
putrefaction. After removal from the incubator, each sample was treated with $10 \mathrm{cc}$. of 40 per cent. formaldehyde solution (commercial formalin), previously neutralized to phenolphthalein by sodium hydroxid, and containing, therefore, some of the indicator (about $1 / 2 \mathrm{cc}$. of 0.5 per cent. solution of phenolphthalein in 50 per cent. alcohol to each $10 \mathrm{ce}$. of formalin). The formaldehydepeptone mixture was then immediately titrated.

In our experience, the average formol titration index in 87 instances of operatively proved gastric cancer was 22.3; the average index in 22 cases of ulcus carcinomatosum 19.8; the average index in 99 cases of duodenal ulcer, 12.4; of 57 cases of benign gastric ulcer 11.6 ; of 32 cases of benign achylia gastrica, 14.1; of 16 instances of pernicious anemia, 14.5 and 5 cases of cancer of the liver, 4.25 . It would appear that, in certain cases, the estimation of the ereptic power of gastric juice toward peptone solutions is of considerable value when interpreted in the light of other clinical findings.

Glycyltryptophan Test.-Various workers, notably Müller, Fischer, and Abderhalden have reported that malignant neoplasmata contain certain peptidolytic enzymes. This discovery appeared to have clinical value when Neubauer and Fischer announced that simple peptids, particularly the dipeptid, glycyltryptophan, were hydrolyzed by cancerous ferments. In the case of glycyltryptophan the amino-acid tryptophan, which is liberated by this cleavage, can be recognized readily in acid solution by the rose-pink color occurring on the addition of bromine. This reaction forms the basis of the "glycyltryptophan test" for cancer of the stomach, advanced by Neubauer and Fischer.

Clinicians generally have disagreed widely on the 
actual value of the test. The reaction's sponsor's, together with Lyle and Kober and Weinstein, early reported enthusiastically on the procedure. Later observers, especially Warfield, Oppenheim, Kohlenberger and, most recently, Sanford and Rosenbloom, declare that the test is of dubious value. They admit that while certain cases of cancer of the stomach undoubtedly give the reaction, many non-malignant gastric disturbances give similar tests. Factors claimed to influence the reliability of the reaction are swallowed saliva and bacteria, bile or blood in the gastric extracts, low or absent free hydrochloric acid and regurgitated duodenal contents.

In October, 1911, Weinstein announced that he had improved on the Neubauer and Fischer test. He stated that in extracts from cases of carcinoma ventriculi there exist free amino-acids, notably tryptophan, and that the latter can be tested for directly with bromine. This procedure appeared to render unnecessary the addition of glycyltryptophan to such gastric contents, with search for its clearage products subsequently. This so-called "tryptophan test" was claimed as a reaction pathognomonic of eancer of the stomach. Weinstein did not, howerer, go so far as to state just how early in the progress of the disease this test could be regarded as pathognomonic. Certainly, in the clinical cases which he briefly quoted, when the tryptophan test was positive, other evidences of cancer were not lacking. Recently Hall and Williamson and Sanford and Rosenbloom have recorded observations which appear to indicate that Weinstein's test has even less value than, in their experience, had the glycyltryptophan test.

We have tested more than 1,400 gastric extracts for the glycyltryptophan and the tryptophan reactions. On 
1,175 different individuals, the gastric extracts were tested according to the modification of the glycyltryptophan and the tryptophan tests recently suggested by me. This modification appear's to have the advantages of requiring less of the test ingredients than the Neubauer and Fischer method, of being a controlled procedure, and one in which the end-reaction may be easily determined. It is our purpose at this place to include our experience with the cases tested by this uniform method. The test is set up as follows:

Test-tubes of 10 ce. capacity are employed. These should be carefully cleaned with boiling water and dried inside. They are numerically marked for identification with a wax pencil. Into each test-tube is carefully measured, by means of a sterile graduated pipette, $0.5 \mathrm{ce}$. of the glycyltryptophan solution. Five ec. of the recently secured filtered gastric extract are then measured by a clean, graduated pipette and poured into the correspondingly numbered test-tubes to which glycyltryptophan solution has already been added. Two control tubes are used. In one is placed 0.5 ce. of glycyltryptophan solution and 5 cc. of normal salt solution, and into the other is placed $5 \mathrm{cc}$. of normal salt solution, without adding glycyltryptophan solution. In the entire series, each tube next receives 0.5 cc. of toluol (toluene, Merck). The contents of the tubes are then mixed by inverting several times. The tubes are next placed in a water-bath (an incubator may be used) at $37^{\circ} \mathrm{C}$. for 24 hours.

At the expiration of the incubation period, the testtubes are removed from the water-bath. Clean test-tubes of $10 \mathrm{cc}$. capacity and numbered to correspond with the gastric extracts tested, as well as the controls, are set in racks. Into each of these tubes is measured by means of a graduated pipet, 2 cc. of the glycyltryptophan-gastricextract mixture lying below the toluol in the recently incubated tubes. To each tube are then added 3 drops of a 3 per cent. glacial acetic acid in distilled water solution. The tubes are well shaken. Bromine vapor is allowed to 
flow in each tube until it appears amber-yellow above the contained fluid. The tubes are again shaken. Examination by daylight (preferred) or by white, artificial light is now made for evidences of the characteristic rose-pink reaction between the amino-acid (tryptophan) and the bromine.

Tryptophan Test.-As suggested by Weinstein, this is made, as routine, on the fresh gastric extracts, inasmuch as occasionally, swallowed saliva, amino-acids, regurgitated duodenal contents and the like may give the bromine vapor reaction, before incubation or without the addition of a dipeptid such as glycyltryptophan. Five cc. of each fresh, filtered gastric extract are poured into testtubes of 10 cc. capacity acidulated with the 3 per cent. acetic acid solution and treated with bromine vapor as above. If no characteristic rose-pink color results, the tubes are incubated with the corresponding specimens that have been mixed with glycyltryptophan solution. For accurate work it has seemed best to us to cover these "tryptophan test" contents with a layer of toluol. At the end of 12,24 and 48 hours, note is made of changes in color, and these results are compared with those obtained with the preparations in the first series.

Certain precautions taken in the manipulation of the reaction might be mentioned briefly. All glassware was boiled in distilled water and dried before using. The solution of glycyltryptophan employed was obtained, in bulk and unopened, direct from the makers. To guard against its tendency to crystallize out in cold solution, the preparation was kept in a water-bath at $37^{\circ} \mathrm{C}$. until used. All gastric extracts were carefully filtered before testing, and the tests were set up within 2 hours, at the outside, from the time the contents were taken from the patients. In testing for tryptophan, before or after incubation, bromine vapor was preferred over bromine water. It is more readily controlled quantitatively and permits of better color determination. All end reactions were read by daylight. 
Typical Reactions. - When bromine vapor is used for the detection of amino-acid (tryptophan), its presence is indicated, even in small amounts, by lilac-violet to rose-pink shades. The color is usually a lively one, and appears quickly. Admixtures of much blood and bile produce, respectively, dirty, brownish-yellow and muddy green to drab. In such, gradations in shade are impossible. High organic acidity often gives rich purple or magenta hues. When the color change is opalescent, with bluish or delicate lilac cast, the results may be classed safely as negative.

Results.-The gross results of our obseryations are as follows: Of 1,175 gastric extracts from individuals with gastric symptoms, clinically, 110, or 9.36 per cent., were

Table 18

\begin{tabular}{|c|c|c|c|}
\hline Diagnosis & $\begin{array}{l}\text { Number } \\
\text { of cases }\end{array}$ & Diagnosis & $\begin{array}{l}\text { Number } \\
\text { of cases }\end{array}$ \\
\hline Carcinoma ventriculi..... & 31 & Achylia gastrica......... & 6 \\
\hline Ulcus ventriculi......... & 9 & Appendicitis........... & 8 \\
\hline Carcinoma of the liver.... & 3 & Primary anemia ......... & 3 \\
\hline Uleer of duodenum...... & 3 & Syphilis-stomach....... & 1 \\
\hline $\begin{array}{l}\text { Non-malignant pyloric ob- } \\
\text { struction............... }\end{array}$ & 1 & $\begin{array}{l}\text { Various (gastritis, gastric } \\
\text { neurosis, chronic diarrhœa, }\end{array}$ & \\
\hline Cholecystitis.......... & 11 & epilepsy.............. & 10 \\
\hline Gall-stones............ & 6 & & \\
\hline Hypochlorhydria......... & 7 & & \\
\hline Achlorhydria.......... & 11 & Total..... & 110 \\
\hline
\end{tabular}

Summary of Cases giving Positive Glycyltryptophan Test.-(Author.)

Table 19

\begin{tabular}{|c|c|c|c|}
\hline Diagnosis & $\begin{array}{l}\text { Number } \\
\text { of cases }\end{array}$ & Diagnosis & $\begin{array}{l}\text { Number } \\
\text { of cases }\end{array}$ \\
\hline Carcinoma ventriculi..... & 7 & Appendix lesions......... & 1 \\
\hline Ulcus ventriculi.......... & 3 & Various (neuroses, achlor- & \\
\hline Uleer duodenum......... & 3 & hydria, arteriosclerosis)... & 6 \\
\hline Carcinoma of the liver.... & 1 & & \\
\hline Gall-stones............. & 3 & Total... & 24 \\
\hline
\end{tabular}

Summary of Cases giving Positive Tryptophan Test.-(Author.) 
Table 20

\begin{tabular}{|c|c|c|c|c|c|c|c|}
\hline $\begin{array}{l}\text { Case } \\
\text { No. }\end{array}$ & Diagnosis & $\begin{array}{c}\text { Total } \\
\text { acidity }\end{array}$ & $\begin{array}{l}\text { Free } \\
\mathrm{HCl}\end{array}$ & Blood & Bilc & $\begin{array}{l}\text { Lactic } \\
\text { acid }\end{array}$ & $\begin{array}{c}\text { Degree } \\
\text { of re- } \\
\text { action * }\end{array}$ \\
\hline 61563 & Gall-bladder infect........ & 46 & 36 & + & 0 & 0 & + \\
\hline 9532 & Anemia (post. mort.)...... & 0 & 0 & + & 0 & 0 & + \\
\hline 61743 & Duodenal ulcer-opr........ & 86 & 80 & 0 & ++ & 0 & + \\
\hline 61000 & Gall-bladder ............. & 46 & 40 & 0 & 0 & 0 & + \\
\hline 61795 & Gastritis chr............ & 14 & 6 & + & 0 & 0 & + \\
\hline 61857 & Carcinoma stom.-opr..... & 0 & 0 & ++ & 0 & 0 & ++ \\
\hline 61802 & Gastritis; chr. append...... & 4 & 0 & + & + & $?$ & + \\
\hline $3 \$+06$ & Gall-bladder infect........ & 6 & 0 & 0 & 0 & 0 & ++ \\
\hline 53228 & Gastric neurosis........... & 18 & 18 & + & 0 & 0 & ++ \\
\hline 61852 & Carcinoma of stomach ..... & 4 & 0 & + & 0 & 0 & ++ \\
\hline 61910 & Carcinoma of stomach-opr. & 0 & 0 & ++ & 0 & ++ & + \\
\hline 61812 & Epilepsy.............. & 10 & 10 & + & + & 0 & ++ \\
\hline 61940 & Gastritis-chr.......... & 24 & 24 & 0 & 0 & 0 & + \\
\hline 61862 & Gastric neurosis........... & 40 & 30 & 0 & $++t$ & 0 & + \\
\hline 61974 & Gastritis-aleoholic........ & 0 & 0 & + & + & + & +++ \\
\hline 62100 & Carcinoma of stomach-opr. & 20 & 0 & + & 0 & + & + \\
\hline 62171 & Carcinoma of stom.-opr. . & 48 & 0 & + & 0 & ++ & + \\
\hline 62086 & Gastritis-chr........... & 10 & 0 & 0 & ++ & 0 & + \\
\hline 62089 & Gastric ulcer........... & 40 & 40 & 0 & + & 0 & + \\
\hline 62219 & Carcinoma of stom.-opr. . . & 24 & 12 & + & 0 & 0 & + \\
\hline 62154 & Carcinoma of stom.-opr. . & 8 & 0 & 0 & + & 0 & ++ \\
\hline 62233 & Carcinoma of liver-expl. .. & 24 & 24 & + & + & 0 & ++ \\
\hline 62260 & Pyloric obstr., non-malignant & 40 & 32 & ++ & + & 0 & + \\
\hline 62399 & Careinoma of stom.-opr. . . & 0 & 0 & + & 0 & 0 & + \\
\hline 53032 & Duodenal ulcer一opr........ & 50 & 50 & Tr. & + & 0 & + \\
\hline 62562 & Carcinoma of gall-bl. opr... & 6 & 0 & + & + & 0 & + \\
\hline 62665 & Gastric ulcer............ & 28 & 28 & Tr. & + & 0 & ++ \\
\hline 37124 & Carcinoma of stom. гесur. .. & 8 & 0 & 0 & + & 0 & + \\
\hline 61072 & Gall-stones-opr.......... & 56 & 40 & 0 & $\mathrm{Tr}$. & 0 & + \\
\hline 62876 & Cholecystitis............. & 10 & 0 & Tr. & ++ & 0 & +++ \\
\hline 62912 & Chr. ap. opr............. & 22 & 18 & Tr. & + & 0 & + \\
\hline 62971 & Chr. ap. opr............. & 22 & 0 & 0 & + & 0 & + \\
\hline 62977 & Ulcer of stomach ......... & 48 & 40 & 0 & 0 & 0 & + \\
\hline 63026 & Chr. appendicitis........... & 32 & 32 & + & + & 0 & + \\
\hline 63051 & Neg. stom. ap........... & 36 & 32 & 0 & + & 0 & + \\
\hline 63093 & Chr. diarrh. stom.-neg..... & 4 & 0 & 0 & 0 & 0 & + \\
\hline 63129 & Stom. ulcer and G. B.-opr.. & 32 & 30 & + & 0 & 0 & 0 \\
\hline 63130 & Chr. app--opr........... & 0 & 0 & 0 & 0 & 0 & + \\
\hline 62699 & Cholangitis............ & 0 & 0 & Tr. & 0 & 0 & + \\
\hline 63030 & Carcinoma of stomach ..... & 8 & 0 & 0 & 0 & 0 & + \\
\hline $632+1$ & Carcinoma of stomach-opr. & 56 & 18 & + & 0 & 0 & + \\
\hline 63292 & Gastric ulcer............ & 46 & 46 & + & + & 0 & + \\
\hline 63197 & Multiple sclerosis.......... & 20 & 6 & 0 & + & 0 & + \\
\hline 63335 & Duod. ulcer; cholecyst...... & 48 & 40 & 0 & + & 0 & + \\
\hline 52034 & Recurrent ca. of stomach ... & 10 & 0 & + & + & 0 & + \\
\hline 62876 & 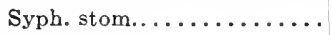 & 0 & 0 & 0 & 0 & 0 & + \\
\hline 63506 & 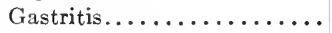 & 38 & 22 & 0 & + & 0 & + \\
\hline 63547 & Resect. stom. ca.......... & 0 & 0 & + & + & $?$ & + \\
\hline 63562 & Arteriosclerosis........... & 0 & 0 & 0 & 0 & 0 & + \\
\hline 63600 & Appendicitis-neg........ & 44 & 40 & 0 & + & 0 & + \\
\hline 63616 & Appendix and G. B.-opr. .. & 12 & 0 & 0 & + & 0 & + \\
\hline 63653 & Ulcer stom.-opr. ........ & 32 & 28 & $\operatorname{Tr}$. & 0 & 0 & + \\
\hline 63636 & Gall-stones-opr........... & 22 & 0 & 0 & 0 & 0 & + \\
\hline 63778 & Carcinoma of stom. ......... & 14 & 0 & + & + & + & + \\
\hline 36634 & Ulcer of stom.......... & 40 & 30 & + & 0 & 0 & + \\
\hline 63383 & 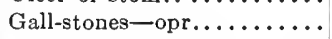 & 6 & 6 & 0 & 0 & 0 & + \\
\hline 64057 & Hypochlorhydria.......... & 8 & 0 & + & ++ & 0 & + \\
\hline
\end{tabular}


Table 20-(Continued)

\begin{tabular}{|c|c|c|c|c|c|c|c|}
\hline $\begin{array}{l}\text { Case } \\
\text { No. }\end{array}$ & Diagnosis & $\begin{array}{l}\text { Total } \\
\text { acility }\end{array}$ & $\begin{array}{l}\text { Free } \\
\text { HCl }\end{array}$ & Blood & Bile & $\begin{array}{c}\text { Lactic } \\
\text { acid }\end{array}$ & $\begin{array}{c}\text { Degree } \\
\text { of re- } \\
\text { action* }\end{array}$ \\
\hline
\end{tabular}

\begin{tabular}{|c|c|c|c|c|c|c|}
\hline 64039 & Aclilorhydria............. & 0 & 0 & 0 & 0 & 0 \\
\hline 61915 & Hypochlorlydria.......... & 4 & 4 & 0 & 0 & 0 \\
\hline 64270 & Ilerr of stom.-opr......... & 50 & 46 & + & 0 & 0 \\
\hline 64330 & Carcinomat of stomach...... & 11 & 0 & + & 0 & + \\
\hline 61360 & Neurosis................. & 54 & 50 & 0 & + & 0 \\
\hline 61452 & Carc. of stom-resect...... & $S$ & 0 & + & 0 & 0 \\
\hline 64155 & IIypochlorhydria.......... & t & 0 & + & 0 & 0 \\
\hline $6+4251$ & Achylia gastr.............. & 0 & 0 & + & 0 & 0 \\
\hline 34078 & Neuroses................ & 42 & 40 & 0 & 0 & 0 \\
\hline 64877 & Cholecystitis............ & 4 & 0 & 0 & 0 & 0 \\
\hline 65179 & Appendicitis............ & 36 & 26 & 0 & Tr. & 0 \\
\hline 65229 & Ca. of stom. and liver....... & 0 & 0 & + & 0 & 0 \\
\hline 65293 & Achlorhydria $\ldots \ldots \ldots \ldots \ldots$ & S & 0 & 0 & + & 0 \\
\hline 65337 & Cholecystitis, appendix...... & 16 & 0 & 0 & Tr. & 0 \\
\hline 37059 & Appendicitis-opr.......... & 12 & 12 & Tr. & 0 & 0 \\
\hline 65693 & Gall-stones, and appendix- & & & & & \\
\hline & 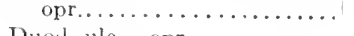 & 38 & 11 & 24 & + & 0 \\
\hline 22528 & Duoul. ule.-opr........... & $s$ & 0 & 0 & 0 & 0 \\
\hline 65703 & Degen. gast. ule.......... & $s$ & 0 & 0 & 0 & 0 \\
\hline 65835 & Gen. carc. prin. stom........ & 0 & 0 & 0 & 0 & 0 \\
\hline 65901 & Carcinoma of stom........ & 6 & 0 & + & 0 & 0 \\
\hline 5231 & Achlorhydria............ & 8 & 0 & 0 & + & 0 \\
\hline 65953 & 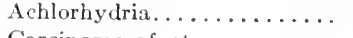 & 12 & 0 & 0 & + & 0 \\
\hline 66017 & Carcinoma of stom........ & 30 & 0 & + & 0 & 0 \\
\hline 66108 & Gall-stone-opr.......... & 8 & 0 & 0 & + & 0 \\
\hline 66225 & Pernicious anemia......... & 40 & 0 & + & 0 & 0 \\
\hline $6+9 \cdot 12$ & Second. anemia........... & 10 & 0 & 0 & 0 & 0 \\
\hline 66314 & Cholecystitis............ & 30 & 30 & 0 & + & 0 \\
\hline 66333 & Gall-stones,-opr.......... & 10 & 0 & + & 0 & 0 \\
\hline 66409 & Achlorhydria............. & 20 & 0 & + & 0 & 0 \\
\hline 66462 & Gastric ulcer.............. & 26 & 26 & + & 0 & 0 \\
\hline 56586 & Carcinoma of stom.-recur... & $4 S$ & 48 & 0 & 0 & 0 \\
\hline 66166 & Achlorhydria.............. & 45 & 0 & 0 & 0 & 0 \\
\hline $635+7$ & Carcinoma of stom.-resect... & 12 & 0 & Tr. & 0 & 0 \\
\hline 66511 & Achlorhydria............ & 4 & 0 & 0 & 0 & 0 \\
\hline $665 \$ 3$ & 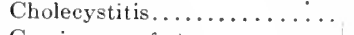 & 50 & 50 & 0 & 0 & 0 \\
\hline 66614 & Carcinoma of stom......... & 12 & 0 & 0 & 0 & 0 \\
\hline 60757 & Achlorhydria............. & 20 & 0 & $\operatorname{Tr}$ & 0 & 0 \\
\hline 66904 & Achlorhydria............ & 34 & 0 & $\operatorname{Tr}$ & 0 & 0 \\
\hline 66855 & Carcinoma of stom.-opr..... & 26 & 26 & + & 0 & 0 \\
\hline 66864 & Hypochlorhydria.......... & 12 & 12 & + & 0 & 0 \\
\hline 67000 & Deg. gast. ulc............ & 26 & 26 & + & 0 & 0 \\
\hline 45833 & Hypochlorhydria.......... & 8 & 8 & + & 0 & 0 \\
\hline 67110 & Cancer of stomach......... & 20 & 0 & + & 0 & + \\
\hline 67112 & Gastric neurosis........... & $3 \mathrm{~s}$ & 24 & 0 & 0 & 0 \\
\hline 67077 & Care. of liver and spleen..... & 80 & 80 & 0 & 0 & 0 \\
\hline 67206 & Carcinoma of stom......... & 14 & 0 & 0 & + & 0 \\
\hline 67295 & Hypochlorhydria.......... & 18 & $\$$ & Tr. & + & 0 \\
\hline 67368 & Carcinoma of stom......... & 50 & 50 & + & 0 & 0 \\
\hline 67690 & Pernicious anemia.......... & 4 & 0 & 0 & + & 0 \\
\hline 67298 & Carcinoma of stom. P.A. (?). & 14 & 0 & + & 0 & 0 \\
\hline 67562 & 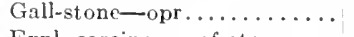 & 12 & 0 & 0 & 0 & 0 \\
\hline 67537 & Expl, carcinoma of stom..... & 20 & 0 & 0 & 0 & 0 \\
\hline
\end{tabular}

Clinical and Laboratory Data of the Cases Returning Positive Glycyltryptophan Test (Author).

* Degree of reaction: Lilac equals + ; rose-pink equals ++ ; rose-purple equals +++ . 
glycyltryptophan positive. In the same cases, 24, or 2.04 per cent. were tryptophan positive, either before or after incubation. Tables 18 and 19 show, respectively, the number of positives with each test, associated with different diseases. Tables 20 and 21 , respectively, consider the clinical and laboratory data.

It will be noted that one of the valuable features of the tables is the fact that the majority of the cases exhibiting positive reactions were treated surgically; hence, the conclusions derived from consideration of the figures returned have a fairly definite pathologic basis.

Table 21

\begin{tabular}{|c|c|c|c|c|c|c|c|}
\hline No. & Diagnosis & $\begin{array}{c}\text { Total } \\
\text { acidity }\end{array}$ & $\begin{array}{l}\text { Free } \\
\mathrm{HCl}\end{array}$ & Blood & Bile & $\begin{array}{l}\text { Lactic } \\
\text { acid }\end{array}$ & $\begin{array}{c}\text { Degree } \\
\text { of re- } \\
\text { action* }\end{array}$ \\
\hline 67644 & Achlorhydria.............. & 4 & 0 & 0 & 0 & 0 & + \\
\hline $6150 \mathrm{~s}$ & 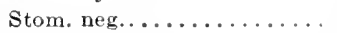 & 36 & 36 & 0 & + & 0 & + \\
\hline 61496 & Duod. ulcer-opr........... & 56 & $3 \mathrm{~s}$ & 0 & 0 & 0 & + \\
\hline 61567 & Gastric ulcer.............. & 42 & 20 & 0 & + & 0 & + \\
\hline 61552 & Carcinoma of stom.-inop.. & $3 \mathrm{~s}$ & $1 \mathrm{~s}$ & + & 0 & + & ++ \\
\hline 62223 & Carcinoma of liver and G.B. & 24 & 24 & + & + & 0 & ++ \\
\hline 62784 & Gastric ulcer-clin......... & 12 & 12 & + & 0 & 0 & $\div$ \\
\hline 62876 & Achlorhydria and G.B...... & 10 & 0 & + & $+t$ & 0 & + \\
\hline $62 \$ 65$ & Appendix, chronic......... & 24 & 18 & $\operatorname{Tr}$. & + & 0 & + \\
\hline 63051 & Gastric neurosis........... & 36 & 32 & 0 & + & 0 & + \\
\hline 63230 & Carcinoma of stom. (mass).. & $\mathrm{s}$ & 0 & 0 & 0 & 0 & + \\
\hline 63241 & Carc of stomach (resect.).... & $5 s$ & 18 & + & 0 & 0 & + \\
\hline 63221 & Gall-stone empyema G.B... & 20 & 20 & 0 & Tr. & 0 & + \\
\hline 63414 & Duodenal ulcer-opr.......... & 80 & so & 0 & + & 0 & + \\
\hline 63408 & Carcinoma of stom.-opr... & 66 & 60 & 0 & 0 & 0 & + \\
\hline 63653 & Gastric ulcer-clin......... & 32 & $2 S$ & $\mathrm{Tr}$. & 0 & 0 & + \\
\hline 63354 & Tabes-crises.............. & 1 & 0 & + & 0 & 0 & + \\
\hline 63563 & Arteriosclerosis-gen........ & 0 & 0 & 0 & 0 & 0 & + \\
\hline 63536 & Gall-stone-opr............ & 22 & 0 & 0 & 0 & 0 & + \\
\hline $6+394$ & Duodenal ulcer-opr......... & 66 & 60 & 0 & 0 & 0 & + \\
\hline 64294 & Carcinoma of stom.-opr... & 14 & 4 & + & 0 & + & + \\
\hline 65693 & Gall-stones and append-opr. & $3 S$ & 14 & + & 0 & 0 & + \\
\hline 5231 & Gastric ulcer degen. post-opr. & 8 & 0 & 0 & + & 0 & ++ \\
\hline $565 \$ 6$ & Carcinoma of stom.-recur.. & 45 & 0 & 0 & 0 & 0 & + \\
\hline 67112 & Gastric neurosis.......... & 38 & 24 & 0 & + & 0 & $+t$ \\
\hline
\end{tabular}

Clinical and Laboratory Data of the Cases Returning Positive Tryptophan Test (Author).

* Degree of Reaction: Iilac equals + ; Rose-pink equals ++ ; rose-purple equals +++ .

Cancer.-The total number of proven cases of cancer of the stomach, primary or secondary, in this series is 87 . 
Of this number, 31 or 35.6 per cent., gave positive glycyltryptophan tests, while 7 , or 8.04 per cent. were tryptophan positive. Of the 31 cases of cancer, in which the glycyltryptophan test was positive, the tryptophan test was positive but 7 times. In 3 cases in which the tryptophan test was positive, the glycyltryptophan test was negative.

Of 9 gastric ulcers with fair evidence of carcinomatous degeneration (of the type described by MacCarty), two, or 22.2 per cent., gave the glycyltryptophan reaction. In these same cases there was no positive tryptophan test. If we combine the returns from these cases with those from the specimens of advanced carcinoma, we noted that the glycyltryptophan test is positive in 39.09 per cent. and the tryptophan in 7.28 per cent., or the glycyltryptophan test is positive approximately $51 / 3$ times as frequently as is the tryptophan test.

Gastric Ulcer.-In none of 35 operated gastric ulcers (microscopically carcinoma-free) was the glycyltryptophan test positive. The tryptophan reaction was obtained once.

Thirty-nine cases were clinically diagnosed as gastric ulcer. Three of these (7.4 per cent.) were glycyltryptophan-positive, and two (5.2 per cent.) were tryptophanpositive.

Duodenal Ulcer.-Operations were performed on 78 patients with duodenal ulcers. Of this number, three (2.6 per cent.) gave glycyltryptophan and tryptophan tests. They were not identical cases and the reactions were not always associated with low acidity.

Fifty-seven individuals had duodenal ulcer, clinically. One (1.7 per cent.) was glycyltryptophan-positive. None gave the tryptophan test. 
Table 22

\begin{tabular}{|c|c|c|c|c|c|}
\hline Group & $\begin{array}{l}\text { Number } \\
\text { of posi- } \\
\text { tives }\end{array}$ & $\begin{array}{l}\text { Number } \\
\text { of nega- } \\
\text { tives }\end{array}$ & Group & $\begin{array}{l}\text { Number } \\
\text { of posi- } \\
\text { tives }\end{array}$ & $\begin{array}{l}\text { Number } \\
\text { of nega- } \\
\text { tives }\end{array}$ \\
\hline $\begin{array}{r}\text { Extracts having no } \\
\text { acidity .......... }\end{array}$ & 14 & 20 & $\begin{array}{l}\text { Extracts having de- } \\
\text { creased T. A...... }\end{array}$ & $8 S$ & 515 \\
\hline $\begin{array}{l}\text { Extracts having no } \\
\text { free } H c l . . . \ldots . .\end{array}$ & 52 & 31 & $\begin{array}{l}\text { Extracts having } \\
\text { normal } \mathrm{T} . \mathrm{A} . . . .\end{array}$ & 17 & 213 \\
\hline $\begin{array}{c}\text { Extracts having di- } \\
\text { minished Hcl.. ... }\end{array}$ & 15 & 214 & $\begin{array}{c}\text { Extracts having in- } \\
\text { creased 'T. A..... }\end{array}$ & 5 & 337 \\
\hline $\begin{array}{c}\text { Extracts having nor- } \\
\text { mal Hcl............ }\end{array}$ & 22 & 369 & Totals... & 110 & 1,065 \\
\hline $\begin{array}{l}\text { Extracts having in- } \\
\text { creased Hcl........ }\end{array}$ & $7^{\circ}$ & 431 & having & & \\
\hline Totals. & 110 & 1,065 & & & \\
\hline
\end{tabular}

The Relation of Gylcyltryptophan Test to Acidity.-(Author.)

Other Gastric Conditions.-It has been advanced by Weinstein, Warfield and Sanford and Rosenbloom that positive glycyltryptophan reactions are usually obtained in gastric extracts exhibiting achylia or low hydrochloric acid. These reactions are claimed to result from the presence of a peptid-splitting enzyme (Warfield) existing in saliva. Gies thinks that mouth-bacteria may be capable of splitting simple peptids under these conditions. In order to determine the results in our cases from the viewpoint of acidity, we have compiled Tables 22 and 23 . It will be seen that about 60 per cent. of the positive glycyltryptophan tests were obtained from extracts showing no free hydrochloric acid, while in an additional 13.6 per cent., the free hydrochloric acid was low. In other words, nearly three-fourths of the positives occurred in gastric extracts showing diminished acidity. Table 22 also brings out the interesting fact that approximately 80 per cent. of the glycyltryptophan reactions were returned by contents in which the total acidity was low.

The support which these figures apparently give to 
Warfield's saliva ferment action on peptids is qualified when one considers the negative glycyltryptophan tests in Table 22. Fifty-one of these extracts showed no free hydrochloric acid. In 214 extracts the free hydrochloric content was diminished. The combination of these results demonstrates that about one-fourth (24.8 per cent.) of the negatives were associated with low free hydrochloric acid. It could scarcely be maintined that all these extracts were saliva-free. Table 20 shows that some of the extracts were from cancerous patients. Approximately one-half ( 48.3 per cent.) of the negative glycyltryptophan tests were on extracts with diminished total aciclity.

Table 23

\begin{tabular}{|c|c|c|c|c|c|}
\hline Group & $\begin{array}{l}\text { Number } \\
\text { of posi- } \\
\text { tives }\end{array}$ & $\begin{array}{l}\text { Number } \\
\text { of nega- } \\
\text { tives }\end{array}$ & Group & $\begin{array}{l}\text { Number } \\
\text { of posi- } \\
\text { tives }\end{array}$ & $\begin{array}{l}\text { Number } \\
\text { of nega- } \\
\text { tives }\end{array}$ \\
\hline $\begin{array}{l}\text { Extracts having no } \\
\text { acidity........... }\end{array}$ & 1 & 33 & $\begin{array}{l}\text { Extracts having de- } \\
\text { creased } T \text {. }\end{array}$ & 17 & 586 \\
\hline Extracts having no & & & Extracts having & & \\
\hline free $\mathrm{Hcl} . . . . . . .$. & 6 & $\pi$ & normal T. A..... & 2 & 228 \\
\hline $\begin{array}{l}\text { Extracts having di- } \\
\text { minished Hcl...... }\end{array}$ & 10 & 219 & $\begin{array}{l}\text { Extracts having in- } \\
\text { creased T. A. .... }\end{array}$ & 5 & 337 \\
\hline Extracts having nor- & & & & & \\
\hline mal Hel......... & 4 & 357 & Totals... & 24 & 1,151 \\
\hline $\begin{array}{l}\text { Extracts having in- } \\
\text { creased Hel......... }\end{array}$ & 3 & 435 & Extracts & & \\
\hline Totals.... & 21 & $1,1.51$ & & & \\
\hline
\end{tabular}

The Relation of Tryptophan Test to Acidity.-(Author.)

A consideration of the relation of the tryptophan test to acidity is of interest. Of the positives 7 , or 28.9 per cent. of the contents contained no free hydrochloric acid. In 17 ( 75 per cent.) of the positives the free hychrochloric acid was diminished or absent. This combined figure is practically identical with that returned by the glycyltryptophan positives, although the percentage of extracts con- 
taining no free acid is much lower. In the tryptophan positives it will be seen that 75 per cent. showed diminished total acidity as against 80 per cent. in the case of glycyltryptophan positives (Table 23).

Studying the negative tryptophan reactions, we note that in 329 instances (28.6 per cent.) there was absent or diminished free acid, while in 586 cases ( 50.8 per cent.) the total acidity was low. These figures closely approximate those shown by the tabulations from the negative glycyltryptophan reactions.

It would appear that Weinstein's contention that his tryptophan test removes the consideration of contaminating saliva as a source of error is not borne out by our study. Further, the presence of negative glycyltryptophan reaction, in so large a percentage of extracts with low acidity, leads one to the opinion that the significance of the peptidase, said to exist in saliva, as a factor in hydrolyzing glycyltryptophan added to gastric extracts, is quite questionable. This opinion is substantiated by our work on saliva. (Arch. Int. Med., Dec., 1912, p. 1.)

Organic Acid.--Ten per cent. of the positive glycyltryptophan tests were associated with the presence of lactic acid. With the exception of one, the cases rere carcinoma. Thirty-three negative reactions (3.9 per cent.) were in contents containing lactic acid. Eight and one-third per cent. of the positive tryptophan tests were present in lactic-acidcontaining extracts, while 42 (3.6 per cent.) negative tryptophan contents contained lactic acid. It would seem that organic acids have little bearing on the relative variation of the two tests.

Of the entire number of gastric extracts $(1,175)$ analyzed in this series, 44 , or 3.7 per cent., contained lactic acid by the controlled Uffelmann test. Of the cases proved 16 
Table 24

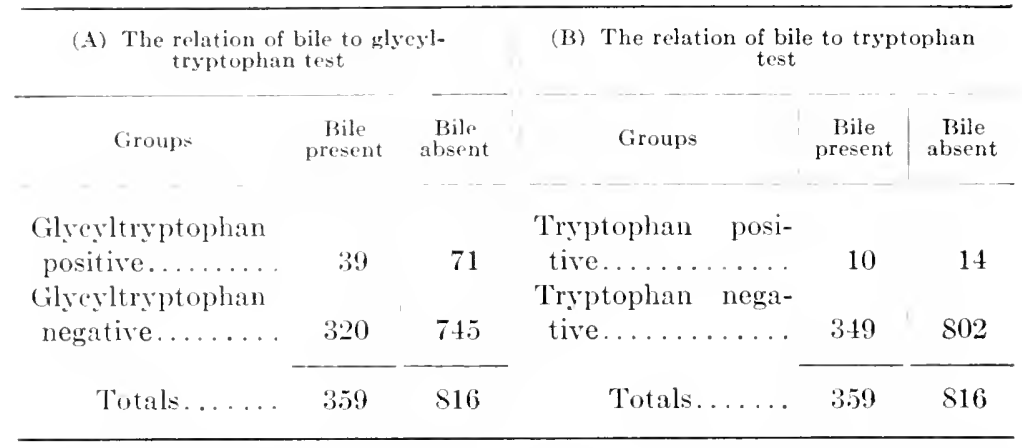

The Influence of the Presence of Bile upon the Glycyltyptophan and the Tryptophan Tests-(Author).

to be carcinoma rentriculi, lactic acid was present in 25 (28.7 per cent.). As we have shown in these cases, the glycyltryptophan reaction was positive in 31 (35.6 per cent.) and the tryptophan tests in 7 (8.04 per cent.). The relatively low percentage of extracts containing lactic acid may be explained on the basis of early diagnosis, many cases being operated on before marked obstruction and retention had dereloped. Emerson states that in his series of cases of carcinoma ventriculi, lactic acid was present in approximately 90 per cent. From our experience, it would appear that the great majority of his cases were far advanced, and exhibited marked retention. High mixed organic acidity frequently gives confusing Uffelmann reactions.

It has been held that the chyle in gastric extracts vitiates the glycyltryptophan test, but need not be considered when making the tryptophan test. 'The presence of bile or evidences of tryptic digestion has been used as proof that duodenal contents have been mixed with gastric juice.

The significance of this supposition is shown by analysis of Table 24. The gastric extracts were judged macroscopically as to the presence of bile, and were also tested 
by means of the Pettinkofer or the fuming nitric acid reaction. It will be seen $(a)$ that of 110 positive glycyltryptophan reactions, 39 (35.4 per cent.) contained bile; of 1,065 negative reactions, 320 (20.4 per cent.) showed bile; of the 24 positive tryptophan tests; (b) 10 (41.6 per cent.) were in bile-containing extracts, while 349 ( 30.4 per cent.) negative tryptophan tests were bile positive. These figures do not demonstrate that the tryptophan test is uninfluenced by chyle in the extracts. It is worthy of note that a relatively high number of both glycyltryptophan and tryptophan reactions are found in bile-containing chyme.

Table 25

\begin{tabular}{|c|c|c|c|c|c|}
\hline \multicolumn{3}{|c|}{$\begin{array}{c}\text { (A) The relation of blood to glycyl- } \\
\text { tryptophan test }\end{array}$} & \multicolumn{3}{|c|}{$\begin{array}{c}\text { (B) The relation of blood to tryptophan } \\
\text { test }\end{array}$} \\
\hline Groups & $\begin{array}{c}\text { Bile } \\
\text { present }\end{array}$ & $\begin{array}{l}\text { Bile } \\
\text { absent }\end{array}$ & Groups & $\begin{array}{c}\text { Bile } \\
\text { present }\end{array}$ & $\begin{array}{c}\text { Bile } \\
\text { absent }\end{array}$ \\
\hline $\begin{array}{l}\text { Glycyltryptophan } \\
\text { positive........... }\end{array}$ & 56 & 54 & $\begin{array}{l}\text { Tryptophan posi- } \\
\text { tive............ }\end{array}$ & 10 & 14 \\
\hline $\begin{array}{l}\text { Glycyltryptophan } \\
\text { negative........... }\end{array}$ & 236 & 829 & $\begin{array}{l}\text { Tryptophan nega- } \\
\text { tive............. }\end{array}$ & 282 & \$69 \\
\hline Totals........ & 292 & SS3 & Totals......... & 292 & S\$3 \\
\hline
\end{tabular}

The Influence of Blood upon the Glycyltryptophan and the Tryptophan Tests-(Author).

The effect of blood, traumatic or "occult," in gastric extracts has at least two points worthy of consideration with regard to the glycyltryptophan and tryptophan tests. Traumatic blood of itself gives a tan or definitely red cast to filtrates. A color reaction such as we are discussing is readily affected by such shades. The second point of note is the possibility of tryptophan resulting from split digestion products of the blood itself, particularly in those cases in which there is marked gastric retention with much flora. 
Table 25 furnishes interesting data on the above points. In 56 (50.9 per cent.) of the glycyltryptophan positive extracts, blood, traumatic or altered (benzidin test), was present. Of the glycyltryptophan negative extracts, in 236 (22 per cent.) blood was demonstrated. Of the tryptophan positive extracts 10 (41.6 per cent.) contained blood. In 282 (24.6 per cent.) tryptophan negatives, blood was proved. These figures for both tests so closely approximate that it does not seem possible to state that adrantage lies with either. The relatively high percentage of positives in extracts containing blood should, however, be borne in mind.

Summary.-1. In our series, more than one-third of the proved cases of cancer of the stomach gave positive glycyltryptophan reactions; more than one-fourth were lactic-acid positive and about one-thirteenth of the number exhibited the tryptophan test. Diagnosis of malignant disease of the stomach was in each case quite possible independent of the above chemical reactions. As a test associated with cancer of the stomach, it will be seen that in our series the glycyltryptophan reaction proved more consistent than test for existing free amino-acid (tryptophan).

2. While gastric conditions other than cancer exhibit positive glycyltryptophan reactions, in no single class of disease of the stomach is this test obtained so frequently as in cancer. This fact is of considerable significance chemically, and, perhaps, etiologically. While cancer of the stomach can doubtless be diagnosticated clinically without the glycyltryptophan test, one camnot state that the study of this and allied reactions will prove valueless.

3. Our work does not show that the tryptophan test is, as has been advanced, pathognomonic of cancer. 
4. Low free hydrochloric or total acidity is frequently determined in gastric contents exhibiting positive glycyltryptophan, lactic acid and tryptophan reactions. One cannot state positively that this diminished acidity is causative. Many cases of low acidity were negative to the above tests.

5. Approximately one-half of the positive glycyltryptophan and tryptophan reactions were in gastric extracts containing bile and blood elements. Approximately onefourth of the negative extracts contained blood and bile elements.

Wolff-Junghans' Test for Soluble Albumin.-Methods for the estimation of the soluble albuminous products of digestion have frequently been devised with the hope that such might prove of practical service in the differential diagnosis of gastric ailments. Of these methods, the well-known procedure advanced by Salomon had for a time the greatest vogue. Esbach's reagent and tubes proved, however, unsatisfactory and inaccurate from a clinical viewpoint. More recently the problem has been approached from the practical quantitative side and encouraging work recorded.

Wolff and Junghans report a method for estimation of the amount of soluble albumin in gastric extracts which they claim have given excellent clinical information in Ewald's service at the Augusta Hospital, Berlin.

Theoretically, their procedure has the following basis: In the normal aspirated test-meal there are demonstrable relatively large quantities of soluble albumin by means of precipitating reagents. This soluble albumin appears only through the agency of the gastric enzymes. This fact is proved by testing for soluble albumin a similar test-meal which has been chymified but not swallowed. In 
such erent, only minute quantities of dissolved albumin are present.

Acting on these observed facts, Wolff and Junghans fed similar meals to sets of individuals revealing malignant and benign achylias. Their work appeared to show that in the malignant achylias, aspirated test-meals were rich in soluble albumin, while in benign achylias very little of the albumin could be demonstrated.

Three suppositions have been adranced to explain this increased volume of dissolved albumin in the malignant achylias. It has been suggested that the excess of albumin is due $(a)$ to interference with albuminous resorption; (b) to a "cancer milk" rich in albumin which exudes from malignant growths, and $(c)$ to a specific, peptid-splitting ferment from the neoplasm, capable of carrying protein digestion as far as the completely soluble albumin stage.

Clinically, the reaction was shown to be positive in 18 of a series of 20 gastric cancers and negative in 14 of a series of 15 cases of simple achylia in Ewald's serrice. Recently, Rolph has reported positive tests in all of 7 cases where cancer was present in the stomach or secondarily involved that viscus. In 8 cases of benign achylia the test proved negative. Rolph states that gastric contents contaminated with blood beyond a dilution of 1 to 3,000 may give the reaction and cautions against positive interpretation in instances where there is high combined acid present. In such event peptone is usually present. He claims that cancer of the cardia is not so likely to give positive reaction as is cancer in other parts of the stomach.

Author's Study.--In the last 3,950 patients presenting themselves for test-meal examination of gastric function in his service at the Mayo Clinic, three were 747 instances 
where gastric extracts showed achylia or were associated with conditions confusable with malignancy. These gastric extracts were all tested by him for soluble albumin by the Wolff-Junghans' method. Records were kept of the association of the results of this test with other testmeal and clinical findings. When the tabulations were completed the diagnoses were entered on the daily sheets. In 78.4 per cent. of cases it was possible to obtain check upon diagnoses by operation.

Preparation for Test.--The day previous to the examination of his gastric extract the patient was given 1 ounce of castor oil at 4 p.m. This was followed at 6 p.m. by a motor test-meal consisting of mixed food. At 7 p.m. twenty raw, seedless raisins were given. Twelve hour's later $(7$ a.m. the following morning) the patient was fed 60 grams of second-day bread and 200 ec. of water. This secretory test-meal was removed from 50 to 60 minutes after administering. The specimen secured was thoroughly mixed, filtered through double hydrochloric-acidwashed papers, and tested for dissolved albumin within an hour of its being obtained from the stomach. On account of the fact that, as had been shown in this clinic, but 52.2 per cent. of cases of gastric cancer yield gastric extracts revealing absence of free hydrochloric acid, and that in 15.7 per cent. of cases, free hydrochloric acid ranges between 20 and 50 per cent., we deemed it advisable to apply the test for soluble albumin not only to achylias but also to gastric extracts where the free hydrochloric acid was below 20 per cent. In a few instances of suspected malignant ulcer we have performed the test upon gastric extracts with higher free hydrochloric acid content. In such we have been fully alive to the possibilities of error, but for the purpose of gaining informa- 
tion and fol comparison we have deemed it wise to make the test.

Mode of Procedure.- Six absolutely clean test-tubes are required for each test. Those of the narrow type and of 20 cc. capacity answer very well. The tubes are numbered serially from 1 to 6 . They receive respectively 1 cc., 0.5 cc., 0.25 ce., 0.1 cc., 0.05 ce. and 0.025 ce. of the filtered gastric extract. These amounts are readily measured by means of a 1 cc. pipette, graduated into 1/100's cc. By means of a 10 cc. pipette, graduated into 1/100's ce., the rolume in each test-tube is next consecutively brought up to $10 \mathrm{cc}$. volume with distilled water. This gives from the tubes 1 to 6 dilutions of gastric juice varying respectively from 1 to 10 to 1 to 400 (viz., 1 to 10,1 to 20 , 1 to 40, 1 to 100,1 to 200 , and 1 to 400). These figures we have termed "units" of precipitable albumin. The tubes are then inverted several times to insure complete mixture of their contents. One cc. of the reagent to precipitate the albumin in solution is then carefully layered upon the contents of each tube. The precipitating reagent suggested by Wolff has proved satisfactory with us.

It has the following formula:

Phosphotungstic acid (puriss) ................... $3 \mathrm{ce}$

Hydrochloric acid (concentrated) ... . . . . . . . . . . 10 ce.

Alcohol $(96$ per cent.). ........................ 200 ce.

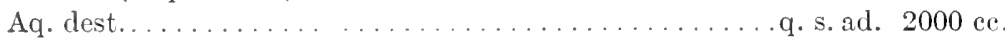

Mix and keep in a glass or rubber-stoppered flask in a cool place.

Manifestation and Interpretation of the Test.-If there has been dissolved albumin in any of the tubes, the junction of the Wolff reagent with the diluted gastric extracts is marked by a pearly white zone or "ring." This is better brought out if the tubes are inspected against a black background. (We have used a piece of black cloth such as photographers employ when focussing cameras). The 
tubes should be inspected at once after adding the Wolff solution. Prolonged standing allows cloudy zones to form which render comparative interpretation dubious.

We have interpreted our results after Wolff and Junghans' suggestion. If the white ring of precipitated albumin appears in tubes 1,2 and 3 (namely, units of albumin from 10 up to 50) and no further manifestations are present in the remaining three tubes we have called the test negative. If tubes 1, 2, 3 and 4 exhibit rings (units of albumin from 10 to 100) we have considered the reaction suspicious. The presence of white rings in tubes $1,2,3,4,5$ and above (units of albumin ranging from 10 to 200 to 400) we have taken to denote a positive test.

Results.-The gross results of our work were as follows: Of 747 gastric extracts of the class described above, 318 (42.6 per cent.) gave 200 to 400 units of precipitable albumin; 112 ( 15.7 per cent.) exhibited 100 units, and 317 (42.4 per cent.) showed less than 100 units. In this grouping 71.5 per cent. of the gastric extracts were from cases showing some degree of gastric retention.

Consideration of Cancer Cases. - There were 215 cases of operatively and pathologically demonstrated gastric carcinoma in this series. In 141 (65.1 per cent.) units of precipitable albumin ranged from 200 to 400. In 29 instances (13.4 per cent.) there were 100 units of albumin shown. Combining the returns it is evident that 170 (78.5 per cent.) of the proved cases of gastric cancer gave either undoubtedly positive or suspiciously positive WolffJunghans' test. In 45 cases (21 per cent.) the test was negative, less than 100 units of precipitable albumin being demonstrated. Of this group of 215 cases of gastric cancer, 73.2 per cent. exhibited some grade of motor stagnation. 
Gastric extracts from 15 cases of ulcus carcinomatosum were tested. In 11 instances (73.3 per cent.) units of precipitable albumin ranged between 200 to 400. In 3 (20 per cent.) 100 units were shown. In other words, of the 15 cases of malignant gastric ulcer, 14 (93.3 per cent.) were either definitely positive or suspiciously so to the Wolff-Junghans' test. One case (6.6 per cent.) exhibited below 100 units of albumin. In this group, motor stagnation of some degree was present in 86.6 per cent.

Combining the results from the cases of frank gastric carcinoma and those of ulcus arcinomatosum, it is seen that of a total of 230 cases, 184 ( 80 per cent.) returned positive or suspicious Wolff-Junghans' test.

Relation of Manifestations of Test to Location of Malignant Proccss.- We examined gastric extracts from 10 cases of cancer involving the cardia. Six cases (60 per cent.) gave positive test, 1 (10 per cent.) was suspicious, and 3 (30 per cent.) were negative. Thus 70 per cent. of our cases of cancer at the cardia showed units of precipitable albumin ranging from 100 to 400 .

There were 5 cases of cancer of the fundus in our series; 1 (20 per cent.) was positive, 1 (20 per cent.) was doubtful, and 3 (60 per cent.) were negative.

We have records of $t 4$ cases where the neoplasm involved mainly the lesser curcature of the stomach. Of this group, 33 cases ( 75 per cent.) gave clearly positive Wolff-Junghans' tests, 4 (9.1 per cent.) were supicious, and 7 ( 15.8 per cent.) were negative. It is evident that $\$ 4.1$ per cent. of cancers involving the lesser curvature show units of precipitable albumin ranging from 100 upward.

In our series there were 3 cases of cancer of the greater curcature. Two cases (66.6 per cent.) were positive and 
the remaining case suspicious. Thus all showed 100 plus units of albumin.

Eight of our cases were proved to have cancer involving mainly the posterior wall of the stomach. Of this group but 3 cases ( 37.5 per cent.) were positive to the test, while 5 cases (62.5 per cent.) were negative.

The pars media was involved 14 times. Of this number, 11 cases (78.5 per cent.) gave positive tests and 3 cases $(21.5$ per cent.) were negative.

In 93 instances the malignant growth was at the pylorus and antrum. In this class, 59 cases (63.4 per cent.) showed units of precipitable albumin from 200 upward, 8 cases (\$.6 per cent.) were suspicious, revealing 100 units, and 25 cases (26.9 per cent.) were negative. In other words, 72 per cent. of the cancers at the pyloric region gave positive or suspicious Wolff-Junghans' tests.

Our series includes $3 S$ cases where the stomach showed general or extensive malignant involvement. In 26 instances (68.5 per cent.) the test was positive, in 3 cases (7.9 per cent.) it was suspicious, while 9 times (23.6 per cent.) negative results were obtained.

Comparison of Other Test-meal Findings in the Cancer Cases with the Wolff-Junghans' Test.-It might be profitable here to emphasize the diagnostic relation of other tests associated with that for dissolved albumin in the gastric extracts from our malignant cases. It will be noted above that of the 230 cancer and malignant ulcer cases the WolffJunghans' test was positive or suspicious in 184 ( $\$ 0$ per cent.). In this same group of cases, free hydrochloric acid was absent, in 52.2 per cent., lactic acid was demonstrated in 48.8 per cent., "occult" or altered blood shown in 75 per cent., glycyltryptophan test present in 40 per cent. (141 cases), the average formol index (method of Sorenson and 
Schiff) was 21 (57 cases), and organisms of the OpplerBoas group were demonstrated in 93.8 per cent. (146 cases) by the colored agar method. Some degree of gastric retention was shown in nearly 74 per cent. of the entire group of cancer cases, irrespective of the location of the growth.

The Wolff-Junghans' Test in Extragastric Cancer: Liver and Gall Tract.--Our series includes 15 instances of malignancy in these locations. In 5 cases ( 33.3 per cent.) the test was positive, in 3 cases ( 20 per cent.) it was suspicious, and in 7 cases (46.6 per cent.) it was negative. Thus 8 cases (53.3 per cent.) of extragastric malignancy showed units of albumin from 100 upward. Some degree of motor stagnation was evidenced in 26.6 per cent. of these cases.

The pancreas was the seat of malignant processes 3 times. In no instance was a positive Wolff-Junghans' test obtained. Motor defect was not noted in any of these cases.

There was 1 case of cancer of the transverse colon. It gave a negative test. There was normal gastric motility in this case.

Gastric Syphilis.-We have tested gastric extracts for dissolved albumin from 5 cases. The reaction was positive in 2 instances (40 per cent.), suspicious in 1 (20 per cent.), and negative in 2 (40 per cent.). In one of the positive cases the specific process in the stomach was associated with multiple and exuberant ulceration. Gastric motility was interfered with in 1 case (20 per cent.) of this group.

Primary Anemias (Mainly Pernicious).-Twenty-four cases of achylia in severe anemia comprise this class. In none of them was gastric stagnation present. Twentythree $(95.6$ per cent.) of this group were negative to the Wolff-Junghans' test. In but 1 instance (3.3 per cent.) were the units of perceptible albumin above 200 . 
Simple Achylia Gastrica.-We examined gastric extracts from 35 such cases. Gastric stagnation was proved in 4 cases (11.9 per cent.). In 22 instances ( 63 per cent.) of this type of achylia the test was negative, in 9 instances (25.9 per cent.) suspicious, and positive but 4 times (11.9 per cent.).

Achylorhydria.-In addition to the cases of absent free hydrochloric acid mentioned in the above groups there were 212 cases of non-malignant disease showing achylorhydria. Gastric motility was impaired in 22 cases (10.3 per cent.). In this group 136 cases (64.1 per cent.) were Wolff-Junghans' negative, 41 cases (19.3 per cent.) were doubtful, and 35 cases (16.5 per cent.) were positive.

Simple Gastric Ulcer.-A number of cases of this affection and of duodenal ulcer were studied for purposes of comparison with malignant disease. Their gastric extracts generally showed low free hydrochloric acid content. We tested extracts from 33 cases of operatively demonstrated gastric ulcer for dissolved albumin. In 16 cases ( 48.4 per cent.) units of albumin ranged above 200, in 6 instances (1S.1 per cent.) the units ran as high as 100 , while in 11 cases (30.3 per cent.) units of albumin were below 100. It is thus apparent that 66.5 per cent. of the proved cases of simple gastric ulcer were positive or suspicious to the Wolff-Junghans' test. Gastric motility was delayed in 39.4 per cent. of this group.

Duodenal Ulcer.-Gastric extracts from 18 cases of duodenal ulcer were tested. In 12 cases (66.6 per cent.) units of albumin ranged above 200, in 2 cases (11 per cent). at least 100 units were present, while 4 times $(22.7$ per cent.) less than 100 units were demonstrated. It is thus evident that 78 per cent. of our cases of duodenal ulcers were Wolff-Junghans' positive or suspicious. In this 
group, gastric stagnation was present in 55.5 per cent. of the cases.

Nephritis and Cardiovascular Disease--Our series includes 12 cases of cardiorenal affections associated with obscure gastric complaint and anemia. The gastric extracts showed achylia. In 6 instances (50 per cent.) the Wolff-Junghans' test was doubtful, while in an equal number it was negative. The doubtful cases were associated with some degree of gastric motor insufficiency.

Cases Exhibiting Low Gastric Acidity.-In this group we include 159 instances where gastric acidity ranged from 2 to 70 . The average was 18.7. This group furnished what might be regarded as controls on our reactions in other groups, as well as demonstrated what results might be expected from the Wolff-Junghans' test in extragastric, malignant, and non-malignant ailments. It should be emphasized that all of the patients examined complained of some gastric disturbance. The finding of the low free hydrochloric acid in some instances might have led to suspicions of malignancy by those who hold gastric acidity as a strong index of such condition. This might have been especially so when we recall that the average age of our patients is above 40 years.

Clinically, this group was raried as to diagnosis. Among the affections were appendicitis, cholecystitis, cholelithiasis, alcoholic gastritis, gastric neuroses, pulmonary tuberculosis, tabes, multiple sclerosis, tuberculous peritonitis, nephrolithiasis, pancreatitis, cirrhosis of the liver, pregnancy, malaria, diabetes, aneurysm of the abdominal aorta, chronic constipation, hemophilia, cancer of the breast, cancer of the lip.

Of this heterogeneous group of low gastric acidity cases, 40 (25.1 per cent.) were Wolff-Junghans' positive, 38 
(23.9 per cent.) were doubtful, and $\$ 1$ (50.9 per cent.) were negative. In other words, of this class nearly 50 per cent. of cases showed units of precipitable albumin from 100 upward. Gastric motility was interfered with in some degree in 25 cases ( 15.7 per cent.).

Relation of the Wolff-Junghans' Test to the Presence of Blood in Gastric Extracts.-We have frequently tested gastric contents that were discolored bright red by traumatic blood without getting positive Wrolff-Junghans' tests. Of our entire series of $74 \tau$ cases herewith detailed, "occult" blood was demonstrated by the benzidin test in 43.2 per' cent. Reference to the gross summary of our work above will reveal the fact that we obtained positive tests for precipitable albumin in 42.6 per cent. of the gastric extracts of the entire series, while in 15 per cent. the test was doubtful. There may be more than a curious relationship between these groups of figures.

Summary.-Our work appears to justify the following conclusions:

1. When carefully performed and interpreted the WolffJunghans' test for demonstration of dissolved albumin in gastric extracts was positive or suspicious in $\$ 0$ per cent. of our series of gastric cancer. In this series it was a more constant finding in gastric extracts than were absent free hydrochloric acid, the presence of lactic acid, and the glycyltryptophan test. It was rather more constant than tests for occult blood and the demonstration of gastric motor inefficiency. It was not so consistent in its manifestation as the demonstration of organisms of the Oppler-Boas group or the increase in the formol index.

2. In extragastric malignancy, gastric syphilis, and nephritis the Wolff-Junghans' test seems inconstant.

3 . In the differentiation between malignant and non- 
malignant achylias the Wolff-Junghans' test, when interpreted in connection with other clinical and laboratory data, is of considerable value. Positive reactions are rarely obtained in the achylias of primary anemia, simple achylia gastrica, and simple achlorhyclrias, when such are unassociated with gastric motor inefficiency.

4. Simple gastric and duodenal ulcers, especially when accompanied by pyloric stenosis or gastric atony, may give confusing responses to the Wolff-Junghans' test.

5. The presence of blood in gastric extracts may be a factor in the production of certain atypical positive tests.

\section{(B) EXAMINATION OF THE FECES IN GASTRIC CANCER}

The routine examination of freshly passed feces should never be neglected. While in the majority of instances of the disease, analysis of the stool reveals little characteristic, not infrequently cases occur that are clinically suspected of being gastric cancer where stool analysis reveals not cancer of the stomach, but malignant disease of the large bowel, rectum or anus, anomalies of the biliary tract or pancreas, or the presence of intestinal parasites.

Macroscopic clinical reference has been previously made (vide Chapter III) respecting the incidence of diarrhea and constipation in gastric cancer. The macroscopic examination of the stool is of much value. Where constipation exists, its degree may be roughly ascertained by marking of the stools with two tablets of charcoal or 5 grains of carmine. With the patient upon diet as nearly normal as possible, the time of administration of the coloring substance is compared with that when the stool is passed stained black (charcoal) or brick-red (carmine). We have found the above simple method of considerable clinical value. 
Roentgen ray examination of the degree of stomach or bowel stasis is of but relative service. The large mass of opaque medium is not physiologically a food. It is not without its effect upon gastric and intestinal secretions, peristalsis or digestive-tract flora. Its weight is out of all proportion to its bulk as a food. We not rarely see instances where Roentgen plates show bismuth in the intestinal tract, or even in the stomach from 3 to 5 days following its administration, and yet charcoal or carmine colored stools are passed within 12 to 24 hours. It, therefore, appears that Roentgen estimation of gastrointestinal motility when such substances as bismuth or barium form the basis of the test-meal is at present of dubious service. Its value can only be placed upon a firm, clinical footing when actinologists and clinicians, after years of co-operative work, have followed a large series of similar and different cases through medicinal or surgical treatment, or examined such at autopsy. Then it will be proper to draw conclusions from facts and not have such depend upon fancy or individual enthusiasm.

The gross appearance of sluggish stools in instances of gastric cancer may differ little from the appearance of constipation stools in other ailments. If little food is passing into or out of the stomach, then the stools may be small, hard and often covered with mucus. In cases where the bile tract has been invaded by the neoplasm claycolored or putty-like movements may be noted. Where there has been involvement of the pancreas, the stool may be large in amount, putty or gun-metal colored, pasty, greasy, foamy or fatty, contain much unaltered food (gobs of fatbutter-like or egg-yolk appearing-or chunks of poorly digested meat or vegetable fiber) and have a penetrating, pungent, acrid, musty or sour odor. Fistulous com- 
munication between stomach and bowel may cause the stool to contain macroscopic blood, necrotic tissue, pus, undigested food or much mucus. Black or red stools occur when sudden extensive bleeding has resulted from the local growth in the stomach, or from necrosis which opens up a large blood-ressel as a consequence of secondary invasion of other viscera.

Diarrheic stools may be expected at any time during the course of gastric cancer. Frequently they are caused by faulty diet, insufficiency of the digestive glands (stomach, liver, pancreas, bowel), asthenia, nervous disturbances, hemorrhage, colitis associated with cardio-renal complications, abnormal intestinal microorganisms, or by pressure or actual invasion of the central nerrous system by the neoplasm or by medicines.

The stools may be small, frequent and watery, large and mush-like, foamy, sticky, slimy, gelatinous or custard-like. The odor is often very offensive. The color varies widely. Green, tan, cream, yellow, red or black movements are not uncommon. Occasionally fleshy clots or spongy or friable chunks of tissue may be intermixed.

\section{CHEMICAL EXAMINATION}

Reaction.-Stools in gastric cancer are commonly amphoteric or alkaline to litmus unless there is a marked involvement of the pancreas or the bile ducts. In such event, the stools have acid reaction in a high proportion of cases.

Hydrobilirubin is usually present, unless the free flow of bile is interfered with or there is marked cancerous change in the liver.

Bile coloring matter is readily recognized by intimately mixing 10 grams (or $10 \mathrm{cc}$. in case the stool is fluid) 
of stool with about 4 times its volume of saturated normal salt solution of bichloride of mercury in a mortar. The fluid portion is poured off into a Petri dish, covered and allowed to stand for from 2 to 24 hours. If hydrobilirubin is present a salmon to deep red color appears. Not infrequently, olive green color changes occur due to the presence of biliverdin. When this is present, chlorophylaceous moulds or certain bacilli associated with "green diarrhea" must be excluded by spectroscopic examination of alcoholic extracts of the feces. Biliverdin shows no absorption bands spectroscopically. All stools of light yellow to putty color should routinely be examined for bile pigments. Very often, chalky or cream, pale yellow or black stools render it difficult to observe whether or not bile elements are present. Such stools are common when patients are on milk diet, have been examined by the Roentgen ray, or are taking certain medicines (bismuth, tannic acid, iron, etc.).

Blood.-After severe hemorrhage, there is usually no difficulty in recognizing blood macroscopically in the stools. It is sometimes necessary to be sure that an ulcer suspected of being malignant is bleeding, or it is desirable to ascertain roughly how much seepage of blood is occurring in a known gastric neoplasm. For this purpose chemical tests for altered ("occult") blood are useful.

It should be strongly emphasized that positive chemical tests for blood only indicate that blood pigment is in the feces. The clinician must, therefore, exclude all possible sources of error before he can state definitely that a chemical reaction positive for blood indicates that such blood comes from the suspected gastric focus. It should be strictly seen that no likely source for these reactions lies in a lesion existing in the mouth, nasopharynx, respiratory 
tract, or parts of the digestive canal outside of the stomach. Dependable reagents must be employed in making the test. Many preparations of phenolphthalein, guaiac, or orthotoluidin are chemically useless for the reaction. Benzidin is less likely to be unreliable, provided the pinkishgray powder be used. If hydrogen peroxide or turpentine is employed, it is very essential that such possess dependable oxidizing properties. Thorough dietetic preparation is necessary before chemical tests for blood in the stools can be interpreted clinically. It is our custom first to give the patient 2 ounces of castor oil and thereby empty the gastro-intestinal canal of material likely to be a source of error. He is then placed upon soft lactovegetable diet (aroiding excess of greens and fresh garden truck) for 3 days. A saline cathartic is then administered. For the following 24 hours nothing but parboiled milk is permitted. The bowels are then allowed to move naturally, or by a mild saline laxative. The second stool passed is examined. This furnishes the specimen to be tested for altered blood.

The method of procedure has been described above (see Gastric Analyses). Care should be taken that at least 5 grams of stool are employed and that its breaking up with acetic acid and its extraction with ether are thoroughly carried out.

Clinical Interpretation of "Occult" Blood Tests.-If the reaction has been carefully checked as outlined above, a positive test means only that there is a bleeding point somewhere between the lips and the external anal ring. It is the physician's business to find where this point is located. Given a history of a malignant gastric ailment or of chronic dyspepsia of the ulcer type, it is most likely that 
persistent seepage or intermittent bleeding come from such focus of disease.

While a positive chemical reaction for blood in a given specimen of feces has a certain value diagnostically, yet when such are obtained in gastric cancer, other more easily and more dependable signs and symptoms of the existence of the disease are not lacking. Of the stools of gastric cancer patients where we have performed benzidin tests, the reaction was positive in approximately $\$ 9$ per cent. Of this group of cases, abdominal tumor was palpated in nearly 75 per cent., gastric acidity was below 15 in 83 per cent., bacilli of the Oppler-Boas type were present in stomach extracts in nearly 94 per cent., Roentgen findings were positive or suspicious in about 90 per cent., and the clinical history was that of malignancy in about 92 per cent. of instances.

The negative test for "occult" blood in the feces is a considerable aid in excluding gastric cancer where, with a doubtful clinical history, an atypical abdominal tumor is made out.

Ferment Tests.- The main worth of such lies in gaining knowledge respecting the function of the pancreas. Tests for amylase (diastase) and trypsin appear to have clinical value. While not infrequently low figures are returned when amylolytic and tryptic digestion are estimated in the event of involvement of the pancreas by a growth, primarily gastric, we have found that in cases of achylia or hypoacidity of extragastric origin, similar results may be obtained.

Wohlgemuth's Method of Determining Diastase in the Stools. - Wohlgemuth has adopted the following quantitative method for determining the diastase in the stools: The fresh feces are well mixed, and 5 grams are thoroughly 
ground in a mortar with $20 \mathrm{ce}$. of 1 per cent. solution of sodium chloride, added a small quantity at a time. The emulsion is then left for half an hour at the room temperature, stirring it frequently meanwhile. It is now divided into two equal portions of $10 \mathrm{cc}$. each, and is transferred to graduated centrifuge tubes, which are centrifugalized until all the solid material is collected at the bottom and stands at the same height in both tubes. The quantities of sediment and supernatant fluid are noted. Nine test-tubes are now taken. Into the first three, $1.0 \mathrm{cc} ., 0.5 \mathrm{cc} ., 0.25 \mathrm{cc}$. of the undiluted extract; into the next three, 1.0 cc., 0.5 cc., 0.25 cc. of an eightfold dilution of the original extract, made with 1 per cent. sodium chloride; and in the last three, 1.0 cc., 0.5 cc., 0.25 cc. of a sixty-four-fold dilution are placed, so that each tube contains half the fecal extract of the preceding:

1st tube 1.0

2nd tube 0.5

3rd tube 0.25 4th tube 0.125

5th tube 0.0625

6th tube 0.0312 7th tube 0.0156

8th tube 0.0078

9th tube 0.0039

To each tube 5 cc. of a 1 per cent. solution of starch are then added. The tubes are now plugged with wool, or closed with corks, and placed in the incubator at $38^{\circ}$ C. for 24 hours. At the end of that time they are filled to within a finger breadth of the brim with cold distilled water, one drop of a decinormal iodine solution is added to each, and the lowest dilution giving a blue reaction looked for. It is then assumed that the tube next lowest in order contains sufficient diastase to convert all the added starch; and from this the quantity of 1 per cent. starch solution fermented by 1 cc. of the fecal extract can be calculated. Knowing the proportion of solid residue to liquid extract in the 5 grams of feces the quantity of ferment corre- 
sponding to $1 \mathrm{cc}$. of this residue can be determined and from this the diastatic power of the total daily mass of feces can be determined. According to Wohlgemuth and Wynhausen, the average diastatic value of the feces lies between 470 and 500. To obtain satisfactory results, the feces must be homogeneous and alkaline in reaction, as diastase does not act in an acid medium. It is advisable to place the patient on a simple mixed diet, calculated to stimulate the functions of the pancreas to normal activity, for a couple of days before the feces are collected for examination (Cammidge).

Gross-Wynhausen's Method of Determining Tryptic Digestion in the Stools. - Wynhausen has suggested the following method for carrying out Gross' test quantitatively: Twelve test-tubes are taken. Into the first two are placed 0.25 cc. and $0.1 \mathrm{cc}$. of the undiluted fecal extract; into the next five, 0.6 cc., 0.4 cc., 0.25 cc., 0.16 cc. and 0.1 cc. of a 10 times dilution; into the next three, 0.05 cc., $0.25 \mathrm{cc}$., and $0.1 \mathrm{cc}$., of a 100 times dilution; and into the last two, $0.5 \mathrm{cc}$. and $0.25 \mathrm{cc}$. of a 1000 times dilution. To each is now added $5 \mathrm{cc}$. and 0.1 per cent. casein solution. The tubes are closed with corks or wool and incubated for 24 hours. At the end of this time they are tested with 1 per cent. acetic acid. The digestion of 1 cc. of 0.1 per cent. casein solution by $1 \mathrm{cc}$. of the filtrate is taken as the tryptic unit. Normally, the value exceeds 200 (Cammidge).

\section{MICROSCOPIC EXAMINATION OF FECES IN GASTRIC CANCER}

The stools should be examined fresh and preferably warm. It is our practice to routinely have stools collected in warm pots, placed at once in an incubator built for that purpose (Fig. 59) and examined on a warm stage, with high power amplification. Stools should be 
examined within 2 hours after their being passed if one desires to learn facts of dependable worth regarding the flora of the intestinal tract or to make representative cultures. Whenever possible stools should travel from patient to microscope by the most direct route. Stools
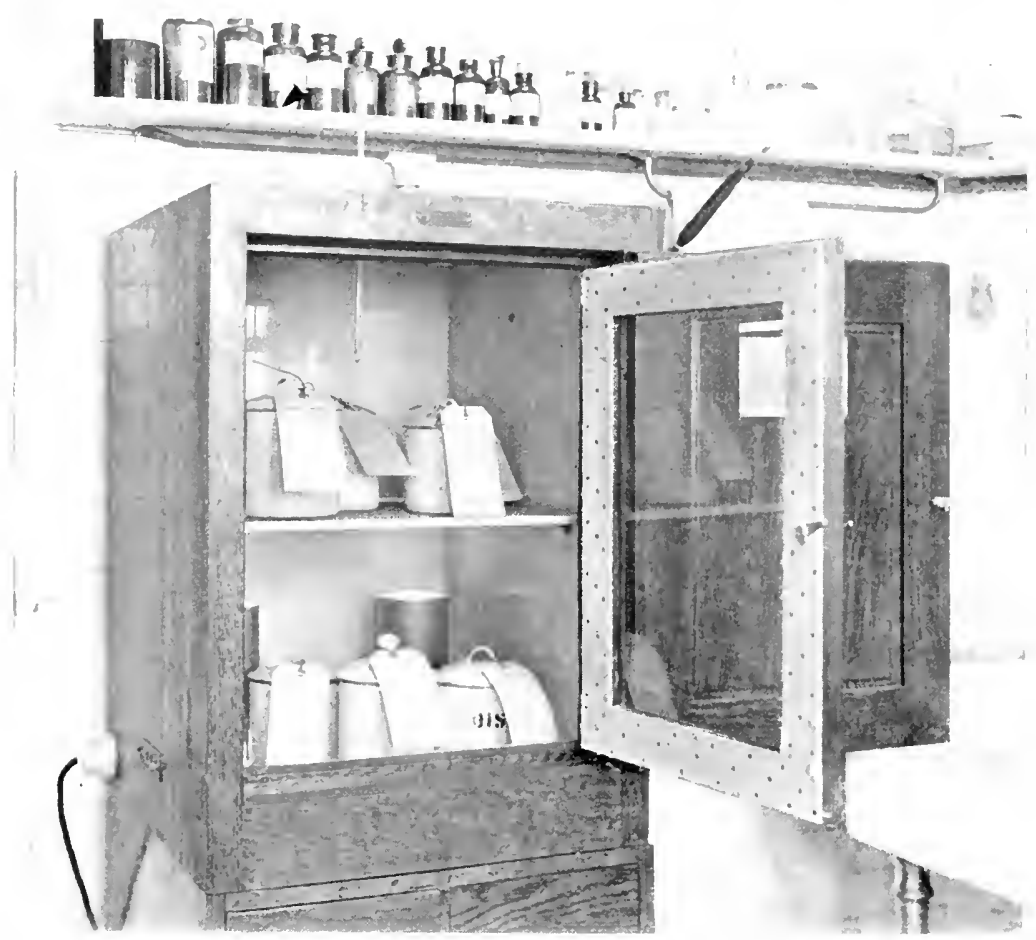

FIG. 59.-Electric incubator for preserving stools warm until ready for examination.-(Author.)

which have stood about for hours, grown cold or allowed to "ferment" return wholly unreliable findings upon microscopic examination. Food rests may have become digested or autolysized, motile bacteria or protozoa have died or encysted, or abnormal proliferation of such organisms as facultative aerobes taken place.

Method of Microscopic Examination.-Much can be learned from examination of the fresh stool unstained but 
diluted about 3 times with warm normal salt solution. At least one preparation should be viewed in this manner, using all 3 regular objectives. This examination permits the recognition of motile bacteria, protozoa, parasite eggs, crystals and the like, relatively unchanged. Wet or dry stained preparations may next be observed. Wet preparations are conveniently made by adding a drop of Lugol's solution or solution of osmic acid to a like quantity of fresh, fluid feces upon a slide. By this method food digestion (starches, fats) can be made out. Dry preparations are available by making thin smears of fluid feces upon cover slips, passing through a Bunsen flame rapidly, and staining with Wright's solution, Unna's polychrome methylene blue, Loeffler's blue, hematoxylin and eosin or Gram's stain. In carcinoma of the stomach, staining by polychrome methylene blue or Gram's method is a satisfactory routine procedure. Where many specimens are to be examined in a short time, the agar staining technique which I suggested several years ago is very useful (see under Gastric Analysis).

\section{Microscopic Findings in the Feces in Gastric Cancer.-} Where the malignant process is early or developing in a pre-existing ulcer, nothing characteristic is seen in the stools. If there is marked gastric stagnation of the benign type, an abundant growth of yeasts and sarcinæ may be observed. Where gastric malignancy is well established, bacilli of the Oppler-Boas type may be recognized in as high as 60-85 per cent. of cases by the Gram stain. Associated with these long acid-fast bacilli, there are commonly large colonies of streptococci and staphylococci.

Protozoa may be recognized in unstained specimens examined on a warm microscope stage. We have seen amœbæ, trichomonads, cercomonads, balantidium coli, 
and megastoma entericum in the feces of gastric cancer patients. Occasionally tape-worms, pin worms or flukes are seen.

L'ndigested food is common in the stools of patients affected with cancer of the stomach. Large pieces of striated muscle fiber, regetable reticula, or unaltered fat may be abundant.

Red blood corpuscles and leucoeytes (usually polymorphonuclears or of the large mononuclear type) are often present in sloughing gastric cancers, or where fistulæ have dereloped. We have nerer seen mitotic cells in the feces of such cases.

Crystals, particularly of cholesterin, ammonium phosphate, fatty acids, leucin or tyrosin are not infrequently increased in the stools of late gastric malignancy.

\section{REFERENCES}

Suithies: "A New Motor-meal and Lavage Tube," Jour. of Am. Med. Assoc., 1914, Feb. 7, p. 453.

Schryter and Singer: The Quarterly Jour. of Med., London, 1912, Oct., p. 71, and 1913, April, p. 310.

Ruttax Axd Hardisty: Canadian Ned. Assn. Jour., 1912, Nov., p. 1.

Suithies: Arch. Int. Med., 1912, June, p. 736.

Kauffmanx: Berl. klin. Wchnschr., 1595, No. 6, p. 1.

Pelosi: Gazz. deg. osped. e de. Clin., Milan, 1912, Oct. 22.

Fuld ANd Levisox: Biochem. Ztschrft., Berlin, 1907, Bd. vi, Hft. 5 and $6, \mathrm{p} .473$.

Sorensox And Schiff: Ztschrft. f. physiol. Chem., 1909, xiii, p. 27.

MIuller: Ztschrft. f. klin. Med., 1S\$9, xvi, p. 496.

Fischer: Deutsch. Arch. f. klin. Med., 1902, lxxii, p. 415.

Abderhalden: Ztschrft. f. physiol. Chem., 1909, lxii., p. 136.

Necbater axd Fischer: Deutsch. Arch. f. klin. Mled., 1909, xciii, p. 499.

Lyle ANd Kober: New York Med. Jour., 1910, xci, p. 1151.

Weinsteix: Jour. Am. Med. Assn., 1910, lv, p. 1085.

Warfield: Bull. Johns Hopkins Hosp., May, 1911, p. 150.

Oppexhen: Deutsch. Arch. f. klin. Med., 1910-11, ci, p. 293.

Kohlenberger: Deutsch. Arch. f. klin. Med., 1910, xcix, p. 148. 
Sanford and Rosenbloom: Arch. Int. Med., 1912, ix, p. 445.

Weinstein: Jour. Am. Med. Assn., 1911, lvii, p. 1420.

Hall and Willianson: Lancet, London, 1911, clxxxi, p. 731.

Suithies: Archiv of Int. Med., 1912, x, p. 357.

Smithies: Jour. Am. Med. Assoc., 1912, April 6, p. 100 S.

Giss: Quoted by Weinstein, Jour. Am. Med. Assn., 1911, lvii, p. 1420.

Emerson: "Clinical Diagnosis," 1906.

Wolff and Junghans: Berl. klin. Wehnchrft., 1911, May 29, and 1912, March 1S; Medizin. Klinik. 1912, March 24, also Taschenbuch Magen und Darm Krankheiten, 1912.

Rolph: Medical Record, New York, 1913, May 10, p. $\$ 49$.

Smithies: Am. Jour. Med. Sc., 1914, May, p. 713.

Suithies: Jour. Am. Med. Assn., 1913, Nov. 15, p. 1793.

Wohlgenuth: Biochem. Ztschrft., 1909, xxi.

CAmmidge: "Feces of Children and Adults," London, 1913.

Wynhausen: Berl. klin. Wchnschrft., 1910, No. 11. 


\section{ROENTGEN EXAMINATION IN GASTRIC CANCER}

During the past 5 years, such advances have been made in the technique of examining hollow viscera (which have been rendered opaque with such substances as bismuth or barium), through the medium of the Roentgen ray, as to permit of the methods being of certain clinical value.

Class of Cases in which Roentgen Examination is of Value.-It is well to admit that much of the positive information which $x$-ray examinations give us concerning the function or abnormalities of the stomach can be as well obtained by routine clinical examination. This especially applies to gross lesions of the stomach or bowel. In the large majority of instances, $x$-ray findings only concern what might be termed accidents in the progress of the disease process. By this we mean that such features as stenosis at either orifice, or on the course of the gastric lumen, alterations in shape or position of the viscus with relation to adjacent organs, or deformities in outline are determined Roentgenographically. Until such complications occur, it is not unusual for Roentgen findings to be entirely negative. The diagnosis must be made clinically or chemically. This applies particularly with regard to the $x$-ray diagnosis of malignant gastric ulcer and latent gastric cancer.

Both by $x$-ray plate and flouroscopic screen it is quite impossible to accurately localize or even diagnose the majority of uncomplicated peptic ulcers. Until there has been such accumulation of scar-tissue as to make a lesion 
1 to $3 \mathrm{~cm}$. in diameter, very little of definite value is derived from $x$-ray examination.

In these instances, the so-called $x$-ray diagnosis of early gastric cancer is really based upon other clinical and laboratory data. Not infrequently valuable service is rendered by the Roentgen ray in actually demonstrating to us that, in a patient with ulcer symptoms, the disease is located in the stomach and not in the duodenum. This is a valuable fact, because it has been shown by the work of the surgeon and the cellular pathologist that only rarely does malignancy occur upon the site of a duodenal ulcer. In our experience, carcinoma of the duodenum, where there had been previously ulcer history, happened but 7 times in an analysis of the records of 1,000 duodenal ulcers.

If the chronic, recurrent, calloused ulcer is located well within the stomach, as shown by the $x$-ray examination, then, from a knowledge of the facts that Wilson and MacCarty have found cancerous tissue in 71 per cent. of such ulcers resected, the actinologist can quite reasonably entertain the suspicion that such ulcers may be cancerous and recommend surgical intervention. Moreover, if the duodenal ulcer, which is quite apt itself to be benign, is proved to extensively involve the pylorus at the $x$-ray examination, it is well to suspect that such ulcer may be or is potentially carcinomatous.

Similar observations may also be made regarding ulcers or scar-tissue about the ulcer causing local deformity or local irritation of groups of muscle fibers in the stomach wall. Here the various so-called signs are quite indefinite and uncertain, unless the facts which clinical history or chemical examination of gastric function return are considered.

When a large accumulation of callus has formed, or when the ulcer breaks through by perforation, or where the 
surface mucous membrane has been lost as the result of necrosis, thus forming a crater-ulcer, or if, in the attempt to heal, fibrous tissue has caused contraction at the cardia or in the stomach lumen (hour-glass or narrowing at the pylorus, Figs. 60 and 61) the Roentgen examination of the stomach which has been filled with opaque medium gives testimony corroborative of the clinical opinion that such complication has occurred.

We have already shown that the early beginnings of many gastric cancers are essentially microscopic alterations in epithelium lying in the areolar tissue at the edges or bases of gastric ulcer. When this pathologic fact is borne in mind, it is to be readily understood how the $x$-ray examination in these instances can only give information with regard to the presence or absence of calloused ulcer. It is not possible to state whether or no such is a benign ulcer or a malignant ulcer. Actinologists have learned from the clinical pathologists, however, that complicated ulcers of the calloused type which have crater diameters or scartissue infiltration broader than 1 to $3 \mathrm{~cm}$. are very likely to be malignant. This applies especially to ulcers located at or near the cardia or the pylorus.

With regard to ulcer in the antrum, pars media, or the body of the stomach, it is frequently impossible to make any Roentgen diagnosis whatever if such do not involve one or both curvatures and produce malformations of the stomach outline.

Inasmuch as we have shown that the early diagnosis of gastric cancer is essentially that of the diagnosis of chronic, calloused ulcer, it will be seen that in such the $x$-ray evidence is wholly inefficient with regard to absolute diagnosis, and only of limited value with respect to presumptive diagnosis. It is to be regretted that in these cases which 


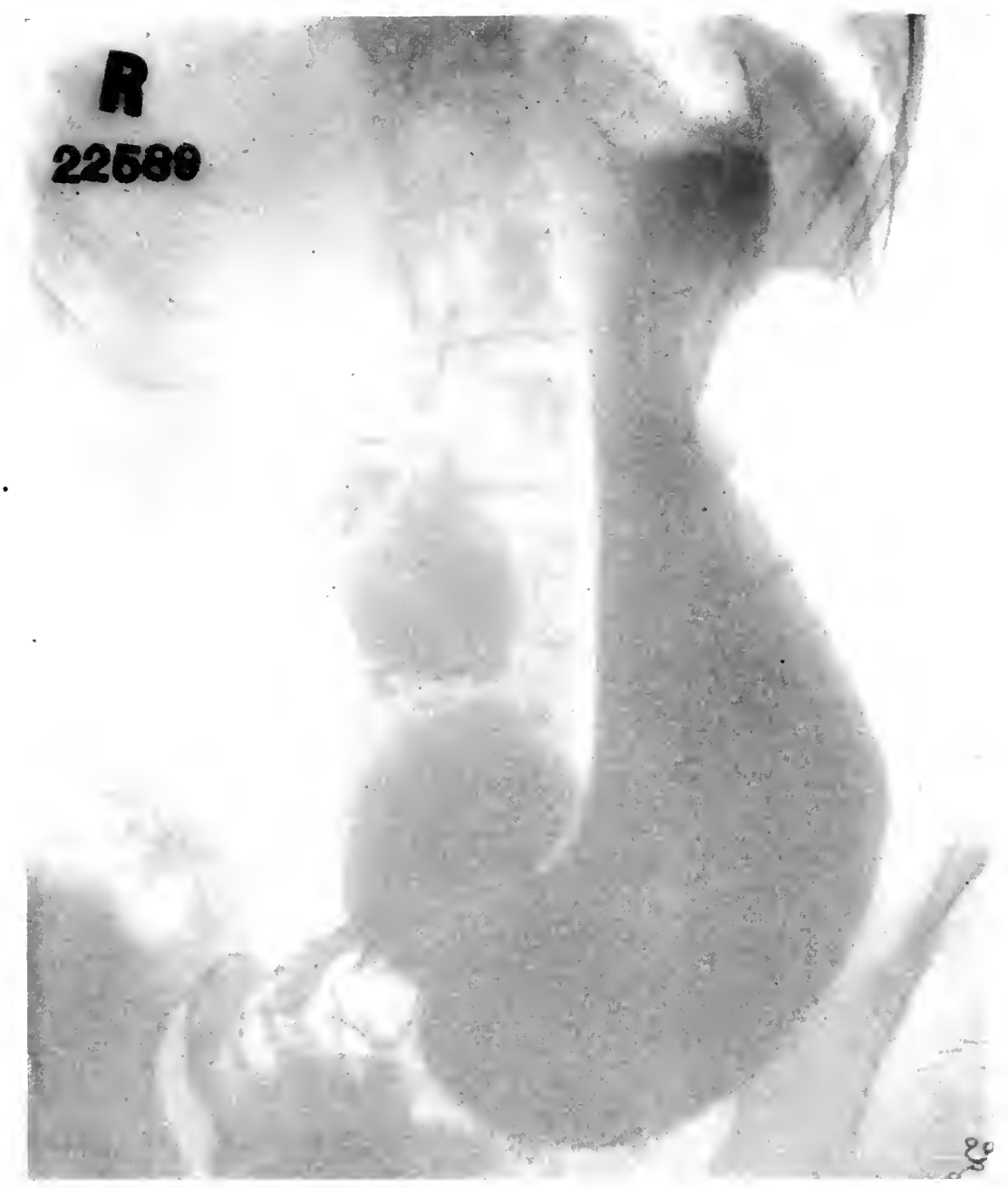

Fig. 60.-Benign pyloric uhstruction.

Case No. 22,583-Male--Age 63.

History of progressive gistric ulcer with recent obstruction; dilatation of stomach and weight luss.

Abdominal Examination.-Marked tenderness to right of navel.

Test-meal.-Pronounced 12-hour retention; tan color extracts with yeasty odor. Total acidity, 68 ; free liydrochloric acid, 63 ; altered blood + (Benzidin test).

Microscopic Examination.-Great numbers of yeasts and sarcince.

Clinical Diagnosis.-Pyloric obstruction, subacute perforating ulcer.

X-Ray Diagnosis.-Pyloric obstruction.

Plate shows "fish-hook" stomach; vigorous peristalsis; bulbous antrum and dilated duodenum.

Surgical Diaynosis.-Pyloric ulcer with obstruction. 


\section{0}

FIG. 61.-Prloric obstruetion; duodemal ulver: deferent visualization of pylorus and dumlentum; malignaney fquestionalse.

Mr. A. Z. - Ige 40.

Frmily History. - Negative.

Previous History. - Premunonia as a routh.

Comes on acemut of chronic indigestion and ahomic eonstipation.

I)

These attarks always eime on grablually and were characterized hy epigastrie distress 2 to 3 hours after meals and particularly at night. The distress was usually a burning sensation and occasionally he has hat ramps. The pain usually lasted several hours and was frequently transmitted to the precordia. The pain was aggavated ly body movement and relieved by limiting the amount of his diet, gastric lavage, rest or by pear juice. Vomiting would cone on regularly at night if the stomach was not lavaged 
make up the bulk of instances of surgically curable cancer, Roentgen examination fails as a clinical method of precision.

With regard to early so-called "primary" gastric cancer - that is gastric cancer developing in stomachs that had previously functionated normally-the $x$-ray gives practically no information. At the time when anomalies of the stomach, such as obstruction, filling defects, malignant adhesions or perforation, are exhibited, the disease process is well under way.

There is a class of case, however, in which $x$-ray examination aids us in confirming the clinical suspicion that a gastric growth exists. Such instances are where the neoplasm is located on the lesser currature near the cardia, at the fundus, greater curvature or posterior wall. This group comprised not more than 6 per cent. of all instances, in a study of 187 consecutive cases. In such instances it is usually impossible to feel an abdominal tumor, the history is obscure and such thing as an atypical blood picture, diarrhea, or regurgitant romiting, may make it necessary to exclude malignant involvement of organs other than the stomach. In no instance of this type has the $x$-ray examination returned positive information, however, where the presumptive diagnosis of gastric

and romitus would consist of sour food. Never suffered any nausea, but belching and water-brash were fairly constant.

A ppetite.-Food desire excellent.

Boucls.- Markedly constipated, sometimes do not move for a weekbleeding. Had bloody "flux" lasting 3 days, age 22. Had no recurrence.

Weight.-Six months ago, weight 130; present, weight 100; height, 6 ft. $t$ in.

Temperament.-Markedly neurotic-says, "he is very delicate and sensitive."

Clinical Diagnosis.-Peptic ulcer; enormously dilated stomach; mentally deficient.

X-ray Diagnosis.-Perforating duodenal uleer. Plate shows dilated ptosed stomach of the fish-hook type; hyperperistalsis; pylorus shows deficient filling with bismuth; irregular outline with a residue in the duodenum.

Surgical finding.--Extensive duodenal uleer extending to and involving the prlorus; adhesions to pancreas but no perforation. 
malignancy had not been strongly considered clinically. In many cases the stomach-tube examination alone has been quite sufficient to establish positive diagnosis in a fer minutes and at little expense to the patient.

In the great number of instances where the $x$-ray examination showed positive diagnosis, clinical facts had already established this. For example: in 147 consecutive cases of proved gastric cancer, there was palpable epigastric ridge or tumor present in 72 per cent. In this group, gastric retention of the malignant type was demonstrated in 74 per cent.; occult blood existed in the gastric extracts in 92 per cent. and in the stools in 86 per cent. The microscopic examination of gastric extracts showed bacilli of the Oppler-Boas type in 93.8 per cent. In this group of cases, the $x$-ray diagnosis was "positive" for carcinoma, "probably" or "possibly" carcinoma in 92 per cent.

The information of the greatest value which the Roentgen examinations give us is that of roughly locating the position of a growth, determining the extent of such, visualizing the deformity existing in the stomach, and sometimes indicating the invasion of adjacent viscera. Roentgen examinations have a not-to-be neglected psychic value. They make it much easier to convince the patient or medical attendant that carcinoma exists, or does not exist, than if such an examination had not been made. They also permit of interesting visualization of diseased organs. In this way, valuable service may be rendered by enabling one to regard the different phases of the disease in a patient under various types of surgical or non-surgical treatment.

METHODS OF EXAMINATION BY MEANS OF THE ROENTGEN RAY

There are two useful methods in vogue: the examination by making plates or films and the examination by means of 
the fluoroscopic screen. Our experience, from the study of the records of more than 2,000 gastro-intestinal $x$-ray examinations, would indicate that neither method is allsufficient, but that the greatest amount of information is to be obtained by a combination of both procedures. It is such method that we deem best to describe.

\section{EXAMINATION BY FLUOROSCOPIC SCREEN}

This examination has for its object two main ends. The first is to demonstrate the ability of the stomach to empty itself of ingested material. The second is the examination of the stomach filled with a medium opaque to the Roentgen rays for the purpose of noting abnormalities, with and without the aid of abdominal palpation or change in the patient's position.

To test the emptying power of the stomach it is our practice to first wash out the stomach so as to free it from an accumulation of food that may have resulted from improper diet. The next procedure is to empty, as far as possible, the gastro-intestinal tract by means of a cathartic. For this purpose, we use 2 ounces of castor oil in a half glass of beer or malt extract, or mixed with a small amount of iced water, lemon or orange juice. The patient is then kept on a liquid diet for from 8 to 12 hours. He is then fed a so-called "motor-meal." We have found it useful to feed this in the form of bismuth subcarbonate or barium sulphate in Cream of Wheat. The proportions are 2-4 ounces of either of these drugs to 8 ounces (cooked weight) of Cream of Wheat. This mush may be flavored with fruit juices. A little cream is permissible. The patient eats the mush slowly, and is not usually told its ingredients.

Six hours after this motor-meal, the stomach is examined by means of the fluoroscopic screen and $x$-ray tube. The 
patient is stripped of all excepting a light surgical gown. We prefer to examine our patients standing, but there is no objection to conducting the examination with the patient seated (say upon a high bicycle seat) or even lying upon a proper table. The form of apparatus used is of little consequence so long as one has arailable a first-rate fluoroscope screen (the best are imported from Germany or France) and a number of high-grade water-cooled $x$-ray tubes. Many good tubes are made. Those which have a cooling arrangement in the form of a continuous current of water (after the suggestion of Albers Schoenberg) are very satisfactory. The new tube derised by Coolidge is most excellent.

Negative Findings.- - If there is no mechanical interference to food entering the stomach (stricture at the cardia), to its passage through the stomach (tumor mass, malignant hour-glass, perforation, adhesions) or to its exit from the stomach (malignant stenosis at the pylorus), at the end of 6 hours the "motor-meal" of bismuth or barium mush will have left the stomach. The mass of it will be located in the lower ileum or some part of the large bowel.

Positive Findings. - If malignant obstruction has occurred, in the above-named fashion, one may see varying amounts of bismuth in the esophagus, in a loculus of the stomach or lying at the most dependent part of the viscus. In but few extensive gastric cancers does the stomach empty itself completely of the motor-meal. Eren if the orifices are wide open, it is not uncommon to find "rests" of the opaque-meal lying between projections or in the irregularities formed by the neoplasm. The absence of all the motor-meal from the stomach is not a conclusive proof that the orifices are not involved. It is not uncommon to discover at laparotomy, in such instances, a hard gristle- 
like neoplasm extensively involving the pylorus and antrum. This may so stiffen the gastric wall that the lumen remains patent and allows the opaque meal to pass rapidly into the small gut after the fashion of liquid flowing through a funnel.

In our experience 6-hour opaque "rests" of a motormeal are seen in approximately 2 out of 3 cases of gastric cancer where there is moderately extensive involvement.

Fluoroscopic Examination of Abnormalities in Gastric Outline.-For this purpose it is necessary to almost fill the stomach with a puree-like mixture. In this manner, one can then observe shape, size, position, contour, emptying power and tender points of the stomach. One can also determine the relation of the stomach to adjacent viscera, and rapidly note the effect of peristalsis or the influence upon the above factors brought about by change of position or by abdominal palpation.

The opaque liquid which we have found useful consists of 6 to 8 ounces of bismuth subcarbonate or barium sulphate in 1,000 cc. of buttermilk, "fermilac," or thin starch-paste. These mixtures may be flarored with chocolate, vanilla or fruit juices. Usually little flaroring is necessary.

After examining the stomach for a 6-hour "rest" described under estimation of motor function, the patient is next permitted to drink 500 to $1500 \mathrm{cc}$. of the bismuth subcarbonate or the barium sulphate puree. While this is being taken, the esophagus is scrutinized with the patient in both the anterior, posterior and lateral positions. This maneuver permits of one gauging the size and patency of the esophagus and also makes it possible to note the freedom with which the opaque mixture passes the cardia. Note should be carefully made regarding these points and if there is abnormality observed, repeated examination should 
demonstrate its permanency or its transientness. If such anomaly be permanent, a record should be obtained of it upon plates.

In cases where there is no abnormality noted in the esophagus or at the cardia, the stomach should be observed as the opaque meal enters. Depending upon the thickness of the abdominal parietes, the excellence of the tube and screen, and the success with which the opaque meal has been made, one usually observes in gastric cancer, first, that there is some fault in the gastric outline. This fault consists most generally of a diminution in the size of the gastric lumen. This is due to the fact that the majority of malignant growths extend into the stomach cavity and hence, at these points, there is a defect of normal gastric filling due to the intrusion into the lumen of solid tissue. This may be of greater or less extent. Generally, the most marked "filling-defects" are seen toward the pylorus, that is, in the region where gastric cancer is most common. They may occur, however, at any part of the stomach. They result in atypical irregularities of the lumen. In some instances they totally occlude the gastric cavity. On account of the fact that gastric cancers are generally of the medullary type, one not infrequently sees that the outline of the gastric lumen is irregular, or has a worm-eaten or old cheese appearance. This condition exists because the nodular projections of the growth permit of varying amounts of bismuth lodging between them. Hence, the Roentgen ray penetrates greater quantities of bismuth at some parts than at others.

Whatever may be the situation of the gastric neoplasm, if it has advanced far enough to be recognized by the $x$-ray, there is always encroachment upon the lumen of the organ at some point. In cases of scirrhus growth in- 
vading largely the submucosa, there is a smaller lumen than ordinarily shown. The lumen may be quite uniform in such instances, but due to the thickness of the gastric wall, the resultant cavity is contracted (so-called "leatherbottle stomach").

When the growth is at the cardia, not infrequently such is difficult to recognize due to the left lobe of the liver, the lower ribs, the breast or the sternum lying in front of it. Careful examination, however, usually discloses some outline defect or some atypical way in which the opaque medium enters the stomach. In normal cases, a fluid passing the cardia seems to closely hug the lesser curvature (magen$w e g)$. Where there is some fault at the cardia, one not infrequently sees that the bismuth is delayed in the esophagus. When it does enter the stomach, it may pass in in several streams, may not hug the lesser curvature, or it falls through in large drops or gobs. Above neoplasms at the cardia there may be extensive dilatation or the esophagus may even be infiltrated by the tumor.

When the growth is high in the lesser curvature, the lumen is usually encroached upon in a characteristic way. If, however, the growth is not very soft or papillomatous the outline of the lesser curvature may be fairly regular. The resultant appearance then may be difficult to differentiate from a normal lesser curvature pushed to the left by an extragastric tumor (liver, gall-bladder, pancreas, retroperitoneal sarcoma). In this event an examination of the patient in different positions will often serve to delimit the esophagus. It may even be infiltrated by the tumor.

When the growth is situated in the body or the pars media of the stomach, fluoroscopic examination usually makes evident local narrowing of the gastric lumen. The extent of this depends upon the size of the neoplasm. Often, hour- 
glass loculation is seen. Occasionally, several gastric chambers are apparent, partly or wholly filled with the shadow-casting substance. Not infrequently, in extensive tumor involvement, all that remains of the stomach's lumen is a narrow, usually tortuous, canal, through which the bismuth or barium mixture passes in an attenuated stream and is visible with difficulty. If such canal has between it and the screen a great mass of cancer tissue, opaque media in it may not be evident. The test-meal may then be seen in an upper (usually larger) loculus and appear again in a carity toward the antrum or pylorus.

Grouths involving the antral or the pyloric region generally manifest their presence by such intrusion upon the gastric carity as to produce partial or nearly complete obstruction to the onward passage of the bismuth or barium meal. In these instances gastric emptying power may be greatly deficient, delayed or lost. Consequently, even bimanual massage of the epigastrium cannot force the opaque mixtures forward in such amount as to permit the risualization of the pyloric channel or the duodenum. If the growth in these locations is firm or large, there is, commonly, compensatory dilatation of the stomach. Such neoplasms intrude so greatly into the narrow distal end of the viscus as to render much of its channel invisible or only recognizable as a wary canal wandering between indefinitely delimited cancer masses. Not rarely, the entire pyloric third of the stomach appears chopped or torn off and only a few rests of bismuth or barium, in its general direction or lodged in the viscera beyond, indicate that some canalization of the mass exists.

There is, as we have mentioned above, a certain type of tumor (usually of the class styled "scirrhus") which may extensively infiltrate large sections of the pyloric end or 
eren the entire wall of stomach, without producing obstruction. In such cases, the pylorus may gape and gastric contents rapidly escape into the small intestine. Usually, howerer, the general thickness of the stomach wall results in narrowing of the gastric lumen, locally, toward the pylorus or, generally, throughout the stomach. The lumen thus appears small, often tapering in a fumnel-like manner toward the duodenum. Its boundaries may be slightly fringed, wary or irregular. In any event, a relatively small gastric shadow is visible upon the fluorescent screen. The shadow may be pyriform, gourd-shape or bottle-like.

Cancers directly upon the posterior or anterior wall, unless large enough to produce deformity of the stomach, may reveal little evidence of their presence unless various portions of the stomach are successively silhouetted either by abdominal palpation, or by observing the filled riscus at different angles (anterior-posteriorly, standing and reclining; laterally, from both sides, standing and lying, etc.). In the case of growths on the posterior wall, great difficulty may be experienced in differentiating such from tumors of the liver, pancreas, retroperitoneal tissues or left kidney.

In general gastric carcinosis, occlusion of the norma! lumen may be so extensive that very little may be recognized. Instead, "flecks" or larger "rests," the shadowcasting media may be intermingled with gas bubbles and solid tissue, the whole giving indefinite shadows (mottling, dappling, sponge-like, or thick soap suds appearance) in the region where we normally locate the stomach, or where abdominal palpation permits the recognition of a tumor.

In gastric cancer, where early or late ulceration has occurred, one can frequently delimit definite craters, with ragged edges, and these often retain rests of bismuth or barium. If such have become adherent to adjacent viscera 
or perforated into these, then the adhesion or the false channel can sometimes be established.

Peristalsis is generally not evidenced by vigorous gastric contractions in cancer of the stomach, unless the process is implanted on an ulcer and not far advanced, or unless a small (often ring-like) growth causes hour-glass contraction or pyloric stenosis. The invasion of the stomach wall by malignant disease appears to early bring about varying grades of gastric atony or palsy. In non-obstructive growths, where the stomach's emptying time is better than normal, it would seem that weakness of the walls of the viscus rather than strength or frequency of peristaltic waves permit the heavy, opaque mixtures to, roughiy speaking, "fall through" into the duodenum. This is particularly marked where there are few nodulations to the growth or where the neoplasm is located proximal to the antrum.

Palpation is one of the most valuable maneuvers during fluoroscopy. By its use, one is able to establish the movability of the stomach, to delimit the lumen completely, to fill or empty ulcer craters, to test the patency of the pylorus or cardia, to outline the boundaries of the duodenum, to prove the relationship of the stomach to other viscera, to locate a palpable abdominal mass with respect to its being gastric or extragastric, to test its mobility, to estimate the relative involvement of the stomach by such mass, or to uncover gastric neoplasms (as on posterior wall) which direct view, without palpation, does not reveal. Tender areas can be roughly located with respect to their association with parts of the stomach. Thus, one may sometimes gauge the presence or absence of perforation and peritoneal invasion. In instances, where free fluid exists in the abdominal cavity, palpation enables one to recognize its 
presence and extent and by observing the limits of such with the patient in different postures to demonstrate its situation with respect to the abdominal riscera. Of course, common clinical tests frequently return information equally valuable. There seems to be a peculiar satisfaction, however, in the risualization of these phenomena. The psychic effect of these maneuvers upon the patient or his friends appears to be highly valued in some quarters.

\section{THE EXAMINATION BY MEANS OF ROENTGENOGRAPHIC PLATES OR FILMS}

The perfection of the fluoroscope has rendered this method largely obsolete, tedious, of little added clinical worth and umnecessarily expensive. There are few cases of gastric cancer where any Roentgenographic evidence is at all possible, that cannot be quite as successfully recognized by aid of the fluoroscopic screen as by a series of plates or films. The fine distinctions which certain observers demonstrate upon 18 to 40 successive plates, while they may have artistic worth have little practical, clinical value. The multiplication or diminution of the number of shadows in an area in which already one knows that a tumor exists, while entertaining to certain minds is clinically as meaningless and non-essential as are the multitudinous incongruities of a cubistic canvas.

The physical effort demanded of a desperately ill and generally weak patient in the making of a large series of plates is often a serious consideration. The expense of the maneuver is out of all reasonable proportion to the worth of the information returned. As we have mentioned above, in a series of 147 gastric cancers where the $x$-ray examination gave information of possible or positive 
diagnostic worth in 92 per cent. of instances, by such inexpensive and simple procedure as microscopic examination of stained smears of gastric extracts or vomitus, bacilli of the Oppler-Boas type were demonstrated in 93.8 per cent. But rarely are bacilli of this type observed in nonmalignant conditions.

Certain value is found in the making of a few radiograms in gastric cancer. Such enable one to keep a record of the progress of a non-operated case, to recognize recurrences after excision of a tumor, to corroborate fluoroscopic observations and as an aid in advancing knowledge. It is thought by some that prints from plate series are not without advertising value, even if of little scientific worth, provided they be sent abroad appropriately bedecked. The unique scientific spirit which prompts this course is productive of much unwarranted cancerphobia. However successful a trade venture it may be, it cannot be too strongly condemned as a professional act.

Mode of Procedure.-Ordinarily, after the fluoroscopic examination of gastric function, it is possible to proceed with the plate making. If records of an opaque-meal "rests" remaining after 6 hours is desired, plates may be made before the complete fluoroscopic examination is made.

After the fluoroscopic examination, the stomach should be filled again with bismuth or barium puree. This is to be especially urged if the gastric emptying time has been rapid. Radiograms of the partially filled stomach do not enable one to delimit its outline accurately.

When the stomach has been filled, plates are made in the usual manner. In uncommon cases, it is advisable to expose the plates with the patient successively standing, recumbent or lying on one or the other side. In this 


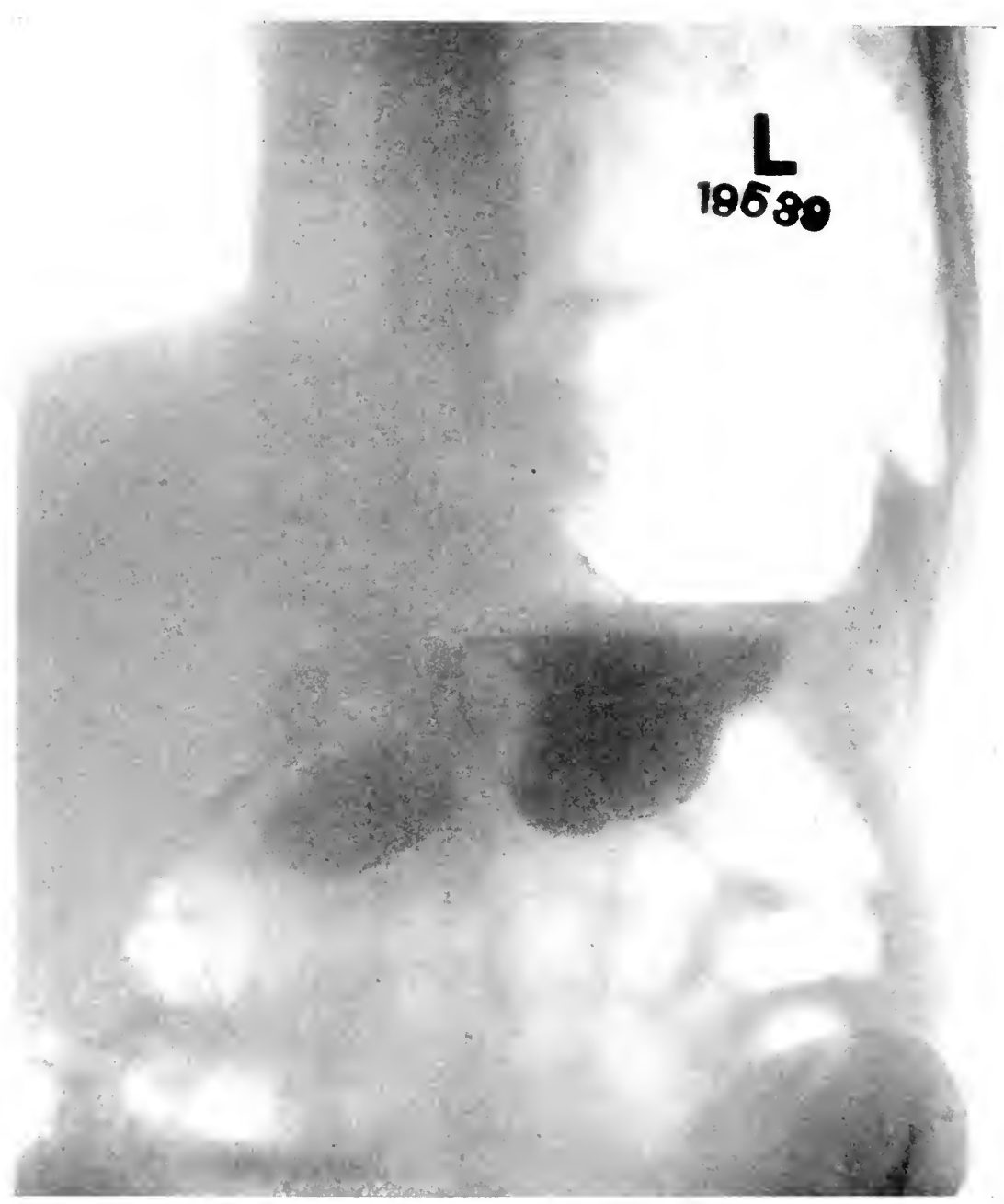

Fig. 62.-C ( ase No. 19,539).-Malignant hour-glass stomach with antecedent history of ulcer; partial obstruction at cardia, with bismuth in dilated esophagus. Tuberculosis of stomach (patient also had pulmonary tuber(culosis). 


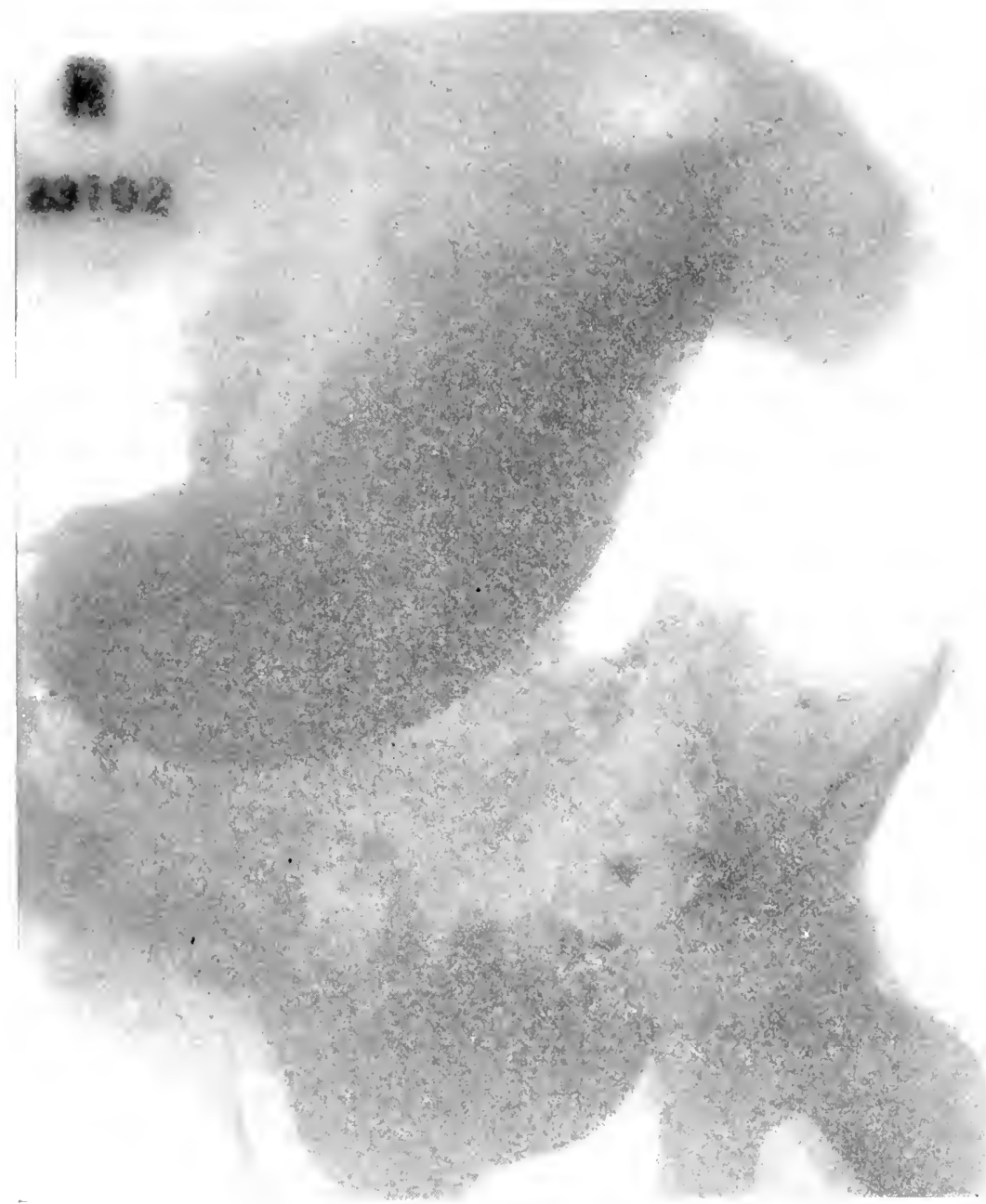

Fic, 63.-Irregular primary cancer history: involvement of cardia and Mr. W. K.-Age 62. pylorus.

Personal History.-Negative.

Duration of Present Ailment.- About 1 year, trouble has been continuous, with recent aggravation.

Comes on account of dyspepsia, abdominal pain, weight and strength loss and anemia.

Discomfort is located in the epigastrium, is described as a "dull ache" and "shuts off wind." It is more or less constant, but worse immediately following food ingestion. It is transmitted to rib edges. Distress relieved by limiting diet, by vomiting and rest.

Patient vomits two or three times a week (usually in the afternoon). Vomitus consists of sour liquid and food. The emesis is brought on by pain. Water-brash and pyrosis are annoying. 
way, one is least likely to fail in the recognition of a gastric deformity. Its extent can be better calculated by such procedure.

If a series of plates is desired, such may be made rapidly, as, for example, 15 to 25 within 20 minutes followed by plates made at from 1 - to 6 -hour intervals until the gastro-intestinal tract has freed itself of bismuth or barium mixtures. There are numerous ingenious mechanical devices for making such a plate series. In admiration of the perfection of these contrivances one may readily lose sight of the fact that the information they return to us has little actual, clinical worth.

The Information Derived from Roentgenograms in Gastric Cancer.-In early cancer there are no positive $x$-ray signs beyond those of complicated ulcer or obstruction, whatever may be the location of the growth. Where wellestablished carcinoma is located at the cardia permanent record may be obtained of dilatation, involvement or displacement of the esophagus, alterations in outline, size, position and patency of the cardia and, rarely, evidences of perforation to adjacent viscera.

A ppetite is variable.

Bowels have been constipated for 7 months.

Weight.-Loss of 23 pounds in past year.

Abdominal Examination.- Tender ridge in upper right epigastrium, moving to region of navel on inflation of stomach.

Test-meal. - Stomach tube meets no resistance at cardia. No 12-hour retention. $75 \mathrm{cc}$. of cream yellow, rancid contents removed. Total acidity, 8; free hydrochloric acid, 0 ; altered blood + ; lactic acid,?; Wolff-Junghans' test, 0 ; formol index, 24.

Microscopic Examination.-Few food bits, Oppler-Boas bacilli ?, sarcinæ (large form); numerous deeply staining, lance-shaped rods; leptothrix +; many polynuclear leucocytes.

Stool.- Altered blood + (benzidin test).

Clinical Diagnosis.- Achlorhydria; ulcus carcinomatosum.

$X$-ray Diagnosis.-Filling defect at cardia and pylorus, probable carcinoma, scoliosis. Plate shows a blunted "steer-horn" stomach, with moderate dilatation, peristalsis is almost absent. On high lesser curvature appears a filling-defect of the crater-ulcer type; the chopped-off pylorus appears to be due to a tumor. The duodenum is dilated.

Surgical Diagnosis. - Inoperable carcinoma. 


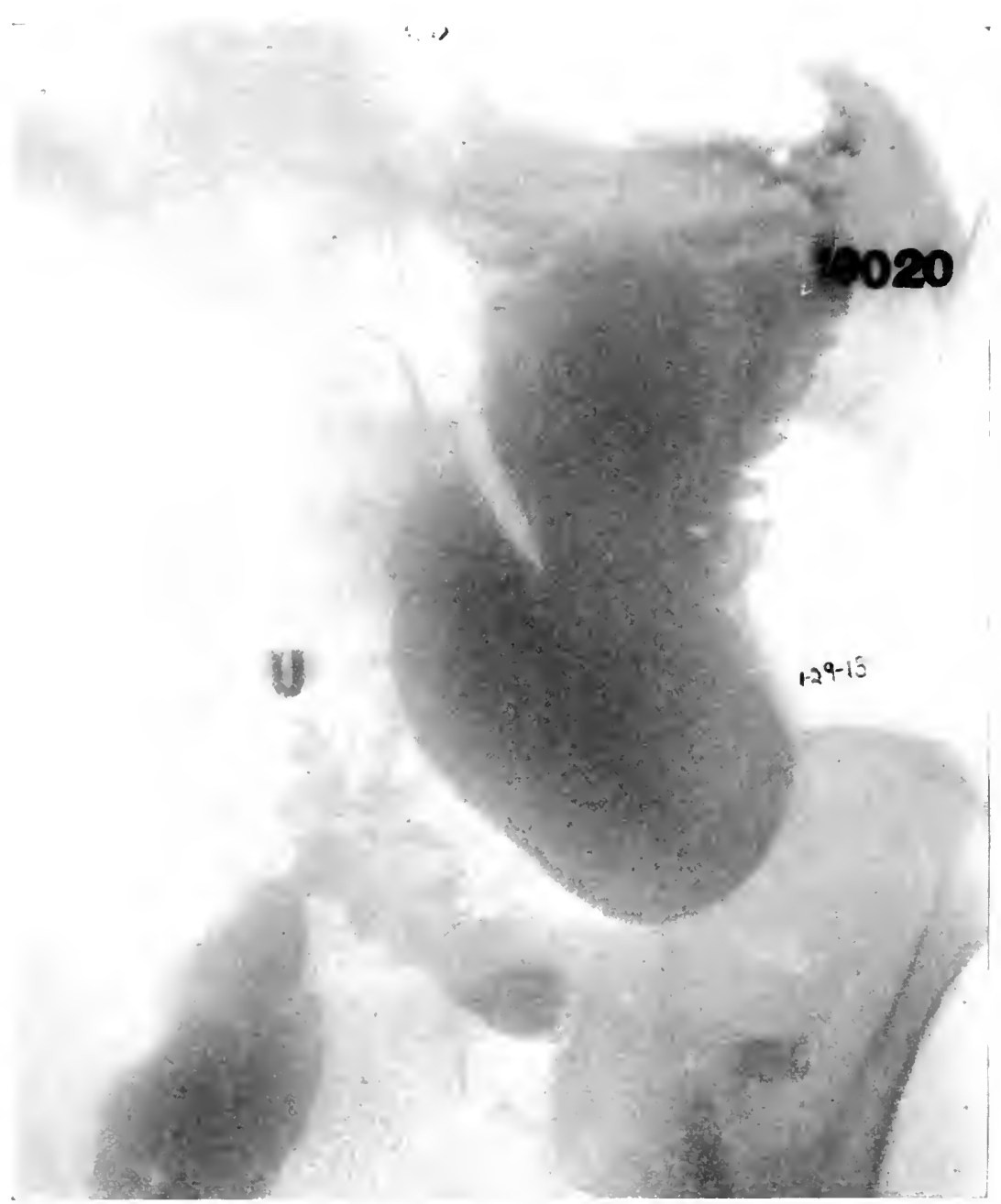

Fig. 64.-(Case No. 19,020), - Inoperable gastric cancer, involving cardia and pars media. 


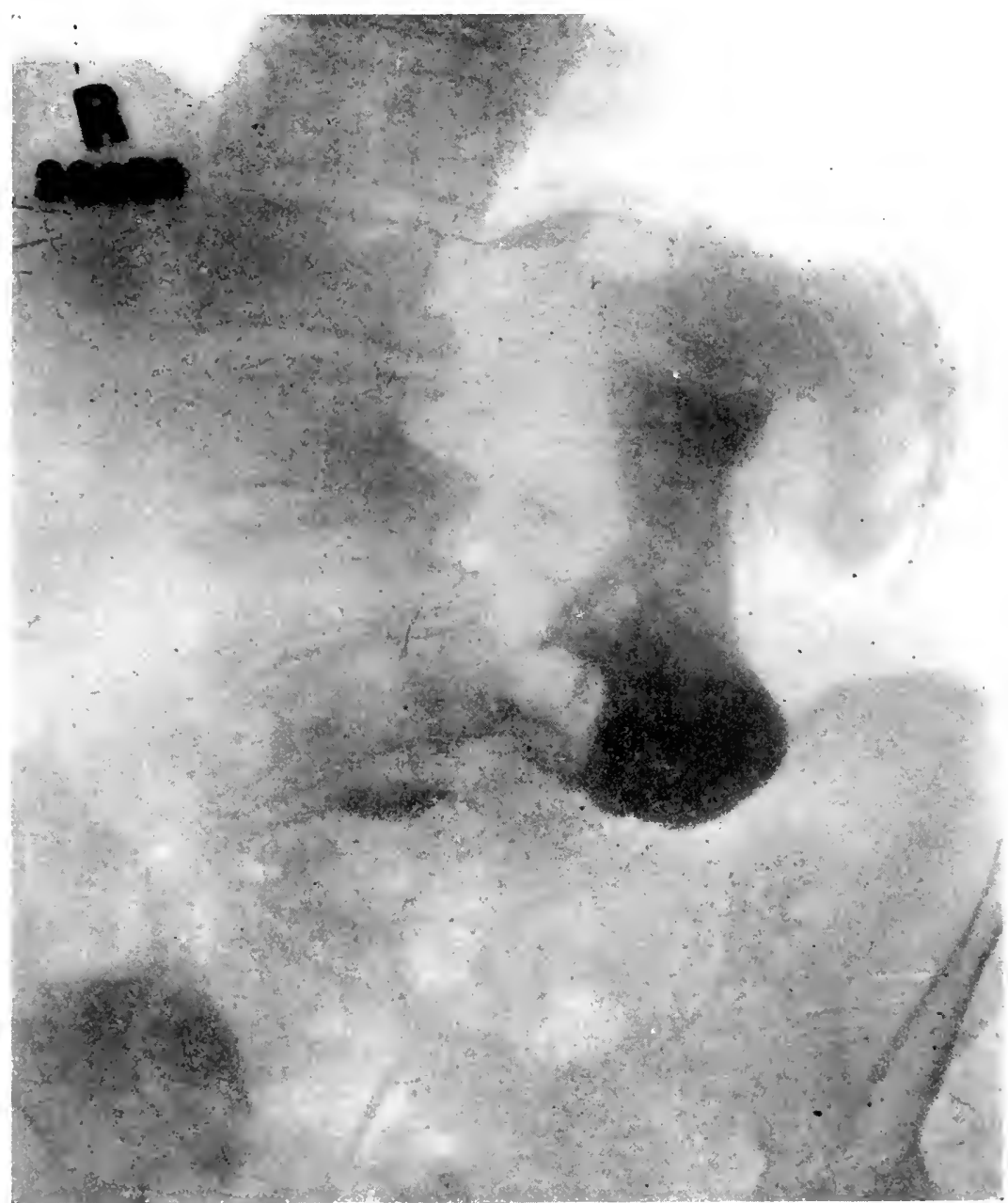

F1G. 65.-Pyloric obstruction; gastric carcinosis.

Case No. 23,650-Mr. J. H.-Age 78.

Abdominal Examination.- Movable mass in mill-epigastrium; stomach moderately dilated.

Test-meal.-Twelve-hour retention; gastric extracts; tan color; rancid oclor. Total acidity, 10 ; free hydrochloric acid, 0 ; altered blood, ? (benzidin test ); lactic acid +; Wolff-Junghans' test, 0 .

Microscopic Examination.-Numerous food rests; great numbers of bacilli of Oppler-Boas type; few budding yeasts.

C'linical Diagnosis.-Pyloric obstruction due to inoperable gastric eancer. I-ray Diagnosis.-Carcinoma of stomach; marked hypertrophic arthritis of spine.

Plate shows pyloric half of stomach and lesser curvature invaded by nodular growth; lumen nearly obliterated in antrum and pylorus; no visualization of duodenum or distal viscera.

Surgical Diagnosis.-Irremovable gastric tumor. 


\section{(6) \\ 201}

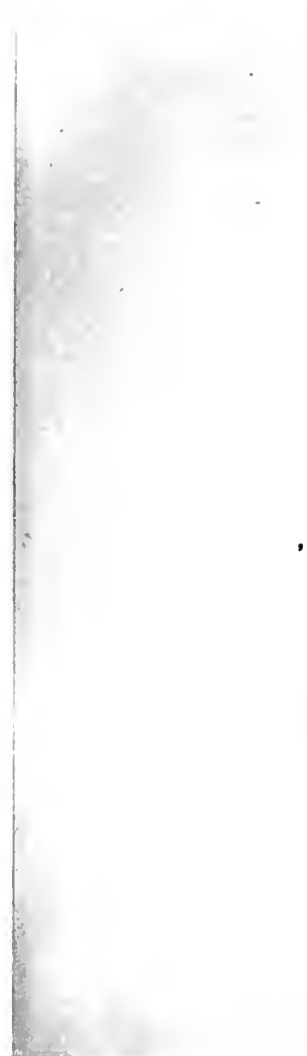

Fig. 66.-(Case No. 23,147).-Perforating ulcer (malignant ?) on lesser curvature; gaping pylorus. 


\section{P \\ 23000}

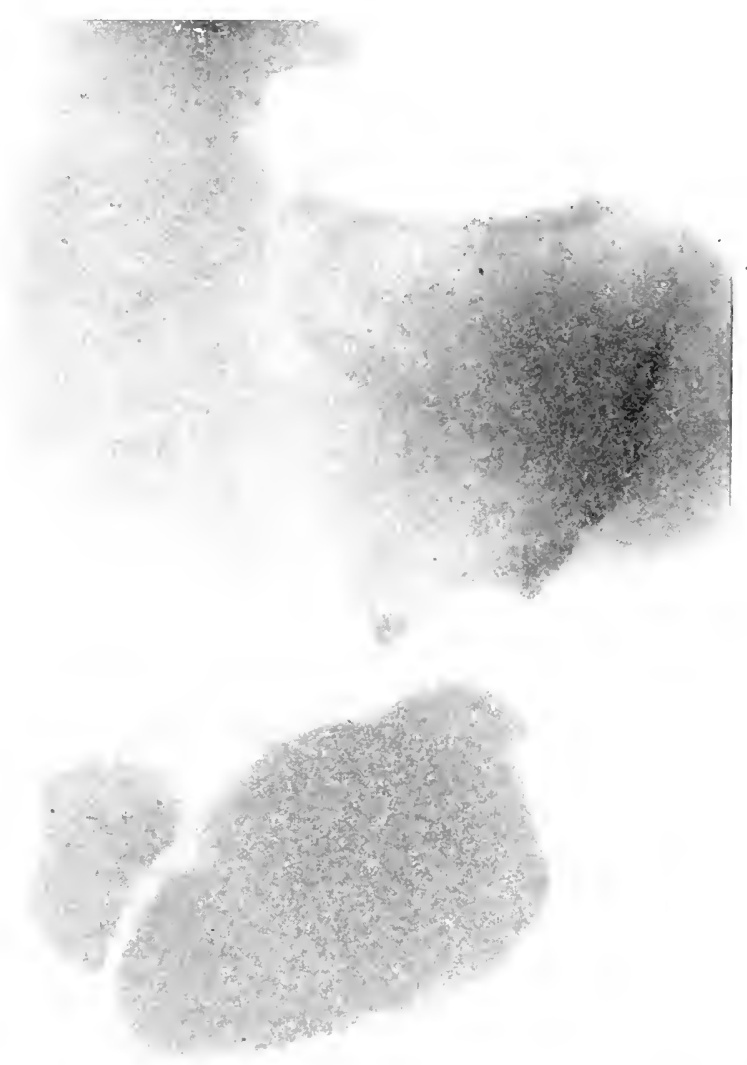

FIG. 67.-Chronic perforating gastric ulcer; malignancy questionable;

Mr. H. C. C.--Age 36.

Previous History.-Negative.

Comes on Account of chronic intermittent indigestion and chronic constipation.

Duration of Disease.-Attacks of dyspepsia for the past 1s years; has three or four attacks a year. Is usually worse in spring and fall. Trouble has been constant during the last 3 months. The attacks are characterized by pain in the upper left epigastrium, come on usually 3 to 4 hours after eating and after he gets to bed at night. Pain is a soreness, a hard ache or a colic. At present he is never free from distress, but relief is obtained by food ingestion, vomiting, rest or alkalies. Pain is usually transmitted to the left costal margin and to the tip of the left scapula. Yomiting is of almost daily 
If the fundus or cardiac portion of the lesser curvature is affected, irregularities in outline with lagging of the opaque-meal, narrowing of the lumen or signs of adhesion or perforation may be determined.

When the neoplasm has invaded the body of the stomach marked irregularities in the outline of the gastric channel are not rarely seen. There may be such constriction of the lumen as to produce hour-glass form. Peristaltic activity is usually diminished. It may be almost absent. Dilatation of the stomach or of one or both loculi (in the event of hour-glass type), with misplacement due to perigastric adhesions (often malignant) or perforation are not uncommonly visualized. In some instances definite crater formation upon the top of nodule projecting into the stomach lumen may be made out. When the growth is limited in extent or confined to the anterior or posterior walls, few radiographic signs, apart from alterations in peristalsis or rate of onward progression of the opaque-meal, may be evident. Observation made with the patient in different positions sometimes helps to elucidate a seemingly negative

occurrence but usually follows almost immediately after food ingestion. It is brought on by abdominal pain and the sensation of food sticking "at the entrance to the stomach." Eructations, belching, water-brash and pyrosis are very marked.

A ppetite.-Capricious.

Weight Loss. - None.

Hemorrhage.- Melena $11 / 2$ years ago followed by severe hematemesis. Nine days ago had severe hemorrhage from stomach and bowels and fainted.

Bowels. - Constipation for the past 18 years.

Abdominal Examination.-Tenderness and dulness very marked in the mid-epigastrium.

Test-meal.-Moderate 12-hour retention. Gastric contents, tan brown in color and of sour odor. Total acid, 70; free hydrochloric acid, 70; altered blood + .

Microscopic Examination.-Teasts; sarcinæ.

Clinical Diagnosis. - Subacute perforating lesser curvature ulcer with obstruction.

X-ray Diagnosis. - Perforating ulcer of the stomach, lesser curvature; hourglass stomach. X-ray plate shows bi-loculation of the stomach due to constriction in the pars media. The channel is very narrow at this point and just above it on the lesser curvature is an ulcer crater containing a bismuth rest. The incomplete filling of the lesser curvature of the upper loculus makes it impossible to say whether this process is malignant or benign. 

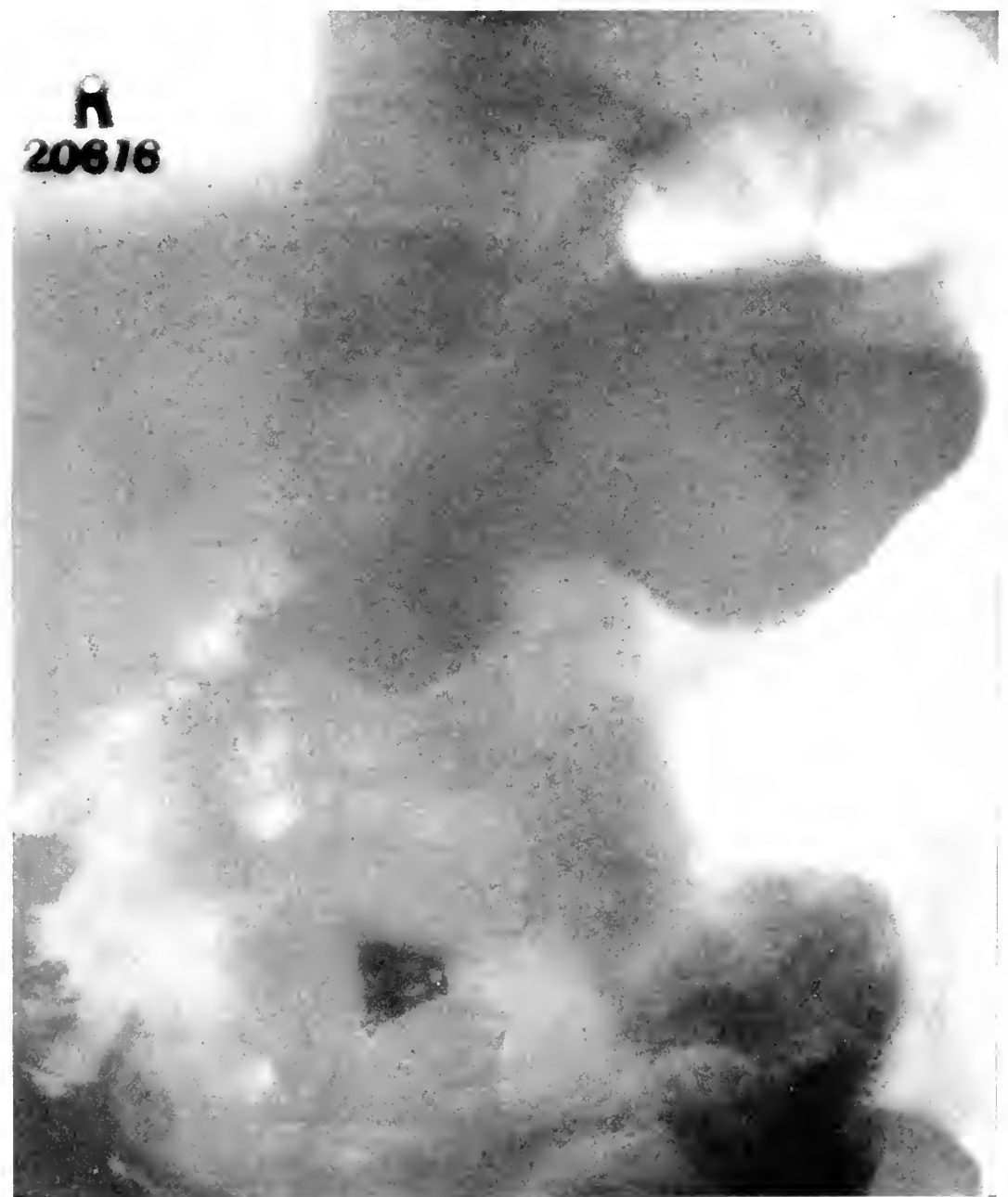

FIg. 68.-(Case No. 20,616).-Hour-glass stomach; saddle ulcer pars media; gaping pylorus; spasm at cardia. 


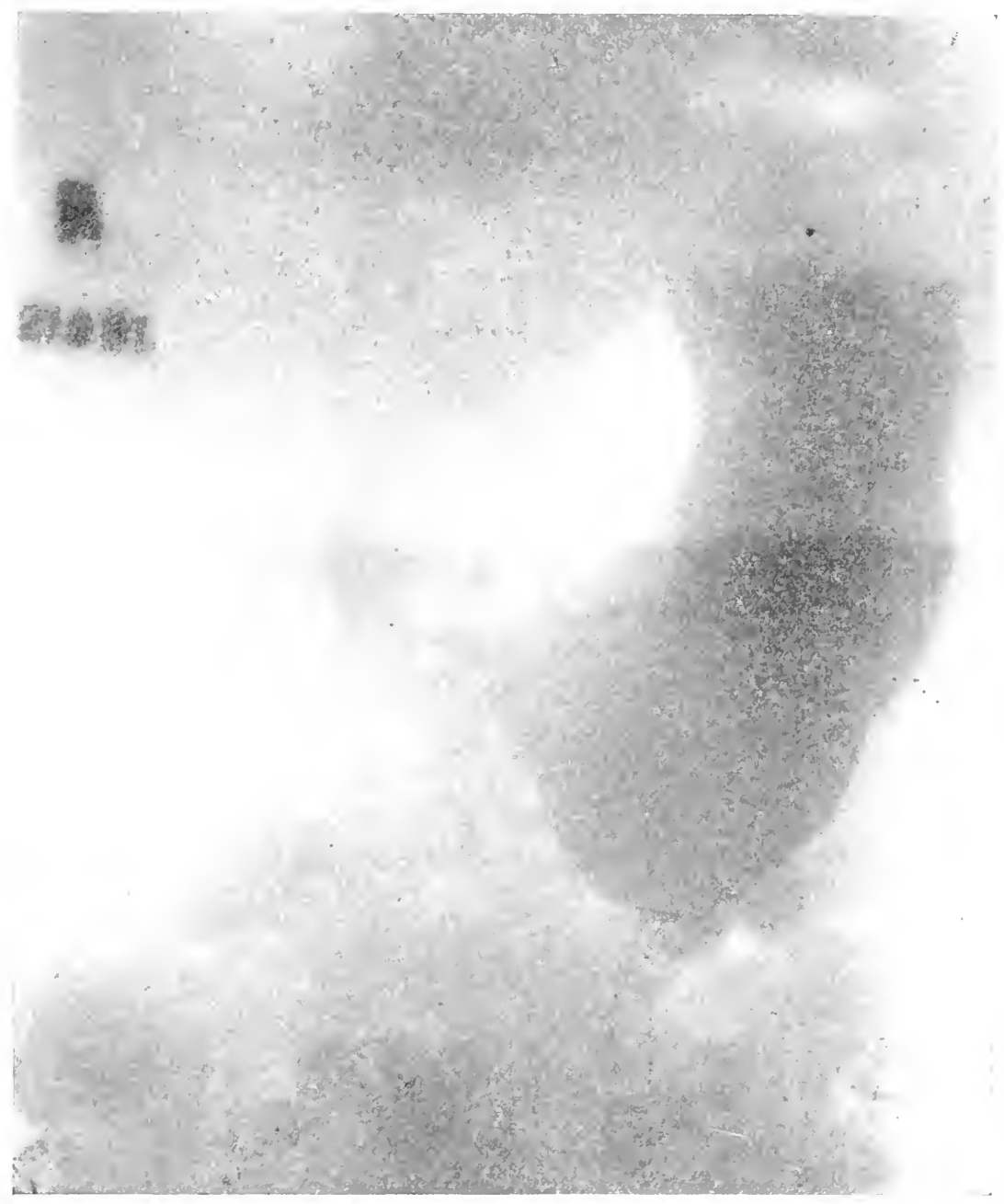

FIG. 69.-Achlorhydria; indefinite clinicai picture. Filling defect of pars media. 


\section{DESCRIPTION OF FIG. 69}

Mr. P. P.-Age 62-Farmer.

Past History. - Typhoid fever, 19 years previously.

Comes on account of anorexia, weight and strength loss, anemia, mild dyspepsia. Patient felt perfectly well up to 4 weeks ago. Gradually lost appetite and experienced vague, bloated sensations in the upper abdomen. These have been continuous, but are most marked soon after food ingestion. Recently has vomited in the late afternoon, and obtained relief of abdominal discomfort. Never vomited blood or food retained more than a half day Suffers nausea soon after eating. Occasionally experiences regurgitation of food. Has been growing paler during past fortnight.

Appetite.-Very poor. Bowels moderately constipated. Never noted melena.

Weight.-Loss of 33 pounds in past 2 months.

Abdominal Examination.-Dulness and area of deep tenderness above the navel. Negative otherwise.

Test-meal.-No 12-hour retention; stomach slightly dilated. Gastric extract is cream colored and food poorly chymified. Total acidity, 10; free hydrochloric acid, 0; altered blood + (benzidin test); lactic acid, 0; WolffJunghans' test + .

Microscopic Examination.-Shows a few budding yeast cells.

Stool.-Altered blood.

Clinical Diagnosis.-Achlorhydria; carcinoma.

$X$-ray Diagnosis.-Apparent filling defect in pylorus, antrum and lesser curvature. Pylorus gapes. Suspicious of malignancy.

Plate reveals a modified "fish-hook" stomach; peristalsis fairly active, diodenum well visualized, apparent canalization of a growth toward pyloric fifth of the stomach.

Surgical Diagnosis.-Cancer at the pyloric end of the stomach. 


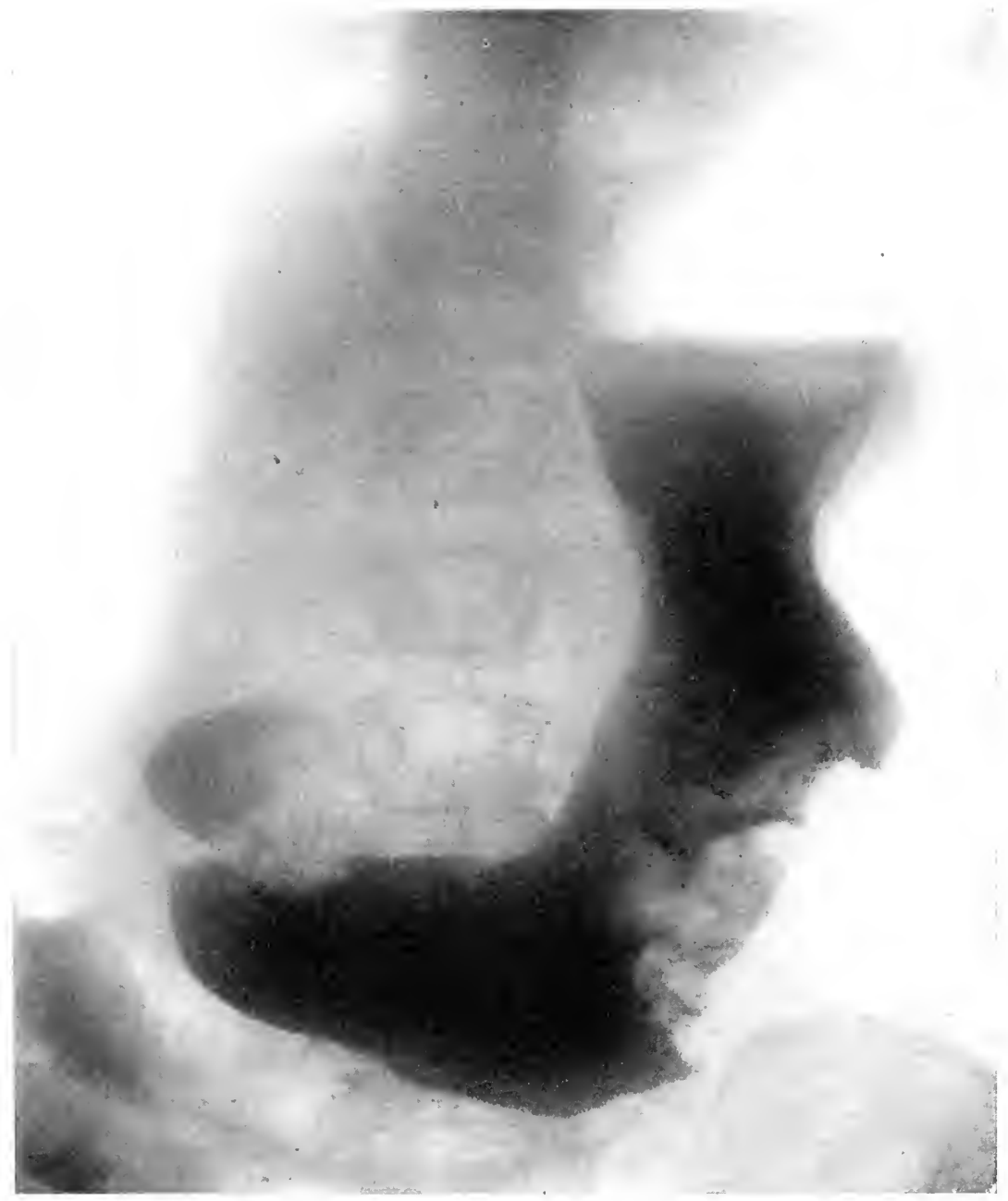

FIG. 70.-Carcinoma involving greater curvature and posterior wall. 


\section{DESCRIPTION OF FIG. 70}

Mrs. C. C. W. - Age 53.

Previous History.-Hysterectomy 13 years ago.

Comes on account of chronic dyspepsia, weight and strength loss, anemia.

Duration of Disease. - Spells of dyspepsia on and off for the past 20 vears at infrequent intervals. Attacks have been characterized by pain in the upper left abdominal quadrant. Would last for days; were colicky and gassy in character; were most marked when the patient had an attack of diarrhcea. Pains did not seem to be aggravated by dietetic variations. Were usually relieved by rest or by vomiting, belching. pyrosis or regurgitation.

A ppetite.-Food desire very good.

Bowels. - Four to seven movements a day for the past 10 months. Light in color.

Weight Loss.-Thirty-five pounds in 1 year.

Abdominal Examination.-Dulness in Traube's space. In upper midepigastrium a large thick tumor is palpated. Stomach holds 23 ounces, greater curvature is 3 finger breadths below the navel line.

Test-meal.-100 ce. of tan and brown poorly chymified material removed after 57 minutes. Marked bleeding on manipulation of tube. Moderate amount of previous evening meal recovered. Very free hemorrhage on lavage. Total acidity, 22; free hydrochloric acid, 0 ; altered blood + (benzidin test;) Wolff-Junghians' test + ; lactic acid, 0.

Vicroscopic Examination.-Oppler-Boas bacilli. Many short bacilli in pairs. Numerous red blood cells and food remains.

Clinical Diagnosis. - Extensive inoperable gastric cancer with involvement of cardia.

I-ray Diagnosis.-Probable carcinoma of the stomach.

Plate shows steer-horn stomach with moderate ptosis and extensive filling defects in the region of the borly, antrum and pylorus.

Surgical Diagnosis.-Inoperable carcinoma. 


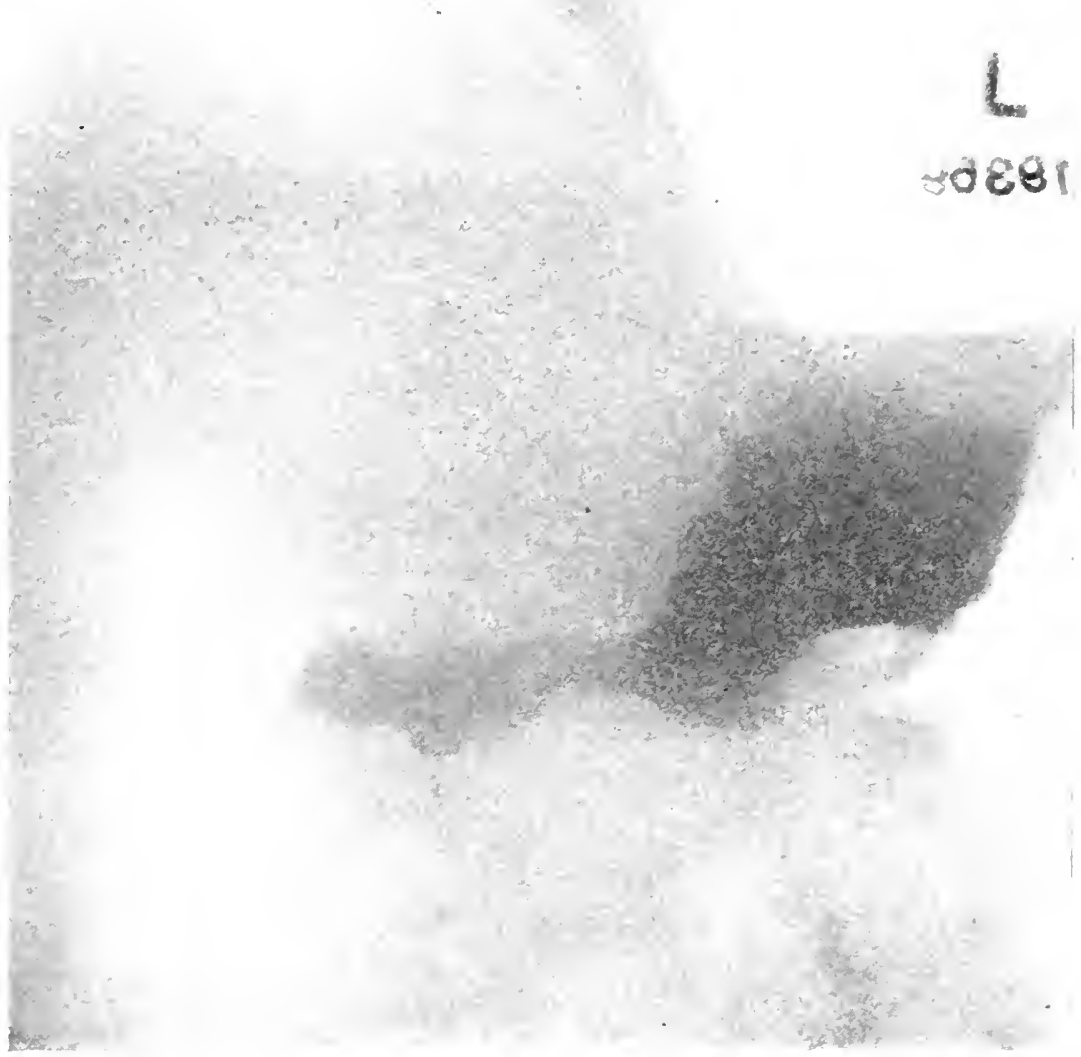

FIG. 71.-(Case No. 19,359).-Extensive cancer of stomach with filling defects at pars media and pyloric end. Pylorus is displaced to the left. It is patent. Duodenum visualized and dilated. 


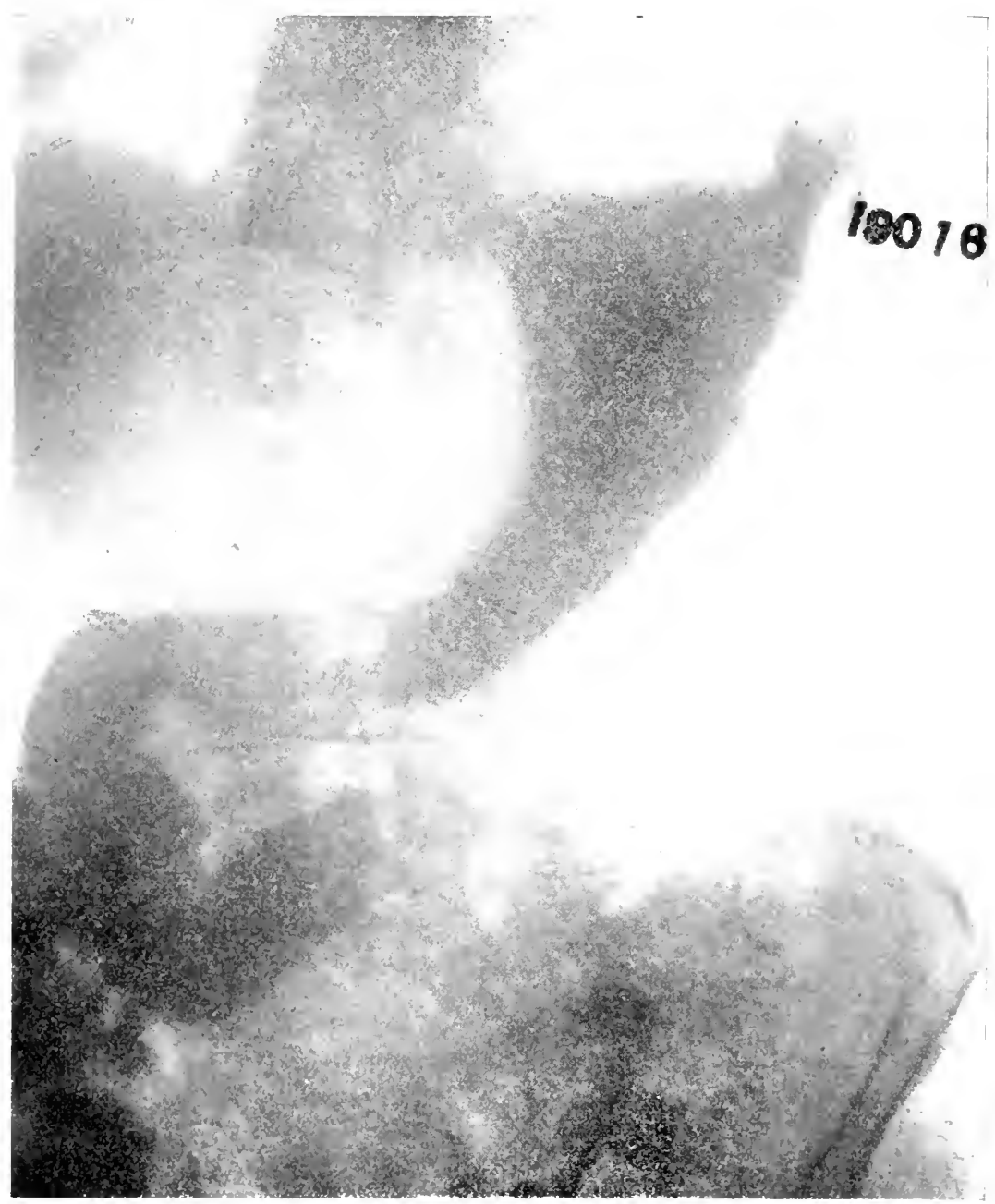

Fig. 72.-(Case No. 1,906).-Scirrhus cancer of stomach; funnel-shaped stomach; pylorus displaced to eft and patent; duodenum visualized. 


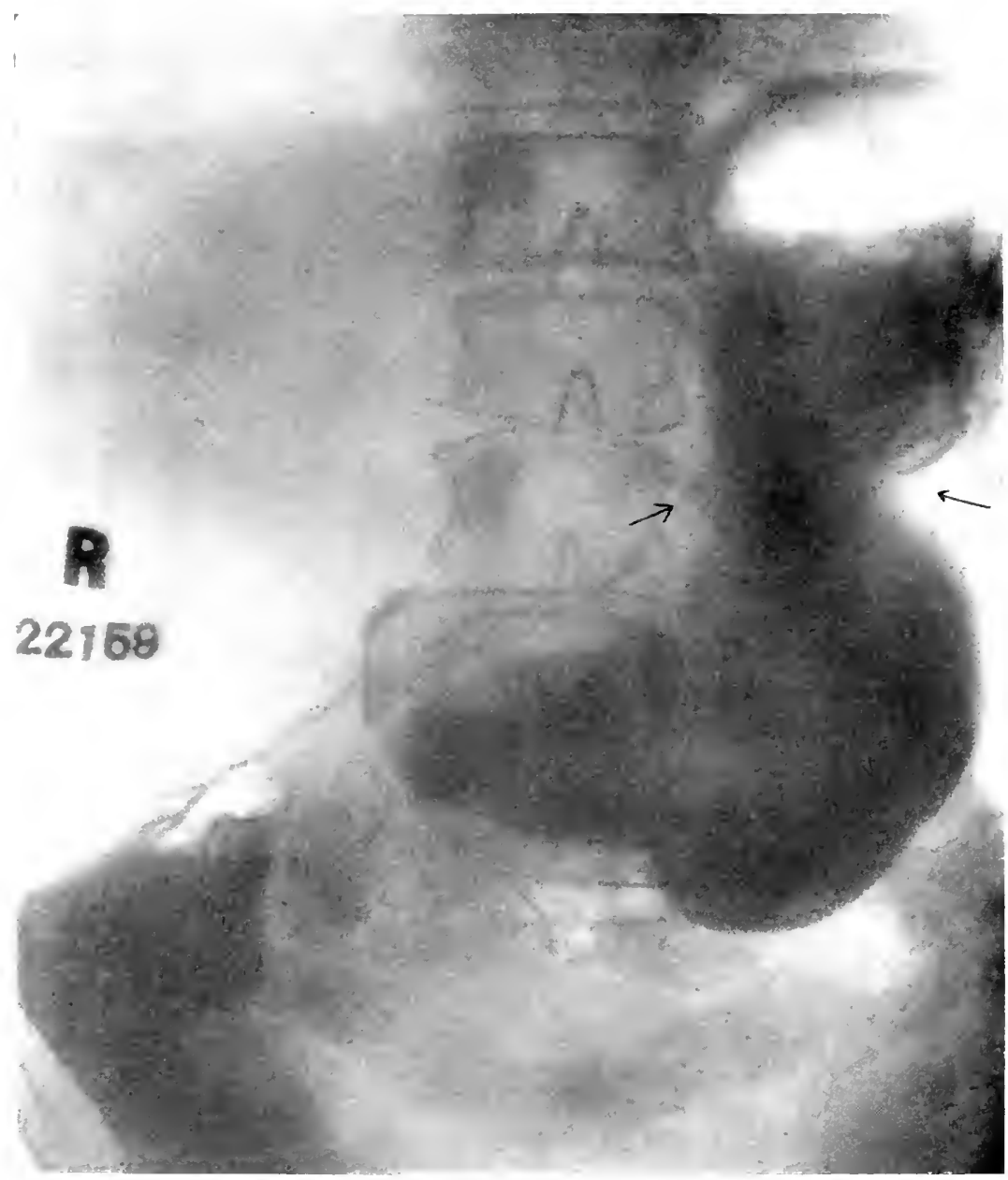

FIG. 73.-History suggesting chronic perforating peptic ulcer; syphilis (?); lesser curvature ulcer (malignant?); demonstrated. 


\section{DESCRIPTION OF FIG. 73}

Mr. Wr. P.-Age 5S-Farmer.

Personal and Family Historics.-Syphilis.?

Duration of Present Ailment. - Infrequent attacks of dyspepsia for 3 years. Trouble has been constant for past montly.

Complains of abdominal pain, indigestion and recent weight loss. Pain is marked in the mid-epigastrium, is of severe aching and "doubling up" type and usually transmitted to the right costal margin. Pain comes on usually 4 to 5 hours after meals and lasts until something is eaten. Is less comfortable on light diet. Relief of distress is also obtained by peppermint water and rest.

Fomiting-usually brought on as result of sudden movement, occurs irregularly. Pyrosis is annoying at irregular intervals, but usually relief is obtained by drinking peppermint water.

Appetite-Fair.

Bowels.-Constipation altermating with diarrlioea.

Abdominal Examination. - To right and above navel is an indefinite, fixed, tender ridge. Stomach splashy.

Test-meal.- No 12-hour retention. Gastric extract is of light green color. Test food is well chymified. $175 \mathrm{cc}$. recovered. Total acidity, 30; free hydrochloric acid, 30 ; altered blood, 0 ; lactic acid, 0; Wolff-Junghans' test, ?; formol index, 19.2 .

Microscopic Examination.-Food remains, veasts and diplocoeci.

Clinical Diagnosis.-Subacute perforating ulcer lesser curvature. Malignant change.? Syphilis.?

X-ray Diagnosis.-Calloused ulcer of the stomach.

Plate shows ulcer crater on lesser curvature with deep incisura on greater curvature at about the same level. It is impossible to say whether or not the ulcer is malignant.

Stomach empties freely, pyloric end is in state of moderate contraction; duodenum is poorly visualized.

Surgical Diagnosis. - Ulcer on lesser curvature with chronic perforation. 


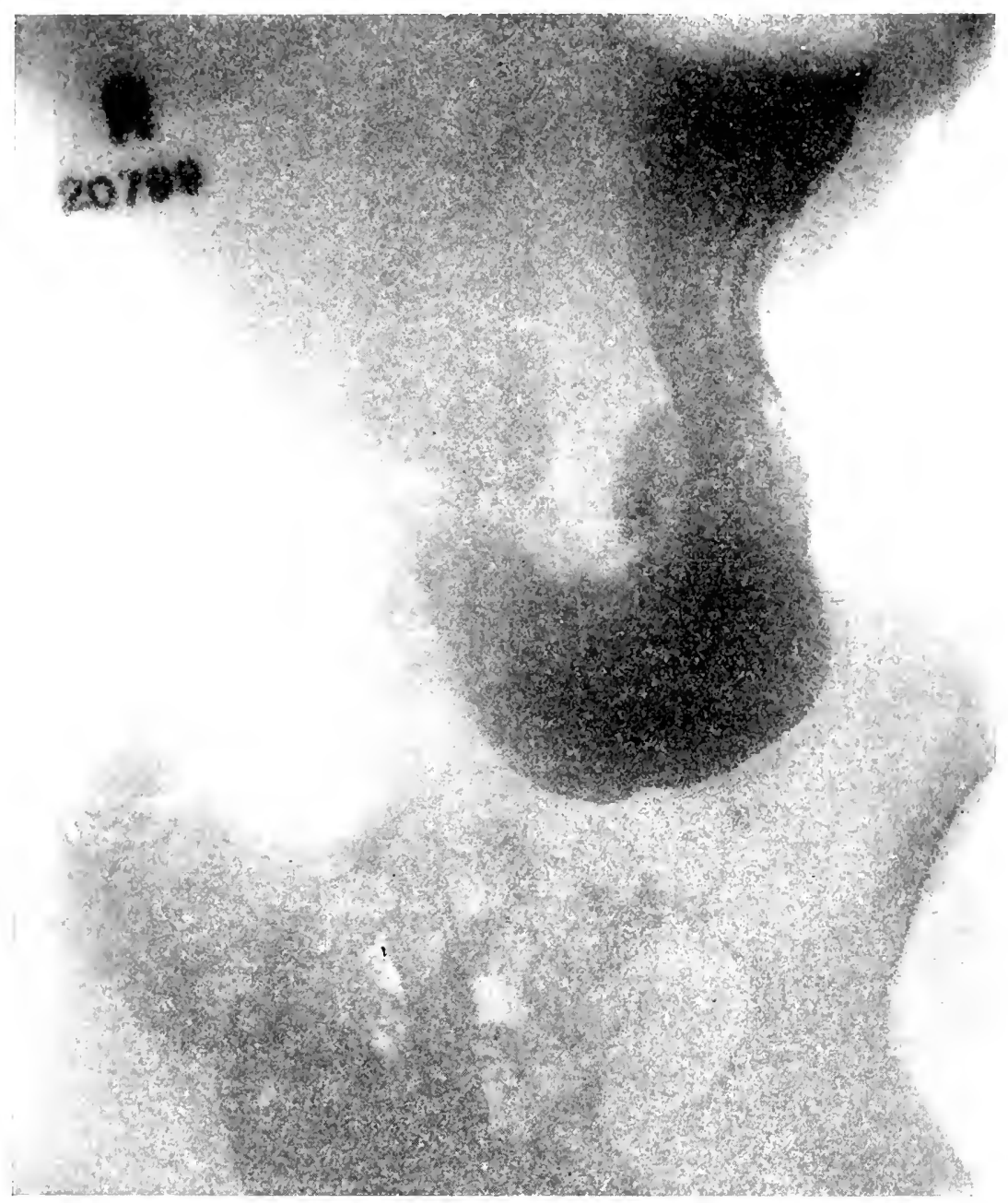

FIG. 74.

Case No. 20,799-Male-Age 62

History that of chronic perforating gastric ulcer with suspicion of recent malignant change.

X-ray Diagnosis.-Perforating ulcer on lesser curvature.

Operative Findings.-Large ulcus carcinomatosum on lesser curvature. 
case as shown by routine technique where in such case test-meal findings and clinical examinations strongly point toward gastric cancer.

Carcinoma at the pyloric end of the stomach is commonly associated with some grade of gastric retention and quite often with compensatory dilatation of the viscus. This is not always demonstrated because occasionally the stomach is involved locally in a tough, gristle-like growth that stiffens the wall, thereby maintaining a widely patent lumen, with not infrequently a gaping pylorus. Little detail of the cancer, Roentgenographically, may be possible in such instances on account of the rapid emptying of the stomach. There may be few or no opaque-meal "rests." Ordinarily, invasion of the antrum or pylorus results in great change in the caliber of the lumen. This may be narrowed, generally or locally, with resultant tortuous, irregular, bulbed, lobulated, funnel-shaped, or almost invisible gastric channel. Perforation or ulceration may produce accessory chambers filled with bismuth or barium, or reveal evidences of involvement of liver, pancreas, adjacent bowel or the peritoneal cavity.

There are instances where ring cancers at or near the pylorus produce striking local constriction of the lumen with marked obstruction. If such cancer occurs directly at the pylorus, the blunt, abruptly cut-off termination of the stomach, with dilatation of the viscus, generally, or the antrum, locally, associated with failure of visualization of the duodenum, is a fairly characteristic picture. In this type of neoplasm, peristalsis may be quite active.

With the object of demonstrating the relative worth of Roentgenographic evidence in gastric cancer, we have arranged a series of Roentgenograms in association with other clinical and surgical findings. It will be observed that in 


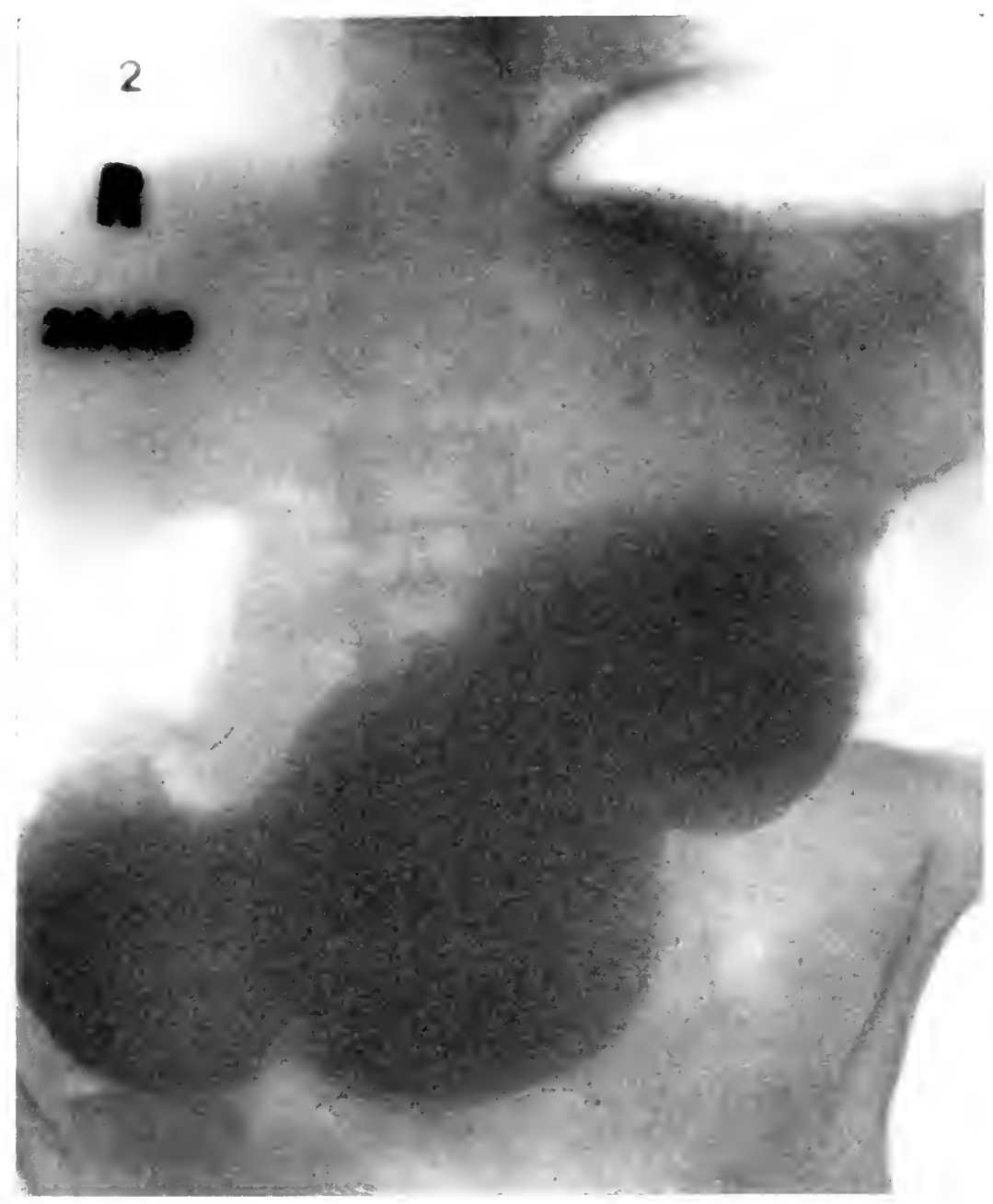

Fic. 75.-PYloric obstruction; malignant ulcer (?). 


\section{DESCRIPTION OF FIG. 75}

Mr. G. H. S.-Age 37-American.

Comes on account of chronic constipation and chronic intermittent dyspepsia.

Previous History.-Typhoid ferer when a routh.

Duration of Present Complaint.-For past 12 years has had attacks of painful dyspepsia, lasting for several weeks and coming on usually in the fall. These attacks have been characterized by soreness or gnawing pains in the pit of the stomach, whieh distress usually came on 1 to 2 hours after meals and at night. The distress would last until food was ingested or romiting occurred. Alkalies and limited diet have been frequently required. Pain was generally referred to the back, between the shoulders. Fomiting occurred irregularly and was most common in the evening. Belching and pyrosis have been annoying. Dyspepsia constant and more marked past 6 weeks.

Appetite.-Good desire.

Bocels. - Very constipated for 10 years. During the past 3 months this has been aggravated.

Bleeding.-Two weeks ago severe hematemesis.

Weight.-Lost 10 pounds during past 2 months.

Abdominal Examination. - Tery tender in right upper quadrant. Stomach splashy.

Test-meal.-Narked 12-hour retention; $300 \mathrm{cc}$. recovered; extracts are tan-brown and with yeasty odor. Total acidity, 4S; free hydrochlorie acid, 46 ; altered blood, 0 (henzidin test).

Microscopic Examination.-Many food rests. Yeasts and sarcine in excess.

Clinical Diagnosis.-Prloric obstruction; duodenal ulcer.

X-ray Diagnosis.-Pyloric obstruction; probable duodenal uleer.

Plate shows large "steer-horn" type stomach with hyperperistalsis and irregular filling out of pytorus; duodenum is but faintly visualized.

Surgical Findings.-Chronic "saddle-ulcer" on lesser curvature at pylorus.

Without microscopic examination it was impossible to tell whether ulcer was malignant or benign. 


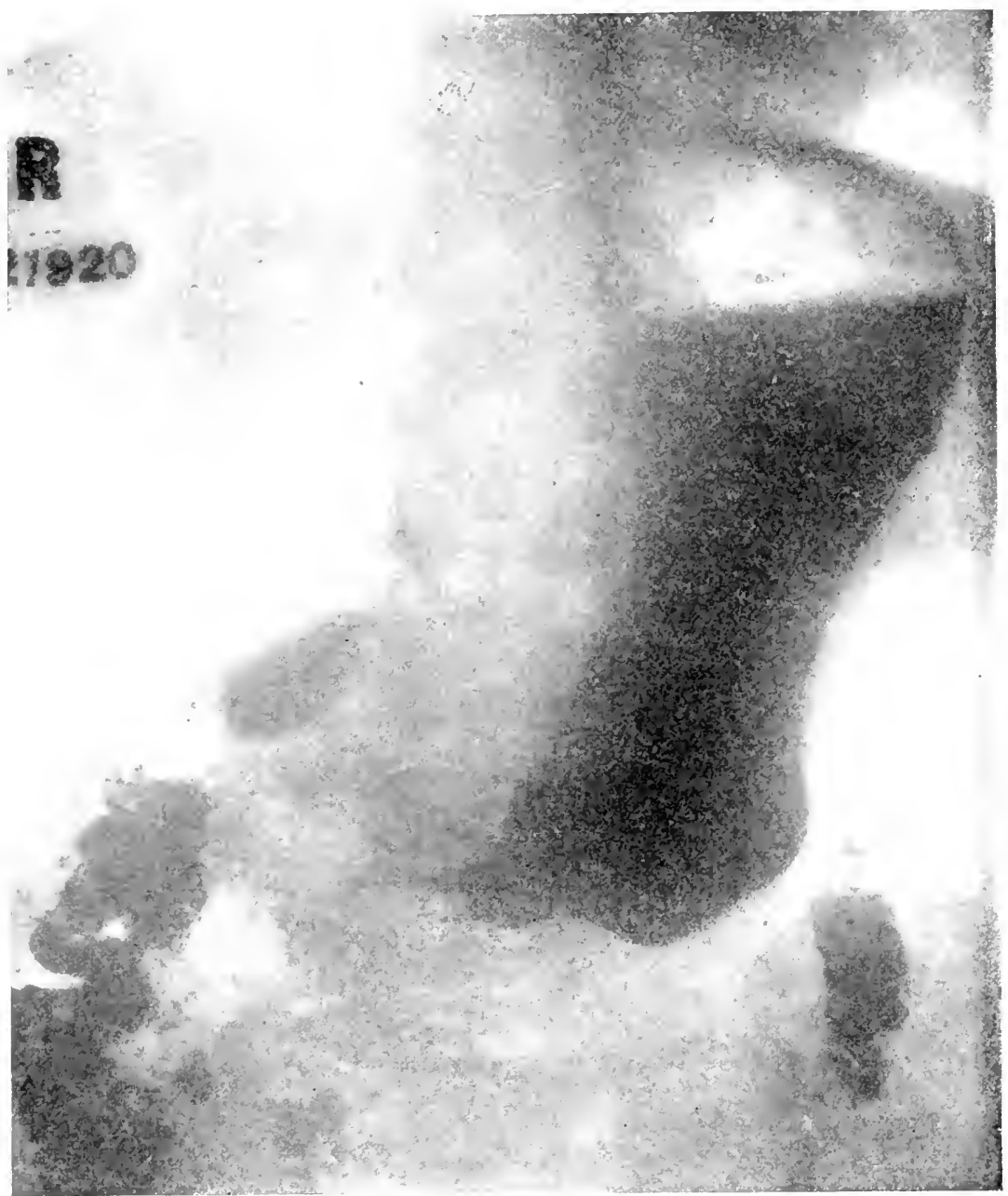

FIG. 76.-Achlorhydria: Nervous patient; irregular ulcer history with recent exacerbation; pyloric cancer. 


\section{DESCRIPTION OF FIG. 76}

Mr. P. F. C.-Age 45-Manual laborer.

Previous History.-Typhoid fever as a youth.

Comes on account of abdominal pain, dyspepsia, nervousness, recent weight and strength loss.

Duration of Disease.-Intermittent dyspepsia for past 5 years. The present attack has persisted for 3 weeks.

The disturbance is characterized by distress across the mid-epigastrium and by belching and water-brash. These symptoms are moderately constant, but more pronounced when the stomach is empty, or immediately after eating. Distress is often transmitted to the precordia. It is relieved, partially, by food intake, catharsis or rest. Belching and water-brash come on usually soon after eating. Alkalies relieve the distress occasioned thereby.

Mentality.-Below par, patient highly psychic and excitable.

Appetite.-Poor.

Weight.-Lost 11 pounds past 3 weeks and 29 in past year.

Test-meal.- No 12-hour food retention; gastric contents cream tan in color. Total acidity, 10 ; free hydrochloric acid, 0 ; altered blood, 0 (benzidin test); lactic acid, 0; glycyltryptophan test + .

Microscopic Examination.-Numerous small lymphocytes and cocci in short stains and small groups.

Stool.-Altered blood + .

Clinical Diagnosis. - Gastric achlorhydria (carcinoma ?); psychasthenia.

$X$-ray Diagnosis. - Carcinoma of pyloric end of stomach.

Plate demonstrates "steer-horn" type of stomach, with extensive filling defect of the antrum and pylorus. Tumor is irregularly canalized. Pylorus is patent. Bulbus duodeni is deformed. Motor meal rests in ascending, transverse and descending portion of the colon.

Surgical Diagnosis. - Cancer of pyloric end of stomach. 


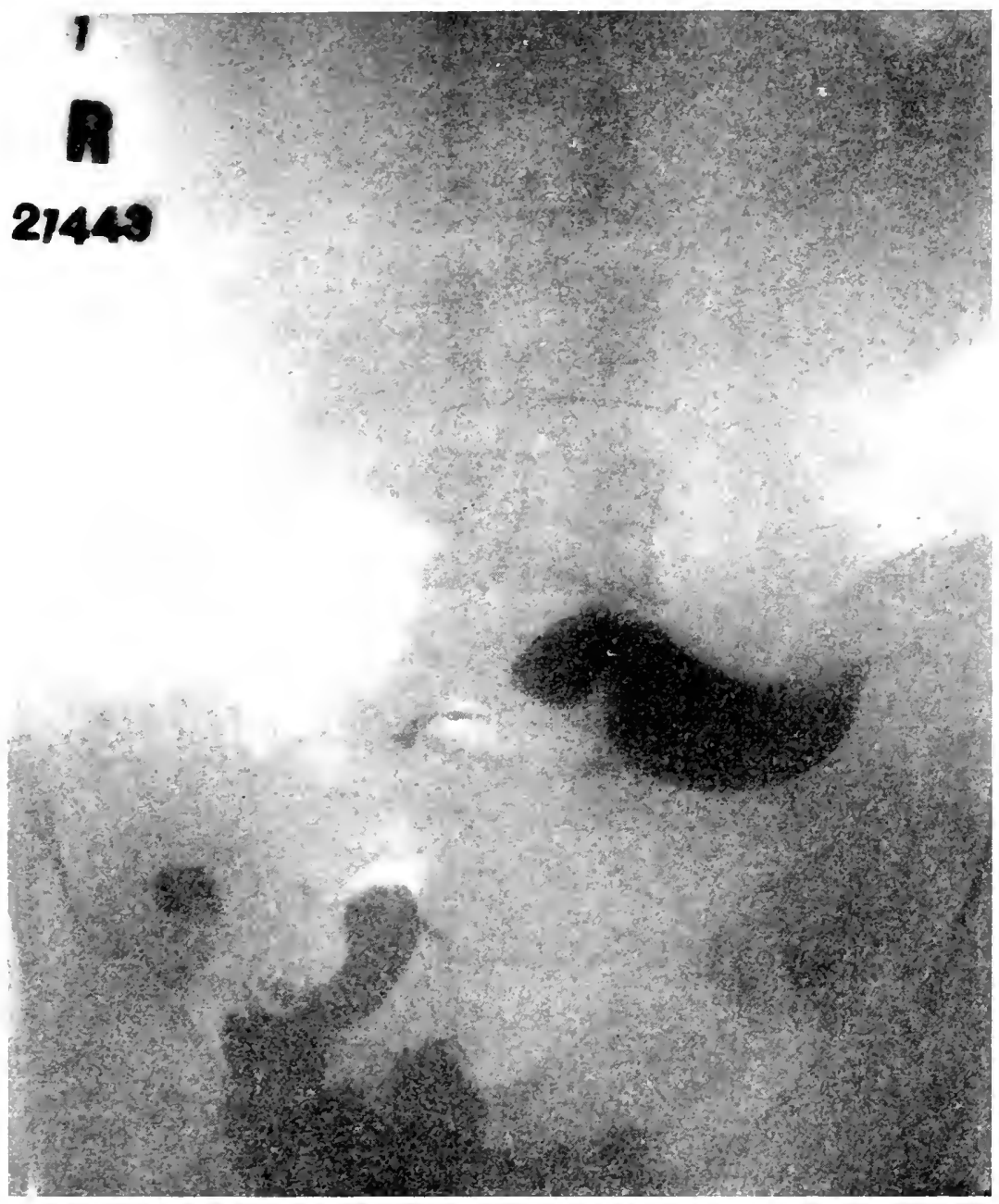

Fig. 77.-Pyloric ulcer; motor meal residue-perforating ulcer. 


\section{DESCRIPTION OF FIG. it}

Mr. P. W.-Age 48-Irish.

Family History.-Degative.

Comes on account of chronic intermittent dyspepsia. Duration of disease 20 rears in spells or attacks. These attacks hare been characterized by marked epigastric pain coming on 1 to 12 hour after meals or at night. Pain is usually very sharp but sometimes a gnawing and burning sensation. Csually lasts several hours and is transmitted to the back between the shoulder blades. Pain is relieved by food intake, romiting and alkalies. Has several tines required morphine. During the last 3 weeks the distress has been constant and romiting has been an annoring srmptom. Vomiting usually oecurs about $1 \frac{1}{2}$ hours after meals and seems to be brought on by abdominal pain. The romitus is sour water and sometimes food. There has been no delayed romiting. Causea has been marked especially in the evening. Eructations, water-brash and pyrosis are particularly distressing at night. Ther are relieved by alkalies.

A ppetite.-Poor.

Bowels.-Constipated; never any bleeding.

Theight Loss. - Eighteen pounds in 6 months.

Clinical Diagnosis.-Partial prloric obstruction, due to peptic uleer (malignaney questionable), moderate dilatation of the stomach.

I-ray Findings.-Molerate to-hour retention (Rieder meal): peristalsis active, incomplete visualization of prlorus and duodenum: tenderness orer region of pylorus, possible ulcer of the stomach or duodenum.

Surgical Findings.-Chronic perforating ulcer of the duodenum to liver and chronic appendieitis. 


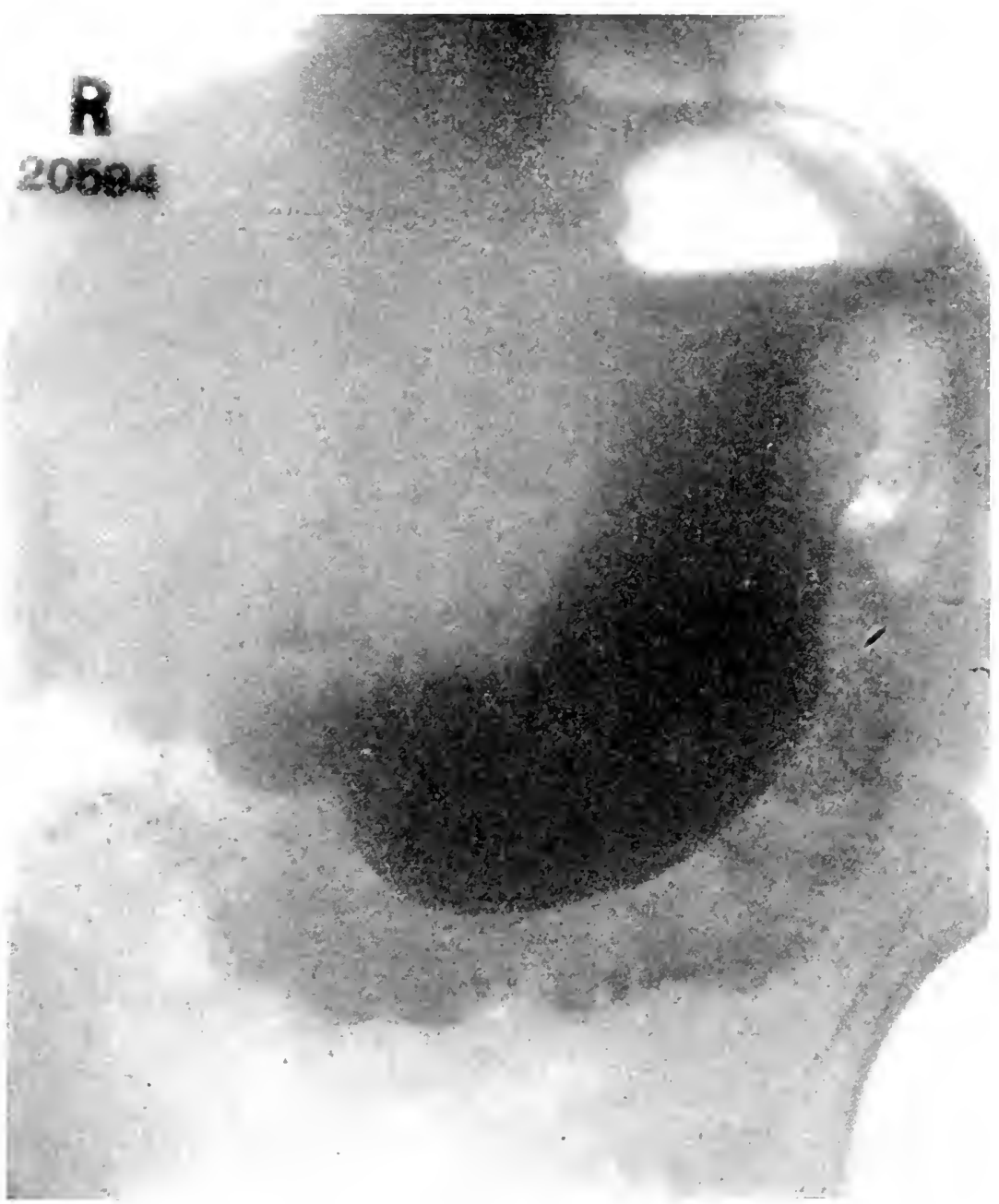

Fig. 78.-(Case No. 20,594).-Chronic, recurrent ulcer (malignant ?) at pylorus with partial obstruction. 
Fig. 79.-(Case No. 22,157).-Pyloric obstruction. Plate shows dilated stomach with extensive neoplastic involvement of antrum and pylorus; pylorus partly patent; fair gastric peristalsis. 


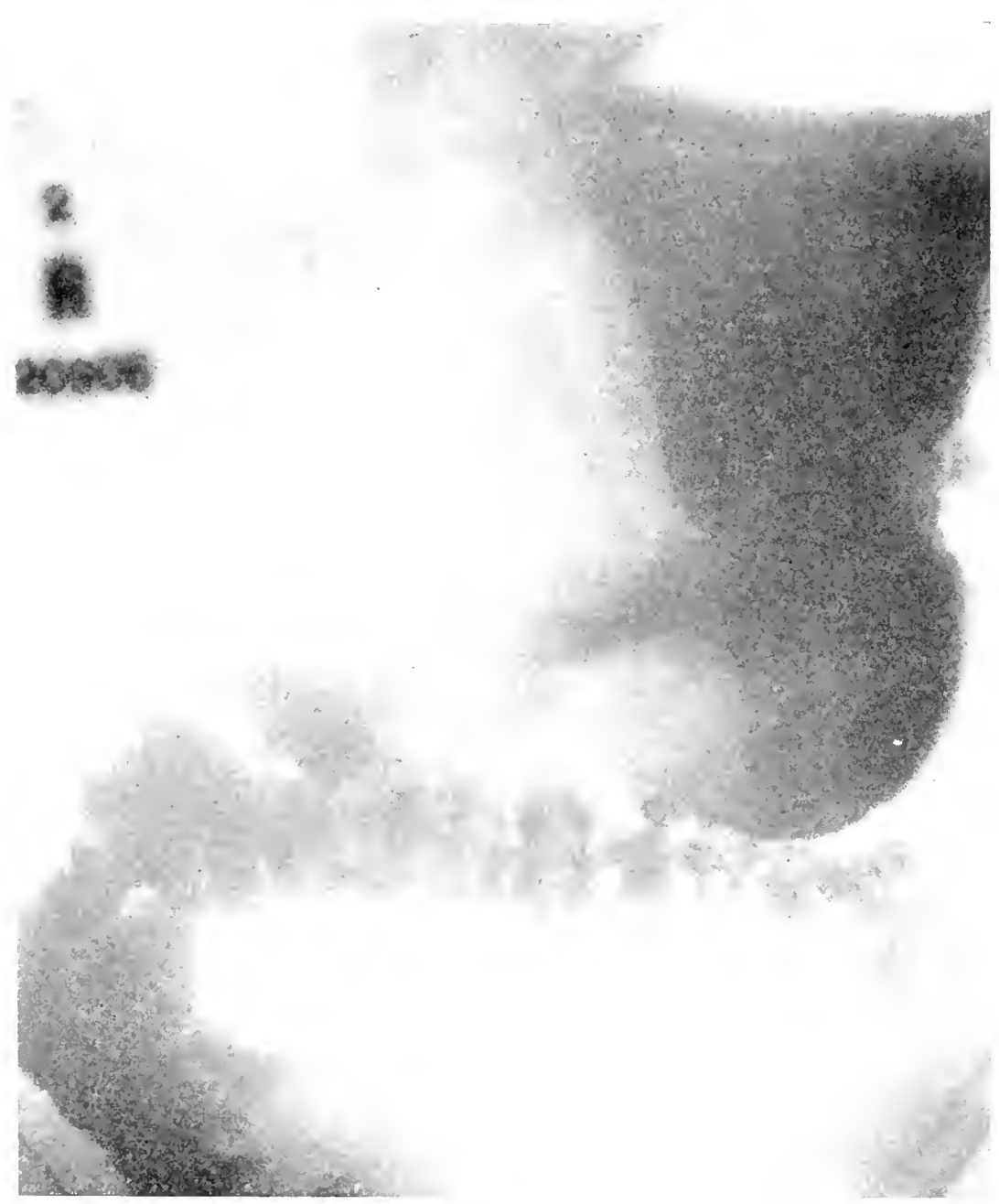

FIG. S0.

Case No. 20,90.5-Male-Age 41.

Involvement of pyloric third of stomach by cancer following history clinically that of ulcer. Rests of motor-meal seen below stomach in colon. 
ROENTGEN EXAMINATION IN GASTRIC CANCER 313

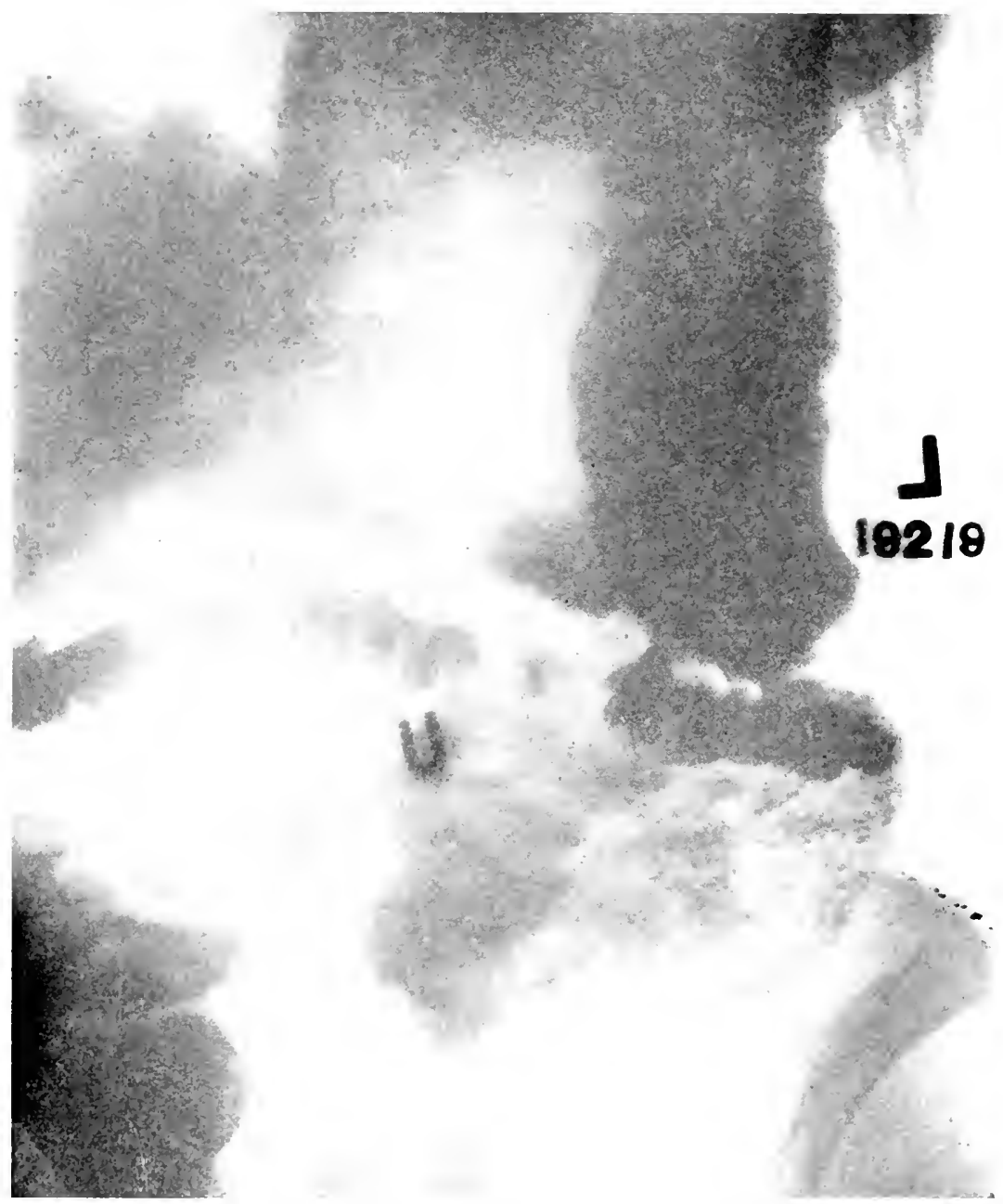

Fig, s1.- (Case No. 19,219).-Cancer of pars pylorica following history clinically that of ulcer; partial prloric obstruction to physiologic motormeal. Dilated small bowel partly filled with bismuth, suggesting an obstruction distally. 


\section{${ }_{\text {mino }}^{\mathrm{R}}$}

Fig. S2.-Pyloric obstruction, malignant.

Case No. 23,652-Male-Age 48.

Previous History. - Clinically that of chronic, recurrent peptic ulcer. Two previous operations demonstrated such.

Abdominal Examination. - Indefinite ridge in mid-epigastrium.

Test-meal.-Slight 12-hour food retention. Gastric extracts cream-tan color. Poorly chymified. Total acidity, 10; free hydrochloric acid, 0; altered blood, ? (benzidin test); lactic acid +; Wolff-Junghans' + test.

Microscopic Examination. - Numerous bacilli of Oppler-Boas type; few budding yeasts.

Clinical Diagnosis.-Carcinoma following ulcer; questionably operable. X-ray Diagnosis. - Carcinoma of stomach.

Plate shows tumor mass involving pyloric third, with irregular canalization of mass, but gaping pylorus, permitting rapid exit of bismuth into duodenum and snall bowel (which are visualized). Lesser curvature is also infiltrated;

Surgical Diagnosis.-Gastric cancer. 


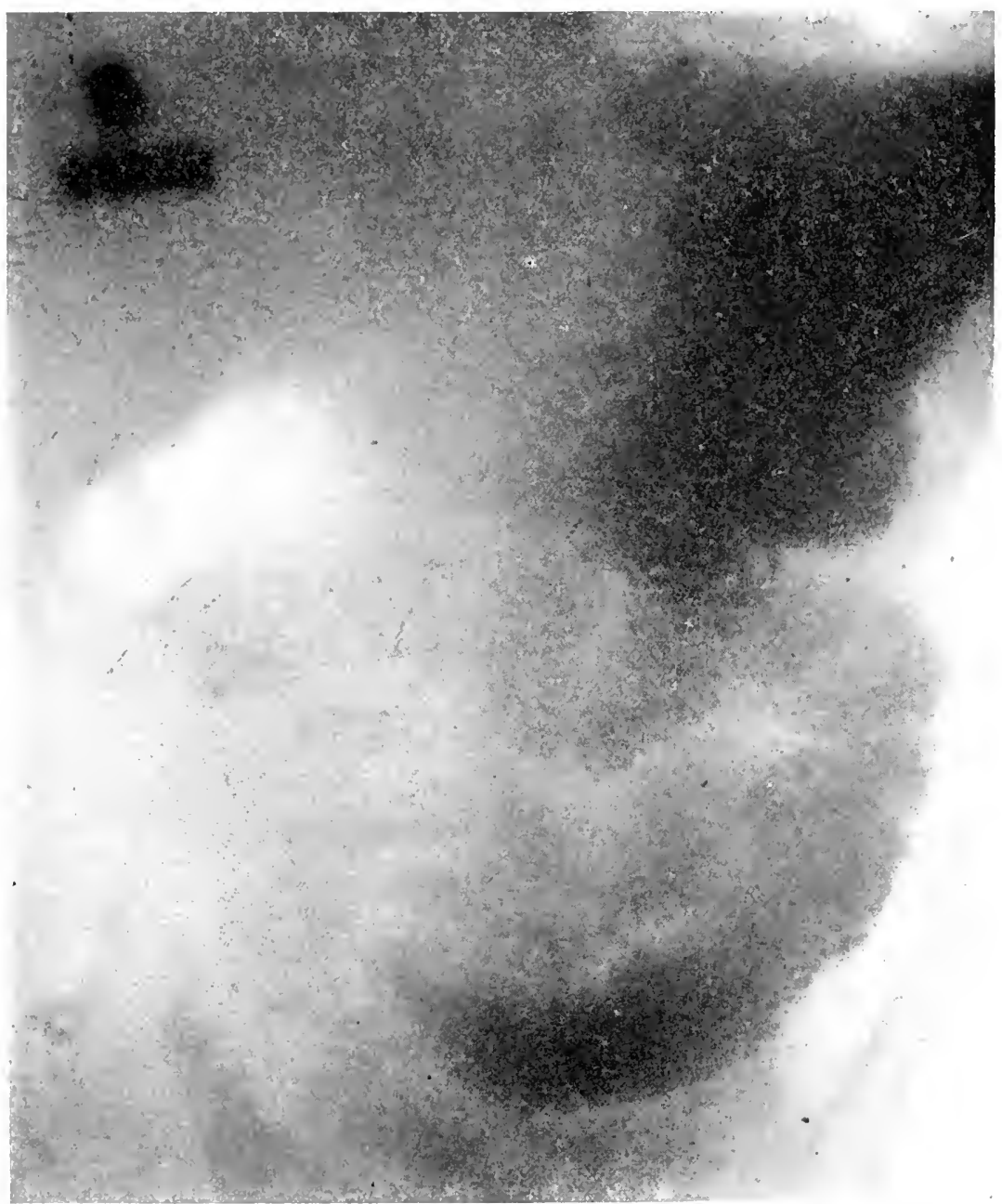

FIG. 83.-(Case No. 20,730).- Recurring gastric cancer, with partially patent gastro-enterostomy stoma. 


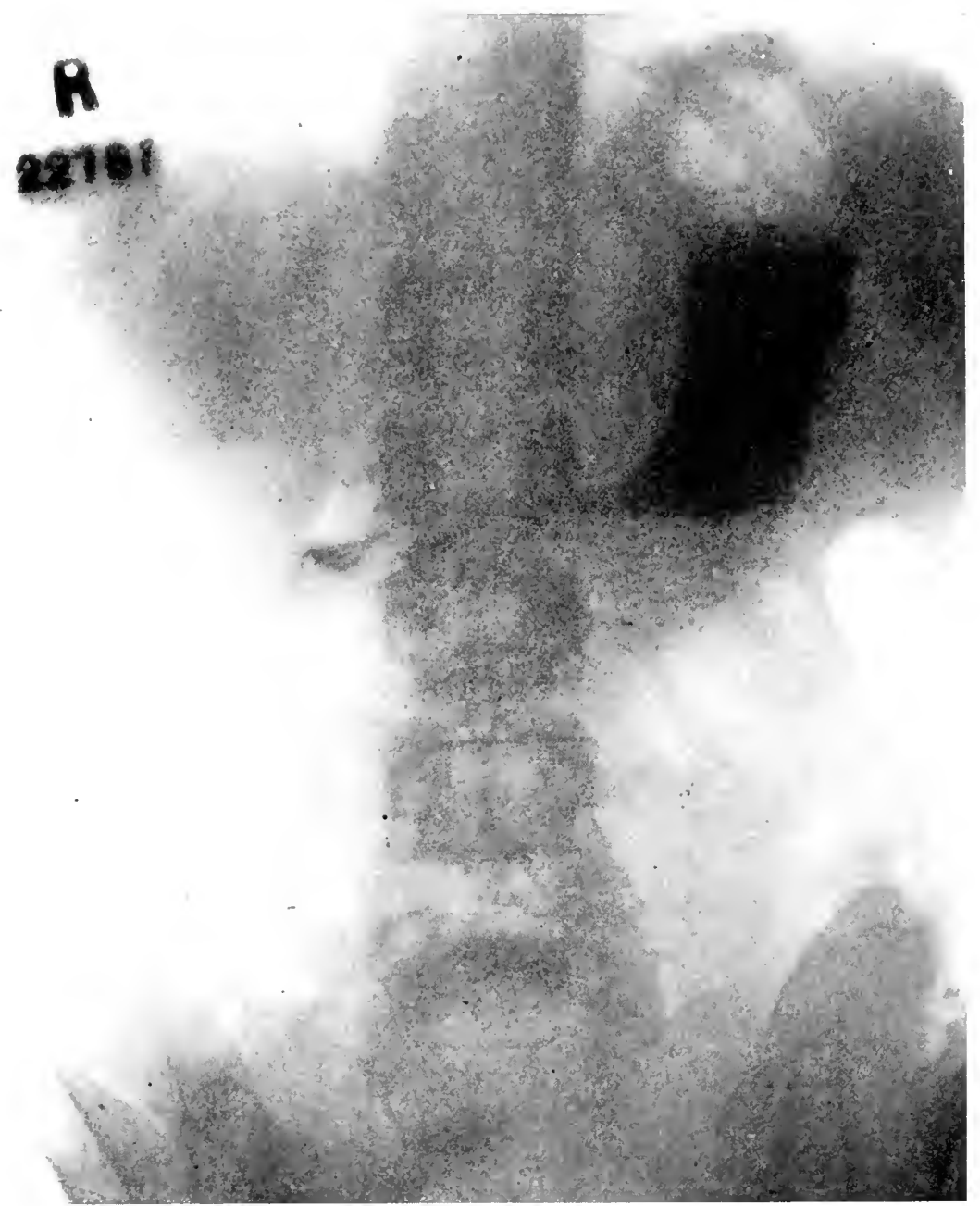

FIg. S4.-Pyloric obstruction; previous irregular peptic uleer history; irremovable gastric tumor; srphilis. 


\section{DESCRIPTION OF FIG 84}

Mr. M. S.-Age 40-German-Merehant.

Previous History.-Genital sore in youth. Abdominal section (elsewhere)

1 year ago; gastric ulcer?.

Comes on account of chronic indigestion, weight and strength loss, anemia.

Duration of Disease.-For past 12 years has had attacks of indigestion. These were characterized by epigastric distress several hours after meals, belching, pyrosis and loss of appetite. Distress and associated symptoms were relieved by foud, alkalies or diet. Eighteen months ago the symptoms became so annoving that patient was unable to work. A year ago had laparotomy and thinks ulcer and adhesions were found. Did fairly well up to 3 months ago. Since then has had constant epigastric pain, worse immediately after meals and at night, and only partially relieved by rest and limited diet. Eructations and prosis have been annoying.

Appetite.-Poor.

Bowels.-Unexplainable diarrhea for past 4 days. Never bled.

Weight.-Lost 11 pounds last 2 nionths.

Abdominal Examination.-Healed laparotomy scar. In mid-epigastrium to right of scar and adherent to it is an oval, fixed, tender nodule.

Test-meal-Moderate 12-hour retention. Gastric extract cream color, with sour odor. Total acidity, 14; free hydrochloric acid, 0 ; altered bloodt (benzidin test); Wolff-Junghans' test+; formol index, 20; lactic acid, 0.

Microscopic Examination.-Numerous bacilli of the Oppler-Boas type, buckding yeasts and small form sarcinse, few streptococci.

Clinical Diagnosis.-Pyloric obstruction; partial hour-glass; achlorhydria ; gastric cancer on old ulcer. Syphilis? Inoperable.

I-ray Diagnosis.-Probable carcinoma or syphilis of the stomach.

Plate shows small modified "steer-horn" type of stomach. The lumen of the pyloric half is encroached upon by a mass so extensively as to almost obliterate it; nodular infiltration is denoted by the irregular outline of the curvatures, particularly the greater; obstruction is shown by failure to visualize the duodenum or parts of the bowel distal to it.

Surgical Diagnosis.-Irremovable gastric tunor. 
the majority of cases, clinical diagnosis was quite possible without the added $x$-ray examination; that in clinically doubtful cases, the Roentgenographic diagnosis was similarly dubious. The plates form, however, an interesting accessory record to the histories, and aid in selection of cases likely to be benefited by surgical procedures, by indicating, roughly, the location of the growths and the extent that they invade the gastric wall or involve other viscera. 


\section{CHAPTER VII}

\section{THE BLOOD IN GASTRIC CANCER}

The terms "malignancy" and "cachexia" have become so closely associated as result of years of clinical investigation, that the mention of one suggests the other. Of the numerous evidences of cachexia with neoplastic foundation, anemia is one most commonly recognized by both layman and physician. Certain gross manifestations of the cancerous anemia are often appreciated upon even casual examination.

The skin is usually dry, loose, scaly, muddy, grayishwhite or of fawn or lemon-yellow tint. Were the early decrease in the amount of subcutaneous fat not of frequent occurrence, the skin-tint might readily be mistaken for that observed in such constitutional fault associated with that clinical picture which we call pernicious anemia. The sclera are commonly pearly white, but may be discolored or faintly yellow. Mucous surfaces exhibit varying degrees of pallor. They are generally dry and often shiny. Those of the lips and tongue may be fissured or herpetic. The tongue is frequently pale, dry, roughened and coated. Capillary edema accumulates about the malleoli. Sometimes puffiness beneath the eyes indicates a hematopoietic dyscrasia or kidney malfunction.

It should be emphasized here, however, that the above popularly accepted indications of the anemia in malignant disease only appear when the neoplasm is well advanced. Latent or early cancer may exist a considerable time before readily recognized systemic damage results. This is especially the case in carcinoma of the stomach. Well 
confined ulcera careinomatosa or incipient, primary cancerous change in the gastric lining may produce few external signs indicative of their presence. Moreover, in such erent, eren most carefully performed blood analyses may fail to demonstrate any suspicious cellular or serologic anomaly.

The causes of anemia in gastric cancer appear to be multiple. It is only an exhibition of our lack of exact knowledge which permits the statement that the growth of a neoplasm exerts deleterious influences upon normal metabolism. When physiologic and biologic chemists have discovered what factors are concerned in normal metabolism, it may then be possible to estimate the precise, constitutional significance of what are now considered by-products of a malignant tumor's cellular activity. That foreign substances emanate from cancer tissue seems quite probable. The researches of Gay, Ascoli, Abderhalden, Weil, Jobling, IV. Hamburger and others appear to demonstrate that in the derelopment of a neoplasm some ferment-like body or bodies are manufactured or increased locally. These may exert pernicious effects by stimulating cell proliferation, by overflowing into the blood or the lymph streams, and thus acting as potential, if not actual, foreign substances, or, by acting, locally or generally upon already damaged or biologically immature tissue, they may liberate end-digestion products, which affect the body economy either by their presence in excessive amounts or by their entrance into the body fluids at a non-physiologic point. While there is no direct evidence that products of malignant growths directly injure the blood-forming centers, there is considerable proof that when cancer has become well established, the products of the hematopoietic organs are of inferior quality, and not rarely, of diminished quantity. 
Other factors likely to be of importance in the causation of anemia in gastric cancer are secondary infection, starvation, exhaustion (from vomiting, diarrhea or prolonged pain), hemorrhage, certain psychic abnormalities, or deficient function of the kidneys, liver or the pancreas. The development of extensive metastases sometimes exerts pernicious effects upon the blood picture.

Blood changes observed in gastric cancer resolve themselves into a consideration of the deviations from the normal of (1) formed elements (erythrocytes, leucocytes) and (2) the blood plasma.

\section{(1.) DEVIATIONS FROM THE NORMAL OF THE FORMED BLOOD CONSTITUENTS}

Quantitative Changes in Erythrocytes.-The average red blood cell count of 129 cases of gastric cancer examined by Cabot is given as 4,018,000 cells. In more than 75 per cent. of these cases the diagnosis was confirmed by operation or by autopsy. The cases remaining were clinically cancer of the stomach.

The average red count from 59 cases studied by Osler and McCrae was $3,712,186$. Their cases were clinically, or at post-mortem, cancerous.

In our series, there were 267 instances of the disease where accurate red cell counts were made. Table 26 shows the summary of these investigations.

It will be noted that the average erythrocyte count was $4,380,000$. This is a figure rather higher than the average given by other investigators. It is to be explained partly on the basis that many of our cases were diagnosed very early (often at laparotomy and then, microscopically) or the large number of patients returns a fairer average than does a relatively small list. The minimum erythrocyte 


\begin{tabular}{|c|c|c|}
\hline Red cell count & $\begin{array}{c}\text { Number of } \\
\text { cases }\end{array}$ & Per cent. \\
\hline Below $1,000,000 \ldots \ldots \ldots \ldots \ldots \ldots \ldots$ & 2 & 0.75 \\
\hline From $1,000,000$ to $2,000,000 \ldots \ldots \ldots \ldots$ & 3 & 1.1 \\
\hline From $2,000,000$ to $3,000,000 \ldots \ldots \ldots \ldots \ldots$ & 17 & 6.3 \\
\hline From $3,000,000$ to $4,000,000 \ldots \ldots \ldots \ldots$ & 51 & 19.2 \\
\hline From $4,000,000$ to $5,000,000 \ldots \ldots \ldots \ldots$ & 132 & 49.3 \\
\hline From $5,000,000$ to $6,000,000 \ldots \ldots \ldots \ldots \ldots$ & 59 & 22.0 \\
\hline From $6,000,000$ to $7,000,000 \ldots \ldots \ldots \ldots$ & 3 & 1.1 \\
\hline Average $4,380,000$ for $\ldots \ldots \ldots \ldots \ldots$ & 267 & 99.7 \\
\hline
\end{tabular}

Showing Variations in Erythrocyte Count in 267 Cases of Gastric Cancer (Author).

count was $\$ 60,000$ cells. This was returned in a case of inoperable tumor with ascites and extensive general metastases. The maximum red cell count was $6,328,000$ cells. This was from a patient with inoperable cancer of the lesser curvature and the body of the stomach. There were metastases to the rectal shelf and the peritoneum. It is thus seen that while low erythrocyte counts may be returned in instances where there are hopeless gastric neoplasms, high counts may likewise be obtained late in the progress of the disease.

In more than 91 per cent: of our cases the red cell count lay between $3,000,000$ and $7,000,000$ cells. In nearly 50 per cent. the counts fell between $4,000,000$ and 5,000,000 cells.

The Influence of Metastases.--Our experience is summarized in Tables 27 and 28.

Study of these two tables demonstrates that of the 267 cases of gastric cancer in which erythrocyte counts are available, in 79.9 per cent. there were gross or microscopic metastases; of those instances in which metastases were present the red cell count was included between limits of from $3,000,000$ to $6,000,000$ cells in 90.4 per cent. where 
Table 27

\begin{tabular}{|c|c|c|c|c|}
\hline $\begin{array}{l}\text { Range of erythrocyte } \\
\text { counts }\end{array}$ & $\begin{array}{c}\text { Metastases } \\
\text { (number of } \\
\text { cases) } \\
+\end{array}$ & $\begin{array}{c}\text { Metastases } \\
\text { (number of } \\
\text { cases) } \\
++\end{array}$ & $\begin{array}{l}\text { Metastases } \\
\text { (number of } \\
\text { cases) } \\
+++\end{array}$ & $\begin{array}{l}\text { Free Fluid } \\
\text { (number of } \\
\text { cases) }\end{array}$ \\
\hline Below $1,000,000 \ldots \ldots$ & $\cdots$ & & & 2 \\
\hline $1,000,000$ to $2,000,000$. & 3 & & . & $\ldots \ldots \ldots$ \\
\hline $2,000,000$ to $3,000,000$. & 6 & $\ldots \ldots \ldots$ & 2 & \\
\hline $3,000,000$ to $4,000,000$. & 23 & 9 & 1 & . \\
\hline $4,000,000$ to $5,000,000$. & 45 & 34 & 20 & 5 \\
\hline $5,000,000$ to $6,000,000$ & 17 & 17 & 16 & 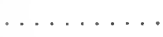 \\
\hline $6,000,000$ to $7,000,000$ & & . & 3 & \\
\hline Summaries. & 94 & 60 & 42 & 7 \\
\hline
\end{tabular}

To Demonstrate Relation of Erythrocyte Count to Metastasis in Gastric Cancer (Author).

Table $2 \mathrm{~s}$

\begin{tabular}{|c|c|c|}
\hline Range of erythrocyte counts & $\begin{array}{c}\text { Number of } \\
\text { cases }\end{array}$ & Per cent. \\
\hline \multirow{2}{*}{\multicolumn{3}{|c|}{$\begin{array}{l}\text { Below } 1,000,000 \ldots \ldots \\
1,000,000 \text { to } 2,000,000\end{array}$}} \\
\hline & & \\
\hline $\begin{array}{l}2,000,000 \text { to } 3,000,000 \ldots \ldots \ldots \ldots \ldots \ldots \ldots \ldots \ldots \ldots \ldots \ldots \ldots \ldots \ldots \ldots \\
3,000,000 \text { to } 4,000,000 \ldots \ldots \ldots \ldots \ldots \ldots \ldots\end{array}$ & $\begin{array}{r}9 \\
18\end{array}$ & $\begin{array}{l}13.9 \\
28.1\end{array}$ \\
\hline $\begin{array}{l}3,000,000 \text { to } 4,000,000 \ldots \ldots \ldots \ldots \ldots \ldots \ldots \ldots \ldots \ldots \ldots \\
4,000,000 \text { to } 5,000,000 \ldots \ldots \ldots \ldots\end{array}$ & 18 & 28.1 \\
\hline $4,000,000$ to $5,000,000 \ldots \ldots \ldots \ldots \ldots \ldots \ldots$ & $\begin{array}{r}38 \\
0\end{array}$ & 43.1 \\
\hline $5,000,000$ to $6,000,000 \ldots \ldots \ldots \ldots \ldots \ldots \ldots$ & 9 & 13.9 \\
\hline \multicolumn{3}{|l|}{$6,000,000$ to $7,000,000 \ldots$} \\
\hline Summary. & $6+$ & 99.0 \\
\hline
\end{tabular}

Demonstrating Erythrocyte Counts in Cases where no Mletastases Existed (Author).

metastases were limited in extent; in 100 per cent. where metastases were moderate in degree; in 88 per cent. where metastasis to lymph glands was very extensive, and in 71 per cent. where free abdominal fluid occurred with or without other evidences of metastasis. The average percentage of cases showing metastasis where the red cell count lay between $3,000,000$ and $6,000,000$ cells was 87.3 .

In the 64 cases in which no metastases could be demonstrated, in 85.1 per cent. the erythrocyte count lay between $3,000,000$ and $6,000,000$ cells. 
It would seem from the above analysis of 267 proved cases of gastric cancer, that diminution in red cell count depends not wholly upon the development of metastases. On the contrary, a large group of cases with extensive metastases have higher average erythrocyte counts than have those free from metastases.

Quantitative Changes in Hemoglobin.-Osler and McCrac (loc. cit.) record hemoglobin estimations in 52 instances of cancer of the stomach. These cases returned an average hemoglobin of 49.9 per cent. and an average color index of 0.63 (Cabot).

We have records of hemoglobin estimations (Dare method for the majority) upon 454 cases. Table 29 shows the variations in the readings.

Table 29

\begin{tabular}{|c|c|c|}
\hline Hemoglobin reading, per cent. & Number of cases & Per cent. \\
\hline \multicolumn{3}{|l|}{ Below 20.} \\
\hline Between $20-30 \ldots \ldots \ldots \ldots \ldots \ldots \ldots \ldots$ & 9 & 1.5 \\
\hline Between $30-40 \ldots \ldots \ldots \ldots \ldots \ldots \ldots \ldots$ & 31 & 6.8 \\
\hline Between $40-50 \ldots \ldots \ldots \ldots \ldots \ldots \ldots \ldots$ & 76 & 16.7 \\
\hline Between $50-60 \ldots \ldots \ldots \ldots \ldots \ldots \ldots \ldots$ & 64 & 14.6 \\
\hline Between $60-70 \ldots \ldots \ldots \ldots \ldots \ldots \ldots \ldots$ & $\$ 2$ & 18.1 \\
\hline Between $70-80 \ldots \ldots \ldots \ldots \ldots \ldots \ldots \ldots \ldots \ldots$ & 77 & 16.9 \\
\hline Between $80-90 \ldots \ldots \ldots \ldots \ldots \ldots \ldots \ldots \ldots$ & 106 & 23.4 \\
\hline Between $90-100 \ldots \ldots \ldots \ldots \ldots \ldots \ldots \ldots$ & 9 & 1.9 \\
\hline Total. & 454 & 99.9 \\
\hline
\end{tabular}

Showing the Variation of Hemoglobin Reading in 454 Cases of Gastric Cancer (Author).

It will be noted that in 72.2 per cent. of the patients, the hemoglobin ranged between 50 per cent. and 100 per cent., that in 42.2 per cent. it was above 70 per cent. and more than 1 out of every 3 cases (38.5 per cent.) had a hemoglobin reading between 50 per cent. and 80 per cent. The average hemoglobin percentage for the series was 64.3 per cent. 
The minimum was 25 per cent. and the maximum 95 per cent.

The Effect of Metastases.-In this respect we have studied hemoglobin records in a manner similar to that above described when considering the erythrocyte counts. Tables 30 and 31 demonstrate the rariations in hemoglobin when metastases were present and when they were absent.

Table 30

\begin{tabular}{|c|c|c|c|c|}
\hline Hemoglobin percentage & $\begin{array}{c}\text { Metastases } \\
+\end{array}$ & $\begin{array}{c}\text { Metastases } \\
++\end{array}$ & $\begin{array}{c}\text { Metastases } \\
+++\end{array}$ & Free fluid \\
\hline $10-20$ & & & & $\cdots$ \\
\hline $20-30$ & $\ldots \ldots \ldots$ & 5 & 3 & 1 \\
\hline $30-40$ & 12 & 5 & 8 & 1 \\
\hline $40-50$ & 20 & 26 & 6 & 0 \\
\hline $50-60$ & 24 & 12 & 11 & 4 \\
\hline $60-70$ & 43 & 15 & 11 & 0 \\
\hline $70-80$ & 13 & 14 & 18 & 2 \\
\hline $80-90$ & 46 & 32 & 12 & 1 \\
\hline $90-100$ & 5 & 2 & ...... & $\ldots$ \\
\hline Summary....... & 163 & 111 & 69 & 9 \\
\hline
\end{tabular}

Demonstrating Variations in Hemoglobin in Gastric Cancer Percentage when Metastases are Present (Author).

\begin{tabular}{|c|c|}
\hline \multicolumn{2}{|c|}{ Table 31} \\
\hline Htemoglobin percentage & Number of cases \\
\hline $10-20$ & \\
\hline $20-30$ & \\
\hline $30-40$ & 5 \\
\hline $40-50$ & $2 t$ \\
\hline $50-60$ & 13 \\
\hline $60-70$ & 13 \\
\hline $70-80$ & 30 \\
\hline so-90 & 15 \\
\hline $90-100$ & 2 \\
\hline Total...... & 102 \\
\hline
\end{tabular}

Demonstrating Variations in the Hem oglobin in Gastric Cancer when Metastases are Abșent (Author). 
Of the cases in which metastases were proven, hemoglobin was above 50 per cent. in 131 cases or 80.4 per cent. of those where there was slight lymph-gland invasion; in 75 cases or 67.5 per cent. where there were moderate metastases; in 52 cases or 75.3 per cent. where there was extensive lymphatic involvement, and in 7 cases or 77.7 per cent. where there was ascitic fluid. In other words, of 352 cases ( 77.5 per cent.) showing metastases the hemoglobin was 50 per cent. or higher in 75.2 per cent.

There were 102 cases (22.5 per cent.) where there were no metastases. In this group hemoglobin was 50 per cent. or higher in 73 instances or 71.5 per cent.

It would seem from the above analysis that low hemoglobin percentages have relatively little value toward indicating the presence or absence of metastases.

Color-index.--In the blood examinations of 267 consecutive cases the average color index was 0.73 . The minimum index was 0.32 and the maximum 0.97 .

Shape and Size of Erythrocytes.-In well-advanced gastric cancer, when the hemoglobin is below 75 per cent., it is not uncommon to find red blood cells that exhibit wide variations in shape and, occasionally, in size. The studies of Osler and McCrae and Cabot (loc. cit.) seem to show that in malignant disease macrocytes are unusual findings and that the cancer anemia is of the type common to chlorosis. While this holds generally true, there are interesting exceptions. Our records detail 17 blood studies where macrocytes were found and eight instances in which megaloblasts are recorded. In 23 cases normoblasts were noted. We never observed macrocytes or nucleated erythrocytes where the hemoglobin was higher than 70 per cent. or the red cell count above $3,500,000$.

Of 204 blood analyses where definite record of red-cell 
variations in shape and size were recorded, in 82 instances (40.3 per cent.) the variation was noted as being "slight," in 92 cases ( 45.09 per cent.) as of "moderate degree," and in 30 instances ( 14.7 per cent.) as "marked."

Poikilocytosis, blood "shadows," or degenerative forms are quite common in the blood smears from well-established gastric cancer. From the blood of early cases they may be wholly absent. Not infrequently, these variations in erythrocyte structure seem out of all reasonable proportion to the red cell count, or the hemoglobin. We have not been able to establish any definite cause for this phenomenon. "Cancerous poisoning" of the blood-forming centers does not furnish a satisfactory explanation. In view of work done upon isolysins in malignant disease, it might be suggested that the rate of hemolysis was accelerated in gastric cancer.

\section{CHANGES IN THE LEUCOCYTES}

Quantitative.-Summarizing leucocyte counts in 23 cases of gastric cancer, DaCosta (quoted by Cabot) found an average count of 8,100 cells. The minimum was 1,000 cells and the maximum 14,000 cells. Cunliffe's records of 10 cases of the disease reveal an average white cell count of 17,280 , with a minimum of 5,200 cells and a maximum of 36,800 cells. Cabot (loc. cit.) analyzed the leucocytes in 235 cases of cancer of the stomach. His study shows an average count of 10,600 cells. The minimum count was between 3,000 and 4,000 cells and the maximum between 30,000 and 40,000 cells.

A review of the literature demonstrates that high leucocyte counts are not uncommon in rapidly growing gastric neoplasms. The ratio between white and red cells may be greatly reduced. Cabot reports a case of Welch's in which 
the ratio of white to red cells was as 1:25 instead of the normal ratio of as $1: 750$.

Of our series of gastric cancers, we have leucocyte counts in 261 instances. The arerage count was 11,270 cells. The minimum was 4,200 cells and the maximum 36,200 cells. Table 32 groups the cases according to cell-count range.

Table 32

\begin{tabular}{|c|c|c|}
\hline Leucocytes & Number of cases & Percentage \\
\hline Between $\quad 4,000-5,000 \ldots \ldots \ldots \ldots \ldots$ & 15 & 5.7 \\
\hline Between $5,000-6,000 \ldots \ldots \ldots \ldots \ldots$ & 17 & $6 . \overline{5}$ \\
\hline Between $6,000-7,000 \ldots \ldots \ldots \ldots \ldots$ & 33 & 12.7 \\
\hline Between $7,000-8,000 \ldots \ldots \ldots \ldots \ldots$ & 56 & 21.4 \\
\hline Between $\$, 000-9,000 \ldots \ldots \ldots \ldots$ & 31 & 11.5 \\
\hline Between $9,000-10,000 \ldots \ldots \ldots \ldots \ldots \ldots$ & 27 & 10.3 \\
\hline Between $10,000-11,000 \ldots \ldots \ldots \ldots \ldots \ldots$ & 18 & 6.9 \\
\hline Between $11,000-12,000 \ldots \ldots \ldots \ldots \ldots$ & 15 & 5.7 \\
\hline Between 12,000-13,000.............. & 6 & 2.3 \\
\hline Between $13,000-14,000 \ldots \ldots \ldots \ldots \ldots$ & 16 & 6.1 \\
\hline Between $14,000-15,000 \ldots \ldots \ldots \ldots \ldots$ & 9 & 3.4 \\
\hline Between $15,000-16,000 \ldots \ldots \ldots \ldots \ldots$ & 3 & 1.1 \\
\hline Between $16,000-17,000 \ldots \ldots \ldots \ldots \ldots$ & 3 & 1.1 \\
\hline Between 17,000-18,000.............. & 2 & 0.76 \\
\hline Between $18,000-19,000 \ldots \ldots \ldots \ldots \ldots$ & 3 & 1.1 \\
\hline Between $19,000-21,000 \ldots \ldots \ldots \ldots \ldots \ldots$ & 5 & 1.9 \\
\hline Between $21,000-37,000 \ldots \ldots \ldots \ldots \ldots$ & 2 & 0.76 \\
\hline Average $11,270 \ldots \ldots \ldots \ldots \ldots \ldots$ & 261 & 99.22 \\
\hline
\end{tabular}

Demonstrating Tariations in Leucocyte Count of 261 Cases of Gastric Cancer (Author).

Study of the table shows that 55.9 per cent. of all cases had leucocyte count between 6,000 and 10,000 cells; that more than 4 out of 5 of all the cases had leucocytes between 6,000 and 14,000 cells; that not quite 1 out of every 4 cases (24.22 per cent.) had white cell count above the arerage of the series, and that approximately 3 out of 4 cases had white cell count below the average for the series. 
The complete blood count in the case with minimum leucocyte count was as follows:

White blood corpuscles, 4,200; red blood corpuscles, 1,830,000; hemoglobin, 30 per cent.

Differential count of leucocytes: Polymorphonuclears, 67.7 per cent.; small lymphocytes, 27.3 per cent.; large lymphocytes, 3.0 per cent.; eosinophiles, 2.0 per cent.; transitionals, 0.7 per cent.

There was noted "moderate" variation in the size and shape of the individual red blood cells. Pathologically, the case was one of inoperable cancer of the lesser curvature and the body of the stomach with extensive metastases and free abdominal fluid.

The complete blood count in the case with the maximum leucocyte count was as follows:

White blood corpuscles, 36,200; red blood corpuscles, 4,980,000; hemoglobin, 82 per cent.

The differential count of leucocytes: polymorphonuclears, 81.3 per cent.; small lymphocytes, 13.3 per cent.; large lymphocytes, 3.0 per cent.; eosinophiles, 1.0 per cent.: transitionals, 0.3 per cent.; myelocytes, 1.3 per cent.

There was recorded "marked" variation in the size and shape of the individual erythrocytes.

Surgically, this case was one of extensive carcinoma of the posterior wall of the stomach, with invasion of the pancreas and extensive lymph-gland metastases.

It has been held that leucocytosis in gastric cancer is an index to the extent of the metatases, the rapidity of growth of the tumor, or such complications in the course of the ailment, as hemorrhage or perforation. The validity of these claims has been questioned by Osler and MeCrae and by Cabot.

For the purpose of studying the effect of metastases upon 
the leucocyte count, we have compiled from our records Tables 33 and 34 .

Table 33

\begin{tabular}{|c|c|c|c|c|}
\hline Leucocyte variations & $\begin{array}{l}\text { Metastases } \\
\text { (number of } \\
\text { cases) } \\
+\end{array}$ & $\begin{array}{l}\text { Metastases } \\
\text { (number of } \\
\text { cases) } \\
++\end{array}$ & $\begin{array}{c}\text { Metastases } \\
\text { (number of } \\
\text { cases) } \\
+++\end{array}$ & $\begin{array}{c}\text { Ascites } \\
\text { (number of } \\
\text { cases) }\end{array}$ \\
\hline $4,000-5,000 \ldots \ldots$ & 7 & 3 & 3 & $\ldots \ldots \ldots$ \\
\hline $5,000-6,000 \ldots \ldots$ & 8 & 0 & 3 & $\ldots \ldots$ \\
\hline $6,000-7,000 \ldots \ldots$ & 5 & 12 & 2 & $\ldots \ldots$ \\
\hline $7,000-8,000 \ldots \ldots$ & 13 & 13 & 16 & $\ldots \ldots$ \\
\hline $8,000-9,000 \ldots \ldots$ & 2 & 3 & 10 & 3 \\
\hline $9,000-10,000 \ldots \ldots$ & 3 & 12 & 10 & $\ldots \ldots$ \\
\hline $10,000-11,000 \ldots \ldots$ & 8 & 3 & 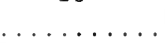 & ( \\
\hline $11,000-12,000 \ldots \ldots$ & 7 & 5 & & $\ldots \ldots$ \\
\hline $12,000-13,000 \ldots \ldots$ & 5 & & $\cdots$ & $\ldots \ldots$ \\
\hline $13,000-14,000 \ldots \ldots$ & 11 & $\ldots \ldots \ldots$ & 5 & $\ldots \ldots$ \\
\hline $14,000-15,000 \ldots \ldots$ & 9 & $\ldots \ldots \ldots$ & $\ldots$ & $\ldots \ldots \ldots \ldots$ \\
\hline $15,000-16,000 \ldots \ldots$ & 2 & 1 & & $\ldots \ldots \ldots$ \\
\hline $16,000-17,000 \ldots \ldots$ & 1 & & 2 & $\ldots \ldots \ldots \ldots$ \\
\hline $17,000-18,000 \ldots \ldots$ & $\ldots \ldots$ & 2 & . & $\ldots \ldots \ldots \ldots$ \\
\hline $18,000-19,000 \ldots \ldots$ & $\ldots \ldots$ & 3 & & $\ldots \ldots \ldots \ldots$ \\
\hline $19,000-21,000 \ldots \ldots$ & 2 & 1 & 2 & $\ldots$ \\
\hline $21,000-37,000 \ldots \ldots$ & 1 & (n) & 1 & \\
\hline Summar & 84 & 58 & 54 & 3 \\
\hline
\end{tabular}

Showing the Relation of Leucocyte Counts to Metastases in Gastric Cancer (Author).

Table 34

\begin{tabular}{cc} 
Leucocyte variations & Number of cases \\
$4,000-5,000$ & 2 \\
$5,000-6,000$ & 6 \\
$6,000-7,000$ & 14 \\
$7,000-8,000$ & 14 \\
$8,000-9,000$ & 13 \\
$9,000-10,000$ & 2 \\
$10,000-11,000$ & 7 \\
$11,000-12,000$ & 3 \\
$12,000-13,000$ & \\
\hline
\end{tabular}

Showing Variations in Leucocyte Counts in Cases of Gastric Cancer without Metastases (Author). 
Certain facts of interest are brought out by a study of these tables. It will be observed that there are 199 cases where metastases occurred, and 62 instances free from metastases. Of the cases showing metastases there were 84 instances (42.2 per cent.) where the involvement was small. Of this number the leucocyte count was 10,000 cells or below in 45.2 per cent. or 19 per cent. of the entire group showing metastases. There were 58 cases (29 per cent.) showing moderate degree of metastasis. Of this number 74 per cent. exhibited white cell count of 10,000 cells or below (21.6 per cent. of entire group). There were 54 cases (22 per cent.) with extensive metastases. Of this group 81 per cent. had leucocyte count of 10,000 cells or below (26 per cent. of metastasis class). Of the cases where ascites was demonstrated 100 per cent. (1.6 per cent. of group) had white cell count of 10,000 or below. Of all the cases showing metastases 13.6 per cent. had leucocytosis of 15,000 or above.

Of the entire number of cases with metastases, leucocytes averaged 10,000 cells or below in 22.6 per cent. In this figure the cases classed under ascites are not included. The comparative average for the different groups is 66.5 per cent.

In the group of gastric cancers without metastases (62 cases) the leucocytes were at or below 10,000 in 82 per cent. There were no instances where leucocytes were higher than 13,000 cells.

From a study of the above analysis, it will be seen that while more than 4 out of $\dot{5}$ cases of gastric cancer without metastases making up our series had leucocyte count of 10,000 cells or below, an average of but 2 out of 3 cases of the different groups showing grades of metastasis had leucocyte count so low. Of the whole number exhibiting 
metastases the white cell count was 10,000 cells or below in but rather more than 1 out of 5 cases.

Digestion Leucocytosis.- We have no original observations to record. Müller and Capps state that digestion leucocytosis is absent in more than 80 per cent. of gastric cancers. Osler and McCrae (loc. cit.) claim that "the presence of digestion leucocytosis is too uncertain to be of much assistance in diagnosis." Recently, Bonhoff has made a study of this phase of the leucocyte count and claims that the observation of digestion leucocytosis is a valuable clinical aid in the differentiation between benign peptic ulcer and ulcus carcinomatosum.

The Qualitative Variation in the Leucocytes.-In our series we have records of differential leucocyte counts in 160 cases. The following facts are of interest:

Polynuclear leucocytes averaged 73.2 per cent. The minimum was 56 per cent. and the maximum 86.4 per cent.

Small lymphocytes averaged 19.1 per cent. The minimum was 9.7 per cent. and the maximum 37.5 per cent.

Large lymphocytes averaged 3.41 per cent. The minimum was 0.7 per cent. and the maximum 13 per cent.

Basophile leucocyles averaged 1.28 per cent. The minimum was 0.3 per cent. and the maximum 5 per cent.

Transitional leucocytes averaged 1.58 per cent. The minimum was 0.3 per cent. and the maximum 6 per cent.

Myelocytes were observed in 43 cases (26.8 per cent.) where differential counts were made. The average was 1.27 per cent. The minimum was 0.3 per cent., and the maximum 3.3 per cent. Myelocytes were frequently noted where the red cell count was above 4,500,000 cells, but were observed in no instance where the hemoglobin was higher than 75 per cent. The lowest myelocyte percentage $(0.2$ per cent.) was seen when the leucocyte count was 7,200 . 
The hemoglobin was 30 per cent. and the red cell count $1,830,000$. The highest myelocyte count was observed where the leucocytes totaled 6,900. In this case the hemoglobin was 40 per cent. and the red cell count 4,110,000.

Eosinophiles were noted in 109 of the differential counts (6r per cent.). The average was 4.01 . The minimum was 0.3 per cent. The maximum was 7.7 per cent.

In the case with the minimum percentage of eosinophiles, the leucocytes were 5,600, the erythrocytes $3,260,000$ and the hemoglobin 38 per cent.

In the case with the maximum percentage of eosinophiles, the leucocytes were 7,200 , the red blood cells 4,180 ,000 and the hemoglobin 65 per cent.

In none of our cases of relatively high eosinophilia could we attribute the increase to such ills as lung affections, skin disease, high fever, medication, severe hemorrhage, or disease of the genito-urinary tract.

\section{(2) DEVIATIONS FROM THE NORMAL AS SHOWN BY THE BLOOD SERUM}

The gastric neoplasm can quite properly be regarded as itself being a parasite with the patient a host. From etiologic, histologic and clinical study, it would seem that the host has a form of protective mechanism. If such did not exist, it would be inconceivable why all members of the human family do not die of cancer, or why certain individuals live longer than do others when affected with the same histologic type of cancer in relatively the same part of the stomach. It would appear that the body's protective mechanism is similar in kind against all histologic forms of cancer. The strength of this defensive power seems not only to vary in different indiriduals, but may exhibit alterations in degree in the same individual at different times. 
In the human, at least, the body's defensive ability appears to rest mainly upon ferment-like agents. These agents are evidently essential components or products of protoplasm. They represent the most highly refined endresults of intercellular or intracellular biochemic change. These cell products, while apparently rigidly specific in their function, appear to act in a similar manner. These defensive substances are doubtless present in both cellular and fluid structures of the body. There is reason to suppose that in blood serum, lymph and spinal fluid protective agents against cancer are constantly present. If malignant neoplasms behave as do other parasites or foreign bodies when they invade their hosts, it would seem that the added demand for a specific defensive agent against such might be measurable by biochemic study of the body fluids, particularly of the blood serum and the blood cells. Much research along these lines has been carried on. While thus far there has been isolated no absolutely specific defensive agent against cancer, it would seem that certain biologic phenomena of blood sera indicate a broad, basic principle of defence, the exact significance of which has not yet been established. But a few aspects of the mechanism of this protective agent against cancer in general can be considered in a special monograph upon cancer of the stomach.

The Hemolytic Reaction.--In 1902, Lang showed that in advanced cases of cancer, the erythrocytes were very resistant to anisotonic solutions of sodium chloride. In 1908, Weil demonstrated that the blood serum of dogs harboring experimental lymphosarcoma was lytic for the erythrocytes of non-sarcomatous dogs, but not for the red blood cells of other dogs harboring like malignant tumors. Under certain limitations this observation appeared to be of diagnostic worth in human cancer. To Krida, we 
are indebted for a detailed description of the method of performing the test, and for a résumé of its diagnostic worth.

Hemolysis in the Diagnosis of Cancer.-Krida employs the following technique: The blood for the test is obtained by puncturing one of the superficial veins after tying a tight bandage around the arm above the point of puncture. About $10 \mathrm{cc}$. are withdrawn; of this about $1 \mathrm{cc}$. is added to a centrifuge tube which has been previously half filled with a normal salt solution to which 1 per cent. sodium citrate. has been added, and immediately centrifuged to throw down the cells. Or the cells may be obtained by defibrinating a small quantity of blood by shaking with glass beads. The rest of the blood is placed in a sterile test-tube, slanted and placed in the ice chest to clot and allowed to remain there for 12 to 24 hours. The cells as previously obtained are washed 2 or 3 times in salt solution, and then made up to a 5 per cent. emulsion with salt solution and placed in the ice chest until ready for use. Cells and serum are obtained in a similar manner from two normal individuals to act as controls and are also placed in the ice chest until ready for use. Several pathological sera may of course be tested with these two controls.

When the test is ready to set up, the hemolysis tubes are sterilized, then washed in normal salt solution. As many different combinations of cells and serum as possible are made, using 5 cc. of each, and the plan of the mixtures noted. The simplest procedure is to begin with one cell emulsion, placing 5 cc. in as many tubes as there are kinds of sera, and adding to each tube $5 \mathrm{cc}$. of the various sera. As an additional control, a preparation with cells and salt solution and cells and sterile water might be made; the hemolysis 
in the tube containing the sterile water will, of course, be complete.

The rack containing the test is now placed in the incubator at $37^{\circ} \mathrm{C}$. for 2 hours. Some make an entire duplicate set and heat it in the water-bath for 10 minutes at $55^{\circ} \mathrm{C}$. before incubating (to destroy complement). At the end of 2 hours, the tests are placed in the ice chest for 12 hours, and the results are then read off by some person who does not know the key to the plan of mixtures. Hemolysis is apparent by a pink or red discoloration of the supernatant fluid in the test-tubes.

If the hemolysis test for cancer is positive the cancer serum should have hemolyzed the cells of both the normal controls. A reverse hemolysis, i.e., normal serum hemolyzing pathologic cells is considered characteristic of tuberculosis by Crile, but in this the results of the other observers are almost uniformly negative.

The following table presents a summary of the results with the hemolytic reaction:

Table 35

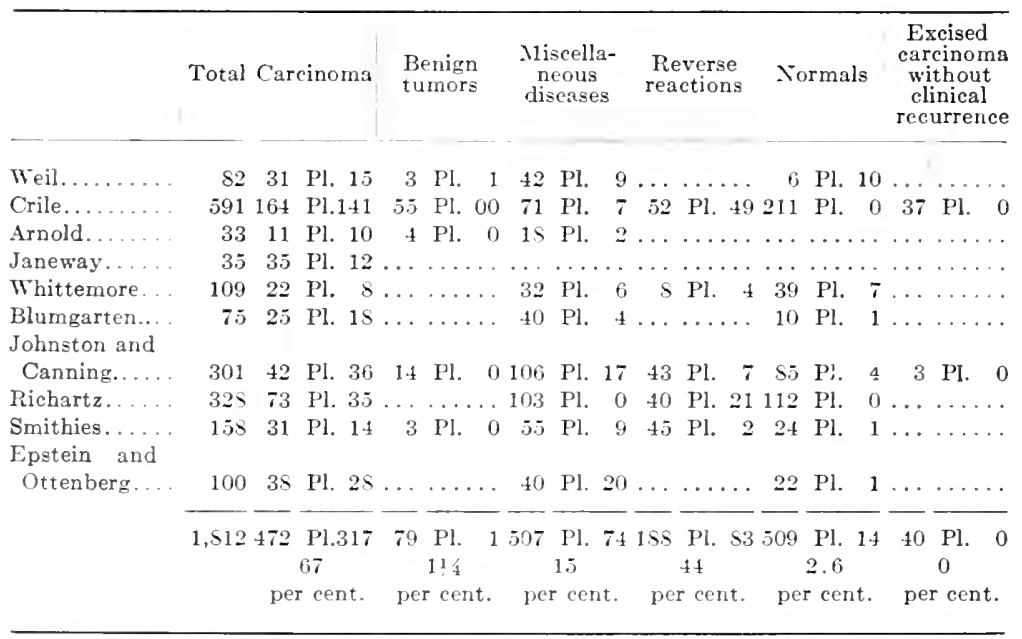

Showing the Diagnostic Significance of the Hemolytic Test in Cancer (Kirida). 
Of a total of 1,812 observations reported by ten different workers, 472 cases were carcinoma; of these, 317 or 67 per cent. gave positive hemolytic reactions. Seventy-nine were benign tumors, of which 1 or $1 \frac{1}{4}$ per cent. were positive.

Five hundred seven observations were made in a variety of diseases, 74 or 15 per cent. of which presented positive reaction.

Five hundred nine observations were made on normal individuals, 14 or 2.6 per cent. were positive.

In 40 post-operative carcinoma cases without clinical recurrence, the reaction was uniformly negative.

One hundred eighty-eight tests were performed on tuberculosis patients; of these, $\$ 2$ or 44 per cent. presented "reverse" hemolysis.

Skin Reaction.-The basis of the skin reactions for carcinoma is the subcutaneous injection into the forearm of about 5 minims of a 20 per cent. suspension in physiological salt solution of red blood cells obtained from a normal, healthy individual.

The quantity of blood necessary varies, of course, with the number of reactions to be carried out. For 10 or 12 reactions, 2 to $3 \mathrm{cc}$. are obtained as described previously. Sufficient blood for two or three tests maybe obtained by simply puncturing the finger and allowing a dozen drops to flow into a centrifuge tube half full of salt-citrate solution. This is immediately centrifuged, washed 3 times as previously described, and made into a 20 per cent. suspension in physiological salt solution. The suspension is placed at $0^{\circ} \mathrm{C}$. for 24 to 48 hours and is then ready for use. A convenient method of keeping this suspension until ready for use is to draw up a sufficient quantity for individual tests into pipettes and seal the ends. The suspension does"not keep longer than 5 days, and one should see that no hemol- 
ysis has occurred previous to using it for injection (Krida).

If the patient's serum is hemolytic for normal cells, an oral area of discoloration about 2 by $5 \mathrm{~cm}$. will appear at the site of injection in from 5 to $S$ hours.

The discoloration is described as "brownish red to a maroon, with rarely a bluish tinge. The lesion is distinctly raised from the surrounding surface." The discoloration persists from 1 to 3 hours, and usually begins to fade at the end of $S$ hours, leaving a greenish ecchymosis at about the end of 12 hours. The element of time here is variable, howerer, as we have found. If the patient is in a hospital, he should be observed about once an hour, beginning about 5 hours after the injection, and making 3 or 4 observations. If the patient be at home, he may be conreniently seen about 6 hours after the injection. If the observation then made be inconclusive, he may be instructed to notify the physician should a reaction appear within 3 or 4 hours.

Elsberg, Neuhoff and Geist have classified the results of their tests as follows:

Carcinoma, positive or probable, 69 cases, 62 or $\$ 9.9$ per cent. plus and 2 or 2.9 per cent. doubtful.

No carcinoma, 325 cases, 15 or 4.6 per cent. plus and 3 or 1.1 per cent. doubtful.

Possible carcinoma, 9 cases, 7 or 77.8 per cent. plus.

Carcinoma, advanced or miliary, 11 cases, 0 or 100 per cent. negative.

Krida states that of 12 cases of carcinoma, the reaction was positive in 9 , or 75 per cent. He states further that the reaction is not uniformly absent in the cachectic cases. Elsberg, Neuhoff and Geist have made such a subdivision and found the results negative in their 11 cases so classified. Warfield thinks the test of dubious worth. 
It is certainly interesting from a biochemic, and perhaps anaphylactic standpoint.

Cancer Diagnosis of Freund and Kaminer.-Müller states that the blood serum of normal individuals sometimes has the power to dissolve cancer cells. The blood serum of patients suffering from cancer frequently lacks this power, and has the power to inhibit the destruction of such cells by normal serum.

When the blood serum of cancer patients is mixed with a cancer extract, a precipitate forms.

Ingredients of the Test.-1. An Emulsion of Cancer Cells.-Grind in a mortar the necrotic portions of the tumor, freed so far as possible of fat and extraneous cells, with about five rolumes of 1 per cent. sodium biphosphate. Squeeze the suspension through several thicknesses of gauze. Allow the cells to settle, and remove the supernatant fluid with a pipette. Wash the residue with 0.6 per cent. sodium chloride solution. Allow the cells to settle again, pipette off the supernatant fluid and cover the residue with 1 per cent. sodium fluoride. The lastnamed fluid should be first neutralized against alizarin till only a trace of violet color remains. This emulsion will keep for several weeks in the ice-box.

2. An Extract of Cancer Cells.-Fresh tumor tissue obtained at autopsy, or tumor tissue preserved in alcohol may be used. Portions as free as possible of necrotic areas and fat are cut into small pieces and worked through a coarse-meshed cloth by the gradual addition of ten volumes of 0.6 per cent. acid sodium phosphate solution. Allow the cells to separate by settling or by careful centrifugation. Wash sereral times with the same fluid, and preserve at $0^{\circ}$. Sodium fluoride, up to 1 per cent., may be added as a preservative, provided care is taken 
to see that it does not render the fluid alkaline. Thymol may also be used.

For use add 5 cc. of 5 per cent acetic acid to 100 cc. of the fluid. Heat the mixture in the water-bath for 15 minutes at $80^{\circ} \mathrm{C}$., filter, cool, and neutralize to litmus with sodium carbonate. Heat again as above, cool and filter. Heating at $100^{\circ} \mathrm{C}$. or over the free flame must be avoided. The activity of the extract is determined by testing with 10 drops of known normal and cancerous sera whether the extract, undiluted, and diluted 10, 50, and 100 times, gives a precipitate with cancer serum, plainly visible in test-tubes held against the window, while the normal serum gives no such precipitate. The extract keeps for only 2 or 3 dlays. The extract if used in too concentrated form causes a precipitate with normal serum, but if diluted to opalescence gives a dismal serum, or of an ether extract of horse serum.

3. The Patient's Serum.-This is obtained in any of the customary ways. The serum must be separated from the cells within a few hours after the blood is drawn and cannot be used when it is more than 48 hours old.

4. A blood-counting chamber.

5. Small test-tubes or small test dishes with parallel sides.

6. Capillary pipettes, and volumetric pipettes, 1 cc. in $1-100$ 's.

7. An incubator at $37^{\circ} \mathrm{C}$.

S. A 0.5 per cent. solution of sodium fluoride.

Technique.-To 10 drops of the patient's serum add 1 drop of the 0.5 per cent. solution of sodium fluoride. Then add 1 drop of the cancer cell emulsion so diluted that when 1 drop of the mixture is placed in the blood-counting chamber, about 10 to 20 tumor cells will be found in a 
large field (25 of the smallest squares) of the apparatus. Close the counting chamber carefully and place in the incubator for 24 hours. Count the number of cells again.

It is said that a material reduction in the number of cells will be found when the serum is derived from a healthy individual, whereas, if the serum is derived from a cancer patient such a reduction does not take place.

A second test is made by diluting normal serum with an equal amount of 0.6 per cent. sodium chloride solution, and also with an equal volume of the patient's serum. To each of these fluoride and the cell emulsion is added as above. If the patient's serum prevents the solution of the cells by the normal serum, this again constitutes a positive reaction.

The test for a precipitate is made as follows: Place 10 drops of the patient's serum in a small test-tube or test dish with parallel sides and add $2 \mathrm{cc}$. of the extract properly diluted as described above. Controls are made by adding to a separate quantity of serum a fluid identical with the tumor extract with the exception of the tumor tissue. In other words, if the extract was prepared from fresh tumor tissue this fluid is prepared by adding to $100 \mathrm{ce}$. of 1 per cent. acid sodium phosphate 5 cc. of 5 per cent. acetic acid, and neutralizing with sodium carbonate. When the extract is prepared from tissues preserved in alcohol, use 0.25 per cent. acetic acid neutralized with sodium carbonate.

The precipitate forms at once and must be viewed by transmitted, not by reflected light. A distinct clouding under these conditions constitutes a positive reaction. Freund and Kaminer recommend that all three tests should be made when enough serum is arailable. This 
calls for about $2.5 \mathrm{cc}$. of serum, representing about $6 \mathrm{cc}$. of whole blood (Müller-Whitman).

The Antitryptic Reaction.-In 1902 Petry demonstrated that cancers contain a proteolytic ferment. He showed that such proteids as gelatin or casein could be digested by an extract of the malignant tumor. The agent causing this digestion appeared to have the properties of a ferment. It was later shown that the passage of such proteolytic ferments into the circulation resulted in the formation of anti-ferments in the body fluids, especially in the blood serum. This anti-ferment was named "antitrypsin." It was thought that a method of measuring the strength or the amount of the antitrypsin would indirectly serve as a method of diagnosing the existence of malignancy, inasmuch as it had been demonstrated that normal blood serum contains relatively small amounts of antitrypsin. In 1908, Bergmann and Meyer announced a method whereby the antitrypsin content of blood sera could be estimated clinically. Their method is as follows:

Estimation of the Antitrypsin Content of the Blood.Principle: One determines what amount of trypsin is neutralized, as to its digestive power, by a certain amount of serum or blood. The substance to be digested may be either Loeffler's blood serum, as ordinarily used by bacteriologists, or a solution of casein. In the former case the criterion for the occurrence of digestion is the formation of a dimple on the surface of the serum; in the latter the non-appearance of a cloud, due to the precipitation of casein, on the addition of acetic acid.

Practical Application.-Whitman states that the antitrypsin of the blood is most markedly increased in cancer. Hence the method has been used chiefly for cancer diagnosis. 
Method of Bergmann and Meyer. Apparatus.-1. A 1 per mille solution of trypsin sicc. (Gruebler): dissolve 0.5 gram trypsin in 50 cc. physiological salt solution, add 0.5 cc. normal soda solution, and make up to $500 \mathrm{cc}$.

2. Casein solution: 1 gram casein (rhenania) is dissolved in 100 cc. $n / 10 \mathrm{NaOH}$ solution, with the aid of gentle heat. Neutralize to litmus with $n / 10 \mathrm{HCl}$ solution and make up to $500 \mathrm{cc}$.

3. Acetic acid solution: 5 cc. acetic acid, $45 \mathrm{cc}$. alcohol, and 50 cc. water.

4. The patient's serum, diluted 20 times with salt solution; 0.1 to 0.2 cc. suffices.

5. Small test-tubes.

6. Pipettes, 2 cc. graduated into 1-10's.

Technique.-Titration of the trypsin solution. This must precede the test proper. In each of several test-tubes place $2 \mathrm{cc}$. of the casein solution and decreasing amounts of the trypsin solution as, for example, 1.0, 0.9, 0.8, 0.7,0.2 . Shake carefully and place in the incubator for $1 / 2$ hour. Then add the acetic acid solution drop by drop, observing which tube shows cloudiness after a few minutes. The tube containing the smallest amount of trypsin, and which remains perfectly clear, contains the "completely digesting dose," which is used for the test proper.

Estimation of the Antitrypsin.-In each of 6 tubes place 0.2 cc. of the patient's serum, and increasing amounts of the trypsin solution, beginning with the completely digesting dose, and increasing by $0.1 \mathrm{cc}$. Add 2 cc. casein solution to each tube and bring all the tubes to a like volume. Incubate as before for $1 / 2$ hour at $37^{\circ} \mathrm{C}$. Acidify as before, and again note the tube containing the smallest amount of trypsin in which cloudiness can be seen. The amount of trypsin paralyzed by the antitrypsin of the 
serum is thus determined. If, for example, the completely digesting dose is 0.7 cc. and if, in the presence of serum, 2.0 cc. of trypsin are required to bring about complete digestion, then:

$$
2.0 \text { cc. }-0.7 \text { cc. }=1.3 \text { cc., }
$$

or the amount of trypsin paralyzed by the amount of serum used (in this case 0.2 cc. of a 5 per cent. dilution, or 0.01 cc. whole serum). One cc. of serum, therefore, paralyzes 130 cc. of the trypsin solution. Furst, expresses the result as follows:

$$
\text { Antitrypsin } \frac{37^{\circ} \mathrm{C}}{1 / 2 \text { hour }}=130 \text {. }
$$

Comparison of the values thus obtained with those given by normal serum shows whether the antitryptic power of the serum tested is altered (Müller-Whitman).

The method of Bergmann and Meyer was later improved by Weil and Feldstein, by the introduction of the "viscosimeter," an instrument permitting accurate determination of the end point of digestion of a pure proteid by blood serum.

From the perusal of a huge literature, it would appear that the "antitryptic reaction" in the blood serum is positive in from 75 to 95 per cent. of all cancers. It is of comparative rarity in the blood serum of non-cancerous patients. That it has a definite place as an aid in differential diagnosis is attested by Stockton, Bergmann and Meyer, Roche and others.

Roche emphasizes the great importance of negative antitryptic reactions in excluding the diagnosis of malignancy.

Abderhalden Method for the Detection of Specific Ereptases.-Numerous investigators have demonstrated the presence of ereptic ferments in blood serum. It remained for Abderhalden, however, to show that specific 
ereptases were developed or increased by the presence of a foreign proteid (parenterally) or by the growth of neoplasms in the body. The protective mechanism of the organism appears to take the form of a highly specialized ereptic ferment capable only of digesting the proteid composing the type of tumor exciting its production.

Principle.-The blood serum of cancer individuals contains a specific ferment which digests cancer cells.

Apparatus.-1. Cancer tissue.-Cut a fresh human cancer into small pieces, place in a wide dish, and wash in running (tap) water. The external portions may be separated and discarded, but this is not necessary. While the washing, which is for the purpose of removing blood, is going on, a large enamel basin or evaporating dish containing 1 to 2 liters of water, to which 1 drop per liter of glacial acetic acid is added, is heated to boiling. Throw the washed cancer tissue into the boiling water, and continue the boiling for 5 minutes. Pour off the boiling water through a gauze strainer or plaited filter, taking care that the coagulated bits of cancer remain as far as possible in the dish. Pour on more water and boil again for 5 minutes. Now test a portion of the water for the biuret reaction as follows: To about $10 \mathrm{cc}$. of the water, add $5 \mathrm{cc}$. of a 33 per cent. caustic soda solution, mix and layer enough of a rery dilute (0.25 per cent.) solution of cupric sulphate on top to make a layer $0.25-0.5 \mathrm{~cm}$. deep. A red color at the zone of contact constitutes a positive reaction. If the process has been carried out rapidly, the test will always be negative.

In place of the biuret reaction for peptone the more delicate triketohydrinden hydrate (Hoechst), may be used. This gires a blue color with compounds which have a $\mathrm{COOH}$ group and an amino-acid in the "a" position. Albumin, peptone, polypeptids and amino-acids react. Place $10 \mathrm{cc}$. 
of the fluid in a large test-tube, add exactly 0.2 cc. of a 1 per cent. aqueous solution of triketohydrinden hydrate, bring quickly to boiling and continue the boiling for 1 minute without interruption. When the reaction is positive, a beautiful violet-blue color develops in a short time. When negative, the solution remains colorless, or takes on a yellowish tone. The use of the triketohydrinden hydrate is recommended, but it must not be used for the test proper unless the cancer tissue, as prepared above, has been shown to react negatively with it.

If the water in which the cancer is cooked gives one or other of these reactions for peptone, the boiling must be continued as before till the reaction is negative. When this occurs pour the last cooking water, together with the cancer tissue, into a wide-mouthed flask. Cover with a layer of toluol, and cork tightly. The entire process requires not more than $1 / 2$ to 1 hour, and furnishes material for several hundred tests.

2. Extraction thimbles. (S. and S. 579.) These should be kept under water and a layer of toluol, and should be tested for permeability to Witte's peptone before use. After being used, they may be carefully washed in running water and used again. They should never be used dry.

3. The patient's serum. This must not be shaken. It is recommended to allow about $10 \mathrm{cc}$. of blood to flow directly into a centrifugal tube. As soon as the clot forms centrifugate, pour off the serum, and use at once for the test. If the serum contains any hemoglobin it cannot be used.

4. Small beakers, Erlenmeyer flasks or especially designed cylinders of such a size that the thimbles above mentioned fit into them leaving not more than $0.25 \mathrm{~cm}$. between the shell and the wall. 
5. Reagents for the biuret reaction, or triketohydrinden hydrate, or both.

6. Incubator at $37^{\circ} \mathrm{C}$.

7. Distilled water.

Technique.-In one of the extraction thimbles, previously tested as to its permeability, washed, and kept as above described, place about 1 gram of the cancer tissue, crumbling it between the fingers into pieces the size of a wheat grain while doing so. Pour about 2 or $3 \mathrm{cc}$. of the patient's serum over the tissue in the bottom of the thimble. Add a few drops of the toluol to prevent putrefaction. Now hold the thimble at the top, and rinse the outside thoroughly under the tap. Place the thimble in one of the beakers, described above, and add 15 cc. of water, outside the thimble. A thin layer of toluol is placed also on the outside. Controls should be prepared in the same way, consisting of the patient's serum alone, and of the cancer tissue alone, respectively. Place in the incubator for 12-16 hours. Now remove $10 \mathrm{cc}$. of the fluid outside of the thimble, by means of a pipette, thrust below the level of the toluol, and test this for peptone with one or both of the reagents described above. When triketohydrinden hydrate is to be used, it is especially important that as little toluol as possible be transferred to the test-tube, as overheating is then apt to occur. A glass rod may be placed in the test-tube to prevent bumping.

A positive result is indicated when the dialysate gives a positive reaction for peptone.

The method has been verified by R. Franz and by Frank and Heimann.

The polariscope method of Abderhalden is sometimes used. It is too complicated for general clinical application (Müller-IVhitman). 
Ransohoff's Anaphylaxis Test for Cancer.-Guinea-pigs are sensitized by the intraperitoneal injection of bloodserum from (a) a normal patient and $(b)$ a patient affected with adranced cancer. At the end of 10 days, each group of guinea-pigs is injected with known malignant serum, intraperitoneally. When such serum is introduced into guinea-pigs sensitized with cancer serum, typical anaphylactic reactions are claimed to develop. The injection of serum into guinea-pigs into whom non-cancer serum had been preriously introduced causes very slight local or systemic disturbance or none at all.

Ransohoff states that in his hands the test proved positive for 92 per cent. of 26 cancer cases tested.

The Miostagmin Reaction.-Ascoli and his pupils have presented a blood-serum reaction based upon certain principles of physical chemistry. They claim that when the essential principles of a specific antigen are extracted, the resultant extract, if added to a suspected blood serum, produces such alteration that rariations in surface tension may be measured. These alterations are estimated by a Traube stalagmometer before and after incubation of the antigen-extract-serum mixture. Ascoli claims that such changes in surface tension occur when antigen and specific antibody unite, that the decrease in surface tension may result in stalagmometric readings showing an increase of from 2 to 5 drops.

The Ascoli reaction is very complicated. While in its originator's hands it seems to be a valuable aid in the diagnosis of cancer, numerous competent investigators (Kelling, Bernstein and Simons and other's) claim that the reaction has little clinical worth. 


\section{REFERENCES}

Cавот: "A Guide to the Clinical Examination of Blood," New York, 1904.

Oster ANd McCrae: "Cancer of the Stomach," Philadelphia, 1900.

Cunliffe: Medical Chronicle, Sept.. 1903.

Bonhoff: Beitr. z. klin. Chir., Tubingen, 1914, June, xeii.

LANG: Ztschrft. f. klin. Med., 1902, xiii, p. 106.

WeIL, R.: Arch. Int. Med., 1908, i, p. 2 S.

Krida: Albany Medical Amnals, 1910, May, p. 259.

Elsberg, Neuhoff and Geist: Am. Jour. Med. Sc., 1910, Feb., p. 264.

Warfield: Arch. Int. Med., 1911, Nov., p. 621.

Müller: "Serodiagnostic Methods," 1913, Philadelphia and London.

Translated by R. C. Whitman.

Freund \& Kaminer: Biochem. Ztschrft., 1910; also Wien. klin.

Wehnrft., 1910, xiii., pp. 378 and 1221, and 1911, xxiv., p. 1759.

Bergmany and Merer: Berl. klin. Wschnschrft., 190S, xir, p. 1673.

Weil, R. Axd Feldsteix: Proc. Soc. Exper. Biol. and Med., 1910, vii, p. 61 .

Stockton: "Diseases of the Stomach," New York, 1914.

Roche: Arch. Int. Med., 1909, April, p. 1.

Abderhaldex: "Schutzfermente des tierischen Organismus," Berlin,

1912; also Münch. med. Wchnrft., 1912, lix., pp. 1305, 1939 and 2172

Franz: Münch. med. Wschnschrft., 1912, lix., p. 1702.

Frank and Hemand: Berl. klin. Wschnschrft., 1912, lix, p. 1706.

Ransohoff, Jour. Am. Med. Assn., 1913, July 5, p. S.

Ascoli: Münch. med. Wschnschrft., 1910, Jan. 11, p. 63.

Ascoli and Izar: Ibid., 1910, Feb. 22, p. 403.

Ascoli: Ibid., 1910, Oct. 11, p. 2129.

Ascour: Deutsch med. Wschnschrft., 1910, Oct. 27, p. 1997.

Kelling: Wien klin. Wschschrft., 1911, Jan., No. 3.

Bernstein And Simons: Am. Jour. Med. Sc., 1911, Dec. 


\section{THE SIGNIFICANCE OF GASTRIC ULCER WITH RESPECT TO GASTRIC CANCER}

During the past decade there has been a growing conviction among clinical and laboratory workers that there exists an increasing number of cases clinically admitting a diagnosis only of chronic gastric ulcer which do not pursue an orthodox course of chronicity, but often rapidly assume aspects of malignant disease. If such cases come to laparotomy or necropsy, the surgeon or pathologist demonstrates cancer.

Conversely, surgically and pathologically proved cases of gastric cancer frequently reveal an early clinical history, which at any stage prior to the terminal period of evident malignancy might logically be interpreted clinically as chronic gastric ulcer.

The subject has etiologic, diagnostic and prognostic aspects. Inasmuch as this group of cases satisfies our present day diagnostic requirements for gastric ulcer and this process, of whatever nature it may be, later assumes characteristics that we associate with malignancy, it would appear imperative to determine how frequently this type of affection exists, in what manner, if any, it manifests itself, the possibilities of its recognition and the indicated treatment when demonstrated.

At the present state of our knowledge it is impossible to prove how frequently gastric ulcers become cancers. Whatever individual opinion may be, this statement holds true. There are many reasons why conclusive facts cannot yet be given. Some of these we would emphasize: 
(a) Regional variation in the incidence of gastric ulcer. In 1864 Brinton claimed that peptic ulcer was found at necropsy in from 2 to 13 per cent. of persons dying from all causes. The average incidence was approximately 5 per cent. These figures have been much quoted and indiscriminately compared. It will be recalled that they were collected at a time when pathological methods were poorly developed. Modern writers have shown that not only is there a wide range in incidence of peptic ulcer in different countries, but that there is also striking variation in its occurrence in different parts of the same country. In a recent and careful survey of the subject Bolton points out that pathological statistics collected from Europe and America indicate that this variation ranges between 0.5 per cent. and 20 per cent. Bolton gives the average figures as follows: Denmark 16.7 per cent., England 5 per cent., Germany 5 per cent., Austria 4 per cent., Switzerland 2.6 per cent., North America 1.3 per cent., Russia 0.8 per cent. It is said that in North Germany the disease is about as frequent as it is in Denmark, while parts of South Germany approximate the average for North America. Whatever may be the estimated incidence based upon clinical diagnoses, postmortem, or laparotomy statistics are the only figures upon which we can base dependable comparisons. We have already shown (Chapter I) that our 921 instances of operatively and pathologically demonstrated cases of gastric cancer occurred in about \$2,000 hospital admissions. This indicates an incidence of 1.12 per cent. In approximately the same number of admissions there were operatively demonstrated 1250 chronic or acute gastric ulcers, or a percentage of 1.51. It is seen that the incidence of proved gastric cancer and gastric ulcer in 
our hospital admissions did not rary greatly. Comparing the two percentages, it is remarkable to note that the cancer incidence is approximately 74 per cent. of that of gastric ulcer. When the appended analysis of the relation of ulcer and cancer is considered, it must be admitted that this figure is striking. The above is an American experience and, hence, scarcely comparable with that in other countries. MIoreover, it is an experience largely from the Central and Northwest United States and Southern Canadi.

(b) Tariation in pathologic opinion as to the life history of gastric ulcer and of gastric cancer. While the literature contains many excellent monographs descriptive of gastric ulcer, the researches of Bolton supply us with practically our only dependable evidence of such elementary knowledge as that an acute gastric ulcer may progress to chronicity and is not a different clinical entity. Bolton's investigations appear to be warranted by both histologic and clinical facts. This observer has not yet demonstrated carcinomatous changes in any of his chronic gastric ulcers. Certainly if some of the photomicrographs submitted were examined by oil-immersion lens, it would be rather difficult to exclude the presence of hyperplasia approaching the malignant form described by MacCarty.

With respect to the histologic demonstration of the beginnings of so-called "primary" gastric cancer, we are more uncertain than with respect to known cell change in gastric ulcer. No one has ever seen the actual transition of gastric mucosa from benignness to malignancy. One can only say of a given specimen that eancer is present or it is absent. It would appear, however, that careful study of fresh tissue with highest magnification reveals certain undifferentiated cells which aid in the segregation of 
benign from malignant hyperplasia (MacCarty). When such is present a "precancerous" state not infrequently seems to exist (vide infra).

(c) The variation in an accepted clinical complex of gastric ulcer. We refer in full to this below. Modern investigations would certainly warrant the statement that the clinical diagnosis of gastric ulcer is by no means simple and according to well-marked symptoms and signs. It is now generally admitted by those who have followed the progress of any considerable group of cases that many of the instances which were formerly classed as gastric ulcer are not such, but are gastric eridences of extragastric disease. This applies especially to cases below age 30 , who present many of our so-called ulcer symptoms, clinically.

(d) The tendency to cloud our present-day knowledge with ancient statistics and imperfect pathologic and histologic descriptions. To those familiar with the literature, it is not necessary to point out the hindrances to the advance of knowledge that have resulted from the constant quoting of work clone by investigators of a half century ago. Such researches were remarkably acute for their day, but modern science would indeed have cause for regret if it were not able to claim new facts with its added armamentarium for research. The developments of laboratory methods for the preservation and study of tissue, the improvement in microscopes, the checking up of clinical material pathologically, the advance in hospital facilities for examination of disease, and the systematization and standardization of records, have all contributed toward rendering extremely questionable the points upon which classifications of gastric ulcer and cancer were made 20 to 70 years ago. Except for the historical interest, one would hardly think of burdening himself with the pathology, his- 
tology and bacteriology of syphilis, meningitis, malaria or typhoid fever as set down three decades ago, yet strenuous attempts are still made to fit twentieth century gastric ulcer and gastric cancer to the conception of it a half century ago. It is quite possible that these diseases have altered in many ways as succeeding generations have harbored them, or that environment has had such effect. That variations are within the range of probability is evidenced by a study of the clinical history of syphilis, leprosy, typhus fever, poliomyelitis, gout, etc.

For the purpose of returning facts regarding the relationship between gastric cancer and gastric ulcer, the author recently made an analysis of 566 operatively and pathologically demonstrated cases of gastric cancer.

\section{CLINICAL CONSIDERATION}

Significance of History.-Sex.-In the 566 cases there were 436 males, and 130 females, or 3.1 males to each female. We have shown that this is very near the sex ratio existing in non-malignant, chronic gastric ulcer.

Age.-The youngest patient in the series was 20 years of age; there were 26 patients aged over 70 . More than three-fourths of the cases came between the ages of 40 and 70 years. A comparative study of 134 cases of nonmalignant chronic gastric ulcers shows that rather more than one-half were in the 40- to 70 -year period.

Etiologic Factors.-A history of trauma was obtained in 3.4 per cent. of the cases. In 2.9 per cent. the traumatism occurred in the early history of the affection, and frequently appeared to cause or precipitate symptoms. There were three instances in which husband and wife became affected with cancer within a few months of each other. A family or blood-relationship history was obtain- 
able in 9.2 per cent., and a history of tuberculosis in 1.2 per cent.

\section{Previous Disorders of Digestion (the "Precancerous} History).-More than 10 years ago Graham called attention to the significance of the early clinical history in patients presenting themselves in his service for evident gastric cancer. He stated that more than 47 per cent. of his operatively demonstrated cases of cancer had had previous histories which strongly suggested that the cancer had followed a chronic gastric ulcer, existing variously from 3 to 37 years. Graham also emphasized the fact that nearly 40 per cent. of his cases of cancer were not associated with the previous so-called "ulcer history," but that in this group of cases cancer developed in stomachs which formerly functionated normally.

The value of early history as indicating that succeeding cancer of the stomach has its origin in such hypothetic ulcer has been justly questioned. The weakness of the argument appears to be at least threefold: (1) the clinical variation in an ulcer symptom-complex, (2) the indefinite ideas existing as to the time element in the development of "chronic" ulcer and of cancer, and (3) the difficulties in actually proving whether or not a process which is later shown to be malignant was ever anything else. I shall consider these points seriatim.

1. To admit the indefiniteness of an ulcer symptomcomplex is to grant at once that mistakes in diagnosis are readily possible. That this is a fact has come within the experience of all gastrologists who have handled either a few or a large number of cases. In spite of personal opinions, we must admit that the only gastric ulcers that we can positively say exist are those which we can see or feel. While it may be clinically safer to treat a given 
case as gastric ulcer, as recommended by Shutz, nevertheless such uncertain procedures have undoubtedly led to error, confusion and injury to patients. The prognostic aspect of the case is of greater import than the question of type of treatment based on uncertain diagnosis.

In endearoring to gauge the importance of the previous (the precancerous) gastric history of the cases in our series we found it necessary to group them according to accepted clinical symptom-complexes, of ulcer and of cancer, respectively. This mode of procedure has many faults, but it should be emphasized that this method of classification furnishes the bulk of the literature on the subject.

The clinical symptom-complex considered for gastric ulcer is based on Friedenwald's recently analyzed 409 cases, while that for cancer is compiled from the work of Osler and McCrae. If the value of such grouping is questioned, then the value of much that makes up accepted clinical knowledge of the diagnosis and the treatment of the two ailments must also be questioned. If the symptomcomplexes indicated mean clinically ulcer or cancer of the stomach, then the facts that we have to present are not without significance.

The have taken the following symptom-complex to mean gastric ulcer clinically: a form of gastric malfunction occurring usually between the ages 10 and 70 years, characterized by periodic or continuous abdominal discomfort or pain, frequently bearing definite relation to food ingestion, and often associated with epigastric or dorsal tenderness, romiting, loss of blood (hematemesis or melena) and with hyperacid gastric contents.

We have considered as "primary" cancer, clinically a form of gastric malfunction of a downwardly progressive 
nature, usually occurring in persons between the ages of 40 and 70 years, who have been previously normal gastrically, the imperfect function being characterized by abdominal distress or pain, usually associated with cachexia, loss of blood, epigastric tumor, vomiting and with gastric contents revealing motor defects, low free hydrochloric acid and the presence of organic acids and of foreign microorganisms.

In grouping our material under these accepted clinical symptom-complexes, we find that of the 566 proved cases of gastric cancer, 239 or 41.8 per cent. fall into the cancerfollowing-ulcer classification, while 182 or 32.1 per cent. are in the "primary" cancer division. There is, in addition, a group which may be termed cases of "irregular ulcer" that numbers 106, or 18.7 per cent. Twenty-two patients (3.9 per cent.) had a previous clinical history of gall-bladder affection, while 17 or 3 per cent. had early symptoms pointing to primary processes in the appendix, the pancreas or the bowel. Combining the returns from the two "ulcer" groups, it is seen that precancerous history indicates that 60.5 per cent. of the subsequently demonstrated cases of cancer gave those clinical evidences which we associate with chronic gastric ulcer, prior to the time when the ailment assumed the clinical picture that we associate with gastric malignancy. In but 32.1 per cent. was the disease, from its inception, continuous and progressively downward, and in persons who had been previously sound gastrically. These figures are not to be taken as they stand to indicate that nearly two-thirds of all chronic gastric ulcers later become malignant, because we know that ulcers frequently heal spontaneously or continue as chronic, inflammatory processes. Added significance, however, is given to the figures by the observation of the surgical pathologist that more than two-thirds of all excised chronic 
calloused gastric ulcers show early evidences of malignant metamorphosis (Wilson and MacCarty).

2. The analysis of any considerable material, ulcer or cancer, reveals many striking variations in the duration of the morbid process. Both clinical and pathologic differentiation should be made between the terms "old" and "chronic" as applied to ulcer and cancer. Chronicity, pathologically, does not necessarily mean that the disease is old, that is, of long duration in terms of months or years. Large, excavated, calloused ulcers may apparently develop in a few weeks, while many small, indurated round ulcers may give even obstructive symptoms for years. This also applies to cancer. Within 2 weeks of the onset of disability we have seen a patient exhibit general carcinosis, with a large primary mass in the stomach. Another patient may have noticed an epigastric nodule for a year, and yet laparotomy demonstrates a small mass well confined to the wall of the stomach.

The average length of time of all symptoms in our 182 cases clinically satisfying the symptom-complex of cancer was 7.1 months. Of this group the shortest history extended over but 2 weeks, and the longest was about 3 years. In 9 cases ( 1.6 per cent.) cancer of the stomach was found at exploration, when there had been no indications of gastric disorder. Such cases have been described by Osler, Chesnel and others.

Of the 239 cases clinically furnishing the symptomcomplex of a chronic gastric disorder previous to the period of erident malignancy, the average duration of all symptoms was 11.4 years. In this group the average duration of the supervening malignant course was 6.1 months. It seems thus manifest that the periods of downward progression closely approximate in the two classes of cases, wholly inde- 
pendent of the earlier gastric history of the case. From our knowledge of malignant processes in general, it would seem scarcely possible that the "primary" cases of cancer, mentioned before, existed for any considerable length of time without giving clinical evidences of their presence. Especially is this emphasized when we are aware that between 60 and 70 per cent. of all proved cases of chronic gastric ulcer and of gastric cancer are so located in the visceral wall as to early interfere with the stomach's emptying power. We have been frequently impressed by the fact that many so-called "primary" gastric cancers in the early weeks of their disturbance gave such clinical symptoms as are commonly ascribed, clinically, to chronic ulcer.

3. The demonstration that a long-standing gastric -disturbance which is later shown to be malignant was ever benign leads largely into realms of speculation. The chief arguments in support of this supposition appear to be the following:

(a) After gastro-enterostomy for chronic ulcer, when the ulcer is not excised, it is stated that such patient rarely develops gastric cancer (Paterson, Gressot and others). The argument loses much of its force when we recall that in such subject the entire physiology of the stomach and related viscera may have been upset. It is well recognized by able surgeons and physiologists that gastro-enterostomy is more than a simple procedure of "drainage." In the large majority of gastric extracts from stomachs where gastro-enterostomy has been performed, it is possible to demonstrate, chemically or microscopically, both duodenal and jejunal contents. Just what effect these foreign substances have on gastric ulcers or gastric cancers we have yet no means of knowing. We do know, how- 
ever, that the parts of the alimentary tract from which they come are rarely affected with cancer. In our series of cases of gastric cancers, there are four patients which later developed cancer following gastro-enterostomy for ulcer. It also seems to hold that in cases of gastric cancer in which no pyloric obstructions are demonstrable gastro-enterostomy grants a longer lease of life than when such operations have not been performed. After gastro-enterostomy, stomach emptying is more rapid than before. This freeing the viscus of irritant food and secretions, and the cast-off material from ulcers or cancers, might be a not inconsiderable factor in the after course, histologically, of such lesions.

(b) Duodenal ulcer of the indurated type is a relatively more common affection than is gastric ulcer, yet carcinoma of the duodenum is a rarity. It is held that if cancer develops on chronic ulcer a great frequency of its duodenal incidence should be expected. That the duodenum has a protective mechanism against malignancy appears to be shown by the surgical observation that only rarely does cancer at the pylorus, on the stomach side, pass to the duodenum by direct extension. In our series but four such instances were noted. The difference in the character of the tissue in which the chronic ulcer is implanted is also demonstrated by the fact that it is not uncommon to find that primary ulceration of the duodenum which extends up to the pylorus, assumes malignant characteristics on its gastric side while the ulcer on the duodenal side remains benign. There are 6 such cases in our series. Recurrences after removal of cancer are extremely rare in the duodenum. We have also 5 cases in which malignant gastric ulcer was demonstrated together with benign calloused duodenal ulcer. Cancers of the duodenum occur in the 
great majority of instances, at or about the papilla of Vater. It is well known that this region of the viscus suffers traumatism from gall-stones, altered secretion of the liver and pancreas and from infective processes of the gall-tract. The upper part of the duodenum, where ulcer is common, is relatively immune to these influences, and from the intense acidity and the associated peptolytic power of the gastric juice, which may irritate gastric ulcers. Further, food remains but a brief time in the duodenum. It is rapidly passed on into the jejunum, where it stays but briefly. This region is also almost cancerfree. The chyle, however, passes more slowly through the ileum, and as the ileo-cecal valve is approached, malignant processes again appear. The cecum and ascending colon are common grounds of cancer.

(c) Pathologists readily grant that there is a type of gastric affection which they class as "ulcus carcinomatosum." They demonstrate this generally at post-mortem. They do not, however, explain why this type of ulceration exists, by revealing any characteristic changes in the gastric mucosa in which it occurs, nor do they show that from its beginning it was not anything else than malignant. They are willing to grant that it is something different from primary cancer which later ulcerates, but just what this difference is they do not state. This type of affection appear's curiously to exist as an isolated entity with no explanation of its existence or prophecy as to its future course.

(d) Clinicians hold that malignant ulcer exists in from 3 to 6 per cent. of all ulcers of the stomach (Fenwick, Rosenheim and others). The various observers, however, do not detail just how to segregate this group clinically from benign chronic gastric ulcer, nor do they offer suggestions of 
guidance for the determination of just what chronic ulcers are destined to pursue this course.

In our series there were 239 cases which, up to within an average time of 6.1 months before being microscopically demonstrated as cancer, showed nothing to indicate that were a laparotomy to be performed in that period anything other than benign chronic calloused gastric ulcers would be found. Only the subsequent course or the examination of fresh tissue, using high magnification, at operation, revealed the true nature of the affection. In this group of cases cancerous ulcers were found on laparotomy in 105 (43.9 per cent.) and extensive carcinomas, with or without ulceration, in 134 ( 56.1 per cent.). Of the 183 cases with a clinical history of primary carcinoma, ulcus carcinomatosum was demonstrated in 28 (15.8 per cent.), while in 154 ( 84.2 per cent.) extensive growth was found. Of the 106 cases with clinical history or irregular gastric ulcer in the precancerous stage, ulcus carcinomatosum was shown in 22 (20.7 per cent.) and in $\$ 4$ (79.2 per cent.) extensive involvement, with or without secondary ulceration.

These observations suggest several points. Development and careful interpretation of the early-the precancerous-history permits patients coming to laparotomy at a stage when in more than one-half of the instances the maximum advantage of localization of the disease is available, and hence the maximum benefit accrues to the individual case. In about one-fifth of the cases of so-called primary and gastric cancer ulcus carcinomatosum can be demonstrated at operation, and these appear to be generally favorable cases for operative procedure, compared to their fellows. This is especially to be emphasized inasmuch as it has been shown by MacCarty and Blackford that timeduration of symptoms bears no proportionate relation to 
the size and extent of involvement of the lymph nodes, and that the operative and the ultimate mortality are in direct proportion to the amount of involvement of the lymph nodes.

It would appear from the brief consideration of the objections to the interpretation of the so-called "precancerous" history with respect to the succeeding neoplasm having developed an earlier benign chronic gastric ulcer, that so far as we can judge, clinically, the careful development of such history furnishes extremely valuable diagnostic and prognostic information. It would appear from the facts submitted that such interpretation allows the greatest degree of operative benefit with the minimum of operative risk. These facts have especially to be considered when we recall that we have no better clinical guide and that those who object to the significance of the "precancerous" history have nothing better to offer.

\section{SIGNIFICANCE OF CLINICAL SYMPTOMS}

Periodicity of Symptoms.-One of the strongest clinical evidences in the diagnosis of chronic gastric ulcer is the periodic recurrence of dyspeptic symptoms with perfect or fair health between the attacks. Graham, Friedenwald and ourselves have pointed this out. In 239 of the cases furnishing the material for this report in which there was a precancerous history of digestive disturbance, 81 per cent. complained of attacks of discomfort in that period; in 13.3 per cent. the attacks were of rare occurrence, and 4.7 per cent. had had continuous disturbance. When the period of malignancy supervened on the intermittent dyspeptic storm the affection was continuous and progressive in more than 99 per cent., irrespective of the earlier history. In the 182 cases making up the "primary" cancerous group, 
continuous disturbance was noted in 95.1 per cent. and frequent periodic attacks in 4.8 per cent.

Types of Pain.--In the group of cases comprising cancer following previous dyspepsia, severe pain and colic were noted in 23 per cent., steady ache in 48 per cent. and abdominal discomfort in 28 per cent. In the primary cancer group colicky pain was noted in but 6.6 per cent., steady ache in 32.4 per cent., and vague discomfort or "bloat" in 56 per cent., while in 4.3 per cent. there was no abdominal discomfort whatever. Opiate relief of pain was required in 6.5 per cent. of the first group of cases, and in 2 per cent. of the second group.

Food Relation of Abdominal Pain or Distress. - In the diagnosis of chronic gastric ulceration, the relief of discomfort by the ingestion of food (if this form of relief be fairly constant) is granted to be a most valuable diagnostic sign. In a chronic dyspeptic its continuous presence is often almost pathognomonic for ulcer. At the stage when the cases in our series came under observation, of the dyspepsia-preceding-malignancy group, food ease was present in 20.9 per cent., food aggravation in 46.4 per cent., food of negative significance in 27.4 per cent., and uncertain in 5.1 per cent. In the group of primary cancers, food ease was noted in but 3.2 per cent., food aggravation in 57.6 per cent., food of negative significance in 36.8 per cent., and of uncertain effect in 2.1 per cent.

Hemorrhage.--Intermittent bleeding (melena, hematemesis) is considered as almost conclusive evidence that peptic ulcer exists. While only but from 22 to 40 per cent. of gastric ulcers bleed (Friedenwald, Smithies), yet when hemorrhage occurs, other things being equal, it is clinically assumed that ulcer is present. In the series of cancers here analyzed, hemorrhage was noted in 97 cases. Of the group 
designated by history symptomatology as malignancyfollowing-ulcer, hemorrhage occurred in 62.9 per cent.; in the group styled from early history "irregular" ulcer hemorrhage occurred in 19.5 per cent., while in the group of "primary" cancers hemorrhage occurred in 16.5 per cent. Of the whole number bleeding, 52 per cent. bled at least 2 years prior to their coming under observation, while 42 per cent. had bled within 2 years of that time. In 6 per cent. the time of hemorrhage was not determined. Of those who bled within the 2-year period, 77.5 per cent. were cases comprising the non-primary cancerous group.

Anemia.-Of one or more estimations of hemoglobin in 250 of the cases, the arerage hemoglobin was 69.6 per cent. for the primary cancerous group and 67.2 per cent. for the dyspepsia-before-cancer class. The average for the series was 68.1 per cent.

Vomiting.-Of the entire series 326 , or 57.5 per cent., of the cases vomited, and of this number 57 per cent. vomited daily. Of the series, 132 (40.5 per cent.) exhibited delayed romiting. In but $5 \mathrm{~s}$ ( 15 per cent.) was the vomitus dark or "coffee-ground."

\section{SIGNIFICANCE OF PHYSICAL EXAMINATION}

Tumor or ridge in the abdomen (generally epigastric) was demonstrated in 411 instances (72.6 per cent.). It was morable in 63.6 per cent. Of the primary cancerous group tumor was present in 39.8 per cent., while in the ulcer-cancer class it was shown in 60 per cent.

Metastases were demonstrated in $\$ 6$ cases (14.7 per cent.) before laparotomy, and were, in the order of frequency, rectal and pelvic, cervical and axillary, navel and abdominal wall, and in the groin. In the primary cancerous group, metastases were present in 20.5 per 
cent. and in the non-primary class in 13 per cent. When such metastases were present, the cases were generally inoperable.

\section{SIGNIFICANCE OF TEST-MEAL FINDINGS}

The secretory function of the stomach was determined by means of the Ewald breakfast. It was preceded 12 hours by a motor-meal after the suggestions of Strauss and Hansmann. Routine quantitative and qualitative estimations were performed by the Töpfer method.

There is not space here to go into elaborate detail of the information derived from examination of gastric contents in our series of 566 cases of cancer. A few of the more important points will be detailed, and the complete report reserved for a subsequent paper.

Food Remnants.-Motility was interfered with in 73.9 per cent. of the entire series. Of the primary cancerous group, remnants were present in 64.8 per cent. and in the non-primary class in 74.2 per cent.

Acidity of Gastric Extract.-In the primary cancerous group there were 55.4 per cent. of cases in which hydrochloric acid was absent, in 11.5 per cent. hydrochloric acid was between 20 and 50 . In this group 79 per cent. had total acidity under 50 , and $\$ 4$ per cent. had combined acidity and acid salts under 50 .

In the non-primary cancerous group, free hydrochloric acid was absent in 49 per cent., in 20 per cent. it was between 20 and 50 , and in 46.3 per cent. it was under 50 . Total acidity was below 50 in 78 per cent., and combined acidity and acid salts below 50 in 90 per cent.

Lactic Acid.-In the primary cancerous group this was demonstrated in 52.2 per cent., while in the non-primary class it was present in 44.9 per cent. 
Occult Blood.-This was shown (benzidin or guaiac tests) in 73 per cent. of the primary cancerous class, and in 77 per cent. of the non-primary group.

Microscopic Examinations of Gastric Extracts.-These were made on the last 146 cases of gastric carcinoma by the agar-differential-stain method devised by the author.

Oppler-Boas Bacilli.--This form of organism was demonstrated in 93.8 per cent. of all the cases. In this same series, yeasts were shown in 50.7 per cent. and sarcines in 17 per cent. Oppler-Boas bacilli and yeasts were combined in 30 per cent., Oppler-Boas bacilli and sarcines in 10 per cent. and Oppler-Boas bacilli together with yeasts and sarcines in 9.2 per cent. Cells showing atypical mitoses were present in 5 cases.

Special Tests. - In 141 instances of demonstrated cancer the glycyltryptophan test was made. It was positive in 40 per cent. We have already analyzed much of the material at another place in this book.

In 31 cases the hemolytic reaction to alien erythrocytes in vitro was positive in 47.2 per cent.

Woodyatt and Jacques have recently pointed out that in gastric cancers an ereptic ferment, as estimated by the modified formaldehyde titration method suggested by Sorenson and Schiff, can be demonstrated in excess in the gastric extract that has been passed through a Berkefeld filter. Our experience with the original procedure is briefly summarized as follows: The average formaldehyde index of 57 cases of gastric cancer was 21 . The average index of 40 cases of benign gastric ulcer was 10.8 and the average index in 75 cases of duodenal ulcer was 11.9 . In 17 cases of achylia gastrica, the average formaldehyde titration index was 14.1, of 10 cases of pernicious anemia, 14.5 , and in 5 cases of carcinomas of the liver, 4.25 . It 
would appear that in some instances, the estimation of the ereptic power of gastric juice toward peptone solutions is of some value when taken in consideration with clinical history and symptomatology.

Wolff-Junghans' Test for Soluble Albumin.-By this quantitative estimation method we in this series have made 260 tests on gastric extracts showing achylia or free hydrochloric acid below 20 from cases in this series. There were 83 cases of gastric cancer. The reaction was positive in 86 per cent. In this group there were 20 cases of lesser curvature and cardiac malignancy, and the reaction was positive in 75 per cent. Of 11 cases of ulcus carcinomatosum without gastric retention the reaction was positive in 10 cases, or 90.9 per cent. In 11 cases of extragastric carcinomas (liver and gall-tract) the reaction was positive in 10 per cent.; in 17 cases of achylia gastrica, positive in 17 per cent. It would seem that this test is of value when taken in consideration with other evidence in carcinomas not associated with pyloric obstruction or palpable tumor, for example, growths at the cardia, the fundus, high on the lesser currature, and on the posterior wall, and in cases in which a large carcinoma, with considerable induration, holds a pylorus open.

\section{SURGICAL CONSIDERATION}

Location.-In 210 cases (39 per cent.) the ulcus carcinomatosum or the growth was at the pylorus; in 27.1 per cent. on the lesser currature near the pylorus; in 19.3 per cent. general; in 7.2 per cent. on the posterior wall, and in 4.2 per cent. at the cardia. The greater curve was involved in 1.1 per cent., the fundus in 0.75 per cent., and the anterior wall in 0.37 per cent. In 9 cases there were simple and malignant ulcers associated in the same stomach, 
and in 5 cases simple duodenal ulcer and malignant gastric ulcer.

The figures for location are to be contrasted with those of Welch, Brinton, Lebert and others. These authorities observed generally the terminal results of cancer of the stomach; nor were their observations always in a consecutive series, examined by uniform procedures. To any one who has had access to post-mortem material the difficulties connected with primary localization of gastric neoplasms need not be explained. The localization figures of our series, however, closely correspond to the location of chronic, calloused gastric ulcer, as shown by the tables of Welch and myself.

Lymph nodes were involved in 71 per cent., irrespective of the early or late history. In 22.2 per cent., there was no lymph-node involvement and these cases were, as a rule, favorable for operation. Free fluid in the abdomen was present in 3.9 per cent. These were inoperable cases.

There were 16 cases in which a carcinomatous ulcer had been previously excised, but in which the involvement of the lymph nodes (often microscopic only) had been noted-in which the subjects later returned with huge inoperable masses in the epigastrium.

Twelve per cent. of the patients died within 6 months following operation, but 36.6 per cent. remained well for more than 3 years, and 22 per cent. remained well over 5 year's.

Pathology.-It is not feasible to give here a detailed description of the specimens secured at laparotomy in this series of gastric cancers, and only a gross summary will be made.

The specimens were first examined in frozen section within a few minutes after their removal from the patients. 
They were next fixed in Melinkow's modification of Kaiserling's fluid and again sectioned and examined.

Full reports have been made by MacCarty and recently some of the specimens furnish part of the study of Wilson and McDowell.

Types of Growth.-Adenocarcinomas were demonstrated in 556 instances (98.2 per cent.); colloid carcinomas, 5 times; fibromas, 4 times, and sarcoma once. In 155 instances (27.4 per cent.) ulcera carcinomatosa were shown. These may have been primarily such, had formed from previous chronic ulcer or had resulted from surface proteolytic ulceration of preceding cancer. It is often impossible to say, without clinical history or test-meal findings, whether an ulcus carcinomatosum developed as such or whether it is a secondary result of a "primary" cancer. At the present stage of our knowledge, the surgical pathologist can positively say only that in a given specimen of chronic, indurated gastric ulcer, cancer is, or is not present. There appears to be a border-line class, however, in which surgical pathologists of the widest experience in the examination of fresh or fixed tissue can often distinguish cellular arrangement or intracellular change of such nature as to warrant their stating that the process is "precancerous." Not infrequently the subsequent course of the ailment bears out the histologic prophecy.

In the experience of Wilson and MacCarty, 71 per cent. of 153 cases of undoubted gastric carcinomas presented gross and microscopic evidence of previous ulcer. These observers also demonstrated that 68 per cent. of resected chronic ulcers of the stomach and duodenum (the latter furnishing a very small proportion of cases) were associated with eancer. In several instances, MacCarty noted that 
the presence of erosions, simple round ulcer and ulcus carcinomatosum in the same specimen suggested possibilities of transition corresponding to that shown by Wooley in cases of adrenal tumor. MacCarty has emphasized the difficulties of always differentiating between simple hyperplasia and malignant hyperplasia. He suggests that hyperplasia is a forerunner of malignancy, that hyperplasia varies in degree, that cancer is malignant hyperplasia, which also varies in degree, and that some degrees of both processes are indistinguishable histologically. This view is well within the opinion of Adami. It seems to be partially substantiated by the recent work of Drew and of Levin in experimental tissue proliferation and inoculation of malignant tumors.

Association of Malignant and Benign Processes.-In this series of gastric cancers there were five instances of simple ulcer of the duodenum associated with malignant gastric ulcer. In 9 cases, simple and malignant ulcers were found in the same stomach. Independent cancer of the stomach and of the duodenum was demonstrated once. There were 2 cases of multiple gastric cancers. Six times it was observed that malignant gastric ulcers stopped sharply when duodenal mucosa was reached, but in seven instances of extensive gastric cancers it was shown that the duodenum was secondarily involved by direct extension.

\section{REFERENCES}

Brinton: Diseases of the Stomach, 2nd edition, pp. 124, 133.

Bolton, C.: Ulcer of the Stomach, 1913, London, p. 6.

Bolton: Quarterly Journal of Medicine, Vol. v, No. 20, p. 438; Brit.

Med. Jour., 1910, Vol. i, p. 1222; Waus. Path. Sect. Ray. Soc. Med. (London), 1910, Vol. iv, No. 2, p. 57; Jour. of Path. and Bact., 1910, Vol. xiv, p. 418.

1. Sirthies: Am. Jour. Med. Sc., 1913, March, p. 340.

2. Graham: Collected Papers by the Staff of St. Mary's Hospital, i,'111. 
3. Suutz: Wien. klin. Wehnsehr., 1912, Oct. 10, p. 1513.

4. Friederwald: An. Jour. Med. Sc., 1912, August, p. 157.

5. Osler axd MicCrae: Cancer of the Stomach, Practice of Medicine, 1912 .

6. Wilsox and MacCarty: Am. Jour. Med. Sc., 1909, December, p. 846 .

7. MacCarty: surg., Gynec. and Obst., 1910, May, p. 449.

S. Osler : Philadelphia Med. Jour., 1900, p. 245.

9. Chessel: Thése de Paris, 1Sti.

10. Paterson: Surgery of the Stomach, 1913, p. 248.

11. Gressot: Berl. klin., Wehnschr., 1912, xlix, 22.

12. Fenwick: Quoted by Paterson, Surgery of the Stomach, 1913, p. $2 \pm 5$.

13. Rosenhema: Ztschr. f. klin. Med., Breslau, 1890, vii, 116.

15. Grahan: Prominent Symptoms in the Diagnosis of Gastric and Duodenal Ulcers, The Journal A. M. A., 1908, Aug. 22, p. 651.

16. Surthies: A Method for the Microscopic Examination of Gastric Extracts and of Feces, Arch. Int. Med., 1912, June, p. 736.

17. Woodyatt, R. T. and Jaceues, J. L.: The Peptolytic Power of Gastric Juice and Saliva with Special Reference to the Diagnosis of Cancer, Arch. Int. Med., 1912, December, p. 560.

1S. Sorenson And Schiff: Zeitschr. f. physiol. Chem., 1909, xiii, 27.

19. WelciI: Cancer of the Stomach, American System of Medicine, ii.

20. Brintox: Brit. and For. Med.-Chir. Rev., 1S57, January.

21. Lebert: Traite pratique des maladies cancereuses, Paris, 1851, p. 97 .

22. Welch: Simple Ulcers of the Stomach, Pepper's System of Medicine, ii.

23. Wooley: Tr. Assn. Am. Phys., 1902, No. 17, p. 627.

24. AdAmi: Malignancy; Principles of Pathology, p. 616.

25. Drew: Jour. Path. and Bacteriol., 1912, July, p. 42.

26. Levin: Jour. Exper. Med., 1912, No. 12, p. 149. 


\section{CHAPTER IX}

\section{GASTRIC CANCER IN THE YOUNG}

We have tabulated 18 instances of gastric cancer in individuals under age 31. These occurred in my study of the records of 921 pathologically demonstrated cases of cancer of the stomach. It is thought that their analysis will demonstrate certain facts of value in anticipating or detecting this grave malady at an age when it is not commonly suspected.

Those who wish to familiarize themselves with the literature on this subject may refer to the excellent monographs of Welch, Osler and McCrae, and Dock.

A summary of these previous reports on gastric cancer in the first three decades may be of service. Six instances (often rather dubious as to cancer) have been recorded below the age of 10 . In the second decade 15 cases are described. In but 9 of these there were reliable pathologic reports. In the thirteen instances occurring in the third decade, few descriptions are accompanied by pathologic findings. Many of the patients in this group, however, appeared to have been affected with a gastric malady, seemingly malignant.

Incidence.-The relative frequency of gastric cancer in the young varies with the individual series studied. In a small series as, for example, the beautifully analyzed 150 cases of Osler and McCrae, the proportion was 4 per cent. In a large series, collected for statistical purposes from various non-related sources throughout the world, as in the 2,038 instances tabulated by Welch, the ratio was 
2.5 per cent. In my own group of 921 consecutive cases, examined by a uniform method (and a distinctly American experience), the percentage was 2.17 per cent. Sex: There were 12 females and $S$ males. Age: The youngest patient was aged 18 ; the oldest just past 30 years. The average age of the series was 27.2 years. Status: Twelve patients were married. In two instances (12 per cent.) there was a family or blood-relationship history of cancer. Only twice were more than ordinary indulgence in tobacco or alcohol noted. Apparently occupation had no etiologic significance.

Duration of all Gastric Complaints. - This data concerns but sixteen instances. Most useful facts are brought out by study of this phase (see Table 36 ). It will be noted that the shortest time was 4 months, while in one instance the gastric disturbance had persisted for 15 years. The average length of time of the disability was 4.2 years.

Consideration of the earlier part of those histories in patients exhibiting protracted gastric complaints not infrequently reveals the fact that there is in the early stages a different clinical presentation of the disease than is exhibited toward its end. It would seem that the observation of occasional cases of cancer in which the duration of the gastric history had been long drawn-out, doubtless led such clinicians as Dock and Nathieu to venture the opinion that cancer of the stomach in early life runs a somewhat slow course. This opinion appears well-founded if one considers both the "pre-cancerous" and the evidently cancerous portions of the history.

Types of Gastric Histories.-In respect to types the histories divide themselves quite sharply into two groups: Group 1 includes cases in which there appeared a gastric affection, pernicious in nature and progressively downward 


\begin{tabular}{|c|c|c|c|c|c|c|c|c|c|c|c|c|c|c|c|c|c|c|c|}
\hline \multirow[b]{2}{*}{ 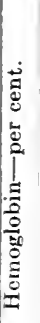 } & \multirow[b]{2}{*}{ 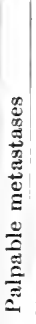 } & \multicolumn{11}{|c|}{ Test-meal } & \multicolumn{7}{|c|}{ Surgical and pathologic findings } \\
\hline & & . & $\frac{D}{D}$ & 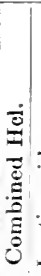 & 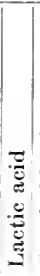 & 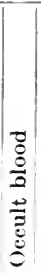 & 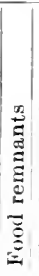 & 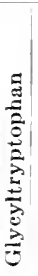 & $\frac{1}{3}=$ & 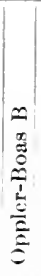 & $\underset{-}{\stackrel{\infty}{\pi}}$ & 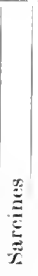 & 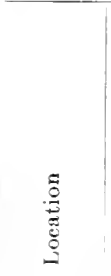 & $\frac{\stackrel{5}{0}}{0}$ & 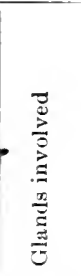 & 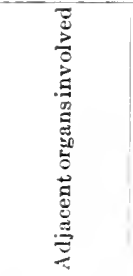 & 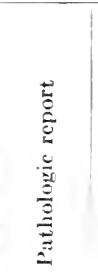 & 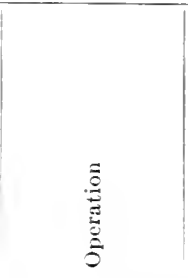 & 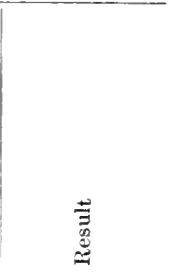 \\
\hline 0 & + & 64 & $64 !$ & 0 & 0 & + & + & $?$ & $?$ & 0 & + & + & Pylorus & $\begin{array}{l}\text { Infiltra- } \\
\text { ting mass. }\end{array}$ & + & $\begin{array}{l}\text { Pancreas } \\
\text { and liver. }\end{array}$ & $\begin{array}{l}\text { Carcin } \\
\text { oma. }\end{array}$ & $\begin{array}{l}\text { Posterior gast.- } \\
\text { ent. }\end{array}$ & Died in 6 mos. \\
\hline $0^{3}$ & 0 & 28 & 16 & 8 & $?$ & + & + & 0 & + & $?$ & + & + & General & $\begin{array}{l}\text { Infiltra- } \\
\text { ting nass. }\end{array}$ & ++ & $\begin{array}{l}\text { Pancreas, } \\
\text { liver and } \\
\text { perito- } \\
\text { neun. }\end{array}$ & $\begin{array}{l}\text { Carcin- } \\
\text { oma. }\end{array}$ & Explored & $\begin{array}{l}\text { Died } 1 \text { day } \\
\text { after oper. }\end{array}$ \\
\hline$?$ & 0 & 35 & 181 & 12 & + & $t$ & + & $t$ & $?$ & + & + & 0 & Pylorus & $\begin{array}{l}\text { Nodule (ul- } \\
\text { cer?) size } \\
t \text { in. }\end{array}$ & $\begin{array}{l}\text { t+ } \\
\text { Peri- } \\
\text { to- }\end{array}$ & $\begin{array}{l}\text { Liver and } \\
\text { perito- } \\
\text { neum. }\end{array}$ & $\begin{array}{l}\text { Carcin- } \\
\text { oma. }\end{array}$ & $\begin{array}{l}\text { Anterior gast.- } \\
\text { ent. }\end{array}$ & Alive 3 years \\
\hline 5 & + & $5 \tilde{5}$ & & & + & + & + & ? & $?$ & + & 0 & 0 & $\begin{array}{l}\text { Lesser } \\
\text { curve } \\
\text { and py- } \\
\text { lorus. }\end{array}$ & $\begin{array}{l}\text { Huge ma- } \\
\text { lignant } \\
\text { ulcer. }\end{array}$ & $\begin{array}{l}\text { neum } \\
++t\end{array}$ & 0 & $\begin{array}{l}\text { Carcin- } \\
\text { oma. }\end{array}$ & Erplored & Died in 6 mos. \\
\hline 0 & 0 & 64 & 451 & 19 & 0 & 0 & 0 & $?$ & $?$ & 0 & + & + & $\begin{array}{l}\text { Malig. } \\
\text { ulcer } \\
\text { on less. } \\
\text { cury. }\end{array}$ & $\begin{array}{l}\text { Inop. large } \\
\text { adherent } \\
\text { mass. }\end{array}$ & + & 0 & $\begin{array}{l}\text { Carcin- } \\
\text { oma. }\end{array}$ & $\begin{array}{l}\text { Posterior gast.- } \\
\text { ent. }\end{array}$ & Lived 5 years \\
\hline 5 & 0 & $60 \mathrm{c}$ & 60 & 0 & 0 & + & 0 & $?$ & $?$ & 0 & 0 & 0 & $\begin{array}{l}\text { curv. } \\
\text { Lesser } \\
\text { curva- } \\
\text { ture. }\end{array}$ & $\begin{array}{l}\text { Malignant } \\
\text { ulcer. }\end{array}$ & 0 & Pancreas & $\begin{array}{l}\text { Carcin- } \\
\text { oma. }\end{array}$ & $\begin{array}{l}\text { Witzel jejunos- } \\
\text { tomy. }\end{array}$ & Alive. \\
\hline 0 & 0 & 402 & 281 & 10 & 0 & 0 & 0 & $?$ & $?$ & 0 & 0 & 0 & Pylorus & $\begin{array}{l}\text { Inop. infil- } \\
\text { trating }\end{array}$ & 0 & $\begin{array}{l}0 \text { Gall- } \\
\text { stones. }\end{array}$ & $\begin{array}{l}\text { Carcin- } \\
\text { oma. }\end{array}$ & $\begin{array}{l}\text { Posterior gast.- } \\
\text { ent. }\end{array}$ & Alive 2 years \\
\hline ) & 0 & $2 S$ & 02 & 20 & + & + & + & $?$ & $?$ & + & 0 & 0 & $\begin{array}{l}\text { Lesser } \\
\text { curve } \\
\text { and } \\
\text { post. } \\
\text { wall. }\end{array}$ & $\begin{array}{l}\text { Inass. } \\
\text { maperable } \\
\text { mass. }\end{array}$ & ++ & Pancreas & $\begin{array}{l}\text { Carcin- } \\
\text { oma. }\end{array}$ & Explored & Died in $1 \mathrm{yr}$. \\
\hline & 0 & 453 & $3+1$ & 11 & 0 & + & 0 & $?$ & $?$ & 0 & + & + & $\begin{array}{l}\text { Lesser } \\
\text { curve } \\
\text { and } \\
\text { ant. } \\
\text { wall. }\end{array}$ & $\begin{array}{l}\text { Malignant } \\
\text { ulcer. }\end{array}$ & + & 0 & $\begin{array}{l}\text { Carcin- } \\
\text { oma. }\end{array}$ & $\begin{array}{l}\text { Miculicz-Hart- } \\
\text { man- Billroth } \\
\text { No. } 2 \text { and } \\
\text { post. gast.- } \\
\text { ent. }\end{array}$ & Alive 2 years. \\
\hline & 0 & 33 & 52 & 26 & 0 & + & + & + & $?$ & + & 0 & 0 & $\begin{array}{l}\text { Post. } \\
\text { wall. }\end{array}$ & $\begin{array}{l}\text { Malignant } \\
\text { ulcer. }\end{array}$ & + & 0 & $\begin{array}{c}\text { Carcin- } \\
\text { onia. }\end{array}$ & $\begin{array}{l}\text { Miculicz-Hart- } \\
\text { man-Billroth } \\
\text { No. } 2 \text { and } \\
\text { post. gast.- } \\
\text { ent. }\end{array}$ & Died $S$ mos. \\
\hline$?$ & 0 & 46 & 143 & 32 & + & + & + & $?$ & $?$ & 0 & + & 0 & $\begin{array}{l}\text { Lesser } \\
\text { curva- } \\
\text { ture }\end{array}$ & $\begin{array}{l}\text { Large ma- } \\
\text { lignant } \\
\text { ulcer. }\end{array}$ & + & 0 & $\begin{array}{l}\text { Carcin- } \\
\text { oma. }\end{array}$ & $\begin{array}{l}\text { Pylorectomy } \\
\text { and post. } \\
\text { gast.-ent. }\end{array}$ & Alive 5 years \\
\hline & + & 56 & 421 & 14 & 0 & 0 & + & $?$ & $?$ & 0 & + & 0 & $\begin{array}{l}\text { Post. } \\
\text { wall. }\end{array}$ & $\begin{array}{l}\text { Huge ma- } \\
\text { lignant } \\
\text { ulcer. }\end{array}$ & + & 0 & $\begin{array}{l}\text { Carein- } \\
\text { onia. }\end{array}$ & $\begin{array}{l}\text { Milculicz-Hart- } \\
\text { man-Billroth } \\
\text { No. } 2 \text { and } \\
\text { post. gast.- } \\
\text { ent. }\end{array}$ & $\begin{array}{l}\text { Patient died } \\
112 \text { years la- } \\
\text { ter with ova- } \\
\text { rian metas- } \\
\text { tases. }\end{array}$ \\
\hline & 0 & 60 & 461 & 14 & 0 & + & + & $?$ & $?$ & 0 & + & + & $\begin{array}{l}\text { Lesser } \\
\text { curva- } \\
\text { ture. }\end{array}$ & $\begin{array}{l}\text { Early ma- } \\
\text { lignant } \\
\text { ulcer. }\end{array}$ & 0 & 0 & $\begin{array}{l}\text { Carcin- } \\
\text { oma. }\end{array}$ & $\begin{array}{l}\text { Miculicz-Hart- } \\
\text { man-Billroth } \\
\text { No. } 2 \text { and } \\
\text { post. } \\
\text { ent. }\end{array}$ & $\begin{array}{l}\text { Died } 1 \text { year } \\
\text { ovarian and } \\
\text { uterine metas- } \\
\text { tases. }\end{array}$ \\
\hline & 0 & $\begin{array}{r}\mathrm{Nc} \\
\mathrm{n} \\
\mathrm{c}\end{array}$ & $\begin{array}{l}\text { o c } \\
\text { not } \\
\text { card }\end{array}$ & lia. & be & nts. & pas & & & & $\begin{array}{l}\text { coul } \\
\text { yon }\end{array}$ & & $\begin{array}{l}\text { High on } \\
\text { lesser } \\
\text { curve } \\
\text { and at } \\
\text { cardia. }\end{array}$ & $\begin{array}{l}\text { Malignant } \\
\text { ulcer. }\end{array}$ & + & $\begin{array}{l}\text { Liver, ova- } \\
\text { ries, peri- } \\
\text { toneum. }\end{array}$ & $\begin{array}{c}\text { Carcin- } \\
\text { oma. }\end{array}$ & Explored & Alive 1 year \\
\hline 55 & 0 & 40 & & & $t$ & + & + & + & + & + & 0 & 0 & $\begin{array}{l}\text { Lesser } \\
\text { curva- } \\
\text { ture. }\end{array}$ & $\begin{array}{l}\text { Huge ma- } \\
\text { lignant } \\
\text { ulcer. }\end{array}$ & ++ & $\begin{array}{l}\text { Liver, pan- } \\
\text { creas, } \\
\text { periton. }\end{array}$ & $\begin{array}{l}\text { Carcin- } \\
\text { oma. }\end{array}$ & $\begin{array}{l}\text { Anterior gast.- } \\
\text { ent. }\end{array}$ & $\begin{array}{l}\text { Died in } 216 \\
\text { months. }\end{array}$ \\
\hline 60 & 0 & 38 & 62 & 26 & + & & + & + & + & + & 0 & 0 & Pylorus & $\begin{array}{l}\text { Large } \\
\text { mass. }\end{array}$ & $+t$ & Pancreas & $\begin{array}{l}\text { Carcin- } \\
\text { oma. }\end{array}$ & $\begin{array}{l}\text { Anterior gast.- } \\
\text { ent. }\end{array}$ & $\begin{array}{l}\text { Died in } \\
\text { days. }\end{array}$ \\
\hline
\end{tabular}

ally. 


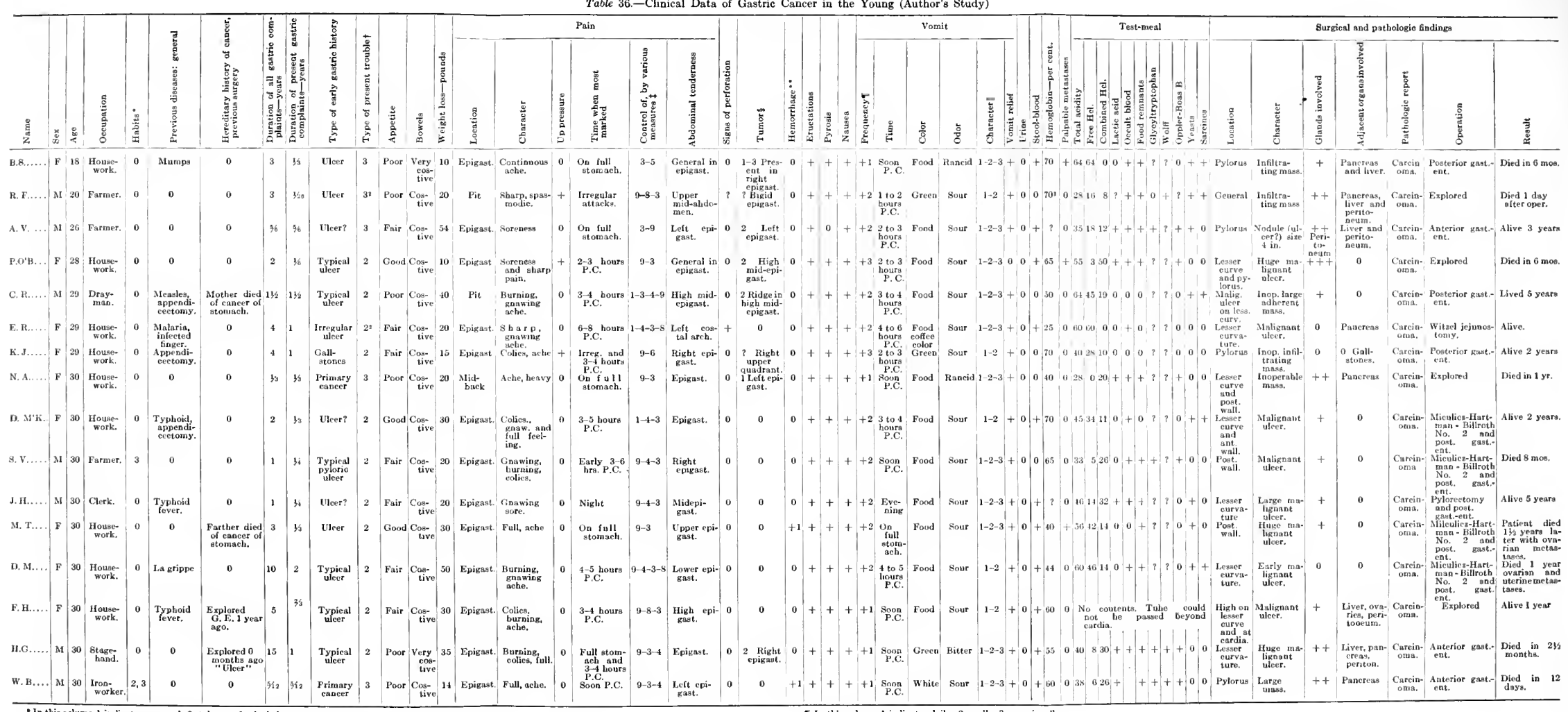


in course, in patients in whom there had been no previous gastric ailment. Group 2 comprises a number of cases in which, previous to the time at which they came under observation for a gastric disease of a malignant kind, there had been gastric complaints which frequently conformed to the type which we call clinically "peptic ulcer."

Group 1.-Two of the 16 cases studied fall into this class. The average duration of all symptoms was 4.5 months. In these 2 cases the disease was from its inception progressively and perniciously downward in course and type, and there was no alteration in the character of the affection. There were, clinically, rapid emaciation, continuous abdominal discomfort, early onset of vomiting, development of epigastric tumor and departures from the normal in the test-meal analysis (see Table 36). Laparotomy revealed large, inoperable growths, with extensive involvement of the lymph nodes and pancreas. One patient died within 12 days following operation; the other lived a year.

Group 2.-This includes 14 cases. These patients had been affected for an average time of 4.8 years with some gastric malfunction. In type the early part of their affection had been roughly classed among the "dyspepsias." A careful analysis of the character of these dyspepsias reveals useful facts. In five instances (36 per cent.) the gastric complaint and course had fully satisfied the symptomcomplex which we ascribe clinically to peptic ulcer. In 4 cases (25 per cent.) so-called ulcer features were definite at some stage in the early period (in one instance in this group there was frank gastric hemorrhage). In 4 other cases (25 per cent.) the long-term history previous to onset of evident malignancy was that of peptic ulcer of irregular type. In the remaining case, gall-stone attacks were 
typical through 4 years, and the stones were later found at laparotomy.

The second part of the histories of cases making up Group 2 was typical of gastric malignancy. While this phase differed widely in character from the early manifestations, yet it could in nowise be distinguished clinically from the whole course of the ailment in the 2 cases making up Group 1. If one had not carefully analyzed the early histories of these 14 cases in Group 2 he could have in no way shown their clinical variation from cases in Group 1. The period of evident malignancy in Group 2 averaged 7.8 months in duration. The most rapid progress was 3 weeks; the most protracted nearly 3 years.

It might be useful to emphasize the fact here that of this group of gastric cancer cases in the young with longtime histories, 86 per cent. satisfied or approximated our present-day clinical complex of peptic ulcer at some time during a period averaging 4.8 years, previous to the time when the course became malignant; that during this time medical treatment arailed nothing and the surgical opportunity for cure was usually neglected; that the cases in this group, due to some unknown cause, did not pursue a course of chronicity of benign type, but later evidenced malignant pointings, and that the malignant course of the cases in this group exhibited an average duration nearly half again as long as occurred in cases of Group 1 in which no previous gastric disorder had been observed.

Clinical Data.-Appetite.-In six instances (38 per cent.) the appetite was poor. In the remaining cases it was usually fair.

Bowels.-All cases exhibited some degree of constipation.

Weight Loss.-In the two instances of short-term cancer (the so-called "primary cancers"), the average loss of 
weight was 17 pounds. In the early period of the group of cases with long-term histories, weight loss generally occurred during the "spells" of the disability, but was rapidly regained during the succeeding quiescent stages. In some of these spells the weight loss was as much as 35 pounds. In the late stage of cases of this type (the malignant period) the weight loss averaged 31 pounds. It was continuous and accompanied by other evidences of cachexia. Some rapid weight losses were observed, e.g., 20 pounds in 2 weeks.

Abdominal Pain or Distress.--In some degree, this was noted in all patients. It was commonly located indefinitely in the epigastrium, but occasionally the complaint was of distress in the back or the high "pit." In two instances pains of so severe a degree and of such spasmodic character were noted that perforation was believed to have taken place.

In the 2 cases comprising Group 1 above ("primary cancers") the pain was never severe, never definitely localized, was continuous, but was generally aggravated by the ingestion of food or drink. In these instances the common pain relief was obtained by limiting the amount of ingested food, vomiting at the height of distress, and, occasionally, by alkalies.

Of the 14 cases included in Group 2 above (those in which the patients had had gastric distress before malignancy became evident), 12 (85 per cent.) had pain in "spells" or "attacks." In 7 instances, the pain at such times had borne fairly definite relation to food ingestion; in 4 instances, even when malignancy had supervened, the food relief of pain persisted. In 10 cases former food relief of pain had changed to food aggravation of pain, as the malignant process became manifest. In this group of 
14 cases the common modes of pain relief were romiting, lavage, limited or varied diet, alkalies, food intake and some form of opiate.

Abdominal Tenderness. - This was exhibited on palpation by all patients. In 38 per cent. it was located generally in the epigastrium. In 3 instances, the maximum tenderness was in the left epigastrium. In rare cases was abdominal tenderness, such as is characteristic of chronic ulcer of the perforative type, noted even when laparotomy showed that the peritoneum had become extensively involved.

Epigastric Tumor.-Tumor was palpated in 6 cases (3S per cent.) in the entire series (see Table 36 ). In but one instance was a large mass demonstrable. In the cases presenting no definite tumor, peculiar stiffness of the abdominal wall with a sense of deep resistance was frequently observed.

Four times free fluid was shown to be present in the abdominal cavity before laparotomy. These were inoperable cases. In three metastases, as in the left supraclavicular region and pelvis, were noted before operation. These patients had hopeless involvement.

Eructations and Pyrosis.- Such were commonly noted particularly when gastric motility had been interfered with.

Vomiting.-Vomiting, with or without nausea, was observed in all the cases at some time (usually in the terminal stage) in the course of the disease. In the two "primary cancers," vomiting came on daily soon after taking food, was large in quantity, rancid in odor, but never "coffee colored."

In the fourteen instances in which malignancy had terminated a long-term gastric history, vomiting occurred from 1 
to 8 hours after meals; in but one instance was it "coffee colored," in all instances it contained food particles, was of a rancid color and relieved abdominal distress. In 8 cases "delayed" vomiting was observed. Such sign was usually associated with pyloric stenosis and huge, dilated stomach.

Laboratory Data.-Blood.- In the fourteen instances in which the hemoglobin was estimated, it areraged 66 per cent. The range was from 25 per cent. to 70 per cent.

Stool.-In ten instances (62.5 per cent.) altered blood was chemically demonstrated by the benzidin or the guaiac tests.

Test-meals.-In fifteen instances facts of much interest were obtained by this mode of examination of gastric function. One case (No. 14, see Table 36) returned no information by test-meal analysis because the stomach tube could not be advanced beyond the cardia. There was obstruction due to extensive local growth.

Gastric Emptying Power.-In 11 cases (73 per cent.) motility was interfered with and there was retained food at the end of 12 hours. In such instances, dilation of the stomach of various degrees had invariably occurred.

Gastric Acidity.-In no case was achylia shown. The average total acidity was 59 . It ranged from 28 to 64 .

Free hydrochloric acid was absent in but one instance. This was a case of fulminant, primary cancer. The average free hydrochloric acid estimation for the entire series was 26. It ranged from as low as 3 to as high as 60 . The highest free hydrochloric acidities were noted in association with large, cancerous ulcers.

Combined hydrochloric acid and acid 'salts averaged 18.1 per cent. It ranged from 0 to 50 .

Lactic acid was demonstrated by the modified Uffelmann test in 6 cases (40 per cent.). 
Altered blood was proven by chemical test in gastric contents 12 times ( 80 per cent.).

Microscopic Examination of Unflttered Gastric Extracts.In but 6 instances (40 per cent.) were organisms of the Oppler-Boas group definitely identified. In 8 cases (53 per cent.) yeasts and sarcinæ were present. Microscopic food bits were recognized in nearly 90 per cent. of instances.

The Laparotomy Findings. - Two cases had been explored before coming to us. Both the patients had been operated on within the year. At both abdominal sections the surgeon had made a clinical diagnosis of "benign" gastric ulcer of the chronic type, yet both of these patients were dead from inoperable gastric cancer before a year had elapsed! The resection of the ulcer or the microscopic examination of a bit of extipated tissue at the time of the initial laparotomy doubtless would have made known earlier the prognosis and have prevented further surgery. It might have saved life.

Location of the Malignant Process.-Abdominal section revealed five instances in which the pylorus was involved; 9 cases in which the lesser currature and some part of the surface of the viscus were affected; one instance of infiltration at the cardia, and 1 case of general carcinosis.

Lymph nodes had been invaded in 14 out of the 16 cases in this series. Nine times secondary growths were demonstrated in the liver, pancreas, omenta or pelvic organs.

Character of Neoplasms. - In eight instances, extensive ulcera carcinomatosa were present. They were of the medullary type. In the remaining cases, adeno-carcinomatosa of the common type were demonstrated.

Operative Procedures and Outcome.-In four instances exploration only was possible. In 5 cases some form of resection was performed with or without gastrojejunos- 
tomy. In the remaining seven patients, "drainage operations" to fit the case at hand were devised.

Nine patients died within $11 / 4$ years following operation. To the other patients of this series was granted a lease of life of from 2 to more than 5 years.

\section{REFERENCES}

1. WeLch: Proceedings Boston Society for Medical Improvement, v, Appendix, p. 109.

2. Osler and McCrae: Cancer of the Stomach, Philadelphia, 1900, p. 16.

3. Dock: Am. Jour. Med. Sc., 1897, June, p. 665.

4. Mathiev: Semaine med., 1895, p. 225.

Siıthies: Jour. Am. Med. Assn., 1914, Nov. 21, p. 1839.

Note: We have since had opportunity of observing three other cases. They were a male aged 24 and two females aged 18 and 27 . They are not analyzed in the data herewith. 


\section{DIFFERENTIAL DIAGNOSIS}

General.-Every case of chronic dyspepsia should be broadly considered from two aspects, namely, the surgical and the medical. This applies with especial force to instances of indigestion-chronic or of recent onset-in patients above 35 years of age. The primary principle of differential diagnosis of intraabdominal disease, or disease with intraabdominal pointings, is that of segregating the group likely to be benefited by laparotomy from that where the hope of relief lies in general medical care. It is admitted that this division of cases is not always easily made. Doubtful instances are of common enough occurrence. There is, however, too much unnecessary and usually valueless quibbling over finer points of differential diagnosis in abdominal disease. This is often to the patient's detriment. While learned, pseudo-scientific investigations are being carried on, or while certain medical attendants are awaiting the appearance of their pet differential points, not infrequently the subject of the research passes beyond any form of aid. Rash surgery is to be condemned, but a sharp scalpel is often a more satisfactory differential diagnostician than is the keenest mind, medically.

Exploratory laparotomy has perhaps been needlessly urged in many instances of chronic dyspepsia. In these cases it is not infrequently found that but a limited study has been made of the gastro-intestinal functions before such laparotomy. Not rarely one could have accurately 
prognosed unsatisfactory surgical findings or results from operation before laparotomy, if routine examinations had been systematically carried out. While there is a tendency on the part of many clinicians, particularly the surgeons, to perform abdominal section in order to make optically evident intraabdominal pathology, yet this procedure must not be condemned as a diagnostic method simply because groups of poorly trained surgeons are getting negative results by it.

When every case of chronic dyspepsia has been considered a strictly medical one before it is admitted to be surgical, exploratory laparotomy very often yields results which are satisfactory to both the patient and clinician.

Internists have not been backward in claiming that abdominal surgery is too readily resorted to for the purposes of diagnosis, and that such procedure is essentially the lazy man's way of elucidating a problem which could have been very well worked out along other lines. The internist's position, however, is not wholly an unassailable one. While much may be expected from the medical treatment of gastric ulcer in competent hands, it is a fact that the large majority of malignant gastric ulcers are allowed to progress to the hopeless stage before they are referred to the surgeon.

Mortality in gastric cancer can only be successfully attacked by a greater number of obstinate peptic ulcer cases being treated surgically than is at present the vogue. The physician's waiting until the so-called clinical picture of gastric cancer appears, is practically equivalent to signing the patient's death certificate. From what has already been stated in Chapters II and III, it is quite evident that the early diagnosis of gastric cancer concerns itself with the recognition of chronic calloused peptic ulcer, 
particularly of the recurrent form. We would especially emphasize this point. Inasmuch as it cannot be too strongly urged, we offer the following summary of the essential points in the diagnosis of chronic calloused ulcer of the stomach and the duodenum.

\section{GASTRIC ULCER}

1. Facts Determined from History.-(a) Frequently, dietetic or hygienic irregularities. Males are more frequently affected than are females.

(b) History of recurring acute infections. (La grippe, tonsillitis, exanthemata, etc.). Seasonal relation of distress not uncommon, exacerbations occurring in fall or spring.

(c) Association with disease of appendix or gall-bladder (with which ulcer, especially in subjects below age 30 , is often confused).

(d) Periodicity of complaint occurs in from 75 to 85 per cent. until complications set in. Between "spells" or attacks of indigestion, so-called, there is generally good gastric health. Weight is not infrequently lost during attacks and rapidly gained when such cease.

(e) Epigastric distress is present in more than 95 per cent. It varies in severity, from discomfort to severe, gnawing or cramp-like pains. Discomfort has point of maximum location, subjectively, in practically 3 out of 4 cases. Pain has usually reached its height within 4 hours following meals. Pain comes on sooner past cibo in ulcers located near the cardia than where such are well toward the pylorus.

(f) Food relief of distress occurs in four out of five instances of peptic ulcer of the uncomplicated type. Relief of pain frequently bears relation to amount of food taken, i.e., a large meal gires longer relief than a small one. Pain 
is also relieved by vomiting, the taking of alkalies, by rest, diet and opiates.

(g) Vomiting occurs in more than two-thirds of the instances; romitus usually comes on at the height of gastric distress and when acidity is highest. Tomitus of food that has lain in the stomach longer than 6 hours ("delayed vomit") increases as complications (stenoses or perforation) develop. Pyrosis, water-brash, eructations and sour belching are common on ordinary diet.

(h) Hemorrhage (hematemesis or melena) occurs in from 20 to 40 per cent. of instances. While hematemesis is more frequent than melena, yet melena alone may occur wholly irrespective of the location of the gastric ulcer. Severe hemorrhage is accompanied by signs of shock and collapse.

2. Facts Obtained by Examination.-(a) Patient is usually well nourished without toxic or cachectic appearance, unless pyloric stenosis or "hour-glass" contraction has occurred.

(b) Average hemoglobin about 80 per cent. unless severe hemorrhage has recently taken place.

(c) Area of epigastric tenderness in region of pyloric half of stomach. This is usually in the mid-epigastrium, but not necessarily so. The area is most frequently definitely local where acute or chronic perforation has taken place. A tender ridge may sometimes be palpated where a large chronic ulcer exists.

(d) Dilated, splashy stomach occurs where marked pyloric spasm exists or stenosis has taken place. If this is excessive, visible peristalsis (and rarely, "reverse" peristalsis) may be noted.

3. Facts Secured by Laboratory Examination.-(a) Testmeal.-Motility interfered with in more than 50 per cent. 
of instances. Gastric acidity increased with respect to free hydrochloric acid in the majority of non-stenosing ulcers. In stenosing ulcers, with dilatation of the stomach, while free hydrochloric acidity may not be above normal, the total acidity is increased in greater ratio. Blood may or may not be present in gastric extracts (macroscopically or by chemical test). Lactic acid is a rare finding. Pepsin and rennin are frequently increased.

Microscopically where gastric dilatation has occurred, fermentative changes are proved by the finding of great numbers of budding yeasts and of sarcinæ (large and small types) together with remnants of retained food.

(b) Stool may show nothing pathologic. Recent hemorrhages generally result in the passage of "tarry" stools for several succeeding days. Perforation of an ulcer to the pancreas, not infrequently brings on pancreatic inefficiency with passage of stools containing undigested food.

During periods of the ulcer's activity, if the patient is kept upon meat-free or milk diet for several days, at the end of such time the stool may be shown by chemical tests (benzidin or guaiac) to contain blood. Progressive ulcers, or ulcers undergoing cancerous change, generally show blood constantly in the stools by chemic tests.

(c) X-ray Findings.-In many instances of uncomplicated ulcer no facts are returned after most careful examination by both fluoroscopic or plate methods. Complicated ulcers (stenosing, calloused, "hour-glass" producing, perforating, etc.), are recognizable in nearly three out of four instances by the combined screen and plate method.

1. Positive Signs.-The "niche" or "accessory cavity," indicating calloused, penetrating ulcer.

2. Corroborative Signs.-(a) "Incisura," i.e., local evi- 
dence of halting peristaltic rhythm by spastic contraction of circular muscle fibers in the vicinity of an ulcer. Best brought out on screen examination during or after palpation.

(b) "Hour-glass" stomach (biloculation). This may be permanent (callous ulcer, perforation, adhesion), or transient (local spasm, with or without ulcer); should always be proved by repeated examinations with and without an antispasmodic (atropine, belladonna).

(c) Gastric residue. This may vary in amount. Its constant demonstration after 6 hours means atony or stenosis. Intermittently it may result from extragastric or gastric pathology causing pyloric spasm.

(d) Fixation of all or part of the stomach (perforation, adhesion, fistula).

(e) Area of tenderness to palpation, usually localized at some part of the stomach shadow. Should always be checked by repeated examination before and after an antispasmodic.

(f) Alterations in gastric peristalsis, e.g., exaggerated peristalsis, intermittent, frequently associated with spasmodic closure and relaxation of the pylorus. Antiperistalsis may be seen on rare occasions.

\section{DUODENAL ULCER}

1. Facts Elicited from History.-(a) Males are affected approximately three times as frequently as females. Between the ages of 35 and 50 lie most of the cases.

(b) Patients have generally had acid dyspepsia of the intermittent type for from 5 to 30 years. Seasonal relation of complaint is often pronounced.

(c) Periodicity of the complaint is marked.

(d) Chronic appendix dyspepsia has frequently clouded 
the early symptomatology. The appendix has not rarely been removed for early disability.

(e) Epigastric pain associated with dyspeptic storms occurs in practically 100 per cent. of cases. This pain is often subjectively noted to the right of the mid-line or in the right posterior scapular region. Pain is usually a dull aching, gnawing or sore feeling. In rapidly developing cases or where perforative exacerbations have taken place, pain may be colicky, cramp-like or boring.

( $f$ ) Pain time is most frequently at its maximum when the stomach is nearly or entirely empty. The ingestion of food usually relieves all symptoms, unless perforation or stenoses have supervened. The periodic repetition of this syndrome associated with "food-ease" is characteristic of nearly 90 per cent. of uncomplicated ulcers. Pain relief is also obtained by alkalies, vomiting, rest or opiates.

(g) Tomiting occurs less frequently in duodenal than in gastric ulcer (apart from the extrapyloric type), until perforation or stenosis takes place. When vomiting occurs it is apt to be more copious than in case of the majority of gastric ulcers, inasmuch as duodenal stenoses tend to more rapidly produce extensive dilatation of the stomach. "Delayed" vomiting is common.

(h) Hemorrhage (melena or hematemesis) occurs in about 30 per cent. of instances. Melena is a most frequent happening, but pronounced hematemesis may occur. The reverse is the rule in gastric ulcer. The hemorrhages are generally intermittent. Inasmuch as duodenal ulcers rarely become carcinomatous, and because they have a tendency to heal and to be protected by scar tissue, persistent melena (even by chemical demonstration) is unusual.

(i) Eructations, pyrosis and water-brash are common, especially 3 to 5 hours post cibo or at night (12 m. to 1 a.m.). 
At these times maximum gastric acidity may be demonstrated. Vomiting, gastric lavage or alkalies generally bring about prompt relief.

(j) Appetite is usually good except in attacks, but fear of bringing on pain by eating frequently leads to a poor appetite habit. Weight loss may be rapid during attacks, or when pyloric stenosis has supervened. Bowels are not infrequently constipated during periods of exacerbation.

2. Facts Determined upon Examination.- (a) Patient usually of plethoric type unless starved by diet, recent hemorrhage or stenosis. Body nourishment generally good; toxic evidences or cachexia absent in majority of instances.

(b) Hemoglobin averages 80 per cent. or above in uncomplicated cases.

(c) Epigastric tenderness may be lacking. There is no typical point of tenderness in duodenal ulcer, but usually spasm of the right rectus muscle may be elicited on both superficial and deep palpation. When recent or protected perforation results in peritoneal involvement, then localized tenderness of varying grades may be demonstrated. Occasionally tumor-like, tender ridges may be present where much callous has developed or adhesions have formed.

(d) Dilated, splashy stomach may be outlined even without air inflation. This is common where stenoses have occurred. Visible peristalsis (Kussmaul type) is not uncommon where marked stenosis at the pylorus has developed and where the abdominal parietes is thin.

3. Facts Obtained upon Roentgen Examination.--Upon fluoroscopic examination, uncomplicated ulcers frequently return nothing of definite diagnostic value. Evidence of pyloric spasm associated with hyperperistalsis are not 
infrequently seen. Such conditions, however, are not typical of duodenal ulcer, even though frequently they may be associated with it. Local areas of tenderness are sometimes delimited at the pylorus, or over the duodenum itself. If they are persistent after the hypodermatic administration of atropine sulphate, and the clinical history is that suggesting duodenal ulcer, these areas of tenderness are not without certain relative diagnostic significance.

Plate or film examination in instances of duodenal ulcer may readily demonstrate 6 -hour retention of the opaque meal and over-vigorous peristalsis.

If the duodenal ulcer is of the complicated type (viz., has exuberant callus, perforation, adhesions, or is large enough to form a crater), plate or screen examination may reveal malformations of the duodenum, fixture of the duodenum by adhesions, various grades of obstruction, or even fistulæ.

It is well in suspected cases of calloused ulcer of the duodenum to make plates with the patient not only in the dorsal and prone positions, but after he has been placed in the left or right lateral positions. By this maneuver one not infrequently demonstrates malformations of the bulbus duodeni.

Sometimes the degree of obstruction at the pylorus and the deformity of the duodenum may readily suggest some anomaly at the pyloric end of the stomach, on account of the abrupt termination of the stomach lumen or irregularities in its pyloric outline. In such instances at laparotomy, one may find extensive adhesions, perforation or a duodenal ulcer which has become cancerous on the gastric side.

The comparative worth of the different diagnostic points in gastric and duodenal ulcer and gastric cancer is further emphasized in Table 37. 
Table 37

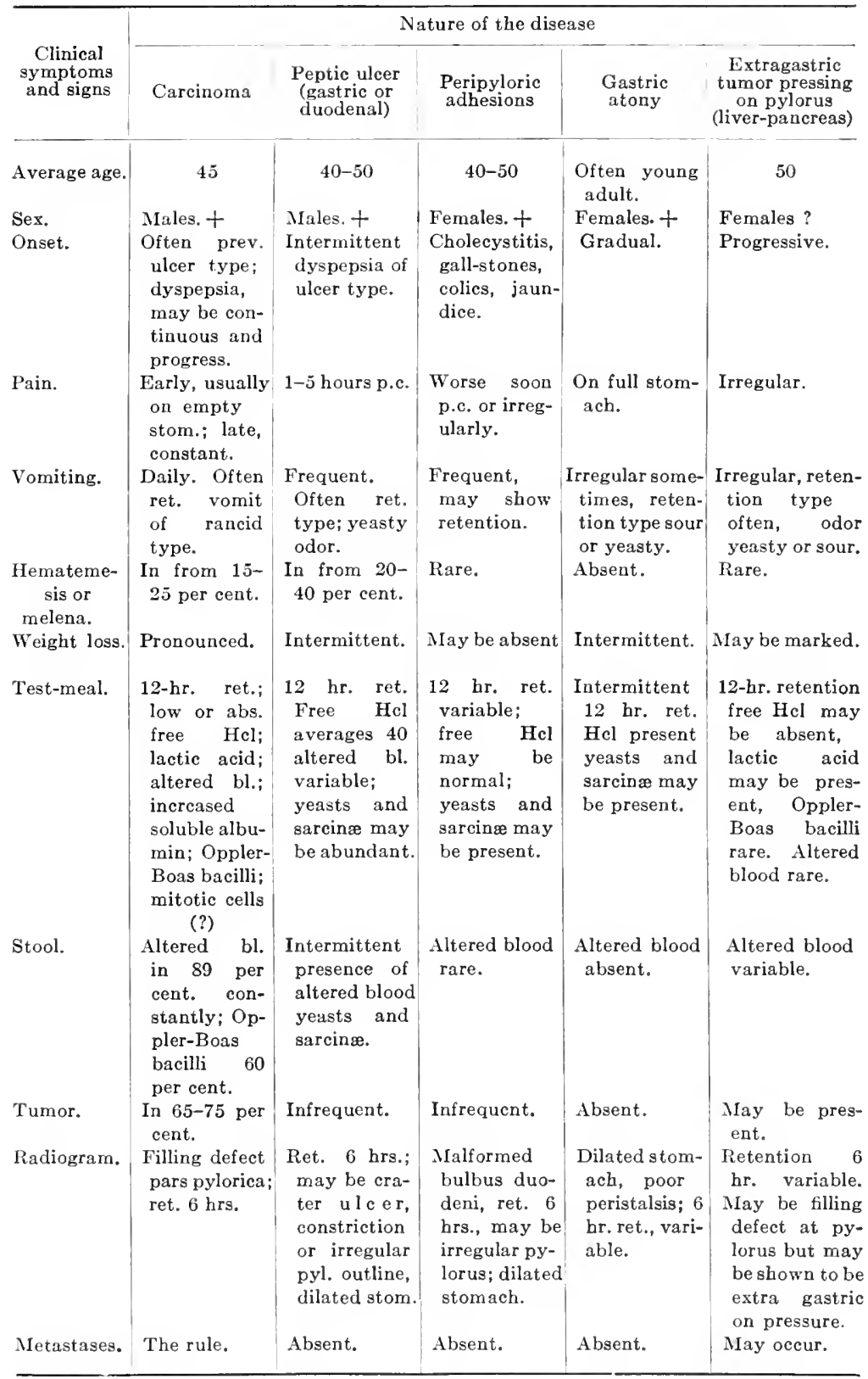

Malignant Pyloric Stenosis and its Differential Diagnosis (Author). 
Not infrequently certain lesions of the esophagus or of structures in the thorax are confused with gastric cancer at or near the cardia. Table 38 summarizes the essential differential points in the diagnosis of these ailments.

Table $3 \mathrm{~s}$

\begin{tabular}{|c|c|c|c|c|c|c|}
\hline \multirow[b]{2}{*}{$\begin{array}{l}\text { Clinical } \\
\text { symptoms } \\
\text { and signs }\end{array}$} & \multicolumn{6}{|c|}{ Nature of discase } \\
\hline & $\begin{array}{l}\text { Carcinoma } \\
\text { at or near } \\
\text { cardia }\end{array}$ & $\begin{array}{l}\text { Car. of } \\
\text { esophagus }\end{array}$ & $\begin{array}{l}\text { Cicatricial } \\
\text { stenosis of } \\
\text { esophagus }\end{array}$ & $\begin{array}{l}\text { Cardio- } \\
\text { spasm }\end{array}$ & $\begin{array}{l}\text { Esopha- } \\
\text { geal diver- } \\
\text { ticulum }\end{array}$ & $\begin{array}{l}\text { Thoracic } \\
\text { tumor- } \\
\text { aneurysm }\end{array}$ \\
\hline Average age & 45 & 45 & $\begin{array}{l}\text { Young } \\
\text { adult. }\end{array}$ & $\begin{array}{l}\text { Young } \\
\text { adult. }\end{array}$ & 45 & 45 \\
\hline Sex. & Male. + & Male. + & $?$ & Fenale. + & Male. + & $?$ \\
\hline Onset. & $\begin{array}{l}\text { Prev. ulcer } \\
\text { or gradual. }\end{array}$ & Gradual. & $\begin{array}{l}\text { Ulcer his. } \\
\text { or corro- } \\
\text { sives. }\end{array}$ & $\begin{array}{l}\text { Sudden, } \\
\text { rarely } \\
\text { gradual or } \\
\text { intermit- } \\
\text { tent. }\end{array}$ & Gradual. & $\begin{array}{l}\text { Usually } \\
\text { gradual. }\end{array}$ \\
\hline Dysphagia. & $\begin{array}{l}\text { Intermit- } \\
\text { tent or } \\
\text { progres- } \\
\text { sive. }\end{array}$ & $\begin{array}{l}\text { Usually } \\
\text { constant } \\
\text { and pro- } \\
\text { gressive. }\end{array}$ & $\begin{array}{l}\text { Progressive } \\
\text { and mark- } \\
\text { ed. }\end{array}$ & $\begin{array}{l}\text { Often in- } \\
\text { termittent. }\end{array}$ & Progressive. & Progressive. \\
\hline Pain. & $\begin{array}{l}\text { Soon after } \\
\text { swallowing. }\end{array}$ & $\begin{array}{l}\text { During } \\
\text { swallowing } \\
\text { usually. }\end{array}$ & $\begin{array}{l}\text { During } \\
\text { swallowing. }\end{array}$ & $\begin{array}{l}\text { May be ab- } \\
\text { sent while } \\
\text { eating. }\end{array}$ & $\begin{array}{l}\text { During deg- } \\
\text { lutition, } \\
\text { but may } \\
\text { be absent. }\end{array}$ & $\begin{array}{l}\text { During deg- } \\
\text { lutition. }\end{array}$ \\
\hline $\begin{array}{l}\text { Vomiting or } \\
\text { regurgita- } \\
\text { tion. }\end{array}$ & $\begin{array}{l}\text { Fairly con- } \\
\text { stant soon } \\
\text { p.c. }\end{array}$ & $\begin{array}{l}\text { Constant } \\
\text { during or } \\
\text { soon after } \\
\text { eating. }\end{array}$ & $\begin{array}{l}\text { Constant } \\
\text { p.c. }\end{array}$ & $\begin{array}{l}\text { Soon after } \\
\text { or many } \\
\text { hours after } \\
\text { eating. }\end{array}$ & $\begin{array}{l}\text { Soon p.c. } \\
\text { or hours } \\
\text { after. }\end{array}$ & Soon p.c. \\
\hline Weight loss. & Progrcssive. & Progressive. & $\begin{array}{l}\text { Progressive } \\
\text { in late } \\
\text { stages. }\end{array}$ & $\begin{array}{l}\text { Intermit- } \\
\text { tent, may } \\
\text { be marked } \\
\text { late. }\end{array}$ & $\begin{array}{l}\text { Little early, } \\
\text { rapid late. }\end{array}$ & $\begin{array}{l}\text { Progressive } \\
\text { but rarely } \\
\text { rapid. }\end{array}$ \\
\hline Cachexia. & Pronounced. & Pronounced. & $\begin{array}{l}\text { Emacia- } \\
\text { tion. }\end{array}$ & $\begin{array}{l}\text { Usually not } \\
\text { marked. }\end{array}$ & $\begin{array}{l}\text { Rarely } \\
\text { marked. }\end{array}$ & $\begin{array}{c}\text { Not marked } \\
\text { until late. }\end{array}$ \\
\hline $\begin{array}{l}\text { Hemor- } \\
\text { rhage. }\end{array}$ & Frequent. & Frequent. & Absent. & Absent. & Absent. & Rare. \\
\hline Test-meal. & $\begin{array}{l}\text { Low acid, } \\
\text { blood, Op- } \\
\text { pler-Boas } \\
\text { bacilli } \\
\text { "cancer } \\
\text { cells." }\end{array}$ & $\begin{array}{l}\text { Food as } \\
\text { eaten, } \\
\text { blood pres. } \\
\text { "cancer } \\
\text { cells." }\end{array}$ & $\begin{array}{l}\text { Food as } \\
\text { eaten. }\end{array}$ & $\begin{array}{l}\text { Food as } \\
\text { eaten, } \\
\text { mucus } \\
\text { often ret. } \\
\text { food rests. }\end{array}$ & $\begin{array}{l}\text { Often ret. } \\
\text { food rests, } \\
\text { much } \\
\text { mucus. }\end{array}$ & $\begin{array}{l}\text { Often acid } \\
\text { stomach } \\
\text { juice; may } \\
\text { be reten. } \\
\text { tion. }\end{array}$ \\
\hline Stool. & Altered bl. & Alt. bl. & No blood. & No blood. & No blood. & $\begin{array}{l}\text { Rarely } \\
\text { blood. }\end{array}$ \\
\hline $\begin{array}{l}\text { Esophago- } \\
\text { scope. }\end{array}$ & $\begin{array}{c}\text { Tumor at } \\
\text { cardia. }\end{array}$ & $\begin{array}{l}\text { Tumor in } \\
\text { esopha- } \\
\text { gus. }\end{array}$ & $\begin{array}{l}\text { Cicatrix in } \\
\text { esopha- } \\
\text { gus. }\end{array}$ & $\begin{array}{l}\text { May be } \\
\text { negative } \\
\text { or dilated } \\
\text { esophagus, } \\
\text { small car- } \\
\text { diac ori- } \\
\text { fice. }\end{array}$ & $\begin{array}{l}\text { Opening to } \\
\text { sac may } \\
\text { be seen. }\end{array}$ & $\begin{array}{l}\text { Prssure on } \\
\text { oesopha- } \\
\text { gus locally. }\end{array}$ \\
\hline
\end{tabular}


Table 35.-Continued

\begin{tabular}{|c|c|c|c|c|c|c|}
\hline \multirow{3}{*}{$\begin{array}{l}\text { Clinical } \\
\text { symptoms } \\
\text { and signs } \\
\text { Radiogram. }\end{array}$} & \multicolumn{6}{|c|}{ Nature of the disease } \\
\hline & $\begin{array}{l}\text { Carcinoma } \\
\text { at or near } \\
\text { cardia }\end{array}$ & $\begin{array}{l}\text { Car. of } \\
\text { esophagus }\end{array}$ & $\begin{array}{l}\text { Cicatricial } \\
\text { stenosis of } \\
\text { esophagus }\end{array}$ & $\begin{array}{l}\text { Cardio- } \\
\text { spasm }\end{array}$ & $\begin{array}{l}\text { Esophageal } \\
\text { diver- } \\
\text { ticulum }\end{array}$ & $\begin{array}{l}\text { Thoracic } \\
\text { tumor- } \\
\text { aneurysm }\end{array}$ \\
\hline & $\begin{array}{l}\text { Opaque } \\
\text { meal rests } \\
\text { in esopha- } \\
\text { gus; fill- } \\
\text { ing defect } \\
\text { at cardia. }\end{array}$ & $\begin{array}{l}\text { Obst. to } \\
\text { opaque } \\
\text { meal, local } \\
\text { dilatation } \\
\text { of esoph.; } \\
\text { irregular } \\
\text { lumen out- } \\
\text { line. }\end{array}$ & $\begin{array}{l}\text { Obst. to } \\
\text { opaque } \\
\text { meal; de- } \\
\text { formity of } \\
\text { esopha- } \\
\text { gus. }\end{array}$ & $\begin{array}{l}\text { Usually dif- } \\
\text { fuse dila- } \\
\text { tation of } \\
\text { esophagus; } \\
\text { tapering } \\
\text { cardiac } \\
\text { end. }\end{array}$ & $\begin{array}{l}\text { Dilated } \\
\text { esophagus } \\
\text { or local sac- } \\
\text { culation. } \\
\end{array}$ & $\begin{array}{l}\text { Rarely op. } \\
\text { meal rests; } \\
\text { local stric- } \\
\text { ture of } \\
\text { esophagus, } \\
\text { tumor or } \\
\text { aneurysm. }\end{array}$ \\
\hline Duration. & $3 \mathrm{mo}-4 \mathrm{yr}$. & $\begin{array}{l}\text { Rarely } \\
\text { more than } \\
2 \text { years }\end{array}$ & $\begin{array}{l}\text { Nay be } \\
\text { many yrs. }\end{array}$ & Many yrs. & Many yrs. & Varies. \\
\hline Metastases. & Late in pit. & None. & None. & None. & $\begin{array}{l}\text { Local in tho- } \\
\text { rax some- } \\
\text { times. } \\
\text { None. }\end{array}$ & $\begin{array}{l}\text { Local at } \\
\text { times. } \\
\text { None. }\end{array}$ \\
\hline
\end{tabular}

1. Table of Differential Diagnosis where Cancer Involves Cardiac Orifice.(Author.)

Gall-bladder Disease.-Certain cases of chronic cholecystitis or cholecystitis with cholelithiasis are associated with gastric achylia, pancreatic inefficiency and deficient function on the part of the liver. Not infrequently anemia is present and marked alterations of the biliary tract may occur without jaundice. Loss of flesh, diarrhœa, vomiting and epigastric pain may produce a symptom-complex which is difficult to distinguish from gastric malignancy. In the majority of these cases, the essential guide to the diagnosis is a careful anamnesis, including particular inquiry into the earlier years of the patient's dyspeptic ailment. One not infrequently learns by such inquiry that the disease is more common in females, that there has been antecedent typhoid fever or malaria, that the earlier dyspeptic history is not the type which we associate clinically with chronic peptic ulcer; that obstruction has arisen late, or is entirely absent, and that certain acute attacks of indigestion have been associated with biliary symptoms, 
namely, slight jaundice, gaseous abdominal distension, perhaps vomiting of bile, acute colic of a passing stone, irregular pain usually without definite relation to food intake, and not unusually transitory rises in temperature. Gastric analysis may show some retention of the 12-hour type. Retained contents are rarely of an obnoxious odor, and but infrequently contain lactic acid, altered blood or bacilli of the Oppler-Boas group. Free hydrochloric acid may be absent or very low, but is not so uniformly decreased as it is in gastric cancer. Stools may be diarrhœic, they rarely contain blood or long acid-fast bacilli. The $x$-ray examination may show 6 -hour retention of the opaque meal, dilated stomach, deficient peristaltic activity, and at times irregularities toward the pylorus. In these cases, however, filling defects of the gastric lumen with irregular outline, which outline is indistinct, are uniformly uncommon. Sometimes the screen or plate reveals gall-stones, or upon fluoroscopic examination one can fully delimit the boundaries of the pyloric channel and show that such are of normal contour.

Cancer of the Gall-tract.-Malignant disease of the biliary passages, particularly of the gall-bladder, is associated with a history of cholecystitis or the presence of gall-stones in from 18 to 40 per cent. of cases. In such patients there is commonly an extensive precarcinomatous history of gall-bladder disease. - The patient may even have passed gall-stones. The antecedent dyspepsia has been of irregular occurrence, and has not rarely been associated with jaundice or fever. At the time that the patient comes under observation, jaundice is not an uncommon finding.

Cachexia, associated with clinical evidence of pyloric obstruction, or the presence of an epigastric mass, may 
render it difficult to exclude a neoplasm involving the stomach. In fact, the stomach is not rarely involved by contiguity in these cases. Cachexia is not, as a rule, so pronounced as in cases of gastric cancer. Vomiting is not so constant or copious as in gastric malignancy. Free hydrochloric acid may be present in gastric extracts, and at times, though infrequently, lactic acid, altered blood and Oppler-Boas bacilli may be found. Blood is rarely a constant finding in the properly prepared stool. The abdominal tumor is usually well to the right, in the midline, or beneath the edge of the ribs. It may move up and down upon respiration, but rarely changes position when the stomach or colon is inflated. The tumor cannot be moved during palpation, and can rarely be held down by the hands while the patient is in expiration. These tumors are apt to be of less irregular form than are the tumors of gastric cancer. They are apt to be more tender upon palpation.

Metastases develop more slowly than in the case of gastric cancer, particularly metastasis to the pelvis, navel and left supraclavicular space. Ascites may develop much earlier than where the neoplasm involves the stomach, and the accumulation of ascitic fluid is likely to be of much greater quantity than in the average instance of pyloric cancer.

Roentgen examination may show at once that the tumor is extragastric. While not infrequently adhesions rapidly develop between the diseased gall-tract and the stomach, in many instances the pylorus remains free and can be pushed away from the gall-tract tumor and shown to be of regular outline. There are some cases, however, in which extensive adhesions about the gall-bladder and the head of the pancreas may immobilize the pyloric end of the stomach, 
and even give the appearance Roentgenographically of a canalized tumor involving the pylorus and antrum.

Tumors of the liver are of comparative rarity. The left lobe of the liver is apt to be the seat of the disease. The early history of the infection is rarely that of a longcontinued dyspepsia. Jaundice may appear early. Cachexia is a comparatively late manifestation of the disease. Persistent romiting is relatively uncommon. Weight loss may be of extremely gradual onset, and may not be associated with marked evidences of systemic cancerous intoxication. Anemia may be marked. Not infrequently the Wassermann reaction is positive, owing to the fact that primary tumors of the liver are not uncommonly syphilitic. Additional blood examination may show eosinophilia if the liver tumor is of parasitic origin (echinococcus). In the early stages of the disease, alteration in gastric emptying power is uncommon. Free hydrochloric acid is apt to be normal and not diminished or absent until systemic cancer poisoning occurs. The stools rarely show the presence of "occult" blood. Diarrhœa may develop, and microscopic examination but infrequently shows the presence of long, acid-fast bacilli. A chemical examination of the stool may show the absence of hydrobilirubin.

The abdominal tumor usually lies high in the epigastrium. It is rarely localized definitely. It is not usually so tender as is a neoplasm involving the stomach. It cannot be moved freely upon palpation. It moves with respiration. Secondary metastases are rare. X-ray examination usually enables one to demonstrate that the tumor is extragastric, insomuch as an apparent filling defect in the stomach will disappear upon change of position of the patient or upon one's moving the stomach. 
Disease of the Pancreas.-Instances of chronic interstitial pancreatitis, associated with enlargement of the gland, dyspepsia and cachexia, are not uncommonly confused with gastric cancer. The following case illustrates this type of ailment.

Chronic Interstitial Pancreatitis; Emaciation and Epigastric Tumor:

Mrs. M. B.-Age 41, American, housewife.

Family History.-Negative.

Personal History.-Typhoid fever at age of 20. Recently had laparotomy performed for cholecystitis and pericholecystitis. At laparotomy the pancreas was found to be 3 times normal size, was hard and nodular.

Comes on account of romiting, weight loss, abdominal distress, and diarrhœa.

Duration of Disease.-Twenty years off and on; has had several acute attacks of dyspepsia; characterized by abdominal pain, vomiting, chilly sensations and jaundice.

At present has mid-epigastric distress constantly; the feeling is of "soreness and weakness;" it is vaguely transmitted to the back; it is only relieved by opiates. Tomiting of food and foul-tasting, green liquid occurs daily and is brought on by food intake. Belching, water-brash and eructations are nearly constant.

Bouels.-Diarrhœa for 6 months; stools are watery, small in amount, contain undigested food and have a disgusting, penetrating odor.

Weight.-Normal 100 pounds; one year ago 96 pounds; 6 months ago 88 pounds; present 55 pounds.

Examination.-Thin, cachectic, bed-ridden female, afebrile; constantly belches gas and raises small quantity of watery fluid.

Throat.-Infected tonsils.

Thorax.-Small heart, with weak muscle sounds.

Abdomen.-Stomach splashy below navel; slight Kussmaul peristalsis seen; areas of tenderness over gall-bladder region and just above navel. In low epigastrium is a tender, nodular, sausage-shaped mass, slightly movable on palpation, but not upon inflation of the stomach.

Rectal Examination.-Negative. 
Laboratory Findings: 7,000 .

Blood.-Hg., 60 per cent.; r.b.c., 3,900,000; w.b.c.,

Urine.-Few hyaline casts.

Stools.-Thin, watery, greenish brown; much undigested regetable and meat; microscopically enormous numbers of motile and non-motile rods, numerous yeasts and cocci.

Chemical.-Alkaline reaction; altered blood, trace; trypsin absent; amylase over 600 units (in normal range).

Test-meal.-Contents of stomach greenish-yellow. No 12-hour retention. Total acidity, 0; free Hcl., 0; lactic acid, 0 ; altered blood, 0 ; Wolff, 0 .

Microscopically.-Few yeasts and small type sarcinæ are seen.

Roentgen Examination.-Narkedly ptosed stomach of fish-hook form; atonic; pylorus and antrum well visualized, duodenum seems regular. Epigastric tumor is seen to lie 3 f.b. above lesser curvature of stomach.

Wassermann.-Negative.

Laparotomy.-Large, hard nodular pancreas; chronic cholecystitis and pericholecystitis.

Pathology.-Tissue (gland) inflammatory.

After Course.-Patient alive after nearly 3 years; weighs 99 pounds; still has epigastric nodule.

Important differential points are summarized in Table 39. Emphasis is to be especially placed upon the anamnesis of the precachectic stage. This usually exhibits symptoms clinically referable to gall-tract malfunction. Test-meal findings are but rarely characteristic of gastric cancer at a stage when epigastric tumor can be palpated. While free hydrochloric acid may be low or absent, 12-hour retention, altered blood, lactic acid, positive Wolff-Junghans' test, increase in the formol index, or bacilli of the Oppler-Boas type are commonly absent or irregularly found. The abdominal tumor is not generally movable upon gastric or colon inflation. The stools rarely exhibit altered blood. Tests for pancreatic ferments usu- 
ally reveal absence or diminution of amylase or trypsin, or both. Roentgen examination usually shows regular gastric outline, with the stomach perhaps at a distance from the palpable abdominal tumor.

Table 39

\begin{tabular}{|c|c|c|c|c|c|c|}
\hline \multirow[b]{2}{*}{$\begin{array}{l}\text { Signs and } \\
\text { symptoms }\end{array}$} & \multirow[b]{2}{*}{$\begin{array}{l}\text { Gastric } \\
\text { cancer } \\
\text { (body or } \\
\text { fundus) }\end{array}$} & \multicolumn{4}{|c|}{ Nature of the disease } & \multirow[b]{2}{*}{$\begin{array}{l}\text { Retroperi- } \\
\text { toneal } \\
\text { tumor }\end{array}$} \\
\hline & & $\begin{array}{l}\text { Tumor of } \\
\text { liver }\end{array}$ & $\begin{array}{l}\text { Tumor of } \\
\text { pancreas }\end{array}$ & $\begin{array}{l}\text { Tumor of } \\
\text { colon }\end{array}$ & $\begin{array}{l}\text { Tumor of } \\
\text { kidney }\end{array}$ & \\
\hline Age. & 45 & 40 & Variable. & 40 & 35 & Variable. \\
\hline $\begin{array}{l}\text { Sex. } \\
\text { Onset. }\end{array}$ & Males. + & Variable. & Women? & Males? & Males? & Variable. \\
\hline & $\begin{array}{l}\text { Previous } \\
\text { ulcer his- } \\
\text { tory or } \\
\text { primary } \\
\text { progres- } \\
\text { sive dys- } \\
\text { pepsia. }\end{array}$ & $\begin{array}{l}\text { Gradual } \\
\text { and pro- } \\
\text { gressive. }\end{array}$ & $\begin{array}{l}\text { Gradual } \\
\text { and prog- } \\
\text { gressive; } \\
\text { trauma? }\end{array}$ & $\begin{array}{l}\text { Gradual } \\
\text { and pro- } \\
\text { gressive } \\
\text { acute with } \\
\text { obstr. }\end{array}$ & $\begin{array}{l}\text { Gradual; } \\
\text { may have } \\
\text { early he- } \\
\text { maturia. }\end{array}$ & $\begin{array}{l}\text { Gradual } \\
\text { and pro- } \\
\text { gressive. }\end{array}$ \\
\hline Pain. & $\begin{array}{l}\text { Pres. in } 95 \\
\text { per cent. }\end{array}$ & Indefinite. & $\begin{array}{l}\text { Rarely } \\
\text { marked. }\end{array}$ & $\begin{array}{l}\text { Intermit- } \\
\text { tent usu- } \\
\text { ally; may } \\
\text { be con- } \\
\text { stant. }\end{array}$ & $\begin{array}{l}\text { Indefinite, } \\
\text { sometimes } \\
\text { colics. }\end{array}$ & Indefinite. \\
\hline Vomiting. & $\begin{array}{l}\text { Common } \\
\text { and pro- } \\
\text { gressive. }\end{array}$ & $\begin{array}{l}\text { Irregular; } \\
\text { may occur } \\
\text { from pres- } \\
\text { sure. }\end{array}$ & $\begin{array}{l}\text { Irregular; } \\
\text { may occur } \\
\text { from pres- } \\
\text { sure. }\end{array}$ & $\begin{array}{c}\text { Rarely } \\
\text { early. }\end{array}$ & Infrequent. & $\begin{array}{l}\text { Irregular; } \\
\text { may occur } \\
\text { from pres- } \\
\text { sure. }\end{array}$ \\
\hline Weight loss. & $\begin{array}{l}\text { Constant } \\
\text { and pro- } \\
\text { gressive. }\end{array}$ & $\begin{array}{l}\text { Progres- } \\
\text { sive. }\end{array}$ & Irregular. & Constant. & $\begin{array}{l}\text { Constant } \\
\text { as a rule. }\end{array}$ & $\begin{array}{l}\text { Constant } \\
\text { but grad- } \\
\text { ual. }\end{array}$ \\
\hline Cachexia. & Pronounced & $\begin{array}{l}\text { Fronounced } \\
\text { late. }\end{array}$ & Variable. & $\begin{array}{l}\text { May be } \\
\text { marked. }\end{array}$ & $\begin{array}{l}\text { Often pro- } \\
\text { nounced. }\end{array}$ & Present. \\
\hline $\begin{array}{l}\text { Appetite. } \\
\text { Bowels. }\end{array}$ & $\begin{array}{l}\text { Poor. } \\
\text { Constipa- }\end{array}$ & $\begin{array}{l}\text { Variable. } \\
\text { Constipa- }\end{array}$ & $\begin{array}{l}\text { Variable. } \\
\text { Diarrhœa }\end{array}$ & $\begin{array}{l}\text { Variable. } \\
\text { Obstipation }\end{array}$ & $\begin{array}{l}\text { Variable. } \\
\text { May be }\end{array}$ & $\begin{array}{l}\text { Variable. } \\
\text { May be }\end{array}$ \\
\hline & $\begin{array}{l}\text { tion the } \\
\text { rule. }\end{array}$ & $\begin{array}{l}\text { tion fre- } \\
\text { quent. }\end{array}$ & $\begin{array}{l}\text { not un- } \\
\text { common. }\end{array}$ & $\begin{array}{l}\text { later diar- } \\
\text { rhœa. }\end{array}$ & normal. & normal. \\
\hline Bleeding. & $\begin{array}{l}\text { Hemate- } \\
\text { mesis or } \\
\text { melena } 15- \\
25 \text { per } \\
\text { cent. }\end{array}$ & $\begin{array}{l}\text { Melena at } \\
\text { times. }\end{array}$ & Absent. & $\begin{array}{l}\text { Common, } \\
\text { especially } \\
\text { late. }\end{array}$ & Absent. & Absent. \\
\hline Urine. & $\begin{array}{l}\text { Often nega- } \\
\text { tive. }\end{array}$ & $\begin{array}{l}\text { Often nega- } \\
\text { tive. }\end{array}$ & $\begin{array}{l}\text { Often nega- } \\
\text { tive. }\end{array}$ & $\begin{array}{l}\text { Often nega- } \\
\text { tive. }\end{array}$ & $\begin{array}{l}\text { Local he- } \\
\text { maturia in } \\
\text { about } 50 \\
\text { per cent. }\end{array}$ & $\begin{array}{l}\text { Often nega- } \\
\text { tive. }\end{array}$ \\
\hline $\begin{array}{l}\text { Cystoscopic } \\
\text { and pyelo- } \\
\text { gram. }\end{array}$ & Negative. & Negative. & Negative. & Negative. & $\begin{array}{l}\text { Blood or } \\
\text { pus from } \\
1 \text { ureter; } \\
\text { deformed } \\
\text { pelvis on } \\
\text { collargol } \\
\text { injection. }\end{array}$ & Negative. \\
\hline
\end{tabular}


Table 39.-Continued

\begin{tabular}{|c|c|c|c|c|c|c|}
\hline \multirow[b]{2}{*}{$\begin{array}{l}\text { Signs and } \\
\text { symptoms }\end{array}$} & \multicolumn{6}{|c|}{ Nature of the disease } \\
\hline & $\begin{array}{l}\text { Gastric } \\
\text { cancer } \\
\text { (body or } \\
\text { fundus) }\end{array}$ & $\begin{array}{l}\text { Tumor of } \\
\text { liver }\end{array}$ & $\begin{array}{l}\text { Tumor of } \\
\text { pancreas }\end{array}$ & $\begin{array}{l}\text { Tumor of } \\
\text { colon }\end{array}$ & $\begin{array}{l}\text { Tumor of } \\
\text { kidney }\end{array}$ & $\begin{array}{l}\text { Retroperi- } \\
\text { toneal } \\
\text { tumor }\end{array}$ \\
\hline Test-meal. & $\begin{array}{l}\text { Is ow or ab- } \\
\text { sent frce } \\
\text { IIcl; } 12 \\
\text { hr. ret.; } \\
\text { Oppler- } \\
\text { Boas ba- } \\
\text { cilli; lactic } \\
\text { acid; al- } \\
\text { tered } \\
\text { blood; } \\
\text { Wolff test } \\
\text { in } 80 \text { per } \\
\text { cent. }\end{array}$ & $\begin{array}{l}\text { II m may } \\
\text { be abst.; } \\
12 \text { hr. ret. } \\
\text { var.; rare- } \\
\text { ly Oppler- } \\
\text { Boas ba- } \\
\text { cilli or } \\
\text { lactic acid } \\
\text { Wolff test } \\
\text { in } 30-50 \\
\text { per cent. }\end{array}$ & $\begin{array}{l}\text { Hel may } \\
\text { be low or } \\
\text { normal; } 12 \\
\text { lir. ret. } \\
\text { variable; } \\
\text { Oppler- } \\
\text { Boas bac- } \\
\text { illi, lactic } \\
\text { acid or } \\
\text { altered } \\
\text { blood rare. }\end{array}$ & $\begin{array}{l}\text { Hel often } \\
\text { low but } \\
\text { may be } \\
\text { normal; in- } \\
\text { freq. } 12 \text { hr. } \\
\text { ret.; lactic } \\
\text { acid, al. } \\
\text { blood or } \\
\text { Oppler- } \\
\text { Boas un- } \\
\text { common. }\end{array}$ & $\begin{array}{l}\text { Hel may } \\
\text { be low to } \\
\text { normal; } \\
12 \text { hr. ret. } \\
\text { rare, lactic } \\
\text { and alt. } \\
\text { b l o o d, } \\
\text { Oppler- } \\
\text { Boas ba- } \\
\text { cilli very } \\
\text { rare. }\end{array}$ & $\begin{array}{l}\text { Hel usually } \\
\text { low; } 12 \text { hr. } \\
\text { ret. vari- } \\
\text { able, lactic } \\
\text { acid, alter- } \\
\text { ed blood } \\
\text { or Oppler- } \\
\text { Boas ba- } \\
\text { cilli infre- } \\
\text { quent. }\end{array}$ \\
\hline Stool. & $\begin{array}{l}\text { Altered } \\
\text { blood } 89 \\
\text { per cent. }\end{array}$ & $\begin{array}{l}\text { Altered } \\
\text { blood un- } \\
\text { common. }\end{array}$ & $\begin{array}{l}\text { Altered } \\
\text { blood un- } \\
\text { common. }\end{array}$ & $\begin{array}{l}\text { Altered } \\
\text { blood in } \\
70-90 \text { per } \\
\text { cent. }\end{array}$ & $\begin{array}{l}\text { Altered } \\
\text { blood rare. }\end{array}$ & $\begin{array}{l}\text { Altered } \\
\text { blood rare. }\end{array}$ \\
\hline $\begin{array}{l}\text { Abdominal } \\
\text { tumor. }\end{array}$ & $\begin{array}{l}\text { In about } 60 \\
\text { per cent., } \\
\text { of ten } \\
\text { movable. }\end{array}$ & $\begin{array}{l}\text { In from } 60- \\
90 \quad \text { per } \\
\text { cent. } \\
\text { moves } \\
\text { with res- } \\
\text { piration. }\end{array}$ & $\begin{array}{l}\text { In } 50 \text { per } \\
\text { ccnt. } \\
\text { moves } \\
\text { with res- } \\
\text { piration or } \\
\text { with stom- } \\
\text { ach. }\end{array}$ & $\begin{array}{l}\text { In } \quad 30-50 \\
\text { per cent. } \\
\text { moves on } \\
\text { respiration } \\
\text { or on colon } \\
\text { inflation. }\end{array}$ & $\begin{array}{l}\text { In } 75 \text { per } \\
\text { cent. may } \\
\text { move on } \\
\text { respir. }\end{array}$ & $\begin{array}{l}\text { In } \quad 70-90 \\
\text { per cent. } \\
\text { usually } \\
\text { fixed. }\end{array}$ \\
\hline Radiogram. & $\begin{array}{l}12 \text { hours } \\
\text { retention } \\
\text { not un- } \\
\text { common.; } \\
\text { filling de- } \\
\text { fects. }\end{array}$ & $\begin{array}{l}12 \text { hours } \\
\text { ret. un- } \\
\text { common; } \\
\text { palpation; } \\
\text { on fluoro- } \\
\text { scopic } \\
\text { exam. } \\
\text { shows } \\
\text { mass to be } \\
\text { extra- } \\
\text { gastric. }\end{array}$ & $\begin{array}{l}12 \text { hours } \\
\text { ret. var.; } \\
\text { palpation } \\
\text { on fluoro- } \\
\text { scopy } \\
\text { shows } \\
\text { tumor to } \\
\text { be extra- } \\
\text { gastric. }\end{array}$ & $\begin{array}{l}12 \text { hours } \\
\text { stom. ret. } \\
\text { var. tumor } \\
\text { mass ex- } \\
\text { tragastric; } \\
\text { colon } \\
\text { exam. } \\
\text { shows ret. } \\
\text { of opaque } \\
\text { meal and } \\
\text { filling de- } \\
\text { fects of } \\
\text { bowel. }\end{array}$ & $\begin{array}{l}12 \text { hours } \\
\text { stom. ret. } \\
\text { variable; } \\
\text { tumor ex- } \\
\text { tragastric. }\end{array}$ & $\begin{array}{l}12 \text { hours } \\
\text { ret. vari- } \\
\text { able tumor } \\
\text { mass extra- } \\
\text { gastric. }\end{array}$ \\
\hline Ascites. & Occurs late. & $\begin{array}{l}\text { May occur } \\
\text { early. }\end{array}$ & Uncommon. & $\begin{array}{l}\text { Very un- } \\
\text { common. }\end{array}$ & $\begin{array}{l}\text { Very un- } \\
\text { common. }\end{array}$ & May occur. \\
\hline Jaundice. & $\begin{array}{l}\text { Late, due } \\
\text { to metas- } \\
\text { tases. }\end{array}$ & $\begin{array}{l}\text { May be } \\
\text { early and } \\
\text { constant. }\end{array}$ & $\begin{array}{l}\text { Variable } \\
\text { according- } \\
\text { ly as gall- } \\
\text { ducts are } \\
\text { obstructed. }\end{array}$ & Rare. & Rare. & Infrequent. \\
\hline
\end{tabular}

Differentiation of Gastric Cancer of Body or Fundus from Tumors in Adjacent Structures.-(Author.)

Cyst of the pancreas is of infrequent occurrence. The history should be searched for facts relative to gall-stones, 
trauma or perforating peptic ulcer. Pancreatic cysts often attain great size without giving marked gastric symptoms. Cachexia develops slowly. The tumor can generally be shown to be distinct from the stomach by air inflation of the stomach and large bowel. Gastric examination by test-meals, commonly reveals free emptying power, normal free hydrochloric acid, and absence of altered blood, lactic acid or long acid-fast bacilli. The stools may be diarrheic; they rarely exhibit altered blood. Pancreatic ferments may be lacking. Roentgen examination may demonstrate that the tumor has no connection with the stomach.

Abscess of the pancreas is commonly associated with history of infection of the gall-tract, penetrating ulcer, acute pancreatitis, or trauma. Shock may be marked early. Temperature and leucocytes are increased. Vomiting may be uncontrollable, and be bloody in kind. The abdominal tumor is rarely localized. It is usually fixed and tender upon palpation. The test-meal findings are variable. They may be negative unless previous gastric ulcer has existed. Stools rarely contain altered, blood, but pancreatic ferments may be lacking. Glycosuria may be demonstrated. Roentgen examination shows the tumor to be extragastric, or reveals crater ulcer or malformations due to adhesions. If the abdomen is very tense, locally, exploratory puncture may reveal an abscess cavity that has no communication with a viscus. Carmine (gr. v) administered by the mouth may prove this lack of a fistulous tract.

Carcinoma of the pancreas is generally secondary to malignant disease of the stomach, gall-tract or liver. The history of the primary affection is usually characteristic and suggestive. It is impossible clinically, to 
separate some primary malignant pancreatic tumors from gastric cancers until laparotomy is performed. On account of the early invasion of the stomach wall the symptoms of the disease may closely mimic gastric cancer. Tumors of the pancreas early become fixed or move only on respiration, and not upon gastric or colonic inflation with air. Diarrhea, often prostrating, may be an early manifestation. The stools rarely contain blood, macroscopically or chemically. Tryptic and amylolytic digestion may be reduced or lacking. Roentgen examination may show that the focus of disease is extragastric.

Enlargement of the spleen may at times present a clinical picture that is confused with cancer of the greater currature or fundus of the stomach. In splenic enlargements the anamnesis usually discloses history of a chronic, general infection, of malaria, trauma, or of blood dyscrasia. Abdominal examination generally demonstrates a tumor with a well delimited, rather sharp edge; the spleen "notches" are recognized; the mass is smooth and moves on respiration but not upon inflation of the stomach or colon. Blood analyses often establish a leukemia. Secondary nodules are rare in disease of the spleen. When such occur, they are in the blood-forming organs or lymphatics. The liver may show enlargement nearly as great as that of the spleen. The test-meal rarely exhibits gastric retention. Low free hydrochloric acid may be observed, but organic acids, altered blood or bacilli of the Oppler-Boas type are rarely found. The stool contains blood chemically in almost 50 per cent. of cases. Roentgen examination may prove that the tumor is extragastric.

Tumors of the kidney, large bowel, omentum and retroperitoneal tissues not infrequently offer problems in the 
proof of their being extragastric. Table 39 summarizes the essential features of their differentiation.

Primary tumors of the small intestine are of very uncommon occurrence. Their most common location is in the first 3 feet of the jejunum or the terminal 3 feet of the ileum. They may give rise to only vague dyspepsia until stenosis occurs. On account of the occurrence of colicky pains, this dyspepsia is frequently mistaken for gastric ulcer or appendicitis until diarrhea, hemorrhage, emaciation or abdominal tumor derelop. If the jejunum is involved just distal to the duodenum, gastric contents exhibiting retention (really duodenal) or low free hydrochloric acid may be obtained. Rarely are organic acids, altered blood or Oppler-Boas bacilli noted. Early tenderness of the tumor is observed. The bowel proximal to it is distended. The tumor shows little, if any, relation to the stomach upon that viscus being distended with air. Roentgen examination may show halting of the opaque meal with anomaly in outline of the bowel in the region of the tumor. When the ileum is the seat of the disease, stenosis may be the earliest sign, apart from cachexia. This eridences itself by constipation (occasionally bloody stools with diarrhea), colicky abdominal pains, tender, distended abdomen or irregular "false tumors" due to accumulations of air and chyle in the small intestine. Roentgen examination may demonstrate the dilated ileum proximal to an alteration in outline of its lumen.

Achylia gastrica (primary or associated with the anemias coincident to pernicious anemia, Addison's disease and cardio-renal malfunction) not infrequently occurs at the "cancer age" and may, on casual examination, be confused with gastric cancer. The essential differential points are summarized in Table 40. 
Table 40

\begin{tabular}{|c|c|c|c|c|c|}
\hline \multirow{2}{*}{$\begin{array}{l}\text { Clinieal } \\
\text { signs and } \\
\text { symptoms }\end{array}$} & \multicolumn{5}{|c|}{ Nature of the direase } \\
\hline & $\begin{array}{l}\text { Gastric } \\
\text { eancer }\end{array}$ & $\begin{array}{l}\text { Achylia } \\
\text { gastrica }\end{array}$ & $\begin{array}{l}\text { Pernicious } \\
\text { anemia }\end{array}$ & $\begin{array}{l}\text { Cardio-renal } \\
\text { disease }\end{array}$ & $\begin{array}{l}\text { Addison's } \\
\text { disease }\end{array}$ \\
\hline Age. & 15 & 40 & 35 & 45 & 30 \\
\hline Sex. & Males + & $\begin{array}{l}\text { About equally } \\
\text { divided. }\end{array}$ & Nales. + & Males. + & $\begin{array}{l}\text { About equally } \\
\text { divided, }\end{array}$ \\
\hline Onset. & $\begin{array}{l}\text { Often malig. } \\
\text { after years of } \\
\text { dyspepsia. If } \\
\text { no prev. his- } \\
\text { tory of dysp. } \\
\text { prog. and } \\
\text { continuous } \\
\text { indigestion. }\end{array}$ & $\begin{array}{l}\text { Gradual on- } \\
\text { set; may be } \\
\text { old history } \\
\text { of gastritis, } \\
\text { usually in- } \\
\text { definite "dys- } \\
\text { spepsia." }\end{array}$ & $\begin{array}{l}\text { Slow and in- } \\
\text { sidious; gas- } \\
\text { tric synp- } \\
\text { toms often } \\
\text { secondary. }\end{array}$ & $\begin{array}{l}\text { History of in- } \\
\text { fectious ail- } \\
\text { ment or } \\
\text { cardio-renal } \\
\text { overstrain. }\end{array}$ & $\begin{array}{l}\text { Insidious with } \\
\text { progressive } \\
\text { weakness. }\end{array}$ \\
\hline Pain. & $\begin{array}{l}\text { Present in } 95 \\
\text { per cent. }\end{array}$ & $\begin{array}{l}\text { Indefinite dis- } \\
\text { tress. }\end{array}$ & $\begin{array}{l}\text { Rare unless } \\
\text { card. symp- } \\
\text { toms de- } \\
\text { velop. }\end{array}$ & $\begin{array}{l}\text { Rare or vague. } \\
\text { Occasionally } \\
\text { abdominal } \\
\text { "cramps." }\end{array}$ & $\begin{array}{l}\text { Irregular } \\
\text { cramps at } \\
\text { times. }\end{array}$ \\
\hline Vomiting. & $\begin{array}{l}\text { Common } \\
\text { early and } \\
\text { late in dis- } \\
\text { ease. }\end{array}$ & Irregular. & Infrequent. & Irregular. & Irregular. \\
\hline Weight loss. & $\begin{array}{c}\text { Constant and } \\
\text { progressive. }\end{array}$ & Variable. & $\begin{array}{l}\text { Variable or } \\
\text { intermittent. }\end{array}$ & Variable. & Progressive. \\
\hline Cachexia & Pronounced. & $\begin{array}{l}\text { Rarely } \\
\text { marked. }\end{array}$ & $\begin{array}{l}\text { Not often } \\
\text { pronounced. }\end{array}$ & Infrequent. & $\begin{array}{l}\text { May be pro- } \\
\text { nounced; } \\
\text { weakness out } \\
\text { of all propor- } \\
\text { tion. }\end{array}$ \\
\hline $\begin{array}{l}\text { Blood pres- } \\
\text { sure. }\end{array}$ & Reduced. & $\begin{array}{l}\text { May be re- } \\
\text { duced. }\end{array}$ & Reduced. & $\begin{array}{l}\text { Increased } \\
\text { usually. }\end{array}$ & $\begin{array}{l}\text { Markedly re- } \\
\text { duced. }\end{array}$ \\
\hline Anemia. & $\begin{array}{l}\text { Erythrocytes } \\
\text { average } \\
\text { above } 3,500,- \\
\text { 000, Hg. } \\
\text { above } \quad 60 \\
\text { per cent. }\end{array}$ & $\begin{array}{l}\text { May be slight } \\
\text { secondary } \\
\text { anemia. }\end{array}$ & $\begin{array}{l}\text { Erythroeytes } \\
\text { usually be- } \\
\text { low 2,500,- } \\
\text { 000; Hg. re- } \\
\text { latively high; } \\
\text { nucleated, } \\
\text { red cells, } \\
\text { variations in } \\
\text { size and } \\
\text { shape. }\end{array}$ & $\begin{array}{l}\text { Slight secon- } \\
\text { dary anemia } \\
\text { the rule. }\end{array}$ & $\begin{array}{l}\text { May be mark- } \\
\text { ed. Rarely } \\
\text { high color } \\
\text { index. Nu- } \\
\text { cleated ery- } \\
\text { throcytes may } \\
\text { be found. }\end{array}$ \\
\hline Erine. & $\begin{array}{l}\text { Often nega- } \\
\text { tive. }\end{array}$ & $\begin{array}{l}\text { Often nega- } \\
\text { tive. }\end{array}$ & $\begin{array}{l}\text { Usually nega- } \\
\text { tive or mild } \\
\text { albumin- } \\
\text { uria. }\end{array}$ & $\begin{array}{l}\text { Albumin } \\
\text { casts, ery- } \\
\text { throcytes or } \\
\text { alteration in } \\
\text { daily quant. } \\
\text { Phenosul- } \\
\text { phona ptha- } \\
\text { lein test. }\end{array}$ & $\begin{array}{l}\text { May be nega- } \\
\text { tive. }\end{array}$ \\
\hline Hemorrhage & $\begin{array}{l}\text { In from } 15- \\
25 \text { per cent. }\end{array}$ & Very rare. & Very rare. & Rare. & Rare. \\
\hline
\end{tabular}


Table 40.-Continued

\begin{tabular}{|c|c|c|c|c|c|}
\hline \multirow{2}{*}{$\begin{array}{l}\text { Clinical } \\
\text { signs and } \\
\text { symptoms }\end{array}$} & \multicolumn{5}{|c|}{ Nature of the disease } \\
\hline & $\begin{array}{l}\text { Gastric } \\
\text { cancer }\end{array}$ & $\begin{array}{l}\text { Achylia } \\
\text { gastrica }\end{array}$ & $\begin{array}{l}\text { Pernicious } \\
\text { anemia }\end{array}$ & $\begin{array}{l}\text { Cardio-renal } \\
\text { disease }\end{array}$ & $\begin{array}{l}\text { Addison's } \\
\text { disease }\end{array}$ \\
\hline Test-meal. & $\begin{array}{l}\text { Low free } \mathrm{Hcl} \text {; } \\
\text { often } 12 \mathrm{hr} \text {. } \\
\text { food reten- } \\
\text { tention; Op- } \\
\text { pler-Boas } \\
\text { bacilli;lactic } \\
\text { acid; altered } \\
\text { blood. }\end{array}$ & $\begin{array}{l}\text { Absent free } \\
\text { Hcl; total } \\
\text { acidity low; } \\
12 \text { hr. ret. } \\
\text { infrequent; } \\
\text { lactic acid } \\
\text { and altered } \\
\text { blood rare. }\end{array}$ & $\begin{array}{l}\text { Low or abs. } \\
\text { acidity; rare- } \\
\text { ly } 12 \mathrm{hr} \text {. ret.; } \\
\text { lactic acid } \\
\text { rare; altered } \\
\text { blood often } \\
\text { in trace; } \\
\text { streptococci } \\
\text { abundant; } \\
\text { Oppler-Boas } \\
\text { bacilli very } \\
\text { rare. }\end{array}$ & $\begin{array}{l}\text { Acid may be } \\
\text { normal or } \\
\text { low, not } \\
\text { usually ab- } \\
\text { sent; } 12 \text { hr. } \\
\text { ret. infre- } \\
\text { quent. }\end{array}$ & $\begin{array}{l}\text { Free Hcl and } \\
\text { total acidity } \\
\text { absent or low; } \\
12 \text { br. ret. } \\
\text { very rare; } \\
\text { lactic acid or } \\
\text { altered blood } \\
\text { uncommon. }\end{array}$ \\
\hline Stool. & $\begin{array}{l}\text { Altered blood } \\
89 \text { per cent. }\end{array}$ & Infrequent. & Often traces. & Infrequent. & Variable. \\
\hline Bowels. & $\begin{array}{l}\text { Constipation } \\
\text { the rule. }\end{array}$ & $\begin{array}{l}\text { Diarrhœa not } \\
\text { uncommon. }\end{array}$ & $\begin{array}{c}\text { Diarrhcea not } \\
\text { uncemmon. }\end{array}$ & $\begin{array}{l}\text { Alternating } \\
\text { diarrhoea } \\
\text { and consti- } \\
\text { pation. }\end{array}$ & $\begin{array}{l}\text { Diarrhœa not } \\
\text { infrequent. }\end{array}$ \\
\hline $\begin{array}{l}\text { Appetite. } \\
\text { Tempera- } \\
\text { ture. }\end{array}$ & $\begin{array}{l}\text { Usually poor } \\
\text { Infrequent. }\end{array}$ & $\begin{array}{l}\text { Capricious. } \\
\text { Normal. }\end{array}$ & $\begin{array}{l}\text { Variable. } \\
\text { Often eleva- } \\
\text { tions of } 1^{\circ} \\
\text { to } 3^{\circ} \mathrm{F} \text {. }\end{array}$ & $\begin{array}{l}\text { Often good. } \\
\text { Normal or } \\
\text { subnormal. }\end{array}$ & $\begin{array}{l}\text { Capricious. } \\
\text { Frequently ele- } \\
\text { vations of } 1^{\circ} \\
\text { to } 3^{\circ} \mathrm{F} \text {. }\end{array}$ \\
\hline $\begin{array}{l}\text { Tuberculin } \\
\text { test (sub- } \\
\text { cutaneous). }\end{array}$ & $\begin{array}{l}\text { Negative, the } \\
\text { rule. }\end{array}$ & $\begin{array}{l}\text { Negative, the } \\
\text { rule. }\end{array}$ & $\begin{array}{l}\text { Negative, the } \\
\text { rule. }\end{array}$ & $\begin{array}{l}\text { Negative, the } \\
\text { rule. }\end{array}$ & $\begin{array}{l}\text { Frequently } \\
\text { positive. }\end{array}$ \\
\hline Radiogram. & $\begin{array}{l}\text { Gastric reten- } \\
\text { tion; filling } \\
\text { defects. }\end{array}$ & $\begin{array}{cc}\text { Rarely } & \text { ret.; } \\
\text { atony, } & \text { no } \\
\text { filling } & \text { de- } \\
\text { fects. } & \end{array}$ & $\begin{array}{l}\text { No retention } \\
\text { or flling de- } \\
\text { defects; } \\
\text { gastric atony }\end{array}$ & $\begin{array}{l}\text { Cardiac } \\
\text { changes } \\
\text { stomach } \\
\text { may be } \\
\text { normal. }\end{array}$ & $\begin{array}{l}\text { Gastric atony } \\
\text { marked. }\end{array}$ \\
\hline $\begin{array}{l}\text { Abdominal } \\
\text { tumor. }\end{array}$ & $\begin{array}{l}\text { In } 3 \text { out of } 4 \\
\text { cases. }\end{array}$ & Absent. & Absent. & Absent. & Absent. \\
\hline $\begin{array}{l}\text { Metastases. } \\
\text { Ascites. }\end{array}$ & $\begin{array}{l}\text { Common. } \\
\text { Not infre- } \\
\text { quent; late } \\
\text { in disease. }\end{array}$ & $\begin{array}{l}\text { Absent. } \\
\text { Absent. }\end{array}$ & $\begin{array}{l}\text { Absent. } \\
\text { Absent. }\end{array}$ & $\begin{array}{l}\text { Absent. } \\
\text { May occur } \\
\text { with per- } \\
\text { ipheral } \\
\text { edema. }\end{array}$ & $\begin{array}{l}\text { Absent. } \\
\text { Absent. }\end{array}$ \\
\hline Edema. & $\begin{array}{l}\text { Small amt. } \\
\text { at ankles. }\end{array}$ & Rare. & $\begin{array}{l}\text { Small amount } \\
\text { in extrem- } \\
\text { ities. }\end{array}$ & Often marked. & May occur \\
\hline
\end{tabular}

Differentiation of Gastric Cancer from Other Ailments Associated with Low Gastric Acidity, Anemia and Cachexia.-(Author.)

Malignant peritonitis may result from lymphatic spreading of a primary gastric focus, cancer of the liver, the galltract, neoplasm of the bowel or the pelvic organs. It is rarely primary. Sometimes the clinical picture presented is confusing on account of its association with 
ascites. In its early stage it not infrequently resembles tubereulous peritonitis. It is rarely febrile, is apt to be less painful and is not, as a rule, associated with ulcerations of the bowel. The various differential facts have been brought out in Table 41 .

Ascites is of not. infrequent occurrence in late gastric cancer. If the symptoms of the primary disease have been obscure, or have not been inquired into clinically, the presence of free intrabdominal fluid may render the diagnosis difficult. These facts have also been emphasized in Table 41.

Usually careful anamnesis, examination of the gastric extract, search for "occult" blood in a properly prepared stool, or Roentgen examination of the stomach, enable one to locate the stomach as the original seat of trouble.

A study of the ascitic fluid is not infrequently of much value in the differentiation between malignant, infectious or benign abdominal ailments. These facts have been fully emphasized by Dock. This investigator has shown that fluid, obtained from the abdomen by puncture, can sometimes be demonstrated to be malignant by a careful study of its cellular elements. These may be obtained from the aspirated fluid by successive centrifugalization. The making of smears from the precipitate in the tubes, drying them, fixing and staining with Unna's polychrome methylene blue, Wright's stain, or hematoxylin and eosin enable study of the different types.

In cancer of the stomach, and not infrequently in other malignant intraabdominal diseases, large, swollen, irregularly shaped endothelial cells may be recognized. 'These show atypical mitoses, the nuclei being frequently large or of extremely bizarre shapes. 
Table 41.

\begin{tabular}{|c|c|c|c|c|c|}
\hline \multirow[b]{2}{*}{$\begin{array}{l}\text { Signs and } \\
\text { symptoms }\end{array}$} & \multicolumn{5}{|c|}{ Nature of the disease } \\
\hline & $\begin{array}{c}\text { Gastric cancer } \\
\text { peritoneal or } \\
\text { hepatic metas- } \\
\text { tases }\end{array}$ & $\begin{array}{l}\text { Cancer of } \\
\text { peritoneum }\end{array}$ & $\begin{array}{c}\text { Tuberculous } \\
\text { peritonitis }\end{array}$ & $\begin{array}{l}\text { Cirrhosis of } \\
\text { liver }\end{array}$ & $\begin{array}{l}\text { Cardio-renal } \\
\text { disease }\end{array}$ \\
\hline Age. & 45 & $?$ & Young adults. & 40 & 40 \\
\hline Sex. & Males. + & Females. + & & Males. + & Males? \\
\hline Onset. & $\begin{array}{l}\text { Prev. peptic } \\
\text { ulc. or pri- } \\
\text { mary malig- } \\
\text { nant gastric } \\
\text { disease. }\end{array}$ & $\begin{array}{l}\text { Secondary to } \\
\text { pelvic or } \\
\text { gradual and } \\
\text { atypic. }\end{array}$ & $\begin{array}{l}\text { Pulmonary or } \\
\text { abdominal } \\
\text { tuberculosis } \\
\text { (kidney, } \\
\text { ovaries). }\end{array}$ & $\begin{array}{l}\text { Gastritis, } \\
\text { alcoholic } \\
\text { excesses. }\end{array}$ & $\begin{array}{l}\text { Infectious ex- } \\
\text { posure or } \\
\text { cardio-renal } \\
\text { overstrain. }\end{array}$ \\
\hline Pain. & $\begin{array}{l}\text { Present in } 95 \\
\text { per cent. }\end{array}$ & $\begin{array}{l}\text { Colics may } \\
\text { occur early } \\
\text { or late. }\end{array}$ & $\begin{array}{l}\text { Colics or ache } \\
\text { early. }\end{array}$ & Distress late. & $\begin{array}{l}\text { Irreguler } \\
\text { cramps at } \\
\text { times. }\end{array}$ \\
\hline Vomiting. & $\begin{array}{l}\text { Common early } \\
\text { or late. }\end{array}$ & Infrequent. & Not the rule. & Variable. & Irregular. \\
\hline Weight loss. & Progressive. & Progressive. & $\begin{array}{l}\text { Progressive } \\
\text { but often } \\
\text { very gradual. }\end{array}$ & $\begin{array}{l}\text { Marked in } \\
\text { late stages. }\end{array}$ & Variable. \\
\hline Cachexia. & Pronounced. & Pronounced. & $\begin{array}{l}\text { Pronounced } \\
\text { late. }\end{array}$ & $\begin{array}{l}\text { Sometimes } \\
\text { late. }\end{array}$ & Infrequent. \\
\hline $\begin{array}{l}\text { Appetite. } \\
\text { Bowels. }\end{array}$ & $\begin{array}{l}\text { Poor. } \\
\text { Constipation }\end{array}$ & $\begin{array}{l}\text { Poor. } \\
\text { Variable. }\end{array}$ & $\begin{array}{l}\text { Capricious. } \\
\text { Diarrhœa }\end{array}$ & $\begin{array}{l}\text { Variable. } \\
\text { Constipation, }\end{array}$ & $\begin{array}{l}\text { Often good. } \\
\text { Diarrhœea and }\end{array}$ \\
\hline & often. & & variable. & $\begin{array}{l}\text { diarrhœa at } \\
\text { times. }\end{array}$ & $\begin{array}{l}\text { constip. al- } \\
\text { ternating not } \\
\text { infrequently. }\end{array}$ \\
\hline $\begin{array}{l}\text { Blood pres- } \\
\text { sure. }\end{array}$ & Reduced. & Reduced. & Reduced. & $\begin{array}{l}\text { May be in- } \\
\text { creased. }\end{array}$ & $\begin{array}{l}\text { Usually in- } \\
\text { creased. }\end{array}$ \\
\hline Jaundice. & Late if at all. & Rare. & Rare. & May occur. & Infrequent. \\
\hline Abdomen. & $\begin{array}{l}\text { Often scaphoid } \\
\text { but may be } \\
\text { distended in } \\
\text { flanks or } \\
\text { wholly. }\end{array}$ & $\begin{array}{l}\text { Distended } \\
\text { late. }\end{array}$ & $\begin{array}{l}\text { May be early } \\
\text { tense or dis- } \\
\text { tended. }\end{array}$ & $\begin{array}{l}\text { Tense or dis- } \\
\text { tended. }\end{array}$ & Distended. \\
\hline Tumor. & $\begin{array}{l}\text { In } 3 \text { out of } 4 \\
\text { cases. }\end{array}$ & $\begin{array}{l}\text { Early multi- } \\
\text { ple nodules. }\end{array}$ & $\begin{array}{l}\text { Numerous } \\
\text { ridges often } \\
\text { above navel; } \\
\text { slow devel- } \\
\text { opment. }\end{array}$ & $\begin{array}{l}\text { Local enlarge- } \\
\text { ment in right } \\
\text { upper abd. } \\
\text { (liver). }\end{array}$ & $\begin{array}{l}\text { Liver may en- } \\
\text { large or no } \\
\text { tumor pres- } \\
\text { ent. }\end{array}$ \\
\hline Fluid. & $\begin{array}{l}\text { Usually free; } \\
\text { serous or } \\
\text { bloody "can- } \\
\text { cer cells" (?). }\end{array}$ & $\begin{array}{l}\text { Often in loculi } \\
\text { bloody, chy- } \\
\text { lous, or ser- } \\
\text { ous; "cancer } \\
\text { cells" (?). }\end{array}$ & $\begin{array}{l}\text { Serous or } \\
\text { cloudy, many } \\
\text { small lympho- } \\
\text { cytes, bacilli } \\
\text { rarely found. }\end{array}$ & $\begin{array}{l}\text { Serous, rarely } \\
\text { bloody but } \\
\text { may be bile- } \\
\text { stained. }\end{array}$ & Serous. \\
\hline Test-meal. & $\begin{array}{l}\text { Low or absent } \\
\text { Hcl; } 12 \text { hr. } \\
\text { ret. lactic } \\
\text { acid; altered } \\
\text { blood or Op- } \\
\text { pler-Boas ba- } \\
\text { cilli. }\end{array}$ & Low Hel. & $\begin{array}{l}\text { Often low } \\
\text { Hel. }\end{array}$ & $\begin{array}{l}\text { Hel may be } \\
\text { uormal. }\end{array}$ & $\begin{array}{l}\text { Hel may be } \\
\text { normal. }\end{array}$ \\
\hline
\end{tabular}

Table continued on page 408. 
Table 41.-Continued

\begin{tabular}{|c|c|c|c|c|c|}
\hline \multirow[b]{2}{*}{$\begin{array}{l}\text { Signs and } \\
\text { eymptoms }\end{array}$} & \multicolumn{5}{|c|}{ Nature of the disease } \\
\hline & $\begin{array}{l}\text { Gastric cancer } \\
\text { peritoncal or } \\
\text { hepatic metae- } \\
\text { tases }\end{array}$ & $\begin{array}{l}\text { Cancer of } \\
\text { peritoneum }\end{array}$ & $\begin{array}{l}\text { Tuberculous } \\
\text { peritonitis }\end{array}$ & $\begin{array}{l}\text { Cirrhosis of } \\
\text { liver }\end{array}$ & $\begin{array}{l}\text { Cardio-renal } \\
\text { disease }\end{array}$ \\
\hline Stool. & $\begin{array}{l}\text { Altcred blood } \\
\text { in } 89 \text { per cent. }\end{array}$ & Blood rare. & Blood late. & $\begin{array}{l}\text { Blood may } \\
\text { occur late. }\end{array}$ & $\begin{array}{l}\text { Blood infre- } \\
\text { quent. }\end{array}$ \\
\hline Urine. & May be normal. & $\begin{array}{l}\text { May be nor- } \\
\text { mal. }\end{array}$ & Albumin late. & Albumin late. & $\begin{array}{l}\text { Large volumes } \\
\text { of albumin } \\
\text { and casts } \\
\text { often early. } \\
\text { Phenolsul- } \\
\text { phonphtha- } \\
\text { lein test. }\end{array}$ \\
\hline Radiogram. & $\begin{array}{l}\text { Gastric reten- } \\
\text { tion variable; } \\
\text { filling defects. }\end{array}$ & $\begin{array}{l}\text { Usually nega- } \\
\text { tive. }\end{array}$ & $\begin{array}{l}\text { Usually nega- } \\
\text { tive. }\end{array}$ & $\begin{array}{l}\text { Negative or } \\
\text { pressure de- } \\
\text { fect from } \\
\text { liver. }\end{array}$ & $\begin{array}{l}\text { Usually nega- } \\
\text { tive. }\end{array}$ \\
\hline Metastases. & Common. & $\begin{array}{l}\text { Common to } \\
\text { serous sur- } \\
\text { faces or belly } \\
\text { wall. }\end{array}$ & $\begin{array}{l}\text { Involvement } \\
\text { of } 1 \mathrm{ungs} \text {, } \\
\text { liver, intes- } \\
\text { tines or pel- } \\
\text { vic organs. }\end{array}$ & Absent. & Absent. \\
\hline Edema. & $\begin{array}{l}\text { Late and } \\
\text { limited. }\end{array}$ & $\begin{array}{l}\text { L a te and } \\
\text { limited. }\end{array}$ & $\begin{array}{l}\text { Late and } \\
\text { limited. }\end{array}$ & $\begin{array}{l}\text { Late and not } \\
\text { often mark- } \\
\text { ed. }\end{array}$ & $\begin{array}{l}\text { May be mark- } \\
\text { ed early. }\end{array}$ \\
\hline $\begin{array}{l}\text { Tempera- } \\
\text { ture. }\end{array}$ & $\begin{array}{l}\text { Infreque nt } \\
\text { increase. }\end{array}$ & $\begin{array}{l}\text { Infrequent } \\
\text { increase. }\end{array}$ & $\begin{array}{l}\text { Irregular } \\
\text { clevation. }\end{array}$ & $\begin{array}{l}\text { Elevation } \\
\text { late. }\end{array}$ & $\begin{array}{l}\text { May be sub- } \\
\text { normal. }\end{array}$ \\
\hline
\end{tabular}

Differential Diagnosis between Gastric Cancer with Metastases to the Peritoneal or Portal System and Diseases Associated with Ascites.-(Author.)

In tuberculosis, colorless or straw-colored fluid is usually obtained. Its cellular constituents are few. A study of the cell forms reveals excess of small lymphocytes. In extensive malignancy, bloody ascitic fluid may be present. In such instances red blood cells may be found together with endothelial cells showing atypical division.

In a few cases of gastric cancer, particularly where there has been involvement of the large lymphatic channels, as for example the receptaculum chyli, or the thoracic duct, the ascitic fluid may be milk-like or creamy. This occurred in three instances in our series. A microscopic study of this fluid is not infrequently negative, with the exception of demonstrating a large number of lymphocytes and fat droplets. 
The Incidence of Ascites in Gastric Cancer.-Free fluid is usually an evidence of late, hopelessly inoperable gastric cancer. When free fluid is present other signs of the disease are not lacking. It most commonly occurs with secondary involvement of the peritoneum itself, the liver, pelvic organs, the ovary, transverse colon, gastrocolic omentum and pancreas. In our series of cases, ascites was present in 86 instances or 9.3 per cent. These were all inoperable cases, and laparotomy usually disclosed extensive glandular invasion wholly irrespective of the size or position of the local growth in the stomach.

Gastric cancer secondary to malignancy in organs other than the stomach occurred in sixteen instances in our series. It was secondary to tumors of the breast, the liver, female genitalia, colon and the pancreas. Invasion of the stomach was either by lymph-gland metastases, or by contiguous involvement. Usually in these cases anamnesis and general physical examination disclosed a primary tumor. Not rarely operative procedures had already been performed upon the primary growth.

As we have already mentioned, it would seem quite necessary to differentiate many so-called secondary gastric cancers from primary malignant processes wholly independent of an extra-gastric lesion, to which they are sometimes considered secondary.

Syphilis of the Stomach.- Syphilis of the stomach is becoming more readily recognized since the perfection of blood-serum tests for lues. In the majority of instances of gastric syphilis anamnesis discloses an early specific lesion. Physical examination may demonstrate certain local or general evidences of secondary or tertiary manifestation of the disease.

Before the onset of a definite tumor growth in the 
stomach, the history is not infrequently that which we associate with chronic peptic ulcer. Inasmuch as ulceration of the stomach in gastric syphilis is apt to be multiple, epigastric pain and a continuance of the dyspepsia, instead of having it occur at intervals, may be quite markedly evidenced.

Gummata of the wall of the stomach or extensive invasion of the mucous membrane may develop in time in the course of the disease and may be readily mistaken for cancer. A large growth may press upon the pylorus, may produce malignant hour-glass, bring about multiple tumors in the stomach, or, as a result of necrosis of a portion of these tumors, cause the development of numerous, seemingly malignant ulcers.

Whenever a case of multiple gastric tumor or multiple chronic gastric ulcer is demonstrated, the Wassermann test should be made as routine. If this test is positive, then specific treatment should be pushed. This therapeutic differentiation is an important one. We have seen large multiple ulcers of the stomach and even gummata disappear under proper antiluetic treatment.

Tuberculosis of the stomach is extremely uncommon. While tubercle bacilli are frequently swallowed, they do not seem to retain their viability in the presence of normal gastric juice. While it is conceivable that initial trauma in the gastric lining may furnish a lodging point for tubercle bacilli, and as a consequence ulcer may develop, it is more than likely that tuberculous ulcers or granulomata develop in the gastric wall as a consequence of bacilli being carried by the blood or lymphatic streams.

Primary tuberculosis of the stomach is of such great rarity that when a large chronic tuberculous ulcer is found in this viscus, search for the primary focus of the disease 
should be made. It is usually found in the lungs, kidneys or the peritoneum. The symptoms of the disease are similar to those associated with non-tuberculous, benign gastric ulcer. The presence of these ulcer symptoms in an individual with no tuberculosis should be sufficient to suggest proper diagnosis. Extreme emaciation, the presence of fever, abdominal tenderness and the positive tuberculin reaction should render recognition of the condition apparently easy if the fact is borne in mind that such an affection is likely to occur.

Gastric granuloma is a rare ailment and is not usually diagnosed until laparotomy or at the post-mortem table. Interesting cases have been reported by Meyer and Hardy. A syphilitic etiology not infrequently underlies the condition. Epigastric pain, weight loss, vomiting, hemorrhage and sometimes the finding of a characteristic bit of tissue in either the vomitus or the stools are facts which are of service in diagnosing the existence of the disease in the stomach.

Roentgen examination may reveal multiple gastric tumors, a gastric outline of small size and irregular form or occasionally the actual evidences of polypoid growths.

The importance in recognizing this condition is that of preventing patients coming to operation for a condition which, of course, is not curable surgically.

Linitis plastica or cirrhosis of the stomach is a disease in which there is an enormous fibroid thickening of the gastric wall. This is usually general, although it appears in certain cases to progress from the pyloric end toward the cardia. This enormous thickening of the stomach wall results in a contraction of the stomach with the production of a small lumen. Obstruction with romiting, retention, absent hydrochloric acid, emaciation, anemia are usually clinical manifestations. A full account of this rather uncommon 
condition has been given by Lyle. Clinically, the affection is usually considered as extensive, scirrhus cancer of the stomach, or as sarcoma. Laparotomy and the examination of sections of extirpated tissue furnish the only reliable differential points in this disease.

Sarcoma of the stomach is comparatively rare. It was present in 4 cases in our series. Excellent analyses of this condition have been made by Clendemning, Yates and Campbell. Clinically, there is not infrequently a previous history of gastric ulcer. The supervening malignant period is in every way similar to that of gastric cancer. Hemorrhage is perhaps more common. Extensive metastases may occur early.

We have scen one instance where invasion of the left supraclavicular lymph nodes resulted in a tumor the size of a grape fruit 2 months after the onset of a gastric disease apparently malignant, and following upon a previous ulcer.

Foreign Bodies in the Stomach.-These are of rare occurrence in the human, but may attain great size. There is usually the history of swallowing of such things as nails, keys, hair, thread and the like. The patients are not infrequently neurotic or of the circus class. The foreign bodies may form recognizable abdominal tumors. Their traumata may bring about hemorrhage. Weight loss may be constant on account of obstruction or dread of producing pain on food ingestion. There is rarely associated with it cachexia coming from systemic poison as a consequence of malignant disease.

Roentgen examination may definitely recognize the tumor as being intragastric, or demonstrate metal, stones and the like.

Foreign Bodies in the Large Bowel.-_"Bezoar stones" are uncommon. They may result from inspissated feces, 
calcareous deposits, gall-stones or parasites. They not infrequently occur in individuals who have worked on farms or have been associated with cattle and horses. The gastric findings are rarely confused with those of cancer of the stomach. Unless there is obstruction, emaciation and cachexia are uncommon. The abdominal tumor is atypically situated and in the large majority of cases below the navel. Roentgen examination may definitely prove that the tumor is extragastric. Sometimes its character may be determined by plate or screen examination. The following history is suggestive.

Tumor in Left Hypochondrium; Constipation; Bezoar Stone.

Dr. J. C.-Age 46, American, veterinarian.

Family History.-Unimportant.

Personal History.-Several severe attacks of la grippe. Intermittent dyspepsia of type, clinically, duodenal ulcer for 6 to 10 years.

Present Trouble.-Gradually developing constipation for past $11 / 2$ years; at present has marked obstipation; associated with this are abdominal pains (usually about navel) coming on when bowels have not moved for several days, sensations of gurgling in abdomen, bloating and slight nausea. Several months ago, noted painless tumor just to the left of the navel. This has increased in size gradually since when first noted.

Obstipation.-Very marked; can now get bowels to move only with great difficulty; never noted blood or pus in stools.

Appetite.-Fair.

Weight.-Lost 15 pounds in past 3 months.

Examination.-Stocky, well-nourished, thick-set male; skin pale, sallow.

Throat.-Peritonsillar redness.

Thorax.-Negative.

Abdomen.-Flat, panniculus moderately thick. Stomach slightly splashy and reaches to navel in mid-line. Deep tenderness in right upper abdominal quadrant (pylorus?). In the left hypochondrium, extending from the rib margin 
to the level of the iliac crest is a nodular, firm, slightly movable, not tender mass. It is about 3 inches long and 1 to 2 inches wide. On inflation of the colon the tumor goes apparently posteriorly.

Proctoscopic Examination.-Negative. Gastric inflation seems to indicate that the tumor is extragastric.

Laboratory Examinations:

Blood.-Hg., 90 per cent.; r.b.c., 5,000,000; w.b.c., 8,400 .

Stool.-Small, hard, firm, no blood.

Test-meal.-Slight gastric stagnation. Total acidity, 62; free $\mathrm{Hcl}, 40$.

X-ray.-Stomach moderately dilated. Outline negative; peristalsis active.

Colon.-On fluoroscopy a dense mass is seen in the lower half of the descending colon; it has fairly regular outline and appears fixed to deep structures. The mass is lost on filling the colon with bismuth.

Laparotomy.-Calcified bezoar stone, densely adherent to the posterior wall of the descending colon; chronic perforation.

Chronic obstipation occurs in patients past middle life. When associated with weight loss, anemia, and irregular gastric disturbance, it is sometimes confused with gastric malignancy. In these instances routine anamnesis, physical examination, analysis of the gastric contents, fluoroscopic examination of the stomach and Roentgen plates made of the abdomen, after the colon has been injected with barium sulphate, are usually sufficient to exclude gastric cancer and show malformation or malposition of portions of the large bowel.

Protozoic Infections of the Bowel.-Even in the temperate zone there is a definite class of toxic, anemic, dyspeptic individuals who present themselves for diagnosis and in whom cancer may be suspected. Intermittent diarrhœa is a not infrequent finding. Altered blood may be present in the stools. The patients have not rarely lost consider- 
able weight. The anamnesis in such instances discloses frequently the long-continued drinking of contaminated water or the eating of farm produce contaminated by water or the discharges of farm cattle, ducks, etc. The disease seems to be periodic in a large number of instances. Attacks of diarrhœa last for several weeks, alternating with weeks, or even months, of fair health. As an end result anemia develops. It is usually of the secondary type, but in many cases there is an excess of eosinophiles. The gastric secretions often exhibit absent or low free hydrochloric acid. Pancreatic functional tests show diminution in ferments. The examination of a fresh stool on a warm microscopic stage reveals protozoa associated with great numbers of motile and non-motile bacilli, chains of streptococci, yeasts and undigested food. From such cases we have been able to isolate endamœba, trichomonas, cercomonas, balantidium coli and megastoma entericum.

In the average case, ridding the bowel of these parasites goes a long way toward getting the patient well.

$X$-ray examination of the stomach and bowel in these patients is negative.

It should be emphasized that the above type of case is more frequently seen than is recognized in current textbooks. These protozoic infections are chronic and usually intermittent. They seem to be due to precisely the same type of organisms which, under proper circumstances (climate, diet, change of water, etc.), may be capable of causing acute colitis, with or without hemorrhage, ulceration, abscess formation, and the like.

Certain constitutional faults are occasionally mistaken for gastric cancer, and in fact may be associated with it. Among such we would mention, gout, diabetes and nephritis. The clinical history of these cases is different from 
that of cancer. Analyses of the test-meal, feces, blood and urine generally bring out the essential differential points. Examination of the stomach and bowel by Roentgen ray aids in establishing the diagnosis of non-malignant disease.

Certain drug addictions produce a systemic change resembling that of gastric cancer. Among such are the habitual use of morphine, heroin, codein, bromides or the smoking of opiates. In these cases, however, careful examination usually demonstrates needle-punctures, alterations in the pupil, in the mental state, in the urine. To such the gastro-intestinal findings are entirely subordinate.

In this class of patients especially, observations must be made with the clothing removed, and in doubtful cases the patient should be isolated and watched until the definite cause of the anomaly is established.

Certain tumors of the abdominal wall may be considered, upon casual examination, as having connection with the stomach. Such tumors are lipomata, enchondromata, cysts, abscesses, or fibro-sarcomata. Careful anamnesis, physical, chemical and $x$-ray examinations generally differentiate such conditions quite readily. When doubt exists, sections of the growth may be removed, or the tumor may be aspirated or incised in order to permit microscopic study of tissue or pus.

\section{REFERENCE}

Dock, George: Amer. Jour. Med. Sciences, 1902. 


\section{CHAPTER XI}

\section{SURGICAL TREATMENT OF CANCER OF THE STOMACH}

BY

Albert J. Ochsner, B.S., M.D., LL.D., F.R.C.S., F.A.C.S.

The value of surgical treatment of cancer of the stomach primarily depends: first, upon an early diagnosis; second, upon the complete excision of all of the cancerous tissue; third, upon the fact that the traumatism caused by the operation is within the margin of safety of the individual patient; fourth, upon a satisfactory mechanical result which will enable the patient to live comfortably with his changed digestive apparatus, and fifth, upon careful aftertreatment.

Fortunately, patients suffering from cancer of the stomach come for surgical treatment earlier in the course of the disease than in former times, and as a result of this condition it is possible to make a complete excision in a larger number of cases. There is a class in which it may not be possible to make a differential diagnosis positively between cancer of the stomach, ulcer of the stomach or duodenum, disease of the gall-bladder, adhesions in the region of the pylorus, or obstruction to the pylorus due to cicatricial contraction resulting from a previous ulcer. It is in this class of case that the surgeon has more frequently committed errors than in any other group. It is without doubt, in this type of case, that an operation should always be performed, because whatever condition may be found after the abdomen has been opened, it is proper that the condition be treated surgically. 
The pride or personal conceit of the surgeon may suffer because of his inability to make a positive diagnosis in these cases and he may wish to continue his observations for a number of weeks or months in order to be certain before operating, so that he may be able to demonstrate the correctness of his diagnosis at the time of the operation. This plan is, howerer, exceedingly bad because in case of cancer, the condition may have progressed to such an extent that while a permanent cure might have been accomplished with an early operation, the late operation will result at best only in a temporary improvement. Consequently, after a very thorough examination has been made, if there is still doubt, an operation should invariably be performed.

\section{COMPLETE EXCISION}

By far the greatest number of cases in which the complete excision of all malignant tissue is feasible, belong to a class in which it was not possible to make a positive differential diagnosis between cancer and ulcer. There is, however, a class of cases in which the cancer seems to be almost entirely confined to the mucous membrane, and does not penetrate the entire wall of the stomach and is of sufficient firmness to prevent the loosening of cells to be carried to distant points for a considerable period of time. In this group of cases it is also possible to remove all of the cancerous tissue.

In planning the operation, one should constantly bear in mind the distribution of lymph channels and lymph nodes described by Cuneo, because in this way it will be possible to remove the tissue which is likely to be infected with cancer beyond the tumor itself, together with the lymph nodes which are most likely to contain cancerous infection. 
Preparation for Operation.-The preparation for all operations for the relief of cancer of the stomach is the same, consequently it may be given here and then it may be applied to all of the operations upon the stomach which will be described through this Chapter.

First, an attempt must be made to prevent as much as possible the introduction into the stomach of septic material. For this reason it is important to free the mouth and the nasal passages as thoroughly as possible of all infective material before the operation is undertaken. If the patient has decayed teeth or roots, or both, or suffers from pyorrhea, or from catarrh, or from tonsillar infection, all of these conditions should be disposed of by proper treatment, provided this treatment will not consume a sufficient amount of time to endanger the patient's condition from progression of his disease. In case of such danger, it is better to thoroughly spray the nasal cavities and the pharynx repeatedly. with some mild antiseptic solution for 24 hours preceding the operation, giving the patient a good antiseptic gargle to thoroughly cleanse the teeth a number of times during the 24 hours preceding the operation, and to paint the roots of the teeth in case of pyorrhea with tincture of iodine; also to cleanse the tongue thoroughly and to get the cavities of the nose and mouth in as nearly an aseptic condition as we are able to get them.

No nourishment should be given that contains any septic material. It is best to give these patients broth only for 24 hours preceding the operation. The stomach should be thoroughly irrigated until the return fluid is perfectly clear. The patient should be given 2 ounces of castor oil the day before the operation, because this will carry away a great amount of foul material. It is well to place this castor oil in the stomach through the 
stomach tube, after thorough irrigation of the viscus the day before the operation after all of the irrigating fluid has been siphoned out. By doing this, even though the passage between the stomach and the duodenum may be almost completely occluded because of the presence of the tumor, a sufficient amount of the oil will pass through this space, as a rule, to completely cleanse the intestines. The stomach should be washed out again within an hour preceding the operation. It is well to thoroughly cocainize the pharynx with a spray of 2 per cent. cocaine and to permit the patient to swallow this in order that the pharynx and the esophagus will become properly anesthetized by this substance. It is best to wait about 12 minutes after the spraying has ceased in order that the cocaine may have completely removed all sensation from the parts over which the stomach tube is to be passed. Not more than 2 drams of a 2 per cent. solution of cocaine should be used for this purpose, this amount being quite sufficient and there being no danger from cocaine poisoning if no larger amount is used.

The patient should, of course, have all of the other ordinary preparations, such as a warm bath previous to the operation, etc. The abdominal wall should be treated in the manner which is usually employed in preparing for all abdominal sections.

Cancers not Located in or Near the Pylorus. - The rare cases of cancer located away from the pylorus which come under surgical treatment sufficiently early to permit complete excision, are usually encountered accidentally either in the form of sarcoma of which we have had a few cases, or in the form of cancer developing at the bottom or in the edge of a gastric ulcer. In the case of sarcoma, metastatic tumors are not common early in the development of these 
growths and consequently it is possible to make a complete excision by remoring tissue $3 \mathrm{~cm}$. in every direction from the primary growth and then closing the wound left in the stomach in a manner to prevent obstruction from cicatricial contraction.

Usually the line of suture should be in a transverse or an oblique direction, but the location and the extent of the growth in the individual case must determine the direction of the closure. In case the growth involves the lower end of the esophagus, the chances of recovery after removal are so slight that the operation need scarcely be considered at the present time.

Willy Meyer is developing a technique in the use of his differential air-pressure cabinet, with which he hopes in time to succeed in satisfactorily removing such growths and by reuniting the esophagus and stomach, by a tube constructed from a portion of the stomach with the remmant of the esophagus, prolong life. At the present time it is too early to discuss this operation.

Carl Beck has devised an operation in which he utilizes the lower portion of the stomach for the purpose of constructing a tube, which can be used as an artificial esophagus, to be carried upward underneath the skin of the chest, in cases of carcinoma of the esophagus or of the cardiac end of the stomach involving the lower end of the esophagus. Unfortunately these cases usually die so soon after the operation from the recurrence of the malignant growth that it is doubtful whether so severe and so extensive an operation is indicated in a patient whose life expectancy is relatively short. In case one does not feel warranted in performing so extensive an operation in this class of cases which have advanced so far that food can no longer 
be taken through the esophagus, it is well to perform a gastrostomy.

Technique of Operation.-(Witzel's Gastrostomy).-An incision $5 \mathrm{~cm}$. long is made through the edge of the left rectus abdominis muscle just below the costal margin and 3 $\mathrm{cm}$. to the left of the median line. The incision is carried through all layers into the peritoneal cavity. The stomach is then examined to determine the exact extent and location of the tumor. A fold of the stomach-wall is drawn through the abdominal opening, the fold being chosen far enough away from the growth to prevent its involvement in the adrance of the malignant growth. The skin outside of the deep fascia is then undermined upward over the costal margin for a distance of $4 \mathrm{~cm}$. and a transverse incision is made through the skin. The undermined space is loosened to a width of $3 \mathrm{~cm}$. in order that the fold of the stomach, which is to be drawn through this space, may not be unduly compressed and thus produce gangrene of the stomach wall. The tip of this fold, $1 \frac{1}{2} \mathrm{~cm}$. long, is permitted to project through the transverse opening and is sutured in place with six or eight fine, silk sutures. Asecond row of fine, silk sutures is placed so as to unite the wall of the stomach with the peritoneum and transversalis fascia and a few additional sutures are placed between the stomach wall and the edges of the rectus abdominis muscle and the anterior sheath of the rectus abdominis muscle. The wound is then closed.

If the patient is suffering from starvation, the fold of stomach projecting beyond the transverse incision is at once opened and a Jacobson retention catheter is passed into the stomach and some concentrated food and water are at once administered through this catheter whose distal end is closed by means of a cork or a clamp during 
the intervals between feedings. In these cases the patient should receive nourishment every 2 hours. If the patient's condition is not so serious it is well to postpone opening the stomach for 24 or 48 hours, until a strong adhesion between the stomach and surrounding tissue has been formed, in order that there may not be any infection from the stomach wound. The danger from such an infection is so slight that it need not interfere with the completion of the operation if the patient is in need of nourishment. This arrangement will prevent leakage from the stomach perfectly and permanently.

There are many other operations equally as satisfactory as the one described (which was introduced by Professor Witzel) but none of them are simpler or more satisfactory than the above.

In case the entire cardiac end of the stomach has been involved in a malignant growth, then it may become necessary to make the gastrostomy to the right of the median line, and in that case the amount of stomach wall is usually not sufficient for this operation and the operation introduced by Senn will give fairly satisfactory results.

Senn's Operation.-This operation consists in making a small puncture in the available stomach wall and introducing through this a Jacobson's retention catheter, which has been stretched upon a probe so that the diameter of the rubber tubing is greatly reduced in order that it may thoroughly occlude the puncture opening in the stomach wall upon contraction. A purse-string suture is then passed through the stomach wall $1 / 2 \mathrm{~cm}$. from the rubber tubing and tied snugly about the tubing. It is best to use fine silk for this purpose. This is introduced with a fine, curved needle in order that all of the layers of the stomach wall down to, but not through, the mucous membrane, may be engaged in 
the suture in order that it may have a substantial hold, because of its passing through the submucous connective tissue. A second, third and a fourth purse-string suture of the same kind are introduced successively and then the stomach is attached by means of fine silk sutures to the peritoneum and transversalis fascia and the tube is permitted to pass out through the abdominal wound. This is closed by means of sutures on each side of this tube. In case there is leakage around the tubing, which may occur if the patient lives for a number of months after the operation, it is well to increase the size of the rubber tube sufficiently to produce a perfect closure of this canal.

In order that the patient may have the pleasure of eating, he may chew the food he takes very thoroughly, place it in the barrel of a large glass syringe and then inject it into his stomach through this tube. Aside from the satisfaction the patient has in chewing the food, there is the advantage of having the food mixed with saliva. Until the patient becomes accustomed to being fed in this manner it is well to continue the administration of concentrated food by means of enemata given every 3 hours, and also by the administration of normal salt solution by the drop method of proctoclysis. In most of these cases, the administration of from 5 to 10 drops of eucalyptus oil, in a little cream, by mouth from 3 to 6 times a day, serves to disinfect the malignant growth in the lower end of the esophagus and to increase the comfort of the patient.

\section{EXCISION OF CIRCUMSCRIBED GROWTHS NOT LOCATED IN THE REGION OF THE PYLORUS OR THE ESOPHAGUS}

In order to reduce the traumatism to a minimum, it is well to make a very large abdominal incision, so that the stomach may be brought out into view without unneces- 
sary tension or injury. A long stomach clamp with blades sufficiently thin and covered with rubber tubes to prevent injury from pressure, is applied at a distance of $10 \mathrm{~cm}$. above and below the growth, in order to compress the bloodvessels supplying the region of the stomach to be operated. A thin, silk suture is then applied around each individual vessel $4 \mathrm{~cm}$. away from the growth in every direction. (Before beginning the operation the stomach is thoroughly irrigated until the returning water is perfectly clear. It is well to use normal salt solution at a temperature of $105^{\circ} \mathrm{F}$. for this purpose.) The stomach is then opened directly to the outer side of the line of sutures controlling the bloodressels and fine tenaculum forceps are applied in such a manner that later they may be utilized as land-marks in closing the defect in the stomach. In case there is bleeding from the mucous membrane, the bleeding point is caught and ligated. After the entire growth, together with $3 \mathrm{~cm}$. of the surrounding tissue, has been remored, the tenaculum forceps are utilized for bringing the edges together. A row of sutures of thin, chromicized catgut threaded double is used for uniting the mucous membrane. A second row of fine silk sutures is used for uniting the muscular layer and over this a row of fine, silk sutures is applied grasping all of the layers down to but not through the mucous membrane. In this way a perfect union can be accomplished without much infolding of the stomach wall. In case the tumor is small, the Connell suture may be used instead of this method. The suture is passed through all of the layers of the edge of the wound on either side, so that the serous surface is placed in apposition throughout. A second row of sutures corresponding to the third row which has just been described is placed over this and in this way the Connell suture is reinforced. In either of these cases there can be 
no hemorrhage because all of the blood-ressels are thoroughly controlled by the various rows of sutures.

In case the growth is so near the pylorus that it will be necessary to constrict the pyloric end of the stomach by the application of the sutures closing the defect, it is best to plan a gastro-enterostomy in addition to the excision of the growth. This will, however, be different in no way from the operation to be described in connection with the treatment of carcinoma of the pylorus. It is consequently not necessary to explain it at this point.

\section{CARCINOMA OF THE PYLORUS}

Nearly all of the cases of cancer of the stomach which come early enough to make it reasonable to expect a permanent cure following an operation, are located in the region of the pylorus. This is also true of a fairly large class of cases which can be much benefited by an operation although there is no possibility of a permanent cure. In nearly all of these cases the obstruction to the pylorus is so marked that practically no food can pass into the small intestines. This condition causes starvation, and also makes a foul retention cavity of the remaining portion of the stomach, in which the remnants of food and the mucus secreted by the stomach and the broken-down ulcerating carcinomatous tissue, produce a decomposing substance, portions of which will be absorbed through the stomach walls and will cause more or less severe symptoms of cachexia.

On the one hand these patients are starved and on the other hand they are poisoned by these foul accumulations. Moreover, the vomiting which is caused by the latter serves to irritate the cancerous growth so that these patients are in a most deplorable condition. Even with 
repeated lavage, it is not possible to keep the stomach even fairly clean. These patients gain enormously by a properly planned and executed gastro-enterostomy, because this will permit nourishment from the stomach to reach the intestines and will remove the suffering from starvation. It will prevent the accumulation of decomposing material in the stomach and will thus remove the auto-intoxication. It will in the same way alleviate the nausea and romiting and the irritation from the latter to the tumor. The ulcerated surface of the tumor is likely to become clean as a result of this improved condition.

The patient's life is naturally prolonged and his comfort is enormously increased by these changed conditions. Moreover, a patient may gain so much in strength that although the removal of the growth at the primary operation would undoubtedly have resulted in death from shock, the increase in strength as a result of the improved nutrition and the absence of the other distressing conditions may make a secondary operation fairly, if not perfectly, safe.

This leads us to the plan of operating for the relief of cancer of the pylorus in two stages in these greatly reduced patients. In order to perform the operation satisfactorily in two stages, however, it is of the greatest importance that the first operation be so performed that the intraabdominal condition will be favorable for performing the second operation at a later date. This presupposes that the first operation will not result in extensive adhesions, but simply in a union between the jejunum and the stomach at a point sufficiently distant from the malignant growth to make its complete excision possible at the second operation. If the first operation is followed by extensive 
adhesions, then the second operation is liable to fail from two causes. In the first place the extensive loosening from adhesions is likely to produce a degree of shock so severe that the patient will succumb to the operation a short time after it has been performed. In the second place the traumatism eausing these adhesions is likely to result in transplantation of the cancerous tissue, so that the adhesions will be filled with secondary carcinomatous growths.

The location of the gastro-enterostomy opening in the stomach must be chosen so that practically the lowest portion of the stomach will contain this opening, provided it is possible by doing this to remain far enough away from the original growth to make its excision possible or to prevent its encroaching upon this opening in cases which are so far adranced that complete remoral is not possible at a later period.

The operation of gastro-enterostomy is the same without regard to the other operation, consequently one description will suffice as well for cases that are simply to have this operation for temporary relief, for those who are later to have the excision of the growth, and for those in whom the gastro-enterostony and the excision are to be performed at the same time.

Posterior Gastro-enterostomy.-During the early period of stomach surgery we performed over 200 anterior gastro-enterostomies, always choosing the lowest point in the stomach in accordance with a suggestion made by $\mathrm{W}$. J. Mayo 15 years ago. During the past years we have, however, chosen the posterior gastro-enterostomy with a short loop of the jejunum in all cases in which this was not prevented by the location of the tumor, not that the results are any better, but because it has seemed to be the opera- 
tion which impressed one as being more nearly correct from a mechanical standpoint. In cases in which it is likely that a secondary operation must be performed, the incision should be made through the median line from the ensiform cartilage of the sternum to a point $3 \mathrm{~cm}$. below the umbilicus. This will provide an abundance of space for bringing

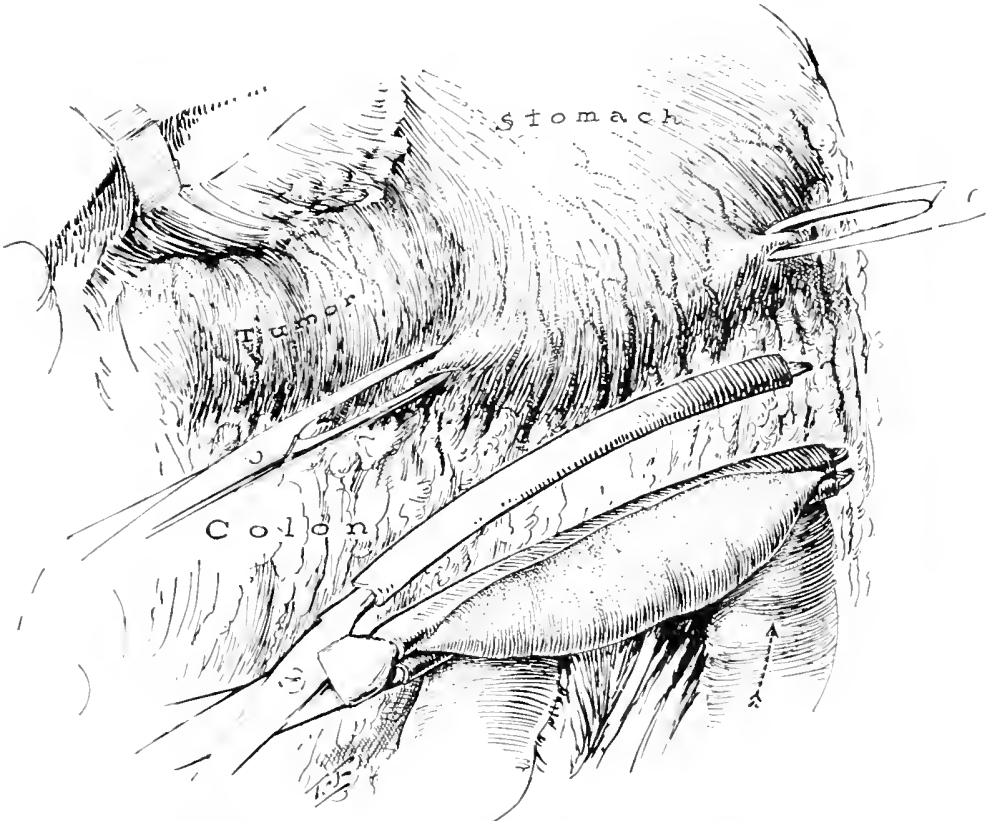

Fig. 85.-Anterior gastrojejunostomy made with gastrie clamp for inoperable carcinoma of pyloric third of stomach with obstruction. A long loose loop of jejunum is carried over the greater omentum and colon. This is held in one half of the rubber covered gastric clamp, the other half of which is open to receive the portion of stomach held by the mouse-tooth clamps. The anastomosis is made just anterior to the greater curvature of the stomach and as close to the tumor as possible. Arrow indicates the direction of flow in the bowel.

the stomach and intestine into view with the slightest amount of traumatism.

The skin to each side of the abdominal incision is covered with towels and then the omentum and transverse colon are carried upward in order to expose the jejunum where it emerges from beneath the transverse mesocolon. It is 
well to have the mesocolon spread out so that one can fairly see its blood-ressels, in order that these will not be injured during the next step of the operation, which consists of the tearing of a hole in this structure sufficiently large to permit the union between the lower posterior wall of the stomach and the loop of the jejunum. The stomach wall is drawn through this opening and a point as far away

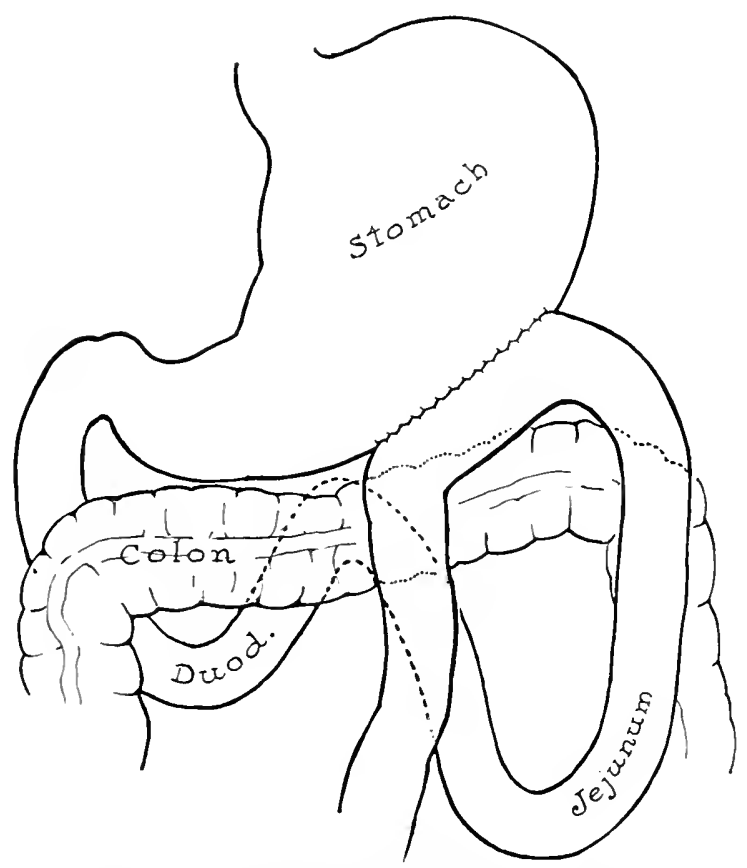

Fig. 86.-Diagram to show the relations in a completed anterior gastro-enterostomy.

as possible from the malignant growth is chosen. A pair of fine-toothed clamps is applied to the lower margin of this space in close proximity to the gastro-epiploic ressels. A second pair of forceps is applied upward and a little to the right at a distance of $5 \mathrm{~cm}$. from the first pair. A straight line between these two forceps indicates the location of the gastro-enterostomy opening to be made presently. 
This fold of stomach wall is now drawn through a pair of gastro-enterostomy forceps and the transverse colon and omentum are replaced in the abdominal cavity and held in

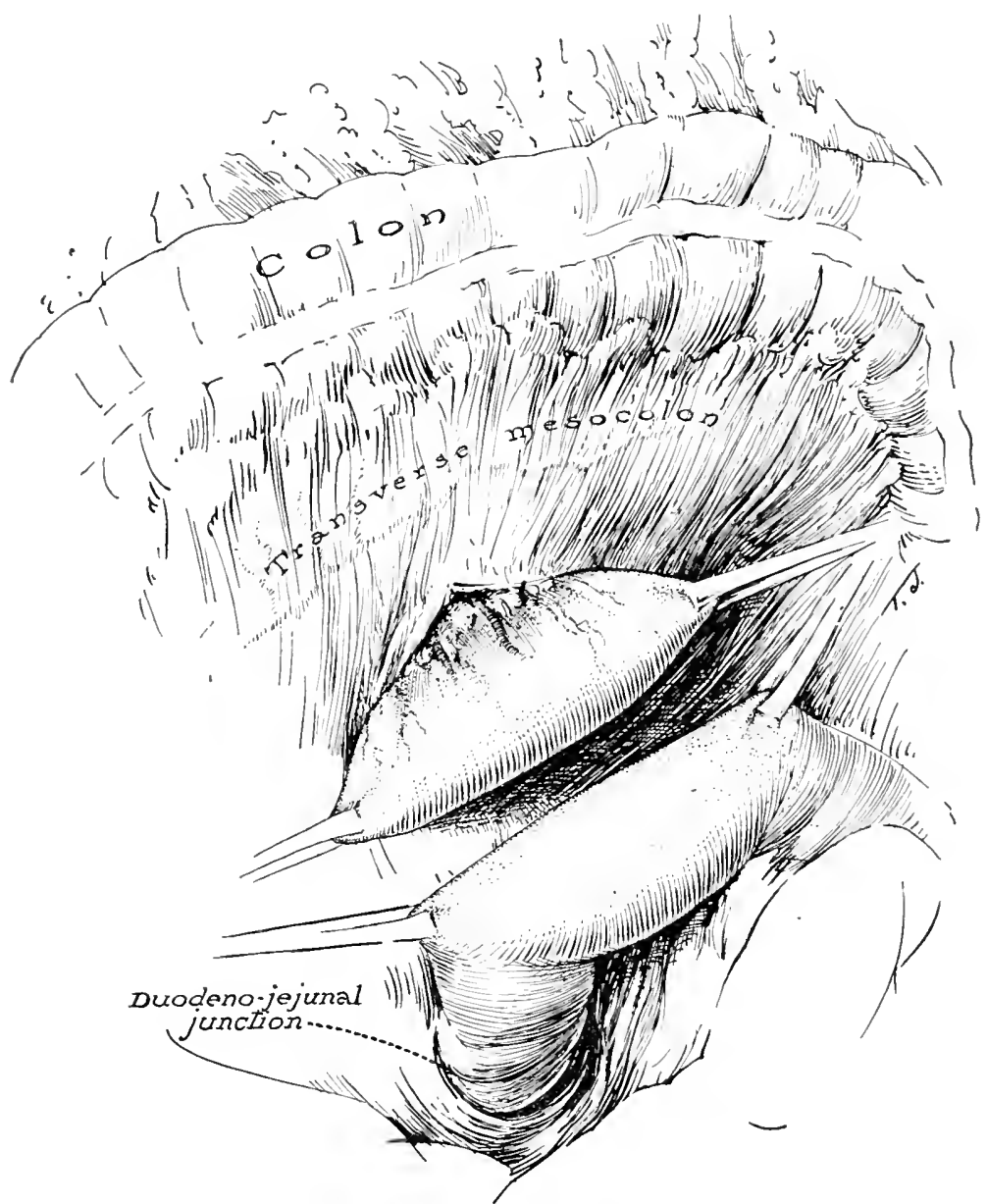

FIG. 87.- Steps in the making of posterior gastrojejumostomy by means of the gastric clamp. Portion of greater curvature nearest the pylorus brought through a tear in transverse mesocolon. Loop of jejunum in position for application of clamp.

position by means of gauze pads saturated with warm normal salt solution. The loop of jejunum nearest the transverse mesocolon is then brought up into view and a 


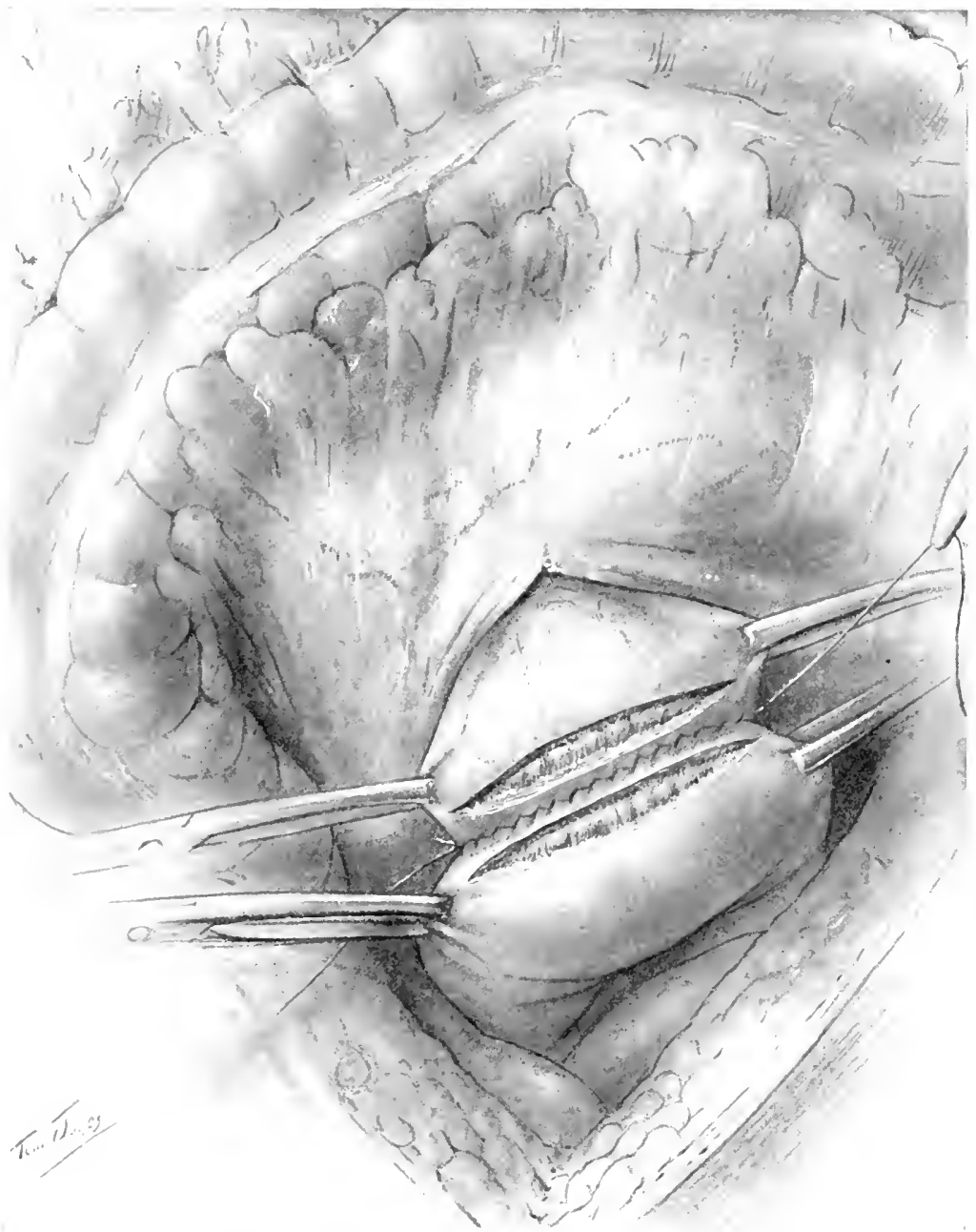

Fat. SS.-Posterior gastrojejunostomy for inoperable earrinoma of lesser arvature with obstruction of pyorus. Crastrie clamp not used. The colon and omentum have been reflected upwarls, an opening made into the transverse meso-colom, the greater curvature of the stomach brought through the opening and held with mouse-tooth clamps. The loop of jejumum, 5 em. from its beginning, is attached with a continuous row of silk sutures to the stomach. Initial openings through both stomach and jejunum, $8 \mathrm{~cm}$. long and extending to but not through the mucose, have been made. 
pair of fine-toothed clamps is placed upon the wall of the intestine farthest away from its mesentery, $5 \mathrm{~cm}$. from the mesentery of the transverse colon. A second pair of fine-toothed clamps is applied in the same manner $6 \mathrm{~cm}$. distally from this first pair, the distance being slightly greater than that between the two forceps on the stomach wall, because the longitudinal fibers of the jejunum will contract and bring these forceps near together. The fold of the jejunum is then drawn through the second blade of the gastro-enterostomy forceps in such a manner that the

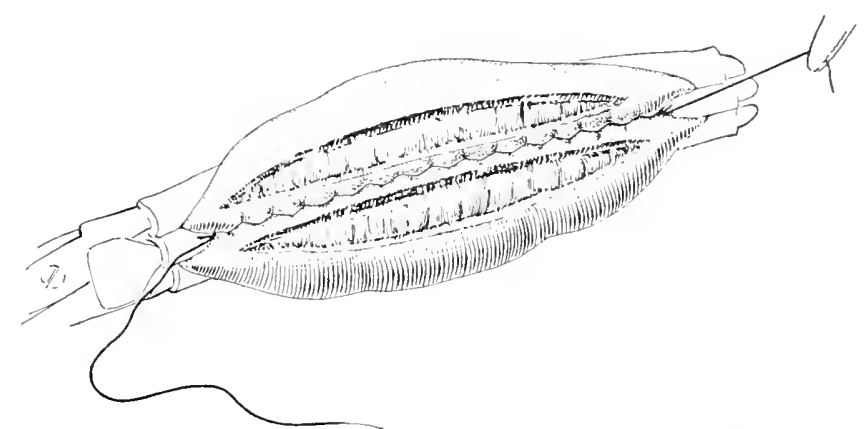

FIg. 89.-The gastric clamp applied, the posterior row of Lembert sutures in place and the incisions through walls of stomach and jejunum made as close to Lembert sutures as possible and extending to the mucosæ.

forceps nearest the transverse mesocolon will be opposite the forceps farthest away from the gastro-epiploic vessels, and the other two forceps will also be in apposition.

It is well now to make a straight incision through the peritoneum and muscle down to but not through the mucous membrane of the stomach wall between the two fine-toothed clamps which have been applied, and also between the two fine-toothed clamps on the wall of the jejunum. The incision will mark the location of the gastro-enterostomy opening. A fine, silk suture threaded in an ordinary cambric needle is used to unite the serous surfaces. The thread 
should be about $75 \mathrm{~cm}$. long and should be used double so as to prevent slipping and twisting. It is well to begin this suture at a point opposite the distal end of the gastro-enterostomy clamp and to make the sutures so that the serous surface will be in perfect coaptation, learing a fair margin of 2 or $3 \mathrm{~mm}$. between this row of sutures and the edge of the incision. This row of sutures is carried $1 / 2 \mathrm{~cm}$. beyond the end of the incision. The needle and thread are then placed to one side for future use. A fine, double

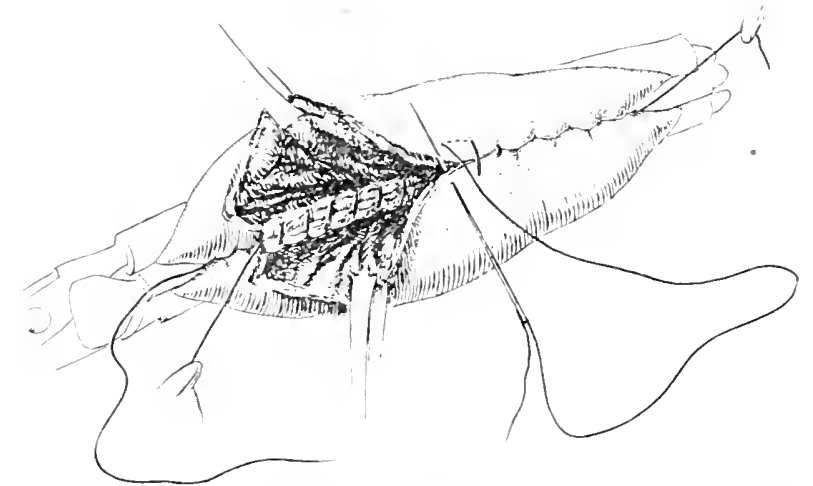

Fig. 99.-Row of connell ratgut sutures being appliexl.

chromicized catgut suture, the surface of which has been rendered smooth by rubbing with raseline is next employed as a deep suture. This suture is begun at a point opposite to the conclusion of the serous suture in order that the knots of this suture may not be opposite the knots of the silk suture previously described. This suture is carried into the lumen of the small intestine, then across the edge of the incision taking a bite of about $1 \mathrm{~mm}$. of the small intestine and the stomach so that it includes all of the layers of both structures. The first suture is tied and then a continuous suture is applied with the stitches near enough to each other to absolutely prevent hemorrhage. This suture is carried to the distal end of both incisions and then 


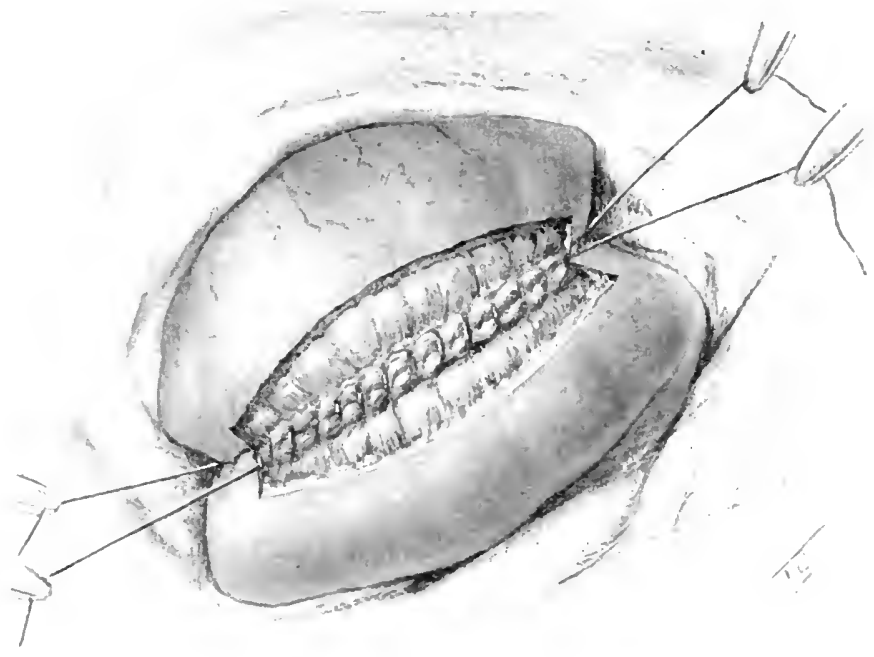

Fug. 91.- A continuous row of fine chromicizel catgut sutures extenling through and through the mucose of stomach and of jejunum. This catgut suture is begun at the opposite end from which the silk suture was started and the ends of both of these sutures are left long.

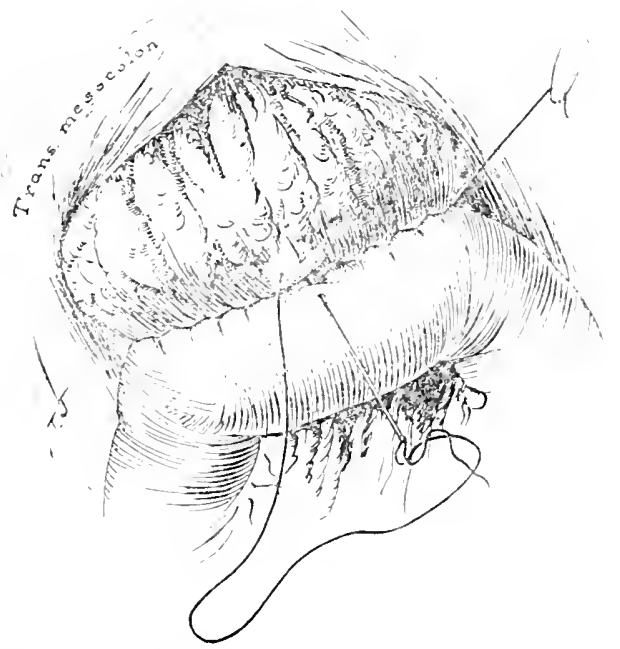

Fig. 92.-Clamp removed and outer Lembert sutures in place. 
pasied out through the wall of the stomach at a point opposite the distal end of the gastro-enterostomy clamps. The lumen of the stomach is now opened by means of scisorsts, are being taken to so cut the mucous membrane along the edge of the incision which has already been made, that all the layers of the stomach wall present a smooth surface.

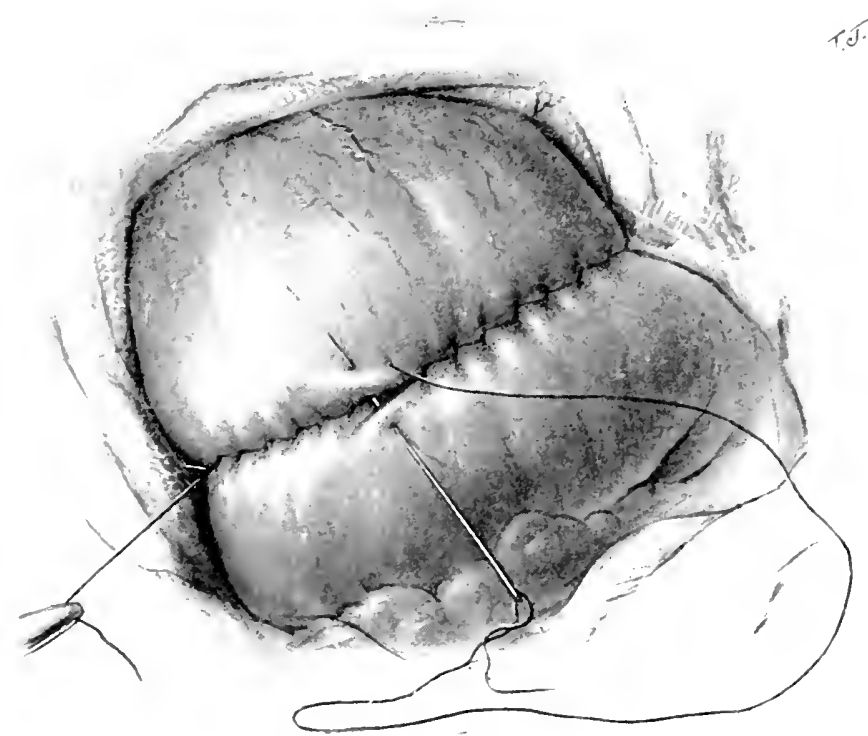

Ficr. 93.--Showing the anterior Lembert silk stitch, which entirely surrounds the inner Comell row of sutures, and which is a continuation of the first row of silk sutures applier.

It is important to be careful to sponge away any mucus which may be emptied upon the wound surface when the stomach is opened, but in doing this one should be careful not to loosen the mucous membrane from the overlying muscle and in no way to traumatize the delicate tissues composing the edge of this wound. The edge is then caught at three points with delicate fine-toothed clamps constructed for this purpose, so that these layers may be held in their proper 
position during the remaining steps of the operation. The same steps are then carried out in opening the jejunum, care being taken to place the fine-toothed clamps opposite those on the stomach wound edge. In case the mucous membrane overlying the posterior two rows of sutures does not fall into absolutely close apposition, it is best to place a fine silk, continuous suture uniting this mucous layer which, however, is not necessary in case there is perfect coaptation.

The anterior opening is now closed by applying the suture introduced by C. H. Mayo. This is a continuation of the continuous catgut suture already applied. The suture is carried through all the lavers of the intestine from without

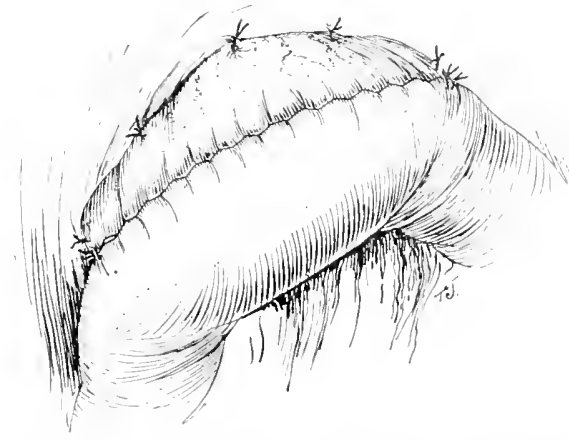

Fig. 94-Extra, stay, silk-stitehes placed at each end of the anastomosis. Edges of opening in transverse mesucolon sutured to stomark $1 \mathrm{~cm}$. from anastomosis, with interupter silk sutures.

inward, then from within outward, then across the space and then through the stomach wall in the same manner from without inward grasping all layers, then from within outward, then across the space to the jejunum. These steps are repeated until the entire anterior wound has been closed and the point is reached at which the catgut suture was begun. The end of the suture containing the needle is then tied to the portion of the catgut at the beginning of the suture which was left long for this purpose. This suture inverts every portion of the stomach and intestinal 
wall so that the serous surfaces are in apposition. The needle containing the original suture is then taken up and a row of Lembert sutures is applied covering up the eatgut suture line, which is continued at the point at which this suture was started and there tied to the ends which were left long for this purpose. It this point there is some danger of strain and in order to overcome this, two or three

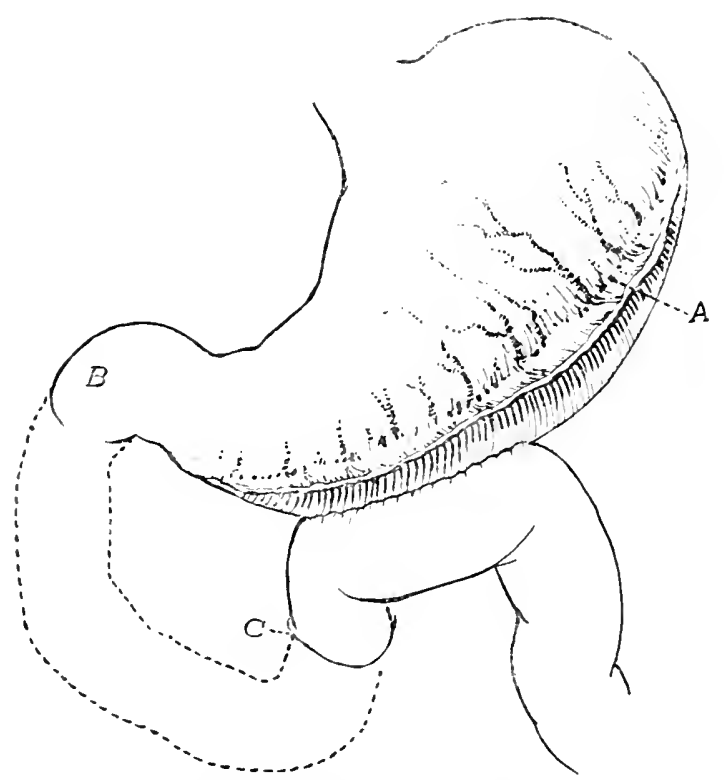

FIG. 95.-Diagram to show relations in a posterior gastro-jejunostomy. $A$, Greater curvature; $B$, pylorus; $C$, duodeno-jejunal flexure.

interrupted silk sutures are applied, attaching the stomach wall to the jejunum. It is well to apply the same number of sutures at a point directly opposite this, because that point is also likely to require a little additional protection.

At the time of tearing the opening in the transverse mesocolon from four to six Kocher hemostatic forceps are placed upon the edge of the opening in the transverse mesocolon at a uniform distance from each other. After the anastomosis between the stomach and the jejunum 
has been completed, a fine silk suture is passed through the wall of the stomach down to but not through the mucous membrane or mesocolon from the suture line at points opposite to those indicated by the Kocher forceps. The edge of the tear in the transverse mesocolon is then brought down to the point at which this suture has been placed and the latter is tied about the small portion of tissue grasped by the Kocher forceps. This is repeated so that the territory in the transverse mesocolon is held in apposition with the posterior stomach-wall at six points $1 \mathrm{~mm}$. from the line of suture. This will prevent a hernia through this opening and it will also prevent the tissues of this opening from causing an obstruction due to contraction following the operation, because union will take place between the raw surfaces of this opening and the posterior wall of the stomach immediately after these sutures have been tied. The stomach is then laid down in a normal position, the jejunum is replaced, the transverse colon and the omentum are placed over the jejunum and the abdominal wall is closed.

Practically no unnecessary manipulation has been made during the entire operation so that the tissues have not been bruised and consequently it is not likely that adhesions will occur. In closing the abdominal wall great care is taken to erert the peritoneum in order that there may not be contact of raw surfaces from the line of incision with any of the intraabdominal structures. In this way a further precaution can be taken against the formation of peritoneal adhesions.

It is well to perform gastric lavage immediately after the operation has been completed, using normal salt solution at a temperature of $105^{\circ} \mathrm{F}$. in order to remove any mucus and blood which may have accumulated in the 
cavity of the stomach. The warm water has a tendency to stimulate the patient and to orercome shock by heat, and by the absorption which will take place of a certain portion of the normal salt solution. The cleansing of the stomach from mucus and blood will prevent the occurrence of nausea and romiting and the patient will be greatly benefited because of this.

After Treatment.-In case the patient suffers from nausea or romiting, or both, after the operation, gastric lavage is employed at once in order to again relieve the irritation. The temperature of the lavage fluid should be the same as that mentioned above. Not more than 1 pint of water should be passed into the stomach at one time, and then this should be evacuated again in order not to overdistend the organ or place an unnecessary strain upon the sutures. Usually it is not necessary to perform lavage more than once or twice, but occasionally it may become necessary to repeat gastric lavage several times a day for a number of days. In the meantime the patient receives a nourishing enema every 3 hours. This consists of 1 ounce of some concentrated predigested food dissolved in 3 ounces of normal salt solution, and is introduced through a small catheter which is inserted into the rectum for a distance of not more than 2 or 3 inches. It is best to attach a funnel or the barrel of a good sized glass syringe to the catheter, and to permit the fluid to enter the rectum by its own weight. Several minutes should be consumed each time during the introduction of this fluid.

In the meantime it is well for the patient to chew gum vigorously in order to produce saliva whose alkaline reaction has a beneficial effect upon the healing of the wound in the stomach. It will also prevent the patient from suffering from parotitis. The patient may take 
small sips of very hot water from the first and increase the amount constantly from day to day. After the second day the patient may take small quantities of broth or water gruel every 2 or 3 hours. It is best to give him a half teaspoonful of milk of magnesia in a little water before taking the gruel in order that the stomach may remain alkaline.

After the first week small quantities of buttermilk and cream ( 3 parts of the former to 1 part of the latter) will be borne very well; also egg albumin in sweet orange juice. Later on a general liquid diet is permissible. It is best not to begin giving solid food for about 6 weeks. After the operation, if milk is given, it is well to add a teaspoonful of milk of magnesia or $1 / 2$ ounce of lime water to 4 ounces of milk. If a patient is suffering from constipation, 2 ounces of castor oil may be given in the foam of beer or malt extract any time after the third day. Usually the milk of magnesia prevents constipation, howerer.

\section{EXCISION OF THE PYLORIC END OF THE STOMACH FOR THE REMOVAL OF CANCER}

The preparatory treatment is the same as that which has already been described. In planning the operation it is best to take a general survey not only of the extent of the growth, but also of the direction in which it has made the greatest amount of progress and the distribution of lymph nodes which show some degree of enlargement. If these enlarged lymph nodes extend behind the stomach into the space behind the duodenum so that they can be found below the transverse mesocolon, then their remoral is of no use. In many of these cases the enlarged lymph nodes are inflammatory in appearance and may contain no malignant elements. They are due to the infection from 
the ulcerated stomach, the infectious material haring traveled farther than the malignant elements. In cases then in which the cancer is sererely ulcerated, the presence of enlarged lymph nodes, to points beyond which it is safe to remove these structures, does not absolutely contraindicate the excision of the malignant growth. There is, howerer, always a strong probability that these glands contain elements of the cancer infection aside from the infection due to other microorganisms, and for this reason while it is important that a bad prognosis be made, under no condition should a hopeless prognosis be given.

We have patients alive at the present time operated more than $S$ years ago in whom many of the greatly enlarged glands could not be removed, but from the post-operative history it is plain that there were no malignant elements present in these enlarged glands, or that if they were present they hare since been destroyed. The latter theory is quite as likely to be the correct one as the former, because there can be no doubt but that a large proportion of the malignant elements in every cancer which are carried away from the original growth are destroyed and do not succeed in causing secondary growths. By removing one of these lymph nodes and splitting it through the center, one can see the little white areas which are due to cancer with the naked eye, if the lymph nodes are at all severely infected with the malignant growth. So if there is any doubt, one can satisfy himself very readily. Moreover, the enlargement due to the infiltration with cancerous tissue is much harder than the enlargement due to other infections. In cases in which the lymph nodes are hard and large along the coronary artery, we have never seen a case that has permanently recorered after gastrectomy, either partial or entire. But it is theoretically possible for these glands 
also to be enlarged in these cases without containing cancerous elements.

The next important point is to determine the adhesions of the malignant growth. There may be inflammatory adhesions in comnection with these growths in cases in which the cancer itself has not invaded the surrounding tissues, but these cases are not numerous, and as a rule if there are adhesions the excision is not likely to increase the life of the patient. The liver should be carefully examined. If this organ contains secondary growths the removal of the primary cancer in the stomach will be of no benefit.

In some cases, there is an involvement of the transverse colon and this may be quite as circumscribed as is the involvement of the pylorus. It is doubtful, however, whether any of these patients can be permanently cured by the removal of the pyloric end of the stomach together with the involved portion of the transverse colon, although theoretically this is possible. It is consequently proper that this condition should be considered from a surgical standpoint. After completing this examination which can be carried out without traumatizing any of the tissues, and without forcing any of the cancer infection into the lymphatics by manipulating the tissues with a proper degree of gentleness (especially if this examination is performed with the bare hands without gloves), the operation should be selected which seems most suitable for the indiridual case.

It is well to bear in mind that violence either during the manipulations necessary for making this examination, or during the operation is of great harm and no benefit to the patient. First, because it greatly increases the shock, and second, because it, undoubtedly, serves to disseminate 
the cancerous infection. At this point, one must decide whether it is best to make a gastro-enterostomy, with the plan of relieving the obstruction and the irritation of the diseased portion of the stomach, and improving the nutrition of the patient during the remainder of his life, without interfering with the malignant growth itself, or whether it is best to perform a gastro-enterostomy as a preliminary operation with the plan of removing the pyloric end of the stomach at a later operation. The latter should be performed 2 or 3 weeks after the patient has recovered from the traumatism and shock of the preliminary operation and has gained a little because of his improved nutrition. Lastly, whether both operations, gastro-enterostomy and the excision of the pyloric end of the stomach, should be performed at the same time.

It is in these cases in which experience and judgment are of the greatest value. Both of these elements will enable the surgeon to reduce the traumatism and the shock to a minimum, and consequently an experienced surgeon with good judgment can perform a more extensive operation than it would be safe for a less experienced surgeon to undertake. For example, in a case in which it would seem proper to remove the pyloric end of the stomach and the transverse colon together with the omentum, the operation might last an hour in the hands of a surgeon with great experience, while it would surely last two or three times as long in the hands of a less experienced surgeon. The patient would consequently be exposed to traumatism two or three times as severe in the latter case. The exposure of the intraabdominal organs to the atmosphere during this time would greatly increase the shock. Many unnecessary manipulations would be made by the latter surgeon. In most instances, an inexperienced surgeon will manipulate 
tissues more violently, especially in periods during which some uncertainty arises as to the best method of accomplishing certain ends, and at times when he is trying to think. During all of this time the patient is under the influence of an anesthetic, the bad effects of which increase greatly with the length of time during which it is being administered. There can be no doubt but that many of the deaths following operations upon the stomach during the early period of stomach surgery were due to the fact that at that time no one had acquired any special skill and judgment in these operations; inasmuch as it was not possible to acquire this skill and judgment except through personal experience. The frequency with which these early cases died from pneumonia was, undoubtedly, due to unnecessary traumatism, to long-continued anesthesia, and to long exposure of the diaphragm and the intraabdominal tissues to this unnecessary irritation and, of course, also to the fact that these patients were placed in bed in a horizontal position instead of having the upper end of the body elevated after the operation.

Having then considered all of the conditions present, together with the elements connected with the individual patient, the further steps of the operation must be chosen. If it seems wise to make an excision of the pyloric end of the stomach, the question will arise as to whether it is best to perform a gastro-enterostomy first or whether it is better to make an excision and later make the gastroenterostomy. Theoretically, both plans are equally good so that this may be left to the individual inclination of the operator. If the gastro-enterostomy is performed first, the method which has already been described should be followed. If the excision of the pylorus is made first, there remains a choice between making a posterior gastro-enteros- 
tomy or using a portion of the end of the stomach for the purpose of making this anastomosis. Both of these methods will be described later.

Excision of the Pyloric End of the Stomach.-If one bears in mind the blood supply of the stomach, it is an easy matter to exrise the pyloric end without the loss of more than a few drams of blood. This is because the blood supply comes from four definite points: the coronary artery above to the left, the pyloric artery above to the right, the gastroepiploic artery to the right and left below. If these four points are grasped between two pairs of forceps at each point and eut and ligated immediately, the principal blood supply of the portion to be removed is completely under control. Communicating branches of blood-ressels are then controlled by the application to the greater and lesser omenta of strong hemostatic forceps placed in pairs, the intervening tissue being cut and the distal portion being carefully ligated. A sufficient amount of the lesser and greater omentum is permitted to project beyond the ligation to make slipping impossible. These ressels may be ligated with catgut or fine silk. In this way, the portion of the stomach to be removed is entirely separated from its attachment from the greater and lesser omenta. It should include all of the tissues containing enlarged lymph nodes, and in early cases it should extend up to the coronary arteries and in late cases beyond these arteries. On the side of the duodenum, the forceps should be applied to a point at least $3 \mathrm{~cm}$. beyond the pyloric end of the malignant growth, but even in cases in which the growth does not approach the pylorus, the excision should still include this structure, because recurrence is not uncommon in the tissues belonging to the pylorus, while it almost never occurs in the tissues belonging to the duodenum. 
If there are inflammatory adhesions to the pancreas these are removed with great care in order to prevent injury to the pancreatic tissues. If the tumor extends into the pancreas a portion of this organ may be removed, but inasmuch as there have been no permanent recoveries in our

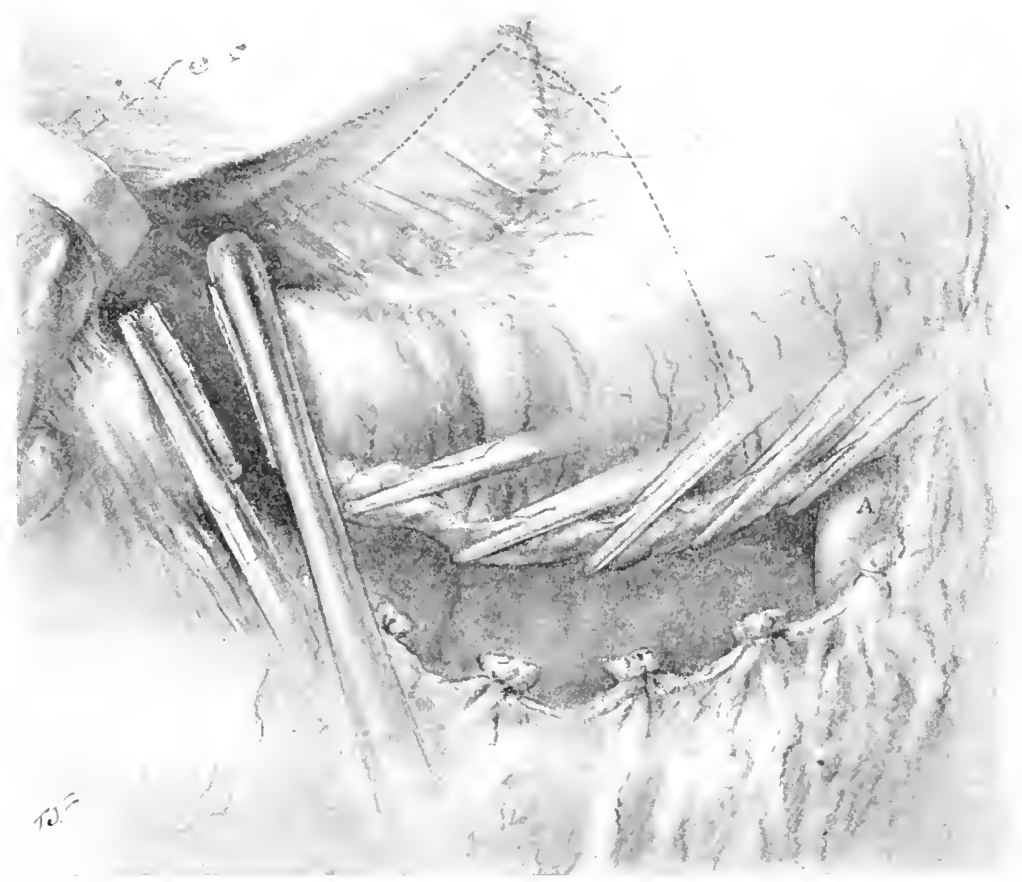

FIG. 96.-Resection of two-fifths of the stomach for carcinoma at the lesser curvature with posterior gastro-jejunostomy $(A)$ made as close a possible to the line of excision of the stomach. The anterior layer of the greater omentum has been divided close to the greater curvature and the pylorus has been doubly clamped and sectioned. Portion to be exeised delimited by dotted lines.

experience in any of these cases, it is doubtful whether the additional danger to the life of the patient caused by cutting into the pancreas will be compensated for by the probability of the permanency of a cure.

The entire portion of the stomach containing the cancer, together with at least $3 \mathrm{~cm}$. of apparently normal tissue in 
the direction of the duodenum including the pylorus and at least $6 \mathrm{~cm}$. toward the cardiac end of the stomach of apparently normal tissue is in this manner entirely loosened from all surrounding tissues.

Especial attention should be directed toward the importance of preventing injury to the arteries supplying the transverse colon. In order that the latter viscus may not become necrotic as a result of interference with its blood supply, a strong stomach clamp is next applied to the

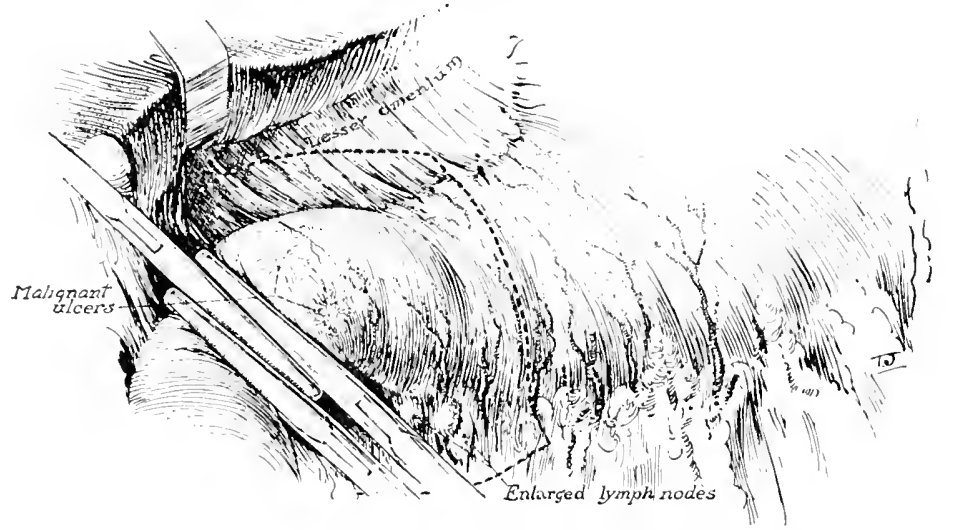

FIG. 97.-Excision of pylorus and antrum of stomach with end-to-side gastrojejunostomy for multiple malignant ulcers just proximal to the pylorus. Dotted line shows projected line of excision. Clamps in position for section of pylorus.

upper end of the duodenum. A second clamp is applied to the stomach at a point chosen for resection, then two other clamps are applied to keep the contents of the portion to be removed from soiling the peritoneal cavity when the portion is cut away. The intervening portion is then removed by cutting along the edge of the two clamps which were applied first.

The treatment of the two stumps must now be considered. If enough of the duodenal end is left to invert the cut end of this structure, this may be accomplished in various ways. A purse-string suture of silk or linen should be 
applied $1 \mathrm{~cm}$. below the clamp and the crushed end should be inverted into the lumen of the duodenum while this purse-string suture is being tied. The surface which has been puckered in by the tying of this suture is then covered by the use of two rows of interrupted Lembert sutures of silk or linen, care being taken not to leave any portion which is not covered with peritoneum. A gauze pad is
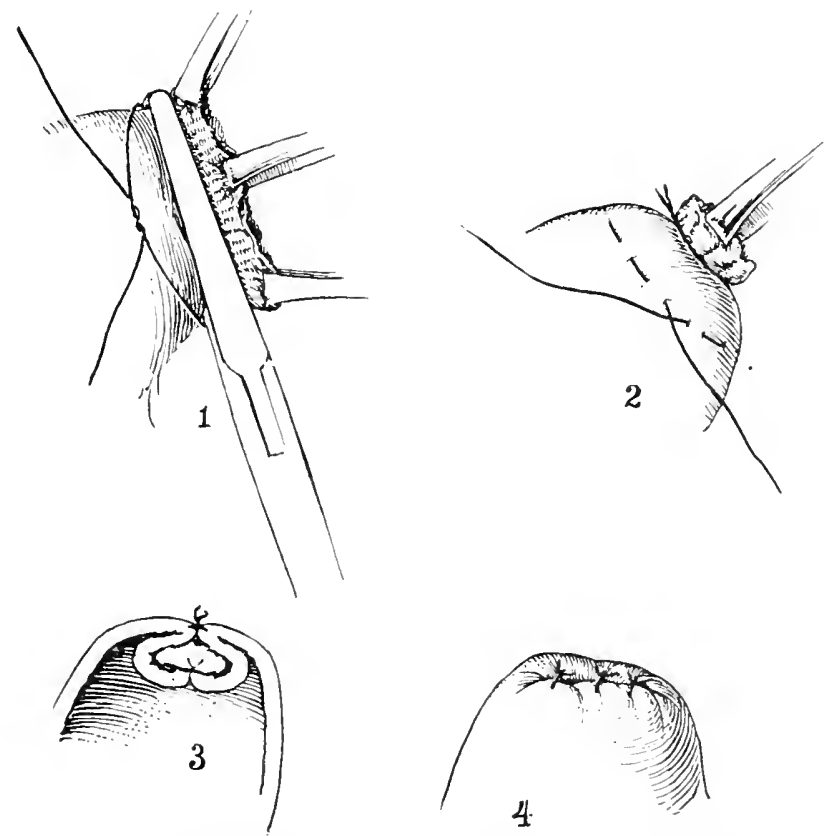

FIG. 9S.- Shows method of inverting stump of duodenum.

then laid over this surface while the stump of the stomach is being disposed of. It seems to be a good practice to cauterize the portion of the stomach-wall grasped in the heavy clamps with the actual cautery. A strong, silk or linen suture or a fine, double, chromicized catgut suture is then used to fold in the portion of the stomach-wall which has been crushed into the lumen of the stomach by placing Lembert sutures, grasping the tissues on each side of the 
clamps successively and passing them orer the clamps passing throughout the distance until the entire defect has been covered with these sutures. Then the clamps are loosened and the continuous sutures are tightened. This causes the crushed portion to fold into the lumen of the stomach, while the serous surfaces on the two sides come into accurate apposition. An additional stitch is added at the end of this line of sutures for the purpose of tying the free end upon which traction has been made to bring the surfaces in apposition. It is important to fold in the corners carefully with additional sutures, because these points represent the weak points in the closure. A fine silk Lembert suture is then placed orer the entire line of these deep sutures to serve as a provision for safety in case some one of the stitches might not be absolutely reliable.

We now have the stomach entirely separated from the intestine and it recomes necessary to make a gastro-jejunostomy in order to reunite these structures. It is best to do this on the posterior surface of the remaining portion of the stomach at a sufficient distance from the suture line just described, to insure a satisfactory blood supply so that there may be a rapid and perfect union between the stomach and the intestine. It is well to thoroughly irrigate the portion of the stomach with normal salt solution at $105^{\circ} \mathrm{F}$. before this last step is carried out. If there is any doubt about the safety of using the gastro-enterostomy clamps which were used in the gastro-enterostomy operation described above, then the operation can be performed without clamps quite as conveniently as with them. A loop of the jejunum just below the transverse mesocolon is placed in the same relative position that was described in the gastro-enterostomy operation above, then the posterior serous suture is applied, uniting the jejunum to the pos- 
terior surface of the stomach for a distance of $5 \mathrm{~cm}$. These sutures should grasp all the tissues down to the mucous membrane but not through this tissue. An incision $4 \mathrm{~cm}$. long is then made parallel to and $1 / 2 \mathrm{~cm}$. from this row of sutures extending down to the mucous membrane but not through it, both on the stomach and the small intestine. A fine, double, chromicized catgut thread in an ordinary sewing needle is then passed through all the layers of the

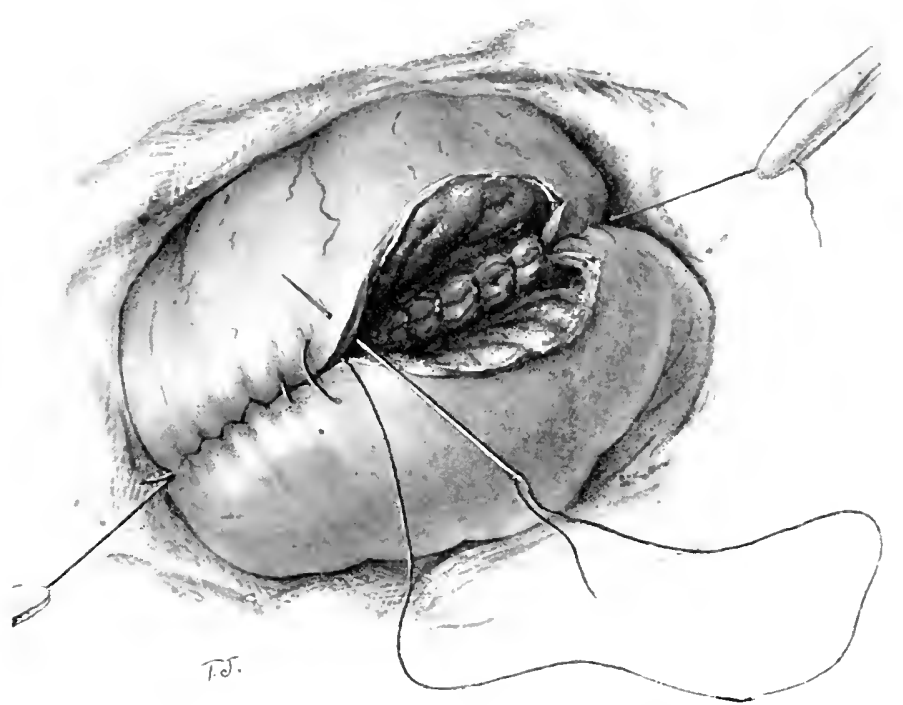

FIG. 99.-Showing the anterior Connell catgut stitch.

stomach and the intestine. The line of sutures is begun at the point at which the silk suture was ended and carried to the point at which the silk suture was begun.

The mucous membrane is then cut, care being taken to first place moist gauze bands around the tissues so as to prevent soiling of the peritoneal cavity. Any substance which may come from the cavity of the intestine and the stomach is carefully sponged away and then the catgut suture is continued around the remaining portion of the 
opening. The Connell suture is employed for this purpose. It is applied in the following manner. The suture is parsed through the layers of the stomach wall from within outward, then carried over to the wall of the intestine, passed through all the layers from without inward, then from within outward and then it is carried across to the stomach again. These steps are repeated until the entire

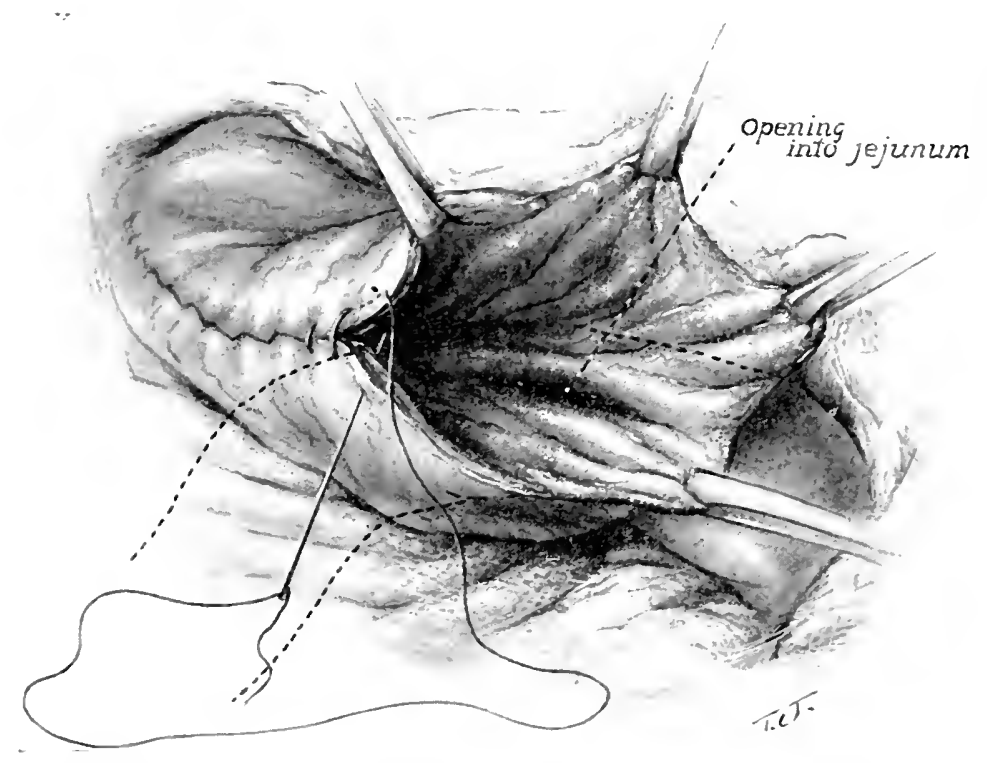

Fig. 100.- - Showing method of closure of end of the stomach by a Connell hine of catgut sutures which is then covered with a row of Lembert silk slitures.

opening is closed, care being taken to remain within $2 \mathrm{~mm}$. from the edge of the wound. As these sutures pass through all of the layers, there can be no hemorrhage from the edges of the incision. The catgut suture is then tied to the end which was left long at the beginning. It is well to oil the catgut suture with raseline so as to have it perfectly smooth in order that it may not tear the tissues as it passes through them. 
The original silk suture is now taken up again and is carried on in the form of a Lembert suture around the anterior surface of the anastomosis to the point of its beginning where it is tied to the end which was left at the beginning of this suture. The edges of the opening in the transverse mesocolon are now sutured to the stump of the

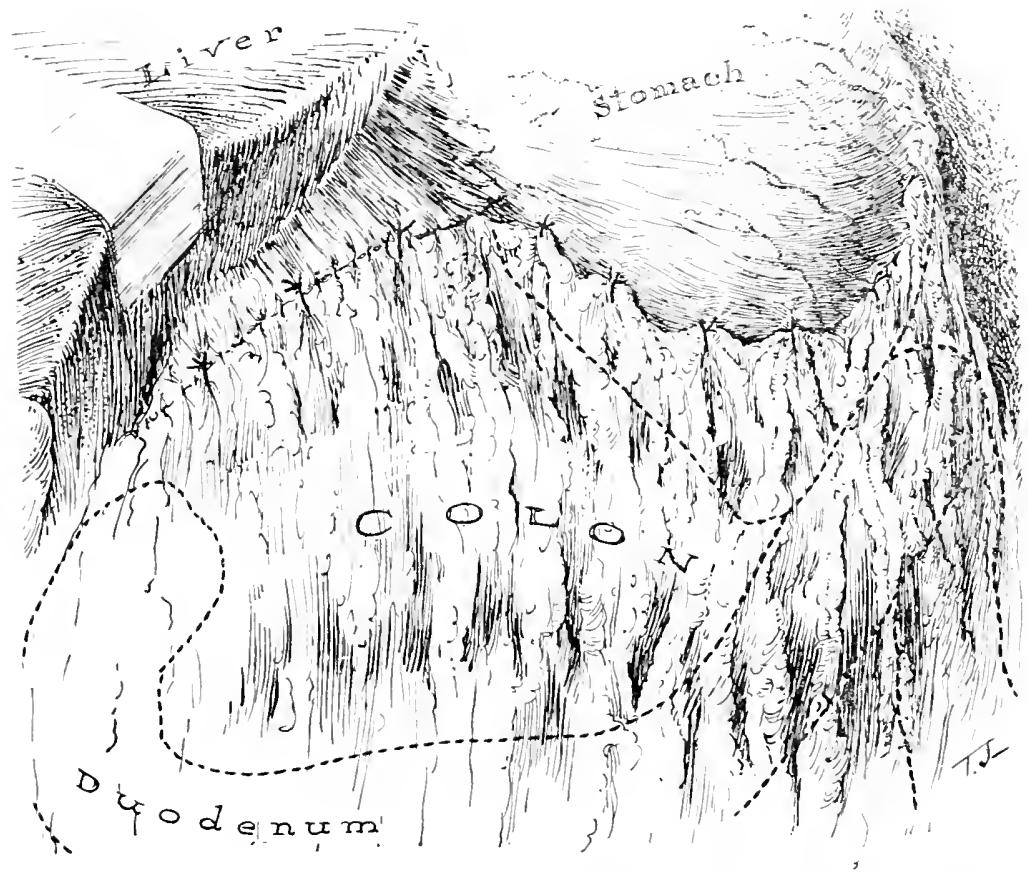

Fig. 101.-The greater omentum sutured with interrupted silk stitches to the stomach and lesser omentum. Dotted lines indicate cut end of stomach and the anastomosis.

stomach and the entire surface left bare by the removal of the pyloric end of the stomach is covered by uniting the greater and lesser omenta, or by carrying the stump of the duodenum up to the stump of the stomach and uniting these by means of fine silk sutures. At all erents, every portion of the raw surface must be carefully covered with peritoneum. 
In some cases the amount of available tissue for closing the duodenum is not so plentiful as might be desired to obtain a closure in whose permanency one can have complete confidence. In such cases it is best to provide for the posible occurrence of a leakage some days after the operation. By carrying one or two cigarette drains down to the closed end of the duodenum, passing these out of the upper end from the abdominal wound one can provide for this complication. These cigarette drains are constructed by rolling a pad of gauze within a piece of rubber tissue so that the rubber tissue overlaps the gauze. The end of the gauze should project a little from each end of the rubber tissue. It may be well to add to this a glass drainage tube which will serve to keep the abdominal wound sufficiently open to permit any accumulations to pass out of the wound.

In case the cancer involves the pylorus so that it will become necessary to remove 2 or $3 \mathrm{~cm}$. or more of the upper end of the duodenum, it is often better to utilize a rubber drainage tube $30 \mathrm{~cm}$. long and $1 \mathrm{~cm}$. in diameter, and to draw over this a second rubber drainage tube just large enough to prevent the inner drainage tube from slipping through the outer one unless quite a little force is applied. The outer drainage tube should have the length of $15 \mathrm{~cm}$. so that the inner tube projects beyond it at each end about $71_{2}, \mathrm{~cm}$. At one end the inner tube should have a number of small perforations; this end should then be inserted into the duodenum for a distance of $15 \mathrm{~cm}$. and the free end of the duodenum should be closed by means of several purse-string sutures about this drainage tube so as to make a complete closure and so as to guard against leakage. The serous surface should be inverted and after three or four purse-string sutures have been applied, the 
edge of the duodenum should be sutured by means of from four to six fine, silk sutures to the outer rubber tube. Then this should be carried out of the upper angle to the abdominal wound or through a small stab wound at a point directly in front of the end of the duodenum. It is possible for bile and duodenal secretion to escape through this tube and, on the other hand, one may introduce into the duodenum through this tube a quantity of normal salt solution by the drop method, to which some form of concentrated food has been added. Adhesions will take place between the end of the duodenum and the abdominal wall, and after 1 or 2 weeks the sutures holding the rubber tubing in place will cut through the edge of the duodenum by causing pressurenecrosis and then the walls of the duodenum will close spontaneously. Occasionally, considerable quantities of bile and pancreatic fluid will escape through the wound and this may cause excoriation of the skin. This, however, can be readily corrected by giving the patient the white of one egg every 2 hours night and day for several days and by covering the raw surface freely with white vaseline or with castor oil. The ingestion of egg albumin seems to prevent the formation of this irritating secretion. In patients who are very much depressed as a result of their disease and the operation, it is wise to treat the end of the duodenum in this way, because this enables one to supply the necessary amount of nourishment almost immediately after the operation without in any way interfering with the healing of the gastro-enterostomy wound. Usually it is better to pass this duodenal rubber tube out through a separate opening and to suture the stump of the duodenum by means of a few fine, silk sutures; thus one can provide an additional safeguard against soiling of the peritoneal cavity with fluid coming from the duodenum. The closure 
of the end of the chodenum about the rubber tubing should, however, be so complete that leakage cannot occur for a considerable period of time. By that time adhesions will have formed which will protect the general peritoneal cavity. It is of course, of the greatest possible importance throughout this operation that at no point will there be tension upon any of the tissues because all of the tissues are so delicate that the sutures may not hold if subjected to tension. By carefully bearing in mind this additional principle, one can invariably provide some means of aroiding such difficulty. Of course, the smaller the amount of tissue which it is necessary to remore in the individual case, the less the danger from tension upon the sutures, but eren in cases in which a very large amount of the stomach has been removed, it is possible to guard against this difficulty. Under no condition should any suspicious tissue be left in order to prevent tension. One can always improvise some other means of accomplishing this end.

\section{COMPLETE GASTRECTOMY}

From a technical standpoint, a complete gastrectomy is but slightly more difficult than is the operation which has just been described. From a practical standpoint, on the other hand, the operation may be looked upon as almost, if not entirely useless, not because of the difficulty of the operation itself, but because of the fact that in cases in which the operation can be performed with permanent success, it is not required, because success can be obtained by means of the simpler operation which has just been described. On the other hand, in cases in which complete gastrectomy is the only operation which gives any possibility of permanent success, there are practically always complications which will make it either impossible for the 
patient to recover permanently from the operation or certain that recurrence will cause the death of the patient in a relatively short time.

There are certain advertising advantages in performing a complete gastrectomy, occasionally, because no matter how carefully one guards the secret of having performed such an operation, it is certain to be carried from mouth to mouth until the surgeon who has performed it gains a certain degree of notoriety for unusual surgical skill. But so far as the patient is concerned, it is doubtful whether in any case in which the operation was necessary, he had secured any real benefit.

Operative Technique for Gastrectomy.-The technique described in the previous operation will also serve for the operation of complete gastrectomy with the exception that the entire stomach is laid bare instead of only its pyloric end. This is done by applying artery forceps behind the entire greater and lesser curvature to a point within 3 or $4 \mathrm{~cm}$. of the esophagus. Stomach clamps are then applied as in the previous operation, with the exception, however, that the clamp nearest the esophagus must be applied in such a direction that the stump which is left above it is sufficient to permit its attachment to a loop of the jejunum. The esophagus and small remnant of the stomach are then thoroughly irrigated with normal salt solution through a stomach tube which has previously been inserted into the stomach and has been withdrawn within a point just above the application of the upper clamp. All of the liquid must be siphoned out carefully so that none of it remains in the esophagus or the little remnant of stomach and so causes soiling. The portion of the stomach between the two clamps is then removed in the manner described in the previous operation. The end of 
the duodenum is treated after the method which has just been described by inserting the double drainage tube so that there can be no pressure of fluid from the duodenum upon the jejunum.

A loop of the jejunum, sufficiently long to be carried up to the stump of the stomach without tension, is passed through an opening in the transverse mesocolon, or it may be passed in front of the transverse colon. A longitudinal incision is made in this loop at its upper end. This incision should be $5 \mathrm{~cm}$. long and the edge of the opening carefully sutured to the stump of the remnant of the stomach, one suture grasping all of the layers with the exception of the peritoneum, and a second row of Lembert sutures grasping all the layers down to, but not through, the mucous membrane. It is absolutely necessary that there should be no tension. The edge of the opening in the transverse mesocolon is then carefully sutured about the duodenum. The entire defect in the peritoneum caused by the remoral of the stomach must be covered by uniting the edges of the greater and lesser omenta.

In these cases it is well to place a glass drainage tube and one or two cigarette drains dorn to the space from which the stomach has been removed, in order that any liquid which may accumulate will be carried away from the peritoneal carity. In these cases it is best to pass the rubber drainage tube entering the duodenum through a separate stab-wound, in order that the distance between the end of the duodenum and the abdominal wall may be as small as possible so that the peritoneum about the stab-wound may be sutured to the duodenum.

If there is any doubt about the safety of the suture line between the stump of the stomach and the jejunum, this may be strengthened by suturing orer it a piece of omentum 
or a strip of fascia lata $3 \mathrm{~cm}$. wide and long enough to encircle the gastro-enterostomy without causing any compression.

The after-treatment in these cases is the same as in the case just described. A small, soft-rubber tube may be carried through one nostril and down through the esophagus and the remnant of the stomach into the jejunum so that the end extends for a distance of 15 to $30 \mathrm{~cm}$. into the jejunum. Through this tube small quantities of predigested food may be introduced into the intestine every 2 hours. Water may also be given through this tube. It is well to administer liquid nourishment by rectum in the form of nourishing enemata every 3 hours in order to support the patient as well as possible; normal salt solution may be given by the drop method.

In case the flow of bile and pancreatic fluid does not become established normally through the loop of the jejunum which has been carried up to the stump of the stomach, it may become necessary later on to plan an entero-enterostomy between the two branches of the jejunum. This can best be done at a point from 3 to $5 \mathrm{~cm}$. beyond the transverse mesocolon. This can be accomplished by the application of a Murphy button or by performing an anastomosis $3 \mathrm{~cm}$. in length after the method described for performing anastomosis between the stomach and the jejunum.

\section{GASTRO-ENTEROSTOMY |FOLLOWING PARTIAL GASTRECTOMY BY UNITING THE JEJUNUM DIRECTLY TO THE LOWER END OF THE STOMACH WOUND}

Some surgeons with great experience in intestinal surgery prefer to make the partial excision of the stomach first and then to make the anastomosis between the remaining portion of the stomach and the jejunum directly to the 
lower end of the wound remaining in the stomach. In performing this operation there is great danger of leakage at the point at which the jejunum is united with the suture line of the stomach. It is necessary to bring the opening in the stomach down to the opening in the jejunum, but if this leakage is guarded against by the proper application of sutures at this point, the total amount of traumatism required by the operation is considerably less than if the operation is performed which has already been described. But it must be borne in mind that it requires a much greater amount of technical skill to execute this operation than the one which has been described.

Technique of Operation.-The technique of the operation is exactly the same as in the previous operation. After the clamps have been applied the remnant of the stomach is carefully irrigated and all of the liquid is siphoned out. Then a pad is placed behind the stomach; the portion of the stomach to be sacrificed is cut away and fine-toothed clamps are applied to the edge of the remnant of the stomach grasping all of its layers. If there is a bleeding point, this is caught and a fine, silk suture is passed through the wall of the stomach to compress the bleeding ressel. The carity of the remaining portion of the stomach is carefully sponged until it is perfectly dry and then a suture is applied uniting both wound edges together sufficiently far from the greater curvature of the stomach to determine the size of the opening which must remain for the purpose of making an anastomosis with the opening in the jejunum. It is important to determine this at this stage, because, otherwise, almost invariably the opening is left too small. All of the layers, with the exception of the peritoneal surface, are united by means of a continuous suture, or in case the 
remnant of the stomach is of considerable size, a Connell suture may be used for this purpose. This passes from the mucous membrane out through all of the layers 2 $\mathrm{mm}$. from the edge of the wound, then across to the other side and through all of the layers from without inward. The suture is then tied within the stomach wall, then it is carried out through one edge and across the wound, then from without inward and from within outward on the

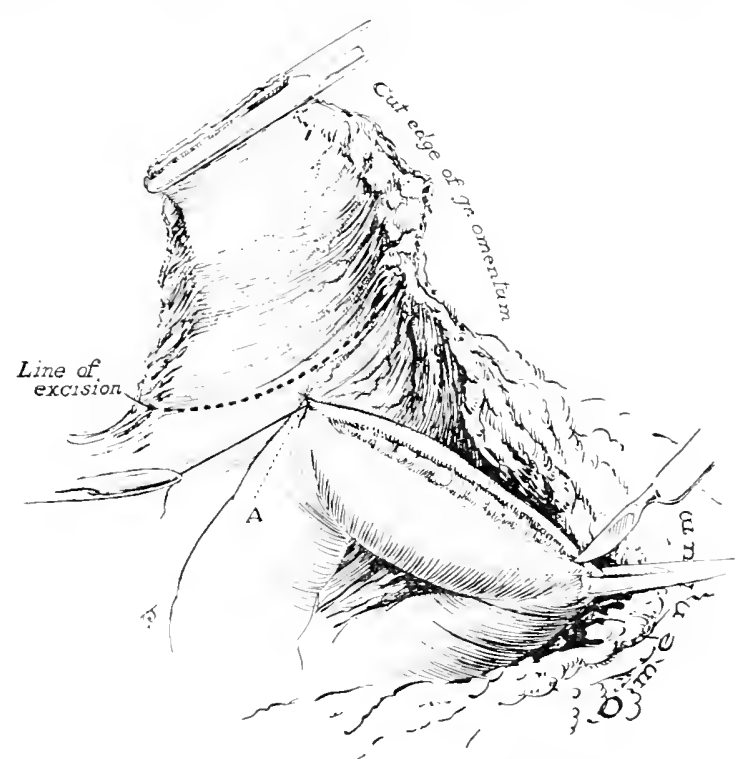

FIG. 102.-The duodenum has been inverted and the lower portion of the stomach reflected upward. A loop of jejumum has been brought through the opening in the transverse mesocolon and point (A) sutured to the stomach.

other side, then across to the other side, then from without inward and from within outward and across again and so on until the entire wound has been closed, especial care being taken to fold in the angle of the upper end. A row of Lembert sutures is now placed over this suture line so that the peritoneum is once more united. The opening at the lower end of the stomach wound is then sutured into a wound of exactly the same size made in the jejunum 
at the point just below the transverse mesocolon unrough which an opening has been torn.

The union between this opening in the stomach and that in the jejunum is accomplished by two rows of sutures, the first grasping all of the layers with the exception of the peritoneum, the latter consisting of a row of Lembert sutures of fine silk completely covering the first row of sutures. From three to six additional sutures are now

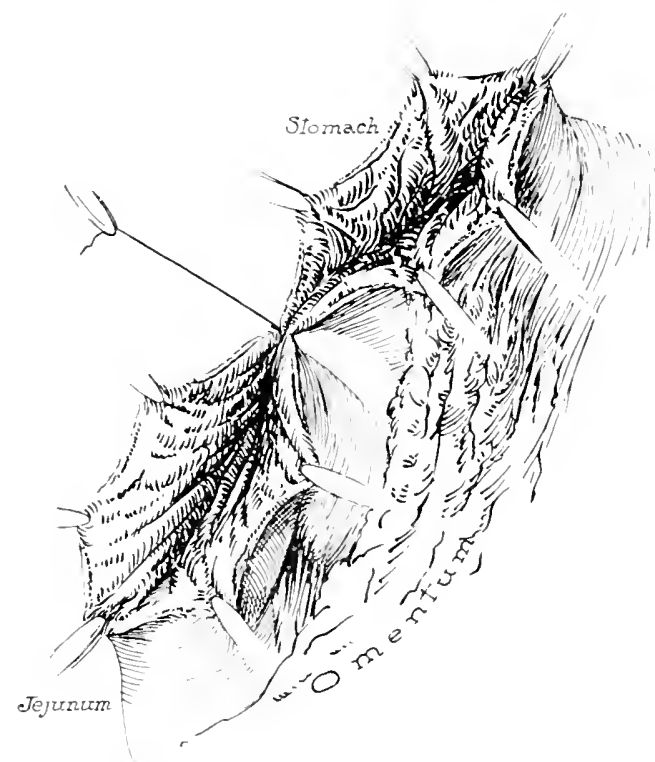

Fig. 103.- Showing method of beginning anastomosis of end of stomach to side of jejunum.

placed at the point at which the jejunum is opposite the suture line which is closed by the upper end of the stomach wound. It is important that these sutures be applied with great accuracy in order that there may be no leakage at this point.

The opening in the transverse mesocolon is then sutured to the wall of the stomach $7 \mathrm{~mm}$. from the gastro-enterostomy opening by means of six or eight fine silk sutures. The ab- 
dominal wall is then closed. The jejunum is treated as in the previous operations.

If this operation is performed with great skill, it has the advantage of placing the gastro-enterostomy opening at the lowest point of the stomach and of providing a mechanical arrangement which is ideal. Were it not for

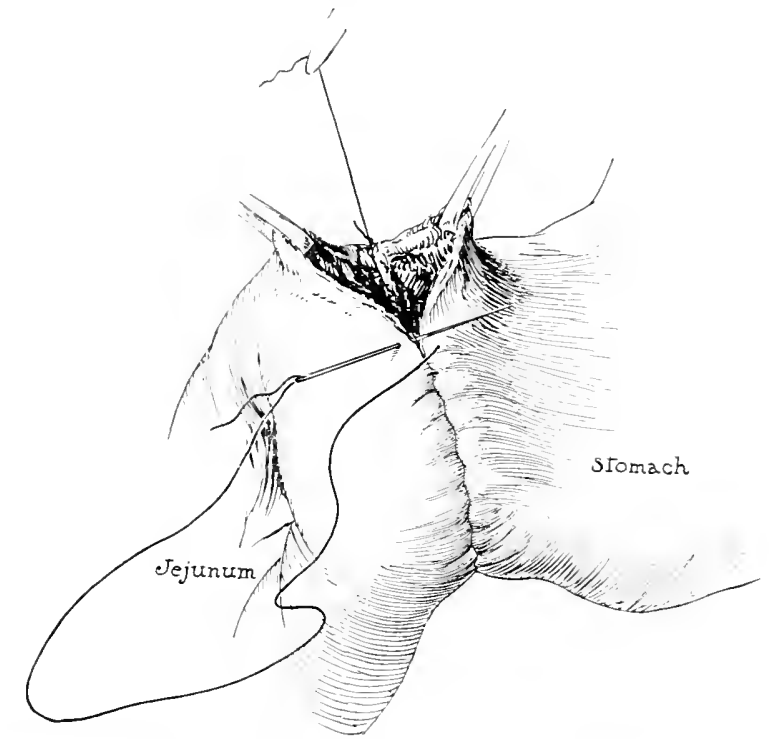

Frg. 104.-The Connell line of suture being applied. A row of Lembert silk sutures later surrounds this.

the great likelihood of faulty union at the one point, this operation would undoubtedly be the operation of choice.

\section{POST-OPERATIVE TREATMENT}

It is important to carry out a definite plan of postoperative treatment in these cases in order to prevent avoidable deaths. During the early times of gastric surgery, there were many deaths from pneumonia and quite a number from acute dilatation of the stomach. The deaths from pneumonia were due to a great extent to unnecessary traumatism during the operation, to infec- 
tion during operation and to anesthesia which was prolonged unnecessarily beeause of the incompetency and inexperience of the surgeon and to lack of judgment. These causes of pneumonia have now, fortunately, been eliminated beeause surgeons can now obtain skill by assisting those who have a vast experience.

Another cause of pneumonia came from aspiration of vomitus. This is eliminated now by performing gastric lavage at the close of the operation and again whenever there is any sign of nausea. Another cause consisted in the tendeney to hypostatic congestion of the lungs by placing the patient in the horizontal recumbent position directly after the operation while the patient was still deeply narcotized, due to the fact that the anesthetie was continued until the close of the operation. By permitting the patient to awaken thoroughly by the time the last stitches are inserted this cause is also eliminated, and by elevating the head of the bed 18 inches and placing pillows under the patient's head and shoulders when he returns to bed and permitting him to move about, the tendeney to hypostatic congestion of the lungs is eliminated. As a result of these ehanges the percentage of cases of pneumonia following operations upon the stomach has been enormously reduced so that we now practically never see a case of this kind.

Acute Post-operative Dilatation of the Stomach.From some mechanical condition which happens occasionally after operations upon the stomach and gall-bladder, the patient suffers from an acute, very rapid dilatation of the stomach which may result in so marked a displacement of the heart that it may prove fatal. It may also cause a rupture of the suture line between the stomach and the intestine. Since we have systematically practised 
gastric lavage at the close of these operations, we have not encountered this complication. It is, however, important to bear the possibility of this condition in mind and to introduce a stomach tube immediately in case this complication occurs. This should be followed by careful gastric lavage with normal salt solution at $105^{\circ} \mathrm{F}$. This should be repeated in case any symptoms recur.

The condition can be recognized by the shortness of breath, by the sudden increase in pulse rate and by the complaint of the patient that he is being smothered. Physical examination will show a marked displacement of the left lung and heart. It is important to act quickly in these cases because death sometimes occurs within an hour of the beginning of the dilatation. It is consequently wise to instruct the nurse to perform lavage whenever there is any doubt. Small sips of water may be given to these patients almost immediately after the operation. Patients receive much comfort from the chewing of gum, because the saliva which they are able to swallow keeps the stomach in an alkaline condition and overcomes the severe thirst which is further controlled by the free administration of water by rectum by the drop method. It is quite as well to use water as normal salt solution by rectum in these cases. The injection of water should be continued very slowly, but very constantly so long as it is being absorbed, then this should be interrupted for 2 full hours in order to give the intestine an opportunity to secure rest. It is best to measure the amount the patient can take comfortably in this manner in order that a quantity slightly less than this may be given during the next proctoclysis. Thus this may be interrupted before the patient's limit has been reached. For instance, if a patient can take 1,000 cc. of water by rectum before feeling any dis30 
comfort during the first administration, only 900 cc. or about 30 ounces should be given during the second administration, always with an interval of 2 hours. It will be found that the saliva will flow much more copiously during the chewing of the gum after the patient has had one or more of these proctoclyses.

Aside from increasing the comfort of the patient, the chewing of gum prevents the infection of the parotid gland. We have had no parotiditis in any case, in which we have directed the patient to chew gum directly after gastric operations, while formerly this condition occurred frequently enough to be very annoying.

Occasionally these patients suffer from acidity of the stomach and eructations of sour substance. Notwithstanding the fact that previous to the operation there may have been no free hydrochloric acid present. In these cases it is well to give from 15 to 30 drops of milk of magnesia or 10 to 20 grains of bicarbonate of soda in 2 to 4 ounces of water in sips every hour, so that it takes the patient about half an hour to take this solution and half an hour to rest from it.

On the third day an ounce or two of broth or water gruel may be given by mouth every 2 hours. This may be continued for 3 or 4 days, when an ounce or two of good buttermilk with an ounce or two of cream may be given once or twice during the day. The white of an egg in 2 to 4 ounces of water may then be given and later a sufficient amount of orange juice may be added to this to disguise the egg. In each instance it is well to give the bicarbonate of soda or the milk of magnesia immediately before giving these articles of food. After 10 days, milk with milk of magnesia may be given regularly and the patient may chew beef or mutton and swallow the juice but not the pulp. 
It is wise to caution these patients after they leave the hospital never to eat any raw fruits or vegetables (especially not those that are likely to be covered with manure), like lettuce, radishes, celery, raw onion, etc. It is also well to give these patients a definite diet list which they may resort to any time when their new digestive apparatus refuses to digest the food contained in the regular diet list which should also be supplied to these patients. Thus it is absolutely necessary to tyrannize over this class of patients in order to keep them in good condition after these operations, because as a rule these patients are primarily erratic eaters and many of them pride themselves upon being able to eat foolish things after such operations. We have had a number of patients who have suffered severely from gastric disturbances as the result of some foolish act of this kind. While many others have been able to digest their food normally for many years by adhering to a reasonable diet similar to the following:

Diet List for Use when Normal.-Drink no water and no other liquid except hot milk during meals, not for 1 hour before or after meals.

Drink an abundance of good water between meals.

Drink no tea or coffee, and nothing containing alcohol.

Eat very slowly and chew all your food for a long time.

Eat nothing very sweet or very sour.

Eat nothing that has been fried.

Eat no hot bread, cake, candy, canned goods, pickles, pancakes, puddings, pie, pastry or pork; no raw vegetables or raw fruits, unless the latter are perfectly ripe and not sour, no bananas or raw apples, no fried onions cabbage, turnips, sweet potatoes, baked beans, corned beef, and no nuts.

You may eat broiled or stewed beef and mutton, breast of chicken, fish, cooked vegetables, cooked ripe fruits, bread, 
butter, toast, well cooked cereals, rice, milk or cream soups and regetable soups; also soft-boiled or poached eggs.

You may drink milk, cream and buttermilk.

In case the milk of magnesia used in connection with the milk in the following diet should act too freely upon the bowels, lime water may be substituted for it, but it is best to add one or the other to the milk in order to prevent such from coagulating in the stomach and in order to alkalinize the milk.

Patients should be instructed to use the following diet the moment they feel any distress and not to wait until the digestive apparatus is badly out of order before giving it the rest which this diet affords.

Diet 2.-Chew all food for a long time. Eat nothing sweet or sour.

Take $1 / 2$ pint of hot milk with 1 or 2 teaspoonfuls of milk of magnesia or 2 to 4 tablespoonfuls of lime water at $6,8,10,12,2,4,6$ and 8 . Later you may take the other articles of food in this list at S, 12 and 6 .

First Week.-Four pints of hot milk daily with milk of magnesia or lime water; if this is not sufficient to keep your strength you may add from 1 to 4 raw eggs.

Second Week.- Same as first, and 2 to 4 raw or soft-boiled eggs in addition.

Third Week.- Same as second, and 2 to 6 pieces of very dry toast in addition.

Fourth Week.- Same as third, and all kinds of milk or cream soup in addition.

Fifth Week.- Same as fourth, and all kinds of mush or boiled rice in addition.

Sixth Week.-Same as fifth, and broiled, stewed or boiled beef or mutton in addition, chew and swallow the juice, but not the fiber. 
Later add cooked vegetables and cooked fruits, adding only one kind each week.

Later you may swallow the beef or the mutton.

Even after recovering fully, do not eat pastry, pie, pancakes, pickles, pork, or puddings. No cake, candy or canned goods. No raw regetables or raw fruits, unless the latter are perfectly ripe and not sour.

\section{EXCISION OF THE COLON IN CASE OF CIRCUMSCRIBED INVOLVE- MENT OF THE TRANSVERSE COLON COMPLICATING CANCER OF THE STOMACH}

Very rarely a cancer of the stomach located in the lowest portion of the greater curvature may become adherent to the transverse colon during the early period of its development and this condition may lead to an abdominal section because of the obstruction of the passage of fecal material through the transverse colon together with the presence of digestive disturbance. These cases are extremely rare but they occur frequently enough to warrant a description of the operation which will be required in this case.

The operation which one would naturally wish to perform would consist in the excision of the transverse colon and the union by means of the suturing of the two ends. This, however, is not a satisfactory operation because the posterior surface of the transverse colon is not covered with the peritoneum and a leakage may occur, moreover there is likely to be an obstruction to the passage of gas and feces at this point of union, and this added to the other conditions is likely to cause trouble. It is consequently far better to remove the cecum, the ascending colon and the transverse colon after the manner practised in Lane's operation; to implant the end of the ileum into the sigmoid flexure and to close the free end of the transverse colon at the point at which the section was made. 
This operation at once removes the cancerous portion of the transverse colon and the portion of the omentum containing the infected lymph nodes and leaves a convenient condition for performing a posterior gastro-enterostomy. The excision of the colon should be made before the operation upon the stomach is begun. The important points in this operation consist, first, in securing perfect hemostasis. This can be accomplished by laying bare the peritoneum covering the inner side of the cecum and grasping the blood-vessels supplying the cecum, and the hepatic flexure of the colon and the ascending colon. The ileum is then clamped between two powerful forceps from 5 to $10 \mathrm{~cm}$. from the cecum at a point at which it will be most convenient to implant the proximal end of the ileum into the sigmoid flexure. The distal end is then removed together with the cecum and the ascending colon.

In the region of the hepatic flexure of the colon it is important to guard against injuring the duodenum, which often projects to the right toward the right kidney pouch and unless this fact is borne in mind it is an easy matter to injure this portion of the duodenum. When the hepatic flexure of the colon has been reached it is best to abandon the operation for a time, packing the space made bare with gauze pads and to implant the proximal end of the ileum into the sigmoid flexure, care being taken to close the space behind these structures to prevent a future hernia. Then the operation is again resumed in the region of the hepatic flexure of the colon, the duodenum is laid bare, the four arteries mentioned in gastrectomy are clamped and the pyloric end of the stomach is removed together with the transverse colon, care being taken to grasp the middle colic artery with two pairs of forceps and to cut it before traction is made upon it. 
The colon is then clamped between two strong clamps at least $7 \mathrm{~cm}$. beyond the tumor and the entire structure up to that point together with the pyloric end of the stomach and the omentum are removed together with all of the lymph nodes contained in this region. The duodenum and the stomach are then treated as in the operations described above. The gastro-jejunostomy is also performed after the method already described and the entire raw surface which has been produced by the removal of the portion of the ileum, the cecum, the ascending colon, the transverse colon and the pyloric end of the stomach are carefully covered with peritoneum and the remnant of omentum is sutured over this surface. The operation is completed after the method described in gastrectomy.

By carrying out this operation systematically according to the method just described, being careful throughout the operation to keep the small intestine packed away from the field of operation and by avoiding all unnecessary manipulation of the intraabdominal organs, it is possible to complete this operation in a relatively short length of time and to reduce the shock to a minimum. Normal salt solution and concentrated predigested food may be given by rectum immediately after the operation and it is wise to give from 1,000 to $1,500 \mathrm{cc}$. of normal salt solution under the breasts directly after the operation and to repeat this twice a day for 2 or 3 days until the patient can take fluid by mouth.

The after-treatment is the same as that which has already been described. 


\section{CHAPTER XII}

\section{NON-SURGICAL TREATMENT}

Prophylaxis.-All patients with obstinate dyspepsia should be advised to submit to laparotomy at the hands of a competent surgeon. While experience has shown that there is no "cancer age," it is a proven fact that individuals above the age of 35 years are more prone to malignant disease than are those below such age. Abdominal exploration is a safe procedure with modern operative aids. Its teachings are of such worth that in suspected gastric cancer or atypical dyspepsia, the procedure should be resorted to more frequently than it now is. It is a curious condition of the professional mind which permits argument against laparotomy, where even expert non-surgical treatment has failed. Patients in America are rarely greatly alarmed when competent authorities recommend laparotomy as a solution of their disturbances of digestion. On the contrary, many individuals are so weary of ineffectual attempts to aid them by types of therapy ranging from crystal-gazing to almost permanent residence in hospitals or sanatoria, that they eagerly look forward to surgical intervention as a way out of their dyspeptic dilemmas.

Individuals in middle life, who have unaccountably developed faulty digestion, should be carefully examined for anomalies of the circulatory system, the kidneys, the blood and the blood-forming organs or the central nervous system. If disease is not located in such organs, and infective processes can be excluded, the patient should be 
told the possibilities of a malignant termination of his ailment and advised to consult a reputable surgeon.

Chronic, recurrent, or frequently bleeding ulcers, should be treated as potential or actual cancers and removed when possible.

In all types of cases, diagnosis should not be withheld until everybody recognizes cancer but the guileless patient or his optimistic physician. When the patient can answer "yes" to all the signs and symptoms of gastric cancer set down in the text-books, he would do well to get his affairs in order, for no form of treatment is then of permanent avail. While scientifically conducted clinical investigations of gastric ailments have returned facts of much diagnostic worth, the doctrine that the physicians must wait until analysis of the gastric extracts from dyspeptics shows absent free hydrochloric acid before they call a disease cancer, has sent more people to the grave than have abdominal explorations, however poorly such may have been performed. When made by trained surgeons, abdominal section is attended by a mortality of about $1 / 2$ of 1 per cent. There is a mortality of 100 per cent. in the non-surgical treatment of gastric cancer.

The first aim of prophylaxis then, should be the eradication from the human family of all potential or actual malignancy by surgical means. So long as we are in ignorance regarding the etiology of all cancer, and inasmuch as the question of its parasitic or its constitutional origin is a debatable one, it behooves medical men everywhere to urge early radical treatment of the disease.

In view of the fact that it has been shown that cancer is not infrequently more prevalent in certain districts, houses or families, it would seem proper to systematically study such localities, dwellings or families with a hope of ascer- 
taining etiologic information or to segregate affected individuals as a preventive measure. Intermarriage of cancerous folk, especially members of cancer families, should be interdicted. While no one can say positively that hereditary transmission of cancer takes place, it is not possible to state that certain tendencies toward malignancy are not transmitted from one generation to another.

Registration of cancer cases should be required of physicians by both local, state and federal authorities. Such reports should fully cover the etiologic, clinical and pathologic features connected with such patients.

General Measures.-There are really three main groups of patients for which medical treatment is indicated. These comprise patients in whom external evidences of metastases (to lymph glands, liver, peritoneum) contraindicate surgery; those on whom operations have been performed and instances where surgical measures are indicated, but complications (hemorrhage, vomiting, exhaustion) prevent immediate operation.

It is not necessary that patients know how seriously they are affected. Some individuals demand a statement of their exact condition. One should use tact in imparting such knowledge. To a member of the family, a bloodrelative, or a trusted friend, the ailment and its consequences should be fully described. While no hope of nonsurgical cure of cases of gastric cancer exists, much may be done to make these unfortunates comfortable.

Care of the Teeth and Oral Cavity.-As we have previously pointed out, many patients with malignant disease of the stomach have imperfect or dirty teeth. While no one has absolutely demonstrated the deleterious effects upon injured gastric mucosæ of swallowed, atypic mouth enzymes, amino-acids (e.g., tryptophan), pus, bits of teeth, 
bacteria or protozoa, it would appear safe to admit that such substances throw an excessive amount of unnecessary labor upon the body's defensive mechanism. Decayed teeth should be extracted and imperfect teeth repaired. Cancer patients usually endure extraction of teeth well. If properly carried out this operation requires no more anesthetic than a few whiffs of ether. In many instances, the work can be done under local anesthesia. Following the extraction of the teeth, hemorrhage is generally controlled by having the patient bite upon a firm gauze roll. If this does not suffice, bits of ice may be allowed to dissolve in the mouth, or the gums may be sprayed with weak adrenalin solution. Hypodermatic administration of morphine sulphate (gr. 1/4) often aids in controlling bleeding, and is a useful medicine to exhibit where pain is annoying or the patient is nervous. If seepage of blood from the torn gums continues for longer than 24 hours, active horse serum should be administered (10 cc. doses, 2 or 3 times during the day usually suffice) intramuscularly or intravenously. Where hemorrhage is prolonged, and symptoms of shock appear, transfusion of human blood from a relative is indicated. In our experience, this maneuver is most satisfactorily performed by the Percy-Cook method (see below).

To contract the gums after the extraction of teeth, a useful mouth-wash is Dobell's fluid to which has been added formalin to make a 1 per cent. solution. This wash has also hemostatic and bactericidal properties. It can be used after each feeding. One need scarcely mention that liquid or very soft food should be given until the gums have healed. Proper fitting artificial teeth should be inserted as soon as the gums are healed and firm.

Oral cleanliness should be sought apart from removal of 
decaying teeth. Oozing, soggy, pus-laden gums should be freely incised. Small abscess cavities may be lightly swabbed with 2 per cent. solution of silver nitrate, 10 per cent. formalin solution or dilute tincture of iodine. Foul tonsils or other infected adenoid tissue should be extirpated, if possible. If this cannot be done, then cauterization of disease foci should be thoroughly performed.

It is often astonishing to note the feeling of well-being which gastric cancer patients exhibit after their mouths have been cleaned up. Appetite may return, food may be eaten in abundance, gain in weight occur, and such general improvement supervene as to arouse hopes of a mistake in diagnosis or of spontaneous regression of the neoplasm.

The toilet of the stomach should be as carefully taken care of as in the case of the oral cavity. This demands attention in all instances of gastric cancer, whether they be of the retention or the non-retention type.

The reduction to a minimum of the swallowing of foul substances from the mouth is an important step toward gastric cleanliness. Even with this source of infection limited, the stomach is yet burdened, as a rule, with obnoxious material in the form of retained food, altered blood, products of tissue necrosis, bacteria and foreign chemical compounds. Nuch can be done by regulating the kind of diet (see below). Other measures are 2 ounces of oleum ricini (in beer, malt extract, acid fruit juices or administered through a stomach tube) at least once weekly; lavage of the stomach with solutions of 2 per cent. formalin, 1 per cent. salicylic acid, 0.5 per cent. thymol, $1-2,000$ quinine, or 1-3,000 alphozone (follow the medicinal lavage by a washing with normal salt solution at $37^{\circ} \mathrm{C}$.); the exhibition of gtt. xxx dilute hydrochloric acid (U.S.P.) $1 / 2$ hour after eating, or the administration of oil of eucalyp- 
tus (gtt. $\mathrm{xy}$-t.i.d. a.c.), radium water (5j to $5 \mathrm{ss}$ on an empty stomach several times daily), or bismuth subcarbonate (gr. xxx, when liquid food is taken or upon the empty stomach, 4 times daily).

Of all the above means of keeping the stomach clean, diet and frequent larage are, undoubtedly, the most satisfactory. Patients readily become accustomed to the daily use of the stomach tube, and cling to it when they discover how great is their relief from annoying symptoms by lavage. The best time to wash out the stomach is when the patient experiences the greatest discomfort. Usually a generous lavage before the morning meal, or the last thing at night, will insure the greatest measure of cleanliness and comfort. Patients with far advanced gastric cancer may require lavage several times daily.

Diet.-Feeding is always a serious problem in this disease. What agrees with one patient is often unbearable to another. Routine diet tables will be found to have but a general application. The type of food will frequently require changing in a given patient. It thus follows that dieting in gastric cancer is a highly individual problem.

Not infrequently, success in feeding these unfortunates rests upon the correct estimation of the position occupied by the stomach tumor. Growths at or near the cardia sometimes call for administration of very soft or liquid food. This may have to be given through a long catheter, or a stomach tube of small caliber passed beyond the constriction. It may be necessary to constantly use this method of feeding, but not rarely growths near the entrance to the stomach slough extensively, and so permit periods of relief from tube life. Extensive, canalized growths in the body of the stomach often allow the exhibition of well-chewed, mixed food for a long time. The patient's desires should 
be humored in such event. When early or late pyloric stenosis has occurred, it may be impossible to get a proper quantity of nourishment beyond the stomach. In mild stenoses where the normal way of food ingestion causes distress or pain, or where gastric atony and dilatation result in the constant presence of foul stagnation products in the stomach, considerable relief from discomfort and sufficient nutriment may be obtained by metagastric (duodenojejunal) or so-called "duodenal" alimentation.

Metagastric alimentation is of service where it is possible for the bulb of the "duodenal tube" or the end of a postgastric catheter to pass beyond the pylorus. It has been our observation (corroborated by fluoroscopic examinations) that in many instances duodenal tubes do not pass through the pylorus. Unfortunately in the very class of case in which this mode of feeding is most desirable, there is mechanical hindrance to its successful application. Not only in pyloric cancer does the bulb fail to pass into the duodenum, but we have learned that not infrequently the end of the tube gets caught between nodular projections of the growth and material forced through it lodges in the stomach. Where the gastric lumen is tortuous, this occurrence is quite common. Ordinary duodenal tubes must remain in situ for long periods of time. This renders the method an inconvenient, as well as one not altogether desirable, from the standpoint of cleanliness.

The form of postgastric catheter that can be passed directly into the small bowel upon a silk-cord guide is, in our experience, the most satisfactory. One can be reasonably sure that food is reaching the desired locality, the amount given can be computed, the patient is less inconvenienced by a thread being constantly present in his throat and mouth than by a rubber tube, and, lastly, it is possible 
to keep tubes clean that need only be present in the alimentary tract at the time food is given.

Various nourishing mixtures may be administered by the metagastric method. Those commonly found useful are parboiled milk, cream, buttermilk, malted milk, koumiss, wines, cocoa, chocolate, thin purees, clear broth, egg-nogg, barley-water, black coffee, tea, carbonated preparations, predigested foods, fruit juices, sugar water, or special formulæ. There is a wide range. From 2 to 8 ounces should be administered at a time. The material should be at $37^{\circ} \mathrm{C}$. and be passed in slowly. If one can be sure that the distal end of the feeding-tube is always patent, then nutrient mixtures may be given by a modified "dropmethod."

In instances where emaciation has been marked, we have found it useful to feed patients by the metagastric method, mixtures of amino-acids and maltose. Such are readily prepared by first digesting Witte-peptone in normal salt by trypsin (Fairchild) under toluol or in 0.4 per cent. alphozone-normal salt solution, and then adding sufficient maltose to make a 5 per cent. mixture. From 100 to 500 cc. may be administered through the post-gastric catheter every 3 hours. If thirst is excessive, similar quantities of normal salt solution at $37^{\circ} \mathrm{C}$. should be given 10 minutes before the amino-acid maltose preparation.

Where the gastric lumen is patent the essential feature of successful dieting is to allow only such food as will cause little irritation and will pass through the stomach leaving the minimum of residue. Lumps of food are likely to injure the diseased gastric lining, produce or keep up a hemorrhage and, by stagnating in the stomach, furnish a constant source of irritation as well as an ideal culture media for a host of inimical bacteria. Care should be taken that 
foods are not too hot or too cold. Hot foods cause gastric congestion or even hemorrhage in cancer, while cold foods may be responsible for painful gastro-spasms.

Uncooked vegetables and fruits should be interdicted, not only on account of the possibility of their being mechanical irritants, but also because they are a common source of infection. It has been frequently pointed out by Ochsner, that farm produce is generally contaminated by manure-impregnated soil. It is not essential to believe that these uncooked foods carry a parasite capable of causing cancer, in order to limit their use. Anyone who has taken the trouble to examine microscopically or chemically, the dung which is ordinarily used to force, ripen or bleach garden products, will admit that the possibilities of such, when taken raw, acting as sources of infection, cannot be denied. It is a rather striking fact that in communities where an excess of uncooked vegetables or "greens" comprises a good share of the diet, gastric cancer is very prevalent. Among city dwellers, of the well-to-do class, who can afford fresh garden products, the cancer rate is likewise high. The interesting and valuable investigations of Smith relative to the production of tumors in daisies from bacilli isolated from "galls" would appear to indicate that raw "greens" or vegetables might be a source of gastric irritation. The researches of Gaylord, upon the development of tumors in fish that live in water highly contaminated by their own discharges, are not without significance in pointing out the dangers attending ingestion of bacterially or chemically filthy food.

With the increase in the population of the globe there has been an increase in cancer generally-particularly in gastric cancer. What effect, through pollution of water supplies and food, this increase has exerted toward causing 
the disease, we can but conjecture. The end-result is worthy of thought.

Rectal Feeding.-This may be required after hemorrhage, on account of pain, in "coma carcinomatosa" where exhaustion has resulted from loss of appetite, nausea, vomiting, in starvation due to stenoses, or to allay thirst.

Rectal alimentation is best administered by the "Murphy drip" method. The patient should lie with hips elevated upon one or two pillows. Cleansing the colon with normal salt solution should be performed before the nutrient enema is given. The clystra should be given at a temperature of $37^{\circ} \mathrm{C}$. In ordinary cases enemata are well retained. If they are expelled promptly, more care should be taken in their administration, or gtt. $\mathrm{v}-\mathrm{xx}$ of tincture opii and gtt. iii to $\mathrm{x}$ of tincture belladonna, may be incorporated in every second enema. From 4 to 8 ounces of nutrient fluid may be given at intervals of from 4 to 6 hours. If these feedings are combined with nourishment per oram, it is well to give but two daily.

Various formulæ for nutrient enemata are in vogue. We have found the following useful:

Maltose

Alcohol (95 per cent.)

Peptonized milk or 30.0 grams

or 20.0 cc.

or $100.0 \mathrm{cc}$.

Normal salt solution q. s. ad viii or 240.0 cc.

Mix.-Heat to $37^{\circ} \mathrm{C}$.

Sig.-Administer slowly.

Other enemata of value are parboiled milk ( $\overline{\mathrm{v}}$ every 3 hours); glucose ( $\mathrm{z}$ ij to 5 viii normal salt solution), egg albumen and maltose (whites of 2 eggs, maltose $\tilde{j} \mathrm{j}$ in normal salt solution to 5 viii). It is doubtful if anything of more use than water, salts or alcohol is absorbed from certain of 
"nutrient" mixtures comprising eggs, starch, proprietary predigested foods, beef extracts and the like.

To two patients we have given enemata composed of amino-acids and maltose with seeming benefit, but the procedure is still on trial.

\section{MANAGEMENT OF SYMPTOMS}

Anorexia is not infrequently a most annoying symptom in gastric cancer. The desire for certain kinds of food is lost. This may amount to an actual aversion. Some patients early lose their appetites for meat products, milk or sweets. The change of food desire for the individual is not infrequently radical. The appetite is best stimulated by lavage, catharsis, the employment of hot baths, massage, attempt at change of mental attitude, or by a vacation.

Early lavage often prevents anorexia. Its effect is both tonic and cleansing. After the stomach has once been relieved of such accumulation as may exist at the time the patient comes under observation, it is not necessary to use medicinal remedies in the wash water. Normal salt solution at $37^{\circ} \mathrm{C}$. acts very well in most instances, and is readily prepared. In cases where the gastric retention is very marked, or where a widely sloughing growth constantly befouls the stomach, lavage with Carlsbad water ( 1 dram of Carlsbad Salts to 1,000 cc. of water at $37^{\circ} \mathrm{C}$.), 1 per cent. salicylic acid solution or 0.5 per cent. solution of lysol are usually of service.

Vomiting.-In many instances vomiting is due to improper food, overfeeding, obstruction or pain.

If the diet is altered so that it is enticing, and care is taken that, with the eager attempt to regain health, the patient does not overload his stomach, much of the 
vomiting may cease. When pain is the cause of the emesis . (see below) simple remedies to relieve such should be promptly instituted.

In cases where stenoses produce early with a constant amount of gastric stagnation, lavage usually takes care of the annoying vomiting. It may be performed fearlessly provided a soft stomach tube is used. Only in instances where there has been severe hemorrhage, is it contraindicated. We have frequently observed, however, that hemorrhage itself may initiate a prolonged attack of vomiting which may not cease until the blood clots have been washed from the stomach. We have often thoroughly lavaged a stomach within a few minutes after a severe hemorrhage without untoward symptoms on the part of the patient.

Medicinal remedies are of but secondary value in the treatment of vomiting unless they attack one of its underlying causes.

We have found that severe retching, with or without vomiting, can be controlled by orthoform (grains 10 every 4 hours; taken on an empty stomach), chloroform (gtt. 10-30) administered after lavage, or potassium bromide (grains 30-60) per rectum. Occasionally there are cases where vomiting is so annoying that it is necessary to administer opiates. These should be given by the physician himself and preferably hypodermatically.

Acidity, Pyrosis and Eructation.- Such are not rarely extremely annoying. Regulation of the diet combined with catharsis and frequent gastric lavage do much to prevent or relieve these symptoms.

The administration of hydrochloric acid (gtt. 15-30 after meals) aids in limiting intragastric fermentation, and in this way acts to relieve pyrosis and eructations, which 
are largely due in this disease to fermentation and organic acids. If gastric lavage is not used to remove stagnant contents from the stomach, or if fermentation of such contents is not prevented, then it may be occasionally necessary to employ alkalies to neutralize the high combined acidity. This is a slovenly way to aid the patient, although at times it may prove of value in relieving symptoms. We have found that alkalies given in the form of bismuth subcarbonate (grains 30) and calcined magnesia (grains 20) prove useful in such emergencies.

Bowels.-If constipation exists - and it frequently does2 ounces of castor oil in beer or fruit juices may be given once or even twice weekly. There is nothing superior to castor oil for emptying the intestinal tract of contaminating and putrefying contents. Where castor oil is not well borne, from 1, 2 to 2 ounces of liquid paraffin may be administered, preferably on an empty stomach. There are instances where from 3 to 5 grains of calomel given at bed time are useful in relieving the bowels, and at the same time overcoming flatulence. Mild cathartics which retain their activity for a long time are phenolphthalein (grains 510), cascara sagrada (gtt. 15-60 at bed time) or compound jalap powder (grains 30-60 at bed time).

Where the stools show an excess of mucus, hot Carlsbad water, or solutions of sodium phosphate or sodium citrate, should be given before the first feeding in the morning.

Not infrequently, diarrhœa is an annoying symptom. There are cases where no medicinal agent, other than opiates, will be found of service. Everything else should be tried, however, before morphine, codein or the like are used.

Keeping the stomach clean, putting the patient for a few days upon a diet of parboiled milk or buttermilk, rest in bed, or hot applications orer the abdomen, some- 
times stop exhausting diarrhœa. Where simple measures fail, bismuth subgallate (grains 10) may be given after each bowel movement. Tannigen, tannic acid, or a pill composed of opium and camphor may also be of service.

When opiates are indicated they may be administered in the form of laudanum (gtt. 10-40) or paregoric, by mouth or in starch-water enemata. Codein or morphine should not be used unless absolutely necessary inasmuch as not infrequently they produce nausea, rapid emaciation, together with physical and psychic unrest.

Pain.-In but 15 per cent. of instances of gastric cancer is the pain sharp, colicky or prostrating. These are cases where ulceration exists or perforation has taken place. In these emergencies, opiates should be administered promptly and preferably by the hypodermic needle. Patients should be kept in bed, and after preliminary lavage, hot applications should be applied to the epigastrium. If hemorrhage complicates, an ice-bag or ice-coil locally should be used.

Much of the abdominal distress in gastric cancer is due to accumulation in the stomach of gas or stagnant material. In such instances the stomach tube should be liberally employed. There are instances where improper diet causes epigastric pain. This error should be corrected. At times it will be found necessary to administer such remedies as chloroform water, orthoform, bismuth, creosote, or even opiates.

Flatulence.-A cold enema given in the morning will be found of considerable value in preventing or treating this sometimes annoying symptom. Where a patient is bedridden, and there is a constant tendency to gaseous distention of the bowel, a rectal tube of fair caliber may be inserted 5 times a day and left in situ for from 10 minutes 
to a ${ }^{1}$. hour. Where pronounced gastro-intestinal fermentation exists, alleviation may be secured by the administration of from one to six tubes of liquid culture of Bacillus bulgaricus (Metchnikoff) daily.

Hemorrhage.-It is impossible to prevent the more or less constant seepage of blood that occurs in certain types of gastric malignancy. The hemorrhage which it is necessary to treat is that which occurs acutely, or where constant seepage has produced marked anemia which prevents surgical intervention in an otherwise operable case. The acute hemorrhage of gastric cancer is best treated by prompt lavage of the stomach with normal salt solution $\left(37^{\circ} \mathrm{C}\right.$.), hypodermic administration of morphine (grains 1/4-1/2), absolute rest in bed, ice-coil to the abdomen, heat externally and the institution of rectal feeding. If hemorrhage does not cease within a few hours, horse serum in from 10 to 100 cc. doses should be given hypodermatically. This can be repeated several times in the course of 24 to 48 hours. The preparation known as "coagulose" devised from the experimental work of Clowes of the Buffalo Cancer Research Laboratory may be found of service where horse serum has proven to be ineffective. We have observed that several instances of persistent hemorrhage were satisfactorily treated by the direct transfusion of human blood after the method of Percy and Cook. The latter describes the technique as follows:

A Simple Method of Blood Transfusion.-A method of blood transfusion which presents many desirable features has been in use at Augustana Hospital long enough to establish its value as a means of relieving anemia and increasing resistance.

The blood is aspirated into a specially prepared transfusion tube from a good sized vein of the donor and injected, 
before it has opportunity to coagulate, into a similar vein of the patient.

The Transfusion Tube.-The transfusion tube is made from soft carefully annealed German glass. It consists of a graduated cylinder about 16 inches long and of about 650 cc. capacity. The lower end of this cylinder is drawn out at right angles to its axis to form the canula which is inserted directly into the vein. The upper end or mouth of the cylinder is drawn out at right angles to its axis but is perpendicular to the canula. This end is made about 4 inches long and about $3 / 8$ inch in diameter. Over it is slipped a rubber tube about 15 inches long which ends in a small glass tip or mouth piece. A glass tee or branch is inserted at about 6 inches from the cylinder, cutting the rubber tube in 2 parts, 6 and 9 inches long. To the side branch of the glass tee an ordinary rubber atomizer bulb is connected by a piece of rubber tubing 2 inches long. This rubber tube is clamped shut with an artery forceps while the blood is being aspirated from the donor. The forceps is removed and clamped above the tee when the transfusion tube is filled and inserted in the vein of the patient.

Sterilizing and Coating Transfusion Tube-Caution: Tube must be dry and free from blood. (After use, wash quickly with cold water adding shot if necessary to loosen clots, then wash with alcohol and ether separately and successively.)

1. Dry Heat Method.-Wrap tube in a white cloth, sterilize in bacteriological sterilizer by dry air, 30 minutes at $175^{\circ} \mathrm{C}$. Caution.-Do not overheat. Before tube becomes cold pour in a couple of ounces of melted and sterilized ordinary hard grocer's paraffin. Turn tube around (handling it with sterile gloves or scrubbed hands) so that 
every part is reached by the melted paraffin. The paraffin is then poured out of the large end of the tube. Examine canula (small end) to make sure that it is not closed by the hardened paraffin. It should be wide open. If not, heat gently over escaping steam or a small flame and tip the tube so that the paraffin runs out into the large end of the tube. The tube may then be safely done with cold air, fanning it or by pouring over it sterile water, first warm then cooler and cooler. (No water should reach the inside of the tube as it will not then coat over evenly.)

2. Steam or Autoclave Method.-Aspirate or pour about 2 ounces of melted grocer's paraffin into tube (dry and free from blood), wrap loosely in a towel or cloth, sterilize 15 minutes only at 15 pounds pressure in the autoclave. Turn the tube around so that every part of the tube is reached by the paraffin, handling it with sterile gloves or scrubbed hands. The process of cooling is as described in the previous method. The tube, properly coated, has a uniform gray color and the canula is wide open. The tube is now ready for use and may be wrapped up in a sterile towel and put away.

Sterilizing rubber tubing, glass tee, and mouthpiece is done by pulling them apart, wrapping them in a towel (careful not to have any kinks in the tubing) and sterilizing in the autoclave 15 pounds pressure for 15 minutes. (Note. -Use no heat for sterilizing rubber bulb. Wash with alcohol before using; this is sufficient.) A small artery forceps is clamped on the rubber tube between the atomizer bulb and the glass tee. A small piece of sterile cotton is inserted into the glass tee, the rubber tube connected with the mouthpiece is then slipped over one branch of the tee, the atomizer bulb is connected to the side branch of the tee and the rubber remaining connects the other branch of the tee 
to the mouth of the transfusion tube. The mouthpiece at the free end of the rubber tube is now placed in the mouth and an ounce of sterile liquid paraffin is then sucked up through the canula tube into the tube. The mouthpiece is then wrapped in sterile gauze so as not to contaminate the sterile tube. The whole is then set aside ready for use. The liquid paraffin floating on top of the blood prevents the access of air, thus tending to delay coagulation.

Procedure.-A constrictor, which may be a rubber tube but better a blood-pressure instrument, for the donor is placed high around the arm. The veins of the arm of donor and recipient are exposed, preferably above the elbow. The incision is best made from 2 to 3 inches long just above the bifurcation of the median vein at the elbow. Two percent. cocain is injected about the vein hypodermically. The vein is carefully dissected out, the small branches being clamped, ligated, and divided.

Up to this point the operation is the same in the case of both donor and patient. The operation should be completed to this extent in both persons before the vein is opened in either case.

A ligature is then placed around the vein of the donor at its upper end, that is centrally, and tied, another ligature is placed at the lower part and not tied but held by the assistant before and while the vein is being opened by the operator, in order to control bleeding while the canula is being inserted. Similarly a ligature is placed around the vein of the patient and tied at the distal part, another being slipped around the vein centrally and held without tying while this vein is opened and the canula inserted. Both veins are now opened between the ligatures placed as above, with a sharp scalpel or fine sharp scissors. A wide and free opening must be made in the vein in order that the canula 
may slip easily into the lumen of the vein. It is very easy to slip the canula between the walls of the rein instead of into the lumen if this precaution is not observed.

The transfusion tube now contains an ounce of liquid paraffin in it and with atomizer bulb clamped off, is taken up by the assistant. The mouthpiece is placed in his

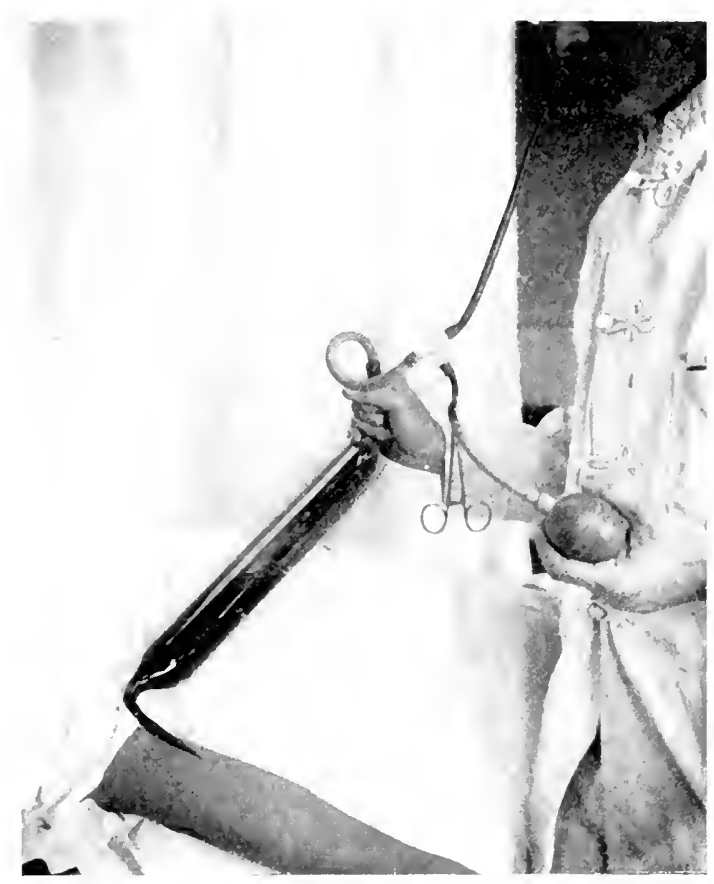

Fig. 105.-Transfusion by Percy-Cook method. Illustration shows end of tube in position for aspirating blood from vein of donor. Note paraffinlined tube, layer of liquid paraffin above blood level. Aspirating tube and clamp on tube leading to compression bulb.

mouth, the upper end of the tube balanced in his hand while the operator slips the canula into the vein of the donor in a distal direction, i.e., toward the hand. If the canula does not slip readily into the vein, this may be facilitated by grasping and holding apart the edges of the cut vein with fine mouse-tooth or small-nosed docking forceps, or the canula may be guided along the channel of a grooved direc- 
tor, which has been slipped into the rein. The canula is pushed home into the rein until its tapering sides just fill the lumen of the vein. The distal ligature is then loosened and the air-pressure constrictor pumped up to 60 to $90 \mathrm{~mm}$. The blood quickly gathers from the anastomosing deep and superficial veins and flows through the open vein into the

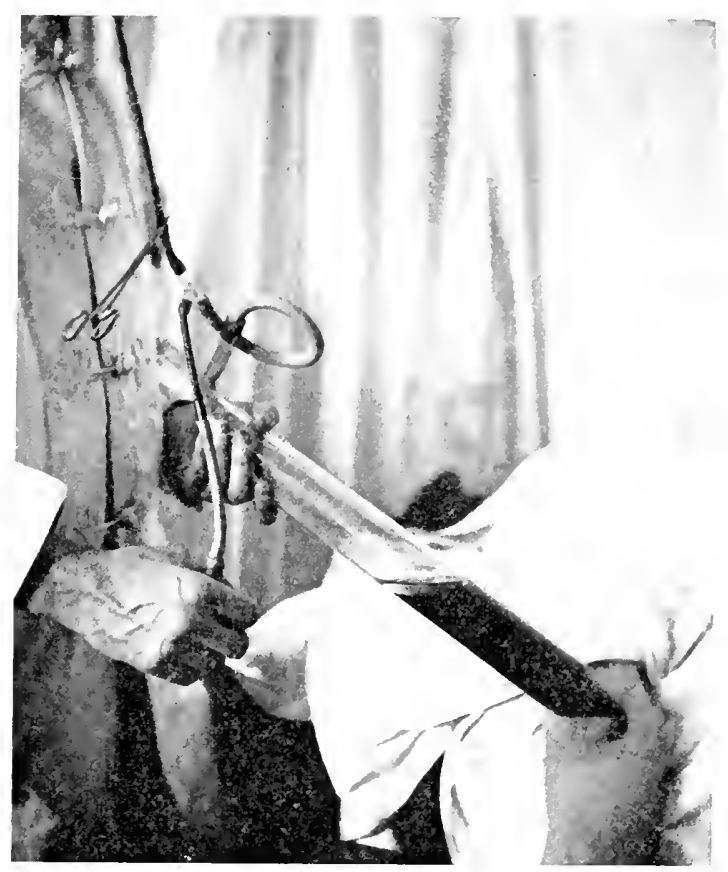

FIG. 106.-Percy-Cook method of blood transfusion. Blood being given to recipient. Note direction of transfusion tip in arm vein; clamp on aspiration tube with compression bulb under control for gradually emptying transfusion tube.

canula. The assistant then sucks gently but continuously and the blood rapidly enters the tube. (Too hard sucking will collapse the vein so that no blood enters the tube.) If the vein has been pulled too hard by the assistant holding the ligature it will collapse much more quickly. If the technique has been good the tube may be filled in 4 or 5 minutes. It is not advisable to leave the canula in the vein 
for a much longer time, however. It is better to be content with taking 200 or 300 cc. of blood than take 6 or 7 minutes to fill the tube. The rubber tube is now clamped with a heavy artery forceps near the mouth of the transfusion tube in order that blood shall not be lost in the transfer from donor to patient. The canula is then taken out and inserted in the vein of the patient in a central direction, i.e., toward the heart. The large clamp is removed from the rubber tube near the mouth of the transfusion tube. The small clamp near the atomizer bulb is taken off and clamped above the glass tee and between it and the mouthpiece. Gentle pressure is now placed upon the atomizer bulb which forces the air into the tube, displacing the blood as it enters the vein. It should take about 4 to 5 minutes to inject the blood as a more rapid injection may cause a dilatation of the right heart with collapse and death. (After taking the canula from the donor's vein an assistant meanwhile removes the constrictor from the arm and ties the distal ligature of the donor's vein.) The canula must be slipped out before any liquid paraffin enters the vein producing fat emboli. When this is done the central ligature on the patient's vein is tied and the skin closed over the vein with horse-hair stitches.

Advantages of the Method.- 1 . The blood is measured and a definite quantity is taken and injected in a short space of time.

2. The donor is protected absolutely from any infection which the patient may have, as there is no contact, direct or indirect. There is no chance for a drop of the patient's blood to get into the donor's vessels.

3. It is a venous operation, so there is no danger from infection to the deep structures lying about the large artery usually used for the purpose. There is no impairment of 
the circulation as from the ligation of such an important artery as the radial.

4. It has proved uniformly successful where care has been taken to insure the proper technique.

In the treatment of the anemia following gastric hemorrhage or gastric seepage, the most satisfactory remedies appear to be salvarsan, arsenious acid, Fowler's solution or cacodylate of sodium. Any of these may be given alone, or with preparations of iron. There are numerous useful iron preparations. We have found to be of service, Blaud's mass or various forms of iron conveniently put up in ampules for hypodermatic administration.

There are many cases of malignant anemia in which ordinary drugs are of little value. In such cases if the stool examination reveals the constant presence of altered blood, the giving of blood-building medicines may become effective after several hypodermic injections of horse serum or a transfusion of from 300 to $600 \mathrm{cc}$. of human blood. Not rarely, attention to these details will enable the patient to come to operation, or will render operative procedures of more marked benefit.

$\mathrm{X}$-rays.-In gastric cancer, the therapeutic value of Roentgen rays is uncertain. The effects appear to be largely influenced by the type of gastric neoplasm, the stage of the disease, the pressure of associated lesions (anemia, metastases, cardiorenal complications and the like), and the skill with which treatments are given.

Cases in which the method appears to be justifiable are those where inoperable growths exist; where operation has been performed and gland involvement discovered; where patients are either too weak for, or refuse, surgical intervention; where recurrences have appeared, and in the interim of two-stage operations. 
It should be especially emphasized that under no circumstances other than absolute refusal of surgical measures, should $x$-ray therapy be advised in instances of suspected or early gastric malignancy. It is the physician's duty to urge the benefits of laparotomy. There is no instance of pathologically demonstrated gastric cancer, on record, that has been cured by $x$-ray treatments.

In suitable instances, $x$-ray exposures may be begun 2 or 3 days following laparotomy. Treatment should be to the deeper tissues, with a hard tube, and there should be (except in very weak patients, or those who have previously been treated by $x$-rays) exposure of from 15 minutes to 1 hour. The parts of the body adjacent to the treatment area must be carefully protected by lead foil. Such procedure insures proper "erythema dose." The treatments should be given daily, or every other day for from 6 to 12 days. A rest of a similar period should be permitted before the patient is again exposed.

During actinotherapy frequent examination of the patient's urine and blood should be made. Vigorous $x$-ray treatment is not infrequently accompanied by the presence of albumin and casts in the urine. Rapid destruction of red blood cells, with a fall in the hemoglobin and an increase of mononuclear leucocytes at the expense of the polynuclear forms may follow prolonged Roentgen exposure.

The precise way in which $x$-ray therapy favorably influences certain malignant growths is not known. It would appear that the $x$-rays may destroy young cancer cells, split cancer-protein into "toxic" and "non-toxic" molecules, cause hemorrhage with liberation of enzymes capable of attacking neoplastic cells, or, by stimulating some yet undiscovered property of the blood serum, formed elements or both, strengthen the body's defensive mechanism. 
Radium, Mesothorium and Allied Substances.-In growths of limited extent, particularly when such are located at or near the cardia, it would seem that radium or mesothorium are of value. Such may be applied directly to the diseased area in protected capsules at the ends of sounds. The capsule should be left in situ for from 1 to 12 hours. Only wealthy folks are able to afford such treatments.

Where there is extensive involvement of the stomach by cancer, we have observed certain interesting results following the drinking of radio-active water. If from 4 to 60 cc. are administered at intervals of from 1 to 4 times daily, pain appears to lessen or subside, the stomach contents become less foul, hemoglobin may increase, and the patient may exhibit an attitude of general well-being. Occasionally, the disease appears to remain quiescent for a time, or it progresses slowly. Massmann reports an instance where cancer at the cardiac orifice was held in check for 2 years by the ingestion of radio-active water. The patient finally succumbed as the result of metastases.

Radio-active water has at least the quality of being comparatively reasonable in price. Its extensive use is thus possible.

It should not be forgotten that, due to much pseudoscientific publicity, and to the cost of treatments by $x$-ray, radium or mesothorium, a not-to-be-neglected benefit that may accrue from their use, has a psychic foundation. If the patient can afford such comfort he should be permitted it. On the other hand, no sacrifice or neglect of simple, proved and accessible therapeutic measures should be permitted in order that a patient enjoy light therapy. Not rarely do we see individuals who have spent their all pur- 
suing phantom cures, die in squalor or become a burden upon the commonwealth, or upon charitable friends.

Serotherapy.--In 1909, Hodenpyl reported striking results in malignancy following the injection into those affected, of ascitic fluid obtained from the peritoneal sac of a woman in whom there seemed to have been a spontaneous cure of an intra-abdominal neoplasm. Unfortunately, both the donor of the serum and the brilliant investigator died when it seemed about to be shown that serum from certain kinds of cancer appeared to possess protective substances. R. Weil made an extensive biochemic examination of the Hodenpyl fluid and showed that it seemed to contain a measurable increase of anti-cancer bodies.

Other antisera have been proposed. The most notable of these is that of Berkeley and Beebe. These investigators claim to have made an extract of cancer tissue removed surgically. By successive injection of the "specific human cancer extract" into alien mammals, a serum may be developed which when injected into the original host in increasing doses, is followed by "rapid regression and disappearance" of what tumor remains. Berkeley and Beebe think the effect is due to cytolysis of the tumor cells. They report encouraging results from the use of their "antiserum" in 16 cases of malignancy.

A utolysates of human embryos have been used by Fischera in the treatment of malignancy. Nearly 50 per cent. of his cases were aided by the treatment.

Vaccine Treatments.- This method of therapy has been frequently suggested, and all types of vaccines have been used. If removal of a metastatic gland shows sarcoma, then a certain beneficial effect may result from the use of Coley's "toxins" (Streptococcus erysipelatis and Bacillus 
prodigiosus). This remedy should be tried whenever it is possible.

Suggestive "split-proteid" vaccination has been carried on by W. Vaughan in cases of cancer. Basing his work upon the investigations of V. C. Vaughan on cellular proteids, W. Vaughan has prepared a "vaccine" consisting of aqueous solutions of the non-toxic radicle of the protein molecule. W. Vaughan describes the preparation of this vaccine as follows:

A small piece of the tissue is first examined by frozen section, in order to ascertain the exact nature of the growth. Next, the malignant tissue is dissected as freely as possible from all surrounding tissues, after which it may be placed in absolute alcohol and kept until opportunity is given to carry out the following steps. All tissue should be dehydrated in absolute alcohol before being split up. Next, the cancer material is ground as finely as possible with an ordinary meat grinder, but if the amount is small, loss may be saved by cutting with scissors and forceps, after which it is placed in a flask which contains about 15 times the volume of material of a 2 per cent. solution of sodium hydrate in absolute alcohol. (It will facilitate the solution of the sodium hydrate in the alcohol if the former is first pulverized finely in a mortar.) The flask containing the material is next heated over a water-bath for from 2 to 3 hours, depending on the amount of tissue that is to be split up. For safety only, the flask is connected with some form of condenser before heating. However, the alcohol should not be heated to such a degree that it actively distils, as too much heat will absolutely destroy the activity of the resulting residue. At the end of 2 or 3 hours the tissue within the flask should appear as a finely divided powder. If this is not the case it is my practice to allow 
the flask to stand over night, after which time the supernatant fluid is decanted and a sufficient amount of the alkaline alcohol solution added to make up the original volume, heat being again applied in the same manner.

Next the solution is filtered off and the residue washed upon the filter with absolute alcohol until its reaction is no longer alkaline. While still somewhat moist the residue is weighed upon the filter, a filter paper of equal weight being placed upon the opposite balance to offset the weight of the one containing the residue. Next it is transferred with a sterile spatula to a sterile bottle and sufficient sterile water added to make the desired solution. With small amounts of material I prefer a 1 per cent. solution, with larger amounts, a 2 per cent. Practically all of the residue is soluble in water, but if such is not the case it may be filtered. Lastly, sufficient phenol is added to make the given amount contain 0.5 per cent. This is kept as stock solution and injections given of the same.

W. Vaughan states that injections of from 3 to $30 \mathrm{cc}$. of his solution may be made frequently. The time for injection is indicated by observing the relative fluctuations of the polymorphonuclear and the mononuclear cells in differential blood counts. An increase in mononuclear cells-particularly of the large mononuclears-appears to be a favorable sign. The injections should be given "only at the periods during which the percentage of polymorphonuclear leucocytes is increasing."

W. Vaughan reports numerous cases illustrative of the effects of using split-proteid vaccine. Some have encouraging pointings. W. Vaughan's contributions to the effect of cancer residue injections upon blood morphology are certainly creditable and perhaps not without practical significance. 
Chemo-therapy.-The amouncement by Wassermann that solutions of an eosin-selenium compound brought about regression or even cure of cancers in mice proved a rare stimulus to workers in experimental laboratories and to clinicians. Notable reports have come from Ehrlich, Caspari, Neuberg and Popov.

These investigators have shown that certain cancers are cured, regress, or have their growth halted when intravenous injections of solutions of colloidal copper, platinum or selenium, eosin-selenium compounds, leech extract or casein are given. Robertson and Burnett have observed that aqueous emulsions of lecithin injected directly into tumors diminish the tendency to form metastases, retard the metastatic growth when it does occur, and in some instances retard the primary growth.

It would appear that treatment of cancer by chemotherapy offers alluring possibilities. The method has not yet proven of absolute worth. It is suggested that direct injection of solutions of colloidal copper or platinum, leech extract, casein or emulsions of lecithin into irremovable gastric cancers or metastases from such might be employed with benefit.

\section{REFERENCES}

Berkeley And Beebe: New York Medical Record, 1912, March 16. Vaughax, W.: N. Y. Med. Jour., 1910, Oct. 15.

Caspari and Neuberg: Deutsch. Med. Wochenschr., 1912, Vol. xxxviii, p. 375 .

Popov: Thèse de Universite de Montpelier, 1913, July 26, No. 24.

Loes, McClurg, and Sweek; Loeb and Fleisher; Loeb, McClurg and Sweek; and Loeb and Fleisher, Jour. A.M.A., 1913, June 14, p. 1857, and The Interstate Medical Journal, 1912, vol. xix, No. 12.

Lовв: Interstate Medical Journal, 1913, xx, No. 5.

Robertson and Burnett; Jour. of Exp. Med., 1913, No. 3, p. 344. 



\section{INDEX OF NAMES}

Abderhalden，230，266，320，344, 347,349

Adami, 371, 372

Aschoff, 31,50

Ascoli, 320, 348, 349

Ascoli and Izar, 349

BASHFoRd, 96

Beck, 421

Beebe and Berkeley, 496, 499

Bergmann and Meyer, 342, 344, 349

Berkeley and Beebe, 496, 499

Bernstein and Simons, 348, 349

Blackford and MacCarty, 105, 109, 362

Blumer, 184, 185, 186

Boas, 205

Boas and Oppler, 211

Bolton, 351, 352, 371

Bonhoff, 332, 349

Brinton, 26, 50, 98, 109, 175, 351, $369,371,372$

Broders and MacCarty, 109

Buday, 18, 50

Burnett and Robertson, 499

CABOT, 24, 50, 324, 326, 327, 329, 349

Cammidge, 263, 267

Campbell, 52, 108, 412

Capps, 332

Carrel, 85, 109

Caspari, 499

Chesnel, 358, 372

Clendenning, 52, 108, 412

Clowes, 486

Coley, 496

Connell, 425, 434, 436, 451, 452, 461, 463

Cook and Percy, 475, 486, 490, 491

Coolidge, 276

Crile, 336

Cuneo, 418

Cunliffe, 327,349
DACosta, 327

Dare, 115, 127, 138, 324

Dittrich, 98

Dobell, 475

Dock, 173, 373, 374, 381, 406, 416

Drew, 371,372

EHRLich, 499

Elsberg, Neuhoff and Geist, 338, 349

Elsner, 190

Emerson, 242, 267

Ewald, 245, 246

Feldstein and Weil, 344, 349

Fenwick, 24, 26, 37, 38, 44, 48, 50, $52,98,107,108,109,134,147$, $175,361,372$

Fibiger, 39, 50, 84, 109

Fischer, 230, 266

Fischer and Neubauer, 230, 231, 232, 266

Fischera, 496

Fleisher and Loeb, 499

Flexner and Jobling, 83

Fonio, 227

Forametti, 190

Fox, 26, 50

Frank and Heimann, 347, 349

Franz, 347, 349

Frazier, 52, 108

Freund and Kaminer, 339, 349

Friedenwald, 26, 28, 50, 175, 356, $363,364,372$

Fuld and Levison, 228, 229, 266

Fütterer, 39, 50

Galton, 44, 50

Gay, 85, 109, 320

Gaylord, 4S0

Geist, Elsberg, and Neuhoff, 338,349

Gies, 239, 267

Gluzinski, 226

Graham, 355, 363, 371, 372 
Gressot, 359. 37\%

Gross, 263

Gunzberg, 202

HAHN, 175

Hall, 190

Hall and Williamson, 231, 267

Hamburger, 320

Hansmann, 366

Hardisty and Ruttan, 205, 266

Hardy and Meyer, 411

Heimann and Frank, 347, 349

Hendly, 37

Hie Ding Lin, 31

Hodenpyl, 496

Hoffman, 20, 21, 23, 25, 28, 29, 34, 35,50

IzAr and Ascoli, 349

JACKSON, 190

Jacques and Woodyatt, 367,372

Janeway, 190

Jobling, 320

Jobling and Flexner, 83

Jones and Rous, 108

Junghans and Wolff, 117, 142, 143, $146,245,246,247,248,250$, $251,252,253,254,255,256$, $267,368,398$

KAmiser and Freund, 339, 349

Kauffmann, 211, 266

Irausch, 190

Kelling, 190, 348, 349

Kober and Lyle, 231, 266

Kocher, 438, 439

Kohlenberger, 231, 266

Krida, 334, 335, 336, 338, 349

Kussmaul, 171, 389, 397

Kuttner, 190

LANE, 469

Lang, 334, 349

Lebert, 98, 175, 369, 372

Lembert, 435, 436, 438, 449, 450, $452,453,458,461,462,463$

Leube, 175

Levin, 83, 108, 371,372

Levison and Fuld, 228, 229, 266
Loeb, 499

Loeb and Fleisher, 499

Loeb, McClurg, and Sweek, 499

Louis, 98

Luton, 98, 109

Lyle, 412

Lyle and Kober, 231, 266

МaCCARTY, 68, 77, 85, 86, 87, 88, 109 , 238, 352, 353, 370, 371, 372

MacCarty and Blackford, 105, 109, 362

MacCarty and Broders, 109

MacCarty and Wilson, 67, 69, 70 , $71,72,73,74,75,76,109,269$, $358,370,372$

Massmann, 495

Mathieu, 374, 381

Mayo (C. H.), 437

Mayo (IV. J.), 428

Mayo-Robson and Moynihan, 50

McClurg, Loeb, and Sweek, 499

MeCrae and Osler, 38, 50, 175, 321, $324,326,329,332,349,356$, $372,373,381$

McDowell and Wilson, 370

Melinkow, 370

Metchnikoff, 486

Neyer, 421

Meyer and Bergmann, 342, 344, 349

Meyer and Hardy, 411

Mikulicz, 190

Miyake, 31, 50

Monichon, 44, 50

Moreschi, 97, 109

Morris, 109

Morris (Roger), 106

Moynihan and Mayo-Robson, 50

Mïller, 230, 266, 332, 339, 349

Müller and Whitman, $342,344,347$

Murphy, 81, 85, 108, 459, 481

Murphy and Rous, 109

Murphy, Rous, and Tytler, 108

Napoleoxi, 123

Neubauer and Fischer, 230, 231, 232, 266

Neuberg, 499

Neuhoff, Elsberg and Geist, 338, 349 
Ochsner, $33,417,480$

Oppenheim, 231, 266

Oppler and Boas, 211

Osler, 4S, 358, 372

Osler and McCrae, 38, 50, 175, 321, $324,326,329,332,349,356$, $372,373,381$

Paterson, 359, 372

Pelosi, 227, 266

Percy and Cook, 475, 486, 490, 491

Petry, 342

Pettinkofer, 243

Popov, 499

RaNSOHOFF, 348,349

Robertson and Burnett, 499

Roche, 344, 349

Rokitansky, $10 \mathrm{~s}$

Rokitansky and Waldever, 52

Rolph, 246, 267

Rosenbloom and Sanford, 231, 239, 267

Rosenheim, 190, 361, 372

Rous, S1, 85, 95, 97, 108, 109

Rous and Jones, $10 \mathrm{~S}$

Rous and Murphy, 109

Rous, Murphy, and Tytler, 108

Ruttan and Hardisty, 205, 266

SAlomox, 245

Sanford and Rosenbloom, 231, 239, 267

Schiff and Sorenson, 138, 142, 229, $252,266,367,372$

Schoenberg, 276

Schryver and Singer, 202, 203, 228, 229,266

Senn, 423

Shutz, 356, 372

Simons and Bernstein, 348, 349

Singer and Schryver, 202, 203, 228, 229,266

Slye, 45,50

Smith, 83, 108, 480

Snow, 83, 108

Sorenson and Schiff, 138, 142, 229, $252,266,367,372$

Stockton, 344, 349
Strauss, 204, 226, 366

Sussmann, 190

Sweek, Loeb and McClurg, 499

Talledist, 115, 118

Tatham, 31, 50

Töpfer, 366

Traube, $18 S$

Tytler, Rous, and Murphy, $10 \mathrm{~S}$

UFFELMANN, 226, 242, 379

VAUGHaN, 147

Vaughan (V. C.), 497

Vaughan (W.), 497, 49S, 499

Virchow, 45, 50, 52, 108, 111, 184

Virchow and Waldeyer, 111

Waldeyer, $52,79,108,111$

Warfield, 231, 239, 240, 266, 338, 349

Wassermann, 396, 410, 499

Weil, 320, 334, 349, 496

Weil and Feldstein, 344,349

Weinstein, 231, 233, 239, 241, 266, 267

Welch, 26, 50, 9S, 100, 109, 327, 369, $372,373,381$

Whitman, 342,349

Whitman and Müller, 342, 344, 347

Williams, 31, 33, 34, 37, 44, 48, 50*

Williamson and Hall, 231, 267

Wilson, 89, 90, 91, 92, 107, 109

Wilson and MacCarty, 67, 69, 70, $71,72,73,74,75,76,109,269$, $35 \mathrm{~S}, 370,372$

Wilson and McDowell, 370

Witzel, 422, 423

Wohlgemuth, 261, 263, 267

Wolff, $24 \mathrm{~S}$

Wolff and Junghans, 117, 142, ${ }^{-143}$, $146,245,246,247,248,250$, $251,252,253,254,255,256$, $267,36 \mathrm{~S}, 39 \mathrm{~S}$

Woodyatt and Jacques, 367,372

Wooley, 371, 372

Wynhausen, 263, 267

YATES, 52, 108, 412 



\section{INDEX}

ABDERHALDEN method for detection of specific ereptases, 344

apparatus for, 345 technique of, 347

Abdominal examination, 166 catharsis before, 167 gastric lavage before, 167 position of patient for, 168 preparation of patient for, 167 inspection, 169

change of position in, 170

enlarged lymph nodes in, 172

local prominences in, 169

pelvic bones in, 169

presence of free fluid in, 169

respiratory movements, in, 170

rib margins in, 169

visible peristalsis in, 170

pain, 132, 149

palpation, 172

after inflation of stomach, 177

appearance of skin in, 173

deep, 174

diminished tension of abdominal wall in, 172

hot bath before, 173

parietes, thickness of, effect on

palpation of abdominal tumors, 183

prominences, local, 169

tumor, 154, 175

effect of gastric inflation on, 182 of respiratory movements on mobility of, 183

incidence, 175

mobility of, 179

influence of complications on demonstration of, 181 of duration of disease on demonstration of, 180

of histologic type on demonstration of, 180
Abdominal tumor, mobility of, influence of location in gastric wall on demonstration of, 181

of position of patient on demonstration of, 181

palpation of, tenderness in, 178

effect of thickness of abdominal parietes on, 183

position of, 176

relation to part of stomach involved, 177

size of, 178

wall, tumors of, gastric cancer and, differential diagnosis, 416

Abnormal proliferation of gland cells, 79

Abnormalities in gastric outline, fluoroscopic examination of, 277

Abscess of pancreas, gastric cancer and, differential diagnosis, 401

Achlorhydria, Wolff-Junghans' test in, 253

Achylia gastrica associated with gastric cancer, 47

gastric cancer and, differential diagnosis, 403

simple, Wolff-Junghans' test in, 253

malignant and benign, WolffJunghans' test in, 246

Acid salts in gastric retention extracts, 203

Acidity, 483

of fasting stomach extracts, 201

of gastric retention extracts, 225, 366

Gluzinski's method of determining, 226

of stomach after operation for gastric cancer, 466

Actinomycosis associated with gastric ulcer, 47 
After-treatment of complete gastrectomy, 459

of posterior gastro-enterostomy, 4.40

Age factor in weight loss, 165

in etiology of gastric cancer, 27 , 354

Air-pressure cabinet, Willy Meyer's, 421

Albumin, precipitible units of, 248 soluble, Wolff-Junghans' test for, 245,368

Alcohol in etiology of gastric cancer, 37

Alimentation, duodenal, 478 metagastric, 478

Altered blood, 204. See also Occult blood.

Amount of gastric retention extracts, 225

Anaphylaxis test, Ransohoff's, 348

Anemia, 125, 154, 319, 365

eanses of, 320

following gastric hemorrhage, treatment of, 493

Anemias, primary, Wolff-Jnnghans' test in, 252

Animals, experimental production of gastric eancer in, 84

Anorexia, 114, 482

Anterior gastro-enterostomy, 430 gastrojejunostomy, 429 wall of stomach, fluoroscopic examination of growth on, 281

Roentgenographic appearance of growth in, 292

Antitrypsin, 342

estimation of, in Bergmann and

Meyer's reaction, 343

Antitryptic reaction, Bergmann and Meyer's, 342

Appearance of mucous membranes, 319

of 'sclera, 319

of skin, 319

Appendix, disease of, association of gastric ulcer with, 384

dyspepsia, chronic, in duodenal ulcer, 387
Appetite, 150

in duodenal uleer, 389

in gastric cancer in young, 376

Ascites, 163

in gastric cancer, 105

incidence of, in gastric cancer, 409

Ascoli's miostagmin reaction, 348

Asiatic cholera with gastric cancer, 49

Asthma with gastric cancer, 50

Anscultation, 189

Autolysates, treatment of gastric cancer by, 496

Bacillus of Oppler-Boas, artificial culture of, 214

characteristics of, 212

cultures in beef bouillon, 216 after forty-eight hours, 220

after seventy-two hours, 221

macroscopic examination, 222 , 223

microscopic examination, 222, 223

frequency of occurrence, 224

in gastric retention extracts, 212

macroseopic examination, 215

mieroseopic examination, 215

staining properties of, 213

Bacteria in gastric cancer, 43

retention extracts, 211

Bacterium tumefaciens, 83

Basophile leucocytes in gastric cancer, 332

Beck's operation in gastric cancer, 421

Benign and malignant growths, association of, 371

gastric cancer, 51

Benzidin test for occult blood in gastric retention extracts, 205

Bergmann and Meyer's antitryptic reaction, 342

antitryptic reaction, apparatus, 343

estimation of antitrypsin, 343

teehnique of, 343 
Bile coloring in gastric retention extracts, 200

effect of, on glycyltryptophan test, 242

on tryptophan test, 242

Biliverdin in feces, 259

Bleeding, 125

Blood, 115, 154

altered, 204. See also Occult blood.

changes in gastric cancer, 321

effect of, in glycyltryptophan test, 243

in tryptophan test, 243

in feces, 259

in gastric cancer, 319

extracts, relation of WolffJunghans' test to, 255

clinical interpretation of tests for, 260

in gastric cancer in young, 379, 380

retention extracts, 204, 226

benzidin test for, 205

instances of, 206

method of determining, 205

negative test for, 261

serum, deviations from normal shown by, 333

shadows, 327

transfusion by Percy and Cook's method, 486

advantages of, 492

technique of, 489

tube used for, 487

coating of, 487

sterilization by dry heat method, 487

by steam method, 488

traumatic, in gastric retention extracts, 200

Blumer's shelf, involvement of, 184

Bright's disease in gastric cancer, 50

Brun's glucose medium for prepar-

ing sections of cancer tissue, 108

Cancer cases, registration of, 474
Cancer, gastric, 17. See also Gastric cancer

milk, 246

of stomach, 17. See also Gastric cancer.

tissue, preparing sections, Brun's glucose medium for, 108

Wilson's rapid method of cutting and staining, 107

Capillary edema, 319

Carcinoma colloides, 58, 94

fibrosum, 52, 94

gastric, 52. See also Gastric cancer. medullare, 53, 94

of pancreas, gastric cancer and, differential diagnosis, 401

of pylorus, 426

gastro-enterostomy for, 427

two-stage operation for, 427

Carcinomatous period, 150

clinical, 130

Cardia, grow ths near, diet in, 477

fluoroscopic examination of, 279

obstruction at, 100

Cardiovascular disease, Wolff-Junghans' test in, 254

Catharsis before abdominal examination, 167

Changes in leucocytes, quantitative, 327

Chemical examination of feces, 258

Chemotherapy in treatment of gastric cancer, 499

Chewing gum after operation, 465

Cholecystitis with gastric cancer, 48

Cholelithiasis with gastric cancer, 48

Chyle, effect of, on glycyltryptophan test, 242

on tryptophan test, 242

Chymification, presence of, in gastric retention extracts, 201

Cirrhosis of liver with gastric cancer, 50

gastric cancer and, differential diagnosis, 411

Classification of gastric cancer, 51 neoplasms, 51

Clinical consideration of gastric cancer, 354

symptoms, significance of, 363 
Coagulose, treatment of hemorrhage by, $4 \$ 6$

Coley's toxins, treatment of gastric cancer by, 496

Colloid gastric cancer, 58

Colon, excision of, in involvement of transverse colon in gastric cancer, 469

Color of gastric retention extracts, 200

Colored agar method for staining gastric retention extracts, 208

Combined acidity in gastric retention extracts, 226 degree of, 203

Complete gastrectomy, 456 after-treatment of, 459 drainage after, 458

Complications, general, of gastric cancer, 100

Conjugal state in etiology of gastric cancer, 35

Connell suture, 425

Constipation, 114, 125, 150, 484 chronic, gastric cancer and, differential diagnosis, 414 in gastric cancer in young, 376

Constitutional discases with gastric cancer, 50

faults, gastric cancer and, differential diagnosis, 415

Contracting gums after extraction of teeth, 475

Cook and Percy's method of blood transfusion, 486

Crystals in feces, 266

Curvature, lesser, fluoroscopic examination of growth in, 279

Cyst of pancreas, gastric cancer and, differential diagnosis, 400

Decayed teeth, 161

Diabetes with gastric eancer, 50

Diagnosis, differential, 382 early, 111

errors in, 24

Diarrhea, 114, 125, 154 green, 259

Diastase in feces, Wohlgemuth's method of determining, 261
Diet after operation for gastric cancer, 466,467

after posterior gastro-enterostomy, 441

in etiology of gastric cancer, 35,477

in growths in body of stomach, 477 near cardia, 477

in patent gastric lumen, 479

in pyloric stenosis, 478

Differential diagnosis, 382

Digestion leucocytosis, 332

previous dlisorders of, in gastric cancer, 355

Digestive disorders, 114

Dilatation of stomach, post-operative, 463,464

Diphtheria with gastric cancer, 49

Disease, duration of, 148

Drainage after complete gastrectomy, 458

after excision of pyloric cancer, 454

Drug addictions, gastric cancer and, differential diagnosis, 416

Duodenal alimentation, 478

ulcer, 387

acid dyspepsia in, 387

appetite in, 389

chronic appendix, dyspepsia in, 387

dilated stomach in, 389

epigastric pain in, 388

tenderness in, 389

eructations in, 388

facts determined upon examination in, 389

from history, 387

upon Roentgen examination in, 389

gastric cancer and, differential

diagnosis, 391

glycyltryptophan test in, 238

hematemesis in, 388

hemoglobin in, 389

hemorrhage in, 388

indurated, 360

melena in, 388

pain time in, $38 s$

periodicity of complaint, 387

plethora in, 389 
Duodenal, uleer, pyrosis in, 388 seasonal relation of complaint in, 387

sex in, 387

tryptophan test in, $23 \mathrm{~S}$

vomiting in, 388

water-brash in, $38 s$

Wolff-Junghans' test in, 253

Duration of disease, 148

influence of, on demonstration of mobility of abdominal tumor, 180

of malignant period, 132

Dyspepsia, acid, in duodenal uleer, 387

chronic laparotomy in, 472

Dysphagia, 114, 147

Eczema with gastric cancer, 50

Edema, 162 capillary, 319

Edestin test of Fuld and Levison, 228

Emptying power, gastric, investigation of, 194

Enlarged lymph nodes in abdominal inspection, 172

Enlargement of liver, 163

Enteritis in gastric cancer, 106

Eosinophiles in gastric cancer, 333

Epigastric pain, 124

in duodenal ulcer, 388

tenderness in duodenal ulcer, 389

tumor in gastric cancer in young, 378

Ereptases, specific, Abderhalden metlrod for detection of, 344

Ereptic ferment in gastric cancer, 367

Eructations, 483

in duodenal ulcer, 388

in gastric cancer in young, 378

Erysipelas with gastric cancer, 49

Erythrocytes, influence of metastases on, 322

quantitative changes in, 321

shape of, 326

size of, 326

Esophageal lesions, gastric cancer and, differential diagnosis, 392
Etiologic factors in gastric cancer, 354

Examination by fluoroscopic screen, 275

of abdomen, 166

of feces, 256. See Feces, examination of.

of gastric function, 193

by test-meal, 194

of secretory function in gastric cancer, 224

physical, significance of, 365

Roentgen, 268

Excision, complete, in gastric cancer, 418

of colon in involvement of transverse colon in gastric cancer, 469

of pyloric cancer, drainage after, 454

end of stomach, 441, 446

care of transverse colon in, 448

treatment of stumps in, 448

Exploratory laparotomy, 382

Extra-esophageal growths, circumscribed, excision of, 424

Extragastric cancer, Wolff-Junghans' test in, 252

disease association of gastric cancer with, 48

Extraglandular structures, hyperplasia of, 90

Extrapyloric cancer, 420

circumscribed, excision of, 424

FACIAL appearance, 161

Family incidence of gastric cancer, 44,45

Farmers, gastric cancer in, 31

Fasting stomach extracts, acidity of, 201

free hydrochloric acidity in, degree of, 202

method of determining, 202

Fasting stomach extracts, total acidity of, 202

determination of, 203

Feces, biliverdin in, 259

blood in, 259

crystals in, 266 
Feces, diastase in, Wohlgemuth's method of determining, 261

examination of. 256

chemical, 25s

macroscopic, 256

inicroscopic, 263

method, 264

ferment tests of, 261

hydrobilirubin in, 258

leucocytes in, 266

microscopic findings in, 265

protozoa in, 265

reaction of, 258

red blood corpuscles in, 266

tryptic digestion in, Gross-Wynhausen's method of determining, 263

undigested food in, 266

Feldstein and Weil's viscosimeter, 344

Ferment tests of feces, 261

Ferments, gastric, tests for, 227

Fetor ex ore, 161

Fibiger's rat tumors, 84

Fibrous gastric cancer, 52

Fistula in gastric cancer, 103

Fixation of stomach in gastric ulcer, 357

Flatulence, 48.5

Fluoroscopic examination in gastric carcinosis, 281

of abnormalities in gastric outline, 277

of growth at cardia, 279

high in lesser curvature, 279

in pars media, 279

in pyloric region, 280

on anterior wall, 281

on posterior wall, 281

of peristalsis, 282

palpation in, 282

screen, examination by, 275

negative findings, 276

positive findings, 276

Food desire, 125

relation of abdominal pain, 364

remnants in gastric extract, 366

Foreign bodies in large bowel, gastric cancer and, differential diagnosis, 412
Foreign bodies in stomach, gastric cancer and, differential diagnosis, 412

Formol index, 229

Free hydrochloric acidity of fasting stomach extracts, degree of, 202

nethod of determining, 202

Frequency of gastric cancer, 17 retention, 200

Freund and Kaminer test, 339

for precipitate, 341

ingredients of, 339

second, 341

technic of, 341

Frozen sections, examination of, for malignaney, 187

Fuld and Levison, edestin test of, 228

Fundus, cancer of, Roentgenographic appearance, 292

Gall-Bladder disease, gastric cancer and, differential diagnosis, 394

association of gastric ulcer with, 384

Gall-stones, 149

Gall-tract, cancer of, gastric cancer and, differential diagnosis, 394

Gastrectomy, complete, 456 operative technique for, 457

partial, gastro-enterostomy following, 459

total, prognosis after, 442

Gastric acidity in gastric cancer in young, 379 ulcer, 386

low, Wolff-Junghans' test in, 254

relation of glycyltryptophan test to, 239

of tryptophan test to, 240

cancer, 17,28

abscess of pancreas and, differential diagnosis, 401

achylia gastrica and differential diagnosis, 403 associated with, 47 
Gastric cancer, acidity of stomach after operation for, 466 actinomycosis associated with, 48

in etiology of, 27,354

alcohol in etiology of, 37

ascites in, 105

Asiatic cholera with, 49

association with other gastric disease, 46

asthma with, 50

bacteria in, 43

Beck's operation in, 421

benign type, 51

blood in, 319

changes, 321

Bright's disease with, 50

cancer of gall-tract and, differential diagnosis, 394

carcinomatous type, 52

care of oral cavity in, $47 \pm$

of teeth in, 474

causes, possible, 17-45

cholecystitis with, 48

cholelithiasis with, $4 \mathrm{~S}$

chronic constipation and, differential diagnosis, 414

cirrhosis of liver with, 50

of stomach and, differential diagnosis, 411

classification of, 51

clinical consideration, 354

differentiation from gastric cancer, 358

clinically carcinomatous period of, 130

developing in patients with years of antecedent dyspepsia of peptic ulcer type, 122

colloid type, 58

complete excision, 418

conjugal state in etiology of, 35

constitutional diseases with, 50

faults and, differential diagnosis, 416

cyst of pancreas and, differential

diagnosis, 401

decayed teeth in, 161

definition, 17
Gastric cancer, diabetes with, 50

diagnostic errors in, 24

diet in, $47 \pi$ after operation for, 466,467 in etiology of, 35

diphtheria with, 49

disease of pancreas and, differential diagnosis, 397

duodenal ulcer and, differential diagnosis, 391

early diagnosis of, 112

Roentgen examination in, 273

eczema with, 50

edema in, 162

enteritis in, 106

ereptic ferment in, 367

erysipelas with, 49

esophageal lesions and, differential diagnosis, 392

etiologic factors in, 354

etiology, possible, 17-45

excision of colon in involvement of transverse colon in, 469

experimental production in animals, 84

facial appearance in, 161

family incidence of, 44,45

fetor ex ore in, 161

fibrous type, 52

fistulæ in, 103

foreign bodies in large bowel and, differential diagnosis, 412

in stomach and, differential diagnosis, 412

gall-bladder disease and, dif-

ferential diagnosis, 394

gastric granuloma and, differential diagnosis, 411

syphilis associated with, 47

ulcer and, differential diagnosis, 391

gelatinous type, $5 \mathrm{~s}$

general complications, 100

distribution, 17

frequency, 17

glycyltryptophan test in, 238

gonorrhea with, 49

gout with, 50 
Gastric cancer, gross deviations from normal in, 52

habits in etiology of, 38

hemorrhage in, 101

heredity in etiology of, 43,474

histologic deriations from normal in, is

hygiene in etiology of, 41

in cases operated for clinically benign gastric ulcer in whom cancer was microscopically diagnosed, 113-122

in farmers, 31

in Jews, 30

in negroes, 28

in patients in whom malignancy followed periods of gastric disturbance of irregular clinical type, 149-153

who presented few clinical evidences of malignant process primary in stomach wall, 153-157

who prior to onset of malignant disease had perfect gastric health, 134-148

in young, 373

abdominal tenderness in, 378

appetite in, 376

blood in, 379

character of neoplasms in, 380

clinical data in, 376

constipation in, 376

duration of gastric complaints in, 374

epigastric tumor in, 378

eructations in, 378

gastric acidity in, 379

emptying power in, 379

incidence of, 373

laboratory data in, 379

laparotomy findings in, 380

location of malignant process in, 380

microscopic examination of unfiltered gastric extracts in, 380

"occult" blood in, 380

operative procedures in, 380

outcome of, 380
Gastric cancer, in young, pyrosis in, 378

stools in, 379

test-meals in, 379

types of histories in, 374

vomiting in, 378

weight loss in, 376

incidence of ascites in, 409

increase in, 26

indications for medical treatment in, 474

infectious diseases with, 48

involvement of transverse colon in, 443

jaundice in, 106

lack of progress in study of, 353

la grippe with, 49

liver metastases in, 105

location of, 97,368

tables of, 99

lung metastases in, 105

malaria with, 49

malignant hour-glass contraction in, 101

peritonitis and, differential diagnosis, 405

type, 51

management of symptoms in, 482

measles with, 49

medullary type, 53

morbid anatomy of, 51

mortality, general increase, 20

table of, 18-23

mucoid type, 58

mucous surfaces in, 161

mumps with, 49

nationality in etiology of, 28

nephritis in, 106

nervous complications in, 106

non-surgical treatment of, 472

nutrition in etiology of, 35

obstruction at cardia in, 100

occupation in etiology of, 31

parasitic infections with, 49

pathologic differentiation from

gastric ulcer, 358

pathology of, 370

perforation in, 101

perigastric abscess in, 102 
Gastric cancer, pneumonia after operation for, 463

precarcinomatous period in, 123, 149

preparation for operation in, 419 previous disorders of digestion in, 355

proctoclysis after operation in, 465

prognosis after operation in, 442 prophylaxis in, 472

proteid cliet in etiology of, 37 protozoal infection with, 50 protozoic infections of bowel and, differential diagnosis, 414

pyloric obstruction in, 100

quinsy with, 49

rate of growth, 95

age factor in, 96

influence of body nutrition on, 96

of location in, 97

ratio of occurrence, 22

raw food in etiology of, 33

rectal feeding in, 481

relation to gastric ulcer, 46

sarcoma of stomach and, dif-

ferential diagnosis, 412

sarcomatous type, 52

scarlet fever with, 49

secondary to extragastric malignant process, 157

to malignancy in other organs, 409

Senn's operation for, 423

seven signs of inoperability of, 186

sex in etiology of, 26

significance of gastric ulcer with respect to, 350

of history in, 354

smallpox with, 49

Smithies' percussion sign in, 188

social status in etiology of, 34

surgical treatment of, 417

symptomatology, 110

symptom-complexes of, 112

syphilis of stomach and, differential diagnosis, 409

33
Gastric cancer, thrombosis in, 106 tobacco habit in, etiology of, 38 toilet of stomach in, 476

tonsillitis with, 49

traumatism in etiology' of, 39,40

treatment of, by autolysates, 496

by chemotherapy, 499

by Coley's toxins, 496

by mesothorium, 495

by radio-active water, 495

by radium, 495

by Roentgen rays, 493

by serotherapy, 496

by vaccines, 496

by Vaughan's split-proteid vaccine, 497

tryptophan test in, 237

tuberculosis of stomach and, differential diagnosis, 410

tumors of abdominal wall and, differential diagnosis, 416

of kidney and, differential diagnosis, 402

large intestine and, differential diagnosis, 402

of liver and, differential diagnosis, 396

of omentum and, differential diagnosis, 402

of retroperitoneal tissues and, differential diagnosis, 402

of small intestine and, dif-

ferential diagnosis, 403

types of, 370

typhoid fever with, 49

ulcerating type, 54

variation in pathologic opinion as to life history of, 352

venereal disease in etiology of, 39

with malignancy primary in other organs, 48

with pleurisy, 49

with pneumonia, 49

with rheumatism, 49

with tuberculosis, $4 \bar{\pi}, 48$

with yellow fever, 49

carcinosis, fluoroscopic examination in, 281 
Gastric disease, association with gastric ulcer, 46

emptying power in gastrie eancer in young, 379

investigation of, 194 normal, time limit for, 196 physiologic method of estimating, 19.5

extract, acidity of, 366

blood in relation of WolffJunghans' test to, 255

food remnants in, 366

lactic acid in, 366

microscopic examination of, 367 occult blood in, 367

Oppler-Boas bacilli in, 367

unfiltered microscopic examination of, 380

ferments, tests for, 227

function, examination of, 193

by test-meal, 193

hemorrhage, treatment of anemia after, 493

inflation, effect of, on abdominal tumor, 182

lavage before abdominal examination, 167

lumen, patent, diet in, 479

mucosa, hyperplasia of elements of, 86

retrograde ehanges in, 93

neoplasms, elassification of, 51

location of, 97

rate of growth, 95

outline, abnormalities in, fluoro-

scopic examination of, 277

residue in gastric ulcer, 387

retention extracts, acid salts in, 203

acidity in, 225

amount of, 225

bacteria in, 211

bile coloring in, 200

chymification of, 201

color of, 200

combined acidity, 226

degree of combined acidity of, 203

of total acidity of, 203

examination of unstained preparations, 210
Gastric retention extracts, free hydrochlorie aeid in, 225

Gluzinski's method of determining, 226

lactic acid in, 226

method of determination of, 204

macroscopic study of, 200, 225

microscopic examination of, 206, 226

method of, 207

significance of, 210

mucus in, 201

"occult" blood in, 204, 226

benzidin test for, 205

instances of, 206

method of determining, 205

odor of, 201, 225

Oppler-Boas bacillus in, 211

staining, by colored agar method, 208

traumatie blood in, 200

frequency of, 200

syphilis associated with gastric cancer, 47

ulcer, 384

actinomycosis associated with, 47

alterations in gastric peristalsis

in, 387

area of tenderness in, 387

association with disease of appendix, 384

of gall-bladder, 384

clinical differentiation from gastric cancer, 358

dietetic irregularities in, 384

epigastric distress in, 384

facts determined from history of, 384

obtained by examination in, 385

secured by laboratory examination in, 385

fixation of stomach in, 387

food relief of distress in, 384

gastric eancer and, differential diagnosis, 391 
Gastric ulcer, gastric cancer, and, residue in, 387

geographic variation in incidence of, 351

glyeyltryptophan test in, 238

hemorrhage in, 385

hour-glass stomach in, 387

lyyperplasia in, 86

in young, abdominal pain in, 377

malignant ulcer in, 361

microscopic examination in, 386

pathologic differentiation from gastric cancer, $35 \mathrm{~s}$

periodicity of complaint in, 384 recurring acute infections in, 384

relation to gastric cancer, 46

Roentgen-ray findings in, 387

significance of, with respect to gastric cancer, 350

simple, Wolff-Junghans' test in, 253

stools in, 386

symptomatology, when disease is well established, 146

symptom-complexes of, 356

test-meal findings in, 385

tryptophan test in, 238

variation in aceepted clinical complex of, 353

in pathologic opinion as to life history of, 352

vomiting in, 385

Gastro-enterostomy, anterior, 430

following partial gastrectomy, 459 technique of, 460

for earcinoma of pylorus, 427

lavage after, 439

opening, location of, 428

posterior, 428

Gastrojejunostomy, 431

anterior, 429

posterior, 431, 432

Gastroscope, Sussmann, 190

Gastroscopy, 189

Gastrostomy, Witzel's technique of, 422

Gelatinous gastric eancer, 58

General appearance of patient, 160
Geographic variation in incidence of gastric ulcer, 351

Gland cells, abnormal proliferation of, 79

Gluzinski's method of letermining acidity of gastric retention extracts, 226

Glycyltryptophan test, 230, 367

effect of bile on, 242

of blood on, $2+3$

of chyle on, 24?

in duodenal uleer, 238

in gastric cancer, $23 \mathrm{~s}$

organic acid in, 241

relation of, to gastric acidity, 239

results, $234-237$

Smithies' modification of, 232

summary, 244

Gonorrhea with gastric cancer, 49

Gout with gastric cancer, 50

Granuloma, gastric eancer and, differential diagnosis, 411

Green diarrhea, 259

Gross-Wynhausen's method of determining tryptic digestion in feces, 263

Growth of gastric neoplasms, rate of, 95

Gum chewing after operation, 465

HaBITs in etiology of gastric cancer, 38

Hematemesis, 364 in duodenal ulcer, 388

Hemoglobin in duodenal ulcer, 389 influence of metastasis on, 325

quantitative changes in, 324

Hemolytic reaction, 334,367 technique of, 335

Hemorrhage, 101, 115, 147, 149, 364, 486

treatment of anemia after, 493

in duodenal ulcer, 388

in gastric ulcer, 385

"occult," 147

treatment by coagulose, 486

Heredity in etiology of gastric cancer, 43,474 
Histologic deviations from normal in gastric cancer, 58

type, influence of, on demonstration of mobility of abdominal tumor, 180

History, precancerous, 355

significance of, in gastric cancer, 354

Hot hath before abdominal palpation, 173

Hour-glass contraction, malignant, in gastric eancer, 101

in gastric ulcer, 387

Hydrobilirubin in feces, 258

Hydrochloric acid, free, in gastric retention extracts, 225

Hygiene in etiology of gastric cancer, 41

Hyperplasia in gastric ulcer, $\mathrm{S6}$ of elements of gastric mucosa, 86 of extraglandular structures, 90

INCDDExce of gastric cancer in young, 373

Index, formol, 229

peptic, 228

Indigestion, 124

Indurated duodenal ulcer, 360

Infectious diseases, gastric cancer with, 48

Ingredients of test-meal, 195

Inguinal nodes, involvement of, 184

Inspection of abdomen, 169 of patient, 160

Investigation of gastric emptying power, 194

Involvement of Blumer's shelf, 184 of inguinal nodes, 184

of liver, 186

of lymph-glands, 184

of pyloric lymph-glands, 186

of supraclavicular glands, 185

JAUNDICE, 149

in gastric cancer, 106

Jews, gastric cancer in, 30

Junghans-Wolff test for soluble albumin, 245,368

Kaminer and Freund test, 339
Kidney, tumors of, gastrie eancer and, differential diagnosis, 402

LABORATORY data in gastric cancer in young, 379

examination, facts secured by, in gastric ulcer, 385

Lactic acid in gastric retention extracts, 226, 366

method of determination, 204

La grippe with gastric cancer, 49

Laparotomy, exploratory, 382

findings in gastric cancer in young, 380

in chronic dyspepsia, 472

Large intestine, tumors of, and gastric cancer, differential diagnosis, 402

Lavage after posterior gastro-enterostomy, 439

best time for, 477

in post-operative dilatation of stomach, 465

tube, 196, 197. See also Stomach tube.

Leucocyte count, maximum, 329 minimum, 329

Leucocytes, basophile, in gastric cancer, 332

changes in, quantitative, 327

in feces, 266

polynuclear, in gastric cancer, 332 qualitative variation in, 332

transitional, in gastric cancer, 332

Leucocytosis, 329

digestion, 332

Levison and Fuld, edestin test of, 228

Liver, enlargement of, 163

involvement of, 186

metastases in gastric cancer, 105

tumors of, gastric cancer and, differential diagnosis, 396

Location of gastric cancer, 97,368 in young, 380

Loss of weight, $150,154,163$

Lungs, metastases to, in gastric cancer, 105

Lymph-glands, involvement of, 184 
Lymphocytes, large, in gastric cancer, 332

small, in gastric cancer, 332

Macroscopic examination of cultures of Oppler-Boas bacillus, $215,222,223$

of feces, 256

of gastric retention extracts, 200,225

"Magenweg," 279

Malaria with gastric cancer, 49

Malignancy primary in other organs, gastric cancer with, 48

Malignant and benign growths, association of, 371

gastric cancer, 51

period, duration of, 132

ulcer in gastric ulcer, 361

Management of symptoms in gastric cancer, 482

Measles with gastric cancer, 49

Medullary gastric cancer, 53

Melena, 364

in duodenal ulcer, $38 s$

Mental attitude factor in weight loss, 166

Mesothorium, treatment of gastric cancer by, 495

Metagastric alimentation, 478

Metastasis, external evidence of, 162 influence on erythrocytes, 322 on hemoglobin, 325

to peritoneum, 163

Meyer and Bergmann's antitryptic reaction, 342

Meyer, Willy, air-pressure cabinet of, 421

Microscopic examination in gastric ulcer, 386

of cultures of Oppler-Boas bacillus, 222,223

of feces, 263

method of, 264

of gastric retention extracts,

206,226

method of, 207

significance of, 210

of Oppler-Boas bacillus, 215

findings in feces, 265
Milk, cancer, 246

Miostagmin reaction, Ascoli's, 348

Mobility of abdominal tumor, 179

Mode of onset, in gastric cancer, 113, 136

Morbid anatomy; of gastric cancer, 51

Mortality, tables of, in gastric cancer, 18-23

Motor-meal tube, Smithies', 196, 197

Mucoid gastric cancer, 58

Mucous membranes, appearance of, 319

surfaces, 161

Mucus in gastric retention extracts, 201

Mumps with gastric cancer, 49

Murphy drip method of rectal feeding, 481

Myelocytes in gastric eancer, 332

Nationality in etiology of gastric cancer, 28

Negative test for "occult" blood, 261

Negroes, gastric cancer in, 28

Nélaton tumor, 174

Nephritis in gastric cancer, 106

Wolff-Junghans' test in, 254

Nervous complications in gastric cancer, 106

signs, 163

Non-pyloric cancers, surgical treatment of, 420

Non-surgical treatment of gastric cancer, 472

Nutrition, 115

in etiology of gastric cancer, 35

"OccolT" blood, clinical interpreta. tion of tests for, 260

in gastric cancer in young, 380 retention extracts, 204, 226, 367

benzidin test for, 205

instances of, 206

method of determining, 205

negative test for, 261

hemorrhage, 147 
Oecupation in etiology of gastric cancer, :31

O) of gastric retention extracts, 201,225

Omentum, tumors of, gastric cancer and, differential diagnosis, 402

Operation, chewing of gum after, 465 in gastric cancer, preparation for, $\$ 19$

Operative technique for gastrectomy, 457

Oppler-Boas hacillus, artificial culture of, 214

characteristics of, 212

cultures in beef bouilon, 216 after forty-eight hours, 220

after seventy-two hours, 221

macroscopic examination, 222,223

microscopic eximination, 222 , 223

frequency of occurrence, 224

in gastric extract, 367

retention extracts, 211,212

macroscopic examination, 215

microseopic examination, 215

staining properties of, 213

Oral cavity, care of, in gastric cancer, 47.4

condition, luad, weight loss from, 165

Organic acid in glyeyltryptophan test, $2 \pm 1$

in tryptoplian test, 241

PAIN, 45is

abdominal, 132, 149

food relation of, 364

in gastric cancer in young, $3 \vec{r}$ epigastric, 124

time in duodenal ulcer, 388

types of, 116,364

Palpation, abulominal, 172

in fluoroscopy, 282

Pancreas, disease of, gastric cancer and, differential diagnosis, 397

Parasitic infections with gastric cancer, 49
Pars media, fluoroseopic examination of growth in, 279

growth in, Rocntgenographic appearance of, 292

Partial gastrectomy, gastro-enterostomy following, 459

Passage of stomach tube, technique of, 199

Pathology of gastric cancer, 370

Patient, general appearance of, 160 after establishment of malignancy, 161

inspection of, 160

preparation of, for operation, 419

Pepsin, tests for, 227

Peptic index, 228

Percussion, 187

Percy and Cook's method of blood transfusion, 486

Perforation in gastric cancer, 101

Perigastric abscess in perforating gastric cancer, case, 102

Periodicity of symptoms, 363

Peristalsis, fluoroscopic examination of, 282

alterations of, in gastric ulcer, 387

visilule, in abdominal inspection, 170

Peritoneal cavity, presence of free fluid in, 185

Peritoneum, metastasis to, 163

Peritonitis, malignant, gastric cancer and, differential diagnosis, 405

Phantom tumor, 171

Physical abnormalities, 160 examination, significance of, 365

Physiologic method of estimating gastric enptying power, 195

Plethora in duodenal ulcer, 389

Pleurisy, gastric cancer with, 49

Pneumonia after operation for gastric cancer, 463 gastric cancer with, 49

Poikilocytosis, 327

Polynuclear leucocytes in gastric cancer, 332

Poor-appetite habit, weight loss from, 166 
Position of abdominal tumor, 176 relation to part of stomach involved, 177

of patient for abdominal examination, 168

Posterior-gastro-enterostomy, 428 after-treatment of, 440

diet after, 441

gastrojejunostomy, 431, 432

wall, fluoroscopic examination of growth on, 281

Roentgenographic appearance of growth in, 292

Post-operative dilatation of stomach, 463, 464

lavage for, 465

treatment, 463

Precancerous history, 355

Precarcinomatous period, 123, 149

Preparation for operation in gastric cancer, 419

of patient for abdominal examination, 167

for operation, 419

for Wolff-Junghans' test, 247

of stomach for operation, 419

Proctoclysis after operation in gastric cancer, 465

Prognosis after operation in gastric cancer, 442

after total gastrectomy, 442

Prophylaxis in gastric cancer, 472

Proteid diet in etiology of gastric cancer, 37

Protozoa in feces, 265

Protozoal infection with gastric cancer, 50

of bowel, gastric cancer and, differential diagnosis, 414

Pyloric cancer, drainage after excision of, 454

end of stomach, excision of, 441, 446

Roentgenographic appearance of growth at, 303

lymph-glands, involvement of, 186 obstruction in gastric eancer, 100 region, fluoroscopic examination of growth in, 280

stenosis, diet in, 478
Pylorus, carcinoma of, 426

Pyorrhea alveolaris, 161

Pyrosis, 483

in duodenal ulcer, $38 s$

in gastric cancer in young, 378

Qualtative variation in leucocytes, 332

Quantitative changes in erythro. cytes, 321

in hemoglobin, 324

Quinsy with gastric cancer, 49

RACE in etiology of gastric cancer, 28

Radio-active water, treatment of gastric cancer by, 495

Radium in treatment of gastric cancer, 495

Ransohoff's anaphylaxis test, 348

Rat tumors, Fibiger's, 84

Raw food in etiology of gastric cancer, 33

Reaction, antitryptic, Bergmann and Meyer's, 342

Ascoli's miostagmin, 348

glycyltryptophan, 231, 367

hemolytic, 334, 367

technique of, 335

of feces, 258

skin, 337

tryptophan, 231

Reagent, Wolff's, 248

Rectal feeding by Murphy drip method, $t 81$

in gastric eancer, 481

Red blood corpuscles in feces, 266

References to literature, 50, 108 , $266,349,371,381,416,499$

Registration of cancer cases, 474

Rennin, tests for, 227

Respiratory movements, effect of, on mobility of abdominal tumor, 183

Retrograde changes in gastric mucosa, 93

Retroperitoneal tissues, tumors of, gastric cancer and; differential diagnosis, 402

Rheumatisn, gastric cancer with, 49 
"Ring cancer," Roentgenographic appearance of, $30: 3$

Roentgen diagnosis in carly gastric cancer, 273

eximination, 268

class of cases for, $26 \mathrm{~s}$

information derived from, 287

methods of, 274

rays, treatment of gastric cancer by, 493

indications for, 494

Roentgenographic appearance of body of stomach, 292

of cancer of fundus, 292

of growth in anterior wall, 292

in pars media, 292

in posterior wall, 292

of ring cancer, 303

plates, examination by, 283

mode of procedure, 284

Roentgen-ray findings in gastric ulcer, 387

Sarcolia, gastric, 52

gastric cancer and, differential diagnosis, 412 .

Scarlet fever with gastric cancer, 49

Scirrhus, 52

Sclera, appearance of, 319

Secretory function of stomach, examination of, 224

Senn's operation for gastric cancer, 423

Serotherapy in gastric cancer, 496

Seven signs of inoperability of gastric cancer, 186

Sex in duodenal ulcer, 387

in etiology of gastric cancer, 26 , 354

Shadows, blood, 327

Shape of erythrocytes, 326

Significance of clinical symptoms, 363

of physical examination, 365

of test-meal findings, 366

Size of abdominal tumor, $17 \mathrm{~s}$ of erythrocytes, 326

Skin, appearance of, 319 in abdominal palpation, 173 reaction, 337
Small intestine, tumors of, gastric cancer and, differential diagnosis, 403

Smallpox with gastric cancer, 49

Smithies' colored agar staining method, 208

method of examination of gastric retention extracts, 210

modification of glycyltryptoplian test, 232

of tryptophan test, 233

motor-meal and lavage tube, 196, 197

percussion sign in cancer of fundus of stomach, $18 \mathrm{~S}$

Social status in etiology of gastric cancer, 34

Soluble albumin, Wolff-Junghans' test for, 245,368

Special tests, 367

Splenic enlargement, gastric cancer and, differential diagnosis, 402

Split-proteid vaccine, Vaughan's, for treatment of gastric cancer, 497

Staining of gastric retention extracts, by colored agar method, $20 \mathrm{~S}$

Stomach, eancer of, 17. See also Gastric cancer.

diet in growths in body of, $4 \pi 7$

dilated, in duodenal ulcer, 389

examination of secretory function of, 224

excision of pyloric end of, 446

post-operative dilatation of, 463 , 464

preparation of, for operation, 419

testing emptying power of, 275

toilet of, in gastric cancer, 476

tube, Smithies', 196, 197

advantages of, 198

description of, 197

faults of ordinary, 197

technique for passage of, 199

Stools, examination of, 256. See also Feces, examination of.

in gastric cancer in young, $\mathbf{3 7} 9$ ulcer, 386

Strength of patients, 115

Supraclavicular glands, involvement of, 185 
Surgical considerations, 368

treatment of gastric cancer, 417 of non-pyloric cancers, 420

Sussmann's gastroscope, 190

Symptomatology of gastric cancer, 110

Symptom-complexes of gastric cancer, 112

of gastric ulcer, 356

Symptoms, clinical, significance, 363 periodicity of, 363

Syphilis, gastric, Wolff-Junghans' test in, 252

gastric cancer and, differential diagnosis, 409

TECHNIQUE of blood transfusion, 489

of gastro-enterostomy following partial gastrectomy, 460

Teeth, care of, in gastric cancer, 474 decayed, 161

extraction of, contracting gums after, 475

in etiology of gastric cancer, 42

Temperature, 147

Tenderness, abdominal, in gastric cancer in young, 378

area of, in gastric ulcer, 387

of palpable abclominal tumors, 178

Test, edestin of Fuld and Levison, 228

glycyltryptophan, 230,244

results, 234-237

Smithies' modification, 232

Kaminer and Freund, 339

Ransohoff's anaphylaxis, 348

tryptophan, 231

results, $23 \pm-237$

Smithies' modification, 233

summary, 244

typical, 234

Test-meal examination of gastric function, 193

findings, 117

comparison of, with WolffJunghans' test, 251

in gastric ulcer, 385

significance of, 366

ingredients of, 195

in gastric cancer in young, 379

Tests, special, 367
Thrombosis in gastric cancer, 106

Tobacco habit in etiology of gastric cancer, 38

Tonsillitis with gastric cancer, 49

Total acidity, degree of, in gastric retention extracts, 203

of fasting stomach contents, 202 determination of, 203

Transfusion of blood, Percy and Cook's method, 486

Transitional leucocytes in gastric cancer, 332

Transverse colon, care of, in excision of pyloric end of stomach, 448

involvement of, in gastric cancer, 443

excision of colon in, 469

Traumatism in etiology of gastric cancer, 39,40

Treatment of anemia after gastric hemorrhage, 493

of gastric cancer by autolysates, 496

by chemotherapy, 499

by Coley's toxins, 496

by mesothorium, 495

by radio-active water, 495

by radium, 495

by Roentgen rays, 493

by vaccines, 496

medical, indications for, 474

non-surgical, 472

post-operative, 463

surgical, 417

Tryptic digestion in feces, GrossWynhausen's method of determining, 263

Tryptophan test, 231

effect of bile on, 242

of blood on, 243

on chyle, 242

in duodenal ulcer, 238

in gastric cancer, 237

ulcer, 238

organic acid in, 241

relation of, to gastric acidity, 240

results, $23 \pm-237$

Smithies' modification, 233

summary, 244 
Tryptophan test, typical, 234

Tube for blood transfusion, 487

for gastric larage, Snithies', 196, 197

Tuberculosis associated with gastric: eancer, 47,48

of stomach, gastric cancer and, differential cliagnosis, 410

Tumor, abdominal, 175

Nélaton, 174

phantom, 171

Tumors of liver, gastric cancer and, differential diagnosis, 396

Two-stage operation for carcinoma of pylorus, 427

Types of gastric cancer, 370 of pain, 364

Typhoid fever with gastric eancer, 49

Ulcer, duodenal, 387. See also Duodenal ulcer.

Ulcerating gastric cancer, 54

Ulcus carcinomatosum, 54, 94, 361

Wolff-Junghans' test in, 250

Uncolored preparations of gastric retention extracts, examination of, by Smithies' method, 210

Uncooked vegetables, danger from, 480

Undigested food in feces, 266

Urine, 148

VACCINE treatment of gastric cancer, 496

Vaughan's split-proteid vaccine for treatment of gastric cancer, 497

Venereal disease in etiology of gastric cancer, 39

Viscosimeter, Weil and Feldstein's, 344

Vomiting, in duodenal ulcer, 388

in gastric cancer, 115, 125, 133, $149,365,482$

in young, 378

ulcer, 385

weight loss from, 165

WATER-BRASH, 114, 149

in duodenal ulcer, 388

Weight loss, 125, 148, 150, 154, 163, 164

age factor in, 165
Weight loss, factor of mental attitude in, 166

from had oral conditions, 165

from poor-appetite habit, 166

from undereating, 165

from vomiting, 165

in gastric cancer in young, 376

Weil and Feldstein's viscosimeter, 344

Wilson's rapid methor of cutting and staining cancer tissue, 107

Witzel's gastrostomy, technique of, 422

Wohlgemuth's method of determining diastase in feces, 261

Wolff-Junghans' test, comparison of other test-meal findings, with, 251

for soluble albumin, 245, 368

in achlorhydria, 253

in cardiovascular disease, 254

in duodenal ulcer, 253

in extragastric cancer, 252

in gastric syphilis, 252

in low gastric acidity, 254

in malignant and benign achylias, 246

in nephritis, 254

in primary anemias, 252

in simple achylia gastrica, 253 gastric ulcer, 253

in ulcus carcinomalosum, 250

interpretation of, 248

manifestation of, 248

manifestations of, relation to location of malignant process, 250

mode of procedure, 248

preparation of patient for, 247

relation of, to presence of blood in gastric extracts, 255

results of, 249

Wolff's reagent, 248

Wynhausen-Gross' method of determining tryptic digestion in feces, 263

X-RAY examination, 268. See also Roentgen examination.

Yellow fever with gastric cancer, 49

Young, gastric cancer in, 373 




\section{SAUNDERS' BOOKS}

\section{on \\ Nervous and Mental \\ Diseases, Children, \\ Hygiene, Nursing, and \\ Medical Jurisprudence}

\section{W. B. SAUNDERS COMPANY WEST WASHINGTON SQUARE PHILADELPHIA}

9. HENRIETTA STREET COVENT GARDEN, LONDON

\section{Dercum's Mental Diseases}

Clinical Manual of Mental Diseases. By Francis X. Dercun, Pн. D., M. D., Professor of Nervous and Mental Diseases at Jefferson Medical College, Philadelphia. Octavo of 425 pages. Cloth, $\$ 3.00$ net.

\section{TWO PRINTINGS IN FIVE MONTHS}

This is a book really useful to the family physician-a book that tells you definitely how to diagnose, how to treat-either at home or in an institution-all classes of mental diseases. First, Dr. Dercum takes up the various primary forms of mental disease, giving emphasis to those you meet in your daily practice as general practitioner-delirium, confusion, stupor. Then melancholia, mania, the insanities of early life, paranoia, the neurasthenic-neuropathic disorders, and the dementias follow. The mental disturbances of the infections (syphilis, tuberculosis, malaria, pellagra, rheumatic fever, etc.), the various forms of intoxicational insanities, those due to metabolic disorders, visceral disease, diseases of the nervous system are all given you-and from your viewpoint. An important section is that devoted to the insanities of pregnancy. An entire part is devoted to the psychologic interpretations of symptoms as evolved by Freud and his disciples. You get a full discussion of the rôle of dreams.

\section{The Medical World}

"This book gives just the information necessary, and gives it in a style studiously adapted to the needs of the general physician." 


\section{Church and Peterson's Nervous and Mental Diseases}

Nervous and Mental Diseases. By Archibald Church, M. D., Projessor of Nervous and Mental Diseases and Medical Jurisprudence, Northwestern University Medical School, Chicago; and Frederick Petersox, M. D., formerly Professor of Psychiatry at the College of Physicians and Surgeons, N. Y. Handsome octavo, 944 pages; 350 illustrations. Cloth, \$5.00 net; Half Morocco, \$6.50 net.

\section{THE NEW (8th) EDITION}

For this new (8th) edition this standard work has undergone a thorough revision. Vertigo and its labyrinthine relations, as developed by Bárány, has received careful consideration; much new matter has been added to the section on Infantile Paralysis; syphilis of the nervous system has been brought into accord with recent epoch-making discoveries. Throughout, references to the new investigations of the spinal fluid, and the relation of spinal fluid changes to the various organic diseases of the brain and cord have been introduced. The bearing of internal secretion upon nervous disorders has been brought right down to date. Tetany has been given its place among nervous diseases associated with glandular disorder. Altogether over 300 interpolations and alterations have been made. It is more than ever the stimlard.

\section{American Journal of the Medical Sciences}

" This edition has been revised, new illustrations added, and some new matter, and really is two books. . . The descriptions of disease are clear, directions as to treatment definite, and disputed matters and theories are omilled. Allogether it is a most useful text-book."

\section{Kaplan's Serology of Nervous and Mental Diseases}

Serology of Nervous and Mental Diseases. By D. M. KAPLAN, M. D., Director of Clinical and Research Laboratories, Neurological Institute, New York City. Octavo of 346 pages, illustrated. Cloth, \$3.jo net.

This is an entirely new work, giving you the indications, contra-indications, preparation of patients, technic, after-phenomena, after-care, and disposal of the fluids obtained by lumbar puncture. You get a full discussion of the serology of all nervous and mental diseases of non-luetic etiology (including disorders of internal secretion), and of every type of luetic nervous and mental disease, giving the IIassemann reaction in detail, the use of salvarsan and neosaivarsan, etc. 


\section{Herrick's Neurology}

Introduction to Neurology. By C. Judson Herrick, Ph. D., Professor of Neurology in the University of Chicago. $12 \mathrm{mo}$ of 360 pages, illustrated.

\section{JUST READY}

Professor Herrick's new work will aid the student to organize his knowledge and appreciate the significance of the nervous system as a mechanism right at the beginning of his study. It is sufficiently elementary to be used by students of elementary psychology in colleges and normal schools, by students of general zoölogy and comparative anatomy in college classes, and by medical students as a guide and key to the interpretation of the larger works on neurology.

\section{Brill's Psychanalysis}

Second Edition

Psychanalysis: Its Theories and Practical Application. By A. A. Brill, Ph. B., M. D., Clinical Assistant in Neurology at Columbia University Medical School. Octavo of 392 pages. "Cloth, \$3.00 net.

To the general practitioner, who first sees these "borderline" cases (the neuroses and the psychoses), as well as to those specially interested in neurologic work, Dr. Brill's work will prove most valuable. Dr. Brill has had wide clinical experience, both in America and in Europe. The results of this experience you get in this book. Here you get the practical application of all Freud's theoriesand from the pen of a man thoroughly competent to write.

\section{Hunt's Diagnostic Symptoms of Nervous Diseases}

Diagnostic Symptoms of Nervous Diseases. By EDward L. Hust, M. D., formerly Instructor in Neurology and Assistant Chief of Clinic, College of Physicians and Surgeons, New York. I I mo of 229 pages, illustrated. Cloth, \$I.50 net.

\section{Stiles on the Nervous System}

The Nervous System and its Conservation. By Percy G. Stiles, Instructor in Physiology at Harvard University. I 2 mo of 230 pages, illustrated. Cloth, \$I.25 net. 


\section{American Illustrated Dictionary}

Just Out-New (8th) Edition-1500 New Words

The American Illustrated Medical Dictionary. A new and complete dictionary of the terms used in Medicine, Surgery; Dentistry, Pharmacy, Chemistry, Veterinary Science, Nursing, and kindred branches; with over 100 new and elaborate tables and many handsome illustrations. By IV. A. Newanan Dorlaxd, M. D. Large octavo, I 37 pages, bound in full flexible leather, $\$ 4.50$ net; with thumb index, $\$ 5.00$ net.

The American Illustrated Medical Dictionary defines hundreds of terms not defined in any other dictionary-bar none. It gives the capitalization and pro. nunciation of all words. It makes a feature of the derivation or etymology of the words. Every word has a separate paragraph, thus making it easy to find a word quickly. The tables of arteries, muscles, nerves, veins, etc., are of the greatest help in assembling anatomic facts. Every word is given its definition-a definition that defines in the fewest possible words.

Howard A. Kelly, M. D., Johns Hopkins Unizersity, Baltimore.

"The American Illustrated Dictionary is admirable. 1t is so well gotten up and of such convenient size. No errors have been found in my use of it."

\section{Goodnow's First-Year Nursing'}

First=Year Nursing. By Misie Goodsow, R. N., formerly Superintendent of the Womer's Hospital, Denver. I 2 mo of $32 S$ pages, illustrated. Cloth, \$1.50 net. Miss Goodnow's work deals entirely with the practical side of first-year nursing work. It is the application of text-book knowledge. It tells the nurse how to do those things she is called upon to do in her first year in the training school-the actual ward work.

\section{Roberts' Bacteriology and Pathology for Nurses}

Bacteriology and Pathology for Nurses. By JAY G. RoBerTs, PH. G., M. D., Oskaloosa, Iowa. 12 mo of 206 pages, illustrated. Cloth, $\$ 1.25$ net.

This new work is practical in the strictest sense. Written specially for nurses, it confines itself to information that the nurse should know. All unessential matter is excluded. "The style is concise and to the point, yet clear and plain. The text is illustrated throughout. 


\section{Kerr's Diagnostics of Diseases of Children}

Diagnostics of the Diseases of Children. By LeGrand Kerr, M. D., Professor of Diseases of Children, Brooklyn Postgraduate Medical School, Brooklyn. Octavo of 542 pages, fully illustrated. Cloth, $\$ 5.00$ net; Half Morocco, $\$ 6.50$ net.

\section{FOR THE PRACTITIONER}

Dr. Kerr's work differs from all others on the diagnosis of diseases of children in that the objective symptoms are particularly emphasized. The constant aim throughout has been to render a correct diagnosis as early in the course of the disease as possible, and for this reason differential diagnosis is presented from the very earliest symptoms. The many original illustrations will be found helpful.

\section{New York State Journal of Medicine}

"The illustrations are excellent and numerous. It will meet the needs of the great mass of physicians who treat the diseases of infancy and childhood."

\section{Kerley's Pediatrics}

Practice of Pediatrics. By Charles Gilmore Kerlex, M. D., Professor of Diseases of Children, New York Polyclinic Medical School and Hospital. Octavo of 878 pages, illustrated. Cloth, $\$ 6.00$ net; Half Morocco, $\$ 7.50$ net,

\section{FOURTH LARGE PRINTING}

This work is not a cut-and-dried treatise-but the practice of pediatrics, giving fullest attention to diagnosis and treatment. The chapters on the newborn and its diseases, the feeding and growth of the baby, the care of the mother's breasts, artificial feeding, milk modification and sterilization, diet for older children-from a monograph of 125 pages. Then are discussed in detail every disease of childhood, telling just what measures should be instituted, what drugs given, bo valuable prescriptions being included. The chapter on vaccine therapy is right down to the minute, including every new method of proved value-with the exact technic. There is an excellent chapter on Gymnastic Therapeutics. Another feature consists of the 165 illustrative cases-case teaching of the most practical sort.

Dr. A. D. Blackader, MCGill University, Montreal

"Dr. Kerley is a pediatrician of large experience who thinks for himself and is never content to accept, without testing, the experiences or statements of previous writers. His book has a very definite value." 


\section{Sanders' Nursing}

Modern Methods in Nursing. By Georglaxa J. Sanders, formerly Superintendent of Nurses at the Massachusetts General Hospital. I 2 mo of SSI pages, with 227 illustrations. Cloth, $\$ 2.50$ net.

\section{THE BEST YET}

Niss Sanders' book gives only modern methods. Then it gives the details of nursing opiration cases, both in the hospital and in the home. The thorough way in which ward work is taken up makes her book indispensable for teaching purposes. In giving directions for mustard baths, poultices, etc., the quantities are given exactly. This is an important point often overlooked.

\section{Stoney's Nursing}

Practical Points in Nursing. By Emily A. M. Stoner. I 2 mo of 495 pages, illustrated. Cloth, \$1.75 net.

\section{THE NEW (4th) EDITION}

In this volume the author explains the entire range of prizate nursing as distinguished from hospital nursing, and the nurse is instructed how best to meet the various emergencies of medical and surgical cases when distant from medical or surgical aid or when thrown on her own resources. An especially valuable feature will be found in the directions how to improzise everything ordinarily needed in the sick-room.

\section{Stoney's Technic for Nurses}

Bacteriology and Surgical Technic for Nurses. By Emily A. M. Stover, formerly Superintendent at Carney Hospital, South Boston. Revised by Frederic R. Griffith, M. D., Surgeon, of New York. I $2 \mathrm{mo}, 3$ I I pages, illustrated. Cloth, \$I.50 net.

\section{Trained Nurse and Hospital Review}

\section{THE NEW 3d) EDITION}

"These subjects are treated most accurately and up to date, without the superfluous reading which is so often employed. . . Nurses will find this book of the greatest value both durin€ their hospital course and in private practice." 


\section{Occupation Therapy for Nurses}

Occupation Therapy: A Manual for Nurses. By WM. Rush DuNTon, Jr., M. D., Assistant Physician at Sheppard and Enoch Pratt Hospitals, Towson, Md. I2mo of 250 pages, illustrated.

\section{JUST READY}

This work treats of a very important subject. It has chapters on hobbies, psychology of occupation, mechanism of recovery by occupation, puzzles, reading, physical exercises, card games, string, paper, wood, and metal work, weaving, picture puzzles, basketry, chair-caning, bookbinding, collecting, gardening, nature study, stenciling and block painting, plastic work, drawing, pyrography, photography, needlework, music. The text is illustrated.

\section{Stoney's Materia Medica for Nurses}

Practical Materia Medica for Nurses, with an Appendix containing Poisons and their Antidotes, with Poison-Emergencies; Mineral Waters; Weights and Measures; Dose-List, and a Glossary of the Terms used in Materia Medica and Therapeutics. By Emily A. M. Stovey, for merly of the Carney Hospital, South Boston. 12mo of 300 pages, Cloth, \$I.5O net.

\section{THE NEW (3d) EDITION}

In making the revision for this new third edition, all the newer drugs have been introduced and fully discussed. The consideration of the drugs includes their sources and composition, their various preparations, physiologic actions. directions for administering, and the symptoms and treatment of poisoning.

\section{Journal of the American Medical Association}

"So far as we can see, it contains everything that a nurse ought to know in regard to drugs. As a reference-book for nurses it will without question be very useful."

\section{Nursing in Diseases of the Eye, Ear, Nose, and Throat}

Nursing in Diseases of the Eye, Ear, Nose, and Throat. By the Committee on Nurses of the Manhattan Fye, Ear, and Throat Hospital. $12 \mathrm{mo}$ of 29 I pages, illustrated.

Cloth, \$r.50 net.

\section{JUST OUT-NEW (2d) EDITION}

This is a practical book, prepared by physicians who, from their experience in the operating amphitheater and at the bedside, have realized the shortcomings of present nursing books in regard to eye, ear, nose, and throat nursing. The scope of the work has been limited to what an intelligent nurse should know, and the style throughout is simple, plain, and definite. 


\section{Hoxie and Laptad's Medicine for Nurses}

New (2d) Edition

Medicine for Nurses and Housemothers. By GEORge Howard Hoxie, M. D., l'hysician to the German Hospital, Kansas City, Mo.; and PEarL L. LAPTAD, formerly Principal of the Training School for Nurses, University of Kansas. I $2 m 0$ of $35^{1}$ pages, illustrated.

Cloth, $\$ 1.50$ net.

This work is truly a practice of medicine for the nurse, enabling her to recognize any signs and changes that may occur between visits of the physician, and, if necessary, to combat them until the physician's arrival. This information the author presents in a way most acceptable, particularly emphasizing the nurse's part.

Trained Nurse and Hospital Review

"This book has our unqualified approval."

\section{McCombs' Diseases of Children for Nurses $_{\text {New (2d) Edition }}$}

Diseases of Children for Nurses. By Robert S. MCCombs, M. D., Instructor of Nurses at the Children's Hospital of Philadelphia. I 2 mo of 470 pages, illustrated. Cloth, $\$ 2.00$ net.

Dr. McCombs' experience in lecturing to nurses has enabled him to emphasize just those points that nurses most need to know. The nursing side has been written by head nurses, especially praiseworthy being the work of Miss Jennie Manly.

\section{National Hospital Record}

"We have needed a good work on children's diseases adapted for nurses' use, and this volume admirably fills the want."

\section{Wilson's Obstetric Nursing}

The New (2d) Edition

A Reference Hand=Book of Otstetric Nursing. By W. REYNoLDS WILsox, M. D., Visiting Physician to the Philadelphia Lying-in Charity. 321 o of $25^{6}$ pages, illustrated. Flevible leather, $\$ 1.25$ net.

Dr. Wilson's work discusses the subject of obstetrics entirely from the nurse's point of view, presenting in detail everything connected with pregnancy and labor and their management. The text is copiously illustrated.

American Journal of Obstetrics

"Every page emphasizes the nurse's relation to the case."

\section{Frühwald and Westcott on Children}

Diseases of Children. A Practical Reference Book for Students and Practitioners. by Professor Dr. Ferdinand Frühwald, of Vienna. Edited, with additions, by Thompson S. Westcotr, M. D., University of Pennsyivania. Octavo, 533 pages, I 76 illustrations. Cloth, $\$ 4.50$ net.

\section{Boyd's State Registration for Nurses New (2d) Edition}

State Registration for Nurses. By Lovie Croft Boyd, R. N., Graduate Colorado Training-school for Nurses. Octavo of I49 pages. \$1.25 net. 
Aikens' Primary Studies for Nurses

New (3d) Edition

Primary Studies for Nurses: A Text-Book for First-year Pupil Nurses. By Charlotte A. Aikens, formerly Director of Sibley Memorial Hospital, Washington, D. C. I 2 mo of 47 I pages, illus. Cloth, \$1.75 net.

This work brings together in concise form well-rounded courses of lessons in all subjects which, with practical nursing technic, constitute the primary studies in a nursing course.

\section{Trained Nurse and Hospital Review}

"It is safe to say that any pupil who has mastered even the major portion of this work would be one of the best prepared first-year pupils that ever stood for examination."

\section{Aikens' Clinical Studies for Nurses}

New (2d) Edition

Clinical Studies for Nurses. By Charlotte A. Aikens, formerly Director of Sibley Memorial Hospital, Washington, D. C. I 2 mo of 569 pages, illustrated. Cloth, $\$ 2.00$ net.

This new work is written along the same lines as Miss Aikens' former work on "Primary Studies," to which it is a companion volume. It takes up all subjects taught during the second and third years and takes them up in a concise, forceful way.

\section{Dietetic and Hygienic Gazette}

"There is a large amount of practical information in this book which the experienced nurse, as well as the undergraduate, will consult with profit. The illustrations are numerous and well selected."

\section{Aikens' Training-School Methods}

Hospital Training-School Methods and the Head Nurse. By Charlotte A. Aikens, formerly Director of Sibley Memorial Hospital, Washington, D. C. I 2 mo of 267 pages. Cloth, \$I.50 net.

\section{Trained Nurse and Hospital Review}

"There is not a chapter in the book that does not contain valuable suggestions."

\section{Aikens' Hospital Management}

Extremely Practical

Hospital Management. By Charlotte A. Aikens, formerly Director of Sibley Memorial Hospital, Washington, D. C. I 2 mo of 488 pages, illustrated. Cloth, $\$ 3.00$ net.

\section{The Medical Record}

"Tells in concise form exactly what a hospital should do and how it should be run, from the scrubwoman up to its financing. A valuable addition to our literature on this subject." 


\section{Bolduan and Grund's Bacteriology for Nurses}

Appleied Bacteriolor; for Norses. By Charles F. Bolduax, M. D., Assistant to the General Medical Officer; and MArie Gruxd, MI. D.. Bacteriologist, Research Laloratory, Department of Health, New York Citr. $12 \mathrm{mo}$ of I 55 pages, illustrated. Cloth, \$1.25 net.

We were fortunate in getting these practical physicians to write this work. It gives particular cmplasis to the immediate application of bacteriology to nursing, only the really practical heing included. A study of all the modes of infection transmission is presented. At the encl of each chapter are suggestions for practical demonstration.

\section{Register's Fever Nursing}

A Text-Book on Practical Fever Nursing. By Edward C. Regrster, M. I., Professor of the Practice of Medicine in the North Carolina Medical College. I 2 mo of 352 pages. Cloth, \$2.50 net.

\section{Hecker, Trumpp, and Abt on Children}

Atlas añd Fitome of Diseases of Childrex. By Dr. R. Hecker and Dr. J. TRLipP, of Munich. Edited, with additions, by ISAAC A. AzT, M.D., Assistant Professor of Diseases of Children, Rush Medical College, Chicago. With 48 colored plates, 144 text-cuts, and 453 pages of text. Cloth, $\$ 5.00$ net.

The many excellent lithographic plates represent cases seen in the authors' clinics, and have been selected with great care, keeping constantly in mind the practical needs of the general practitioner. These beautiful pictures are so true to nature that their study is equivilent to actual clinical observation. The editor, Dr. Isaac A. Abt, has added all new methols of treatment.

\section{Lewis Anatomy and Physiology}

The New (3d) Edition

Axatony and Phrsiologr for Nurses. By I. Roy Lewis, M. D. Formerly Surgeon to and Lecturer on Anatomy and Physiology for Nurses at the Lewis Hospital, Bay City, Michigan. I 2 mo of $3++$ pages, with i6 i illustrations. Cloth, \$1.75 net.

A demand for such a work as this, treating the subjects from the nurses point of view, has long existed. Dr. L'wis has based the plan and scope of this work on the methods employed by him in teaching these branches, making the text unusually simple and clear.

The Nurses Journal of the Pacific Coast

"It is not in any sense rudimentary, but comprehensive in its treatment of the subjects in hand. The application of the knowledge of anatomy in the care of the patient is emphasized."

\section{Friedenwald and Ruhräh's Dietetics}

New (3d) Edition

Dietetics for Nurses. By Julius Friedenwald, M. D., Professor of Diseases of the Stomach, and John RUhr ̈̈H, M. D., Professor of Diseases of Children, College of Physicians and Surgeons, Baltimore. I 2 mo volume of 43 I pages. Cloth, \$I.50 net.

This work has been prepared to meet the needs of the nurse, both in the training school and after graduation, It aims to give the essentials of dietetics, considering briefly the physiology of digestion and the various classes of foods and the part they play in nutrition.

\section{American Journal of Nursing}

"It is exactly the book for which nurses and others have long and vainly sought. A simple manual of dietetics, which does not turn into a cook-book at the end of the first or second chapter. 


\section{Paul's Fever Nursing}

Nursing in the Acute Infectious Fevers. By George P. Paul, M. D., formerly Assistant Visiting Physician to the Samaritan Hospital, Troy, N. Y. I 2 mo of 246 pages. Cloth, \$1.00 net.

Dr. Paul has taken great pains in the presentation of the care and management of each fever. The book treats of tevers in general, then each fever is discussed individually, and the latter part of the book deals with practical procedures and valuable information.

\section{The London Lancet}

"The book is an excelient one and will be of value to those for whom it intended, It is well arranged, the text is clear and full, and the illustrations are good."

\section{Paul's Materia Medica for Nurses}

New (2d) Edition

Materia Medica for Nurses. By George P. Paul, M. D., formerly Assistant Visiting Physician to the Sarnaritan Hospital, Troy. I 2 mo o: 282 pages. Cloth, $\$ 1.50$ net.

Dr. Paul arranges the physiologic actions of the drugs according to the action of the drug and not the organ acted upon. An important section is that on pretoxic signs, giving the warnings of the full action or the beginning toxic effects of the drug, which, if heeded, may prevent many cases of drug poisoning.

The Medical Record, New York

"This volume will be of real help to nurses; the material is well selected and well arranged, and the book is as readable as it is useful."

\section{Pyle's Personal Hygiene}

The New (6th) Edition

A Manual of Personal Hygiene: Proper Living upon a Physiologic Basis. By Eminent Specialists. Edited by Walter L. Pyle, A. M., M.D., Assistant Surgeon to Wills Eye Hospital, Philadelphia. Octaro volume of $54 \mathrm{I}$ pages, fully illustrated. Cloth, $\$ \mathbf{1} .50$ net.

The book has been thoroughly revised for this new edition, and a new chapter on Food Adulteration by Dr. Harey $I I$. Wiley added. There are important chapters on Domestic Hygiene and Home Gymmastics, Hydrotherapy, Mechanotherapy, and First Aid Measures.

\section{Boston Medical and Surgical Journal}

"The work has been excellently done, there is no undue repetition, and the writers have succeeded unusually well in presenting facts of practical significance based on sound knowledge."

\section{Galbraith's Four Epochs of Woman's Life Second Edition}

The Four Epochs of Woman's Life. By Ansa M. Galbraith, M.D. With an Introductory Note by John H. Musser, M. D., University of Pennsylvania. I 2 mo of 247 pages. Cloth, \$I.50 net.

\section{Birmingham Medical Review}

"Ne do not as a rule care for medical books written for the instruction of the public; but we must admit that the advice in Dr. Galbraith's work is in the main wise and wholesome."

\section{Spratling on Epilepsy}

Epilepsy and Its Treatment. By William P. Spratling, M. D., Professor of Physiology and Nervous Diseases, College of Physicians and Surgeons, Baltimore. Octavo of 522 pages, fully illustrated. Cloth, $\$ 4.00$ net.

The Lancet, London

"Dr. Spratling's work is written throughout in a clear and readable style.,. . The work is a mine of information on the whole subject of epilepsy and its treatment.' 


\section{Macfarlane's Gynecology for Nurses}

New (2d) Edition

A Rliference Hand-Book of Gyecology for Nurses. By Catharine Macfarlane, M. I)., Gynecologist to the Woman's Hospital of Philadelphia. $16 \mathrm{mo}$ of 150 pages, with 70 illustrations. Flexible leather, \$1.25 net.

A. M. Seabrook, M. D., Womat's Medical College of Philadelphia.

"It is a most admirable little book, covering in a concise but attractive way the subject from the nurse's standpoint."

\section{Galbraith's Personal Hygiene for Women}

Personal Hygiene and Physical Training for Women. By Anva M. Galbraith, M.D., Fellow New York Academy of Medicine, I $2 \mathrm{mo}$ of $37 \mathrm{I}$ pages, with original illustrations. Cloth, $\$ 2.00$ net.

Dietetic and Hygienic Gazette

"It contains just the sort of information which is very greatly needed by the weaker sex. Its illus. trations are excellent."

\section{De Lee's Obstetrics for Nurses}

Obstetrics for Nurses. By Joseph B. De Lee, M. D., Professor of Obstetrics in the Northwestern University Medical School. I 2 mo volume of 508 pages, fully illustrated. Cloth, $\$ 2.50$ net.

J. Clifton Edgar, M. D.,

Professor of Obstetrics and Clinical Midwifery, Cornell Medical School, N. Y.

"It is far-and-away the best that has come to my notice, and I shall take great pleasure in recommending it to my nurses and students as well."

\section{Davis' Obstetric Nursing}

New (4th) Edition

Obstetric and Gynecologic Nursing. By Edward P. Davis, A. M., M. D., Professor of Obstetrics, Jefferson Medical College and Philadelphia Polyclinic. I 2 mo of 480 pages, illustrated. Buckram, \$I.75 net.

The Lancet, London

" Not only nurses, but even new]y qualified medical men, would learn a great deal by a perusal of this book. It is written in a clear and pleasant style, and is a work we can recommend."

\section{Beck's Hand-Book for Nurses}

New (2d) Edition

A Reference Haxd-Book for Nurses. By Amanda K. Beck, of Chicago, Ill. $32 \mathrm{mo}$ of 200 pages. Flexible leather, $\$ 1.25$ net.

\section{Aikens' Home Nurse's Hand-Book}

Hone Nurse's Haxd-Book. By Charlotte A. Aikexs. I 2 mo of 276 pages, illustrated.

Cloth, \$I.50 net.

The point about this work is this: It tells you and shows you just how to do those little but important things often omitted from other nursing books. "Home Treatments" and "Points to be Remembered "- ter:e, criso reminders-stand out as particularly practical. Just the book for those who have the home-care of the sick. 


\section{Griffith's Care of the Baby}

The Care of the Baby. By J. P. Crozer Griffith, M. D., Professor of Pediatrics in the University of Pennsyivania. I 2 mo of 455 pages, illustrated. Cloth, \$I.5O net.

\section{THE NEW (6th) EDITION}

The author has endeavored to furnish a reliable guide for mothers. He has made his statements plain and easily understood, so that the volume will be of service to mothers and nurses.

\section{New York Medical Journal}

"We are confident if this ltttle work could find its way into the hands of every trained nurse and of every mother, infant mortality would be lessened by at least fifty per cent."

\section{Grulee's Infant Feeding}

Infant Feeding. By Clifford G. Grulee, M. D., Assistant Professor of Pediatrics at Rush Medical College. Octavo of 316 pages, illustrated, including 8 in colors. Cloth, \$3.00 net.

\section{NEW (2d) EDITION}

Dr. Grulee tells you how to feed the infant. He tells you--and show's by clear illustrations-the technic of giving the child the breast. Then artificial feeding is thoughtfully presented, including a number of simple formulas. The colored illustrations showing the actual shapes and appearances of stools are extremely valuable.

\section{Ruhräh's Diseases of Children}

A Manual of Diseases of Children. By John Ruhräh, M. D., Professor of Diseases of Children, College of Physicians and Surgeons, Baltimore. I $2 \mathrm{mo}$ of $55^{2}$ pages, fully illustrated. Flexible leather, $\$ 2.50$ net.

\section{THE NEW (4th) EDITION}

In revising this work for the fourth edition Dr. Ruhräh has carefully incorporated all the latest knowledge on the subject. All the important facts are given concisely and explicitly, the therapeutics of infancy and childhood being outlined very carefully and clearly. There are also directions for dosage and prescribing, and many useful prescriptions are included.

\section{American Journal of the Medical Sciences}

"Treatment has been satisfactorily covered, being quite in accord with the best teaching, yet withal broadly general and free from stock prescriptions." 


\section{Keefer's Military Hygiene}

Military Hygiene and Sanitation. By Lieut.-Col. Frank R. Keefer, Professor of Military Hygiene, United States Military Academy, IVest Point. 12 mo of 305 pages, illustrated. Cloth, \$I.50 net.

\section{ILLUSTRATED}

This is a concise, though complete text-book on this subject, containing chapters on the care of troops, recruits and recruiting, personal hygiene, physical training, preventable diseases, clothing, equipment, water-supply, foods and their preparation, hygiene and sanitation of posts and barracks, the troopship, hygiene and sanitation of marches, camps, and battlefields, disposal of wastes, tropical and arctic service, venereal diseases, alcohol and other narcotics, and a glossary.

\section{Bergey's Hygiene}

The Principles of Hygiene: A Practical Manual for Students, Physicians, and Health Officers. By D. H. Berger, A. M., M. D., Assistant Professor of Bacteriology in the University of Pennsylvania. Octavo volume of 555 pages, illustrated.

Cloth, \$3.00 net.

\section{NEW (5th) EDITION}

This book is intended to meet the needs of students of medicine in the acquirement of a knowledge of those principles upon which modern hygienic practises are based, and to aid physicians and health officers in familiarizing themselves with the advances made in hygiene and sanitation in recent years. This fifth edition has been very carefully revised, and much new matter added, so as to include the most recent advancements.

\section{Buffalo Medical Journal}

"It will be found of value to the practitioner of medicine and the practical sanitarian; and students of architecture, who need to consider problems of heating, lighting, ventilation, water supply, and sewage disposal, may consult it with profit."

\section{Fiske's Human Body}

Structure and Functions of the Body. By Annetre Fiske, A.M. Graduate of the Waltham Training School for Nurses. 12mo of 221 pages, illustrated. Cloth. $\$ 1.25$ net. 


\section{Bohm and Painter's Massage}

Massage. By MAX BöHм, M. D., of Berlin, Germany. Edited, with an Introduction, by Charles F. Painter, M, D., Professor of Orthopedic Surgery at Tufts College Medical School, Boston. Octavo of 9I pages, with 70 practical illustrations.

Cloth, $\$ 1.75$ net.

\section{Draper's Legal Medicine}

A Text=Book of Legal Medicine. By Frank Winthrop Draper, A. M., M. D., Late Professor of Legal Medicine in Harvard University, Boston. Octavo of 573 pages, illustrated. Cloth, $\$ 4.00$ net; Half Morocco, $\$ 5.50$ net.

\section{Golebiewski and Bailey's Accident Diseases}

Atlas and Epitome of Diseases Caused by Accidents. By Dr. ED.

Golebiewski, of Berlin. Edited, with additions, by PeArce Bailey, M. D., Consulting Neurologist to St. Luke's Hospital, New York. With 71 colored illustrations on 40 plates, I 43 text illustrations, and 549 pages of text. Cloth, $\$ 4.00$ net. In Saunders' Hand-Atlas Series.

\section{Hofmann and Peterson's Legal Medicine In Saunders'}

Atlas of Legal Medicine. By Dr. E. vox HofMAxi, of Vienna. Edited by Frederick Peterson, M. D., Professor of Psychiatry in the College of Physicians and Surgeons, New York. With I 20 colored figures on $5^{6}$ plates and 193 half-tone illustrations. Cloth, $\$ 3.50$ net.

\section{Jakob and Fisher's Nervous System Saunders'}

Atlas and Epitome of the Nervous System and its Diseases. By Professor Dr. CHR. JAKOB, of Erlangen. Edited, with additions, by EDWard D. Fisher, M. D., University and Bellevue Hospital Medical College. With 83 plates and copious text. Cloth, $\$ 3.50$ net.

\section{Crothers' Morphinism and Narcomania}

Morphinism and Narcomania. By T. D. CRothers, M. D. I 2 mo of 35 I pages. Cloth, $\$ 2.00$ net.

\section{Peterson and Haines' Legal Medicine and Toxicology}

A thoroughly revised edition of this work is now in press. Every advance in these related subjects will be included, bringing the work right down to date. 


\section{American Pocket Dictionary}

New (9th) Edition

Ayerican Pocket Medical Dictionaky. Edited by W. A. NewMAx Doriant, M. T), Editor "American Illustrated Medical Dictionary." Containing the pronunciation and definition of the principal words used in medicine and kindred sciences, with 75 extensive tables. With 693 pages. Flexible leather, with gold edges, \$1.00 net; with patent thumb index, $\$ 1.25$ net.

"I can recommend it to our students without reserve."-J. H. Holland, M. D., Dean of the Jefferson Medical College, Philadeiphia.

\section{Morrow's Immediate Care of Injured}

New (2d) Edition

Immediate Care of the Injured. By Albert S. Morrow, M. I., Adjunct Professor of Surgery at the New York Polyclinic. Octavo of 360 pages, with 242 illustrations. Cloth, $\$ 2.50$ net.

Dr. Morrow's book on emergency procedures is written in a definite and decisive style, the reader being told just what to do in every emergency. It is a practical book for every day use, and the large number of excellent illustrations can not but make the treatment to be pursued in any case clear and intelligible. Physicians and nurses will find it indispensible.

\section{Powell's Diseases of Children}

Third Edition, Revised

Essentials of the Diseases of Children. By William M. Powell, M. D. Revised by Alfred Hand, Jr., A. B., M. D., Dispensary Physician and Pathologist to the Children's Hospital, Philadelphia. I 2 mo volume of 259 pages. Cloth, \$1.00 net. In Saunders' Question-Compend Series.

\section{Shaw on Nervous Diseases and Insanity Fifth Edition}

Essentials of Nervous Diseases and Insanity: Their Symptoms and Treatment. A Manual for Students and Practitioners. By the late John C. Shaw, M. D., Clinical Professor of Diseases of the Mind and Nervous System, Long Island College Hospital, New York. I 2 mo of 204 pages, illustrated. Cloth, \$1.00 net. In Saunders' Question-Compend Series.

"Clearly and intelligently written; we have noted few inaccuracies and several suggestive points. Some affections unmentioned in many of the large text-books are noted." -Boston Medical and Surgical Journal.

\section{Grafstrom's Mechano-Therapy}

Second Revised Edition

A Text-Book of Mechano-Therapy (Massage and Medical Gymnastics). By Axfr V. Grafstron, B. Sc., M. D., Attending Physician to the Gustavis Adolphus Orphange, Jamestown, New York. r2mo, 200 pages, illustrated. Cloth, \$1.25 net. 



UNIVERSITY OF CALIFORNIA LIBRARY

Los Angeles

This book is DUE on the last date stamped below.

parm $x . \quad \ldots \quad(107)$

Form L9-10m-3,'48 (A7920)444

IHE LIBRARY

UNIVERSITY OF CALIFORNL 

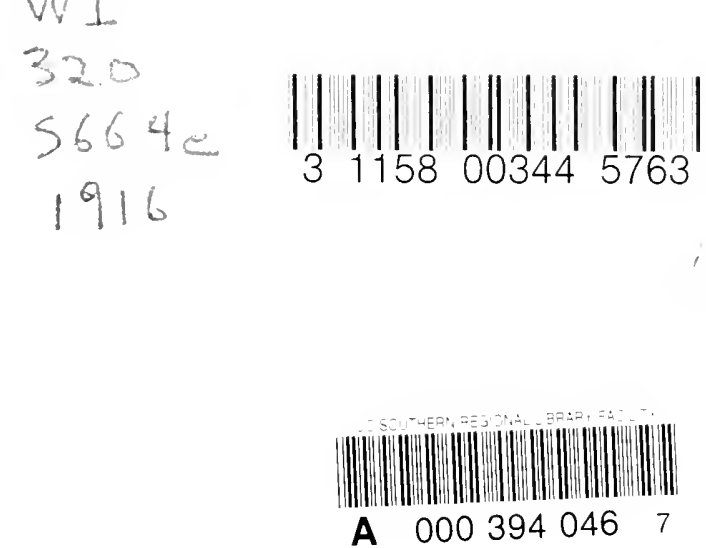


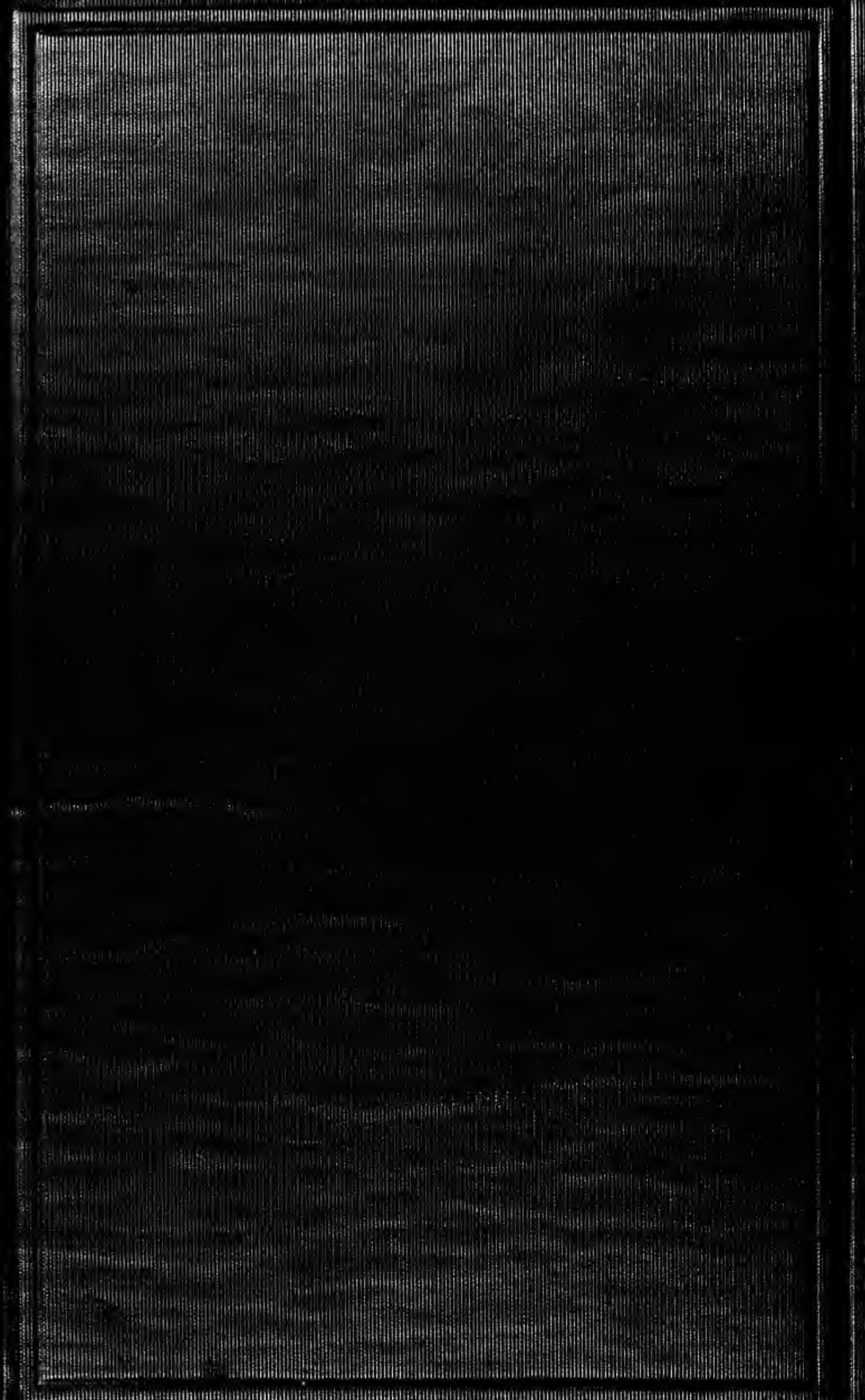

